\title{
Hanford Site Climatological Data Summary 1998 With Historical Data
}

\author{
D. J. Hoitink \\ K. W. Burk \\ J. V. Ramsdell
}

April 1999

Prepared for

the U.S. Department of Energy

under Contract DE-AC06-76RLO 1830

Pacific Northwest National Laboratory

Richland, Washington 99352 


\title{
DISCLAIMER
}

This report was prepared as an account of work sponsored by an agency of the United States Govermment. Neither the United States Government nor any agency thereof, nor Battelle Memorial Institute, nor any of their employees, makes any warranty, express or implied, or assumes any legal liability or responsibility for the accuracy, completeness, or usefulness of any information, apparatus, product, or process disclosed, or represents that its use would not infringe privately owned rights. Reference herein to any specific commercial product, process, or service by trade name, trademark, manufacturer, or otherwise does not necessarily constitute or imply its endorsement, recommendation, or favoring by the United States Government or any agency thereof, or Battelle Memorial Institute. The views and opinions of authors expressed herein do not necessarily state or reflect those of the United States Government or any agency thereof.

\author{
PACIFIC NORTHWEST NATIONAL LABORATORY \\ operated by \\ BATTELLE \\ for the \\ UNITED STATES DEPARTMENT OF ENERGY \\ under Contract DE-AC06-76RLO 1830
}

Printed in the United States of America

Available to DOE and DOE contractors from the

Office of Scientific and Technical Information, P.O. Box 62, Oak Ridge, TN 37831;

prices available from (615) 576-8401.

Available to the public from the National Technical Information Service,

U.S. Department of Commerce, 5285 Port Royal Rd., Springfield, VA 22161 


\section{DISCLAIMER}

Portions of this document may be illegible in electronic image products. Images are produced from the best available original document. 


\section{Summary}

This document presents the climatological data measured at the U.S. Department of Energy's Hanford Site for calendar year 1998. Pacific Northwest National Laboratory ${ }^{1}$ operates the Hanford Meteorology Station and the Hanford Meteorological Monitoring Network from which these data were collected. The information contained herein includes updated historical climatologies for temperature; precipitation, normal and extreme values of temperature and precipitation, and other miscellaneous meteorological parameters. Further, the data are adjunct to and update Hoitink and Burk (1994, 1995, 1996, 1997, 1998); however, Appendix B-Wind Climatology (1994) is excluded.

1998 was much warmer than normal, tying 1992 as the warmest year on record. The average temperature was $56.4^{\circ} \mathrm{F}, 3.1^{\circ} \mathrm{F}$ above normal $\left(53.3^{\circ} \mathrm{F}\right)$. The highest July temperature ever recorded was $112^{\circ} \mathrm{F}$ on July 27,1998 . The first week in May, three daily temperature records were broken or tied. November 1998 was the third warmest on record. For the year 1998, there were 73 days with maximum temperature $\geq 90^{\circ} \mathrm{F}$, the third highest on record. For the 12 -month period, 11 months were warmer than normal and 1 was cooler than normal. The summer (June, July, and August) and autumn (September, October, and November) of 1998 were the fourth warmest on record.

1998 was slightly wetter than normal. Precipitation totaled 6.45 in., $103 \%$ of normal (6.26 in.); snowfall totaled 7.2 in., compared to the normal of 13.8 in. There were eight thunderstorms recorded at Hanford Meteorological Station in July 1998, tying 1983 for the most thunderstorms in July.

The average wind speed during 1998 was $7.9 \mathrm{mph}, 0.2 \mathrm{mph}$ above normal $(7.7 \mathrm{mph})$. There were 32 days with peak gusts $\geq 40 \mathrm{mph}$, compared to a yearly average of $26 \mathrm{mph}$. The peak gust during the year was $56 \mathrm{mph}$ from the south-southwest on November 21 . November 1998 had a record number of days (10) with wind gusts $\geq 40 \mathrm{mph}$.

The heating-degree days for $1997-1998$ were 4,523 (14\% below the 5,231 normal). Cooling-degree days for 1998 were 1,377 ( $38 \%$ above the 994 normal).

For inquiries regarding this report, contact Mr. D. J. Hoitink at Pacific Northwest National Laboratory, P.O. Box 999, Richland, Washington 99352, or by electronic mail at dana.j.hoitink@pnl.gov.

\footnotetext{
${ }^{1}$ Pacific Northwest National Laboratory is operated by Battelle for the U.S. Department of Energy.
} 



\section{Acknowledgments}

The authors wish to acknowledge Dana Ward, the Department of Energy Richland Operations Office Technical Monitor for the Meteorological and Climatological Services Project, for his continued strong support. 



\section{Notes on Units of Measure}

This document mainly uses English units (e.g., miles per hour [mph], inches [in.], degrees Fahrenheit $\left[{ }^{\circ} \mathrm{F}\right]$ ) when presenting all information. This decision to use English units was based on the fact that English units are still the standard in National Oceanic and Atmospheric Administration (specifically, the National Climatic Data Center and National Weather Service) reporting and publications.

Throughout this document the term "normal" is used to indicate climatological normal, defined as an average value over a period of years of any meteorological element such as temperature, pressure, and rainfall. The accepted convention uses a 30-year time period, ending with the first year of each new decade (such as 1951-1980, 1961-1990, 1971-2000). The current time period used for climatological normals is $1961-1990$.

Some useful conversions between English units and metric equivalents are:

$$
\begin{aligned}
& 1 \text { foot }(\mathrm{ft})=0.3048 \text { meter }(\mathrm{m}) \\
& 1 \text { mile }(\mathrm{mi})=1.609 \text { kilometers }(\mathrm{km}) \\
& 1 \text { inch }(\mathrm{in} .)=2.54 \text { centimeters }(\mathrm{cm}) \\
& 1 \text { mile per hour }(\mathrm{mph})=0.447 \text { meter } / \text { second }(\mathrm{m} / \mathrm{s}) \\
& \text { degrees Fahrenheit }\left({ }^{\circ} \mathrm{F}\right)=\left(9 / 5 \times{ }^{\circ} \mathrm{C}\right)+32 \\
& \text { degrees Celsius }\left({ }^{\circ} \mathrm{C}\right)=5 / 9 \times\left({ }^{\circ} \mathrm{F}-32\right) .
\end{aligned}
$$





\section{Contents}

Summary

iii

Acknowledgments

Notes on Units of Measure

vii

1.0 Introduction

2.0 Calendar Year 1998 Summary

3.0 Temperature Climatology.

3.1 Monthly, Seasonal, and Annual Average

3.2 Days with Maximum Temperatures $\geq 100^{\circ} \mathrm{F}, \geq 90^{\circ} \mathrm{F}$, and $\leq 32^{\circ} \mathrm{F}$

3.3 Days with Minimum Temperatures $\leq 32^{\circ} \mathrm{F}$ or $\leq 0^{\circ} \mathrm{F}$

3.4 Monthly Extreme Daily Maximum and Minimum Temperatures

3.5 Average Daily Temperature Range.

3.6 Normal and Extreme Daily Temperatures

3.7 Subsurface Soil Temperatures.

3.8 Heating- and Cooling-Degree Days

4.0 Precipitation Climatology

4.1 Monthly and Annual Totals.

4.2 Seasonal Precipitation

4.3 Average Number of Days with Specified Amounts of Precipitation

4.4 Total Time with Precipitation Observed

4.5 Notable Wet Periods

4.6 Notable Dry Periods

4.7 Snowfall 
4.8 Normal and Maximum Daily Precipitation ............................................................ $\quad 4.12$

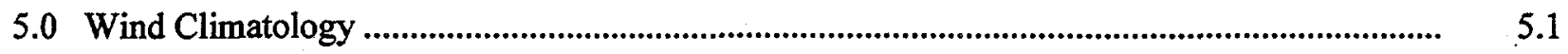

5.1 Monthly and Annual Prevailing Wind Directions, Average Speeds, and Peak Gusts ........ $\quad 5.1$

5.2 Days with Peak Gusts Above or Below Specific Thresholds.........................................

5.3 Frequency of Monthly and Annual Wind Direction and Speed at 50-Foot Level.............. $\quad 5.3$

5.4 Composite Wind Roses and Joint Frequency Distributions for the Hanford Meteorological Monitoring Network

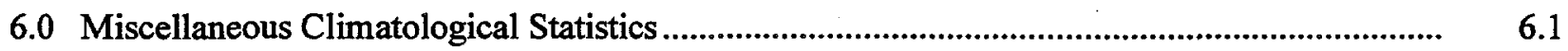

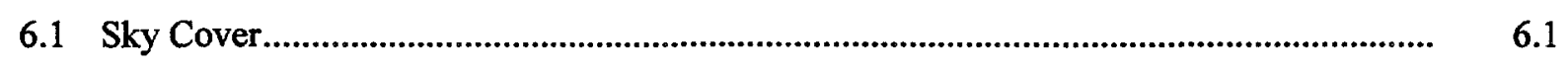

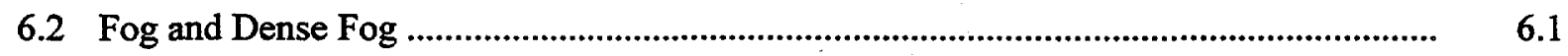

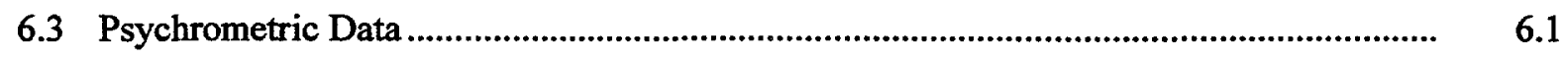

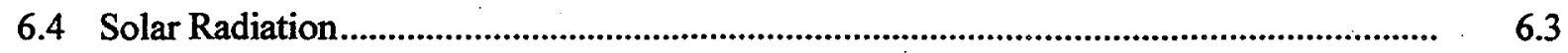

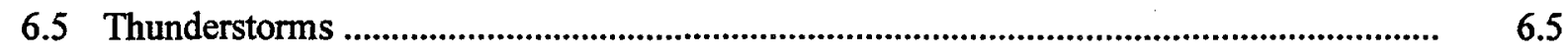

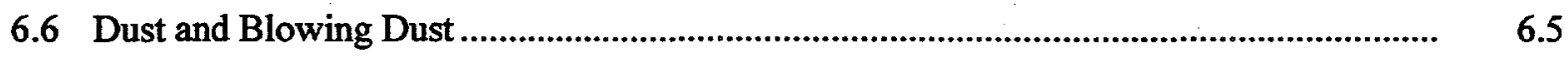

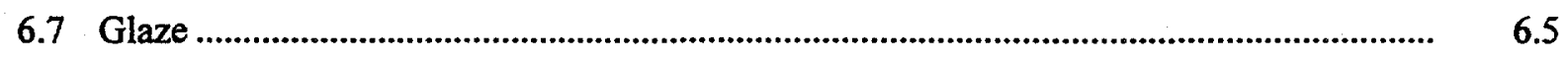

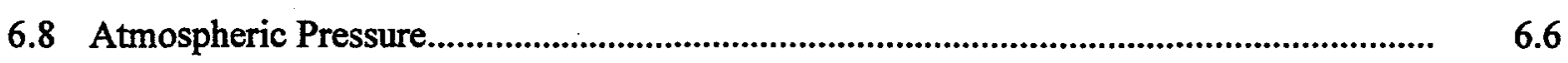

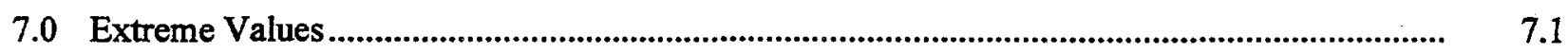

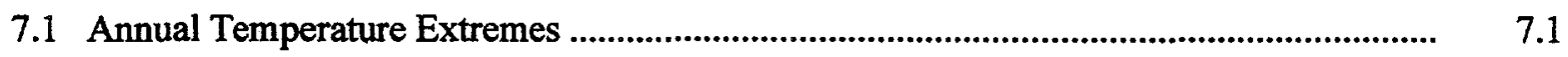

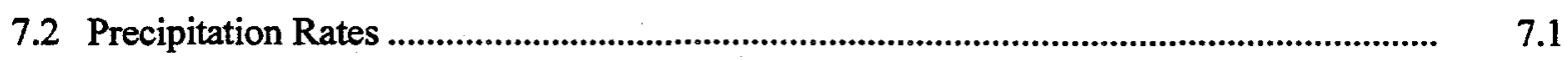

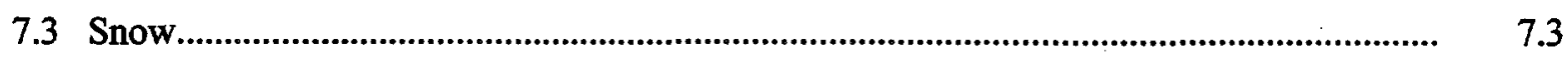

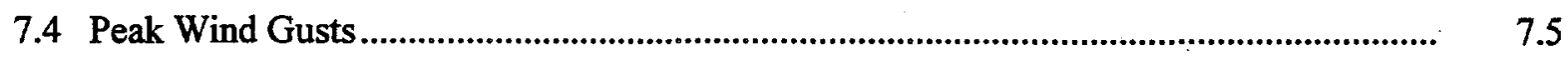

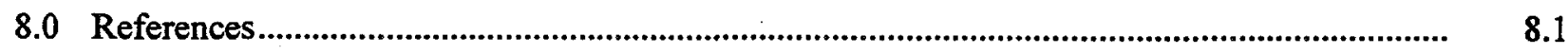

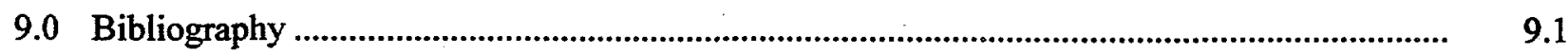

Appendix - 1998 Wind Climatology ............................................................................................ A.1 


\section{Figures}

1.1 Map of the Hanford Site and Surrounding Areas 1.3

2.1 1998 Hanford Meteorological Monitoring Network Wind Roses at 10-Meter Level.........

2.2 1998 Hanford Meteorological Monitoring Network Wind Roses at 60-Meter Level.........

2.3 1998 Observed Daily Temperatures from the Hanford Meteorology Station

5.1 Hanford Meteorological Monitoring Network Wind Roses at 10-Meter Level, 1982 Through 1998

5.2 Hanford Meteorological Monitoring Network Wind Roses at 60-Meter Level, 1986 Through 1998

7.1 Probability of an Annual Maximum Temperature Exceeding a Given Value

7.2 Probability of an Annual Minimum Temperature Being Less Than a Given Value

7.3 Probability of Precipitation Rate Exceeding Given Values by Duration

7.4 Probability of Exceeding a Given Seasonal Snowfall

7.5 Probability of Exceeding a Given Snowfall in a Single Storm

7.6 Probability of Exceeding a Given Snow Depth

7.7 Probabilities of Peak Wind Gusts Exceeding Given Values.

\section{Tables}

1.1 Station Numbers, Names, and Codes for the Hanford Meteorological Monitoring Network

2.11998 Daily Temperature Records.

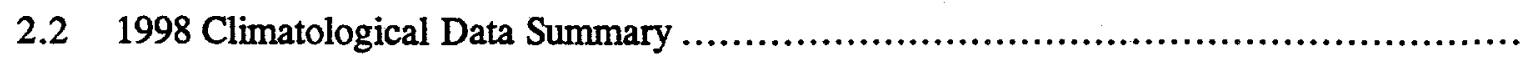

2.3 1998 Monthly and Seasonal Temperature and Precipitation. 
2.4 1998 Monthly and Annual Average Temperatures from the Hanford Meteorological Monitoring Network....

2.5 1998 Monthly and Annual Average Precipitation from the Hanford Meteorological Monitoring Network.

2.6 1998 Monthly and Annual Average Wind Speed from the Hanford Meteorological Monitoring Network

3.1 Monthly and Annual Average Temperatures

3.2 Seasonal Average Temperatures

3.3 Monthly and Seasonal Number of Days with Maximum Temperatures Above or Below Certain Thresholds

3.4 Days with Maximum Temperatures $\geq 104^{\circ} \mathrm{F}$

3.5 Record of Annual First and Last Dates with Maximum Temperatures $\geq 90^{\circ} \mathrm{F}$ and Minimum Temperatures $\leq 32^{\circ} \mathrm{F}$

3.6 Monthly and Annual Maximum Temperatures

3.7 Monthly and Seasonal Number of Days with Minimum Temperatures Below Certain Thresholds

3.8 Days with Minimum Temperatures $\leq 0^{\circ} \mathrm{F}$

3.9 Monthly and Annual Minimum Temperatures

3.10 Monthly Normal Temperatures and Monthly Extremes of Maximum and Minimum Temperatures.

3.11 Average Daily Temperature Range

3.12 Normal and Extreme Daily Maximum and Minimum Temperatures

3.13 Subsurface Soil Temperatures at Depths of $0.5,15$, and 36 Inches

3.14 Monthly and Seasonal Heating-Degree Days

3.15 Monthly and Annual Cooling-Degree Days.

4.1 Monthly and Annual Precipitation 
4.3 Average Number of Days with Precipitation of Specified Amount ......................... 4.4

4.4 Monthly and Annual Averages and Extremes in Total Time with Precipitation Observed: July 1946 Through June 1971, July 1974 Through December 1998

4.5 Total Duration of Precipitation by Month and Year

4.6 Monthly and Seasonal Snowfall, Including First and Last Dates of Both Trace and Measurable Snowfalls

4.7 Snowfall - Greatest Amount from a Single Storm

4.8 Miscellaneous Snowfall Statistics, 1946 Through 1998

4.9 Maximum Precipitation

4.10 Normal and Maximum Daily Precipitation

5.1 Monthly and Annual Prevailing Wind Directions, Average Speeds, and Peak Gusts at 50-Foot Level, 1945 Through 1998.

5.2 Number of Days with Peak Gusts Above or Below Specific Thresholds at 50-Foot Level, 1945 Through 1998.

5.3 Frequency of Monthly and Annual Wind Direction and Speed at 50-Foot Level, 1955 Through 1998

5.4 Joint Frequency Distributions for Hanford Meteorological Monitoring Network Wind Stations at 10-Meter Level, 1982 Through 1998

5.5 Joint Frequency Distributions for Hanford Meteorological Monitoring Network Wind Stations at 60-Meter Level, 1986 Through 1998

6.1 Average Sky Cover, 1946 Through 1998, and Number of Days Clear, Partly Cloudy, and Cloudy, 1954 Through 1998.

6.2 Monthly and Annual Number of Days with Fog and Dense Fog, 1945 Through 1998 ......

6.3 Monthly Averages and Extremes of Psychrometric Data, 1950 Through 1998

6.4 Average and Extreme Solar Radiation Daily Values, 1953 Through 1998.

6.5 Average Number of Days of Various Meteorological Phenomena, 1945 Through 1998 ....

6.6 Average and Extreme Station and Sea-Level Pressure Data, 1955 Through 1998. 
7.1 Return Periods for Annual Maximum and Minimum Temperatures

7.2 Precipitation Rates for 1 to 24 Hours Duration and Return Periods from 2 to 1000 Years .....

7.3 Precipitation Amounts for 1 to 24 Hours Duration and Return Periods from 2 to 1000 Years..

7.4 Snowfall Extremes for Return Periods from 2 to 1000 Years

7.5 Peak Wind Gusts for Return Periods from 2 to 1000 Years.. 


\subsection{Introduction}

The U.S. Department of Energy's Hanford Site lies within the semiarid shrub-steppe Pasco Basin of the Columbia Plateau in southeastern Washington state. The Hanford Site occupies an area of $\sim 560 \mathrm{mi}^{2}$ north of the confluence of the Snake and Yakima Rivers with the Columbia River. The Columbia River flows through the northern part of the Hanford Site and, turning south, forms part of the site's eastern boundary. The Yakima River runs along part of the southern boundary and joins the Columbia River below the city of Richland, which bounds the Hanford Site on the southeast. Rattlesnake Mountain, Yakima Ridge, and Umtanum Ridge form the southwestern and western boundaries. The Saddle Mountains form the northern boundary of the Hanford Site.

The Cascade Range, beyond Yakima to the west, greatly influences the climate of the Hanford Site area by means of its rain shadow effect. This mountain range also serves as a source of cold air drainage, which has a considerable effect on the wind regime on the Hanford Site. The regional temperatures, precipitation, and winds are greatly affected by the presence of mountain barriers. The Rocky Mountains and ranges in southern British Columbia are effective in protecting the inland basin from the more severe winter storms and associated cold polar air masses moving southward across Canada.

This document presents the calendar year 1998 climatological data summary for the Hanford Meteorology Station (HMS) and additional climatologies for temperature, wind, precipitation, and other meteorological parameters for the HMS and the automated stations of the Hanford Meteorological Monitoring Network (HMMN). Climatological normal and extreme values for temperature and precipitation are also presented. Currently, 30 monitoring stations are within and located near the U.S. Department of Energy's Hanford Site (Table 1.1, Figure 1.1). A detailed description of each of the monitoring stations, including photographs of the topography surrounding each site, is provided in Glantz and Islam (1988).

Operation of the HMS is a function of the Meteorological and Climatological Services Project funded by the U.S. Department of Energy. This project, managed by the Pacific Northwest National Laboratory, is responsible for providing the U.S. Department of Energy and Hanford Site contractors ongoing meteorological and climatological services support, primarily for emergency response activities, Hanford Site work scheduling, and general site safety. Detailed, real-time meteorological data are needed in the event of a release of hazardous material to the atmosphere from any of the Hanford Site facilities. These data can be used to model atmospheric dispersion and to estimate the environmental impacts of the release. Meteorological data and weather forecasts are also necessary to ensure that operations and activities on the Hanford Site are conducted safely, particularly where specific weather conditions might impact those operations or activities. The climatological database is also used in environmental studies, environmental impact reports, facility design, and planning operations.

During the period April 1912 through March 1943, cooperative observers for the U.S. Weather Bureau (now the National Weather Service) recorded daily maximum and minimum temperatures and precipitation, including measurements of unmelted snow at the Old Hanford Townsite $\sim 10$ mi eastnortheast of the present HMS. From late 1943 until mid 1944, the U.S. Weather Bureau recorded some 
Table 1.1. Station Numbers, Names, and Codes for the Hanford Meteorological Monitoring Network

\begin{tabular}{|c|c|}
\hline $\begin{array}{l}\text { Station } \\
\text { Number }\end{array}$ & Station Name \\
\hline 1 & Prosser Barricade \\
\hline 2 & Emergency Operations Center \\
\hline 3 & Army Loop Road \\
\hline 4 & Rattlesnake Springs \\
\hline 5 & Edna \\
\hline 6 & 200 East \\
\hline 7 & 200 West \\
\hline $8 \mathrm{~B}$ & Beverly \\
\hline $8 W$ & Wahluke (no longer active) \\
\hline 9 & Fast Flux Test Facility \\
\hline 10 & Yakima Barricade \\
\hline 11 & 300 Area \\
\hline 12 & Wye Barricade \\
\hline 13 & $100-\mathrm{N}$ \\
\hline 14 & WNP-2 \\
\hline 15 & Franklin County \\
\hline 16 & Gable Mountain \\
\hline 17 & Ringold \\
\hline 18 & Richland Airport \\
\hline $19 \mathrm{P}$ & Plutonium Finishing Plant-200W \\
\hline $19 \mathrm{~S}$ & Sagehill (no longer active) \\
\hline 20 & Rattlesnake Mountain \\
\hline 21 & Hanford Meteorology Station \\
\hline 22 & Pasco Airport \\
\hline 23 & Gable West \\
\hline 24 & $100-F$ \\
\hline 25 & Vernita Bridge \\
\hline 26 & Benton City \\
\hline 27 & Tri-City Vocational Skills Center \\
\hline 28 & Roosevelt, WA \\
\hline 29 & $100-K$ \\
\hline 30 & HAMMER \\
\hline
\end{tabular}

\begin{tabular}{lll} 
Station & & \multicolumn{1}{c}{ Period of } \\
Code & & Operation \\
\cline { 3 - 3 } PROS & & $01 / 82$ - Present \\
EOC & $01 / 82$ - Present \\
ARMY & $01 / 82$ - Present \\
RSPG & $01 / 82$ - Present \\
EDNA & $01 / 82$ - Present \\
200E & $01 / 82$ - Present \\
200W & $01 / 82$ - Present \\
BVLY & $08 / 91$ - Present \\
WAHL & $01 / 82$ - 07/91 \\
FFTF & $01 / 82$ - Present \\
YAKB & $01 / 82$ - Present \\
300A & $01 / 82$ - Present \\
WYEB & $01 / 82$ - Present \\
100N & $01 / 82$ - Present \\
WPPS & $01 / 82$ - Present \\
FRNK & $01 / 82$ - Present \\
GABL & $01 / 82$ - Present \\
RING & $01 / 82$ - Present \\
RICH & $01 / 82$ - Present \\
PFP & $02 / 94$ - Present \\
SAGE & $03 / 82$ - 01/93 \\
RMTN & $01 / 82$ - Present \\
HMS & $01 / 82$ - Present \\
PASC & $10 / 87$ - Present \\
GABW & $03 / 86$ - Present \\
100F & $03 / 86$ - Present \\
VERN & $02 / 88$ - Present \\
BENT & $02 / 95$ - Present \\
VSTA & $02 / 91$ - Present \\
SURF & $09 / 94$ - Present \\
l00K & $03 / 96$ - Present \\
HAMR & $01 / 98$ - Present
\end{tabular}

meteorological operations in Richland. Then, in 1944 as part of the Manhattan Project, the HMS was established. Hourly observations began on December 7, 1944.

The HMS and its 410- $\mathrm{ft}$ instrument tower are located near the center of the Hanford Site between the 200-West and 200-East Areas (see Figure 1.1). Hourly observations of wind direction, wind speed, and air temperature are made at multiple levels on the 410-ft tower. Throughout this document, wind measurements from the HMS are made at the 50-ft level and temperature measurements are made at the 3-ft level. A variety of other meteorological parameters are also measured or observed, including current 


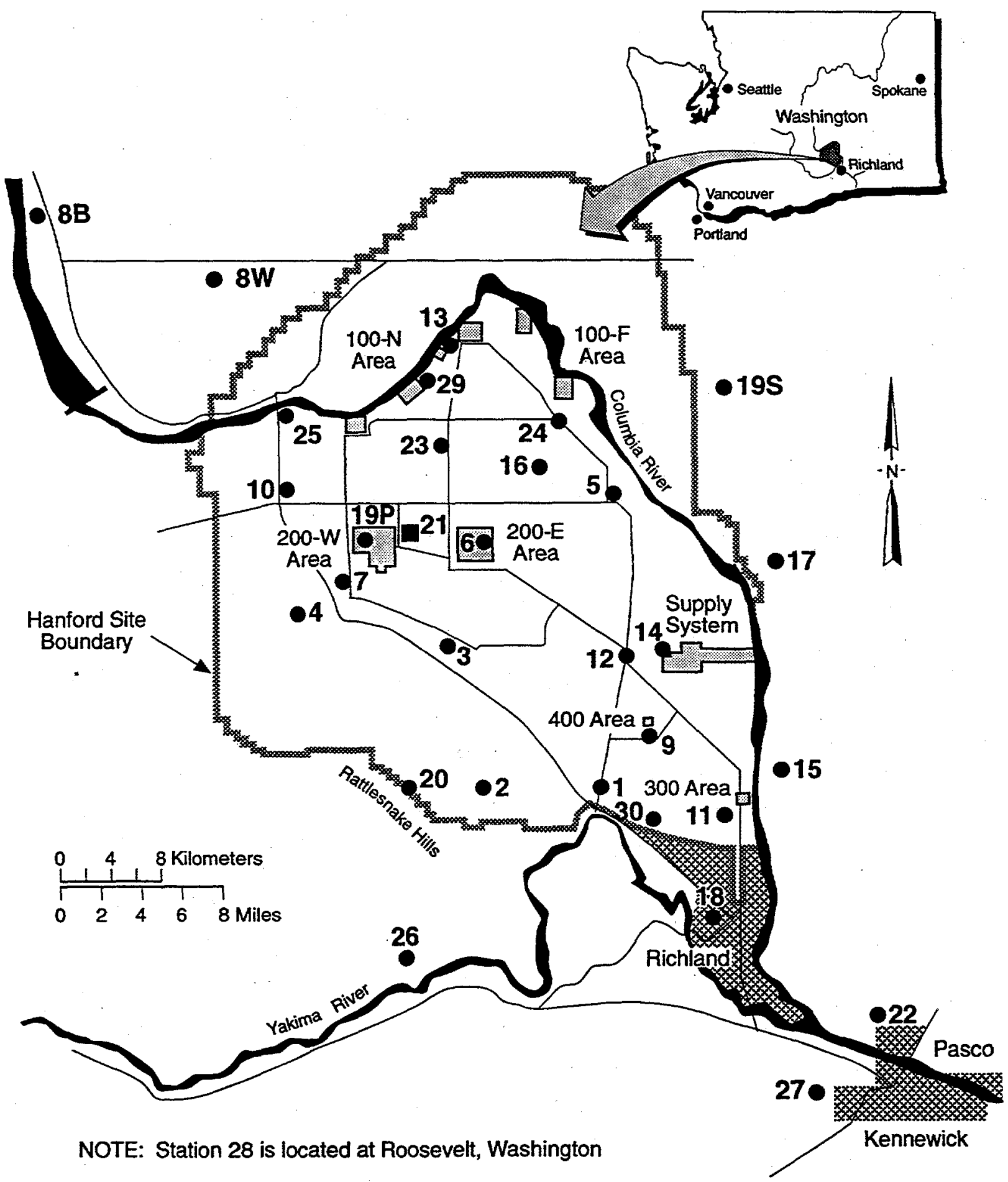

Hanford Meteorology Station

- Hanford Meteorological Monitoring Network Station

RP99010119.1

Figure 1.1. Map of the Hanford Site and Surrounding Areas 
weather, dew point temperature, relative humidity, precipitation, atmospheric pressure, cloud cover, visibility, and solar radiation. Several climatological summaries of data collected at the HMS, at the Old Hanford Townsite, and Richland monitoring locations were published over the past 30 years (Jenne and Kerns 1959; Stone et al. 1972, 1983; Hoitink and Burk 1994, 1995, 1996, 1997, 1998).

This document is composed of the following information. The 1998 calendar year summary of climatological data for the Hanford Site is contained in Section 2.0. Temperature, precipitation, wind, and miscellaneous climatological statistics are contained in Sections 3.0 through 6.0 , respectively. Section 8.0 lists the references cited in the document, and Section 9.0 provides a bibliography of database, computer code, and other pertinent reports. The appendix gives the station-specific wind roses and joint frequency distributions for 1998. 


\subsection{Calendar Year 1998 Summary}

Calendar year 1998 was much warmer than normal, tying 1992 as the warmest year on record. The average temperature was $56.4^{\circ} \mathrm{F}, 3.1^{\circ}$ above normal $\left(53.3^{\circ} \mathrm{F}\right)$. The coldest year on record is 1985 , which averaged $49.6^{\circ} \mathrm{F}$.

Eleven months during 1998 were warmer than normal, 7 of which were at least $2.5^{\circ} \mathrm{F}$ above normal. Only October was cooler than normal, and only by $0.5^{\circ} \mathrm{F}$. July had the greatest positive departure $\left(5.8^{\circ} \mathrm{F}\right)$.

1998 was only slightly wetter than normal. Precipitation totaled 6.45 in., $103 \%$ of normal (6.26 in.). The wettest year was 1995 with 12.31 in.; the driest was 1976 with only 2.99 in. Calendar year snowfall totaled $7.2 \mathrm{in}$., compared to an annual normal of 13.8. The greatest calendar year snowfall was 57.5 in. (1996).

January 1998 was much warmer than normal, averaging $36.2^{\circ} \mathrm{F}, 4.9^{\circ}$ above normal $\left(31.3^{\circ} \mathrm{F}\right)$. Except for a four-day cold spell from January 10-13, which included a minimum temperature of $7^{\circ} \mathrm{F}$ on January 13, the rest of the month was considerably warmer than normal. The warmest January averaged $42.5^{\circ} \mathrm{F}$ (1953); while the coldest averaged $12.1^{\circ} \mathrm{F}(1950)$.

Precipitation for January 1998 totaled 1.24 in., $157 \%$ of normal $(0.79 \mathrm{in}$.). The wettest January, in 1970 , received 2.47 in.; while the driest, in 1977, received 0.08 in. Snowfall for January 1998 totaled 6.3 in., compared to a January normal of 3.9 in. The snowiest January, in 1950, received 23.4 in., while January 1994 received no snowfall.

January 1998's average wind speed was 7.8 miles per hour (mph), compared to a January normal of $6.5 \mathrm{mph}$. The peak wind gust for the month was $43 \mathrm{mph}$ recorded on January 24 . There were 5 days in January 1998 with peak gusts $\geq 40 \mathrm{mph}$, compared to a normal of $3 \mathrm{mph}$ and a high of $11 \mathrm{mph}$ (in 1990 and 1972). The highest wind gust ever recorded during January was $80 \mathrm{mph}$ in 1972. The windiest January averaged $10.3 \mathrm{mph}$ (1972); while the January with the lightest winds (1985) averaged $2.9 \mathrm{mph}$.

February 1998 was much warmer than normal, averaging $42.2^{\circ} \mathrm{F}, 4.2^{\circ}$ above normal $\left(38.0^{\circ} \mathrm{F}\right)$. Only six days during the month had average temperatures below normal. The warmest February averaged $44.5^{\circ} \mathrm{F}$ (1958 and 1991); while the coldest averaged $25.6^{\circ} \mathrm{F}$ (1956).

Precipitation for February 1998 totaled 1.15 in., $186 \%$ of normal ( 0.62 in.). The wettest February, in 1961, received $2.10 \mathrm{in}$.; while the driest, in 1967 and 1988, received only a trace. Only a trace of snowfall was recorded in February 1998, compared to a normal of 2.0 in. The snowiest February, in 1989, received $17.0 \mathrm{in}$., while numerous Februarys (as recently as 1991) received no snowfall.

February 1998's average wind speed was $7.3 \mathrm{mph}$, compared to a February normal of $7.2 \mathrm{mph}$. The peak wind gust for the month was $50 \mathrm{mph}$ recorded on February 21 . The record wind gust for February was $65 \mathrm{mph}$ in 1971. The windiest February averaged $10.8 \mathrm{mph}$ (1976); while the February with the lightest winds (1963) averaged $4.6 \mathrm{mph}$. 
The winter season of 1997-1998 (December, January and February) was much warmer than normal, averaging $37.7^{\circ} \mathrm{F}, 4.1^{\circ} \mathrm{F}$ above normal $\left(33.6^{\circ} \mathrm{F}\right)$. The warmest winter on record (1966-1967) averaged $40.6^{\circ} \mathrm{F}$, while the coldest (1948-1949) averaged only $24.2^{\circ} \mathrm{F}$. The coldest temperature for the winter was $7^{\circ} \mathrm{F}$ on January 13.

Precipitation for the 1997-1998 winter season totaled 2.70 in., $111 \%$ of normal (2.44 in.). The wettest winter season (1996-1997) received 5.45 in., while the driest (1946-1947) received only 0.70 in. Total snowfall for the 1997-1998 winter season totaled 8.1 in., compared to a normal through February of 13.5 in. The snowiest winter on record (1992-1993) received 56.1 in., while the winter with the least snow (1957-1958) received only 0.3 in.

March 1998 was warmer than normal, averaging $48.4^{\circ} \mathrm{F}, 2.8^{\circ}$ above normal ( $\left.45.6^{\circ} \mathrm{F}\right)$. The warmest March averaged $51.5^{\circ} \mathrm{F}(1992)$; while the coolest averaged $39.4^{\circ} \mathrm{F}(1955)$.

Precipitation for March 1998 totaled 0.50 in., 106\% of normal (0.47 in.). The wettest March, in 1957, received 1.86 in.; while the driest, in 1968, received 0.02 in. A trace of snowfall was recorded in March 1998, compared to a normal of 0.3 in. The snowiest March, in 1951, received 4.2 in., while numerous Marches (as recently as 1995) received no snowfall.

March 1998's average wind speed was $7.3 \mathrm{mph}$, compared to a March normal of $8.3 \mathrm{mph}$. The peak gust for the month was $39 \mathrm{mph}$ recorded on March 16 and 26. The record gust for March was $70 \mathrm{mph}$ in 1956. The windiest March averaged $10.7 \mathrm{mph}$ (1977); while the March with the lightest winds (1958) averaged $5.9 \mathrm{mph}$.

April 1998 was warmer than normal, averaging $54.4^{\circ} \mathrm{F}, 1.7^{\circ}$ above normal $\left(52.4^{\circ} \mathrm{F}\right)$. The warmest April averaged $58.2^{\circ} \mathrm{F}(1994)$; while the coolest averaged $47.5^{\circ} \mathrm{F}(1955)$.

Precipitation for April 1998 totaled only 0.07 in., 17\% of normal (0.41 in.). The wettest April, in 1995 , received 1.54 in.; while the driest, in 1986 , received only a trace.

April 1998's average wind speed was $7.6 \mathrm{mph}$, compared to an April normal of $9.0 \mathrm{mph}$. The peak wind gust for the month was $37 \mathrm{mph}$ recorded on April 24. The record wind gust for April was $73 \mathrm{mph}$ in 1972. The windiest April averaged $11.1 \mathrm{mph}$ (1972); while the April with the lightest winds (1989) averaged $7.4 \mathrm{mph}$.

May 1998 was warmer than normal, averaging $62.4^{\circ} \mathrm{F}, 1.1^{\circ}$ above normal $\left(61.3^{\circ} \mathrm{F}\right)$. The first week of the month averaged $14^{\circ}$ above normal, with three daily temperature records broken or tied. May was the tenth consecutive month with above normal average temperatures, dating back to August 1997. The warmest May averaged $68.7^{\circ} \mathrm{F}(1947)$; while the coolest averaged $56.0^{\circ} \mathrm{F}$ (1984).

Precipitation for May 1998 totaled 0.52 in., $102 \%$ of normal ( 0.51 in.). The wettest May, in 1972, received 2.03 in.; while the driest, in 1992 , received only a trace. 
May 1998's average wind speed was $8.0 \mathrm{mph}$, compared to a May normal of $8.8 \mathrm{mph}$. The peak wind gust for the month was $55 \mathrm{mph}$ recorded on May 21. The record wind gust for May was $71 \mathrm{mph}$ in 1948. The windiest May averaged $10.7 \mathrm{mph}$ (1983); while the May with the lightest winds (1957) averaged $5.8 \mathrm{mph}$.

Spring 1998 (March, April, and May) was warmer than normal, averaging $55.1^{\circ} \mathrm{F}, 1.9^{\circ} \mathrm{F}$ above normal $\left(53.2^{\circ} \mathrm{F}\right)$. The warmest spring, in 1992 , averaged $58.2^{\circ} \mathrm{F}$, while the coolest spring, in 1955 , averaged $48.0^{\circ} \mathrm{F}$.

Precipitation for the spring season totaled 1.09 in., $78 \%$ of normal (1.40 in.). The wettest spring, in 1995 , received $3.28 \mathrm{in}$., while the driest, in 1968 , received only 0.09 in. Only a trace of snowfall was recorded during the period.

June 1998 was warmer than normal, averaging $71.0^{\circ} \mathrm{F}, 1.3^{\circ}$ above normal $\left(69.7^{\circ} \mathrm{F}\right)$. There were 7 days in June with maximum temperatures $\geq 90^{\circ} \mathrm{F}$ (normal for June is 9 days). There were no days with maximum temperatures $\geq 100^{\circ} \mathrm{F}$ (normal are 2). The warmest June averaged $76.8^{\circ} \mathrm{F}(1992)$; while the coolest averaged $63.0^{\circ} \mathrm{F}(1953)$.

Precipitation for June 1998 totaled 0.48 in., $126 \%$ of normal (0.38 in.). The wettest June, in 1950, received 2.92 in.; while the driest, in 1986 and 1979 , received only a trace.

June 1998's average wind speed was $9.8 \mathrm{mph}$, compared to a June normal of $9.1 \mathrm{mph}$. The peak wind gust for the month was $50 \mathrm{mph}$ recorded on June 15. The record wind gust for June was $72 \mathrm{mph}$ in 1957 . The windiest June averaged $10.7 \mathrm{mph}$ (1983); while the June with the lightest winds (1950) averaged $7.7 \mathrm{mph}$.

July 1998 was much warmer than normal, averaging $82.0^{\circ} \mathrm{F}, 5.8^{\circ}$ above normal $\left(76.2^{\circ} \mathrm{F}\right)$, and was the second warmest July (and also the second warmest month) on record. The warmest month ever was July 1985 which averaged $82.2^{\circ} \mathrm{F}$. The coolest July was $70.5^{\circ} \mathrm{F}$ in 1993 . There were 26 days in July with maximum temperatures $\geq 90^{\circ} \mathrm{F}$ (normal for July is 19 days), and there were 14 days with maximum temperatures $\geq 100^{\circ} \mathrm{F}$ (normal is 6 ). The maximum temperature of $112^{\circ} \mathrm{F}$ on July 27 was the highest temperature ever recorded in the month of July (the previous highest was $111^{\circ} \mathrm{F}$ on July 22,1994 and July 31,1971 ). This was also the hottest temperature at the HMS since $112^{\circ} \mathrm{F}$ was recorded on August 9 , 1971. The minimum temperature of $81^{\circ} \mathrm{F}$ on July 28 was the second highest minimum temperature ever recorded, the highest was $82^{\circ} \mathrm{F}$ on July $23,1994$.

Precipitation for July 1998 totaled 0.34 in., $189 \%$ of normal ( 0.18 in.). While the HMS received only 0.16 in. of rain on Friday, July 31 , a monitoring station on the north slope of Rattlesnake Mountain reported $1.03 \mathrm{in}$, and the monitoring station in the 300 Area received 0.65 in. The wettest July, in 1993, received 1.76 in.; while the driest, in 1980 and earlier years, received only a trace. There were eight thunderstorms recorded at the HMS in July 1998, tying 1983 for the most thunderstorms in July.

July 1998's average wind speed was $8.1 \mathrm{mph}$, compared to a July normal of $8.8 \mathrm{mph}$. The peak wind gust for the month was $47 \mathrm{mph}$ recorded on July 27 . The record wind gust for July was $69 \mathrm{mph}$ in 1979 . The windiest July averaged $10.7 \mathrm{mph}$ (1983); while the July with the lightest winds (1955) averaged 
$6.8 \mathrm{mph}$. During the thunderstorm of Friday evening, July 10, meteorological monitoring stations at Pasco and Vista Field in Kennewick recorded peak wind gusts of 68 and $66 \mathrm{mph}$, respectively.

August 1998 was warmer than normal, averaging $77.9^{\circ} \mathrm{F}, 2.8^{\circ}$ above normal $\left(75.1^{\circ} \mathrm{F}\right)$. The warmest August occurred in 1967 and averaged $81.5^{\circ} \mathrm{F}$. The coolest August was $69.8^{\circ} \mathrm{F}$ in 1964 . There were 24 days in August with maximum temperatures $\geq 90^{\circ} \mathrm{F}$ (normal for August is 16 days), and there were 9 days with maximum temperatures $\geq 100^{\circ} \mathrm{F}$ (normal is 5 ).

Precipitation for August 1998 totaled only 0.04 in., $15 \%$ of normal ( 0.27 in.). The wettest August, in 1977 , received 1.36 in., while the driest, in 1988, recorded no precipitation.

August 1998's average wind speed was $8.1 \mathrm{mph}$, compared to an August normal of $7.9 \mathrm{mph}$. The peak wind gust for the month was $50 \mathrm{mph}$ recorded on August 15. The record wind gust for August was $66 \mathrm{mph}$ in 1961. The windiest August averaged $9.5 \mathrm{mph}$ (1996); while the August with the lightest winds (1956) averaged $6.0 \mathrm{mph}$.

The summer of 1998 (June, July, and August) was the fourth warmest on record, averaging $77.0^{\circ} \mathrm{F}$, $3.3^{\circ}$ above normal $\left(73.7^{\circ} \mathrm{F}\right)$. The warmest summer, in 1958 , averaged $78.2^{\circ} \mathrm{F}$, while the coolest, in 1980 , averaged $70.2^{\circ} \mathrm{F}$. The hottest maximum temperature of the summer was $112^{\circ} \mathrm{F}$ on July 27 , while the coolest minimum was $47^{\circ} \mathrm{F}$ on June 27.

Precipitation for the summer of 1998 totaled $0.86 \mathrm{in} ., 104$ percent of normal ( $0.83 \mathrm{in}$.). The wettest summer occurred in 1950 when 2.99 in. of precipitation was recorded, while the driest summer (1973) received only 0.03 in.

September 1998 was much warmer than normal, averaging $71.0^{\circ} \mathrm{F}, 5.3^{\circ}$ above normal $\left(65.7^{\circ} \mathrm{F}\right)$, and the fourth warmest on record. The warmest September occurred in 1990 and averaged $72.4^{\circ} \mathrm{F}$. The coolest September was $58.8^{\circ} \mathrm{F}$ in 1985 . September 1998 was the fourteenth consecutive month that averaged above normal. There were 12 days in September with maximum temperatures $\geq 90^{\circ} \mathrm{F}$ (normal for September is 5 days), and there were 3 days with maximum temperatures $\geq 100^{\circ} \mathrm{F}$ (normal is 0 days). For the year, there were 73 days (the third highest on record) with maximum temperatures $290^{\circ} \mathrm{F}$ (normal is 52 days). The most $90^{\circ}$ days were 79 in 1967 and the least were 29 days in 1980 . There were 26 days (also the third highest on record) in 1998 with maximum temperatures $\geq 100^{\circ} \mathrm{F}$ (normal is 13 days). The most $100^{\circ} \mathrm{F}$ days were 28 in 1958 and the least was one day in 1954.

Precipitation for September 1998 totaled only 0.10 in., 32\% of normal ( 0.31 in.). The wettest September, in 1947, received 1.34 in., while the driest, in 1991, recorded no precipitation.

September 1998's average wind speed was $6.7 \mathrm{mph}$, compared to a September normal of $7.4 \mathrm{mph}$. The peak wind gust for the month was $35 \mathrm{mph}$ recorded on September 17. The record wind gust for September was $65 \mathrm{mph}$ in 1953. The windiest September averaged $9.2 \mathrm{mph}$ (1961); while the September with the lightest winds (1957) averaged $5.4 \mathrm{mph}$.

October 1998 was slightly cooler than normal, averaging $52.4^{\circ} \mathrm{F}, 0.5^{\circ}$ below normal $\left(52.9^{\circ} \mathrm{F}\right)$. The warmest October occurred in 1988 and averaged $59.6^{\circ} \mathrm{F}$. The coolest October was $47.9^{\circ} \mathrm{F}$ in 1984 . 
Precipitation for October 1998 totaled 0.28 in., 74\% of normal ( 0.38 in.). The wettest October, in 1957, received 2.72 in., while the driest, in 1987 and earlier years, received only a trace of precipitation.

October 1998's average wind speed was $7.0 \mathrm{mph}$, compared to an October normal of $6.5 \mathrm{mph}$. The peak wind gust for the month was $46 \mathrm{mph}$ recorded on October 8. The record wind gust for October was $72 \mathrm{mph}$ in 1997. The windiest October averaged $9.1 \mathrm{mph}$ (1946); while the October with the lightest winds (1952) averaged $4.4 \mathrm{mph}$.

November 1998 was much warmer than normal, averaging $45.6^{\circ} \mathrm{F}, 5.4^{\circ}$ above normal $\left(40.2^{\circ} \mathrm{F}\right)$, and was the third warmest November on record. The warmest November occurred in 1990 and averaged $46.5^{\circ} \mathrm{F}$, while the coldest was $24.8^{\circ} \mathrm{F}$ in 1985 .

Precipitation for November 1998 totaled 1.29 in., 142\% of normal (0.91 in.). The wettest November, in 1996, received 2.67 in., while the driest, in 1976, received only a trace. There was no snowfall recorded in November 1998, compared to a November normal of 1.8 in.

November 1998 was a very windy month with an average wind speed of $8.8 \mathrm{mph}$, compared to a November normal of $6.4 \mathrm{mph}$. For the period from November 20 through 26 , the average wind speed was $16.2 \mathrm{mph}$, and every day during the period had gusts in excess of $40 \mathrm{mph}$. The peak wind gust for the month was $56 \mathrm{mph}$ recorded on November 21 . There were a record number of days (10) in November 1998 with wind gusts $\geq 40 \mathrm{mph}$. The previous record was 8 days in 1990 (normal is 2 days). There were 4 days during the month with wind gusts $\geq 50$, tying the record of 4 days, also recorded in November 1990. The record wind gust for November was $67 \mathrm{mph}$ in 1993. The windiest November averaged $10.0 \mathrm{mph}$ (1990); while the November with the lightest winds (1956) averaged $2.9 \mathrm{mph}$.

Autumn 1998 (September, October, and November) was much warmer than normal, averaging $56.3^{\circ} \mathrm{F}, 3.4^{\circ}$ above normal $\left(52.9^{\circ} \mathrm{F}\right)$, and was the fourth warmest autumn on record. The warmest was $57.1^{\circ} \mathrm{F}$ in 1990 , while the coolest was $44.5^{\circ} \mathrm{F}$ in 1985 .

Autumn 1998 precipitation totaled $1.67 \mathrm{in} ., 104 \%$ of normal (1.60 in.). The wettest autumn received 4.79 in. (1973), while the driest received only 0.04 in. (1976).

December 1998 was warmer than normal, averaging $33.0^{\circ} \mathrm{F}, 1.6^{\circ}$ above normal $\left(31.4^{\circ} \mathrm{F}\right)$. The warmest December occurred in 1957 and averaged $38.5^{\circ} \mathrm{F}$, while the coldest was $21.0^{\circ} \mathrm{F}$ in 1985 . The minimum temperature of $-1^{\circ} \mathrm{F}$ on December 21 was the coldest temperature in nearly two years.

Precipitation for December 1998 totaled 0.44 in., 43\% of normal (1.03 in.). The wettest December, in 1996, received $3.69 \mathrm{in}$., while the driest, in 1976, received $0.11 \mathrm{in}$. There was $0.9 \mathrm{in}$. of snowfall recorded in December 1998, compared to a December normal of 5.7 in. The snowiest December received 22.6 in. (1996), while December 1957 and 1963 received only a trace.

The average wind speed for December 1998 was $7.8 \mathrm{mph}$ compared to a December normal of $5.9 \mathrm{mph}$. The peak wind gust for the month (at the HMS) was $55 \mathrm{mph}$ recorded on December 26. Monitoring stations in Richland recorded gusts of 67 and $70 \mathrm{mph}$ on December 27. The record wind gust for 
December was $71 \mathrm{mph}$ in 1955. The windiest December averaged $8.3 \mathrm{mph}$ (1968); while the December with the lightest winds (1985) averaged $3.3 \mathrm{mph}$.

The following are some additional statistics for 1998:

\begin{tabular}{|c|c|c|c|c|}
\hline \multirow[b]{2}{*}{ Category } & \multirow[b]{2}{*}{$\begin{array}{l}\text { Number } \\
\text { of days }\end{array}$} & \multirow[b]{2}{*}{ Normal } & \multicolumn{2}{|c|}{ Record } \\
\hline & & & Maximum & Minimum \\
\hline Maximum temperatures $\geq 100^{\circ} \mathrm{F}$ & 26 & 13 & 28 & 1 \\
\hline Maximum temperatures $\geq 90^{\circ} \mathrm{F}$ & 73 & 52 & 79 & 29 \\
\hline Maximum temperatures $\leq 32^{\circ} \mathrm{F}$ & 12 & 24 & 58 & 2 \\
\hline Minimum temperatures $\geq 70^{\circ} \mathrm{F}$ & 8 & 7 & 21 & 0 \\
\hline Minimum temperatures $\leq 32^{\circ} \mathrm{F}$ & 85 & 107 & 139 & 77 \\
\hline Minimum temperatures $\leq 0^{\circ} \mathrm{F}$ & 1 & 3 & 18 & 0 \\
\hline Thunderstorms & 14 & 10 & 23 & 3 \\
\hline Fog (visibility $\leq 6 \mathrm{mi}$ ) & 46 & 45 & 76 & 22 \\
\hline Dense fog (visibility $\leq 0.25 \mathrm{mi}$ ) & 23 & 24 & 42 & 9 \\
\hline Peak wind gusts $\leq 12 \mathrm{mph}$ & 41 & 50 & 87 & 28 \\
\hline Peak wind gusts $\geq 25 \mathrm{mph}$ & 152 & 155 & 190 & 123 \\
\hline Peak wind gusts $\geq 40 \mathrm{mph}$ & 32 & 26 & 57 & 10 \\
\hline Peak wind gusts $\geq 50 \mathrm{mph}$ & 10 & 5 & 18 & 0 \\
\hline
\end{tabular}

Table 2.1 lists the daily temperature records for 1998 along with the previous record and year of occurrence. Table 2.2 lists the monthly and annual totals for numerous meteorological parameters for 1998. Table 2.3 lists the 1998 monthly and seasonal temperature and precipitation compared to normals and extremes. Tables $2.4,2.5$, and 2.6 list the 1998 monthly and annual average temperature, precipitation, and wind speed, respectively, from the HMMN.

Figures 2.1 and 2.2 give a composite of the wind roses (at the 10 - and $60-\mathrm{m}$ levels, respectively) from the HMMN for 1998. The appendix gives the individual 1998 wind roses from the HMMN stations.

Figure 2.3 depicts the 1998 observed daily maximum and minimum temperatures and the normal maximum, minimum, and mean daily temperatures for the HMS. 
Table 2.1. 1998 Daily Temperature Records (previous record and year of occurrence in parentheses)

\begin{tabular}{|c|c|c|c|c|}
\hline \multirow[b]{2}{*}{ Date } & \multicolumn{2}{|c|}{ Maximum } & \multicolumn{2}{|c|}{ Minimum } \\
\hline & High & Low & High & Low \\
\hline Jan 1 & $57^{(\mathrm{a})}(57,1964)$ & & & \\
\hline $\operatorname{Jan} 6$ & & & $42(39,1956)$ & \\
\hline Jan 17 & $57(56,1989)$ & & & \\
\hline Mar 12 & $68^{(\mathrm{a})}(68,1992)$ & & & \\
\hline $\operatorname{Mar} 13$ & $72(70,1992)$ & & $44^{(\mathrm{a})}\left(44,1987^{[\mathrm{b}]}\right)$ & \\
\hline $\operatorname{Mar} 21$ & & & $46^{(\mathrm{a})}(46,1947)$ & \\
\hline Mar 23 & & & $45^{(\mathrm{a})}\left(45,1956^{[\mathrm{b}]}\right)$ & \\
\hline Apr 22 & & & $55\left(52,1981^{[\mathrm{b}]}\right)$ & \\
\hline Apr 30 & $92(91,1981)$ & & $60(56,1981)$ & \\
\hline May 1 & $93(87,1947)$ & & $59^{(a)}(59,1977)$ & \\
\hline May 2 & $89^{(a)}(89,1971)$ & & & \\
\hline May 25 & & $61(62,1984)$ & & \\
\hline May 29 & & $68^{(\mathrm{a})}\left(68,1989^{[\mathrm{b}]}\right)$ & & \\
\hline Jul 26 & $108^{(2)}(108,1996)$ & & & \\
\hline Jul 27 & $112^{(\mathrm{c})}\left(108,1975^{[\mathrm{b}]}\right)$ & & $74^{(a)}\left(74,1973^{[b]}\right)$ & \\
\hline Jul 28 & $108^{(\mathrm{a})}(108,1968)$ & & $81(76,1968)$ & \\
\hline Sep 2 & $102^{(a)}(102,1950)$ & & & \\
\hline Sep 3 & $102(101,1988)$ & & & \\
\hline Sep 14 & $94^{(a)}(94,1955)$ & & & \\
\hline Nov 13 & & & $54(46,199)$ & \\
\hline Nov 14 & & & $47^{(a)}(47,1945)$ & \\
\hline Nov 15 & $67(65,1953)$ & & $47(46,1983)$ & \\
\hline Nov 25 & $67(65,1962)$ & & $50(48,1962)$ & \\
\hline Dec 17 & $57^{(\mathrm{a})}\left(57,1971^{[\mathrm{b}]}\right)$ & & & \\
\hline Dec 28 & $59(57,1997)$ & & $46(38,1949)$ & \\
\hline $\operatorname{Dec} 29$ & $60^{(a)}(60,1949)$ & & $41(39,1962)$ & \\
\hline
\end{tabular}

(a) Ties record.

(b) Most recent of several occurrences.

(c) All-time record for July. Previous record $111^{\circ} \mathrm{F}$ on July 22, 1994 and July 31, 1971. 
Table 2.2. 1998 Climatological Data Summary

\begin{tabular}{|c|c|c|c|c|c|c|c|c|c|c|c|c|c|c|c|c|c|c|c|c|c|c|}
\hline \multirow[b]{3}{*}{ 嶽 } & \multicolumn{8}{|c|}{ Temperatures, ${ }^{\circ} \mathrm{F}$} & \multirow{2}{*}{\multicolumn{4}{|c|}{$\begin{array}{l}\text { Degree Days } \\
\text { Base } 65,{ }^{\circ} \mathrm{F}\end{array}$}} & \multicolumn{8}{|c|}{ Precipitation, in. } & \multirow{2}{*}{\multicolumn{2}{|c|}{$\begin{array}{c}\text { Relative } \\
\text { Humidity, \% }\end{array}$}} \\
\hline & \multicolumn{4}{|c|}{ Averages } & \multicolumn{4}{|c|}{ Extremes } & & & & & & & & & & Snow, & e Pellet & & & \\
\hline & 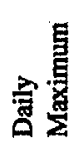 & 竞䂓 & $\begin{array}{l}\text { 窟 } \\
\text { 究 }\end{array}$ & 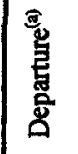 & 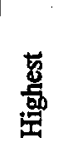 & 苋 & 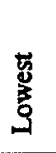 & 茗 & $\begin{array}{l}\text { 层 } \\
\text { 营 }\end{array}$ & 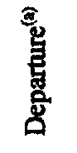 & $\frac{50}{8}$ & 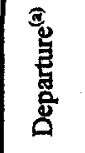 & 褐 & 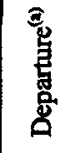 & 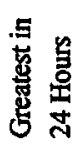 & ڤั & 丞 & 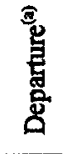 & 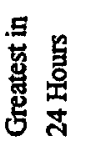 & 䔽 & 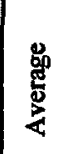 & 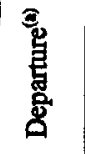 \\
\hline J & 44.1 & 28.2 & 36.2 & +4.9 & 57 & $19^{(\mathrm{c})}$ & 7 & 13 & 892 & .152 & 0 & 0 & 1.24 & +0.45 & 0.40 & $16-17$ & 6.3 & +2.4 & 2.3 & 11 & 76.3 & -0.1 \\
\hline $\mathbf{F}$ & 51.6 & 32.7 & 42.2 & +4.2 & 58 & 21 & 22 & 27 & 642 & -122 & 0 & $\mathbf{0}$ & 1.15 & +0.53 & 0.37 & 12 & $T$ & -2.0 & $\mathrm{~T}$ & 25 & 74.4 & +4.1 \\
\hline $\mathbf{M}$ & 60.2 & 36.5 & 48.4 & +2.8 & 72 & 13 & 23 & 5 & 521 & -81 & 0 & 0 & 0.50 & +0.03 & 0.20 & 23 & $\mathbf{T}$ & -0.3 & $\mathrm{~T}$ & 5 & 58.4 & +2.5 \\
\hline A & 68.7 & 40.1 & 54.4 & +1.7 & 92 & 30 & 29 & 12 & 332 & -40 & 16 & +13 & 0.07 & -0.34 & 0.03 & 3 & 0 & $-T^{(b)}$ & 0 & 0 & 49.4 & +2.2 \\
\hline M & 76.2 & 48.6 & 62.4 & +1.1 & 93 & $6^{(0)}$ & 39 & 15 & 131 & -33 & 55 & +7 & 0.52 & +0.01 & 0.23 & 17 & 0 & 0 & 0 & 0 & 53.2 & +10.5 \\
\hline $\mathbf{J}$ & 85.6 & 56.4 & 71.0 & +1.3 & 99 & 30 & 47 & 27 & 1 & -31 & 183 & +8 & 0.48 & +0.10 & 0.29 & 19 & 0 & 0 & 0 & $\mathbf{0}$ & 41.0 & +2.2 \\
\hline$J$ & 97.3 & 66.7 & 82.0 & +5.8 & 112 & 27 & 58 & $20^{(c)}$ & 0 & -5 & 527 & +176 & 0.34 & +0.16 & 0.16 & $31^{(0)}$ & 0 & 0 & $\mathbf{0}$ & 0 & 33.6 & +0.1 \\
\hline A & 94.5 & 61.3 & 77.9 & +2.8 & 110 & 4 & 50 & 24 & 0 & -5 & 398 & +81 & 0.04 & -0.23 & 0.04 & 23 & 0 & 0 & 0 & 0 & 33.0 & -2.8 \\
\hline $\mathbf{S}$ & 87.0 & 55.1 & 71.0 & +5.3 & 103 & 1 & 43 & 27 & 15 & -63 & 195 & +97 & 0.10 & -0.21 & 0.06 & 25 & 0 & 0 & 0 & 0 & 42.6 & -0.1 \\
\hline 0 & 65.7 & 39.1 & 52.4 & -0.5 & 84 & 1 & 25 & 30 & 393 & +16 & 3 & 0 & 0.28 & -0.10 & 0.22 & 31 & 0 & -0.1 & 0 & 0 & 56.7 & +1.5 \\
\hline $\mathbf{N}$ & 53.7 & 37.4 & 45.6 & +5.4 & 67 & $25^{(\mathrm{c})}$ & 27 & $19^{(c)}$ & 582 & -164 & 0 & 0 & 1.29 & +0.38 & 0.62 & 5 & 0 & -1.8 & 0 & 0 & 72.8 & -0.6 \\
\hline D & 41.8 & 24.2 & 33.0 & +1.6 & 60 & 29 & -1 & 21 & 1000 & 42 & 0 & 0 & 0.44 & -0.59 & 0.16 & $12-13$ & 0.9 & -4.8 & 0.9 & 24 & 72.3 & -8.0 \\
\hline Year & 68.9 & 43.9 & 56.4 & +3.1 & 112 & $\begin{array}{l}\text { Jul } \\
27\end{array}$ & -1 & $\begin{array}{r}\text { Dec } \\
21\end{array}$ & 4,509 & -722 & 1,377 & +382 & 6.45 & +0.19 & 0.62 & $\begin{array}{c}\text { Nov } \\
5\end{array}$ & 7.2 & -6.6 & 2.3 & $\begin{array}{c}\text { Jan } \\
11\end{array}$ & 55.3 & +1.0 \\
\hline
\end{tabular}


Table 2.2. (contd)

\begin{tabular}{|c|c|c|c|c|c|c|c|c|c|c|c|c|c|c|c|c|c|c|c|c|c|}
\hline \multirow[b]{3}{*}{ 点 } & & & \multicolumn{6}{|c|}{ Solar Radiation, Langleys } & \multicolumn{5}{|c|}{$50-f t$ Wind } & \multicolumn{8}{|c|}{ Number of Days } \\
\hline & \multicolumn{2}{|c|}{$\begin{array}{l}\text { Mean Sky } \\
\text { Cover, } \\
\text { Tenths }\end{array}$} & \multirow[b]{2}{*}{ 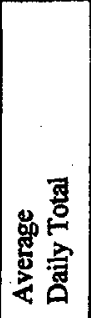 } & \multirow[b]{2}{*}{ 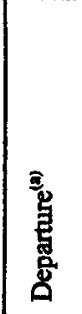 } & \multirow[b]{2}{*}{ 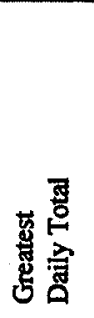 } & \multirow[b]{2}{*}{ 峞 } & \multirow{2}{*}{ 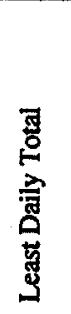 } & \multirow[b]{2}{*}{ 苂 } & \multirow{2}{*}{ 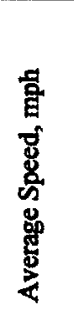 } & \multirow[b]{2}{*}{ 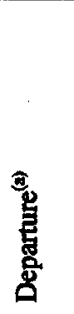 } & \multicolumn{3}{|c|}{ Peak Gusts } & \multirow[b]{2}{*}{ 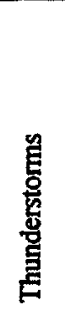 } & \multirow[b]{2}{*}{ 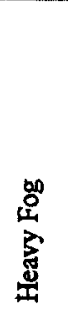 } & \multirow{2}{*}{ 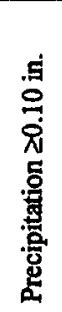 } & \multirow[b]{2}{*}{ 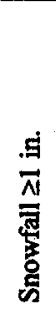 } & \multicolumn{2}{|c|}{$\begin{array}{c}\text { Maximum } \\
\text { Temperature, } \\
{ }^{\circ} \mathrm{F}\end{array}$} & \multicolumn{2}{|c|}{$\begin{array}{c}\text { Minimum } \\
\text { Temperature, }\end{array}$} \\
\hline & 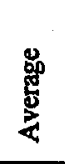 & 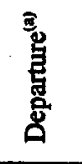 & & & & & & & & & $\begin{array}{l}\text { 总 } \\
\text { 音 } \\
\text { 号 }\end{array}$ & $\begin{array}{l}\text { 总 } \\
\text { 总 } \\
\text { 。 }\end{array}$ & 苋 & & & & & 蓉 & : & ஸั & 嵩 \\
\hline $\mathbf{J}$ & 7.5 & -0.6 & 91 & -18 & 184 & 30 & 19 & 6 & 7.8 & +1.3 & 43 & SW & 24 & 0 & 7 & 6 & 4 & 0 & 5 & 21 & 0 \\
\hline $\mathbf{F}$ & 7.6 & 0.0 & 160 & -27 & 319 & 24 & 42 & 3 & 7.3 & +0.1 & 50 & $\mathbf{S}$ & 21 & 0 & 2 & 3 & 0 & 0 & 0 & 14 & 0 \\
\hline $\mathbf{M}$ & 6.6 & -0.2 & 303 & -21 & 497. & 29 & 107 & 31 & 7.3 & -1.0 & 39 & WSW & 26 & 0 & 0 & 3 & 0 & 0 & 0 & 9 & 0 \\
\hline$A$ & 4.3 & -2.3 & 445 & -7 & 606 & 24 & 151 & 3 & 7.6 & -1.4 & 37 & $\mathbf{W}$ & 24 & 3 & 1 & 0 & 0 & 1 & 0 & 3 & 0 \\
\hline $\mathbf{M}$ & 6.7 & +0.7 & 453 & -107 & 684 & 31 & 156 & 17 & 8.0 & -1.1 & 55 & WNW & 21 & 0 & 1 & 2 & 0 & 3 & 0 & 0 & 0 \\
\hline $\mathbf{J}$ & 3.6 & -1.6 & 608 & -7 & 719 & 28 & 338 & 24 & 9.8 & +0.6 & 50 & WNW & 15 & 3 & 0 & 1 & 0 & 7 & 0 & 0 & 0 \\
\hline A & 1.0 & -2.5 & 557 & +19 & 633 & 6 & 262 & 23 & 8.1 & +0.2 & 50 & NW & 15 & 0 & 0 & 0 & 0 & 24 & 0 & 0 & 0 \\
\hline $\mathbf{S}$ & 3.0 & -1.0 & 394 & -14 & 511 & 5 & 133 & 25 & 6.7 & -0.7 & 35 & Wsw & 17 & 0 & 1 & 0 & 0 & 12 & 0 & 0 & 0 \\
\hline 0 & 3.9 & -1.8 & 256 & -3 & 351 & 5 & 93 & 31 & 7.0 & +0.5 & 46 & SW & 8 & 0 & 0 & 1 & 0 & 0 & 0 & 5 & 0 \\
\hline $\mathbf{N}$ & 8.4 & +0.6 & 109 & -14 & 191 & 1 & 20 & 30 & 8.8 & +2.4 & 56 & SSW & 21 & 0 & 8 & 4 & 0 & 0 & 0 & 10 & 0 \\
\hline D & 7.2 & -0.9 & 87 & +1 & 155 & 4 & 12 & 27 & 7.8 & +1.9 & 55 & WSW & 26 & 0 & 3 & 0 & 0 & 0 & 7 & 23 & 1 \\
\hline Year & 5.2 & -0.9 & 338 & -25 & 719 & $\begin{array}{r}\text { Jun } \\
28\end{array}$ & 12 & $\begin{array}{r}\text { Dec } \\
26\end{array}$ & 7.9 & +0.2 & 56 & SSW & $\begin{array}{r}\text { Nov } \\
21\end{array}$ & 14 & 23 & 22 & 4 & 73 & 12 & 85 & 1 \\
\hline $\begin{array}{l}\text { (a) } \mathrm{D} \\
\text { (b) } \mathrm{T} \\
\text { (c) } \mathrm{L} \\
\mathrm{T}=\mathrm{Tr}\end{array}$ & dat & multi & $\begin{array}{l}\text { cate p } \\
\text { mal; } n\end{array}$ & $\begin{array}{l}\text { ositive } \\
\text { no occu } \\
\text { ences. }\end{array}$ & $\begin{array}{l}\text { negati } \\
\text { ace in }\end{array}$ & $\begin{array}{l}\text { par } \\
\text { l. }\end{array}$ & & & & & & & & & & & & & & & \\
\hline
\end{tabular}


Table 2.3. 1998 Monthly and Seasonal Temperature and Precipitation

\begin{tabular}{|c|c|c|c|c|c|c|c|c|c|c|c|c|c|c|}
\hline $\begin{array}{l}\text { Month/ } \\
\text { Season }\end{array}$ & $\begin{array}{c}\text { Average } \\
\text { Temperature, } \\
{ }^{\circ} \mathbf{F}\end{array}$ & Departure $^{(a)}$ & $\begin{array}{c}\text { Normal, } \\
{ }^{\circ} \mathbf{F} \\
\end{array}$ & $\begin{array}{c}\text { Warmest } \\
\text { of Record, } \\
{ }^{\circ} \mathbf{F} \\
\end{array}$ & Year & $\begin{array}{l}\text { Coolest of } \\
\text { Record, } \\
{ }^{\circ} \mathbf{F} \\
\end{array}$ & Year & $\begin{array}{l}\text { Precipitation, } \\
\text { in. } \\
\end{array}$ & $\begin{array}{l}\text { Percent } \\
\text { of } \\
\text { Normal } \\
\end{array}$ & Normal & $\begin{array}{l}\text { Wettest of } \\
\text { Record, in. }\end{array}$ & Year & $\begin{array}{c}\text { Driest of } \\
\text { Record, } \\
\text { in. } \\
\end{array}$ & Year \\
\hline Jan & 36.2 & +4.9 & 31.3 & 42.5 & 1953 & 12.1 & 1950 & 1.24 & 157 & 0.79 & 2.47 & 1970 & 0.08 & 1977 \\
\hline Feb & 42.2 & +4.2 & 38.0 & 44.5 & 1958 & 25.6 & 1956 & 1.15 & 185 & 0.62 & 2.10 & 1961 & $\mathbf{T}$ & $1988^{(b)}$ \\
\hline Mar & 48.4 & +2.8 & 45.6 & 51.5 & 1992 & 39.4 & 1955 & 0.50 & 106 & 0.47 & 1.86 & 1957 & 0.02 & 1968 \\
\hline Apr & 54.4 & +1.7 & 52.7 & 58.2 & 1994 & 47.5 & 1955 & 0.07 & 17 & 0.41 & 1.54 & 1995 & $\mathbf{T}$ & $1986^{(b)}$ \\
\hline May & 62.4 & +1.1 & 61.3 & 68.7 & 1947 & 56.0 & 1984 & 0.52 & 102 & 0.51 & 2.03 & 1972 & $\mathbf{T}$ & $1992^{(b)}$ \\
\hline Jun & 71.0 & +1.3 & 69.7 & 76.8 & 1992 & 63.0 & 1953 & 0.48 & 126 & 0.38 & 2.92 & 1950 & $\mathrm{~T}$ & $1986^{(0)}$ \\
\hline Jul & 82.0 & +5.8 & 76.2 & 82.2 & 1985 & 70.5 & 1993 & 0.34 & 189 & 0.18 & 1.76 & 1993 & $\mathrm{~T}$ & $1980^{(0)}$ \\
\hline Aug & 77.9 & +2.8 & 75.1 & 81.5 & 1967 & 69.8 & 1964 & 0.04 & 15 & 0.27 & 1.36 & 1977 & 0 & $1988^{(0)}$ \\
\hline Sep & 71.0 & +5.3 & 65.7 & 72.4 & 1990 & 58.8 & 1985 & 0.10 & 32 & 0.31 & 1.34 & 1947 & 0 & 1991 \\
\hline Oct & 52.4 & -0.5 & 52.9 & 59.6 & 1988 & 47.9 & 1984 & 0.28 & 72 & 0.39 & 2.72 & 1957 & $\mathbf{T}$ & $1987^{(b)}$ \\
\hline Nov & 45.6 & +5.4 & 40.2 & 46.5 & 1990 & 24.8 & 1985 & 1.29 & 142 & 0.91 & 2.67 & 1996 & $\mathbf{T}$ & 1976 \\
\hline Dec & 33.0 & +1.6 & 31.4 & 38.5 & 1957 & 21.0 & 1985 & 0.44 & 33 & 1.03 & 3.69 & 1996 & 0.11 & 1976 \\
\hline Winter & 37.7 & +4.1 & 33.6 & 40.6 & $1966-67$ & 24.2 & $1948-49$ & 2.70 & 111 & 2.44 & 5.45 & $1996-97$ & 0.70 & $1946-47$ \\
\hline Spring & 55.1 & +1.9 & 53.2 & 58.2 & 1992 & 48.0 & 1955 & 1.09 & 78 & 1.40 & 3.28 & 1995 & 0.09 & 1968 \\
\hline Summer & 77.0 & +3.3 & 73.7 & 78.2 & 1958 & 70.2 & 1980 & 0.86 & 104 & 0.83 & 2.99 & 1950 & 0.03 & 1973 \\
\hline Autumn & 56.3 & +3.4 & 52.9 & 57.1 & 1990 & 44.5 & 1985 & 1.67 & 104 & 1.60 & 4.79 & 1973 & 0.04 & 1976 \\
\hline $\begin{array}{l}\text { Calendar } \\
\text { year }\end{array}$ & 56.4 & +3.1 & 53.3 & 56.4 & $1998^{(0)}$ & 49.6 & 1985 & 6.45 & 103 & 6.26 & 12.31 & 1995 & 2.99 & 1976 \\
\hline $\begin{array}{l}\text { (a) Depar } \\
\text { (b) Latest } \\
\mathrm{T}=\text { Trace. }\end{array}$ & $\begin{array}{l}\text { e indicates pos } \\
\text { multiple occu }\end{array}$ & $\begin{array}{l}\text { ve or negative } \\
\text { nces. }\end{array}$ & parture f & m 30-year ( & 61-1990) & natological & rmals. & & & & & & & \\
\hline
\end{tabular}


Table 2.4. 1998 Monthly and Annual Average Temperatures ( $\left.{ }^{\circ} \mathrm{F}\right)$ from the Hanford Meteorological Monitoring Network

\begin{tabular}{|c|c|c|c|c|c|c|c|c|c|c|c|c|c|}
\hline Station & $\operatorname{Jan}$ & Feb & Mar & Apr & May & Jun & Jul & Aug & Sep & Oct & Nov & Dec & Annual \\
\hline PROS & 35.8 & 41.2 & 46.5 & 52.2 & 61.0 & 70.1 & 81.3 & 76.7 & 68.2 & 50.1 & 45.0 & 32.8 & 55.2 \\
\hline EOC & 36.2 & 41.7 & 47.1 & 53.2 & 60.3 & 69.2 & 81.1 & 77.5 & 71.0 & 52.8 & 45.0 & 34.2 & 55. \\
\hline ARMY & 35.8 & 41.3 & 46.9 & 53.2 & 61.7 & 70.5 & 82.1 & 78.0 & 69.8 & 51.1 & 45.4 & 33.2 & 55. \\
\hline RSPG & 34.2 & 40.9 & 46.4 & 52.4 & 60.5 & 69.6 & 81.1 & 77.0 & 69.7 & 50.7 & 44.9 & 32.8 & 55.1 \\
\hline EDNA & 37.6 & 44.5 & 50.8 & 55.3 & 62.2 & 70.9 & 81.3 & 76.9 & 68.4 & 50.4 & 45.3 & 33.0 & 56.4 \\
\hline $200 \mathrm{E}$ & 35.6 & 42.3 & 48.1 & 54.8 & 62.6 & 71.4 & 83.2 & 79.5 & 71.7 & 53.1 & 45.8 & 33.8 & 56.5 \\
\hline $200 \mathrm{~W}$ & 34.8 & 40.0 & 45.7 & 52.0 & 60.9 & 69.9 & 81.7 & 77.5 & 69.0 & 49.5 & 44.5 & 32.3 & 55.0 \\
\hline BVLY & 34.4 & 41.8 & 47.4 & 53.9 & 62.7 & 70.1 & 80.8 & 77.4 & 69.8 & 52.8 & 45.7 & 33.5 & 55.9 \\
\hline FFTF & 35.8 & 41.8 & 47.1 & 53.3 & 61.0 & 70.1 & 81.6 & 77.6 & 69.7 & 51.9 & 45.5 & 33.7 & 55.8 \\
\hline 0 YAKB & 34.8 & 41.2 & 47.2 & 53.3 & 61.8 & 70.6 & 82.7 & 78.9 & 71.4 & 52.0 & 44.8 & 33.8 & 56.2 \\
\hline $300 \mathrm{~A}$ & 36.2 & 41.9 & 47.0 & 52.9 & 60.9 & 69.8 & 80.6 & 76.6 & 68.3 & 51.4 & 46.3 & 34.3 & 55. \\
\hline 2 WYEB & 35.4 & 41.4 & 46.9 & 53.2 & 61.2 & 70.4 & 81.9 & 77.8 & 69.5 & 51.4 & 45.1 & 33.1 & 55. \\
\hline $3100 \mathrm{~N}$ & 34.1 & 41.0 & 46.4 & 52.6 & 61.4 & 70.3 & 81.1 & 77.0 & 69.2 & 51.3 & 45.5 & 32.9 & 55.3 \\
\hline 4 WPPS & 35.1 & 40.8 & 46.1 & 52.2 & 61.1 & 70.3 & 81.8 & 77.4 & 68.9 & 50.8 & 45.5 & 33.1 & 55. \\
\hline 5 FRNK & 35.1 & 41.1 & 46.3 & 52.1 & 58.5 & 67.1 & 77.3 & 73.6 & 66.8 & 50.4 & 44.5 & 33.4 & 53. \\
\hline $6 \mathrm{GABL}$ & 35.0 & 42.1 & 47.2 & 54.1 & 60.7 & 69.4 & 81.4 & 78.6 & 71.9 & 53.4 & 45.4 & 33.7 & 56.1 \\
\hline 7 RING & 35.5 & 41.4 & 46.4 & 52.0 & 60.1 & 68.2 & 77.5 & 73.3 & 65.7 & 49.9 & 45.2 & 33.1 & 54.1 \\
\hline $8 \mathrm{RICH}$ & 36.9 & 42.7 & 47.8 & 53.9 & 61.3 & 69.9 & 81.0 & 77.2 & 69.5 & 52.5 & 47.1 & 35.3 & 56. \\
\hline 19 PFP & 34.9 & 41.1 & 47.0 & 53.5 & 61.7 & 70.2 & 82.2 & 78.4 & 70.9 & 51.7 & 45.2 & 32.6 & 55. \\
\hline 0 RMTN & 29.4 & 31.7 & 38.4 & 44.8 & 51.0 & 59.3 & 72.6 & 70.8 & 65.1 & 46.6 & 36.7 & 27.5 & 48.0 \\
\hline $1 \mathrm{HMS}$ & 36.2 & 42.2 & 48.4 & 54.4 & 62.4 & 71.0 & 82.0 & 77.9 & 71.0 & 52.4 & 45.6 & 33.0 & 56. \\
\hline 22 PASC & 36.6 & 42.2 & 47.5 & 53.5 & 61.6 & 70.3 & 81.6 & 77.3 & 68.8 & 51.6 & 46.8 & 35.2 & 56.1 \\
\hline $23 \mathrm{GABW}$ & 34.1 & 40.5 & 45.9 & 52.0 & 61.2 & 70.0 & 81.6 & 77.2 & 68.7 & 50.0 & 44.5 & 31.6 & 54.9 \\
\hline $24100 \mathrm{~F}$ & 34.4 & 40.9 & 46.3 & 52.4 & 61.6 & 70.6 & 81.3 & 77.2 & 68.9 & 50.7 & 45.4 & 32.7 & 55.3 \\
\hline 25 VERN & 35.5 & 41.8 & 47.8 & 53.9 & 62.7 & 70.4 & 82.4 & 79.0 & 71.5 & 53.2 & 46.6 & 34.3 & 56.7 \\
\hline $26 \mathrm{BENT}$ & 34.9 & 40.9 & 46.3 & 52.5 & 59.0 & 68.0 & 78.4 & 74.8 & 68.3 & 50.8 & 44.0 & 33.4 & 54.3 \\
\hline 27 VSTA & 37.5 & 42.9 & 48.3 & 53.6 & 60.6 & 69.7 & 80.8 & 77.4 & 69.9 & 52.6 & 47.5 & 36.5 & 56.5 \\
\hline 28 SURF & 36.8 & 43.1 & 47.4 & 52.7 & 59.6 & 68.3 & 79.0 & 76.1 & 68.9 & 51.1 & 46.6 & 36.9 & 55 \\
\hline $29100 \mathrm{~K}$ & 34.4 & 41.2 & 46.9 & 52.9 & 61.8 & 70.8 & 81.8 & 77.7 & 69.7 & 51.5 & 45.6 & 34.5 & 56.0 \\
\hline 30 HAMR & 35.9 & 42.2 & 47.6 & 53.5 & 61.1 & 69.8 & 81.2 & 77.2 & 69.4 & 52.4 & 46.3 & 32.5 & 55. \\
\hline
\end{tabular}


Table 2.5. 1998 Monthly and Annual Average Precipitation (in.) from the Hanford Meteorological Monitoring Network ${ }^{(a)}$

\begin{tabular}{|c|c|c|c|c|c|c|c|c|c|c|c|c|c|}
\hline Station & Jan & Feb & Mar & Apr & May & Jun & Jul & Aug & Sep & Oct & Nov & Dec & Annual \\
\hline 1 PROS & 0.70 & 1.09 & 0.51 & 0.07 & 0.83 & 0.31 & 0.64 & 0.00 & 0.13 & 0.13 & 1.66 & 0.79 & 6.86 \\
\hline $2 \mathrm{EOC}$ & 1.24 & 1.59 & 0.65 & 0.15 & 0.96 & 0.24 & 1.19 & 0.01 & 0.14 & 0.19 & 2.62 & 1.24 & 10.22 \\
\hline 3 ARMY & 0.84 & 0.80 & 0.37 & 0.13 & 0.56 & 0.39 & 0.50 & 0.00 & 0.22 & 0.22 & 1.29 & 0.29 & 5.61 \\
\hline 4 RSPG & 0.67 & 1.02 & 0.57 & 0.11 & 0.68 & 0.26 & 0.23 & 0.00 & 0.24 & 0.30 & 1.27 & 0.27 & 5.62 \\
\hline $6200 \mathrm{E}$ & 0.99 & 0.99 & 0.50 & 0.17 & 0.45 & 0.52 & 0.71 & 0.01 & 0.22 & 0.23 & 1.20 & 0.28 & 6.27 \\
\hline $7200 \mathrm{w}$ & 1.32 & 0.86 & 0.58 & 0.13 & 0.72 & 0.30 & 0.19 & 0.00 & 0.05 & 0.26 & 1.09 & 0.37 & 5.87 \\
\hline 8 BVLY & 0.67 & 0.49 & 0.54 & 0.04 & 0.79 & 0.05 & 0.40 & 0.02 & 0.08 & 0.07 & 0.92 & 0.33 & 4.40 \\
\hline 9 FFTF & 0.73 & 0.95 & 0.45 & 0.05 & 0.44 & 0.57 & 0.33 & 0.03 & 0.31 & 0.08 & 0.89 & 0.40 & 5.23 \\
\hline 10 YAKB & 0.82 & 0.85 & 0.46 & 0.12 & 0.64 & 0.27 & 0.21 & 0.01 & 0.02 & 0.16 & 1.17 & 0.35 & 5.08 \\
\hline $11300 \mathrm{~A}$ & 0.89 & 0.88 & 0.40 & 0.12 & 0.48 & 0.21 & 0.95 & 0.00 & 0.21 & 0.07 & 1.26 & 0.64 & 6.11 \\
\hline 12 WYEB & 0.56 & 1.25 & 0.57 & 0.11 & 0.58 & 0.28 & 0.55 & 0.00 & 0.43 & 0.06 & 1.14 & 0.41 & 5.94 \\
\hline $13100 \mathrm{~N}$ & 0.89 & 0.68 & 0.41 & 0.04 & 0.30 & 0.30 & 0.13 & 0.01 & 0.16 & 0.27 & 0.75 & 0.34 & 4.28 \\
\hline 14 WPPS & 0.86 & 1.19 & 0.64 & 0.22 & 0.49 & 0.16 & 0.39 & 0.03 & 0.39 & 0.19 & 1.49 & 0.51 & 6.56 \\
\hline $17 \mathrm{RING}$ & 0.86 & 0.82 & 0.31 & 0.20 & 0.45 & 0.07 & 0.50 & 0.03 & 0.44 & 0.28 & 1.65 & 0.55 & 6.16 \\
\hline $18 \mathrm{RICH}$ & 0.85 & 0.67 & 0.43 & 0.09 & 0.68 & 0.13 & 1.08 & 0.00 & 0.05 & 0.14 & 1.34 & 0.75 & 6.21 \\
\hline 20 RMTN & 0.25 & 0.82 & 0.47 & 0.19 & 1.72 & 0.31 & 1.05 & 0.02 & 0.23 & 0.23 & 1.23 & 0.91 & 7.43 \\
\hline $21 \mathrm{HMS}$ & 1.24 & 1.15 & 0.50 & 0.07 & 0.52 & 0.48 & 0.34 & 0.04 & 0.10 & 0.28 & 1.29 & 0.44 & 6.45 \\
\hline 22 PASC & 1.00 & 0.85 & 0.42 & 0.11 & 0.66 & 0.44 & 0.39 & 0.00 & 0.15 & 0.22 & 2.04 & 0.94 & 7.22 \\
\hline $24100 \mathrm{~F}$ & 0.95 & 0.99 & 0.46 & 0.14 & 0.37 & 0.40 & 1.04 & 0.01 & 0.24 & 0.28 & 1.14 & 0.65 & 6.67 \\
\hline 26 BENT & 1.27 & 1.32 & 0.78 & 0.35 & 1.05 & 0.21 & 0.66 & 0.01 & 0.14 & 0.28 & 1.87 & 0.79 & 8.73 \\
\hline 27 VSTA & 0.78 & 0.77 & 0.44 & 0.18 & 0.55 & 0.32 & 0.35 & 0.00 & (b) & (b) & (b) & 0.58 & (c) \\
\hline 28 SURF & 2.08 & 1.64 & 0.57 & 0.21 & 1.40 & 0.28 & 0.45 & 0.38 & 0.76 & 0.24 & 2.08 & 2.05 & 12.14 \\
\hline $29100 \mathrm{~K}$ & 0.96 & 0.87 & 0.43 & 0.17 & 0.33 & 0.51 & 0.15 & 0.02 & 0.04 & 0.27 & 0.89 & 0.51 & 5.15 \\
\hline
\end{tabular}

(a) Stations $5,15,16,19,23$, and 25 are solar powered; therefore, insufficient power is available to operate the heated tippingbucket precipitation gauges.

(b) Missing data

(c) Partial year, no annual average. 
Table 2.6. 1998 Monthly and Annual Average Wind Speed (mph) from the Hanford Meteorological Monitoring Network

\begin{tabular}{|c|c|c|c|c|c|c|c|c|c|c|c|c|c|}
\hline Station & Jan & Feb & Mar & Apr & May & Jun & Jul & Aug & Sep & Oct & Nov & Dec & Annual \\
\hline 1 PROS & 8.6 & 7.5 & 6.8 & 5.8 & 8.9 & 8.4 & 6.7 & 6.0 & 4.9 & 6.4 & 8.6 & 7.7 & 7.2 \\
\hline $2 \mathrm{EOC}$ & 11.4 & 9.6 & 8.9 & 7.6 & 9.1 & 9.0 & 7.2 & 6.4 & 6.6 & 8.3 & 11.2 & 10.6 & 8.8 \\
\hline 3 ARMY & 7.1 & 5.9 & 5.7 & 5.9 & 6.1 & 7.9 & 6.0 & 5.8 & 5.1 & 5.5 & 8.2 & 7.4 & 6.4 \\
\hline 4 RSPG & 6.1 & 6.6 & 6.9 & 7.4 & 6.9 & 7.7 & 6.6 & 7.0 & 7.0 & 7.6 & 8.5 & 7.5 & 7.1 \\
\hline 5 EDNA & 6.2 & 5.4 & 5.4 & 5.3 & 7.2 & 8.1 & 6.1 & 5.8 & 4.8 & 5.1 & 6.9 & 6.4 & 6.0 \\
\hline $6200 \mathrm{E}$ & 7.1 & 6.8 & 6.7 & 6.9 & 7.5 & 9.5 & 7.4 & $7: 4$ & 6.1 & 6.5 & 7.9 & 7.2 & 7.3 \\
\hline $7200 w$ & 5.7 & 4.7 & 5.2 & 5.3 & 5.8 & 7.6 & 5.5 & 5.3 & 4.6 & 5.0 & 6.9 & 5.6 & 5.6 \\
\hline 8 BVLY & 6.5 & 6.2 & 6.3 & 6.8 & 7.5 & 8.3 & 5.9 & 6.3 & 4.8 & 5.1 & 6.6 & 6.2 & 6.4 \\
\hline 9 FFTF & 8.5 & 7.6 & 7.3 & 6.6 & 9.2 & 9.0 & 7.0 & 6.7 & 5.8 & 7.6 & 9.1 & 8.4 & 7.7 \\
\hline 10 YAKB & 7.1 & 6.7 & 7.0 & 7.2 & 7.7 & 9.4 & 7.7 & 7.9 & 6.2 & 6.6 & 8.1 . & 6.7 & 7.4 \\
\hline $11300 \mathrm{~A}$ & 8.3 & 7.4 & 7.1 & 6.1 & 8.9 & 8.6 & 6.6 & 6.0 & 5.1 & 6.8 & 8.9 & 8.7 & 7.4 \\
\hline 12 WYEB & 7.9 & 6.6 & 6.4 & 6.0 & 7.9 & 8.6 & 6.5 & 6.2 & 5.2 & 6.2 & 8.0 & 7.5 & 6.9 \\
\hline $13100 \mathrm{~N}$ & 4.7 & 4.4 & 5.3 & 5.6 & 6.3 & 7.7 & 5.8 & 5.7 & 4.5 & 4.4 & 6.6 & 5.7 & 5.6 \\
\hline 14 WPPS & 7.6 & 6.4 & 6.2 & 5.7 & 8.0 & 8.2 & 6.4 & 5.8 & 4.7 & 5.8 & 7.8 & 7.3 & 6.6 \\
\hline 15 FRNK & 7.7 & 6.9 & 6.7 & 5.8 & 8.0 & $6.9^{\circ}$ & 5.4 & 5.2 & 4.7 & 5.9 & 7.8 & 8.2 & 6.6 \\
\hline $16 \mathrm{GABL}$ & 10.9 & 9.4 & 10.2 & 9.9 & 11.5 & 13.3 & 10.5 & 10.5 & 8.5 & 9.5 & 12.4 & 12.0 & 10.7 \\
\hline 17 RING & 6.4 & 5.6 & 5.8 & 5.5 & 6.9 & 7.1 & 4.8 & 4.8 & 4.1 & 5.2 & 6.8 & 7.0 & 5.8 \\
\hline $18 \mathrm{RICH}$ & 7.3 & 6.0 & 5.9 & 5.2 & 7.7 & 7.1 & 5.5 & 4.6 & 3.9 & 5.3 & 7.5 & 7.3 & 6.1 \\
\hline 19 PFP & 4.7 & 4.0 & 4.3 & 4.4 & 4.9 & 6.0 & 4.8 & 4.4 & 3.7 & 3.8 & 5.6 & 4.6 & 4.6 \\
\hline 20 RMTN & 21.1 & 16.6 & 14.1 & 10.8 & 13.6 & 12.5 & 8.8 & 7.6 & 9.0 & 12.8 & 19.2 & 25.3 & 14.3 \\
\hline 21 HMS & 7.8 & 7.3 & 7.3 & 7.6 & 8.0 & 9.8 & 8.1 & 8.1 & 6.7 & 7.0 & 8.8 & 7.8 & 7.9 \\
\hline 22 PASC & 5.6 & 4.9 & 5.0 & 4.5 & 7.2 & 6.2 & 4.8 & 3.9 & 3.2 & 4.6 & 6.0 & 6.6 & 5.2 \\
\hline $23 \mathrm{GABW}$ & 5.8 & 5.7 & 5.7 & 5.9 & 6.8 & 8.5 & 6.6 & 6.6 & 5.3 & 5.2 & 6.5 & 5.9 & 6.2 \\
\hline $24 \mathrm{l} 100 \mathrm{~F}$ & 5.3 & 4.6 & 5.3 & 5.6 & 7.0 & 7.7 & 5.9 & 5.6 & 4.8 & 5.1 & 6.8 & 5.9 & 5.8 \\
\hline 25 VERN & 7.4 & 7.1 & 7.1 & 7.3 & 8.2 & 9.5 & 8.3 & 8.2 & 6.0 & 6.1 & 7.2 & 6.9 & 7.4 \\
\hline $26 \mathrm{BENT}$ & 6.8 & 6.5 & 6.5 & 5.9 & 6.6 & 6.8 & 5.5 & 5.4 & 5.3 & 5.9 & 7.1 & 7.7 & 6.3 \\
\hline 27 VSTA & 6.6 & 5.5 & 5.8 & 5.2 & 8.8 & 7.0 & 5.8 & 4.9 & 4.2 & 5.6 & 7.2 & 7.4 & 6.2 \\
\hline 28 SURF & 8.0 & 7.8 & 8.6 & 9.4 & 14.2 & 13.6 & 12.5 & 10.8 & 8.3 & 8.3 & 8.6 & $9.0^{\circ}$ & 9.9 \\
\hline $29100 \mathrm{~K}$ & 5.3 & 5.0 & 5.4 & 5.6 & 6.5 & 8.0 & 6.1 & 5.7 & 4.4 & 4.5 & 5.9 & 5.0 & 5.6 \\
\hline 30 HAMR & 7.5 & 6.8 & 6.6 & 5.8 & 8.0 & 7.7 & 6.1 & 5.6 & 4.7 & 6.3 & 7.9 & 7.7 & 6.7 \\
\hline
\end{tabular}




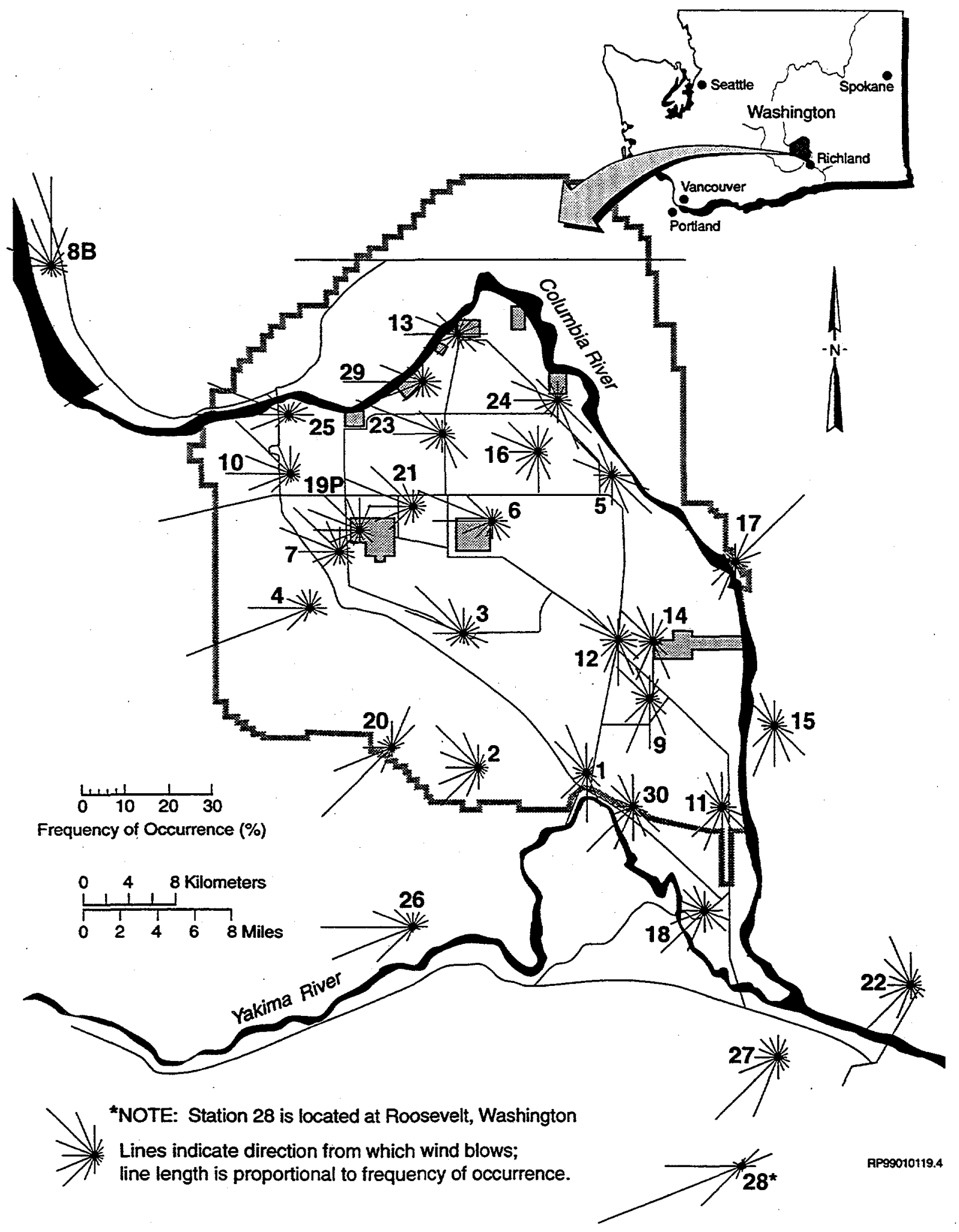

Figure 2.1. 1998 Hanford Meteorological Monitoring Network Wind Roses at 10-Meter Level (see Appendix for station-specific wind rose) 


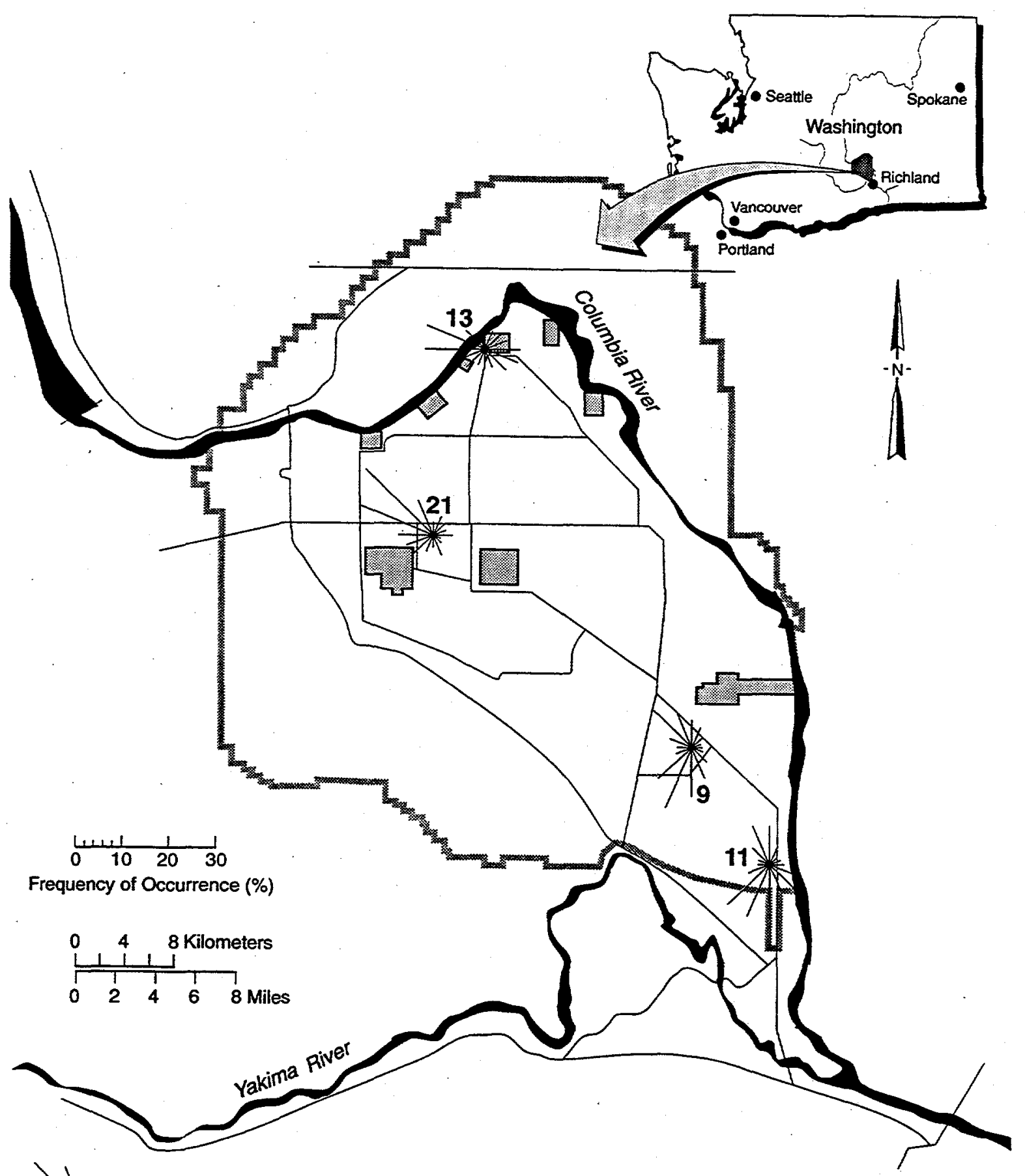

Lines indicate direction from which wind blows; line length is proportional to frequency of occurrence.

RP99010119.2

Figure 2.2. 1998 Hanford Meteorological Monitoring Network Wind Roses at 60-Meter Level (see Appendix for station-specific wind rose) 


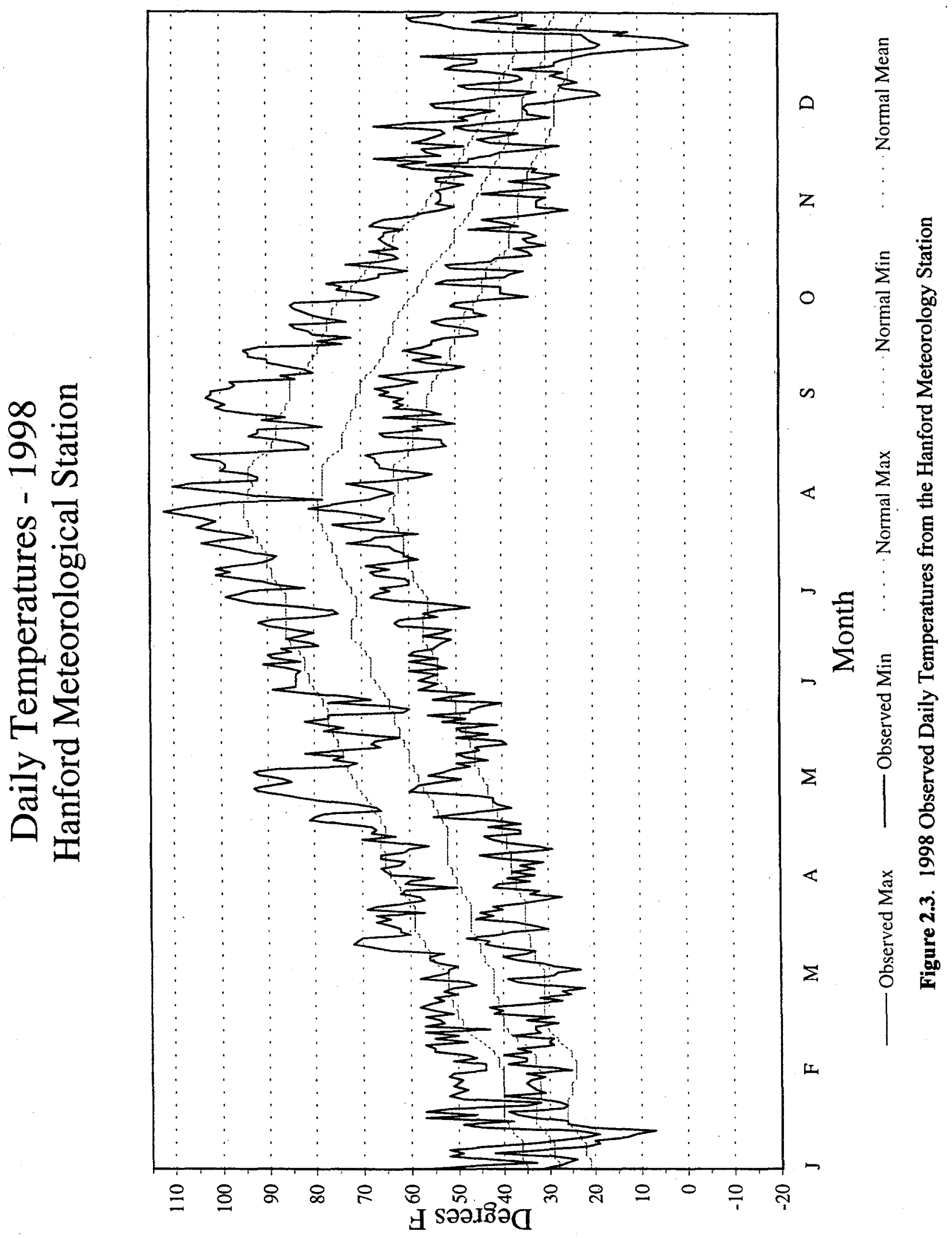




\subsection{Temperature Climatology}

\subsection{Monthly, Seasonal, and Annual Average}

Monthly, seasonal, and annual average temperatures, computed from observed daily maximum and minimum temperatures for the period 1945 through 1998, are presented in Tables 3.1 and 3.2. In these tables, the highest and lowest values, representing the warmest and coldest month, season, or year, are noted. Averages are based on the entire period of record, and climatological normal temperatures are based on the period 1961 through 1990.

As indicated in Table 3.1, much wider ranges and variabilities in temperatures are found during the late autumn and winter months (November through February) than during the rest of the year. The range of average monthly temperatures for January is from $12.1^{\circ} \mathrm{F}(1950)$ to $42.5^{\circ} \mathrm{F}(1953), 30.4^{\circ} \mathrm{F}$; for November $21.7^{\circ} \mathrm{F}$; February $18.9^{\circ} \mathrm{F}$; and December $17.5^{\circ} \mathrm{F}$; whereas for the rest of the year, the monthly range is from a low of $10.5^{\circ} \mathrm{F}$ in April to a high of $13.8^{\circ} \mathrm{F}$ in June. The coldest month recorded was January $1950\left(12.1^{\circ} \mathrm{F}\right)$; the hottest month recorded was July $1985\left(82.2^{\circ} \mathrm{F}\right)$. As shown in Table 3.2, the seasonal range is from $8.0^{\circ} \mathrm{F}$ during the summer (June, July, and August) to $16.4^{\circ} \mathrm{F}$ in winter (December, January, and February). The coldest season was the winter of $1948-1949\left(24.2^{\circ} \mathrm{F}\right)$; the hottest was the summer of $1958\left(78.2^{\circ} \mathrm{F}\right)$.

\subsection{Days with Maximum Temperatures $\geq 100^{\circ} \mathrm{F}, \geq 90^{\circ} \mathrm{F}$, and $\leq 32^{\circ} \mathrm{F}$}

Table 3.3 contains the number of days each year with maximum temperatures in the categories $\geq 100^{\circ} \mathrm{F}, \geq 90^{\circ} \mathrm{F}$, and $\leq 32^{\circ} \mathrm{F}$.

Maximum temperatures $\geq 100^{\circ} \mathrm{F}$ occurred as early as May $5(1966)$ and as late as September $6(1955)$. The annual number of days with maximum temperatures in this category ranged from 1 to 28 (1954 and 1958 , respectively). The greatest number of consecutive days with maximum temperatures $\geq 100^{\circ} \mathrm{F}$ is 11 and occurred 3 times: July 22 through August 1, 1962; August 10 through 20, 1967; and August 6 through $16,1981$.

One particularly notable period of above-normal temperatures occurred July 15 through August 13, 1971. This 30-day period included 27 days with maximum temperatures $\geq 100^{\circ} \mathrm{F}$ in 3 separate periods of 9 consecutive days each. The lowest maximum temperature during the 30 -day period was $98^{\circ} \mathrm{F}$; the highest was $112^{\circ} \mathrm{F}$. The average maximum temperature during this period was $104.7^{\circ} \mathrm{F}$.

Table 3.4 lists the dates of all occurrences of maximum temperatures $\geq 104^{\circ} \mathrm{F}$. 
Table 3.1. Monthly and Annual Average Temperatures $\left({ }^{\circ} \mathrm{F}\right)$

\begin{tabular}{|c|c|c|c|c|c|c|c|c|c|c|c|c|c|}
\hline Year & Jan & Feb & Mar & Apr & May & Jun & JuI & Aug & Sep & Oct & Nov & Dec & Annual \\
\hline 1945 & 33.9 & 38.6 & 42.1 & 50.3 & 61.7 & 67.5 & 78.0 & 77.5 & 64.6 & 56.4 & 40.6 & 32.7 & 53.7 \\
\hline $\begin{array}{l}1946 \\
1947 \\
1948 \\
1949 \\
1950\end{array}$ & $\begin{array}{l}34.4 \\
27.4 \\
32.0 \\
13.9 \\
12.1^{(2)}\end{array}$ & $\begin{array}{l}39.6 \\
40.0 \\
31.8 \\
31.8 \\
30.7\end{array}$ & $\begin{array}{l}45.5 \\
49.6 \\
42.1 \\
45.2 \\
42.3\end{array}$ & $\begin{array}{l}53.7 \\
56.1 \\
49.4 \\
55.5 \\
49.9\end{array}$ & $\begin{array}{l}64.2 \\
68.7^{(a)} \\
58.3 \\
67.0 \\
59.0\end{array}$ & $\begin{array}{l}66.9 \\
67.8 \\
72.4 \\
69.3 \\
66.5\end{array}$ & $\begin{array}{l}76.1 \\
75.3 \\
72.8 \\
74.9 \\
75.4\end{array}$ & $\begin{array}{l}76.6 \\
71.8 \\
71.8 \\
74.8 \\
76.4\end{array}$ & $\begin{array}{l}63.5 \\
65.4 \\
64.4 \\
68.3 \\
67.5\end{array}$ & $\begin{array}{l}49.5 \\
53.4 \\
51.0 \\
50.2 \\
51.1\end{array}$ & $\begin{array}{l}35.8 \\
41.2 \\
40.8 \\
45.2 \\
40.7\end{array}$ & $\begin{array}{l}34.8 \\
33.1 \\
26.9 \\
35.1 \\
36.2\end{array}$ & $\begin{array}{l}53.4 \\
54.2 \\
51.1 \\
52.6 \\
50.6\end{array}$ \\
\hline $\begin{array}{l}1951 \\
1952 \\
1953 \\
1954 \\
1955\end{array}$ & $\begin{array}{l}33.0 \\
25.2 \\
42.5^{(2)} \\
28.9 \\
30.0\end{array}$ & $\begin{array}{l}36.9 \\
36.7 \\
41.2 \\
39.3 \\
35.3\end{array}$ & $\begin{array}{l}40.1 \\
44.1 \\
46.2 \\
41.5 \\
39.4^{(a)}\end{array}$ & $\begin{array}{l}54.1 \\
55.2 \\
51.0 \\
51.4 \\
\mathbf{4 7 . 5}^{(2)}\end{array}$ & $\begin{array}{l}61.1 \\
62.7 \\
58.0 \\
62.9 \\
57.0\end{array}$ & $\begin{array}{l}69.4 \\
67.1 \\
63.0^{(2)} \\
65.5 \\
70.2\end{array}$ & $\begin{array}{l}76.7 \\
77.0 \\
75.8 \\
73.9 \\
73.0\end{array}$ & $\begin{array}{l}74.2 \\
74.0 \\
74.0 \\
71.4 \\
75.5\end{array}$ & $\begin{array}{l}66.8 \\
69.0 \\
67.8 \\
65.1 \\
66.4\end{array}$ & $\begin{array}{l}51.5 \\
59.0 \\
55.4 \\
51.4 \\
53.3\end{array}$ & $\begin{array}{l}39.5 \\
34.0 \\
43.4 \\
46.0 \\
31.3\end{array}$ & $\begin{array}{l}27.4 \\
34.8 \\
37.6 \\
34.0 \\
29.4\end{array}$ & $\begin{array}{l}52.6 \\
53.2 \\
54.7 \\
52.6 \\
50.7\end{array}$ \\
\hline $\begin{array}{l}1956 \\
1957 \\
1958 \\
1959 \\
1960\end{array}$ & $\begin{array}{l}31.8 \\
16.5 \\
37.1 \\
32.0 \\
23.3\end{array}$ & $\begin{array}{l}25.6^{(a)} \\
34.1 \\
44.5^{(a)} \\
35.5 \\
37.4\end{array}$ & $\begin{array}{l}43.8 \\
44.0 \\
43.5 \\
45.1 \\
45.1\end{array}$ & $\begin{array}{l}56.2 \\
55.2 \\
51.3 \\
54.2 \\
52.6\end{array}$ & $\begin{array}{l}65.3 \\
65.9 \\
68.1 \\
57.5 \\
58.5\end{array}$ & $\begin{array}{l}65.7 \\
70.8 \\
73.9 \\
68.6 \\
70.1\end{array}$ & $\begin{array}{l}78.9 \\
74.3 \\
81.2 \\
77.7 \\
81.8\end{array}$ & $\begin{array}{l}75.3 \\
72.9 \\
79.4 \\
71.8 \\
71.4\end{array}$ & $\begin{array}{l}67.3 \\
69.0 \\
65.6 \\
62.6 \\
67.7\end{array}$ & $\begin{array}{l}52.1 \\
50.7 \\
54.4 \\
53.4 \\
54.5\end{array}$ & $\begin{array}{l}36.6 \\
40.4 \\
40.6 \\
36.5 \\
41.2\end{array}$ & $\begin{array}{l}34.6 \\
38.5^{(a)} \\
35.2 \\
33.1 \\
29.0\end{array}$ & $\begin{array}{l}52.8 \\
52.7 \\
56.2 \\
52.3 \\
52.7\end{array}$ \\
\hline $\begin{array}{l}1961 \\
1962 \\
1963 \\
1964 \\
1965\end{array}$ & $\begin{array}{l}35.0 \\
29.8 \\
25.4 \\
35.6 \\
32.3\end{array}$ & $\begin{array}{l}43.7 \\
36.6 \\
38.3 \\
38.1 \\
40.5\end{array}$ & $\begin{array}{l}46.1 \\
42.6 \\
46.4 \\
43.8 \\
42.9\end{array}$ & $\begin{array}{l}52.3 \\
55.6 \\
49.8 \\
50.2 \\
54.8\end{array}$ & $\begin{array}{l}60.0 \\
56.9 \\
61.7 \\
59.7 \\
60.5\end{array}$ & $\begin{array}{l}74.0 \\
68.3 \\
69.4 \\
67.7 \\
69.3\end{array}$ & $\begin{array}{l}79.4 \\
76.0 \\
72.4 \\
74.5 \\
76.5\end{array}$ & $\begin{array}{l}80.2 \\
71.9 \\
75.7 \\
69.8^{(2)} \\
74.7\end{array}$ & $\begin{array}{l}63.8 \\
67.1 \\
71.1 \\
63.0 \\
62.4\end{array}$ & $\begin{array}{l}51.6 \\
52.6 \\
56.0 \\
53.3 \\
57.1\end{array}$ & $\begin{array}{l}35.3 \\
43.2 \\
42.8 \\
38.2 \\
43.1\end{array}$ & $\begin{array}{l}33.7 \\
36.8 \\
30.2 \\
25.5 \\
33.0\end{array}$ & $\begin{array}{l}54.6 \\
53.1 \\
53.3 \\
51.6 \\
53.9\end{array}$ \\
\hline $\begin{array}{l}1966 \\
1967 \\
1968 \\
1969 \\
1970\end{array}$ & $\begin{array}{l}34.0 \\
39.8 \\
35.7 \\
19.8 \\
30.7\end{array}$ & $\begin{array}{l}39.9 \\
43.7 \\
41.8 \\
31.7 \\
40.6\end{array}$ & $\begin{array}{l}45.4 \\
44.3 \\
49.0 \\
45.8 \\
45.0\end{array}$ & $\begin{array}{l}54.6 \\
47.6 \\
51.3 \\
52.2 \\
49.0\end{array}$ & $\begin{array}{l}63.2 \\
60.5 \\
62.4 \\
64.6 \\
61.5\end{array}$ & $\begin{array}{l}66.9 \\
72.5 \\
69.8 \\
75.1 \\
73.6\end{array}$ & $\begin{array}{l}73.3 \\
78.6 \\
79.7 \\
76.0 \\
78.6\end{array}$ & $\begin{array}{l}75.6 \\
81.5^{(2)} \\
71.5 \\
72.8 \\
76.3\end{array}$ & $\begin{array}{l}68.8 \\
71.8 \\
66.8 \\
67.4 \\
61.8\end{array}$ & $\begin{array}{l}53.4 \\
55.1 \\
50.3 \\
51.0 \\
50.9\end{array}$ & $\begin{array}{l}43.7 \\
41.5 \\
41.7 \\
40.2 \\
39.7\end{array}$ & $\begin{array}{l}38.2 \\
33.1 \\
30.6 \\
34.6 \\
30.8\end{array}$ & $\begin{array}{l}54.8 \\
55.8 \\
54.2 \\
52.6 \\
53.2\end{array}$ \\
\hline $\begin{array}{l}1971 \\
1972 \\
1973 \\
1974 \\
1975\end{array}$ & $\begin{array}{l}35.8 \\
30.5 \\
29.1 \\
29.4 \\
32.5\end{array}$ & $\begin{array}{l}39.1 \\
34.8 \\
38.5 \\
40.9 \\
33.7\end{array}$ & $\begin{array}{l}40.7 \\
47.0 \\
47.4 \\
45.2 \\
42.5\end{array}$ & $\begin{array}{l}52.0 \\
49.6 \\
53.6 \\
52.9 \\
48.2\end{array}$ & $\begin{array}{l}64.0 \\
64.3 \\
63.1 \\
57.9 \\
60.2\end{array}$ & $\begin{array}{l}65.3 \\
69.7 \\
68.7 \\
72.6 \\
67.2\end{array}$ & $\begin{array}{l}78.7 \\
76.2 \\
78.2 \\
74.5 \\
79.5\end{array}$ & $\begin{array}{l}80.5 \\
77.6 \\
73.9 \\
75.5 \\
71.0\end{array}$ & $\begin{array}{l}61.5 \\
61.4 \\
65.7 \\
68.0 \\
68.0\end{array}$ & $\begin{array}{l}51.7 \\
52.3 \\
52.4 \\
52.5 \\
52.5\end{array}$ & $\begin{array}{l}40.4 \\
39.9 \\
38.4 \\
41.6 \\
39.5\end{array}$ & $\begin{array}{l}30.6 \\
27.3 \\
38.1 \\
36.2 \\
34.5\end{array}$ & $\begin{array}{l}53.4 \\
52.6 \\
53.9 \\
53.9 \\
52.4\end{array}$ \\
\hline $\begin{array}{l}1976 \\
1977 \\
1978 \\
1979 \\
1980\end{array}$ & $\begin{array}{l}32.0 \\
25.2 \\
32.5 \\
13.9 \\
23.7\end{array}$ & $\begin{array}{l}37.6 \\
40.5 \\
37.9 \\
34.2 \\
34.6\end{array}$ & $\begin{array}{l}41.4 \\
45.4 \\
47.5 \\
46.5 \\
44.5\end{array}$ & $\begin{array}{l}50.8 \\
57.3 \\
51.9 \\
52.8 \\
55.2\end{array}$ & $\begin{array}{l}60.5 \\
56.9 \\
58.6 \\
64.1 \\
61.4\end{array}$ & $\begin{array}{l}65.6 \\
72.6 \\
70.3 \\
70.8 \\
64.7\end{array}$ & $\begin{array}{l}75.1 \\
73.7 \\
75.7 \\
77.2 \\
74.7\end{array}$ & $\begin{array}{l}70.8 \\
79.2 \\
72.7 \\
74.6 \\
71.2\end{array}$ & $\begin{array}{l}69.0 \\
61.5 \\
63.8 \\
69.2 \\
66.0\end{array}$ & $\begin{array}{l}52.4 \\
52.0 \\
52.2 \\
56.5 \\
52.6\end{array}$ & $\begin{array}{l}40.6 \\
38.9 \\
32.3 \\
34.2 \\
41.0\end{array}$ & $\begin{array}{l}30.7 \\
33.8 \\
27.5 \\
36.4 \\
36.6\end{array}$ & $\begin{array}{l}52.2 \\
53.1 \\
51.9 \\
52.5 \\
52.2\end{array}$ \\
\hline $\begin{array}{l}1981 \\
1982 \\
1983 \\
1984 \\
1985\end{array}$ & $\begin{array}{l}38.0 \\
29.8 \\
37.5 \\
31.6 \\
25.0\end{array}$ & $\begin{array}{l}39.7 \\
38.1 \\
40.9 \\
38.7 \\
29.9\end{array}$ & $\begin{array}{l}48.7 \\
45.9 \\
48.5 \\
47.2 \\
44.0\end{array}$ & $\begin{array}{l}54.0 \\
49.4 \\
51.1 \\
50.5 \\
55.5\end{array}$ & $\begin{array}{l}60.5 \\
60.4 \\
63.8 \\
56.0^{(a)} \\
63.2\end{array}$ & $\begin{array}{l}66.0 \\
73.1 \\
65.4 \\
65.7 \\
70.2\end{array}$ & $\begin{array}{l}73.9 \\
74.9 \\
71.3 \\
76.1 \\
\mathbf{8 2 . 2 ^ { ( \mathbf { a } ) }}\end{array}$ & $\begin{array}{l}79.0 \\
75.8 \\
74.4 \\
74.0 \\
70.5\end{array}$ & $\begin{array}{l}66.3 \\
65.4 \\
61.7 \\
62.1 \\
\mathbf{5 8 . 8 ^ { ( a ) }}\end{array}$ & $\begin{array}{l}52.0 \\
51.4 \\
52.6 \\
47.9^{(2)} \\
49.8\end{array}$ & $\begin{array}{l}42.7 \\
36.9 \\
43.6 \\
39.4 \\
\mathbf{2 4 . 8}\end{array}$ & $\begin{array}{l}32.8 \\
32.0 \\
21.2 \\
23.6 \\
21.0^{(a)}\end{array}$ & $\begin{array}{l}54.5 \\
52.8 \\
52.7 \\
51.1 \\
49.6^{(a)}\end{array}$ \\
\hline $\begin{array}{l}1986 \\
1987 \\
1988 \\
1989 \\
1990\end{array}$ & $\begin{array}{l}34.0 \\
30.7 \\
31.9 \\
37.2 \\
40.4\end{array}$ & $\begin{array}{l}39.1 \\
40.1 \\
41.0 \\
27.3 \\
37.6\end{array}$ & $\begin{array}{l}48.6 \\
48.3 \\
45.9 \\
43.8 \\
48.0\end{array}$ & $\begin{array}{l}50.9 \\
58.0 \\
55.2 \\
56.6 \\
57.9\end{array}$ & $\begin{array}{l}62.3 \\
66.2 \\
61.1 \\
61.5 \\
60.7\end{array}$ & $\begin{array}{l}73.0 \\
73.4 \\
69.2 \\
72.0 \\
70.1\end{array}$ & $\begin{array}{l}70.6 \\
74.3 \\
77.3 \\
75.5 \\
80.8\end{array}$ & $\begin{array}{l}79.2 \\
76.6 \\
75.2 \\
73.4 \\
76.8\end{array}$ & $\begin{array}{l}62.2 \\
69.9 \\
65.6 \\
67.4 \\
\mathbf{7 2 . 4} 4^{(2)}\end{array}$ & $\begin{array}{l}54.7 \\
55.5 \\
59.6^{(a)} \\
54.0 \\
52.3\end{array}$ & $\begin{array}{l}42.3 \\
43.6 \\
44.2 \\
44.3 \\
46.5^{(a)}\end{array}$ & $\begin{array}{l}32.4 \\
31.5 \\
31.8 \\
33.3 \\
24.1\end{array}$ & $\begin{array}{l}54.1 \\
55.7 \\
54.8 \\
53.9 \\
55.6\end{array}$ \\
\hline $\begin{array}{l}1991 \\
1992 \\
1993 \\
1994 \\
1995\end{array}$ & $\begin{array}{l}28.7 \\
37.5 \\
24.8 \\
38.6 \\
34.2\end{array}$ & $\begin{array}{l}44.5 \\
42.6 \\
30.8 \\
36.0 \\
43.1\end{array}$ & $\begin{array}{l}44.1 \\
51.5^{(a)} \\
43.2 \\
49.2 \\
46.1\end{array}$ & 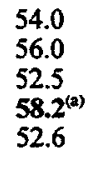 & $\begin{array}{l}60.4 \\
67.2 \\
66.5 \\
64.9 \\
64.5\end{array}$ & $\begin{array}{l}65.6 \\
76.8^{(2)} \\
68.4 \\
69.8 \\
68.1\end{array}$ & $\begin{array}{l}78.0 \\
76.6 \\
70.5^{(a)} \\
81.0 \\
77.1\end{array}$ & $\begin{array}{l}78.9 \\
76.9 \\
73.1 \\
76.6 \\
72.0\end{array}$ & $\begin{array}{l}69.7 \\
64.5 \\
66.4 \\
70.5 \\
69.9\end{array}$ & $\begin{array}{l}52.9 \\
55.7 \\
55.4 \\
54.4 \\
52.1\end{array}$ & $\begin{array}{l}41.3 \\
41.2 \\
34.6 \\
39.6 \\
44.1\end{array}$ & $\begin{array}{l}37.8 \\
30.0 \\
35.4 \\
35.1 \\
32.6\end{array}$ & $\begin{array}{l}54.7 \\
56.4^{(n)} \\
51.8 \\
56.2 \\
54.7\end{array}$ \\
\hline $\begin{array}{l}1996 \\
1997 \\
1998\end{array}$ & $\begin{array}{l}28.8 \\
33.6 \\
36.2\end{array}$ & $\begin{array}{l}32.8 \\
40.2 \\
42.2\end{array}$ & $\begin{array}{l}44.8 \\
47.4 \\
48.4\end{array}$ & $\begin{array}{l}55.0 \\
51.8 \\
54.4\end{array}$ & $\begin{array}{l}58.1 \\
65.0 \\
62.4\end{array}$ & $\begin{array}{l}69.0 \\
68.5 \\
71.0\end{array}$ & $\begin{array}{l}79.5 \\
75.3 \\
82.0\end{array}$ & $\begin{array}{l}75.6 \\
78.0 \\
77.9\end{array}$ & $\begin{array}{l}64.4 \\
66.8 \\
71.0\end{array}$ & $\begin{array}{l}52.4 \\
53.2 \\
52.4\end{array}$ & $\begin{array}{l}38.4 \\
43.2 \\
45.6\end{array}$ & $\begin{array}{l}29.8 \\
34.7 \\
33.0\end{array}$ & $\begin{array}{l}52.4 \\
54.8 \\
56.4^{(x)}\end{array}$ \\
\hline $\begin{array}{l}\text { Average } \\
\text { Normal }\end{array}$ & $\begin{array}{l}30.6 \\
31.3\end{array}$ & $\begin{array}{l}37.6 \\
38.0\end{array}$ & $\begin{array}{l}45.2 \\
45.6\end{array}$ & $\begin{array}{l}52.9 \\
52.7\end{array}$ & $\begin{array}{l}61.9 \\
61.3\end{array}$ & $\begin{array}{l}69.3 \\
69.7\end{array}$ & $\begin{array}{l}76.5 \\
76.2\end{array}$ & $\begin{array}{l}75.0 \\
75.1\end{array}$ & $\begin{array}{l}66.2 \\
65.7\end{array}$ & $\begin{array}{l}53.0 \\
52.9\end{array}$ & $\begin{array}{l}40.1 \\
40.2\end{array}$ & $\begin{array}{l}32.3 \\
31.4\end{array}$ & $\begin{array}{l}53.4 \\
53.3\end{array}$ \\
\hline
\end{tabular}

(a) Highest and lowest averages. 
Table 3.2. Seasonal Average Temperatures ( $\left.{ }^{\circ} \mathrm{F}\right)$

\begin{tabular}{|c|c|c|c|c|}
\hline Year & $\begin{array}{c}\text { Winter } \\
\text { Dec-Feb }\end{array}$ & $\begin{array}{c}\text { Spring } \\
\text { Mar-May } \\
\end{array}$ & $\begin{array}{l}\text { Summer } \\
\text { Jun-Aug }\end{array}$ & $\begin{array}{l}\text { Autumn } \\
\text { Sep-Nov } \\
\end{array}$ \\
\hline 1945 & - & 51.4 & 74.3 & 53.9 \\
\hline $\begin{array}{l}1946 \\
1947 \\
1948 \\
1949 \\
1950\end{array}$ & $\begin{array}{l}35.6 \\
34.1 \\
32.3 \\
24.2^{(\text {a) }} \\
26.0\end{array}$ & $\begin{array}{l}54.5 \\
58.1 \\
49.9 \\
55.9 \\
50.4\end{array}$ & $\begin{array}{l}73.2 \\
71.6 \\
72.3 \\
73.0 \\
72.8\end{array}$ & $\begin{array}{l}49.6 \\
53.3 \\
52.1 \\
54.6 \\
53.1\end{array}$ \\
\hline $\begin{array}{l}1951 \\
1952 \\
1953 \\
1954 \\
1955\end{array}$ & $\begin{array}{l}35.4 \\
29.8 \\
39.5 \\
35.3 \\
33.1\end{array}$ & $\begin{array}{l}51.8 \\
54.0 \\
51.7 \\
51.9 \\
\mathbf{4 8 . 0 ^ { ( a ) }}\end{array}$ & $\begin{array}{l}73.4 \\
72.7 \\
70.9 \\
70.3 \\
72.9\end{array}$ & $\begin{array}{l}52.6 \\
54.0 \\
55.5 \\
54.2 \\
50.3\end{array}$ \\
\hline $\begin{array}{l}1956 \\
1957 \\
1958 \\
1959 \\
1960\end{array}$ & $\begin{array}{l}28.9 \\
28.4 \\
40.0 \\
34.2 \\
31.3\end{array}$ & $\begin{array}{l}55.1 \\
55.0 \\
54.3 \\
52.3 \\
52.1\end{array}$ & 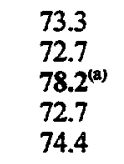 & $\begin{array}{l}52.0 \\
53.4 \\
53.5 \\
50.8 \\
54.5\end{array}$ \\
\hline $\begin{array}{l}1961 \\
1962 \\
1963 \\
1964 \\
1965\end{array}$ & $\begin{array}{l}35.9 \\
33.4 \\
33.5 \\
34.6 \\
32.8\end{array}$ & $\begin{array}{l}52.8 \\
51.7 \\
52.6 \\
51.2 \\
52.7\end{array}$ & $\begin{array}{l}77.9 \\
72.1 \\
72.5 \\
70.7 \\
73.5\end{array}$ & $\begin{array}{l}50.2 \\
54.3 \\
56.6 \\
51.5 \\
54.2\end{array}$ \\
\hline $\begin{array}{l}1966 \\
1967 \\
1968 \\
1969 \\
1970\end{array}$ & $\begin{array}{l}35.6 \\
40.6^{(2)} \\
36.9 \\
27.4 \\
35.3\end{array}$ & $\begin{array}{l}54.4 \\
50.8 \\
54.2 \\
54.2 \\
51.8\end{array}$ & $\begin{array}{l}71.9 \\
77.5 \\
73.7 \\
74.6 \\
76.2\end{array}$ & $\begin{array}{l}55.3 \\
56.1 \\
52.9 \\
52.9 \\
50.8\end{array}$ \\
\hline $\begin{array}{l}1971 \\
1972 \\
1973 \\
1974 \\
1975\end{array}$ & $\begin{array}{l}35.2 \\
32.0 \\
31.6 \\
36.1 \\
34.1\end{array}$ & $\begin{array}{l}52.2 \\
53.6 \\
54.7 \\
52.0 \\
50.3\end{array}$ & $\begin{array}{l}74.8 \\
74.5 \\
73.6 \\
74.2 \\
72.6\end{array}$ & $\begin{array}{l}51.2 \\
51.2 \\
52.2 \\
54.0 \\
53.3\end{array}$ \\
\hline $\begin{array}{l}1976 \\
1977 \\
1978 \\
1979 \\
1980\end{array}$ & $\begin{array}{l}34.7 \\
32.1 \\
34.7 \\
25.2 \\
31.6\end{array}$ & $\begin{array}{l}50.9 \\
53.2 \\
52.7 \\
54.5 \\
53.7\end{array}$ & $\begin{array}{l}\mathbf{7 0 . 5} \\
75.2 \\
72.9 \\
74.2 \\
\mathbf{7 0 . 2}^{(2)}\end{array}$ & $\begin{array}{l}54.0 \\
50.8 \\
49.4 \\
53.3 \\
53.2\end{array}$ \\
\hline $\begin{array}{l}1981 \\
1982 \\
1983 \\
1984 \\
1985\end{array}$ & $\begin{array}{l}38.1 \\
33.6 \\
36.8 \\
30.5 \\
26.2\end{array}$ & $\begin{array}{l}54.4 \\
51.9 \\
54.5 \\
51.2 \\
54.2\end{array}$ & $\begin{array}{l}73.0 \\
74.6 \\
70.4 \\
71.9 \\
74.3 \\
\end{array}$ & $\begin{array}{l}53.7 \\
51.2 \\
52.6 \\
49.8 \\
44.5^{(2)}\end{array}$ \\
\hline $\begin{array}{l}1986 \\
1987 \\
1988 \\
1989 \\
1990\end{array}$ & $\begin{array}{l}31.4 \\
34.4 \\
34.8 \\
32.1 \\
37.1\end{array}$ & $\begin{array}{l}53.9 \\
57.5 \\
54.1 \\
54.0 \\
55.5\end{array}$ & $\begin{array}{l}74.3 \\
74.8 \\
73.9 \\
73.6 \\
75.9\end{array}$ & $\begin{array}{l}\mathbf{5 3 . 1} \\
\mathbf{5 6 . 3} \\
\mathbf{5 6 . 5} \\
\mathbf{5 5 . 2} \\
\mathbf{5 7 . 1} \\
\mathbf{5 a )}\end{array}$ \\
\hline $\begin{array}{l}1991 \\
1992 \\
1993 \\
1994 \\
1995\end{array}$ & $\begin{array}{l}32.4 \\
39.3 \\
28.5 \\
36.7 \\
37.5\end{array}$ & $\begin{array}{l}52.8 \\
58.2^{(a)} \\
54.1 \\
57.4 \\
54.4\end{array}$ & $\begin{array}{l}74.2 \\
76.8 \\
70.7 \\
75.8 \\
72.4\end{array}$ & $\begin{array}{l}54.6 \\
53.8 \\
52.1 \\
54.8 \\
55.4\end{array}$ \\
\hline $\begin{array}{l}1996 \\
1997 \\
1998\end{array}$ & $\begin{array}{r}31.4 \\
34.5 \\
37.7\end{array}$ & $\begin{array}{l}52.6 \\
54.7 \\
55.1\end{array}$ & $\begin{array}{l}74.7 \\
73.9 \\
77.0\end{array}$ & $\begin{array}{l}51.7 \\
54.4 \\
56.3\end{array}$ \\
\hline $\begin{array}{l}\text { Average } \\
\text { Normal }\end{array}$ & $\begin{array}{l}33.5 \\
33.6\end{array}$ & $\begin{array}{l}53.4 \\
53.2\end{array}$ & $\begin{array}{l}73.6 \\
73.7\end{array}$ & $\begin{array}{l}53.1 \\
52.9\end{array}$ \\
\hline
\end{tabular}

(a) Highest and lowest averages. 
Table 3.3. Monthly and Seasonal Number of Days with Maximum Temperatures $\left({ }^{\circ} \mathrm{F}\right)$ Above or Below Certain Thresholds

\begin{tabular}{|c|c|c|c|c|c|c|c|c|c|c|c|c|c|c|c|c|c|c|c|c|c|c|}
\hline \multirow[b]{2}{*}{ Year } & \multicolumn{6}{|c|}{$100^{\circ} \mathrm{F}$ or Above } & \multicolumn{8}{|c|}{$90^{\circ} \mathrm{F}$ or Above } & \multicolumn{8}{|c|}{$32^{\circ} \mathrm{F}$ or Below } \\
\hline & May & Jun & Jul & Aug & Sep & Total & Apr & May & Jun & Jul & Aug & Sep & Oct & Total & Season & Oct & Nov & Dec & Jan & Feb & Mar & Total \\
\hline 1945 & 0 & 0 & 8 & 4 & 0 & 12 & $\mathbf{0}$ & 1 & 7 & 21 & 21 & 5 & 0 & 55 & $1944-45$ & - & -- & - & 12 & 1 & 1 & 14 \\
\hline 1946 & 0 & 0 & 7 & 6 & 0 & 13 & 1 & 0 & 4 & 15 & 18 & 0 & 0 & 38 & $1945-46$ & 0 & 2 & 9 & 0 & 0 & 0 & 11 \\
\hline 1947 & 1 & 0 & 2 & 0 & 0 & 3 & 0 & 8 & 4 & 17 & 11 & 2 & 0 & 42 & $1946-47$ & 0 & 4 & 4 & 14 & 0 & 0 & 22 \\
\hline 1948 & 0 & 2 & 0 & 0 & 0 & 2 & 0 & 1 & 9 & 14. & 7 & 7 & 0 & 38 & $1947-48$ & 0 & 0 & 6 & 8 & 9 & 0 & 23 \\
\hline 1949 & 0 & 1 & 6 . & 2 & 1 & 10 & 0 & 8 & 8 & 15 & 18 & 8 & 0 & 57 & $1948-49$ & 0 & 0 & 13 & 28 & 8 & 0 & 49 \\
\hline 1950 & 0 & 0 & 2 & 3 & 2 & 7 & 0 & 1 & 5 & 20 & 22 & 8 & 0 & 56 & $1949-50$ & 0 & 0 & 5 & 24 & 5 & 1 & 35 . \\
\hline 1951 & 0 & 0 & 8 & 3 & 0 & 11 & 0 & 1 & 8 & 23 & 19 & 5 & 0 & 56 & $1950-51$ & 0 & 0 & 2 & 8 & 2 & 0 & 12 \\
\hline 1952 & 0 & 0 & 9 & 4 & 0 & 13 & 0 & 2 & 5 & 21 & 17 & 12 & 0 & 57 & $1951-52$ & 0 & 0 & 16 & 19 & 0 & 0 & 35 \\
\hline 1953 & 0 & 0 & 4 & 4 & 0 & 8 & 0 & 0 & 0 & 21 & 13 & 11 & 0 & 45 & $1952-53$ & 0 & 9 & 6 & 1 & 0 & 0 & 16 \\
\hline 1954 & 0 & 0 & 1 & 0 & 0 & $\mathbf{1}^{\text {(a) }}$ & 0 & 2 & 3 & 20 & 9 & 3 & 0 & 37 & $1953-54$ & 0 & 0 & 2 & 12 & 4 & 0 & 18 \\
\hline 1955 & 0 & 2 & 5 & 2 & 2 & 11 & 0 & 0 & 9 & 12 & 19 & 8 & 0 & 48 & $1954-55$ & 0 & 0 & 5 & 13 & 2 & 1 & 21 \\
\hline 1956 & 0 . & 0 & 10 & 5 & 0 & 15 & 0 & 7 & 2 & 22 & 16 & 7 & 0 & 54 & $1955-56$ & 0 & 15 & 16 & 7 & 15 & 0 & 53 \\
\hline 1957 & $0^{\circ}$ & 1 & 1 & 0 & 0 & 2 & 0 & 3 & 8 & 14 & 8 & 6 & 0 & 39 & $1956-57$ & 0 & 7 & 10 & 22 & 7 & 0 & 46 \\
\hline 1958 & 1 & 6 & 10 & 11 & 0 & $28^{(n)}$ & 0 & 8 & 11 & 28 & 25 & 5 & 0 & 77 & $1957-58$ & 0 & 0 & 2 & 2 & 0 & 0 & 4 \\
\hline 1959 & 0 & 0 & 8 & 1 & 0 & 9 & 0 & 1 & 7 & 21 & 12 & 3 & 0 & 44 & $1958-59$ & 0 & 3 & 5 & 8 & 2 & 0 & .18 \\
\hline 1960 & 0 & 0 & 16 & 5 & 0 & 21 & 0 & 1 & 12 & 28 & 12 & 5 & 0 & 58 & $1959-60$ & 0 & 5 & 7 & 23 & 1 & 2 & 38 \\
\hline 1961 & 0 & 7 & 8 & 10 & 0 & 25 & 0 & 1 & 15 & 26 & 24 & 1 & 0 & 67 & $1960-61$ & 0 & 0 & 14 & 10 & 0 & 0 & 24 \\
\hline 1962 & 0 & 0 & 10 & 1 & 0 & 11 & 0 & 0 & 11 & 17 & 10 & 8 & 0 & 46 & $1961-62$ & 0 & 0 & 7 & 12 & 2 & 0 & 21 \\
\hline 1963 & 0 & 3 & 0 & 3 & 0 & 6 & 0 & 4 & 7 & 8 & 18 & 11 & 0 & 48 & $1962-63$ & 0 & 0 & 3 & 14 & 3 & 0 & 20 \\
\hline 1964 & 0 & 0 & 6 & 0 & 0 & 6 & 0 & 0 & 5 & 14 & 10 & 2 & 0 & 31 & $1963-64$ & 0 & 1 & 11 & 3 & 0 & 0 & 15 \\
\hline 1965 & 0 & 0 & 6 & 5 & 0 & 11 & 0 & 1 & 7 & 20 & 12 & 1 & 0 & 41 & $1964-65$ & 0 & 0 & 14 & 5 & 0 & 0 & 19 \\
\hline 1966 & 1 & 0 & 2 & 4 & 0 & 7 & 0 & 5 & 2 & 15 & 21 & 7 & 0 & 50 & $1965-66$ & 0 & 1 & 8 & 3 & 0 & 0 & 12 \\
\hline 1967 & 0 & 2 & 6 & 15 & 0 & 23 & 0 & 2 & 13 & 25 & 27 & 12 & 0 & $79^{(a)}$ & $1966-67$ & 0 & 0 & 2 & 0 & 0 & 0 & $2^{(a)}$ \\
\hline 1968 & 0 & 0 & 10 & 3 & 0 & 13 & 1 & 1 & 5 & 22 & 12 & 4 & 0 & 45 & $1967-68$ & 0 & 0 & 10 & 4 & 0 & 0 & 14 \\
\hline 1969 & 0 & 3 & 4 & 2 & 0 & 9 & 0 & 6 & 17 & 20 & 15 & 7 & 0 & 65 & $1968-69$ & 0 & 0 & 7 & 20 & 4 & 0 & 31 \\
\hline 1970 & 0 & 9 & 11 & 5 & 0 & 25 & 0 & 2 & 15 & 22 & 19 & 0 & 0 & 58 & $1969-70$ & 0 & 3 & 9 & 15 & 0 & 0 & 27 \\
\hline 1971 & 0 & 0 & 16 & 11 & $\mathbf{0}$ & 27 & 0 & 2 & 2 & 20 & 26 & 2 & 0 & 52 & $1970-71$ & 0 & 3 & 11 & 9 & 1 & 0 & 24 \\
\hline 1972 & 0 & 0 & 5 & 10 & 0 & 15 & 0 & 5 & 8 & 21 & 19 & 5 & 0 & 58 & $1971-72$ & 1 & 0 & 10 & 9 & 7 & 0 & 27 \\
\hline 1973 & 0 & 2 & 10 & 5 & 0 & 17 & 0 & 6 & 7 & 21 & 18 & 4 & 0 & 56 & $1972-73$ & 0 & 0 & 14 & 10 & 0 & 0 & 24 \\
\hline 1974 & 0 & 6 & 5 & 3 & 0 & 14 & 0 & 0 & 18 & 16 & 18 & 6 & 0 & 58 & $1973-74$ & 0 & 4 & 1 & 12 & 0 & 0 & 17 \\
\hline 1975 & 0 & 0 & 9 & 0 & 0 & 9 & 0 & 2 & 4 & 22 & 12 & 8 & 0 & 48 & $1974-75$ & 0 & 0 & 0 & 6 & 6 & 0 & 12 \\
\hline
\end{tabular}


Table 3.3. (contd)

\begin{tabular}{|c|c|c|c|c|c|c|}
\hline \multirow[b]{2}{*}{ Year } & \multicolumn{6}{|c|}{$100^{\circ} \mathrm{F}$ or Above } \\
\hline & May & Jun & Jul & Aug & Sep & Total \\
\hline 1976 & 0 & 1 & 2 & 0 & 1 & 4 \\
\hline 1977 & 0 & 1 & 2 & 13 & 0 & 16 \\
\hline 1978 & 0 & 1 & 6 & 6 & 0 & 13 \\
\hline 1979 & 0 & 2 & 7 & 1 & 0 & 10 \\
\hline 1980 & 0 & 0 & 3 & 0 & 0 & 3 \\
\hline 1981 & 0 & 0 & 3 & 13 & 0 & 16 \\
\hline 1982 & 0 & 2 & 5 & 3 & 0 & 10 \\
\hline 1983 & 1 & 0 & 1 & 0 & 0 & 2 \\
\hline 1984 & 0 & 0 & 3 & 3 & 0 & 6 \\
\hline 1985 & 0 & 1 & 15 & 0 & 0 & 16 \\
\hline 1986 & 3 & 1 & 0 & 6 & 0 & 10 \\
\hline 1987 & 1 & 5 & 3 & 4 & 1 & 14 \\
\hline 1988 & 0 & 0 & 8 & 3 & 3 & 14 \\
\hline 1989 & 0 & 0 & 2 & 2 & 0 & 4 \\
\hline 1990 & 0 & 0 & 11 & 9 & 0 & 20 \\
\hline 1991 & 0 & 0 & 4 & 8 & 0 & 12 \\
\hline 1992 & 0 & 7 & 5 & 9 & 0 & 21 \\
\hline 1993 & 1 & 0 & 0 & 2 & 0 & 3 \\
\hline 1994 & 0 & 1 & 13 & 7 & 0 & 21 \\
\hline 1995 & 0 & 0 & 5 & 3 & 1 & 9 \\
\hline 1996 & 0 & 0 & 13 & 6 & 0 & 19 \\
\hline 1997 & 0 & 0 & 3 & 7 & 0 & 10 \\
\hline 1998 & 0 & 0 & 14 & 9 & 3 & 26 \\
\hline 1999 & -- & - & - & -. & - & - \\
\hline Average & $<1$ & 1 & 6 & 4 & $<1$ & 12 \\
\hline Normal & $<1$ & 2 & 6 & 5 & $<1$ & 13 \\
\hline
\end{tabular}

\begin{tabular}{rrrrrrrr}
\multicolumn{8}{c}{$90^{\circ}$ F or Above } \\
\hline Apr & May & Jun & Jul & Aug & Sep & Oct & Total \\
\hline 0 & 1 & 4 & 17 & 9 & 4 & 0 & 35 \\
1 & 0 & 13 & 16 & 22 & 0 & 0 & 52 \\
0 & 0 & 12 & 17 & 11 & 2 & 0 & 42 \\
0 & 1 & 13 & 23 & 20 & 7 & 0 & 64 \\
0 & 0 & 0 & 18 & 9 & 2 & 0 & $29^{(a)}$ \\
1 & 0 & 4 & 19 & 22 & 11 & 0 & 57 \\
0 & 0 & 15 & 16 & 17 & 5 & 0 & 53 \\
0 & 8 & 2 & 9 & 13 & 0 & 0 & 32 \\
0 & 1 & 4 & 21 & 16 & 4 & 0 & 46 \\
0 & 3 & 10 & 30 & 7 & 0 & 0 & 50 \\
0 & 6 & 11 & 9 & 27 & 3 & 0 & 56 \\
2 & 6 & 15 & 14 & 19 & 12 & 0 & 68 \\
0 & 4 & 11 & 19 & 20 & 7 & 0 & 61 \\
0 & 0 & 13 & 20 & 9 & 3 & 0 & 45 \\
0 & 1 & 8 & 24 & 15 & 12 & 0 & 60 \\
0 & 0 & 1 & 25 & 23 & 5 & 0 & 54 \\
0 & 8 & 16 & 15 & 17 & 3 & 0 & 59 \\
0 & 7 & 6 & 4 & 15 & 11 & 0 & 43 \\
0 & 5 & 8 & 25 & 18 & 12 & 0 & 68 \\
0 & 4 & 7 & 17 & 11 & 12 & 0 & 51 \\
0 & 0 & 8 & 25 & 18 & 5 & 0 & 56 \\
0 & 5 & 3 & 18 & 22 & 5 & 0 & 53 \\
1 & 3 & 7 & 26 & 24 & 12 & 0 & 73 \\
- & -- & -- & -- & -- & -- & -- & - \\
& & & & & & & \\
$<1$ & 3 & 8 & 19 & 16 & 6 & 0 & 52 \\
$<1$ & 2 & 9 & 19 & 17 & 5 & 0 & 52 \\
& & & & & & &
\end{tabular}

\begin{tabular}{ccrrrrrr}
\multicolumn{10}{c}{$32^{\circ}$ F or Below } \\
\hline Season & Oct & Nov & Dec & Jan & Feb & Mar & Total \\
\hline $1975-76$ & 0 & 3 & 5 & 7 & 0 & 0 & 15 \\
$1976-77$ & 0 & 0 & 12 & 20 & 3 & 0 & 35 \\
$1977-78$ & 0 & 5 & 9 & 6 & 2 & 0 & 22 \\
$1978-79$ & 0 & 7 & 11 & 30 & 4 & 0 & 52 \\
$1979-80$ & 0 & 7 & 3 & 16 & 6 & 0 & 32 \\
$1980-81$ & 0 & 1 & 6 & 0 & 2 & 0 & 9 \\
$1981-82$ & 0 & 0 & 8 & 10 & 2 & 0 & 20 \\
$1982-83$ & 0 & 3 & 10 & 5 & 0 & 0 & 18 \\
$1983-84$ & 0 & 0 & 25 & 12 & 1 & 0 & 38 \\
$1984-85$ & 0 & 2 & 18 & 29 & 9 & 0 & $58^{(a)}$ \\
$1985-86$ & 0 & 15 & 25 & 5 & 1 & 0 & 46 \\
$1986-87$ & 0 & 0 & 7 & 9 & 0 & 0 & 16 \\
$1987-88$ & 0 & 0 & 16 & 11 & 1 & 0 & 28 \\
$1988-89$ & 0 & 0 & 11 & 2 & 8 & 1 & 22 \\
$1989-90$ & 0 & 2 & 6 & 0 & 1 & 0 & 9 \\
$1990-91$ & 0 & 0 & 15 & 13 & 0 & 0 & 28 \\
$1991-92$ & 0 & 0 & 3 & 0 & 0 & 0 & 3 \\
$1992-93$ & 0 & 1 & 11 & 20 & 8 & 2 & 42 \\
$1993-94$ & 0 & 6 & 4 & 1 & 8 & 0 & 19 \\
$1994-95$ & 0 & 0 & 5 & 6 & 2 & 0 & 13 \\
$1995-96$ & 0 & 0 & 8 & 9 & 5 & 0 & 22 \\
$1996-97$ & 0 & 5 & 12 & 8 & 2 & 0 & 27 \\
$1997-98$ & 0 & 0 & 2 & 5 & 0 & 0 & 7 \\
$1998-99$ & 0 & 0 & 7 & -- & -- & -- & -- \\
Average & $<1$ & 2 & 9 & 10 & 3 & $<1$ & 24 \\
Normal & $<1$ & 2 & 10 & 10 & 2 & $<1$ & 24 \\
& & & & & & &
\end{tabular}

(a) Greatest and least seasonal totals. 
Table 3.4. Days with Maximum Temperatures $\geq 104^{\circ} \mathrm{F}$

\begin{tabular}{|c|c|c|c|c|c|c|c|}
\hline $\begin{array}{c}\text { Temperature, } \\
{ }^{\circ} \mathrm{F} \\
\end{array}$ & & & & (s) of Occu & ence & & \\
\hline 113 & $08 / 04 / 61$ & & & & & & \\
\hline 112 & $07 / 27 / 98$ & $08 / 09 / 71$ & & & & & \\
\hline 111 & $07 / 22 / 94$ & $06 / 23 / 92$ & $07 / 31 / 71$ & & & & \\
\hline 110 & $\begin{array}{l}08 / 04 / 98 \\
07 / 17 / 60\end{array}$ & $07 / 12 / 90$ & $07 / 20 / 79$ & $07 / 09 / 75$ & $08 / 08 / 72$ & $07 / 06 / 68$ & $07 / 18 / 60$ \\
\hline 109 & $\begin{array}{l}08 / 10 / 96 \\
08 / 07 / 72\end{array}$ & $\begin{array}{l}07 / 24 / 94 \\
08 / 10 / 71\end{array}$ & $\begin{array}{l}07 / 23 / 94 \\
08 / 01 / 71\end{array}$ & $07 / 21 / 94$ & $08 / 14 / 92$ & $07 / 11 / 90$ & $07 / 19 / 79$ \\
\hline 108 & $\begin{array}{l}07 / 28 / 98 \\
07 / 27 / 75 \\
07 / 08 / 68 \\
06 / 17 / 61\end{array}$ & $\begin{array}{l}07 / 26 / 98 \\
07 / 05 / 75 \\
07 / 04 / 68\end{array}$ & $\begin{array}{l}07 / 26 / 96 \\
08 / 12 / 71 \\
08 / 18 / 67\end{array}$ & $\begin{array}{l}07 / 15 / 96 \\
08 / 11 / 71 \\
08 / 17 / 67\end{array}$ & $\begin{array}{l}06 / 24 / 92 \\
07 / 27 / 71 \\
08 / 16 / 67\end{array}$ & $\begin{array}{l}08 / 05 / 90 \\
07 / 19 / 71 \\
07 / 31 / 65\end{array}$ & $\begin{array}{l}07 / 18 / 79 \\
07 / 28 / 68 \\
07 / 13 / 61\end{array}$ \\
\hline 107 & $\begin{array}{l}08 / 13 / 92 \\
08 / 08 / 81 \\
08 / 03 / 61\end{array}$ & $\begin{array}{l}08 / 01 / 92 \\
07 / 17 / 79 \\
07 / 22 / 59\end{array}$ & $\begin{array}{l}07 / 31 / 92 \\
08 / 18 / 77 \\
07 / 20 / 59\end{array}$ & $\begin{array}{l}06 / 25 / 92 \\
08 / 08 / 71 \\
07 / 19 / 59\end{array}$ & $\begin{array}{l}07 / 14 / 87 \\
07 / 30 / 71 \\
07 / 28 / 58\end{array}$ & $\begin{array}{l}07 / 29 / 82 \\
07 / 28 / 71 \\
07 / 14 / 55\end{array}$ & $\begin{array}{l}07 / 28 / 82 \\
07 / 05 / 68\end{array}$ \\
\hline 106 & $\begin{array}{l}08 / 14 / 98 \\
08 / 18 / 92 \\
07 / 22 / 80 \\
07 / 15 / 73 \\
06 / 16 / 61\end{array}$ & $\begin{array}{l}08 / 05 / 98 \\
07 / 18 / 92 \\
08 / 09 / 78 \\
08 / 06 / 72 \\
06 / 22 / 58\end{array}$ & $\begin{array}{l}08 / 14 / 97 \\
06 / 22 / 92 \\
07 / 23 / 78 \\
07 / 20 / 71 \\
07 / 19 / 56\end{array}$ & $\begin{array}{l}08 / 04 / 97 \\
09 / 01 / 87 \\
08 / 17 / 77 \\
07 / 04 / 70 \\
07 / 09 / 52\end{array}$ & $\begin{array}{l}07 / 27 / 96 \\
06 / 30 / 87 \\
08 / 13 / 77 \\
08 / 01 / 65\end{array}$ & $\begin{array}{l}07 / 14 / 96 \\
07 / 09 / 85 \\
07 / 10 / 75 \\
07 / 12 / 64\end{array}$ & $\begin{array}{l}08 / 02 / 94 \\
07 / 25 / 84 \\
07 / 29 / 73 \\
07 / 24 / 62\end{array}$ \\
\hline 105 & $\begin{array}{l}08 / 13 / 98 \\
07 / 19 / 95 \\
07 / 20 / 85 \\
08 / 03 / 78 \\
07 / 08 / 70 \\
08 / 15 / 67 \\
07 / 13 / 55\end{array}$ & $\begin{array}{l}07 / 22 / 98 \\
07 / 17 / 92 \\
07 / 27 / 82 \\
07 / 04 / 75 \\
07 / 27 / 68 \\
08 / 13 / 67 \\
08 / 04 / 52\end{array}$ & $\begin{array}{l}08 / 06 / 97 \\
07 / 03 / 91 \\
07 / 26 / 82 \\
07 / 21 / 71 \\
07 / 07 / 68 \\
07 / 03 / 67 \\
07 / 30 / 52\end{array}$ & $\begin{array}{l}08 / 05 / 97 \\
07 / 22 / 90 \\
08 / 16 / 81 \\
07 / 18 / 71 \\
07 / 03 / 68 \\
08 / 02 / 61 \\
07 / 10 / 52\end{array}$ & $\begin{array}{l}08 / 25 / 96 \\
07 / 15 / 90 \\
08 / 13 / 81 \\
08 / 23 / 70 \\
08 / 30 / 67 \\
08 / 11 / 60 \\
07 / 20 / 46\end{array}$ & $\begin{array}{l}07 / 28 / 96 \\
07 / 26 / 88 \\
08 / 12 / 81 \\
07 / 19 / 70 \\
08 / 20 / 67 \\
07 / 19 / 60 \\
08 / 16 / 45\end{array}$ & $\begin{array}{l}07 / 24 / 96 \\
08 / 09 / 87 \\
08 / 04 / 78 \\
07 / 16 / 70 \\
08 / 19 / 67 \\
07 / 07 / 60\end{array}$ \\
\hline 104 & $\begin{array}{l}08 / 03 / 98 \\
07 / 18 / 95 \\
05 / 31 / 86 \\
08 / 11 / 81 \\
08 / 20 / 77 \\
06 / 22 / 73 \\
07 / 03 / 70 \\
07 / 25 / 62 \\
08 / 25 / 58 \\
07 / 23 / 56 \\
07 / 31 / 49 \\
07 / 21 / 46\end{array}$ & $\begin{array}{l}07 / 25 / 98 \\
07 / 20 / 94 \\
05 / 30 / 86 \\
08 / 10 / 81 \\
08 / 12 / 77 \\
08 / 28 / 72 \\
06 / 23 / 70 \\
07 / 23 / 62 \\
08 / 24 / 58 \\
07 / 21 / 56 \\
07 / 30 / 49 \\
07 / 11 / 45\end{array}$ & $\begin{array}{l}07 / 17 / 98 \\
07 / 17 / 94 \\
07 / 29 / 85 \\
08 / 07 / 81 \\
07 / 30 / 74 \\
08 / 09 / 72 \\
06 / 21 / 70 \\
08 / 14 / 61 \\
08 / 11 / 58 \\
07 / 22 / 55 \\
07 / 15 / 49 \\
07 / 10 / 45\end{array}$ & $\begin{array}{l}08 / 09 / 96 \\
07 / 10 / 90 \\
07 / 21 / 85 \\
07 / 27 / 81 \\
07 / 28 / 74 \\
08 / 13 / 71 \\
08 / 31 / 67 \\
06 / 18 / 61 \\
07 / 17 / 58 \\
08 / 15 / 53 \\
06 / 29 / 48\end{array}$ & $\begin{array}{l}07 / 25 / 96 \\
07 / 25 / 88 \\
07 / 08 / 85 \\
07 / 04 / 81 \\
08 / 01 / 73 \\
08 / 07 / 71 \\
08 / 11 / 67 \\
08 / 10 / 60 \\
07 / 11 / 58 \\
07 / 23 / 51 \\
08 / 22 / 46\end{array}$ & $\begin{array}{l}07 / 23 / 96 \\
07 / 21 / 88 \\
07 / 04 / 85 \\
08 / 08 / 78 \\
07 / 27 / 73 \\
07 / 16 / 71 \\
07 / 12 / 67 \\
07 / 16 / 60 \\
08 / 22 / 56 \\
07 / 17 / 51 \\
08 / 21 / 46\end{array}$ & $\begin{array}{l}07 / 13 / 96 \\
07 / 20 / 88 \\
08 / 07 / 82 \\
07 / 25 / 78 \\
07 / 19 / 73 \\
07 / 09 / 70 \\
07 / 30 / 65 \\
07 / 18 / 59 \\
07 / 24 / 56 \\
08 / 01 / 49 \\
07 / 28 / 46\end{array}$ \\
\hline
\end{tabular}

Maximum temperatures $\geq 90^{\circ} \mathrm{F}$ occur an average of 52 times per year and vary from a low of $29^{\circ} \mathrm{F}$ (1980) to a high of $79^{\circ} \mathrm{F}$ (1967). The earliest occurrences varied from April 24 (1977) through July 2 (1953), with an average annual date of May 21 (Table 3.5). The latest annual occurrence of maximum temperatures $\geq 90^{\circ} \mathrm{F}$ varied from August 17 (1983) through September 29 (1993 and earlier years). The average date for the period 1946 through 1996 is September 15. The longest period of consecutive maximum temperatures $\geq 90^{\circ} \mathrm{F}$ is 32 days from July 13 through August 13, 1971 .

The average seasonal number of days with maximum temperatures $\leq 32^{\circ} \mathrm{F}$ is 24 . The earliest seasonal occurrence of a day with a maximum temperature $\leq 32^{\circ} \mathrm{F}$ was October 30 (1971) and the latest was March 11 (1950). The number of winter days with maximum temperatures $\leq 32^{\circ} \mathrm{F}$ varied from 
Table 3.5. Record of Annual First and Last Dates with Maximum Temperatures $\geq 90^{\circ} \mathrm{F}$ and Minimum Temperatures $\leq 32^{\circ} \mathrm{F}$

\begin{tabular}{|c|c|c|c|c|c|}
\hline \multirow[b]{2}{*}{ Year } & \multicolumn{2}{|c|}{ Maximum Temperature $\geq 90^{\circ} \mathrm{F}$} & \multicolumn{2}{|c|}{ Minimum Temperature $\leq 32^{\circ} \mathrm{F}$} & \multirow[b]{2}{*}{ Growing Days ${ }^{(a)}$} \\
\hline & First in Spring & Last in Summer & Last in Spring & First in Autumn & \\
\hline 1945 & May 30 & Sep 14 & Apr 10 & Oct 18 & 190 \\
\hline $\begin{array}{l}1946 \\
1947 \\
1948 \\
1949 \\
1950\end{array}$ & $\begin{array}{l}\text { Apr } 25 \\
\text { May 06 } \\
\text { May 26 } \\
\text { May 08 } \\
\text { May 26 }\end{array}$ & $\begin{array}{l}\text { Aug } 24 \\
\text { Sep 12 } \\
\text { Sep } 13 \\
\text { Sep } 27 \\
\text { Sep } 23\end{array}$ & $\begin{array}{l}\text { Apr 07 } \\
\text { Apr 07 } \\
\text { May 02 } \\
\text { May 03 } \\
\text { Apr 27 }\end{array}$ & $\begin{array}{l}\text { Oct } 11 \\
\text { Nov } 04 \\
\text { Oct } 17 \\
\text { Oct } 08 \\
\text { Nov } 08\end{array}$ & $\begin{array}{l}186 \\
210 \\
167 \\
157 \\
194\end{array}$ \\
\hline $\begin{array}{l}1951 \\
1952 \\
1953 \\
1954 \\
1955\end{array}$ & $\begin{array}{l}\text { May } 22 \\
\text { May } 24 \\
\text { Jul 02(b) } \\
\text { May } 17 \\
\text { Jun } 06\end{array}$ & $\begin{array}{l}\text { Sep } 19 \\
\text { Sep } 26 \\
\text { Sep } 15 \\
\text { Sep } 10 \\
\text { Sep } 10\end{array}$ & $\begin{array}{l}\text { Apr } 21 \\
\text { Apr } 29 \\
\text { Apr } 15 \\
\text { May 01 } \\
\text { May } 14\end{array}$ & $\begin{array}{l}\text { Oct } 15 \\
\text { Nov } 01 \\
\text { Oct } 24 \\
\text { Oct } 01 \\
\text { Oet } 31\end{array}$ & $\begin{array}{l}176 \\
185 \\
191 \\
152 \\
169\end{array}$ \\
\hline $\begin{array}{l}1956 \\
1957 \\
1958 \\
1959 \\
1960\end{array}$ & $\begin{array}{l}\text { May } 16 \\
\text { May } 29 \\
\text { May } 18 \\
\text { May } 13 \\
\text { May } 10\end{array}$ & $\begin{array}{l}\text { Sep } 19 \\
\text { Sep } 15 \\
\text { Sep } 10 \\
\text { Sep } 13 \\
\text { Sep } 18\end{array}$ & $\begin{array}{l}\text { Apr 06 } \\
\text { Mar 26 } \\
\text { Mar 19(b) } \\
\text { May 05 } \\
\text { Apr 21 }\end{array}$ & $\begin{array}{l}\text { Oct } 22 \\
\text { Oct } 22 \\
\text { Oct } 21 \\
\text { Oct } 30 \\
\text { Oct } 11\end{array}$ & $\begin{array}{l}198 \\
209 \\
215 \\
177 \\
172\end{array}$ \\
\hline $\begin{array}{l}1961 \\
1962 \\
1963 \\
1964 \\
1965\end{array}$ & $\begin{array}{l}\text { May 25 } \\
\text { Jun 08 } \\
\text { May 20 } \\
\text { Jun 23 } \\
\text { May 28 }\end{array}$ & $\begin{array}{l}\text { Sep } 04 \\
\text { Sep } 26 \\
\text { Sep } 29 \\
\text { Sep } 24 \\
\text { Sep } 01\end{array}$ & $\begin{array}{l}\text { Apr } 19 \\
\text { May 04 } \\
\text { Apr } 16 \\
\text { Apr } 19 \\
\text { May 05 }\end{array}$ & $\begin{array}{l}\text { Oct } 20 \\
\text { Nov 12 } \\
\text { Oct } 25 \\
\text { Oct } 16 \\
\text { Oct } 16\end{array}$ & $\begin{array}{l}183 \\
191 \\
191 \\
179 \\
163\end{array}$ \\
\hline $\begin{array}{l}1966 \\
1967 \\
1968 \\
1969 \\
1970\end{array}$ & $\begin{array}{l}\text { May } 03 \\
\text { May } 20 \\
\text { Apr } 29 \\
\text { May } 09 \\
\text { May } 16\end{array}$ & $\begin{array}{l}\text { Sep } 22 \\
\text { Sep 28 } \\
\text { Sep 09 } \\
\text { Sep 12 } \\
\text { Aug 31 }\end{array}$ & $\begin{array}{l}\text { Apr } 19 \\
\text { Apr } 28 \\
\text { Apr } 22 \\
\text { Apr } 26 \\
\text { May } 11\end{array}$ & $\begin{array}{l}\text { Oct } 14 \\
\text { Oct } 26 \\
\text { Oct } 21 \\
\text { Oct } 13 \\
\text { Oct } 07\end{array}$ & $\begin{array}{l}177 \\
180 \\
181 \\
169 \\
148\end{array}$ \\
\hline $\begin{array}{l}1971 \\
1972 \\
1973 \\
1974 \\
1975\end{array}$ & $\begin{array}{l}\text { May } 11 \\
\text { May } 13 \\
\text { May } 13 \\
\text { Jun } 10 \\
\text { May } 30\end{array}$ & $\begin{array}{l}\text { Sep } 10 \\
\text { Sep } 16 \\
\text { Sep } 11 \\
\text { Sep } 25 \\
\text { Sep } 15\end{array}$ & $\begin{array}{l}\text { Apr } 22 \\
\text { Apr } 30 \\
\text { Apr 08 } \\
\text { May 16(b) } \\
\text { Apr } 29\end{array}$ & $\begin{array}{l}\text { Oct 16 } \\
\text { Sep 25 } \\
\text { Oct 04 } \\
\text { Oct 06 } \\
\text { Oct 23 }\end{array}$ & $\begin{array}{l}176 \\
147 \\
178 \\
142 \\
176\end{array}$ \\
\hline $\begin{array}{l}1976 \\
1977 \\
1978 \\
1979 \\
1980\end{array}$ & $\begin{array}{l}\text { May 16 } \\
\text { Apr 24 } \\
\text { Jun 02 } \\
\text { May 22 } \\
\text { Jun 01 }\end{array}$ & $\begin{array}{l}\text { Sep 29 } \\
\text { Aug 22 } \\
\text { Sep 03 } \\
\text { Sep 20 } \\
\text { Sep 06 }\end{array}$ & $\begin{array}{l}\text { Apr } 23 \\
\text { Apr 14 } \\
\text { Apr 23 } \\
\text { Apr 19 } \\
\text { Apr 11 }\end{array}$ & $\begin{array}{l}\text { Oct } 19 \\
\text { Oct } 27 \\
\text { Oct } 07 \\
\text { Oct } 31 \\
\text { Oct } 22\end{array}$ & $\begin{array}{l}178 \\
195 \\
166 \\
194 \\
193\end{array}$ \\
\hline $\begin{array}{l}1981 \\
1982 \\
1983 \\
1984 \\
1985\end{array}$ & $\begin{array}{l}\text { Apr } 30 \\
\text { Jun 10 } \\
\text { May 23 } \\
\text { May } 29 \\
\text { May 18 }\end{array}$ & $\begin{array}{l}\text { Sep } 18 \\
\text { Sep 08 } \\
\text { Aug 17(0) } \\
\text { Sep } 18 \\
\text { Aug } 29\end{array}$ & $\begin{array}{l}\text { Apr 13 } \\
\text { Apr 21 } \\
\text { Apr 16 } \\
\text { Apr 13 } \\
\text { Apr 21 }\end{array}$ & $\begin{array}{l}\text { Oct } 14 \\
\text { Oct } 18 \\
\text { Oct } 11 \\
\text { Oct } 14 \\
\text { Oct } 07\end{array}$ & $\begin{array}{l}183 \\
179 \\
177 \\
183 \\
168\end{array}$ \\
\hline $\begin{array}{l}1986 \\
1987 \\
1988 \\
1989 \\
1990\end{array}$ & $\begin{array}{l}\text { May } 25 \\
\text { Apr } 27 \\
\text { May } 11 \\
\text { Jun 01 } \\
\text { May 05 }\end{array}$ & $\begin{array}{l}\text { Sep } 04 \\
\text { Sep } 23 \\
\text { Sep } 14 \\
\text { Sep } 24 \\
\text { Sep } 29\end{array}$ & $\begin{array}{l}\text { Apr } 30 \\
\text { Apr 20 } \\
\text { Apr } 09 \\
\text { Mar } 30 \\
\text { Mar } 27\end{array}$ & $\begin{array}{l}\text { Nov } 09 \\
\text { Oct } 16 \\
\text { Oct } 27 \\
\text { Oct } 29 \\
\text { Oct } 17\end{array}$ & $\begin{array}{l}192 \\
178 \\
200 \\
212 \\
203\end{array}$ \\
\hline $\begin{array}{l}1991 \\
1992 \\
1993 \\
1994 \\
1995\end{array}$ & $\begin{array}{l}\text { Jun } 10 \\
\text { May 04 } \\
\text { May 10 } \\
\text { May 07 } \\
\text { May 28 }\end{array}$ & $\begin{array}{l}\text { Sep } 26 \\
\text { Sep } 03 \\
\text { Sep } 29^{(b, c)} \\
\text { Sep } 28 \\
\text { Sep } 17\end{array}$ & $\begin{array}{l}\text { Apr 08 } \\
\text { Apr 08 } \\
\text { Apr 06 } \\
\text { Mar 26 } \\
\text { Apr 15 }\end{array}$ & $\begin{array}{l}\text { Oct } 22 \\
\text { Oct } 15 \\
\text { Oct } 20 \\
\text { Oct } 29 \\
\text { Oct } 29\end{array}$ & $\begin{array}{l}196 \\
189 \\
196 \\
216 \\
196\end{array}$ \\
\hline $\begin{array}{l}1996 \\
1997 \\
1998\end{array}$ & $\begin{array}{l}\text { Jun } 02 \\
\text { May } 12 \\
\text { Apr } 30\end{array}$ & $\begin{array}{l}\text { Sep } 15 \\
\text { Sep } 25 \\
\text { Sep } 17\end{array}$ & $\begin{array}{l}\text { May } 08 \\
\text { May } 02 \\
\text { Apr } 13\end{array}$ & $\begin{array}{l}\text { Oct } 17 \\
\text { Oct } 08 \\
\text { Oct } 19\end{array}$ & $\begin{array}{l}161 \\
158 \\
188\end{array}$ \\
\hline $\begin{array}{l}\text { Average } \\
\text { Normal }\end{array}$ & $\begin{array}{l}\text { May } 21 \\
\text { May } 21\end{array}$ & $\begin{array}{l}\text { Sep } 15 \\
\text { Sep } 14\end{array}$ & $\begin{array}{l}\text { Apr } 19 \\
\text { Apr } 21\end{array}$ & $\begin{array}{l}\text { Oct } 20 \\
\text { Oct } 18\end{array}$ & $\begin{array}{l}183 \\
179\end{array}$ \\
\hline
\end{tabular}

(a) Days between last freezing temperature in spring and first freezing temperature in autumn.

(b) Earliest and latest dates.

(c) Also in previous years. 
2 to 58 days (winters of 1966-1967 and 1984-1985, respectively). The greatest consecutive number of days with maximum temperatures $\leq 32^{\circ} \mathrm{F}$ is 29 days, from December 30,1984 through January 27,1985 . During the period December 27, 1978 through February 4, 1979 (40 days), only 1 maximum temperature $>32^{\circ} \mathrm{F}$ occurred. The average maximum temperature for that period was $21^{\circ} \mathrm{F}$.

Table 3.6 lists the monthly and annual maximum temperatures. Only 6 days were recorded when the daily maximum temperature was $\leq 0^{\circ} \mathrm{F}$. These were:

\begin{tabular}{lc}
\multicolumn{1}{c}{ Maximum Date } & Temperature \\
\cline { 1 - 1 } January 31, 1950 & $-2^{\circ} \mathrm{F}$ \\
February 1, 1950 & $-3^{\circ} \mathrm{F}$ \\
February 2,1950 & $-3^{\circ} \mathrm{F}$ \\
January 27, 1957 & $0^{\circ} \mathrm{F}$ \\
December 29, 1968 & $-2^{\circ} \mathrm{F}$ \\
December 30, 1968 & $-2^{\circ} \mathrm{F}$
\end{tabular}

\subsection{Days with Minimum Temperatures $\leq 32^{\circ} \mathrm{F}$ or $\leq 0^{\circ} \mathrm{F}$}

The monthly and seasonal number of days with minimum temperatures at or below $32^{\circ} \mathrm{F}$ or $0^{\circ} \mathrm{F}$ are listed in Table 3.7.

The seasonal average number of days with minimum temperatures $\leq 32^{\circ} \mathrm{F}$ is 106 ; however, the number ranges from 70 to 143 days (winters of 1991-1992 and 1984-1985, respectively). The greatest consecutive number of days with minimum temperatures of $\leq 32^{\circ} \mathrm{F}$ is 93 , from November 9,1978 through February 9, 1979.

The first autumn temperature $\leq 32^{\circ} \mathrm{F}$ occurred as early as September 25 (1972) and as late as November 12 (1962). The average date is October 20 (see Table 3.5). The last date in spring for minimum temperatures $\leq 32^{\circ} \mathrm{F}$ varied from March $19(1958)$ to May $16(1974)$, with an average date of April 19. The average number of days between last freezing temperature in the spring and first freezing temperature in the autumn is 183 days.

On average, 3 days per winter season have a minimum temperature $\leq 0^{\circ} \mathrm{F}$; however, nearly half of all winters have no minimum temperatures in this category (see Table 3.7). The most of those days in any season was 18 (winter of 1949-1950) and the least of those days was 0 (as recently as the winter of 19971998). The greatest number of consecutive days with minimum temperatures $\leq 0^{\circ} \mathrm{F}$ is 11 days, from January 25 through February 4, 1950. During this same period, 4 consecutive days had minimum temperatures $\leq-20^{\circ} \mathrm{F}$. Table 3.8 lists all days with minimum temperatures $\leq 0^{\circ} \mathrm{F}$. Table 3.9 lists monthly and annual minimum temperatures. 
Table 3.6. Monthly and Annual Maximum Temperatures $\left({ }^{\circ} \mathrm{F}\right)$

\begin{tabular}{|c|c|c|c|c|c|c|c|c|c|c|c|c|c|}
\hline Year & Jan & Feb & Mar & Apr & May & Jun & Jul & Aug & Sep & Oct & Nov & Dec & Annual \\
\hline 1945 & 61 & 65 & 72 & 76 & 90 & 98 & 104 & 105 & 93 & 84 & 72 & 57 & 105 \\
\hline $\begin{array}{l}1946 \\
1947 \\
1948 \\
1949 \\
1950\end{array}$ & $\begin{array}{l}57 \\
57 \\
60 \\
48 \\
50\end{array}$ & $\begin{array}{l}60 \\
68 \\
64 \\
56 \\
63\end{array}$ & $\begin{array}{l}76 \\
76 \\
73 \\
64 \\
64\end{array}$ & $\begin{array}{l}91 \\
88 \\
76 \\
84 \\
78\end{array}$ & $\begin{array}{r}89 \\
101 \\
91 \\
98 \\
90\end{array}$ & $\begin{array}{r}98 \\
97 \\
104 \\
102 \\
99\end{array}$ & $\begin{array}{r}105 \\
103 \\
98 \\
104 \\
102\end{array}$ & $\begin{array}{r}104 \\
98 \\
97 \\
104 \\
103\end{array}$ & $\begin{array}{r}89 \\
94 \\
98 \\
100 \\
102\end{array}$ & $\begin{array}{l}75 \\
83 \\
78 \\
74 \\
76\end{array}$ & $\begin{array}{l}64 \\
63 \\
57 \\
65 \\
62\end{array}$ & $\begin{array}{l}64 \\
55 \\
52 \\
60 \\
55\end{array}$ & $\begin{array}{l}105 \\
103 \\
104 \\
104 \\
103\end{array}$ \\
\hline $\begin{array}{l}1951 \\
1952 \\
1953 \\
1954 \\
1955\end{array}$ & $\begin{array}{l}55 \\
50 \\
63 \\
59 \\
50\end{array}$ & $\begin{array}{l}65 \\
55 \\
65 \\
63 \\
58\end{array}$ & $\begin{array}{l}65 \\
70 \\
69 \\
65 \\
63\end{array}$ & $\begin{array}{l}82 \\
89 \\
78 \\
83 \\
77\end{array}$ & $\begin{array}{l}94 \\
92 \\
88 \\
98 \\
86\end{array}$ & $\begin{array}{r}97 \\
94 \\
86 \\
94 \\
102\end{array}$ & $\begin{array}{l}104 \\
106 \\
103 \\
100 \\
107\end{array}$ & $\begin{array}{r}101 \\
105 \\
104 \\
99 \\
101\end{array}$ & $\begin{array}{r}97 \\
97 \\
97 \\
92 \\
101\end{array}$ & $\begin{array}{l}79 \\
85 \\
81 \\
73 \\
75\end{array}$ & $\begin{array}{l}60 \\
62 \\
65 \\
62 \\
66\end{array}$ & $\begin{array}{l}58 \\
54 \\
59 \\
54 \\
56\end{array}$ & $\begin{array}{l}104 \\
106 \\
104 \\
100 \\
107\end{array}$ \\
\hline $\begin{array}{l}1956 \\
1957 \\
1958 \\
1959 \\
1960\end{array}$ & $\begin{array}{l}59 \\
48 \\
60 \\
59 \\
55\end{array}$ & $\begin{array}{l}56 \\
65 \\
63 \\
60 \\
55\end{array}$ & $\begin{array}{l}64 \\
66 \\
63 \\
65 \\
83\end{array}$ & $\begin{array}{l}85 \\
89 \\
78 \\
79 \\
82\end{array}$ & $\begin{array}{r}96 \\
97 \\
101 \\
91 \\
90\end{array}$ & $\begin{array}{r}95 \\
100 \\
106 \\
97 \\
96\end{array}$ & $\begin{array}{l}106 \\
102 \\
107 \\
107 \\
110\end{array}$ & $\begin{array}{r}104 \\
96 \\
104 \\
103 \\
105\end{array}$ & $\begin{array}{l}94 \\
98 \\
97 \\
92 \\
94\end{array}$ & $\begin{array}{l}79 \\
73 \\
89 \\
77 \\
82\end{array}$ & $\begin{array}{l}64 \\
60 \\
67 \\
70 \\
63\end{array}$ & $\begin{array}{l}59 \\
59 \\
60 \\
64 \\
52\end{array}$ & $\begin{array}{l}106 \\
102 \\
107 \\
107 \\
110\end{array}$ \\
\hline $\begin{array}{l}1961 \\
1962 \\
1963 \\
1964 \\
1965\end{array}$ & $\begin{array}{l}60 \\
63 \\
56 \\
57 \\
60\end{array}$ & $\begin{array}{l}64 \\
60 \\
64 \\
60 \\
67\end{array}$ & $\begin{array}{l}68 \\
70 \\
70 \\
74 \\
71\end{array}$ & $\begin{array}{l}75 \\
85 \\
72 \\
73 \\
82\end{array}$ & $\begin{array}{l}94 \\
81 \\
93 \\
88 \\
91\end{array}$ & $\begin{array}{r}108 \\
98 \\
102 \\
95 \\
96\end{array}$ & $\begin{array}{r}108 \\
106 \\
96 \\
106 \\
108\end{array}$ & $\begin{array}{r}113 \\
100 \\
101 \\
97 \\
106\end{array}$ & $\begin{array}{l}90 \\
97 \\
98 \\
90 \\
91\end{array}$ & $\begin{array}{l}81 \\
76 \\
83 \\
80 \\
84\end{array}$ & $\begin{array}{l}58 \\
67 \\
61 \\
60 \\
64\end{array}$ & $\begin{array}{l}56 \\
56 \\
57 \\
57 \\
56\end{array}$ & $\begin{array}{l}113 \\
106 \\
102 \\
106 \\
108\end{array}$ \\
\hline $\begin{array}{l}1966 \\
1967 \\
1968 \\
1969 \\
1970\end{array}$ & $\begin{array}{l}56 \\
62 \\
66 \\
44 \\
56\end{array}$ & $\begin{array}{l}59 \\
67 \\
64 \\
46 \\
60\end{array}$ & $\begin{array}{l}78 \\
65 \\
68 \\
74 \\
67\end{array}$ & $\begin{array}{l}81 \\
71 \\
90 \\
80 \\
71\end{array}$ & $\begin{array}{r}100 \\
92 \\
90 \\
95 \\
92\end{array}$ & $\begin{array}{r}95 \\
101 \\
99 \\
103 \\
104\end{array}$ & $\begin{array}{l}100 \\
105 \\
110 \\
101 \\
106\end{array}$ & $\begin{array}{l}102 \\
108 \\
102 \\
102 \\
105\end{array}$ & $\begin{array}{l}99 \\
98 \\
97 \\
96 \\
89\end{array}$ & $\begin{array}{l}82 \\
78 \\
73 \\
74 \\
86\end{array}$ & $\begin{array}{l}64 \\
65 \\
60 \\
63 \\
63\end{array}$ & $\begin{array}{l}56 \\
62 \\
59 \\
54 \\
58\end{array}$ & $\begin{array}{l}102 \\
108 \\
110 \\
103 \\
106\end{array}$ \\
\hline $\begin{array}{l}1971 \\
1972 \\
1973 \\
1974 \\
1975\end{array}$ & $\begin{array}{l}72 \\
59 \\
51 \\
61 \\
56\end{array}$ & $\begin{array}{l}66 \\
68 \\
61 \\
59 \\
58\end{array}$ & $\begin{array}{l}65 \\
76 \\
68 \\
69 \\
65\end{array}$ & $\begin{array}{l}76 \\
78 \\
80 \\
77 \\
75\end{array}$ & $\begin{array}{l}92 \\
96 \\
98 \\
86 \\
90\end{array}$ & $\begin{array}{r}99 \\
98 \\
104 \\
103 \\
95\end{array}$ & $\begin{array}{l}111 \\
103 \\
106 \\
104 \\
110\end{array}$ & $\begin{array}{r}112 \\
110 \\
104 \\
103 \\
98\end{array}$ & $\begin{array}{l}91 \\
95 \\
98 \\
92 \\
96\end{array}$ & $\begin{array}{l}85 \\
83 \\
76 \\
80 \\
82\end{array}$ & $\begin{array}{l}64 \\
58 \\
62 \\
64 \\
75\end{array}$ & $\begin{array}{l}50 \\
65 \\
58 \\
60 \\
62\end{array}$ & $\begin{array}{l}112 \\
110 \\
106 \\
104 \\
110\end{array}$ \\
\hline $\begin{array}{l}1976 \\
1977 \\
1978 \\
1979 \\
1980\end{array}$ & $\begin{array}{l}59 \\
61 \\
51 \\
37 \\
51\end{array}$ & $\begin{array}{l}59 \\
70 \\
57 \\
62 \\
59\end{array}$ & $\begin{array}{l}69 \\
73 \\
74 \\
76 \\
68\end{array}$ & $\begin{array}{l}80 \\
94 \\
76 \\
83 \\
87\end{array}$ & $\begin{array}{l}90 \\
82 \\
87 \\
94 \\
87\end{array}$ & $\begin{array}{r}100 \\
100 \\
101 \\
102 \\
88\end{array}$ & $\begin{array}{l}102 \\
101 \\
106 \\
110 \\
106\end{array}$ & $\begin{array}{r}98 \\
107 \\
106 \\
101 \\
98\end{array}$ & $\begin{array}{r}102 \\
87 \\
90 \\
96 \\
95\end{array}$ & $\begin{array}{l}84 \\
75 \\
81 \\
84 \\
89\end{array}$ & $\begin{array}{l}71 \\
68 \\
69 \\
59 \\
65\end{array}$ & $\begin{array}{l}57 \\
64 \\
54 \\
59 \\
69\end{array}$ & $\begin{array}{l}102 \\
107 \\
106 \\
110 \\
106\end{array}$ \\
\hline $\begin{array}{l}1981 \\
1982 \\
1983 \\
1984 \\
1985\end{array}$ & $\begin{array}{l}55 \\
57 \\
61 \\
60 \\
36\end{array}$ & $\begin{array}{l}66 \\
68 \\
62 \\
62 \\
60\end{array}$ & $\begin{array}{l}70 \\
71 \\
64 \\
67 \\
68\end{array}$ & $\begin{array}{l}91 \\
81 \\
77 \\
79 \\
82\end{array}$ & $\begin{array}{r}89 \\
88 \\
103 \\
94 \\
95\end{array}$ & $\begin{array}{r}96 \\
102 \\
92 \\
96 \\
102\end{array}$ & $\begin{array}{l}104 \\
107 \\
100 \\
106 \\
106\end{array}$ & $\begin{array}{r}107 \\
104 \\
99 \\
103 \\
97\end{array}$ & $\begin{array}{l}99 \\
94 \\
87 \\
92 \\
86\end{array}$ & $\begin{array}{l}83 \\
75 \\
78 \\
81 \\
74\end{array}$ & $\begin{array}{l}65 \\
63 \\
67 \\
61 \\
66\end{array}$ & $\begin{array}{l}58 \\
62 \\
46 \\
52 \\
39\end{array}$ & $\begin{array}{l}107 \\
107 \\
103 \\
106 \\
106\end{array}$ \\
\hline $\begin{array}{l}1986 \\
1987 \\
1988 \\
1989 \\
1990\end{array}$ & $\begin{array}{l}57 \\
55 \\
54 \\
67 \\
60\end{array}$ & $\begin{array}{l}72 \\
60 \\
71 \\
53 \\
64\end{array}$ & $\begin{array}{l}74 \\
70 \\
71 \\
67 \\
76\end{array}$ & $\begin{array}{l}84 \\
93 \\
83 \\
80 \\
81\end{array}$ & $\begin{array}{r}104 \\
102 \\
94 \\
88 \\
94\end{array}$ & $\begin{array}{r}103 \\
106 \\
99 \\
97 \\
96\end{array}$ & $\begin{array}{r}99 \\
107 \\
105 \\
101 \\
110\end{array}$ & $\begin{array}{l}103 \\
105 \\
102 \\
103 \\
108\end{array}$ & $\begin{array}{r}95 \\
106 \\
102 \\
94 \\
98\end{array}$ & $\begin{array}{l}84 \\
87 \\
88 \\
80 \\
80\end{array}$ & $\begin{array}{l}63 \\
66 \\
69 \\
73 \\
68\end{array}$ & $\begin{array}{l}52 \\
59 \\
57 \\
58 \\
57\end{array}$ & $\begin{array}{l}104 \\
107 \\
105 \\
103 \\
110\end{array}$ \\
\hline $\begin{array}{l}1991 \\
1992 \\
1993 \\
1994 \\
1995\end{array}$ & $\begin{array}{l}59 \\
60 \\
56 \\
61 \\
67\end{array}$ & $\begin{array}{l}66 \\
62 \\
52 \\
63 \\
68\end{array}$ & $\begin{array}{l}69 \\
78 \\
66 \\
79 \\
69\end{array}$ & $\begin{array}{l}82 \\
85 \\
73 \\
88 \\
80\end{array}$ & $\begin{array}{r}83 \\
98 \\
100 \\
95 \\
95\end{array}$ & $\begin{array}{r}93 \\
111 \\
98 \\
101 \\
98\end{array}$ & $\begin{array}{r}105 \\
107 \\
96 \\
111 \\
105\end{array}$ & $\begin{array}{l}103 \\
109 \\
100 \\
106 \\
102\end{array}$ & $\begin{array}{r}95 \\
91 \\
98 \\
94 \\
101\end{array}$ & $\begin{array}{l}88 \\
87 \\
86 \\
84 \\
74\end{array}$ & $\begin{array}{l}65 \\
62 \\
65 \\
62 \\
69\end{array}$ & $\begin{array}{l}59 \\
53 \\
67 \\
64 \\
57\end{array}$ & $\begin{array}{l}105 \\
111 \\
100 \\
111 \\
105\end{array}$ \\
\hline $\begin{array}{l}1996 \\
1997 \\
1998\end{array}$ & $\begin{array}{l}58 \\
57 \\
57\end{array}$ & $\begin{array}{l}63 \\
64 \\
58\end{array}$ & $\begin{array}{l}68 \\
76 \\
72\end{array}$ & $\begin{array}{l}82 \\
75 \\
92\end{array}$ & $\begin{array}{l}86 \\
94 \\
93\end{array}$ & $\begin{array}{l}98 \\
98 \\
99\end{array}$ & $\begin{array}{l}108 \\
101 \\
112\end{array}$ & $\begin{array}{l}109 \\
106 \\
110\end{array}$ & $\begin{array}{r}94 \\
95 \\
103\end{array}$ & $\begin{array}{l}86 \\
77 \\
84\end{array}$ & $\begin{array}{l}66 \\
63 \\
67\end{array}$ & $\begin{array}{l}52 \\
52 \\
60\end{array}$ & $\begin{array}{l}109 \\
106 \\
112\end{array}$ \\
\hline
\end{tabular}


Table 3.7. Monthly and Seasonal Number of Days with Minimum Temperatures $\left({ }^{\circ} \mathrm{F}\right)$ Below Certain Thresholds

Minimum Temperature $\leq 32^{\circ} \mathrm{F}$

Minimum Temperature $\leq 0^{\circ} \mathrm{F}$

\begin{tabular}{|c|c|c|c|c|c|c|c|c|c|c|c|c|c|c|c|}
\hline \multirow{2}{*}{ Season } & \multicolumn{10}{|c|}{ 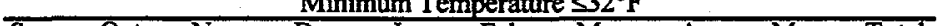 } & \multicolumn{5}{|c|}{ 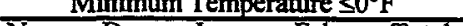 } \\
\hline & Sep & Oct & Nov & Dec & $\underline{\operatorname{Jan}}$ & Feb & Mar & Apr & May & Total & Nov & Dec & $\operatorname{Jan}$ & $\mathrm{Feb}$ & Total \\
\hline $1944-1945$ & - & - & - & -- & 27 & 18 & 12 & 6 & 0 & 63 & - & - & 0 & 0 & 0 \\
\hline $\begin{array}{l}1945-1946 \\
1946-1947 \\
1947-1948 \\
1948-1949 \\
1949-1950\end{array}$ & $\begin{array}{l}0 \\
0 \\
0 \\
0 \\
0\end{array}$ & $\begin{array}{r}5 \\
8 \\
0 \\
8 \\
10\end{array}$ & $\begin{array}{r}14 \\
23 \\
11 \\
15 \\
4\end{array}$ & $\begin{array}{l}25 \\
24 \\
26 \\
30 \\
25\end{array}$ & $\begin{array}{l}27 \\
27 \\
25 \\
31 \\
30\end{array}$ & $\begin{array}{l}20 \\
19 \\
24 \\
25 \\
22\end{array}$ & $\begin{array}{r}10 \\
6 \\
21 \\
11 \\
18\end{array}$ & $\begin{array}{l}2 \\
1 \\
7 \\
4 \\
4\end{array}$ & $\begin{array}{l}0 \\
0 \\
1 \\
1 \\
0\end{array}$ & $\begin{array}{l}103 \\
108 \\
115 \\
125 \\
113\end{array}$ & $\begin{array}{l}0 \\
0 \\
0 \\
0 \\
0\end{array}$ & $\begin{array}{l}0 \\
0 \\
0 \\
2 \\
0\end{array}$ & $\begin{array}{r}0 \\
1 \\
0 \\
9 \\
14\end{array}$ & $\begin{array}{l}0 \\
0 \\
0 \\
0 \\
4\end{array}$ & $\begin{array}{c}0 \\
1 \\
0 \\
11 \\
18^{(a)}\end{array}$ \\
\hline $\begin{array}{l}1950-1951 \\
1951-1952 \\
1952-1953 \\
1953-1954 \\
1954-1955\end{array}$ & $\begin{array}{l}0 \\
0 \\
0 \\
0 \\
0\end{array}$ & $\begin{array}{l}0 \\
6 \\
0 \\
1 \\
6\end{array}$ & $\begin{array}{r}13 \\
19 \\
25 \\
14 \\
6\end{array}$ & $\begin{array}{l}19 \\
26 \\
19 \\
22 \\
26\end{array}$ & $\begin{array}{r}26 \\
31 \\
9 \\
23 \\
30\end{array}$ & $\begin{array}{l}25 \\
24 \\
15 \\
16 \\
25\end{array}$ & $\begin{array}{l}21 \\
20 \\
12 \\
19 \\
22\end{array}$ & $\begin{array}{r}2 \\
6 \\
4 \\
4 \\
10\end{array}$ & $\begin{array}{l}0 \\
0 \\
0 \\
1 \\
1\end{array}$ & $\begin{array}{r}106 \\
132 \\
84 \\
100 \\
126\end{array}$ & $\begin{array}{l}0 \\
0 \\
0 \\
0 \\
0\end{array}$ & $\begin{array}{l}0 \\
0 \\
0 \\
0 \\
0\end{array}$ & $\begin{array}{l}0 \\
0 \\
0 \\
2 \\
0\end{array}$ & $\begin{array}{l}0 \\
0 \\
0 \\
0 \\
0\end{array}$ & $\begin{array}{l}0 \\
0 \\
0 \\
2 \\
0\end{array}$ \\
\hline $\begin{array}{l}1955-1956 \\
1956-1957 \\
1957-1958 \\
1958-1959 \\
1959-1960\end{array}$ & $\begin{array}{l}0 \\
0 \\
0 \\
0 \\
0\end{array}$ & $\begin{array}{l}1 \\
3 \\
2 \\
4 \\
2\end{array}$ & $\begin{array}{l}22 \\
18 \\
17 \\
14 \\
24\end{array}$ & $\begin{array}{l}28 \\
21 \\
16 \\
24 \\
26\end{array}$ & $\begin{array}{l}25 \\
31 \\
19 \\
25 \\
31\end{array}$ & $\begin{array}{r}26 \\
23 \\
5 \\
24 \\
21\end{array}$ & $\begin{array}{l}14 \\
11 \\
16 \\
14 \\
10\end{array}$ & $\begin{array}{l}2 \\
0 \\
0 \\
2 \\
4\end{array}$ & $\begin{array}{l}0 \\
0 \\
0 \\
1 \\
0\end{array}$ & $\begin{array}{c}118 \\
107 \\
75 \\
108 \\
118\end{array}$ & $\begin{array}{l}1 \\
0 \\
0 \\
0 \\
0\end{array}$ & $\begin{array}{l}0 \\
0 \\
0 \\
0 \\
0\end{array}$ & $\begin{array}{r}2 \\
12 \\
0 \\
2 \\
1\end{array}$ & $\begin{array}{l}3 \\
1 \\
0 \\
0 \\
0\end{array}$ & $\begin{array}{r}6 \\
13 \\
0 \\
2 \\
1\end{array}$ \\
\hline $\begin{array}{l}1960-1961 \\
1961-1962 \\
1962-1963 \\
1963-1964 \\
1964-1965\end{array}$ & $\begin{array}{l}0 \\
0 \\
0 \\
0 \\
0\end{array}$ & $\begin{array}{l}4 \\
7 \\
0 \\
5 \\
5\end{array}$ & $\begin{array}{r}15 \\
28 \\
13 \\
8 \\
13\end{array}$ & $\begin{array}{l}29 \\
26 \\
17 \\
31 \\
29\end{array}$ & $\begin{array}{l}23 \\
27 \\
27 \\
26 \\
25\end{array}$ & $\begin{array}{l}10 \\
17 \\
17 \\
26 \\
18\end{array}$ & $\begin{array}{l}7 \\
19 \\
11 \\
16 \\
19\end{array}$ & $\begin{array}{l}5 \\
0 \\
2 \\
4 \\
1\end{array}$ & $\begin{array}{l}0 \\
1 \\
0 \\
0 \\
1\end{array}$ & $\begin{array}{c}93 \\
125 \\
87 \\
116 \\
111\end{array}$ & $\begin{array}{l}0 \\
0 \\
0 \\
0 \\
0\end{array}$ & $\begin{array}{l}0 \\
0 \\
0 \\
0 \\
2\end{array}$ & $\begin{array}{l}0 \\
1 \\
2 \\
0 \\
0\end{array}$ & $\begin{array}{l}0 \\
0 \\
0 \\
0 \\
0\end{array}$ & $\begin{array}{l}0 \\
1 \\
2 \\
0 \\
2\end{array}$ \\
\hline $\begin{array}{l}1965-1966 \\
1966-1967 \\
1967-1968 \\
1968-1969 \\
1969-1970\end{array}$ & $\begin{array}{l}0 \\
0 \\
0 \\
0 \\
0\end{array}$ & $\begin{array}{l}1 \\
3 \\
1 \\
4 \\
5\end{array}$ & $\begin{array}{r}8 \\
11 \\
17 \\
8 \\
19\end{array}$ & $\begin{array}{l}25 \\
18 \\
25 \\
24 \\
21\end{array}$ & $\begin{array}{l}26 \\
20 \\
23 \\
30 \\
28\end{array}$ & $\begin{array}{l}22 \\
17 \\
13 \\
25 \\
13\end{array}$ & $\begin{array}{r}13 \\
18 \\
6 \\
15 \\
16\end{array}$ & $\begin{array}{l}3 \\
9 \\
5 \\
1 \\
7\end{array}$ & $\begin{array}{l}0 \\
0 \\
0 \\
0 \\
1\end{array}$ & $\begin{array}{r}98 \\
96 \\
90 \\
107 \\
110\end{array}$ & $\begin{array}{l}0 \\
0 \\
0 \\
0 \\
0\end{array}$ & $\begin{array}{l}0 \\
0 \\
0 \\
4 \\
0\end{array}$ & $\begin{array}{l}0 \\
0 \\
0 \\
5 \\
0\end{array}$ & $\begin{array}{l}0 \\
0 \\
0 \\
1 \\
0\end{array}$ & $\begin{array}{r}0 \\
0 \\
0 \\
10 \\
0\end{array}$ \\
\hline $\begin{array}{l}1970-1971 \\
1971-1972 \\
1972-1973 \\
1973-1974 \\
1974-1975\end{array}$ & $\begin{array}{l}0 \\
0 \\
3 \\
0 \\
0\end{array}$ & $\begin{array}{l}8 \\
9 \\
6 \\
4 \\
4\end{array}$ & $\begin{array}{l}14 \\
18 \\
13 \\
14 \\
12\end{array}$ & $\begin{array}{l}28 \\
27 \\
23 \\
16 \\
26\end{array}$ & $\begin{array}{l}24 \\
25 \\
30 \\
19 \\
29\end{array}$ & $\begin{array}{l}19 \\
23 \\
23 \\
15 \\
24\end{array}$ & $\begin{array}{l}20 \\
13 \\
10 \\
12 \\
17\end{array}$ & $\begin{array}{l}7 \\
6 \\
4 \\
0 \\
7\end{array}$ & $\begin{array}{l}0 \\
0 \\
0 \\
1 \\
0\end{array}$ & $\begin{array}{c}120 \\
121 \\
112 \\
81 \\
119\end{array}$ & $\begin{array}{l}0 \\
0 \\
0 \\
0 \\
0\end{array}$ & $\begin{array}{l}0 \\
0 \\
7 \\
0 \\
0\end{array}$ & $\begin{array}{l}0 \\
3 \\
1 \\
8 \\
0\end{array}$ & $\begin{array}{l}0 \\
1 \\
0 \\
0 \\
0\end{array}$ & $\begin{array}{l}0 \\
4 \\
8 \\
8 \\
0\end{array}$ \\
\hline $\begin{array}{l}1975-1976 \\
1976-1977 \\
1977-1978 \\
1978-1979 \\
1979-1980\end{array}$ & $\begin{array}{l}0 \\
0 \\
0 \\
0 \\
0\end{array}$ & $\begin{array}{l}2 \\
8 \\
3 \\
7 \\
1\end{array}$ & $\begin{array}{l}23 \\
17 \\
18 \\
26 \\
23\end{array}$ & $\begin{array}{l}28 \\
30 \\
25 \\
31 \\
22\end{array}$ & $\begin{array}{l}30 \\
30 \\
22 \\
31 \\
31\end{array}$ & $\begin{array}{l}22 \\
19 \\
17 \\
21 \\
22\end{array}$ & $\begin{array}{l}19 \\
14 \\
11 \\
13 \\
13\end{array}$ & $\begin{array}{l}6 \\
1 \\
4 \\
2 \\
3\end{array}$ & $\begin{array}{l}0 \\
0 \\
0 \\
0 \\
0\end{array}$ & $\begin{array}{l}130 \\
119 \\
100 \\
131 \\
115\end{array}$ & $\begin{array}{l}0 \\
0 \\
0 \\
0 \\
0\end{array}$ & $\begin{array}{l}0 \\
0 \\
1 \\
3 \\
0\end{array}$ & $\begin{array}{l}0 \\
0 \\
2 \\
8 \\
1\end{array}$ & $\begin{array}{l}0 \\
0 \\
0 \\
2 \\
0\end{array}$ & $\begin{array}{r}0 \\
0 \\
3 \\
13 \\
1\end{array}$ \\
\hline $\begin{array}{l}1980-1981 \\
1981-1982 \\
1982-1983 \\
1983-1984 \\
1984-1985\end{array}$ & $\begin{array}{l}0 \\
0 \\
0 \\
0 \\
0\end{array}$ & $\begin{array}{r}4 \\
5 \\
4 \\
3 \\
14\end{array}$ & $\begin{array}{l}16 \\
13 \\
21 \\
11 \\
20\end{array}$ & $\begin{array}{l}16 \\
23 \\
26 \\
31 \\
31\end{array}$ & $\begin{array}{l}17 \\
27 \\
20 \\
26 \\
31\end{array}$ & $\begin{array}{l}17 \\
17 \\
13 \\
17 \\
25\end{array}$ & $\begin{array}{r}11 \\
12 \\
4 \\
5 \\
20\end{array}$ & $\begin{array}{r}6 \\
12 \\
9 \\
2 \\
2\end{array}$ & $\begin{array}{l}0 \\
0 \\
0 \\
0 \\
0\end{array}$ & $\begin{array}{c}87 \\
109 \\
97 \\
95 \\
143^{(a)}\end{array}$ & $\begin{array}{l}0 \\
0 \\
0 \\
0 \\
0\end{array}$ & $\begin{array}{l}0 \\
0 \\
0 \\
4 \\
4\end{array}$ & $\begin{array}{l}0 \\
2 \\
0 \\
0 \\
0\end{array}$ & $\begin{array}{l}0 \\
0 \\
0 \\
0 \\
3\end{array}$ & $\begin{array}{l}0 \\
2 \\
0 \\
4 \\
7\end{array}$ \\
\hline $\begin{array}{l}1985-1986 \\
1986-1987 \\
1987-1988 \\
1988-1989 \\
1989-1990\end{array}$ & $\begin{array}{l}0 \\
0 \\
0 \\
0 \\
0\end{array}$ & $\begin{array}{l}7 \\
0 \\
3 \\
1 \\
2\end{array}$ & $\begin{array}{l}23 \\
11 \\
11 \\
12 \\
11\end{array}$ & $\begin{array}{l}31 \\
29 \\
25 \\
23 \\
25\end{array}$ & $\begin{array}{l}23 \\
25 \\
29 \\
24 \\
18\end{array}$ & $\begin{array}{l}17 \\
17 \\
22 \\
25 \\
20\end{array}$ & $\begin{array}{r}8 \\
9 \\
13 \\
11 \\
11\end{array}$ & $\begin{array}{l}4 \\
2 \\
2 \\
0 \\
0\end{array}$ & $\begin{array}{l}0 \\
0 \\
0 \\
0 \\
0\end{array}$ & $\begin{array}{c}113 \\
93 \\
105 \\
96 \\
87\end{array}$ & $\begin{array}{l}5 \\
0 \\
0 \\
0 \\
0\end{array}$ & $\begin{array}{l}1 \\
0 \\
0 \\
0 \\
0\end{array}$ & $\begin{array}{l}0 \\
0 \\
0 \\
0 \\
0\end{array}$ & $\begin{array}{l}0 \\
0 \\
0 \\
4 \\
0\end{array}$ & $\begin{array}{l}6 \\
0 \\
0 \\
4 \\
0\end{array}$ \\
\hline $\begin{array}{l}1990-1991 \\
1991-1992 \\
1992-1993 \\
1993-1994 \\
1994-1995\end{array}$ & $\begin{array}{l}0 \\
0 \\
0 \\
0 \\
0\end{array}$ & $\begin{array}{l}2 \\
6 \\
1 \\
5 \\
2\end{array}$ & $\begin{array}{r}11 \\
8 \\
9 \\
26 \\
20\end{array}$ & $\begin{array}{l}27 \\
18 \\
29 \\
22 \\
24\end{array}$ & $\begin{array}{l}27 \\
22 \\
27 \\
21 \\
20\end{array}$ & $\begin{array}{l}14 \\
11 \\
23 \\
21 \\
11\end{array}$ & $\begin{array}{r}14 \\
3 \\
10 \\
12 \\
13\end{array}$ & $\begin{array}{l}2 \\
2 \\
1 \\
0 \\
2\end{array}$ & $\begin{array}{l}0 \\
0 \\
0 \\
0 \\
0\end{array}$ & $\begin{array}{c}97 \\
70^{(a)} \\
100 \\
107 \\
92\end{array}$ & $\begin{array}{l}0 \\
0 \\
0 \\
1 \\
0\end{array}$ & $\begin{array}{l}8 \\
0 \\
0 \\
0 \\
0\end{array}$ & $\begin{array}{l}0 \\
0 \\
2 \\
0 \\
0\end{array}$ & $\begin{array}{l}0 \\
0 \\
0 \\
0 \\
0\end{array}$ & $\begin{array}{l}8 \\
0 \\
2 \\
1 \\
0\end{array}$ \\
\hline $\begin{array}{l}1995-1996 \\
1996-1997 \\
1997-1998 \\
1998-1999\end{array}$ & $\begin{array}{l}0 \\
0 \\
0 \\
0\end{array}$ & $\begin{array}{l}3 \\
7 \\
4 \\
5\end{array}$ & $\begin{array}{l}12 \\
19 \\
12 \\
10\end{array}$ & $\begin{array}{l}25 \\
26 \\
25 \\
23\end{array}$ & $\begin{array}{r}28 \\
24 \\
21 \\
-\end{array}$ & $\begin{array}{l}22 \\
18 \\
14 \\
-\end{array}$ & $\begin{array}{r}13 \\
10 \\
9 \\
-\end{array}$ & $\begin{array}{l}5 \\
7 \\
3 \\
-\end{array}$ & $\begin{array}{l}1 \\
1 \\
0 \\
-\end{array}$ & $\begin{array}{r}109 \\
112 \\
88 \\
-\end{array}$ & $\begin{array}{l}0 \\
0 \\
0 \\
0\end{array}$ & $\begin{array}{l}0 \\
1 \\
0 \\
1\end{array}$ & $\begin{array}{l}2 \\
0 \\
0 \\
-\end{array}$ & $\begin{array}{l}3 \\
0 \\
0 \\
-\end{array}$ & $\begin{array}{l}5 \\
1 \\
0^{(2, b)} \\
-\end{array}$ \\
\hline $\begin{array}{l}\text { Average } \\
\text { Normal }\end{array}$ & $\begin{array}{l}<1 \\
<1\end{array}$ & $\begin{array}{l}4 \\
4\end{array}$ & $\begin{array}{l}15 \\
15\end{array}$ & $\begin{array}{l}24 \\
25\end{array}$ & $\begin{array}{l}26 \\
26\end{array}$ & $\begin{array}{l}19 \\
19\end{array}$ & $\begin{array}{l}13 \\
13\end{array}$ & $\begin{array}{l}4 \\
4\end{array}$ & $\begin{array}{l}<1 \\
<1\end{array}$ & $\begin{array}{l}106 \\
106\end{array}$ & $\begin{array}{l}<1 \\
<1\end{array}$ & $\begin{array}{l}1 \\
1\end{array}$ & 1 & $\begin{array}{l}<1 \\
<1\end{array}$ & $\begin{array}{l}3 \\
3\end{array}$ \\
\hline
\end{tabular}

(a) Greatest and least seasonal totals.

(b) Most recent of numerous occurrences. 
Table 3.8. Days with Minimum Temperatures $\leq 0^{\circ} \mathrm{F}$

\begin{tabular}{|c|c|c|c|c|c|c|}
\hline \multirow{2}{*}{$\frac{\begin{array}{c}\text { Temperature } \\
\left({ }^{\circ} \mathrm{F}\right)\end{array}}{-23}$} & \multicolumn{6}{|c|}{ Date(s) of Occurrence } \\
\hline & $02 / 03 / 50$ & $02 / 01 / 50$ & & & & \\
\hline-22 & $01 / 26 / 57$ & & & & & \\
\hline-21 & $01 / 27 / 57$ & $02 / 02 / 50$ & $01 / 31 / 50$ & & & \\
\hline-18 & $02 / 01 / 96$ & $01 / 31 / 96$ & $01 / 29 / 50$ & & & \\
\hline-15 & $02 / 03 / 96$ & & & & & \\
\hline-14 & $02 / 02 / 96$ & $12 / 30 / 68$ & $01 / 29 / 57$ & $01 / 28 / 57$ & & \\
\hline-13 & $11 / 23 / 85$ & $12 / 22 / 83$ & $01 / 09 / 74$ & $12 / 16 / 64$ & $01 / 30 / 50$ & \\
\hline-12 & $12 / 22 / 90$ & $11 / 24 / 85$ & $02 / 01 / 79$ & $12 / 17 / 64$ & $01 / 25 / 57$ & \\
\hline-11 & $01 / 30 / 96$ & $01 / 01 / 79$ & $01 / 17 / 50$ & $01 / 14 / 50$ & $01 / 25 / 49$ & \\
\hline-10 & $\begin{array}{l}12 / 29 / 90 \\
02 / 02 / 56\end{array}$ & $\begin{array}{l}12 / 21 / 90 \\
02 / 01 / 56\end{array}$ & $02 / 02 / 79$ & $12 / 30 / 78$ & $01 / 06 / 74$ & $12 / 29 / 68$ \\
\hline-9 & $12 / 23 / 83$ & $01 / 06 / 79$ & $12 / 31 / 78$ & $01 / 02 / 78$ & $01 / 08 / 74$ & \\
\hline-8 & $\begin{array}{l}12 / 01 / 85 \\
01 / 16 / 50\end{array}$ & $01 / 06 / 82$ & $01 / 07 / 74$ & $12 / 10 / 72$ & $01 / 23 / 69$ & $01 / 30 / 57$ \\
\hline-7 & $01 / 07 / 79$ & $01 / 31 / 56$ & $01 / 28 / 50$ & $01 / 05 / 50$ & & \\
\hline-6 & $\begin{array}{l}12 / 28 / 96 \\
01 / 29 / 69 \\
01 / 11 / 49\end{array}$ & $\begin{array}{l}11 / 22 / 85 \\
01 / 28 / 69\end{array}$ & $\begin{array}{l}01 / 31 / 79 \\
01 / 18 / 57\end{array}$ & $\begin{array}{l}01 / 05 / 74 \\
01 / 20 / 54\end{array}$ & $\begin{array}{l}12 / 13 / 72 \\
01 / 04 / 50\end{array}$ & $\begin{array}{l}12 / 08 / 72 \\
01 / 24 / 49\end{array}$ \\
\hline-5 & $\begin{array}{l}02 / 05 / 89 \\
01 / 15 / 50\end{array}$ & $02 / 04 / 85$ & $01 / 01 / 78$ & $1 / 10 / 74$ & $12 / 12 / 72$ & $12 / 09 / 72$ \\
\hline-4 & $\begin{array}{l}01 / 13 / 93 \\
01 / 11 / 74 \\
01 / 12 / 49\end{array}$ & $\begin{array}{l}12 / 23 / 90 \\
12 / 11 / 72\end{array}$ & $\begin{array}{l}02 / 04 / 89 \\
01 / 28 / 72\end{array}$ & $\begin{array}{l}12 / 19 / 84 \\
01 / 12 / 63\end{array}$ & $\begin{array}{l}12 / 21 / 83 \\
01 / 28 / 49\end{array}$ & $\begin{array}{l}01 / 27 / 79 \\
01 / 13 / 49\end{array}$ \\
\hline-3 & $\begin{array}{l}02 / 06 / 89 \\
12 / 29 / 78 \\
01 / 11 / 63\end{array}$ & $\begin{array}{l}11 / 25 / 85 \\
12 / 31 / 77 \\
01 / 17 / 57\end{array}$ & $\begin{array}{l}02 / 03 / 85 \\
01 / 31 / 69\end{array}$ & $\begin{array}{l}12 / 18 / 84 \\
01 / 30 / 69\end{array}$ & $\begin{array}{l}01 / 10 / 80 \\
12 / 31 / 68\end{array}$ & $\begin{array}{l}01 / 08 / 79 \\
12 / 28 / 68\end{array}$ \\
\hline-2 & $\begin{array}{l}12 / 31 / 90 \\
01 / 04 / 74 \\
01 / 10 / 49\end{array}$ & $\begin{array}{l}12 / 30 / 90 \\
12 / 14 / 72 \\
12 / 27 / 48\end{array}$ & $\begin{array}{l}12 / 20 / 90 \\
01 / 22 / 62\end{array}$ & $\begin{array}{l}12 / 21 / 84 \\
01 / 31 / 57\end{array}$ & $\begin{array}{l}12 / 20 / 84 \\
01 / 19 / 57\end{array}$ & $\begin{array}{l}01 / 05 / 79 \\
01 / 20 / 49\end{array}$ \\
\hline-1 & $\begin{array}{l}12 / 21 / 98 \\
02 / 01 / 69 \\
01 / 30 / 56\end{array}$ & $\begin{array}{l}11 / 24 / 93 \\
01 / 18 / 60 \\
11 / 14 / 55\end{array}$ & $\begin{array}{l}11 / 26 / 85 \\
01 / 04 / 59 \\
02 / 04 / 50\end{array}$ & $\begin{array}{l}01 / 08 / 73 \\
02 / 02 / 57 \\
01 / 25 / 50\end{array}$ & $\begin{array}{l}02 / 03 / 72 \\
01 / 16 / 57 \\
01 / 13 / 50\end{array}$ & $\begin{array}{l}01 / 26 / 72 \\
02 / 03 / 56\end{array}$ \\
\hline 0 & $\begin{array}{l}01 / 11 / 93 \\
01 / 28 / 79 \\
01 / 26 / 50\end{array}$ & $\begin{array}{l}12 / 24 / 90 \\
01 / 27 / 72 \\
01 / 04 / 49\end{array}$ & $\begin{array}{l}02 / 02 / 89 \\
01 / 03 / 59 \\
12 / 26 / 48\end{array}$ & $\begin{array}{l}02 / 06 / 85 \\
01 / 24 / 57 \\
01 / 15 / 47\end{array}$ & $\begin{array}{l}12 / 27 / 83 \\
01 / 21 / 54\end{array}$ & $\begin{array}{l}01 / 07 / 82 \\
01 / 27 / 50\end{array}$ \\
\hline
\end{tabular}


Table 3.9. Monthly and Annual Minimum Temperatures $\left({ }^{\circ} \mathrm{F}\right)$

\begin{tabular}{|c|c|c|c|c|c|c|c|c|c|c|c|c|c|}
\hline Year & Jan & Feb & Mar & Apr & May & Jun & Jul & Aug & Sep & Oct & Nov & Dec & Annual \\
\hline 1945 & 21 & 14 & 10 & 28 & 38 & 46 & 53 & 47 & 35 & 26 & 16 & 13 & 10 \\
\hline $\begin{array}{l}1946 \\
1947 \\
1948 \\
1949 \\
1950\end{array}$ & $\begin{array}{r}18 \\
0 \\
14 \\
-11 \\
-21\end{array}$ & $\begin{array}{r}18 \\
11 \\
1 \\
3 \\
-23\end{array}$ & $\begin{array}{l}25 \\
23 \\
13 \\
27 \\
20\end{array}$ & $\begin{array}{l}30 \\
32 \\
28 \\
30 \\
27\end{array}$ & $\begin{array}{l}33 \\
42 \\
32 \\
31 \\
38\end{array}$ & $\begin{array}{l}44 \\
45 \\
51 \\
42 \\
44\end{array}$ & $\begin{array}{l}50 \\
53 \\
49 \\
49 \\
49\end{array}$ & $\begin{array}{l}49 \\
50 \\
47 \\
47 \\
51\end{array}$ & $\begin{array}{l}35 \\
40 \\
34 \\
38 \\
38\end{array}$ & $\begin{array}{l}21 \\
34 \\
22 \\
23 \\
34\end{array}$ & $\begin{array}{l}16 \\
22 \\
20 \\
28 \\
21\end{array}$ & $\begin{array}{r}6 \\
16 \\
-2 \\
10 \\
22\end{array}$ & $\begin{array}{r}6 \\
0 \\
-2 \\
-11 \\
-23\end{array}$ \\
\hline $\begin{array}{l}1951 \\
1952 \\
1953 \\
1954 \\
1955\end{array}$ & $\begin{array}{r}6 \\
5 \\
24 \\
-6 \\
18\end{array}$ & $\begin{array}{l}18 \\
16 \\
20 \\
17 \\
15\end{array}$ & $\begin{array}{r}22 \\
24 \\
23 \\
18 \\
6\end{array}$ & $\begin{array}{l}26 \\
27 \\
27 \\
26 \\
26\end{array}$ & $\begin{array}{l}37 \\
37 \\
36 \\
28 \\
31\end{array}$ & $\begin{array}{l}41 \\
42 \\
40 \\
41 \\
42\end{array}$ & $\begin{array}{l}51 \\
49 \\
52 \\
45 \\
43\end{array}$ & $\begin{array}{l}47 \\
46 \\
51 \\
48 \\
48\end{array}$ & $\begin{array}{l}39 \\
42 \\
37 \\
36 \\
37\end{array}$ & $\begin{array}{l}27 \\
34 \\
30 \\
26 \\
32\end{array}$ & $\begin{array}{r}23 \\
7 \\
24 \\
23 \\
-1\end{array}$ & $\begin{array}{r}4 \\
17 \\
20 \\
14 \\
9\end{array}$ & $\begin{array}{r}4 \\
5 \\
20 \\
-6 \\
-1\end{array}$ \\
\hline $\begin{array}{l}1956 \\
1957 \\
1958 \\
1959 \\
1960\end{array}$ & $\begin{array}{r}-7 \\
-22 \\
16 \\
-1 \\
-1\end{array}$ & $\begin{array}{r}-10 \\
-1 \\
29 \\
19 \\
10\end{array}$ & $\begin{array}{l}15 \\
28 \\
23 \\
25 \\
13\end{array}$ & $\begin{array}{l}28 \\
34 \\
34 \\
30 \\
30\end{array}$ & $\begin{array}{l}38 \\
48 \\
38 \\
30 \\
33\end{array}$ & $\begin{array}{l}40 \\
48 \\
47 \\
41 \\
46\end{array}$ & $\begin{array}{l}54 \\
51 \\
49 \\
49 \\
52\end{array}$ & $\begin{array}{l}49 \\
52 \\
53 \\
49 \\
41\end{array}$ & $\begin{array}{l}39 \\
36 \\
34 \\
41 \\
40\end{array}$ & $\begin{array}{l}31 \\
32 \\
30 \\
26 \\
30\end{array}$ & $\begin{array}{r}15 \\
20 \\
9 \\
6 \\
22\end{array}$ & $\begin{array}{r}2 \\
23 \\
21 \\
14 \\
14\end{array}$ & $\begin{array}{r}-10 \\
-22 \\
9 \\
-1 \\
-1\end{array}$ \\
\hline $\begin{array}{l}1961 \\
1962 \\
1963 \\
1964 \\
1965\end{array}$ & $\begin{array}{l}16 \\
-2 \\
4 \\
15 \\
10\end{array}$ & $\begin{array}{r}27 \\
7 \\
8 \\
19 \\
18\end{array}$ & $\begin{array}{l}25 \\
15 \\
22 \\
15 \\
14\end{array}$ & $\begin{array}{l}31 \\
33 \\
28 \\
30 \\
32\end{array}$ & $\begin{array}{l}38 \\
31 \\
36 \\
35 \\
32\end{array}$ & $\begin{array}{l}44 \\
37 \\
45 \\
45 \\
48\end{array}$ & $\begin{array}{l}50 \\
42 \\
49 \\
50 \\
50\end{array}$ & $\begin{array}{l}56 \\
49 \\
49 \\
44 \\
42\end{array}$ & $\begin{array}{l}36 \\
40 \\
45 \\
39 \\
33\end{array}$ & $\begin{array}{l}26 \\
34 \\
23 \\
30 \\
30\end{array}$ & $\begin{array}{l}10 \\
16 \\
17 \\
20 \\
26\end{array}$ & $\begin{array}{r}3 \\
16 \\
7 \\
-13 \\
10\end{array}$ & $\begin{array}{r}3 \\
-2 \\
-4 \\
-13 \\
10\end{array}$ \\
\hline $\begin{array}{l}1966 \\
1967 \\
1968 \\
1969 \\
1970\end{array}$ & $\begin{array}{r}17 \\
23 \\
10 \\
-8 \\
8\end{array}$ & $\begin{array}{l}19 \\
20 \\
15 \\
-1 \\
21\end{array}$ & $\begin{array}{l}19 \\
20 \\
25 \\
22 \\
24\end{array}$ & $\begin{array}{l}26 \\
27 \\
23 \\
31 \\
26\end{array}$ & $\begin{array}{l}37 \\
34 \\
33 \\
38 \\
30\end{array}$ & $\begin{array}{l}38 \\
47 \\
42 \\
52 \\
46\end{array}$ & $\begin{array}{l}48 \\
52 \\
51 \\
53 \\
50\end{array}$ & $\begin{array}{l}50 \\
56 \\
47 \\
45 \\
52\end{array}$ & $\begin{array}{l}43 \\
43 \\
39 \\
41 \\
34\end{array}$ & $\begin{array}{l}29 \\
30 \\
30 \\
29 \\
23\end{array}$ & $\begin{array}{l}22 \\
17 \\
23 \\
19 \\
11\end{array}$ & $\begin{array}{r}22 \\
6 \\
-14 \\
19 \\
8\end{array}$ & $\begin{array}{r}17 \\
6 \\
-14 \\
-8 \\
8\end{array}$ \\
\hline $\begin{array}{l}1971 \\
1972 \\
1973 \\
1974 \\
1975\end{array}$ & $\begin{array}{r}8 \\
-4 \\
-1 \\
-13 \\
14\end{array}$ & $\begin{array}{l}15 \\
-1 \\
21 \\
23 \\
10\end{array}$ & $\begin{array}{l}15 \\
24 \\
26 \\
21 \\
19\end{array}$ & $\begin{array}{l}27 \\
26 \\
27 \\
33 \\
21\end{array}$ & $\begin{array}{l}36 \\
36 \\
34 \\
32 \\
33\end{array}$ & $\begin{array}{l}44 \\
45 \\
45 \\
41 \\
38\end{array}$ & $\begin{array}{l}44 \\
50 \\
46 \\
48 \\
53\end{array}$ & $\begin{array}{l}51 \\
49 \\
46 \\
48 \\
46\end{array}$ & $\begin{array}{l}38 \\
30 \\
43 \\
40 \\
44\end{array}$ & $\begin{array}{l}13 \\
20 \\
31 \\
29 \\
26\end{array}$ & $\begin{array}{l}21 \\
24 \\
16 \\
24 \\
15\end{array}$ & $\begin{array}{r}5 \\
-8 \\
14 \\
17 \\
14\end{array}$ & $\begin{array}{r}5 \\
-8 \\
-1 \\
-13 \\
10\end{array}$ \\
\hline $\begin{array}{l}1976 \\
1977 \\
1978 \\
1979 \\
1980\end{array}$ & $\begin{array}{r}16 \\
4 \\
-9 \\
-11 \\
-3\end{array}$ & $\begin{array}{r}10 \\
21 \\
17 \\
-12 \\
19\end{array}$ & $\begin{array}{l}11 \\
24 \\
25 \\
20 \\
25\end{array}$ & $\begin{array}{l}25 \\
31 \\
30 \\
29 \\
28\end{array}$ & $\begin{array}{l}35 \\
34 \\
37 \\
38 \\
38\end{array}$ & $\begin{array}{l}37 \\
39 \\
44 \\
45 \\
40\end{array}$ & $\begin{array}{l}47 \\
49 \\
50 \\
39 \\
47\end{array}$ & $\begin{array}{l}44 \\
48 \\
47 \\
53 \\
42\end{array}$ & $\begin{array}{l}42 \\
36 \\
41 \\
42 \\
41\end{array}$ & $\begin{array}{l}28 \\
28 \\
21 \\
32 \\
30\end{array}$ & $\begin{array}{r}13 \\
9 \\
7 \\
13 \\
18\end{array}$ & $\begin{array}{r}12 \\
-3 \\
-10 \\
19 \\
9\end{array}$ & $\begin{array}{r}10 \\
-3 \\
-10 \\
-12 \\
-3\end{array}$ \\
\hline $\begin{array}{l}1981 \\
1982 \\
1983 \\
1984 \\
1985\end{array}$ & $\begin{array}{r}23 \\
-8 \\
12 \\
10 \\
5\end{array}$ & $\begin{array}{r}8 \\
9 \\
15 \\
24 \\
-5\end{array}$ & $\begin{array}{l}24 \\
24 \\
29 \\
25 \\
21\end{array}$ & $\begin{array}{l}24 \\
24 \\
27 \\
30 \\
26\end{array}$ & $\begin{array}{l}35 \\
33 \\
37 \\
33 \\
33\end{array}$ & $\begin{array}{l}40 \\
47 \\
40 \\
37 \\
44\end{array}$ & $\begin{array}{l}45 \\
45 \\
49 \\
51 \\
56\end{array}$ & $\begin{array}{l}48 \\
51 \\
50 \\
47 \\
46\end{array}$ & $\begin{array}{l}34 \\
41 \\
35 \\
36 \\
33\end{array}$ & $\begin{array}{l}27 \\
26 \\
29 \\
12 \\
26\end{array}$ & $\begin{array}{r}19 \\
18 \\
22 \\
25 \\
-13\end{array}$ & $\begin{array}{r}8 \\
13 \\
-13 \\
-4 \\
-8\end{array}$ & $\begin{array}{r}8 \\
-8 \\
-13 \\
-4 \\
-13\end{array}$ \\
\hline $\begin{array}{l}1986 \\
1987 \\
1988 \\
1989 \\
1990\end{array}$ & $\begin{array}{r}12 \\
9 \\
14 \\
15 \\
22\end{array}$ & $\begin{array}{r}15 \\
18 \\
9 \\
-5 \\
9\end{array}$ & $\begin{array}{l}29 \\
24 \\
24 \\
14 \\
24\end{array}$ & $\begin{array}{l}28 \\
30 \\
31 \\
35 \\
37\end{array}$ & $\begin{array}{l}37 \\
38 \\
35 \\
39 \\
39\end{array}$ & $\begin{array}{l}43 \\
43 \\
42 \\
46 \\
47\end{array}$ & $\begin{array}{l}48 \\
49 \\
47 \\
49 \\
46\end{array}$ & $\begin{array}{l}54 \\
51 \\
52 \\
52 \\
52\end{array}$ & $\begin{array}{l}38 \\
41 \\
38 \\
44 \\
48\end{array}$ & $\begin{array}{l}33 \\
31 \\
32 \\
27 \\
31\end{array}$ & $\begin{array}{l}16 \\
17 \\
28 \\
21 \\
28\end{array}$ & $\begin{array}{r}18 \\
9 \\
8 \\
19 \\
-12\end{array}$ & $\begin{array}{r}12 \\
9 \\
8 \\
-5 \\
-12\end{array}$ \\
\hline $\begin{array}{l}1991 \\
1992 \\
1993 \\
1994 \\
1995\end{array}$ & $\begin{array}{r}5 \\
19 \\
-4 \\
20 \\
8\end{array}$ & $\begin{array}{r}26 \\
22 \\
3 \\
5 \\
8\end{array}$ & $\begin{array}{l}22 \\
32 \\
17 \\
19 \\
21\end{array}$ & $\begin{array}{l}31 \\
27 \\
32 \\
35 \\
28\end{array}$ & $\begin{array}{l}38 \\
37 \\
35 \\
36 \\
39\end{array}$ & $\begin{array}{l}44 \\
49 \\
46 \\
44 \\
47\end{array}$ & $\begin{array}{l}55 \\
54 \\
50 \\
50 \\
52\end{array}$ & $\begin{array}{l}47 \\
43 \\
43 \\
53 \\
45\end{array}$ & $\begin{array}{l}42 \\
40 \\
37 \\
47 \\
42\end{array}$ & $\begin{array}{l}23 \\
30 \\
29 \\
30 \\
16\end{array}$ & $\begin{array}{l}23 \\
17 \\
-1 \\
19 \\
17\end{array}$ & $\begin{array}{r}20 \\
12 \\
21 \\
8 \\
16\end{array}$ & $\begin{array}{r}5 \\
12 \\
-4 \\
5 \\
8\end{array}$ \\
\hline $\begin{array}{l}1996 \\
1997 \\
1998\end{array}$ & $\begin{array}{r}-18 \\
8 \\
7\end{array}$ & $\begin{array}{r}-18 \\
20 \\
22\end{array}$ & $\begin{array}{l}18 \\
28 \\
23\end{array}$ & $\begin{array}{l}30 \\
25 \\
29\end{array}$ & $\begin{array}{l}29 \\
30 \\
39\end{array}$ & $\begin{array}{l}45 \\
46 \\
47\end{array}$ & $\begin{array}{l}49 \\
49 \\
58\end{array}$ & $\begin{array}{l}48 \\
52 \\
50\end{array}$ & $\begin{array}{l}34 \\
44 \\
43\end{array}$ & $\begin{array}{l}34 \\
29 \\
25\end{array}$ & $\begin{array}{l}17 \\
23 \\
27\end{array}$ & $\begin{array}{l}-6 \\
19 \\
-1\end{array}$ & $\begin{array}{r}-18 \\
8 \\
-1\end{array}$ \\
\hline
\end{tabular}




\subsection{Monthly Extreme Daily Maximum and Minimum Temperatures}

Monthly extreme daily maximum and minimum temperatures are presented in Table 3.10. Note that a greater temperature range exists during the winter than in the summer. February temperatures ranged from $72^{\circ} \mathrm{F}$ to $-23^{\circ} \mathrm{F}$, a range of $95^{\circ} \mathrm{F}$. July temperatures ranged from $112^{\circ} \mathrm{F}$ to $39^{\circ} \mathrm{F}$, a range of $83^{\circ} \mathrm{F}$.

\subsection{Average Daily Temperature Range}

Table 3.11 represents the average daily temperature range by month and year for the period 1945 through 1998. This statistic is compiled by determining each daily temperature range (the difference between the maximum and minimum temperature), totaling for every day of the month, and dividing by the number of days in the month. As can be seen from the table, the average daily temperature ranges for July and August $\left(>30^{\circ} \mathrm{F}\right)$ are more than double the ranges for December and January $\left(<15^{\circ} \mathrm{F}\right)$. The lowest average daily temperature range was $8.0^{\circ} \mathrm{F}$ in December 1985 ; the greatest was $34.5^{\circ} \mathrm{F}$ in August 1967 . The greatest range for any single day was $48^{\circ} \mathrm{F}$ on August 14,1995 (high of $93^{\circ} \mathrm{F}$, low of $45^{\circ} \mathrm{F}$ ) and also on May 11,1946 (high of $86^{\circ} \mathrm{F}$, low of $38^{\circ} \mathrm{F}$ ). The least range for any single day was $1^{\circ} \mathrm{F}$ as recently as December 6, 1997 (high of $30^{\circ} \mathrm{F}$, low of $29^{\circ} \mathrm{F}$ ).

\subsection{Normal and Extreme Daily Temperatures}

Table 3.12 lists the normal and extreme daily maximum and minimum temperatures. Climatological normals are computed every 10 years and are based on a 30-year period, ending with the first year of each new decade. The current normal period is 1961 through 1990; the next will be 1971 through 2000 . The normal temperatures in Table 3.12 are computed using a 7-day running mean, centered about each day.

Four possible temperature extremes are presented for each day, a record high and low maximum and a record high and low minimum. These daily records, plus the year of occurrence for the period 1945 through 1998, are also indicated in Table 3.12.

\subsection{Subsurface Soil Temperatures}

Hourly subsurface soil temperature data at depths of $\sim 0.5 \mathrm{in} ., 15 \mathrm{in}$., and $36 \mathrm{in}$. are available for the period from 1955 through 1998 . The subsurface soil temperature sensors are installed in the natural soil of the area with the vegetation cover removed. The soil is sandy and mixed with large gravel.

Monthly averages and extremes of monthly averages are presented in Table 3.13. The absolute hourly extremes are also indicated in that table. 
Table 3.10. Monthly Normal Temperatures $\left({ }^{\circ} \mathrm{F}\right)$ and Monthly Extremes of Maximum and Minimum Temperatures $\left({ }^{\circ} \mathrm{F}\right)$

\begin{tabular}{|c|c|c|c|c|c|c|c|c|c|c|c|c|c|c|c|}
\hline \multirow[b]{3}{*}{ Month } & \multirow{2}{*}{\multicolumn{3}{|c|}{ Normal (1961-1990) }} & \multicolumn{12}{|c|}{ Daily Extreme } \\
\hline & & & & \multicolumn{6}{|c|}{ Maximum } & \multicolumn{6}{|c|}{ Minimum } \\
\hline & Maximum & Minimum & Mean & High & Day & Year & Low & Day & Year & High & Day & Year & Low & Day & Year \\
\hline Jan & 38.4 & 24.0 & 31.3 & 72 & 31 & 1971 & -2 & 31 & 1950 & 53 & 30 & 1971 & -22 & 26 & 1957 \\
\hline Feb & 47.3 & 28.8 & 38.0 & $\begin{array}{l}72 \\
72\end{array}$ & $\begin{array}{l}25 \\
24\end{array}$ & $\begin{array}{l}1986 \\
1986\end{array}$ & -3 & 1 & 1950 & 60 & 24 & 1986 & $\begin{array}{l}-23 \\
-23\end{array}$ & $\begin{array}{l}3 \\
1\end{array}$ & $\begin{array}{l}1950 \\
1950\end{array}$ \\
\hline Mar & 57.3 & 33.9 & 45.6 & 83 & 25 & 1960 & 24 & 3 & 1960 & $\begin{array}{l}50 \\
50 \\
50\end{array}$ & $\begin{array}{r}15 \\
5 \\
3\end{array}$ & $\begin{array}{l}1992 \\
1987 \\
1987\end{array}$ & 6 & 5 & 1955 \\
\hline Apr & 66.0 & 39.6 & 52.7 & 94 & 24 & 1977 & 41 & 7 & 1945 & 64 & 28 & 1987 & 21 & 5 & 1975 \\
\hline May & 75.2 & 47.4 & 61.3 & $\begin{array}{l}104 \\
104\end{array}$ & $\begin{array}{l}31 \\
30\end{array}$ & $\begin{array}{l}1986 \\
1986\end{array}$ & 51 & 11 & 1967 & 71 & 29 & 1986 & 28 & 1 & 1954 \\
\hline Jun & 83.9 & 55.3 & 69.7 & 111 & 23 & 1992 & 55 & 3 & 1966 & 80 & 24 & 1992 & $\begin{array}{l}37 \\
37 \\
37\end{array}$ & $\begin{array}{l}3 \\
2 \\
1\end{array}$ & $\begin{array}{l}1962 \\
1976 \\
1984\end{array}$ \\
\hline Jul & 91.4 & 60.9 & 76.2 & 112 & 27 & 1998 & 59 & 2 & 1966 & 82 & 23 & 1994 & 39 & 2 & 1979 \\
\hline Aug & 90.2 & 59.9 & 75.1 & 113 & 4 & 1961 & 67 & 20 & 1959 & 81 & 4 & 1961 & 41 & 22 & 1960 \\
\hline Sep & 80.1 & 51.2 & 65.7 & 106 & 1 & 1987 & 52 & 22 & 1984 & 72 & 7 & 1955 & $\begin{array}{l}30 \\
30\end{array}$ & $\begin{array}{l}27 \\
25\end{array}$ & $\begin{array}{l}1972 \\
1972\end{array}$ \\
\hline Oct & 65.7 & 40.3 & 52.9 & $\begin{array}{l}89 \\
89\end{array}$ & $\begin{array}{l}4 \\
3\end{array}$ & $\begin{array}{l}1980 \\
1958\end{array}$ & 32 & 30 & 1971 & $\begin{array}{l}60 \\
60 \\
60\end{array}$ & $\begin{array}{r}25 \\
15 \\
2\end{array}$ & $\begin{array}{l}1945 \\
1988 \\
1988\end{array}$ & 12 & 31 & 1984 \\
\hline Nov & 48.7 & 31.9 & 40.2 & 75 & 3 & 1975 & 6 & 24 & 1985 & 60 & 9 & 1989 & -13 & 23 & 1985 \\
\hline Dec & 38.1 & 24.7 & 31.4 & 69 & 26 & 1980 & $\begin{array}{l}-2 \\
-2\end{array}$ & $\begin{array}{l}30 \\
29\end{array}$ & $\begin{array}{l}1968 \\
1968\end{array}$ & 56 & 2 & 1975 & -14 & 30 & 1968 \\
\hline Annual & & & 53.3 & 113 & & $8 / 4 / 61$ & -3 & & $2 / 1 / 50$ & 82 & & $7 / 23 / 94$ & -23 & & $\begin{array}{l}2 / 3 / 50 \\
2 / 1 / 50\end{array}$ \\
\hline
\end{tabular}


Table 3.11. Average Daily Temperature $\left({ }^{\circ} \mathrm{F}\right)$ Range

\begin{tabular}{|c|c|c|c|c|c|c|c|c|c|c|c|c|c|}
\hline Year & Jan & Feb & Mar & Apr & May & Jun & Jul & Aug & Sep & Oct & Nov & Dec & Annual \\
\hline $\begin{array}{l}1945 \\
1946 \\
1947 \\
1948 \\
1949 \\
1950\end{array}$ & $\begin{array}{c}10.5 \\
17.3 \\
18.4 \\
15.0 \\
18.8^{(2)} \\
16.0\end{array}$ & $\begin{array}{l}17.4 \\
21.0 \\
22.2 \\
17.2 \\
19.3 \\
15.6\end{array}$ & $\begin{array}{l}19.5 \\
21.8 \\
25.5 \\
23.0 \\
20.6 \\
20.0\end{array}$ & $\begin{array}{l}25.1 \\
26.1 \\
27.7 \\
23.2 \\
30.5 \\
25.3\end{array}$ & $\begin{array}{c}25.0 \\
29.6 \\
29.6 \\
22.6^{(a)} \\
28.2 \\
29.6\end{array}$ & $\begin{array}{c}26.1 \\
25.6 \\
25.3^{\text {(a) }} \\
26.4 \\
30.2 \\
25.7\end{array}$ & $\begin{array}{l}31.0 \\
30.4 \\
29.3 \\
29.1 \\
30.5 \\
32.3\end{array}$ & $\begin{array}{l}29.7 \\
29.4 \\
28.8 \\
28.0 \\
30.4 \\
31.6\end{array}$ & $\begin{array}{l}26.9 \\
26.8 \\
27.3 \\
28.7 \\
27.2 \\
32.4\end{array}$ & $\begin{array}{c}27.4 \\
23.6 \\
18.1^{(\mathrm{a})} \\
26.5 \\
26.6 \\
18.2\end{array}$ & $\begin{array}{l}16.3 \\
17.7 \\
15.4 \\
17.8 \\
16.8 \\
14.7\end{array}$ & $\begin{array}{r}11.4 \\
15.8 \\
11.1 \\
15.9 \\
16.7 \\
9.7\end{array}$ & $\begin{array}{l}22.2 \\
23.8 \\
23.2 \\
22.8 \\
24.6 \\
22.6\end{array}$ \\
\hline $\begin{array}{l}1951 \\
1952 \\
1953 \\
1954 \\
1955\end{array}$ & $\begin{array}{l}13.7 \\
12.6 \\
15.6 \\
14.3 \\
9.2^{(2)}\end{array}$ & $\begin{array}{l}18.4 \\
17.0 \\
19.8 \\
13.8 \\
18.9\end{array}$ & $\begin{array}{l}20.8 \\
22.8 \\
24.4 \\
23.7 \\
21.8\end{array}$ & $\begin{array}{l}30.3 \\
30.3 \\
24.0 \\
26.3 \\
24.9\end{array}$ & $\begin{array}{l}30.2 \\
28.1 \\
27.9 \\
28.1 \\
25.3\end{array}$ & $\begin{array}{l}28.9 \\
27.3 \\
26.4 \\
26.8 \\
29.4\end{array}$ & $\begin{array}{c}33.8^{(2)} \\
32.6 \\
32.8 \\
31.4 \\
27.9\end{array}$ & $\begin{array}{l}31.5 \\
32.2 \\
29.1 \\
27.6 \\
31.7\end{array}$ & $\begin{array}{l}30.9 \\
32.8 \\
32.5 \\
26.2 \\
27.5\end{array}$ & $\begin{array}{l}23.3 \\
29.0 \\
27.8 \\
24.5 \\
22.7\end{array}$ & $\begin{array}{l}17.4 \\
16.0 \\
20.3 \\
15.8 \\
16.1\end{array}$ & $\begin{array}{c}13.7 \\
9.4 \\
17.7^{(a)} \\
13.2 \\
12.3\end{array}$ & $\begin{array}{l}24.4 \\
24.2 \\
24.9 \\
22.6 \\
22.3\end{array}$ \\
\hline $\begin{array}{l}1956 \\
1957 \\
1958 \\
1959 \\
1960\end{array}$ & $\begin{array}{l}12.9 \\
15.2 \\
13.3 \\
14.1 \\
14.6\end{array}$ & $\begin{array}{l}15.0 \\
18.3 \\
15.0 \\
16.2 \\
.19 .3\end{array}$ & $\begin{array}{c}20.9 \\
18.4^{(2)} \\
22.0 \\
24.3 \\
23.2\end{array}$ & $\begin{array}{l}28.0 \\
24.7 \\
23.9 \\
26.9 \\
25.7\end{array}$ & $\begin{array}{l}26.6 \\
24.8 \\
29.6 \\
26.5 \\
26.5\end{array}$ & $\begin{array}{l}26.9 \\
27.7 \\
27.3 \\
27.1 \\
31.2\end{array}$ & $\begin{array}{l}30.8 \\
28.7 \\
30.5 \\
31.1 \\
32.8\end{array}$ & $\begin{array}{l}28.8 \\
27.0 \\
33.1 \\
29.9 \\
28.1\end{array}$ & $\begin{array}{l}30.6 \\
29.8 \\
27.0 \\
23.8 \\
28.3\end{array}$ & $\begin{array}{l}22.1 \\
18.4 \\
27.8 \\
24.4 \\
25.7\end{array}$ & $\begin{array}{l}13.9 \\
19.1 \\
17.8 \\
21.9 \\
18.7\end{array}$ & $\begin{array}{l}13.4 \\
14.7 \\
10.2 \\
13.4 \\
10.9\end{array}$ & $\begin{array}{l}22.5 \\
22.2 \\
23.1 \\
23.3 \\
23.8\end{array}$ \\
\hline $\begin{array}{l}1961 \\
1962 \\
1963 \\
1964 \\
1965\end{array}$ & $\begin{array}{l}12.5 \\
18.1 \\
17.2 \\
16.0 \\
12.3\end{array}$ & $\begin{array}{c}17.2 \\
17.4 \\
16.8 \\
24.1^{(2)} \\
20.3\end{array}$ & $\begin{array}{l}20.0 \\
22.0 \\
23.3 \\
23.7 \\
25.6\end{array}$ & $\begin{array}{c}25.3 \\
28.9 \\
21.4^{(a)} \\
27.1 \\
26.4\end{array}$ & $\begin{array}{l}25.5 \\
23.2 \\
28.1 \\
27.9 \\
28.3\end{array}$ & $\begin{array}{l}31.5 \\
29.9 \\
26.6 \\
26.4 \\
28.1\end{array}$ & $\begin{array}{l}30.6 \\
30.2 \\
27.8 \\
31.2 \\
31.1\end{array}$ & $\begin{array}{l}30.9 \\
28.3 \\
30.7 \\
29.4 \\
27.7\end{array}$ & $\begin{array}{l}26.9 \\
29.9 \\
29.8 \\
29.7 \\
29.8\end{array}$ & $\begin{array}{l}25.4 \\
21.4 \\
24.5 \\
26.4 \\
27.4\end{array}$ & $\begin{array}{c}20.4 \\
17.3 \\
16.9 \\
12.2^{(2)} \\
14.2\end{array}$ & $\begin{array}{r}15.0 \\
11.4 \\
9.5 \\
14.8 \\
15.7\end{array}$ & $\begin{array}{l}23.4 \\
23.2 \\
22.7 \\
24.1 \\
23.9\end{array}$ \\
\hline $\begin{array}{l}1966 \\
1967 \\
1968 \\
1969 \\
1970\end{array}$ & $\begin{array}{l}14.3 \\
17.6 \\
17.2 \\
12.2 \\
12.0\end{array}$ & $\begin{array}{l}19.3 \\
24.0 \\
20.4 \\
14.1 \\
16.4\end{array}$ & $\begin{array}{l}24.4 \\
24.6 \\
23.5 \\
25.5 \\
23.8\end{array}$ & $\begin{array}{l}28.2 \\
24.4 \\
27.5 \\
24.5 \\
25.3\end{array}$ & $\begin{array}{l}30.7 \\
27.7 \\
27.5 \\
29.2 \\
29.2\end{array}$ & $\begin{array}{l}27.3 \\
28.7 \\
27.3 \\
27.7 \\
29.3\end{array}$ & $\begin{array}{l}28.7 \\
32.0 \\
31.2 \\
31.3 \\
31.7\end{array}$ & $\begin{array}{c}29.1 \\
34.5^{(a)} \\
26.2^{(a)} \\
33.3 \\
33.1\end{array}$ & $\begin{array}{l}28.4 \\
31.7 \\
28.2 \\
27.6 \\
27.2\end{array}$ & $\begin{array}{l}25.7 \\
25.5 \\
22.8 \\
25.0 \\
26.5\end{array}$ & $\begin{array}{l}18.1 \\
19.9 \\
14.2 \\
17.4 \\
17.8\end{array}$ & $\begin{array}{r}13.4 \\
15.7 \\
13.9 \\
9.2 \\
15.4\end{array}$ & $\begin{array}{c}24.0 \\
25.5^{(\text {a) }} \\
23.3 \\
23.1 \\
24.0\end{array}$ \\
\hline $\begin{array}{l}1971 \\
1972 \\
1973 \\
1974 \\
1975\end{array}$ & $\begin{array}{l}18.4 \\
17.3 \\
15.7 \\
17.8 \\
15.0\end{array}$ & $\begin{array}{l}21.1 \\
18.3 \\
16.6 \\
18.8 \\
17.0\end{array}$ & $\begin{array}{l}22.8 \\
25.2 \\
24.6 \\
23.2 \\
21.2\end{array}$ & $\begin{array}{l}26.8 \\
26.8 \\
29.6 \\
23.4 \\
24.8\end{array}$ & $\begin{array}{l}27.7 \\
27.2 \\
31.1 \\
27.3 \\
29.5\end{array}$ & $\begin{array}{c}26.9 \\
26.9 \\
29.7 \\
32.7^{(a)} \\
28.2\end{array}$ & $\begin{array}{l}32.0 \\
30.1 \\
32.1 \\
29.8 \\
30.3\end{array}$ & $\begin{array}{l}32.3 \\
30.6 \\
32.7 \\
31.9 \\
28.7\end{array}$ & $\begin{array}{l}27.8 \\
30.5 \\
27.0 \\
32.2 \\
32.2\end{array}$ & $\begin{array}{l}25.8 \\
27.5 \\
22.2 \\
28.3 \\
22.0\end{array}$ & $\begin{array}{l}17.8 \\
13.1 \\
12.6 \\
16.3 \\
20.9\end{array}$ & $\begin{array}{l}14.8 \\
17.2 \\
12.5 \\
16.5 \\
14.8\end{array}$ & $\begin{array}{l}24.5 \\
24.2 \\
23.9 \\
24.8 \\
23.7\end{array}$ \\
\hline $\begin{array}{l}1976 \\
1977 \\
1978 \\
1979 \\
1980\end{array}$ & $\begin{array}{l}15.2 \\
10.8 \\
11.4 \\
15.5 \\
13.2\end{array}$ & $\begin{array}{c}21.0 \\
20.7 \\
15.2 \\
18.7 \\
10.5^{(2)}\end{array}$ & $\begin{array}{l}25.3 \\
23.4 \\
23.0 \\
26.0 \\
22.1\end{array}$ & $\begin{array}{c}26.0 \\
30.6^{\text {(a) }} \\
23.8 \\
26.5 \\
27.1\end{array}$ & $\begin{array}{l}30.6 \\
26.0 \\
27.7 \\
29.4 \\
25.8\end{array}$ & $\begin{array}{l}28.8 \\
30.2 \\
31.3 \\
31.1 \\
25.8\end{array}$ & $\begin{array}{l}30.5 \\
30.5 \\
31.0 \\
32.9 \\
31.3\end{array}$ & $\begin{array}{l}28.0 \\
29.1 \\
29.0 \\
32.0 \\
29.9\end{array}$ & $\begin{array}{l}30.5 \\
23.8 \\
25.8 \\
31.1 \\
27.3\end{array}$ & $\begin{array}{c}27.5 \\
26.6 \\
30.3^{(a)} \\
25.6 \\
24.6\end{array}$ & $\begin{array}{l}20.3 \\
19.1 \\
18.2 \\
13.0 \\
15.3\end{array}$ & $\begin{array}{l}16.6 \\
15.1 \\
16.8 \\
12.9 \\
11.4\end{array}$ & $\begin{array}{c}25.0 \\
23.8 \\
23.6 \\
24.6 \\
22.0^{(2)}\end{array}$ \\
\hline $\begin{array}{l}1981 \\
1982 \\
1983 \\
1984 \\
1985\end{array}$ & $\begin{array}{r}9.9 \\
16.0 \\
15.5 \\
13.5 \\
6.8\end{array}$ & $\begin{array}{l}17.5 \\
21.4 \\
17.3 \\
15.2 \\
20.4\end{array}$ & $\begin{array}{l}25.9 \\
24.4 \\
20.7 \\
21.6 \\
25.4\end{array}$ & $\begin{array}{l}27.4 \\
28.2 \\
27.9 \\
23.9 \\
28.7\end{array}$ & $\begin{array}{l}27.1 \\
29.9 \\
28.4 \\
26.3 \\
29.2\end{array}$ & $\begin{array}{l}28.3 \\
28.0 \\
27.9 \\
26.1 \\
29.4\end{array}$ & $\begin{array}{l}31.7 \\
30.6 \\
26.3 \\
32.3 \\
32.0\end{array}$ & $\begin{array}{l}32.9 \\
29.5 \\
28.4 \\
32.0 \\
29.9\end{array}$ & $\begin{array}{l}30.8 \\
27.1 \\
27.5 \\
26.6 \\
24.3\end{array}$ & $\begin{array}{l}26.0 \\
24.9 \\
24.7 \\
25.5 \\
25.4\end{array}$ & $\begin{array}{l}20.0 \\
16.6 \\
15.5 \\
14.0 \\
15.8\end{array}$ & $\begin{array}{c}14.2 \\
13.0 \\
11.0 \\
15.7 \\
8.0^{(a)}\end{array}$ & $\begin{array}{l}24.3 \\
24.1 \\
22.6 \\
22.7 \\
22.9\end{array}$ \\
\hline $\begin{array}{l}1986 \\
1987 \\
1988 \\
1989 \\
1990\end{array}$ & $\begin{array}{l}12.5 \\
11.9 \\
13.4 \\
16.5 \\
15.7\end{array}$ & $\begin{array}{l}17.1 \\
19.0 \\
23.8 \\
17.3 \\
20.5\end{array}$ & $\begin{array}{l}22.1 \\
22.4 \\
25.1 \\
20.2 \\
26.5\end{array}$ & $\begin{array}{l}26.4 \\
28.2 \\
25.3 \\
26.2 \\
27.1\end{array}$ & $\begin{array}{l}26.6 \\
28.9 \\
27.4 \\
26.0 \\
24.4\end{array}$ & $\begin{array}{l}29.1 \\
31.2 \\
26.3 \\
28.5 \\
26.8\end{array}$ & $\begin{array}{l}28.6 \\
28.1 \\
30.0 \\
31.1 \\
28.8\end{array}$ & $\begin{array}{l}31.0 \\
30.1 \\
32.1 \\
27.3 \\
27.3\end{array}$ & $\begin{array}{c}23.5^{(2)} \\
33.0 \\
31.2 \\
31.1 \\
32.2\end{array}$ & $\begin{array}{l}26.8 \\
28.9 \\
26.2 \\
24.0 \\
23.6\end{array}$ & $\begin{array}{l}15.1 \\
19.8 \\
16.9 \\
16.0 \\
18.4\end{array}$ & $\begin{array}{r}8.4 \\
12.0 \\
11.4 \\
9.4 \\
16.0\end{array}$ & $\begin{array}{l}22.3 \\
24.5 \\
24.1 \\
22.8 \\
23.9\end{array}$ \\
\hline $\begin{array}{l}1991 \\
1992 \\
1993 \\
1994 \\
1995\end{array}$ & $\begin{array}{l}16.0 \\
12.5 \\
12.8 \\
15.7 \\
13.2\end{array}$ & $\begin{array}{l}21.4 \\
15.2 \\
15.1 \\
17.2 \\
20.1\end{array}$ & $\begin{array}{c}22.7 \\
25.9 \\
18.6 \\
28.4^{(2)} \\
23.7\end{array}$ & $\begin{array}{c}26 \\
24.6 \\
23.7 \\
26.0 \\
25.2\end{array}$ & $\begin{array}{c}25.1 \\
31.9^{(\ell)} \\
29.1 \\
26.5 \\
28.0\end{array}$ & $\begin{array}{l}25.6 \\
28.5 \\
27.1 \\
29.4 \\
26.3\end{array}$ & $\begin{array}{c}30.2 \\
28.0 \\
25.5^{\text {(a) }} \\
33.0 \\
29.9\end{array}$ & $\begin{array}{l}29.6 \\
31.6 \\
29.5 \\
31.2 \\
30.6\end{array}$ & $\begin{array}{c}31.1 \\
28.1 \\
33.5^{(2)} \\
31.2 \\
30.2\end{array}$ & $\begin{array}{l}26.4 \\
24.4 \\
28.6 \\
25.4 \\
24.3\end{array}$ & $\begin{array}{c}13.1 \\
13.6 \\
23.9^{(a)} \\
17.2 \\
19.5\end{array}$ & $\begin{array}{l}13.5 \\
14.5 \\
10.5 \\
13.6 \\
11.7\end{array}$ & $\begin{array}{l}23.4 \\
23.2 \\
23.2 \\
24.6 \\
23.6\end{array}$ \\
\hline $\begin{array}{l}1996 \\
1997 \\
1998\end{array}$ & $\begin{array}{c}14.0 \\
15.6 \\
15.9\end{array}$ & $\begin{array}{l}21.2 \\
18.8 \\
19.0\end{array}$ & $\begin{array}{l}22.9 \\
22.1 \\
23.7\end{array}$ & $\begin{array}{l}26.0 \\
25.7 \\
28.5\end{array}$ & $\begin{array}{l}26.6 \\
28.1 \\
27.5\end{array}$ & $\begin{array}{l}31.0 \\
27.3 \\
29.2\end{array}$ & $\begin{array}{l}33.7 \\
31.2 \\
30.5\end{array}$ & $\begin{array}{l}34.1 \\
31.2 \\
33.2\end{array}$ & $\begin{array}{l}30.8 \\
26.7 \\
31.9\end{array}$ & $\begin{array}{l}23.9 \\
24.3 \\
26.6\end{array}$ & $\begin{array}{l}17.0 \\
17.6 \\
16.2\end{array}$ & $\begin{array}{l}13.1 \\
14.2 \\
17.7\end{array}$ & $\begin{array}{l}24.5 \\
23.6 \\
25.0\end{array}$ \\
\hline $\begin{array}{l}\text { Average } \\
\text { Normal }\end{array}$ & $\begin{array}{l}14.4 \\
14.4\end{array}$ & $\begin{array}{l}18.3 \\
18.6\end{array}$ & $\begin{array}{l}23.1 \\
23.5\end{array}$ & $\begin{array}{l}26.3 \\
26.5\end{array}$ & $\begin{array}{l}27.7 \\
27.8\end{array}$ & $\begin{array}{l}28.1 \\
28.5\end{array}$ & $\begin{array}{l}30.6 \\
30.5\end{array}$ & $\begin{array}{l}30.3 \\
30.3\end{array}$ & $\begin{array}{l}29.0 \\
28.8\end{array}$ & $\begin{array}{l}25.1 \\
25.6\end{array}$ & $\begin{array}{l}17.0 \\
16.8\end{array}$ & $\begin{array}{l}13.4 \\
13.4\end{array}$ & $\begin{array}{l}23.6 \\
23.7\end{array}$ \\
\hline
\end{tabular}

(a) Greatest and least values. 
Table 3.12. Normal and Extreme Daily Maximum and Minimum Temperatures $\left({ }^{\circ} \mathrm{F}\right)$

\begin{tabular}{|c|c|c|c|c|c|c|c|c|c|c|c|}
\hline \multirow[b]{3}{*}{ Day } & & & & \multicolumn{8}{|c|}{ Extreme (1945-1998) } \\
\hline & \multicolumn{3}{|c|}{ Normal (1961-1990) } & \multicolumn{4}{|c|}{ Maximum } & \multicolumn{4}{|c|}{ Minimum } \\
\hline & Maximum & Minimum & $\overline{\text { Mean }}$ & Figh & Year & Low & Year & High & Year & Low & Year \\
\hline \multicolumn{12}{|c|}{ January } \\
\hline 1 & 35 & 21 & 28 & 57 & $98^{(2)}$ & 8 & 69 & 38 & $81^{(2)}$ & -11 & 79 \\
\hline 2 & 35 & 21 & 28 & 57 & 97 & 15 & 69 & 42 & 63 & -9 & 78 \\
\hline 3 & 36 & 21 & 28 & 63 & 89 & 12 & 50 & 41 & 81 & 0 & 59 \\
\hline 4 & 36 & 21 & 29 & 60 & $94^{(a)}$ & 13 & 59 & 39 & 54 & -6 & 50 \\
\hline 5 & 36 & 22 & 29 & 59 & $90^{(a)}$ & 12 & 50 & 39 & 81 & -7 & 50 \\
\hline 6 & 36 & 22 & 29 & 59 & $90^{(2)}$ & 10 & 82 & 42 & 98 & -10 & 74 \\
\hline 7 & 36 & 22 & 29 & 63 & 62 & 9 & 79 & 45 & 90 & -8 & 74 \\
\hline 8 & 36 & 22 & 29 & 56 & $83^{(2)}$ & 5 & 74 & 44 & 53 & -9 & 74 \\
\hline 9 & 36 & 22 & 29 & 60 & $90^{(a)}$ & 5 & 74 & 41 & 90 & -13 & 74 \\
\hline 10 & 36 & 23 & 30 & 61 & 83 & 10 & 74 & 40 & 83 & -5 & 74 \\
\hline 11 & 37 & 23 & 30 & 60 & $83^{(n)}$ & 13 & 63 & 37 & 90 & -6 & 49 \\
\hline 12 & 38 & 24 & 31 & 59 & 53 & 15 & 63 & 47 & 53 & -4 & $63^{12}$ \\
\hline 13 & 38 & 24 & 31 & 61. & 94 & 10 & 50 & 42 & 66 & -4 & $93^{\text {ta }}$ \\
\hline 14 & 39 & 25 & 32 & 60 & 61 & 7 & 50 & 48 & 61 & -11 & 50 \\
\hline 15 & 39 & 26 & 33 & 60 & $74^{(a)}$ & 5 & 50 & 50 & 74 & -5 & 50 \\
\hline 16 & 40 & 26 & 33 & 61 & 74 & 8 & 50 & 48 & 89 & -8 & 50 \\
\hline 17 & 40 & 26 & 33 & 57 & 98 & 5 & 50 & 40 & 89 & -11 & 50 \\
\hline 18 & 40 & 26 & 33 & 62 & 89 & 10 & 50 & 38 & 89 & -6 & 57 \\
\hline 19 & 40 & 26 & 33 & 63 & 68 & 13 & 50 & 47 & 68 & -2 & 57 \\
\hline 20 & 40 & 26 & 33 & 66 & 68 & 11 & 54 & 47 & 72 & -6 & 54 \\
\hline 21 & 40 & 26 & 33 & 65 & 68 & 14 & 54 & 42 & 72 & 0 & 54 \\
\hline 22 & 40 & 26 & 33 & 56 & 90 & 16 & 69 & 43 & 81 & -2 & 62 \\
\hline 23 & 40 & 26 & 32 & 63 & 53 & 10 & 69 & 43 & 81 & -8 & 69 \\
\hline 24 & 40 & 25 & 32 & 59 & $84^{\text {(a) }}$ & 13 & 57 & 43 & 58 & -6 & 49 \\
\hline 25 & 40 & 25 & 32 & 59 & $92^{(a)}$ & 6 & 50 & 41 & $74^{(a)}$ & -12 & 57 \\
\hline 26 & 40 & 25 & 32 & 61 & 71 & 2 & 57 & 46 & 62 & -22 & 57 \\
\hline 27 & 40 & 25 & 32 & 60 & $84^{(a)}$ & 0 & 57 & 36 & $95^{(a)}$ & -21 & 57 \\
\hline 28 & 40 & 25 & 32 & 61 & 67 & 6 & 57 & 41 & 53 & -14 & 57 \\
\hline 29 & 40 & 24 & 32 & 62 & 67 & 3 & 50 & 44 & $92^{(2)}$ & -18 & 50 \\
\hline 30 & 40 & 24 & 32 & 67 & $89^{(2)}$ & 11 & 57 & 53 & 71 & -13 & 50 \\
\hline 31 & 40 & 24 & 32 & 72 & 71 & -2 & 50 & 45 & 53 & -21 & 50 \\
\hline
\end{tabular}

February

\begin{tabular}{rrrrrrrrrrrr}
\hline 1 & 40 & 24 & 32 & 63 & 71 & -3 & 50 & 42 & 92 & -23 & 50 \\
2 & 41 & 24 & 33 & 61 & $91^{(a)}$ & 0 & 50 & 42 & 68 & -21 & 50 \\
3 & 41 & 24 & 33 & 60 & $67^{(a)}$ & 1 & 50 & 46 & 91 & -23 & 50 \\
4 & 41 & 24 & 33 & 65 & 67 & 11 & 85 & 43 & 68 & -5 & 85 \\
5 & 41 & 25 & 33 & 61 & 65 & 23 & 85 & 39 & 61 & -5 & 89 \\
6 & 42 & 25 & 33 & 61 & 67 & 19 & 85 & 37 & 61 & -3 & 89 \\
7 & 42 & 25 & 34 & 65 & 45 & 20 & 48 & 41 & 55 & 2 & 89 \\
8 & 43 & 26 & 35 & 63 & 96 & 22 & 56 & 45 & 45 & 5 & $94^{(a)}$ \\
9 & 44 & 27 & 35 & 65 & 51 & 23 & 56 & 39 & $61^{(\text {a) }}$ & 4 & 85 \\
10 & 45 & 28 & 37 & 65 & 77 & 30 & $56^{(a)}$ & 50 & 51 & 7 & 85 \\
11 & 46 & 28 & 37 & 66 & 88 & 27 & 54 & 39 & $93^{(a)}$ & 9 & 48 \\
12 & 47 & 29 & 38 & 70 & 77 & 24 & 49 & 40 & 77 & 6 & 48 \\
13 & 48 & 30 & 39 & 66 & 71 & 25 & 49 & 42 & 47 & 3 & 49 \\
14 & 48 & 30 & 39 & 62 & $97^{(2)}$ & 22 & 80 & 54 & 82 & 8 & 95 \\
15 & 49 & 31 & 40 & 67 & 82 & 19 & 56 & 48 & 81 & 5 & 56 \\
16 & 49 & 31 & 40 & 69 & 77 & 20 & 56 & 48 & 81 & 4 & 56 \\
17 & 49 & 31 & 40 & 67 & $77^{(a)}$ & 22 & 56 & 48 & 48 & 9 & 56 \\
18 & 50 & 31 & 40 & 66 & 81 & 24 & 56 & 46 & 81 & 9 & 90 \\
19 & 50 & 31 & 41 & 68 & 95 & 28 & 56 & 56 & 95 & 14 & $90^{(a)}$
\end{tabular}


Table 3.12. (contd)

\begin{tabular}{|c|c|c|}
\hline \multirow[b]{2}{*}{ Day } & \multicolumn{2}{|c|}{ Normal (1961-1990) } \\
\hline & Maximum & Minimum \\
\hline 20 & 50 & 31 \\
\hline 21 & 51 & 31 \\
\hline 22 & 51 & 31 \\
\hline 23 & 51 & 31 \\
\hline 24 & 52 & 31 \\
\hline 25 & 52 & 31 \\
\hline 26 & 52 & 31 \\
\hline 27 & 52 & 31 \\
\hline 28 & 52 & 31 \\
\hline 29 & 52 & 31 \\
\hline
\end{tabular}

Extreme (1945-1998)

\begin{tabular}{|c|c|c|c|c|c|c|c|}
\hline \multicolumn{4}{|c|}{ Maximum } & \multicolumn{4}{|c|}{ Minimum } \\
\hline High & Year & Low & Year & High & Year & Low & Year \\
\hline 68 & 82 & 32 & $57^{(a)}$ & 51 & 61 & 15 & 86 \\
\hline 71 & 88 & 29 & 57 & 45 & 95 & 13 & 57 \\
\hline 62 & $95^{(2)}$ & 29 & 57 & 42 & 58 & 11 & 93 \\
\hline 68 & 47 & 34 & $93^{(2)}$ & 43 & $83^{(x)}$ & 19 & 93 \\
\hline 72 & 86 & 32 & 62 & 60 & 86 & 11 & $93^{(a)}$ \\
\hline 72 & 86 & 28 & 93 & 49 & 86 & 4 & 93 \\
\hline 65 & $57^{(a)}$ & 28 & $93^{(2)}$ & 46 & 92 & 10 & 93 \\
\hline 68 & 72 & 26 & 93 & 44 & 92 & 7 & 62 \\
\hline 67 & 67 & 25 & 93 & 48 & 72 & 3 & 93 \\
\hline 63 & $88^{(a)}$ & 40 & 60 & 43 & 92 & 12 & 60 \\
\hline
\end{tabular}

March

\begin{tabular}{|c|c|c|c|c|c|c|c|c|c|c|c|}
\hline 1 & 52 & 31 & 42 & 67 & 94 & 26 & 93 & 44 & 94 & 15 & $71^{(a)}$ \\
\hline 2 & 52 & 31 & 42 & 66 & 68 & 26 & 60 & 46 & 87 & 14 & 60 \\
\hline 3 & 52 & 31 & 42 & 70 & 94 & 24 & 60 & 50 & 87 & 14 & 89 \\
\hline 4 & 52 & 31 & 42 & 63 & 53 & 26 & 55 & 46 & 87 & 7 & 55 \\
\hline 5 & 53 & 31 & 42 & 68 & 72 & 31 & 45 & 50 & 87 & 6 & 55 \\
\hline 6 & 54 & 32 & 43 & 65 & $67^{(\mathrm{a})}$ & 33 & 57 & 43 & 79 & 18 & 60 \\
\hline 7 & 55 & 32 & 44 & 66 & 53 & 41 & 45 & 42 & $86^{(\mathbf{z})}$ & 21 & $74^{(\mathrm{a})}$ \\
\hline 8 & 55 & 33 & 44 & 67 & 53 & 33 & 51 & 46 & 83 & 20 & 76 \\
\hline 9 & 56 & 33 & 44 & 69 & 53 & 40 & 51 & 47 & 83 & 22 & $94^{(a)}$ \\
\hline 10 & 56 & 33 & 45 & 69 & 72 & 40 & 48 & 45 & $87^{(2)}$ & 13 & 48 \\
\hline 11 & 56 & 33 & 45 & 67 & $95^{(a)}$ & 32 & 50 & 44 & 95 & 21 & 50 \\
\hline 12 & 57 & 34 & 45 & 68 & $98^{(\text {a) }}$ & 37 & 51 & 48 & 87 & 15 & 56 \\
\hline 13 & 57 & 34 & 45 & 72 & 98 & 38 & 51 & 44 & $98^{(2)}$ & 22 & $69^{(a)}$ \\
\hline 14 & 57 & 34 & 46 & 72 & 92 & 40 & 49 & 45 & $61^{\circ}$ & 23 & 53 \\
\hline 15 & 58 & 34 & 46 & 73 & 94 & 37 & 49 & 50 & 92 & 23 & 76 \\
\hline 16 & 58 & 34 & 46 & 76 & 72 & 43 & 89 & 47 & 94 & 23 & 55 \\
\hline 17 & 59 & 35 & 47 & 76 & 72 & 38 & 65 & 48 & 69 & 17 & 65 \\
\hline 18 & 59 & 35 & 47 & 76 & 47 & 41 & 65 & 47 & 90 & 14 & 65 \\
\hline 19 & 59 & 35 & 47 & 76 & 47 & 48 & $65^{(a)}$ & 53 & 97 & 16 & 65 \\
\hline 20 & 59 & 35 & 47 & 76 & 47 & 49 & 50 & 49 & 88 & 22 & 74 \\
\hline 21 & 59 & 35 & 47 & 74 & 60 & 41 & 75 & 46 & $98^{(2)}$ & 26 & $82^{(2)}$ \\
\hline 22 & 59 & 35 & 47 & 74 & $78^{(a)}$ & 47 & 71 & 47 & 78 & 19 & 94 \\
\hline 23 & 59 & 35 & 47 & 77 & 60 & 39 & 64 & 45 & $98^{(a)}$ & 20 & 48 \\
\hline 24 & 59 & 35 & 47. & 78 & 60 & 38 & 55 & 45 & $60^{(2)}$ & 15 & 64 \\
\hline 25 & 59 & 35 & 47 & 83 & 60 & 35 & 55 & 48 & 52 & 21 & 96 \\
\hline 26 & 60 & 35 & 47 & 76 & 46 & 38 & 65 & 49 & $92^{(\mathbf{a})}$ & 21 & 85 \\
\hline 27 & 60 & 35 & 48 & 77 & 94 & 47 & 79 & 46 & 89 & 24 & 75 \\
\hline 28 & 61 & 36 & 48 & 79 & 94 & 42 & 54 & 49 & 78 & 19 & 75 \\
\hline 29 & 61 & 36 & 49 & 78 & $94^{(a)}$ & 49 & 54 & 48 & $94^{(2)}$ & 18 & 54 \\
\hline 30 & 62 & 37 & 49 & 75 & 92 & 52 & 67 & 47 & $92^{(2)}$ & 20 & 54 \\
\hline 31 & 62 & 37 & 49 & 78 & 92 & 45 & 96 & 47 & 61 & 28 & 53 \\
\hline \multicolumn{12}{|c|}{ April } \\
\hline 1 & 63 & 37 & 50 & 80 & 90 & 45 & 76 & 50 & 59 & 24 & 82 \\
\hline 2 & 63 & 37 & 50 & 83 & 92 & 48 & 82 & 50 & 87 & 25 & 76 \\
\hline 3 & 64 & 37 & 50 & 76 & 77 & 50 & $63^{(a)}$ & 48 & 77 & 23 & 75 \\
\hline 4 & 64 & 38 & 51 & 82 & 60 & 45 & 75 & 56 & 91 & 27 & 50 \\
\hline 5 & 64 & 38 & 51 & 78 & $77^{(\mathrm{a})}$ & 51 & 75 & 54 & 60 & 21 & 75 \\
\hline 6 & 65 & 38 & 51 & 82 & 77 & 50 & 82 & 51 & 62 & 25 & 97 \\
\hline 7 & 65 & 38 & 52 & 85 & 77 & 41 & 45 & 53 & 60 & 26 & 54 \\
\hline 8 & 65 & 38 & 52 & 82 & 96 & 51 & 53 & 50 & 96 & 27 & $92^{(a)}$ \\
\hline 9 & 65 & 38 & 52 & 80 & 85 & 48 & 92 & 57 & 96 & 29 & $75^{(\mathrm{a})}$ \\
\hline 10 & 65 & 38 & 52 & 85 & 68 & 52 & 45 & 50 & 96 & 24 & 81 \\
\hline
\end{tabular}


Table 3.12. (contd)

\begin{tabular}{|c|c|c|c|}
\hline \multirow[b]{2}{*}{ Day } & \multicolumn{3}{|c|}{ Normal (1961-1990) } \\
\hline & Maximum & Minimum & Mean \\
\hline 11 & 65 & 38 & 52 \\
\hline 12 & 66 & 39 & 52 \\
\hline 13 & 66 & 39 & 52 \\
\hline 14 & 66 & 39 & 52 \\
\hline 15 & 66 & 39 & 52 \\
\hline 16 & 66 & 39 & 52 \\
\hline 17 & 65 & 40 & 52 \\
\hline 18 & 65 & 40 & 52 \\
\hline 19 & 65 & 40 & 52 \\
\hline 20 & 66 & 40 & 53 \\
\hline 21 & 66 & 40 & 53 \\
\hline 22 & 66 & 41 & 54 \\
\hline 23 & 67 & 41 & 54 \\
\hline 24 & 68 & 41 & 54 \\
\hline 25 & 68 & 42 & 55 \\
\hline 26 & 68 & 42 & 55 \\
\hline 27 & 69 & 42 & 55 \\
\hline 28 & 69 & 42 & 56 \\
\hline 29 & 70 & 43 & 56 \\
\hline 30 & 70 & 43 & 57 \\
\hline
\end{tabular}

\begin{tabular}{|c|c|c|c|c|c|c|c|}
\hline \multicolumn{8}{|c|}{ Extreme (1945-1998) } \\
\hline \multicolumn{4}{|c|}{ Maximum } & \multicolumn{4}{|c|}{ Minimum } \\
\hline High & Year & Low & Year & High & Year & Low & Year \\
\hline 79 & 88 & 52 & 83 & 51 & $56^{(a)}$ & 27 & 83 \\
\hline 83 & 88 & 47 & 95 & 48 & $82^{(a)}$ & 26 & 97 \\
\hline 88 & 47 & 54 & 55 & 59 & 88 & 23 & 68 \\
\hline 85 & $62^{(a)}$ & 47 & 75 & 55 & 85 & 29 & 83 \\
\hline 82 & 88 & 55 & $75^{(\mathrm{a})}$ & 54 & 87 & 27 & 55 \\
\hline 83 & 54 & 53 & 63 & 54 & 92 & 28 & 82 \\
\hline 88 & 94 & 48 & 63 & 52 & 90 & 26 & 55 \\
\hline 88 & 94 & 51 & 67 & 60 & 94 & 29 & 68 \\
\hline 78 & 56 & 50 & 51 & 56 & 94 & 27 & 66 \\
\hline 84 & 56 & 45 & 67 & 53 & $90^{(2)}$ & 28 & 82 \\
\hline 85 & 56 & 52 & 67 & 60 & 56 & 26 & $85^{(a)}$ \\
\hline 81 & $82^{(a)}$ & 53 & 88 & 55 & 98 & 28 & 72 \\
\hline 88 & $81^{(a)}$ & 56 & $79^{(a)}$ & 58 & 77 & 30 & 78 \\
\hline 94 & 77 & 53 & 75 & 56 & 52 & 28 & $86^{(a)}$ \\
\hline 91 & 46 & 56 & 58 & 59 & 52 & 31 & 55 \\
\hline 85 & 92 & 53 & 48 & 57 & 78 & 28 & 48 \\
\hline 90 & 87 & 50 & 90 & 57 & 92 & 27 & 70 \\
\hline 93. & 87 & 54 & 95 & 64 & 87 & 27 & 67 \\
\hline 90 & 68 & 47 & 67 & 60 & 87 & 29 & 52 \\
\hline 92 & 98 & 56 & $67^{(2)}$ & 60 & 98 & 29 & 86 \\
\hline
\end{tabular}

May

\begin{tabular}{|c|c|c|c|c|c|c|c|c|c|c|c|}
\hline 1 & 71 & 43 & 57 & 93 & 98 & 53 & 69 & 59 & $98^{(2)}$ & 28 & 54 \\
\hline 2 & 71 & 43 & 57 & 89 & $98^{(a)}$ & 56 & 88 & 60 & 71 & 30 & 97 \\
\hline 3 & 71 & 44 & 58 & 91 & 66 & 58 & 93 & 60 & 71 & 31 & 49 \\
\hline 4 & 72 & 44 & 58 & 94 & 66 & 56 & 63 & 57 & 46 & 31 & 62 \\
\hline 5 & 72 & 45 & 58 & 100 & 66 & 52 & 61 & 65 & 66 & 30 & 59 \\
\hline 6 & 73 & 45 & 59 & 98 & 92 & $56^{\prime}$ & 86 & 62 & 87 & 34 & 77 \\
\hline 7 & 73 & 45 & 59 & 99 & 87 & 61 & 90 & 66 & 92 & 33 & 84 \\
\hline 8 & 73 & 46 & 59 & 102 & 87 & 56 & 62 & 67 & $94^{(a)}$ & 29 & 96 \\
\hline 9 & 74 & 46 & 60 & 97 & 87 & 56 & 48 & 66 & 49 & 34 & $96^{(2)}$ \\
\hline 10 & 74 & 46 & 60 & 96 & 49 & 53 & 67 & 66 & 49 & 34 & 70 \\
\hline 11 & 74 & 46 & 60 & 98 & 49 & 51 & 67 & 68 & 49 & 30 & 70 \\
\hline 12 & 74 & 46 & 60 & 100 & 93 & 57 & 70 & 66 & 93 & 34 & 85 \\
\hline 13 & 74 & 46 & 60 & 94 & 97 & 57 & 55 & 66 & 97 & 34 & 85 \\
\hline 14 & 75 & 47 & 61 & 98 & 73 & 56 & 55 & 61 & $73^{(3)}$ & 31 & 55 \\
\hline 15 & 75 & 47 & 61 & 97 & 73 & 57 & 59 & 67 & 97 & 35 & 74 \\
\hline 16 & 75 & 47 & 61 & 95 & 73 & 54 & 55 & 60 & 73 & 32 & 74 \\
\hline 17 & 76 & 48 & 62 & 96 & 73 & 61 & 74 & 59 & 85 & 38 & $88^{(\text {a) }}$ \\
\hline 18 & 76 & 48 & 62 & 98 & 54 & 62 & 74 & 67 & 56 & 36 & 72 \\
\hline 19 & 77 & 48 & 62 & 92 & 93 & 56 & 62 & 70 & 56 & 33 & 75 \\
\hline 20 & 77 & 48 & 62 & 93 & 47 & 58 & 60 & 59 & 56 & 36 & 71 \\
\hline 21 & 77 & 49 & 63 & 94 & 58 & 62 & $60^{(2)}$ & 59 & 58 & 37 & $74^{(2)}$ \\
\hline 22 & 77 & 49 & 63 & 98 & 58 & 63 & 64 & 64 & 58 & 33 & 60 \\
\hline 23 & 77 & 49 & 63 & 95 & $85^{(2)}$ & 59 & 62 & 66 & 58 & 35 & 64 \\
\hline 24 & 77 & 49 & 63 & 96 & 92 & 54 & 62 & 63 & 81 & 35 & 75 \\
\hline 25 & 77 & 50 & 63 & 98 & $92^{(a)}$ & 61 & 98 & 65 & 83 & 38 & $91^{(a)}$ \\
\hline 26 & 77 & 50 & 64 & 101 & $58^{(2)}$ & 54 & 80 & 69 & 47 & 38 & 78 \\
\hline 27 & 78 & 50 & 64 & 93 & 83 & 62 & 89 & 69 & 58 & 37 & 73 \\
\hline 28 & 78 & 50 & 64 & 99 & 83 & 61 & 89 & 63 & $72^{(a)}$ & 38 & $79^{(2)}$ \\
\hline 29 & 78 & 51 & 64 & 103 & 83 & 68 & $98^{(a)}$ & 71 & 86 & 35 & 76 \\
\hline 30 & 79 & 51 & 65 & 104 & 86 & 62 & $76^{(2)}$ & 68 & 86 & 41 & $55^{(2)}$ \\
\hline 31 & 79 & 52 & 65 & 104 & 86 & 54 & 71 & 69 & 86 & 40 & \\
\hline
\end{tabular}


Table 3.12. (contd)

\begin{tabular}{|c|c|c|c|}
\hline \multirow[b]{2}{*}{ Day } & \multicolumn{3}{|c|}{ Normal (1961-1990) } \\
\hline & Maximum & Minimum & Mean \\
\hline 1 & 80 & 52 & 66 \\
\hline 2 & 80 & 52 & 66 \\
\hline 3 & 81 & 53 & 67 \\
\hline 4 & 81 & 54 & 67 \\
\hline 5 & 81 & 54 & 68 \\
\hline 6 & 81 & 54 & 68 \\
\hline 7 & 82 & 54 & 68 \\
\hline 8 & 82 & 54 & 68 \\
\hline 9 & 82 & 54 & 68 \\
\hline 10 & 82 & 54 & 68 \\
\hline 11 & 82 & 54 & 68 \\
\hline 12 & 83 & 55 & 69 \\
\hline 13 & 83 & 55 & 69 \\
\hline 14 & 84 & 56 & 70 \\
\hline 15 & 85 & 56 & 70 \\
\hline 16 & 85 & 57 & 71 \\
\hline 17 & 86 & 57 & 72 \\
\hline 18 & 86 & 57 & 72 \\
\hline 19 & 86 & 57 & 72 \\
\hline 20 & 86 & 57 & 72 \\
\hline 21 & 86 & 57 & 72 \\
\hline 22 & 86 & 57 & 72 \\
\hline 23 & 86 & 57 & 72 \\
\hline 24 & 86 & 57 & 71 \\
\hline 25 & 86 & 56 & 71 \\
\hline 26 & 86 & 56 & 71 \\
\hline 27 & 86 & 56 & 71 \\
\hline 28 & 86 & 56 & 71 \\
\hline 29 & 86 & 56 & 71 \\
\hline 30 & 86 & 56 & 71 \\
\hline
\end{tabular}

\begin{tabular}{|c|c|c|c|c|c|c|c|}
\hline \multicolumn{8}{|c|}{ Maximum } \\
\hline High & Year & Low & Year & High & $\overline{\text { Year }}$ & Low & $\overline{\text { Year }}$ \\
\hline 103 & 86 & 64 & 76 & 69 & 86 & 37 & 84 \\
\hline 99 & 70 & 65 & 80 & 69 & $89^{(a)}$ & 37 & 76 \\
\hline 103 & 70 & 55 & 66 & 68 & $86^{(2)}$ & 37 & 62 \\
\hline 103 & 69 & 60 & 74 & 66 & $86^{(a)}$ & 40 & $80^{(a)}$ \\
\hline 101 & 78 & 60 & 88 & 73 & 69 & 43 & $76^{(2)}$ \\
\hline 102 & $70^{(\mathrm{a})}$ & 57 & 95 & 68 & 77 & 46 & 62 \\
\hline 100 & 77 & 56 & 50 & 69 & 77 & 44 & 61 \\
\hline 100 & 48 & 59 & 64 & 69 & 69 & 40 & 53 \\
\hline 98 & 55 & 68 & 59 & 68 & $69^{(2)}$ & 44 & $85^{(a)}$ \\
\hline 98 & 55 & 69 & 72 & 68 & 79 & 41 & 59 \\
\hline 100 & 55 & 64 & 61 & 70 & 55 & 40 & 56 \\
\hline 98 & 74 & 68 & 54 & 67 & $87^{(2)}$ & 42 & 68 \\
\hline 99 & 74 & 59 & 80 & 67 & 89 & 42 & 52 \\
\hline 103 & 74 & 65 & 95 & 68 & 87 & 44 & $78^{(2)}$ \\
\hline 102 & 61 & 70 & 65 & 72 & 63 & 44 & 54 \\
\hline 106 & 61 & 62 & 49 & 70 & 63 & 41 & 54 \\
\hline 108 & 61 & 70 & 73 & 75 & 61 & 40 & 81 \\
\hline 104 & 61 & 69 & 64 & 75 & 58 & 41 & 54 \\
\hline 102 & 85 & 63 & 95 & 73 & 58 & 43 & 86 \\
\hline 102 & 82 & 63 & 91 & 73 & 59 & 42 & 53 \\
\hline 104 & 70 & 62 & 84 & 73 & 58 & 45 & 56 \\
\hline 106 & $92^{(a)}$ & 71 & 93 & 74 & 92 & 46 & $97^{(2)}$ \\
\hline 111 & 92 & 68 & 72 & 75 & 58 & 44 & 52 \\
\hline 108 & 92 & 66 & 72 & 80 & 92 & 40 & 83 \\
\hline 107 & 92 & 70 & 46 & 79 & 92 & 42 & 76 \\
\hline 103 & 87 & 70 & 75 & 74 & 70 & 41 & $76^{(2)}$ \\
\hline 102 & 92 & 68 & 47 & 75 & 87 & 45 & $64^{(2)}$ \\
\hline 102 & $87^{(\mathrm{a})}$ & 64 & 46 & 68 & 87 & 38 & 75 \\
\hline 104 & 48 & 65 & 52 & 74 & 87 & 46 & $71^{(2)}$ \\
\hline 106 & 87 & 71 & 55 & 71 & 87 & 42 & 49 \\
\hline
\end{tabular}

\begin{tabular}{|c|c|c|c|c|c|c|c|c|c|c|c|}
\hline \multicolumn{12}{|c|}{ July } \\
\hline 1 & 86 & 57 & 72 & 103 & 87 & 66 & 66 & 75 & 87 & 46 & $73^{(2)}$ \\
\hline 2 & 87 & 57 & 72 & 103 & 96 & 59 & 66 & 70 & 45 & 39 & 79 \\
\hline 3 & 87 & 58 & 72 & 105 & $91^{(a)}$ & 71 & 66 & 70 & 67 & 46 & 90 \\
\hline 4 & 88 & 58 & 73 & 108 & 68 & 71 & 86 & 75 & 70 & 48 & 66 \\
\hline 5 & 88 & 59 & 73 & 108 & 75 & 66 & 51 & 76 & 75 & 47 & 80 \\
\hline 6 & 89 & 59 & 74 & 110 & 68 & 71 & 55 & 76 & 68 & 44 & 71 \\
\hline 7 & 89 & 59 & 74 & 105 & $68^{(a)}$ & 75 & 81 & 73 & 68 & 45 & 71 \\
\hline 8 & 89 & 59 & 74 & 108 & 68 & 71 & 72 & 74 & 85 & 45 & 81 \\
\hline 9 & 89 & 60 & 74 & 110 & 75 & 76 & 55 & 78 & 75 & 50 & $72^{(x)}$ \\
\hline 10 & 90 & 60 & 75 & 106 & 75 & 67 & 74 & 79 & 75 & 49 & $97^{(a)}$ \\
\hline 11 & 90 & 60 & 75 & 109 & 90 & 76 & 74 & 78 & 75 & 46 & 81 \\
\hline 12 & 90 & 60 & 75 & 110 & 90 & 75 & 88 & 75 & 90 & 50 & 74 \\
\hline 13 & 90 & 60 & 75 & 108 & 61 & 77 & $93^{(2)}$ & 73 & $90^{(2)}$ & 49 & 76 \\
\hline 14 & 90 & 61 & 76 & 107 & $87^{(2)}$ & 77 & 83 & 78 & 61 & 50 & 83 \\
\hline 15 & 91 & 61 & 76 & 108 & 96 & 71 & 82 & 76 & 55 & 45 & 82 \\
\hline 16 & 91 & 61 & 76 & 105 & 70 & 68 & 86 & 74 & 90 & 48 & 74 \\
\hline 17 & 92 & 61 & 76 & 110 & 60 & 73 & 93 & 77 & 58 & 48 & 86 \\
\hline 18 & 92 & 61 & 77 & 110 & 60 & 76 & 96 & 79 & 60 & 49 & $96^{(a)}$ \\
\hline 19 & 92 & 61 & 77 & 109 & 79 & 72 & 49 & 77 & 79 & 51 & 77 \\
\hline 20 & 93 & 62 & 77 & 110 & 79 & 75 & $65^{(2)}$ & 75 & 95 & 53 & $68^{(a)}$ \\
\hline 21 & 93 & 62 & 78 & 109 & 94 & 68 & 65 & 77 & 88 & 49 & 49 \\
\hline 22 & 94 & 62 & 78 & 111 & 94 & 74 & 92 & 75 & $94^{(a)}$ & 47 & 82 \\
\hline 23 & 94 & 63 & 78 & 109 & 94 & 69 & 92 & 82 & 94 & 49 & 63 \\
\hline 24 & 94 & 63 & 79 & 109 & 94 & 78 & 63 & 75 & 62 & 52 & $52^{(a)}$ \\
\hline 25 & 95 & 63 & 79 & 106 & 84 & 73 & 90 & 77 & 62 & 51 & $49^{(2)}$ \\
\hline 26 & 95 & 63 & 79 & 108 & $98^{(a)}$ & 66 & 55 & 76 & 88 & 54 & 48 \\
\hline
\end{tabular}


Table 3.12. (contd)

\begin{tabular}{|c|c|c|c|}
\hline \multirow[b]{2}{*}{ Day } & \multicolumn{3}{|c|}{ Normal (1961-1990) } \\
\hline & Maximum & Minimum & Mean \\
\hline 27 & 95 & 64 & 79 \\
\hline 28 & 95 & 64 & 79 \\
\hline 29 & 95 & 63 & 79 \\
\hline 30 & 95 & 63 & 79 \\
\hline 31 & 94 & 63 & 79 \\
\hline
\end{tabular}

\begin{tabular}{|c|c|c|c|c|c|c|c|}
\hline \multicolumn{8}{|c|}{ Extreme (1945-1998) } \\
\hline \multicolumn{4}{|c|}{ Maximum } & \multicolumn{4}{|c|}{ Minimum } \\
\hline Figh & Year & Low & Year & High & Year & Low & Year \\
\hline 112 & 98 & 74 & 48 & 74 & $98^{(a)}$ & 52 & 86 \\
\hline 108 & $98^{(2)}$ & 77 & $50^{(a)}$ & 81 & 98 & 49 & 59 \\
\hline 107 & 82 & 80 & $93^{(a)}$ & 78 & 82 & 52 & 50 \\
\hline 107 & 71 & 78 & 75 & 74 & 90 & 49 & 50 \\
\hline 111 & 71 & 75 & 85 & 74 & $71^{(a)}$ & 52 & 95 \\
\hline
\end{tabular}

\begin{tabular}{|c|c|c|c|c|c|c|c|c|c|c|c|}
\hline \multicolumn{12}{|c|}{ August } \\
\hline 1 & 94 & 63 & 78 & 109 & 71 & 77 & $76^{(a)}$ & 80 & 49 & 51 & 87 \\
\hline 2 & 94 & 62 & 78 & 106 & 94 & 75 & 56 & 75 & $77^{(a)}$ & 46 & 64 \\
\hline 3 & 94 & 62 & 78 & 107 & 61 & 77 & 62 & 75 & 94 & 52 & 59 \\
\hline 4 & 93 & 62 & 78 & 113 & 61 & 78 & $64^{(a)}$ & 81 & 61 & 48 & 54 \\
\hline 5 & 93 & 62 & 78 & 108 & 90 & 80 & $96^{(2)}$ & 72 & 91 & 45 & 69 \\
\hline 6 & 94 & 62 & 78 & 106 & 72 & 77 & 46 & 77 & 90 & 51 & 47 \\
\hline 7 & 94 & 63 & 78 & 109 & 72 & 70 & 62 & 75 & 45 & 49 & 46 \\
\hline 8 & 94 & 63 & 78 & 110 & 72 & 75 & 62 & 79 & $82^{(k)}$ & 48 & 49 \\
\hline 9 & 94 & 63 & 78 & 112 & 71 & 78 & 47 & 78 & 90 & 51 & $95^{(a)}$ \\
\hline 10 & 94 & 63 & 78 & 109 & $96^{(a)}$ & 76 & 85 & 77 & 71 & 52 & 47 \\
\hline 11 & 93 & 63 & 78 & 108 & 71 & 79 & 83 & 73 & 58 & 50 & 85 \\
\hline 12 & 93 & 62 & 77 & 108 & 71 & 77 & 95 & 77 & 92 & 52 & 57 \\
\hline 13 & 92 & 61 & 77 & 107 & 92 & 74 & 68 & 79 & 92 & 47 & 95 \\
\hline 14 & 91 & 60 & 76 & 109 & 92 & 70 & 68 & 78 & $92^{(a)}$ & 45 & 95 \\
\hline 15 & 90 & 60 & 75 & 105 & 67 & 72 & 60 & 74 & 92 & 51 & 74 \\
\hline 16 & 89 & 59 & 74 & 108 & 67 & 68 & 93 & 76 & 45 & 48 & 76 \\
\hline 17 & 89 & 59 & 74 & 108 & 67 & 76 & 95 & 68 & 91 & 47 & 76 \\
\hline 18 & 89 & 59 & 74 & 108 & 67 & 71 & 80 & 71 & 97 & 47 & 76 \\
\hline 19 & 88 & 59 & 74 & 105 & 67 & 70 & 68 & 76 & 91 & 46 & $80^{(a)}$ \\
\hline 20 & 88 & 59 & 74 & 105 & 67 & 67 & 59 & 77 & 82 & 49 & 52 \\
\hline 21 & 88 & 59 & 74 & 104 & 46 & 70 & 60 & 75 & 46 & 47 & $85^{(2)}$ \\
\hline 22 & 88 & 59 & 73 & 104 & $56^{(a)}$ & 70 & 92 & 76 & $61^{(a)}$ & 41 & 60 \\
\hline 23 & 88 & 58 & 73 & 105 & 70 & 69 & 92 & 76 & 46 & 45 & 92 \\
\hline 24 & 87 & 58 & 73 & 104 & 58 & 70 & 68 & 71 & 66 & 43 & 92 \\
\hline 25 & 87 & 58 & 72 & 105 & 96 & 72 & 77 & 70 & 46 & 43 & 93 \\
\hline 26 & 87 & 58 & 72 & 100 & 84 & 68 & 56 & 70 & 96 & 44 & $93^{(2)}$ \\
\hline 27 & 87 & 57 & 72 & 101 & 72 & 73 & 68 & 71 & 67 & 47 & $78^{(\mathrm{a})}$ \\
\hline 28 & 86 & 57 & 72 & 104 & 72 & 70 & 51 & 74 & 86 & 42 & 80 \\
\hline 29 & 86 & 56 & 71 & 102 & 67 & 72 & 51 & 73 & 67 & 42 & 65 \\
\hline 30 & 86 & 56 & 71 & 105 & 67 & 68 & 51 & 71 & 67 & 44 & 64 \\
\hline 31 & 85 & 56 & 71 & 104 & 67 & 72 & 71 & 73 & 67 & 45 & 65 \\
\hline \multicolumn{12}{|c|}{ September } \\
\hline 1 & 85 & 56 & 71 & 106 & 87 & 61 & 71 & 70 & 87 & 44 & 80 \\
\hline 2 & 85 & 56 & 71 & 102 & $98^{(a)}$ & 70 & 71 & 70 & 49 & 47 & $75^{(\mathrm{a})}$ \\
\hline 3 & 85 & 55 & 70 & 102 & 98 & 71 & 97 & 71 & 95 & 44 & $80^{(a)}$ \\
\hline 4 & 85 & 55 & 70 & 102 & 88 & 68 & 59 & 68 & 55 & 44 & 80 \\
\hline 5 & 85 & 55 & 70 & 100 & 55 & 72 & 60 & 68 & 63 & 43 & 69 \\
\hline 6 & 85 & 55 & 70. & 101 & 55 & 69 & 46 & 65 & $57^{(a)}$ & 41 & 96 \\
\hline 7 & 85 & 54 & 69 & 97 & 58 & 60 & 78 & 72 & 55 & 42 & $92^{(\mathrm{a})}$ \\
\hline 8 & 84 & 54 & 69 & 99 & 81 & 61 & 85 & 69 & 63 & 42 & $76^{(a)}$ \\
\hline 9 & 83 & 53 & 68 & 98 & $81^{(a)}$ & 66 & 85 & 68 & 69 & 40 & 62 \\
\hline 10 & 82 & 53 & 67 & 97 & 93 & 68 & 85 & 65 & 63 & 43 & $82^{(2)}$ \\
\hline 11 & 81 & 52 & 67 & 98 & $90^{(2)}$ & 62 & 85 & 66 & 69 & 41 & 88 \\
\hline 12 & 81 & 52 & 66 & 96 & 69 & 62 & 70 & 67 & 53 & 38 & 49 \\
\hline 13 & 80 & 51 & 66 & 98 & 48 & 59 & 80 & 62 & $60^{(a)}$ & 40 & 74 \\
\hline 14 & 80 & 51 & 65 & 94 & $98^{(2)}$ & 62 & 92 & 61 & $90^{(2)}$ & 38 & 70 \\
\hline 15 & 79 & 51 & 65 & 96 & $79^{(2)}$ & 58 & 59 & 61 & 45 & 35 & 70 \\
\hline 16 & 79 & 51 & 65 & 96 & $81^{(2)}$ & 61 & 46 & 65 & 79 & 35 & 65 \\
\hline 17 & 79 & 51 & 65 & 97 & 81 & 59 & 86 & 62 & 51 & 33 & 65 \\
\hline
\end{tabular}


Table 3.12. (contd)

\begin{tabular}{|c|c|c|}
\hline \multirow[b]{2}{*}{ Day } & \multicolumn{2}{|c|}{ Normal (1961-1990) } \\
\hline & Maximum & Minimum \\
\hline 18 & 78 & 50 \\
\hline 19 & 78 & 50 \\
\hline 20 & 78 & 50 \\
\hline 21 & 78 & 49 \\
\hline 22 & 77 & 49 \\
\hline 23 & 77 & 49 \\
\hline 24 & 77 & 49 \\
\hline 25 & 77 & 48 \\
\hline 26 & 77 & 48 \\
\hline 27 & 76 & 48 \\
\hline 28 & 76 & 48 \\
\hline 29 & 76 & 47 \\
\hline 30 & 75 & 46 \\
\hline
\end{tabular}

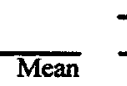

\begin{tabular}{ll}
\multicolumn{3}{c}{ Extreme (1945-1998) } \\
\hline Migh & Mear Low \\
\hline
\end{tabular}

\begin{tabular}{llllllllllllll}
\multicolumn{10}{c}{ October } \\
\hline 1 & 75 & 46 & 60 & 88 & $91^{(a)}$ & 61 & 59 & 59 & 92 & 30 & 54
\end{tabular}

\begin{tabular}{|c|c|c|c|c|c|c|c|c|c|c|c|}
\hline 1 & 75 & 46 & 60 & 88 & $91^{(a)}$ & 61 & 59 & 59 & 92 & 30 & 54 \\
\hline 2 & 74 & 45 & 60 & 86 & $93^{(a)}$ & 56 & 67 & 60 & 88 & 32 & 54 \\
\hline 3 & 73 & 45 & 59 & 89 & 58 & 55 & 50 & 58 & $88^{(a)}$ & 33 & $73^{(a)}$ \\
\hline 4 & 73 & 44 & 58 & 89 & 80 & 55 & 50 & 57 & 88 & 32 & 73 \\
\hline 5 & 72 & 44 & 58 & 87 & 58 & 52 & 57 & 52 & $88^{(2)}$ & 34 & $82^{(\mathrm{a})}$ \\
\hline 6 & 72 & 44 & 58 & 85 & 80 & 53 & $57^{(\mathrm{a})}$ & 57 & 60 & 30 & 74 \\
\hline 7 & 71 & 44 & 57 & 86 & 80 & 48 & 57 & 57 & 88 & 29 & 74 \\
\hline 8 & 70 & 44 & 57 & 84 & 65 & 50 & 97 & 58 & 87 & 26 & 85 \\
\hline 9 & 70 & 43 & 56 & 84 & 45 & 52 & 58 & 56 & 96 & 26 & 85 \\
\hline 10 & 69 & 43 & 56 & 86 & 96 & 50 & 62 & 55 & 96 & 33 & 59 \\
\hline 11 & 69 & 43 & 56 & 84 & 52 & 52 & $68^{(a)}$ & 55 & 63 & 30 & 60 \\
\hline 12 & 68 & 43 & 55 & 84 & 45 & 54 & 66 & 56 & 52 & 34 & $85^{(\mathrm{a})}$ \\
\hline 13 & 68 & 42 & 55 & 79 & 45 & 57 & 69 & 55 & 88 & 31 & 69 \\
\hline 14 & 67 & 41 & 54 & 78 & 45 & 58 & $90^{(a)}$ & 59 & 88 & 24 & 69 \\
\hline 15 & 66 & 40 & 53 & 81. & 63 & 53 & 92 & 60 & 88 & 29 & 70 \\
\hline 16 & 66 & 39 & 53 & 79 & 63 & 54 & $92^{(a)}$ & 56 & 95 & 26 & 46 \\
\hline 17 & 65 & 39 & 52 & 77 & 97 & 45 & 96 & 49 & 55 & 26 & 96 \\
\hline 18 & 65 & 38 & 51 & 76 & 73 & 47 & 49 & 50 & 52 & 27 & 49 \\
\hline 19 & 64 & 38 & 51 & 78 & $92^{(a)}$ & 50 & 45 & 52 & 92 & 27 & $69^{(a)}$ \\
\hline 20 & 63 & 38 & 50 & 74 & 78 & 45 & 47 & 54 & 73 & 23 & 49 \\
\hline 21 & 63 & 38 & 50 & 73 & $91^{(a)}$ & 45 & 96 & 54 & 63 & 20 & 84 \\
\hline 22 & 63 & 38 & 50 & 74 & 59 & 46 & 50 & 51 & 52 & 20 & 84 \\
\hline 23 & 62 & 38 & 50 & 73 & $66^{(2)}$ & 39 & 84 & 51 & 60 & 25 & 84 \\
\hline 24 & 61 & 38 & 50 & 75 & $77^{(a)}$ & 49 & 57 & 49 & 46 & 26 & 75 \\
\hline 25 & 61 & 37 & 49 & 75 & $55^{(a)}$ & 49 & 57 & 60 & 45 & 26 & 78 \\
\hline 26 & 60 & 37 & 48 & 69 & 92 & 49 & 46 & 52 & 94 & 21 & 78 \\
\hline 27 & 59 & 36 & 48 & 74 & 85 & 43 & $93^{(2)}$ & 54 & 81 & 23 & 70 \\
\hline 28 & 58 & 36 & 47 & 68 & $65^{(a)}$ & 35 & 91 & 52 & 49 & 18 & 71 \\
\hline 29 & 58 & 36 & 47 & 70 & 53 & 42 & $91^{(2)}$ & 50 & 97 & 13 & 71 \\
\hline 30 & 57 & 36 & 46 & 75 & 67 & 32 & 71 & 50 & 97 & 20 & 72 \\
\hline 31 & 56 & 36 & 46 & 75 & 67 & 34 & 84 & 54 & 67 & 12 & 84 \\
\hline \multicolumn{12}{|c|}{ November } \\
\hline 1 & 56 & 36 & 46 & 69 & 88 & 31 & 84 & 49 & 87 & 20 & 95 \\
\hline 2 & 56 & 36 & 46 & 70 & 45 & 38 & 91 & 51 & 85 & 17 & 95 \\
\hline 3 & 55 & 36 & 45 & 75 & 75 & 36 & 73 & 53 & 83 & 17 & 95 \\
\hline 4 & 55 & 36 & 45 & 71 & 75 & 32 & 73 & 46 & $89^{(2)}$ & 16 & 73 \\
\hline 5 & 54 & 35 & 44 & 63 & 89 & 31 & 73 & 48 & 88 & 20 & $73^{(a)}$ \\
\hline 6 & 53 & 35 & 44 & 64 & 58 & 32 & 73 & 49 & 89 & 19 & 73 \\
\hline 7 & 53 & 35 & 44 & 69 & 78 & 34 & 45 & 48 & $97^{(2)}$ & 19 & $93^{(2)}$ \\
\hline 8 & 52 & 34 & 43 & 69 & 95 & 34 & 45 & 48 & 89 & 16 & 45 \\
\hline 9 & 51 & 34 & 43 & 73 & 89 & 30 & 45 & 60 & 89 & 18 & 86 \\
\hline
\end{tabular}


Table 3.12. (contd)

\begin{tabular}{|c|c|c|c|}
\hline \multirow[b]{2}{*}{ Day } & \multicolumn{3}{|c|}{ Normal (1961-1990) } \\
\hline & Maximum & Minimum & $\overline{\text { Mean }}$ \\
\hline 10 & 51 & 34 & 42 \\
\hline 11 & 50 & 34 & 42 \\
\hline 12 & 50 & 33 & 42 \\
\hline 13 & 49 & 33 & 41 \\
\hline 14 & 49 & 33 & 41 \\
\hline 15 & 49 & 32 & 40 \\
\hline 16 & 48 & 32 & 40 \\
\hline 17 & 48 & 31 & 40 \\
\hline 18 & 48 & 31 & 39 \\
\hline 19 & 47 & 31 & 39 \\
\hline 20 & 47 & 30 & 38 \\
\hline 21 & 46 & 30 & 38 \\
\hline 22 & 46 & 29 & 38 \\
\hline 23 & 45 & 29 & 37 \\
\hline 24 & 45 & 29 & 37 \\
\hline 25 & 44 & 28 & 36 \\
\hline 26 & 43 & 28 & 36 \\
\hline 27 & 43 & 28 & 35 \\
\hline 28 & 43 & 28 & 35 \\
\hline 29 & 42 & 28 & 35 \\
\hline 30 & 42 & 28 & 35 \\
\hline
\end{tabular}

\begin{tabular}{|c|c|c|c|c|c|c|c|}
\hline \multicolumn{8}{|c|}{ Extreme (1943-1998) } \\
\hline \multicolumn{4}{|c|}{ Maximum } & \multicolumn{4}{|c|}{ Minimum } \\
\hline High & Year & Low & Year & Figh & Year & Low & Year \\
\hline 73 & 89 & 32 & 45 & 56 & 89 & 16 & 86 \\
\hline 66 & 89 & 32 & 85 & 48 & 89 & 14 & 78 \\
\hline 65 & 91 & 20 & 55 & 52 & 49 & 6 & 55 \\
\hline 64 & 87 & 14 & 55 & 56 & 98 & 6 & 59 \\
\hline 65 & 95 & 19 & 55 & 47 & $98^{(2)}$ & -1 & 55 \\
\hline 67 & 98 & 18 & 55 & 47 & 98 & 1 & 55 \\
\hline 65 & 76 & 18 & 55 & 49 & 54 & 7 & 59 \\
\hline 71 & 76 & 22 & 55 & 46 & $83^{(2)}$ & 10 & 61 \\
\hline 64 & 46 & 25 & 55 & 47 & 54 & 11 & 55 \\
\hline 67 & 62 & 22 & 85 & 44 & 54 & 14 & 85 \\
\hline 65 & 58 & 24 & 85 & 47 & 74 & 3 & 85 \\
\hline 63 & 58 & 17 & 85 & 46 & 65 & 3 & 85 \\
\hline 65 & $67^{(a)}$ & 17 & 85 & 53 & 90 & -6 & 85 \\
\hline 70 & 59 & 11 & 85 & 54 & 90 & -13 & 85 \\
\hline 67 & $95^{(2)}$ & 6 & 85 & 57 & 90 & -12 & 85 \\
\hline 67 & 98 & 11 & 85 & 50 & 98 & -3 & 85 \\
\hline 65 & 49 & 15 & 85 & 45 & 49 & -1 & 85 \\
\hline 63 & 49 & 12 & 85 & 46 & 49 & 8 & 85 \\
\hline 64 & 95 & 11 & 85 & 43 & 73 & 7 & 85 \\
\hline 62 & 95 & 14 & 85 & 57 & 95 & 8 & 85 \\
\hline 62 & $95^{(a)}$ & 15 & 85 & 46 & 94 & 6 & 85 \\
\hline & nber & & & & & & \\
\hline
\end{tabular}

\begin{tabular}{rrrrrrrrrrrr}
\hline 1 & 42 & 28 & 35 & 65 & 72 & 14 & 85 & 45 & 81 & -8 & 85 \\
2 & 42 & 28 & 35 & 64 & 77 & 20 & 85 & 56 & 75 & 8 & 85 \\
3 & 42 & 28 & 35 & 62 & $82^{(2)}$ & 27 & $85^{(a)}$ & 54 & 75 & 8 & 85 \\
4 & 42 & 28 & 35 & 60 & $75^{(2)}$ & 23 & 72 & 41 & 52 & 1 & 72 \\
5 & 41 & 28 & 34 & 58 & $91^{(2)}$ & 21 & 72 & 41 & 87 & 7 & 72 \\
6 & 41 & 27 & 34 & 59 & 87 & 16 & 56 & 43 & 87 & 8 & 56 \\
7 & 40 & 27 & 33 & 58 & $73^{(a)}$ & 18 & 56 & 40 & 52 & 2 & 56 \\
8 & 40 & 26 & 33 & 58 & 89 & 17 & 72 & 48 & 46 & -6 & 72 \\
9 & 40 & 26 & 33 & 59 & $87^{(2)}$ & 13 & 72 & 44 & 56 & -5 & 72 \\
10 & 39 & 25 & 32 & 67 & 93 & 13 & 72 & 48 & 46 & -8 & 72 \\
11 & 39 & 25 & 32 & 59 & $91^{(a)}$ & 11 & 72 & 45 & 46 & -4 & 72 \\
12 & 39 & 25 & 32 & 57 & 88 & 22 & 72 & 42 & $77^{(2)}$ & -5 & 72 \\
13 & 38 & 25 & 31 & 59 & 46 & 13 & 72 & 42 & 46 & -6 & 72 \\
14 & 38 & 24 & 31 & 57 & $79^{(a)}$ & 15 & 72 & 48 & 79 & -2 & 72 \\
15 & 37 & 24 & 31 & 64 & 59 & 16 & 72 & 41 & 56 & 2 & 72 \\
16 & 37 & 24 & 30 & 60 & 97 & 4 & 64 & 43 & 73 & -13 & 64 \\
17 & 37 & 24 & 30 & 57 & $98^{(2)}$ & 5 & 64 & 41 & 62 & -12 & 64 \\
18 & 36 & 24 & 30 & 56 & 56 & 13 & 64 & 38 & $94^{(a)}$ & -3 & 84 \\
19 & 36 & 24 & 30 & 59 & 94 & 17 & 84 & 41 & 94 & -4 & 84 \\
20 & 37 & 24 & 30 & 64 & 94 & 11 & 84 & 47 & 94 & -2 & $90^{(a)}$ \\
21 & 37 & 24 & 30 & 61 & 72 & 11 & 90 & 43 & 73 & -10 & 90 \\
22 & 37 & 24 & 30 & 59 & 80 & 7 & 90 & 42 & 72 & -13 & 83 \\
23 & 37 & 24 & 30 & 57 & 63 & 6 & 83 & 40 & 72 & -9 & 83 \\
24 & 37 & 24 & 30 & 55 & $61^{(a)}$ & 15 & $90^{(a)}$ & 39 & $80^{(2)}$ & 0 & 90 \\
25 & 37 & 23 & 30 & 65 & 80 & 16 & 90 & 41 & 72 & 1 & 90 \\
26 & 36 & 23 & 30 & 69 & 80 & 20 & $90^{(a)}$ & 53 & 80 & 0 & 48 \\
27 & 36 & 23 & 29 & 62 & $80^{(a)}$ & 19 & 48 & 40 & $94^{(a)}$ & -2 & 48 \\
28 & 35 & 22 & 29 & 59 & 98 & 11 & 96 & 46 & 98 & -6 & 96 \\
29 & 35 & 22 & 28 & 60 & $98^{(a)}$ & -2 & 68 & 41 & 98 & -10 & $90^{(a)}$ \\
30 & 35 & 21 & 28 & 54 & 70 & -2 & 68 & 39 & $88^{(a)}$ & -14 & 68 \\
31 & 35 & 21 & 28 & 56 & 62 & 4 & 68 & 44 & 80 & -9 & 78
\end{tabular}

(a) Latest of several occurrences. 


\subsection{Heating- and Cooling-Degree Days}

Heating- and cooling-degree day data are generally used by the utility industry and those involved in building design to assess heating and cooling energy requirements. A temperature of $65^{\circ} \mathrm{F}$ is generally used as the basis for this calculation. To determine whether a day has either heating-degree days (HDDs) or cooling-degree days (CDDs), 65 is subtracted from the daily average temperature (computed by adding the daily maximum and minimum temperatures and dividing by two). If the difference is positive, the day has cooling-degree days. If the difference is negative, the day has heating-degree days.

Example Calculations

\begin{tabular}{lll}
\hline Daily high temperature & 90 & 42 \\
Daily low temperature & 60 & 20 \\
Daily average temperature & $75(150 \div 2)$ & $31(62 \div 2)$ \\
Threshold temperature & -65 & -65 \\
Difference & $10(10 \mathrm{CDDs})$ & $-34(34$ HDDs $)$
\end{tabular}

Tables 3.14 and 3.15 provide monthly HDD and CDD data, respectively, for the period 1945 through 1998. The HDDs are traditionally totaled for the 12-month period July through June of the following year. 
Table 3.13. Subsurface Soil Temperatures $\left({ }^{\circ} \mathrm{F}\right)$ at Depths of $0.5,15$, and 36 Inches

\begin{tabular}{|c|c|c|c|c|c|c|c|c|c|c|c|c|c|c|c|}
\hline \multirow[b]{3}{*}{ Month } & & . & & \multicolumn{4}{|c|}{ 0.5-in. Depth } & \multicolumn{4}{|c|}{ 15-in. Depth } & \multicolumn{4}{|c|}{ 36-in. Depth } \\
\hline & \multicolumn{3}{|c|}{ Monthly Averages } & \multirow{2}{*}{$\begin{array}{l}\text { Highest } \\
\text { Monthly } \\
\text { Average }\end{array}$} & \multirow[b]{2}{*}{ Year } & \multirow{2}{*}{$\begin{array}{c}\text { Lowest } \\
\text { Monthly } \\
\text { Average } \\
\end{array}$} & \multirow[b]{2}{*}{ Year } & \multirow{2}{*}{$\begin{array}{l}\text { Highest } \\
\text { Monthly } \\
\text { Average } \\
\end{array}$} & \multirow[b]{2}{*}{ Year } & \multirow{2}{*}{$\begin{array}{l}\text { Lowest } \\
\text { Monthly } \\
\text { Average } \\
\end{array}$} & \multirow[b]{2}{*}{ Year } & \multirow{2}{*}{$\begin{array}{l}\text { Highest } \\
\text { Monthly } \\
\text { Average } \\
\end{array}$} & \multirow[b]{2}{*}{ Year } & \multirow{2}{*}{$\begin{array}{l}\text { Lowest } \\
\text { Monthly } \\
\text { Average } \\
\end{array}$} & \multirow[b]{2}{*}{ Year } \\
\hline & $0.5 \mathrm{in}$. & 15 in. & 36 in. & & & & & & & & & & & & \\
\hline Jan & 32.5 & 36.1 & 42.4 & 39.4 & 1967 & 19.2 & 1979 & 42.7 & 1981 & 25.5 & 1979 & 48.7 & 1975 & 36.3 & 1979 \\
\hline Feb & 38.3 & 38.7 & 41.8 & 45.1 & 1958 & 28.6 & 1989 & 44.9 & 1967 & 29.6 & 1957 & 46.9 & 1967 & 33.5 & 1957 \\
\hline Mar & 48.2 & 46.3 & 46.0 & 54.3 & 1992 & 42.4 & 1955 & 52.6 & 1968 & 37.7 & 1956 & 51.7 & 1968 & 38.0 & 1956 \\
\hline Apr & 59.9 & 55.6 & 53.1 & 69.4 & 1977 & 52.4 & 1984 & 62.1 & 1977 & 48.7 & 1955 & 57.4 & 1966 & 47.3 & 1955 \\
\hline May & 72.1 & 65.5 & 60.8 & 81.4 & 1992 & 63.6 & 1984 & 71.4 & 1992 & 58.7 & 1984 & 65.1 & 1987 & 54.8 & 1955 \\
\hline Jun & 82.7 & 75.1 & 68.8 & 90.4 & 1986 & 75.3 & 1956 & 84.5 & 1966 & 70.2 & 1956 & 73.4 & 1969 & 62.8 & 1984 \\
\hline Jul & 90.9 & 81.8 & 75.3 & 96.2 & 1973 & 81.0 & 1993 & 88.2 & 1967 & 75.4 & 1955 & 81.1 & 1967 & 70.8 & 1955 \\
\hline Aug & 87.7 & 82.7 & 78.7 & 94.9 & 1971 & 81.6 & 1960 & 89.2 & 1967 & 77.5 & 1964 & 83.9 & 1967 & 75.3 & 1978 \\
\hline Sep & 74.0 & 74.6 & 75.0 & 81.0 & 1967 & 65.5 & 1985 & 82.2 & 1967 & 68.8 & 1959 & 81.4 & 1967 & 70.1 & 1978 \\
\hline Oct & 56.5 & 62.4 & 67.3 & 62.6 & 1988 & 52.4 & 1985 & 66.6 & 1967 & 57.9 & 1957 & 72.3 & 1967 & 62.9 & 1959 \\
\hline Nov & 40.6 & 48.0 & 56.6 & 45.2 & 1987 & 31.9 & 1985 & 54.0 & 1974 & 42.5 & 1955 & 62.7 & 1974 & 51.2 & 1955 \\
\hline Dec & 33.3 & 38.9 & 47.6 & 38.7 & 1974 & 26.5 & 1984 & 45.0 & 1974 & 34.1 & 1984 & 54.6 & 1974 & 41.4 & 1955 \\
\hline \multirow[t]{3}{*}{ Annual } & 59.7 & 58.8 & 59.7 & 62.8 & 1967 & 55.9 & 1955 & 63.0 & 1967 & 54.6 & 1955 & 67.3 & 1987 & 55.5 & 1955 \\
\hline & & & & \multicolumn{12}{|c|}{ Absolute Hourly Extremes } \\
\hline & & & & 156.8 & 1996 & -2.0 & 1972 & 93.0 & 1967 & 16.1 & 1979 & 85.3 & 1967 & 32.2 & 1957 \\
\hline
\end{tabular}


Table 3.14. Monthly and Seasonal Heating-Degree Days

\begin{tabular}{|c|c|c|c|c|c|c|c|c|c|c|c|c|c|}
\hline Year & Jul & Aug & Sep & Oct & Nov & Dec & Jan & Feb & Mar & Apr & May & Jun & Season \\
\hline $1944-45$ & - & - & - & - & - & - & 967 & 738 & 709 & 442 & 141 & 38 & - \\
\hline $1945-46$ & 0 & 2 & 97 & 277 & 733 & 1,000 & 949 & 710 & 603 & 331 & 79 & 42 & 4,823 \\
\hline $1946-47$ & 0 & 1 & 101 & 479 & 875 & 935 & 1,168 & 702 & 476 & 266 & $36^{(a)}$ & 25 & 5,064 \\
\hline $1947-48$ & 0 & 7 & 70 & 351 & 714 & 989 & 1,024 & 963 & 709 & 471 & 237 & 5 & 5,540 \\
\hline $1948-49$ & 2 & 0 & 109 & 438 & 725 & 1,184 & 1,581 & 928 & 616 & 281 & 85 & 36 & 5,985 \\
\hline 1949-50 & 1 & 0 & 52 & 456 & 592 & 927 & $1,640^{(a)}$ & 959 & 704 & 452 & 196 & 66 & 6,045 \\
\hline $1950-51$ & 0 & 0 & 64 & 431 & 728 & 895 & 994 & 786 & 773 & 325 & 146 & 45 & 5,187 \\
\hline $1951-52$ & 5 & 19 & 46 & 421 & 763 & 1,164 & 1,235 & 823 & 645 & 311 & 118 & 45 & 5,595 \\
\hline $1952-53$ & 0 & 2 & 34 & $200^{(\mathrm{s})}$ & 929 & 934 & $694^{(a)}$ & 664 & 585 & 419 & 228 & $90^{(2)}$ & 4,779 \\
\hline $1953-54$ & 0 & 3 & 59 & 298 & 649 & 851 & 1,118 & 720 & 722 & 408 & 124 & 77 & 5,029 \\
\hline $1954-55$ & 10 & 4 & 79 & 423 & 567 & 957 & 1,090 & 832 & $794^{(a)}$ & $522^{(a)}$ & 253 & 23 & 5,554 \\
\hline $1955-56$ & $22^{(a)}$ & 0 & 108 & 364 & 1,008 & 1,105 & 1,029 & $1,147^{(2)}$ & 655 & 273 & 110 & 55 & 5,876 \\
\hline $1956-57$ & 0 & 6 & 32 & 399 & 850 & 940 & 1,499 & 862 & 650 & 308 & 50 & 11 & 5,607 \\
\hline $1957-58$ & 0 & 0 & 37 & 443 & 739 & $822^{(a)}$ & 862 & 576 & 666 & 411 & 72 & 3 & 4,631 \\
\hline $1958-59$ & 0 & 0 & 74 & 339 & 731 & 927 & 1,025 & 827 & 617 & 325 & 248 & 29 & 5,142 \\
\hline $1959-60$ & 4 & 6 & 118 & 359 & 855 & 987 & 1,292 & 799 & 616 & 374 & 227 & 21 & 5,658 \\
\hline $1960-61$ & 0 & $32^{(a)}$ & 35 & 330 & 717 & 1,114 & 930 & 598 & 587 & 380 & 179 & 16 & 4,918 \\
\hline $1961-62$ & 0 & 0 & 91 & 418 & 893 & 974 & 1,090 & 797 & 698 & 287 & 255 & 51 & 5,554 \\
\hline $1962-63$ & 12 & 1 & 60 & 385 & 657 & 874 & 1,228 & 747 & 577 & 456 & 170 & 25 & 5,192 \\
\hline $1963-64$ & 1 & 1 & 25 & 285 & 668 & 1,078 & 913 & 784 & 656 & 445 & 195 & 33 & 5,084 \\
\hline $1964-65$ & 0 & 21 & 94 & 360 & 804 & 1,224 & 1,009 & 686 & 685 & 307 & 171 & 16 & 5,377 \\
\hline $1965-66$ & 5 & 14 & 115 & 247 & 660 & 995 & 963 & 702 & 605 & 311 & 133 & 58 & 4,808 \\
\hline $1966-67$ & 17 & 2 & 26 & 362 & 638 & 829 & 782 & 598 & 639 & 519 & 175 & 12 & 4,599 \\
\hline $1967-68$ & 0 & 0 & 13 & 305 & 704 & 993 & 907 & 670 & 495 & 416 & 117 & 23 & 4,643 \\
\hline $1968-69$ & 0 & 13 & 50 & 458 & 702 & 1,064 & 1,399 & 932 & 591 & 384 & 88 & 6 & 5,687 \\
\hline $1969-70$ & 0 & 5 & 39 & 431 & 745 & 941 & 1,064 & 683 & 625 & 480 & 137 & 23 & 5,173 \\
\hline $1970-71$ & 0 & 0 & 122 & 439 & 758 & 1,063 & 906 & 726 & 752 & 392 & 124 & 50 & 5,332 \\
\hline $1971-72$ & 13 & 3 & 133 & 420 & 728 & 1,064 & 1,065 & 878 & 560 & 463 & 112 & 23 & 5,462 \\
\hline $1972-73$ & 1 & 3 & 179 & 397 & 754 & 1,168 & 1,112 & 742 & 544 & 338 & 144 & 38 & 5,420 \\
\hline $1973-74$ & 2 & 9 & 73 & 389 & 798 & 837 & 1,104 & 675 & 611 & 361 & 236 & 27 & 5,122 \\
\hline $1974-75$ & 8 & 0 & 32 & 388 & 698 & 892 & 996 & 880 & 704 & 504 & 174 & 31 & 5,307 \\
\hline $1975-76$ & 0 & 13 & 25 & 388 & 764 & 949 & 1,024 & 796 & 735 & 422 & 159 & 74 & 5,349 \\
\hline $1976-77$ & 5 & 15 & 23 & 392 & 736 & 1,065 & 1,232 & 684 & 608 & 253 & 258 & 22 & 5,293 \\
\hline $1977-78$ & 5 & 7 & 153 & 401 & 783 & 967 & 1,001 & 761 & 550 & 393 & 203 & 22 & 5,246 \\
\hline 1978-79 & 1 & 10 & 76 & 390 & 981 & 1,162 & 1,582 & 861 & 571 & 369 & 94 & 21 & $6,118^{(a)}$ \\
\hline $1979-80$ & 13 & 0 & 10 & 266 & 924 & 887 & 1,277 & 888 & 638 & 302 & 138 & 68 & 5,411 \\
\hline $1980-81$ & 5 & 18 & 53 & 394 & 723 & 883 & 838 & 707 & 503 & 345 & 165 & 51 & 4,685 \\
\hline $1981-82$ & 8 & 0 & 108 & 402 & 668 & 998 & 1,092 & 754 & 590 & 469 & 164 & 17 & 5,270 \\
\hline $1982-83$ & 10 & 0 & 75 & 420 & 844 & 1,023 & 855 & 676 & 511 & 419 & 151 & 50 & 5,034 \\
\hline $1983-84$ & 8 & 0 & 125 & 387 & 643 & 1,357 & 1,035 & 763 & 552 & 432 & $292^{(2)}$ & 70 & 5,664 \\
\hline $1984-85$ & 0 & 3 & 145 & $532^{(a)}$ & 768 & 1,288 & 1,245 & 982 & 651 & 288 & 137 & 21 & 6,060 \\
\hline $1985-86$ & 0 & 10 & $197^{(a)}$ & $475^{\circ}$ & $1,206^{(t)}$ & $1,362^{(a)}$ & 959 & 724 & 509 & 426 & 213 & 10 & 6,091 \\
\hline $1986-87$ & 18 & 0 & 153 & 319 & 680 & 1,009 & 1,066 & 696 & 522 & 239 & 85 & 16 & 4,803 \\
\hline $1987-88$ & 1 & 0 & 32 & 304 & 640 & 1,040 & 1,028 & 695 & 591 & 301 & 166 & 65 & 4,863 \\
\hline $1988-89$ & 3 & 0 & 100 & 208 & 625 & 1,033 & 859 & 1,054 & 658 & 254 & 141 & 6 & 4,941 \\
\hline $1989-90$ & 1 & 0 & 12 & 339 & 621 & 985 & 763 & 767 & 530 & 217 & 149 & 28 & 4,412 \\
\hline $1990-91$ & 4 & $0^{(a)}$ & $\theta^{(a)}$ & 401 & $553^{(a)}$ & 1,269 & 1,124 & $575^{(2)}$ & 649 & 330 & 148 & 55. & 5,108 \\
\hline $1991-92$ & 0 & 0 & 12 & 381 & 710 & 842 & 851 & 648 & $418^{(n)}$ & 278 & 77 & 13 & $4,230^{(2)}$ \\
\hline $1992-93$ & 1 & 18 & 94 & 298 & 716 & 1,084 & 1,247 & 958 & 674 & 374 & 94 & 23 & 5,581 \\
\hline $1993-94$ & 1 & 13 & 89 & 303 & 911 & 914 & 819 & 813 & 490 & $217^{(2)}$ & 97 & 22 & 4,689 \\
\hline $1994-95$ & 0 & 0 & 3 & 332 & 759 & 924 & 954 & 614 & 581 & 372 & 89 & 46 & 4,674 \\
\hline $1995-96$ & 0 & 1 & 24 & 398 & 623 & 1,003 & 1,124 & 935 & 623 & 302 & 225 & 14 & 5,272 \\
\hline $1996-97$ & 3 & 0 & 99 & 401 & 797 & 1,090 & 973 & 692 & 544 & 395 & 95 & 2 & 5,091 \\
\hline $1997-98$ & 2 & 0 & 38 & 367 & 658 & 941 & 892 & 642 & 521 & 332 & 131 & $1^{(\mathbf{a})}$ & 4,523 \\
\hline $1998-99$ & $0^{(2, b)}$ & $0^{(a b)}$ & 15 & 393 & 582 & 1,000 & - & - & - & - & - & - & - \\
\hline Average & 4 & 5 & 71 & 374 & 746 & 1,015 & 1,064 & 774 & 613 & 365 & 152 & 33 & 5,215 \\
\hline Normal & 15 & 5 & 78 & 377 & 746 & 1,042 & 1,044 & 764 & 602 & 372 & $\cdot 164$ & 32 & 5,231 \\
\hline
\end{tabular}

(a) Greatest and least values.

(b) Most recent of numerous occurrences. 
Table 3.15. Monthly and Annual Cooling-Degree Days

\begin{tabular}{|c|c|c|c|c|c|c|c|c|c|c|c|c|c|}
\hline Year & Jan & Feb & Mar & Apr & May & Jun & Jul & Aug & Sep & Oct & Nov & Dec & Annual \\
\hline 1945 & 0 & 0 & 0 & 0 & 39 & 113 & 325 & 388 & 86 & 11 & 0 & 0 & 962 \\
\hline $\begin{array}{l}1946 \\
1947 \\
1948 \\
1949 \\
1950\end{array}$ & $\begin{array}{l}0 \\
0 \\
0 \\
0 \\
0\end{array}$ & $\begin{array}{l}0 \\
0 \\
0 \\
0 \\
0\end{array}$ & $\begin{array}{l}0 \\
0 \\
0 \\
0 \\
0\end{array}$ & $\begin{array}{l}9 \\
3 \\
0 \\
4 \\
0\end{array}$ & $\begin{array}{r}53 \\
153 \\
31 \\
147 \\
13\end{array}$ & $\begin{array}{l}100 \\
108 \\
230 \\
168 \\
112\end{array}$ & $\begin{array}{l}345 \\
321 \\
243 \\
311 \\
321\end{array}$ & $\begin{array}{l}360 \\
217 \\
213 \\
307 \\
353\end{array}$ & $\begin{array}{r}57 \\
86 \\
90 \\
146 \\
139\end{array}$ & $\begin{array}{r}0 \\
11 \\
0 \\
0 \\
0\end{array}$ & $\begin{array}{l}0 \\
0 \\
0 \\
0 \\
0\end{array}$ & $\begin{array}{l}0 \\
0 \\
0 \\
0 \\
0\end{array}$ & $\begin{array}{r}924 \\
899 \\
807 \\
1,083 \\
938\end{array}$ \\
\hline $\begin{array}{l}1951 \\
1952 \\
1953 \\
1954 \\
1955\end{array}$ & $\begin{array}{l}0 \\
0 \\
0 \\
0 \\
0\end{array}$ & $\begin{array}{l}0 \\
0 \\
0 \\
0 \\
0\end{array}$ & $\begin{array}{l}0 \\
0 \\
0 \\
0 \\
0\end{array}$ & $\begin{array}{r}1 \\
16 \\
0 \\
0 \\
0\end{array}$ & $\begin{array}{r}26 \\
46 \\
8 \\
55 \\
4\end{array}$ & $\begin{array}{r}173 \\
110 \\
26^{(a)} \\
90 \\
174\end{array}$ & $\begin{array}{l}370 \\
371 \\
336 \\
289 \\
270\end{array}$ & $\begin{array}{l}303 \\
281 \\
282 \\
204 \\
325\end{array}$ & $\begin{array}{r}123 \\
151 \\
143 \\
91 \\
13\end{array}$ & $\begin{array}{r}0 \\
16 \\
1 \\
0 \\
0\end{array}$ & $\begin{array}{l}0 \\
0 \\
0 \\
0 \\
0\end{array}$ & $\begin{array}{l}0 \\
0 \\
0 \\
0 \\
0\end{array}$ & $\begin{array}{l}996 \\
991 \\
796 \\
729 \\
786\end{array}$ \\
\hline $\begin{array}{l}1956 \\
1957 \\
1958 \\
1959 \\
1960\end{array}$ & $\begin{array}{l}0 \\
0 \\
0 \\
0 \\
0\end{array}$ & $\begin{array}{l}0 \\
0 \\
0 \\
0 \\
0\end{array}$ & $\begin{array}{l}0 \\
0 \\
0 \\
0 \\
0\end{array}$ & $\begin{array}{r}10 \\
12 \\
0 \\
0 \\
2\end{array}$ & $\begin{array}{r}122 \\
77 \\
167^{(\mathrm{a})} \\
15 \\
26\end{array}$ & $\begin{array}{r}78 \\
185 \\
282 \\
137 \\
174\end{array}$ & $\begin{array}{l}430 \\
289 \\
500 \\
397 \\
518\end{array}$ & $\begin{array}{l}322 \\
255 \\
447 \\
218 \\
233\end{array}$ & $\begin{array}{r}106 \\
160 \\
.93 \\
45 \\
118\end{array}$ & $\begin{array}{r}0 \\
0 \\
14 \\
0 \\
3\end{array}$ & $\begin{array}{l}0 \\
0 \\
0 \\
0 \\
0\end{array}$ & $\begin{array}{l}0 \\
0 \\
0 \\
0 \\
0\end{array}$ & $\begin{array}{r}1,068 \\
978 \\
1,503^{(a)} \\
812 \\
1,074\end{array}$ \\
\hline $\begin{array}{l}1961 \\
1962 \\
1963 \\
1964 \\
1965\end{array}$ & $\begin{array}{l}0 \\
0 \\
0 \\
0 \\
0\end{array}$ & $\begin{array}{l}0 \\
0 \\
0 \\
0 \\
0\end{array}$ & $\begin{array}{l}0 \\
0 \\
0 \\
0 \\
0\end{array}$ & $\begin{array}{l}0 \\
5 \\
0 \\
0 \\
0\end{array}$ & $\begin{array}{l}23 \\
3^{(2)} \\
67 \\
30 \\
31\end{array}$ & $\begin{array}{l}288 \\
148 \\
156 \\
115 \\
145\end{array}$ & $\begin{array}{l}447 \\
352 \\
232 \\
299 \\
362\end{array}$ & $\begin{array}{r}469 \\
215 \\
333 \\
171^{(2)} \\
314\end{array}$ & $\begin{array}{r}55 \\
125 \\
205 \\
34 \\
33\end{array}$ & $\begin{array}{l}0 \\
0 \\
5 \\
0 \\
2\end{array}$ & $\begin{array}{l}0 \\
0 \\
0 \\
0 \\
0\end{array}$ & $\begin{array}{l}0 \\
0 \\
0 \\
0 \\
0\end{array}$ & $\begin{array}{r}1,282 \\
848 \\
998 \\
649^{(a)} \\
887\end{array}$ \\
\hline $\begin{array}{l}1966 \\
1967 \\
1968 \\
1969 \\
1970\end{array}$ & $\begin{array}{l}0 \\
0 \\
0 \\
0 \\
0\end{array}$ & $\begin{array}{l}0 \\
0 \\
0 \\
0 \\
0\end{array}$ & $\begin{array}{l}0 \\
0 \\
0 \\
0 \\
0\end{array}$ & $\begin{array}{l}0 \\
0 \\
5 \\
0 \\
0\end{array}$ & $\begin{array}{l}80 \\
34 \\
35 \\
73 \\
29\end{array}$ & $\begin{array}{l}116 \\
237 \\
168 \\
310 \\
281\end{array}$ & $\begin{array}{l}274 \\
419 \\
451 \\
338 \\
421\end{array}$ & $\begin{array}{r}332 \\
\mathbf{5 0 8}^{(\mathbf{a})} \\
213 \\
245 \\
351\end{array}$ & $\begin{array}{r}141 \\
216 \\
104 \\
110 \\
27\end{array}$ & $\begin{array}{l}1 \\
0 \\
0 \\
0 \\
1\end{array}$ & $\begin{array}{l}0 \\
0 \\
0 \\
0 \\
0\end{array}$ & $\begin{array}{l}0 \\
0 \\
0 \\
0 \\
0\end{array}$ & $\begin{array}{r}944 \\
1,414 \\
976 \\
1,076 \\
1,110\end{array}$ \\
\hline $\begin{array}{l}1971 \\
1972 \\
1973 \\
1974 \\
1975\end{array}$ & $\begin{array}{l}0 \\
0 \\
0 \\
0 \\
0\end{array}$ & $\begin{array}{l}0 \\
0 \\
0 \\
0 \\
0\end{array}$ & $\begin{array}{l}0 \\
0 \\
0 \\
0 \\
0\end{array}$ & $\begin{array}{l}0 \\
0 \\
0 \\
0 \\
0\end{array}$ & $\begin{array}{l}94 \\
87 \\
87 \\
12 \\
28\end{array}$ & $\begin{array}{r}59 \\
164 \\
149 \\
264 \\
102\end{array}$ & $\begin{array}{l}437 \\
339 \\
413 \\
303 \\
451\end{array}$ & $\begin{array}{l}481 \\
392 \\
285 \\
326 \\
202\end{array}$ & $\begin{array}{r}28 \\
67 \\
94 \\
125 \\
117\end{array}$ & $\begin{array}{r}10 \\
1 \\
0 \\
0 \\
0\end{array}$ & $\begin{array}{l}0 \\
0 \\
0 \\
0 \\
0\end{array}$ & $\begin{array}{l}0 \\
0 \\
0 \\
0 \\
0\end{array}$ & $\begin{array}{r}1,109 \\
1,050 \\
1,028 \\
1,030 \\
900\end{array}$ \\
\hline $\begin{array}{l}1976 \\
1977 \\
1978 \\
1979 \\
1980\end{array}$ & $\begin{array}{l}0 \\
0 \\
0 \\
0 \\
0\end{array}$ & $\begin{array}{l}0 \\
0 \\
0 \\
0 \\
0\end{array}$ & $\begin{array}{l}0 \\
0 \\
0 \\
0 \\
0\end{array}$ & $\begin{array}{r}0 \\
24 \\
0 \\
1 \\
7\end{array}$ & $\begin{array}{r}22 \\
5 \\
5 \\
65 \\
26\end{array}$ & $\begin{array}{r}91 \\
253 \\
182 \\
197 \\
57\end{array}$ & $\begin{array}{l}319 \\
276 \\
332 \\
394 \\
305\end{array}$ & $\begin{array}{l}195 \\
447 \\
248 \\
299 \\
207\end{array}$ & $\begin{array}{r}141 \\
46 \\
41 \\
138 \\
80\end{array}$ & $\begin{array}{l}3 \\
0 \\
0 \\
5 \\
9\end{array}$ & $\begin{array}{l}0 \\
0 \\
0 \\
0 \\
0\end{array}$ & $\begin{array}{l}0 \\
0 \\
0 \\
0 \\
0\end{array}$ & $\begin{array}{r}771 \\
1,051 \\
808 \\
1,099 \\
6991\end{array}$ \\
\hline $\begin{array}{l}1981 \\
1982 \\
1983 \\
1984 \\
1985\end{array}$ & $\begin{array}{l}0 \\
0 \\
0 \\
0 \\
0\end{array}$ & $\begin{array}{l}0 \\
0 \\
0 \\
0 \\
0\end{array}$ & $\begin{array}{l}0 \\
0 \\
0 \\
0 \\
0\end{array}$ & $\begin{array}{r}16 \\
0 \\
0 \\
0 \\
3\end{array}$ & $\begin{array}{r}25 \\
20 \\
115 \\
11 \\
83\end{array}$ & $\begin{array}{r}82 \\
261 \\
61 \\
88 \\
175\end{array}$ & $\begin{array}{r}287 \\
315 \\
203 \\
340 \\
\mathbf{5 3 2}^{(2)}\end{array}$ & $\begin{array}{l}438 \\
333 \\
291 \\
280 \\
183\end{array}$ & $\begin{array}{r}144 \\
88 \\
26 \\
60 \\
11^{(a)}\end{array}$ & $\begin{array}{l}0 \\
0 \\
1 \\
0 \\
0\end{array}$ & $\begin{array}{l}0 \\
0 \\
0 \\
0 \\
0\end{array}$ & $\begin{array}{l}0 \\
0 \\
0 \\
0 \\
0\end{array}$ & $\begin{array}{r}992 \\
1,017 \\
697 \\
779 \\
987\end{array}$ \\
\hline $\begin{array}{l}1986 \\
1987 \\
1988 \\
1989 \\
1990\end{array}$ & $\begin{array}{l}0 \\
0 \\
0 \\
0 \\
0\end{array}$ & $\begin{array}{r}\mathbf{1}^{(2)} \\
0 \\
0 \\
0 \\
0\end{array}$ & $\begin{array}{l}0 \\
0 \\
0 \\
0 \\
0\end{array}$ & $\begin{array}{r}3 \\
26^{(2)} \\
6 \\
1 \\
3\end{array}$ & $\begin{array}{r}125 \\
125 \\
45 \\
34 \\
16\end{array}$ & $\begin{array}{l}245 \\
265 \\
187 \\
215 \\
182\end{array}$ & $\begin{array}{l}192 \\
289 \\
385 \\
323 \\
491\end{array}$ & $\begin{array}{l}442 \\
359 \\
318 \\
260 \\
367\end{array}$ & $\begin{array}{r}68 \\
179 \\
113 \\
89 \\
222^{(3)}\end{array}$ & $\begin{array}{r}1 \\
11 \\
44^{(2)} \\
0 \\
3\end{array}$ & $\begin{array}{l}0 \\
0 \\
0 \\
1^{(a)} \\
0\end{array}$ & $\begin{array}{l}0 \\
0 \\
0 \\
0 \\
0\end{array}$ & $\begin{array}{r}1,077 \\
1,254 \\
1,098 \\
923 \\
1,284\end{array}$ \\
\hline $\begin{array}{l}1991 \\
1992 \\
1993 \\
1994 \\
1995\end{array}$ & $\begin{array}{l}0 \\
0 \\
0 \\
0 \\
0\end{array}$ & $\begin{array}{l}0 \\
0 \\
0 \\
0 \\
0\end{array}$ & $\begin{array}{l}0 \\
0 \\
0 \\
0 \\
0\end{array}$ & $\begin{array}{r}3 \\
11 \\
0 \\
15 \\
0\end{array}$ & $\begin{array}{r}6 \\
147 \\
139 \\
94 \\
73\end{array}$ & $\begin{array}{r}72 \\
365^{(a)} \\
127 \\
163 \\
142\end{array}$ & $\begin{array}{r}400 \\
362 \\
171^{(2)} \\
501 \\
376\end{array}$ & $\begin{array}{l}427 \\
392 \\
265 \\
358 \\
216\end{array}$ & $\begin{array}{r}155 \\
81 \\
135 \\
167 \\
174\end{array}$ & $\begin{array}{r}7 \\
10 \\
6 \\
3 \\
0\end{array}$ & $\begin{array}{l}0 \\
0 \\
0 \\
0 \\
0\end{array}$ & $\begin{array}{l}0 \\
0 \\
0 \\
0 \\
0\end{array}$ & $\begin{array}{r}1,070 \\
1,368 \\
843 \\
1,301 \\
981\end{array}$ \\
\hline $\begin{array}{l}1996 \\
1997 \\
1998\end{array}$ & $\begin{array}{l}0 \\
0 \\
0\end{array}$ & $\begin{array}{c}0 \\
0 \\
0^{(2, b)}\end{array}$ & $\begin{array}{l}0 \\
0 \\
0\end{array}$ & $\begin{array}{c}4 \\
0^{(x, 0)} \\
16\end{array}$ & $\begin{array}{l}14 \\
96 \\
55\end{array}$ & $\begin{array}{l}134 \\
118 \\
183\end{array}$ & $\begin{array}{l}450 \\
324 \\
527\end{array}$ & $\begin{array}{l}324 \\
404 \\
398\end{array}$ & $\begin{array}{r}79 \\
92 \\
195\end{array}$ & $\begin{array}{r}9 \\
0^{(2,0)} \\
3\end{array}$ & $\begin{array}{r}0 \\
0 \\
0^{(2 \infty)}\end{array}$ & $\begin{array}{l}0 \\
0 \\
0\end{array}$ & $\begin{array}{l}1,014 \\
1,034 \\
1,377\end{array}$ \\
\hline $\begin{array}{l}\text { Average } \\
\text { Normal }\end{array}$ & $\begin{array}{l}0 \\
0\end{array}$ & $\begin{array}{l}<1 \\
<1\end{array}$ & $\begin{array}{l}0 \\
0\end{array}$ & $\begin{array}{l}4 \\
3\end{array}$ & $\begin{array}{l}56 \\
48\end{array}$ & $\begin{array}{l}163 \\
175\end{array}$ & $\begin{array}{l}357 \\
351\end{array}$ & $\begin{array}{l}315 \\
317\end{array}$ & $\begin{array}{r}105 \\
98\end{array}$ & $\begin{array}{l}4 \\
3\end{array}$ & $\begin{array}{l}<1 \\
<1\end{array}$ & $\begin{array}{l}0 \\
0\end{array}$ & $\begin{array}{r}1,003 \\
994\end{array}$ \\
\hline
\end{tabular}

(a) Greatest and least values.

(b) Most recent of numerous occurrences. 


\subsection{Precipitation Climatology}

\subsection{Monthly and Annual Totals}

Table 4.1 shows monthly and annual precipitation totals for the period of record, 1946 through 1998. Normal monthly precipitation amounts for the period 1961 through 1990 and averages for the entire period of record are noted on the table, as are monthly and annual extremes. Normal annual precipitation at the HMS is 6.26 in. The wettest year on record was 1995, with 12.31 in.; the driest was 1976, with 2.99 in.

The months of November through February provide 3.35 in. (54\%) of the normal annual precipitation. December is the wettest month, receiving 1.03 in.; July is the driest, receiving only 0.18 in. The wettest month on record was December 1996, with 3.69 in. September 1991, August 1988, and August 1955 received no precipitation.

\subsection{Seasonal Precipitation}

Table 4.2 provides seasonal precipitation information, with normal and average seasonal data noted. The extremes for each season are also noted. The wettest season was the winter of 1996-1997, with 5.45 in.; the driest received only $0.03 \mathrm{in}$. (summer 1973).

\subsection{Average Number of Days with Specified Amounts of Precipitation}

Table 4.3 presents information on the average number of days per year with precipitation events in six categories. A trace is $<0.005$ in. of precipitation. An average of 124 days per year have a trace or more of precipitation; however, only 23 days receive totals of 0.10 in. or more. During the 53-year period of record, only 4 days had an inch or more of precipitation.

\subsection{Total Time with Precipitation Observed}

The total time during which precipitation was observed at the HMS includes all types of precipitation. Observations of precipitation are recorded in hours and minutes, with the weather observer recording the starting and ending time of each precipitation event. These data are presented in Table 4.4. No record was kept for the hours 1600 through 2400 from July 1971 through June 1974; therefore, a 3-year gap exists in the record for those hours. Also, beginning in late April 1995, operations at the HMS were decreased to $8 \mathrm{~h}(0600$ to 1400$)$ on weekends and holidays. However, a combination of precipitation 
Table 4.1. Monthly and Annual Precipitation (in.)

\begin{tabular}{|c|c|c|c|c|c|c|c|c|c|c|c|c|c|}
\hline Year & Jan & Feb & Mar & Apr & May & Jun & Jul & Aug & Sep & Oct & Nov & Dec & Annual \\
\hline $\begin{array}{l}1946 \\
1947 \\
1948 \\
1949 \\
1950\end{array}$ & $\begin{array}{l}0 . \overline{3} \\
1.36 \\
0.13 \\
1.80\end{array}$ & $\begin{array}{l}0 . \overline{27} \\
0.69 \\
0.68 \\
1.06\end{array}$ & $\begin{array}{l}0 . \overline{2} \\
0.07 \\
1.12 \\
0.87\end{array}$ & $\begin{array}{l}0 . \overline{70} \\
0.95 \\
0.02 \\
0.47\end{array}$ & $\begin{array}{l}0 . \overline{2} \\
1.71 \\
0.16 \\
0.27\end{array}$ & $\begin{array}{r}1.0 \overline{7} \\
1.47 \\
0.01 \\
2.92^{(2)}\end{array}$ & $\begin{array}{l}0.15 \\
0.71 \\
0.40 \\
0.01 \\
0.07\end{array}$ & $\begin{array}{r}0.35 \\
0.68 \\
0.39 \\
0.03 \\
T\end{array}$ & $\begin{array}{r}0.52 \\
1.34^{(2)} \\
0.16 \\
0.23 \\
0.01\end{array}$ & $\begin{array}{l}0.65 \\
2.20 \\
0.45 \\
0.10 \\
2.46\end{array}$ & $\begin{array}{l}0.66 \\
0.81 \\
0.95 \\
1.47 \\
0.55\end{array}$ & $\begin{array}{l}0.11 \\
0.75 \\
1.11 \\
0.16 \\
0.97\end{array}$ & $\begin{array}{r}9 . \overline{-} \\
9.71 \\
4.12 \\
11.45\end{array}$ \\
\hline $\begin{array}{l}1951 \\
1952 \\
1953 \\
1954 \\
1955\end{array}$ & $\begin{array}{l}0.84 \\
0.65 \\
2.16 \\
1.48 \\
0.56\end{array}$ & $\begin{array}{l}0.51 \\
0.50 \\
0.25 \\
0.28 \\
0.22\end{array}$ & $\begin{array}{l}0.46 \\
0.06 \\
0.17 \\
0.59 \\
0.17\end{array}$ & $\begin{array}{l}0.53 \\
0.13 \\
0.77 \\
0.07 \\
0.40\end{array}$ & $\begin{array}{l}0.43 \\
0.58 \\
0.28 \\
0.41 \\
0.59\end{array}$ & $\begin{array}{l}1.38 \\
1.07 \\
0.55 \\
0.10 \\
0.28\end{array}$ & $\begin{array}{r}0.37 \\
\mathrm{~T} \\
\mathrm{~T} \\
0.22 \\
0.57\end{array}$ & $\begin{array}{l}0.15 \\
0.08 \\
0.96 \\
0.42 \\
0_{(2.0)}\end{array}$ & $\begin{array}{l}0.10 \\
0.08 \\
0.13 \\
0.51 \\
0.77\end{array}$ & $\begin{array}{l}0.71 \\
0.04 \\
0.20 \\
0.42 \\
0.40\end{array}$ & $\begin{array}{l}0.82 \\
0.20 \\
0.96 \\
0.86 \\
1.54\end{array}$ & $\begin{array}{l}0.70 \\
0.77 \\
0.49 \\
0.35 \\
2.03\end{array}$ & $\begin{array}{l}7.00 \\
4.16 \\
6.92 \\
5.71 \\
7.53\end{array}$ \\
\hline $\begin{array}{l}1956 \\
1957 \\
1958 \\
1959 \\
1960\end{array}$ & $\begin{array}{l}1.71 \\
0.48 \\
1.74 \\
2.05 \\
0.51\end{array}$ & $\begin{array}{l}0.56 \\
0.23 \\
1.48 \\
1.17 \\
0.58\end{array}$ & $\begin{array}{r}0.10 \\
1.86^{(2)} \\
0.46 \\
0.40 \\
0.67\end{array}$ & $\begin{array}{r}T \\
0.38 \\
0.64 \\
0.20 \\
0.53\end{array}$ & $\begin{array}{l}0.22 \\
0.82 \\
0.74 \\
0.50 \\
0.71\end{array}$ & $\begin{array}{l}0.86 \\
0.47 \\
0.81 \\
0.23 \\
0.14\end{array}$ & $\begin{array}{r}\mathrm{T} \\
0.05 \\
0.02 \\
\mathrm{~T} \\
\mathrm{~T}\end{array}$ & $\begin{array}{r}0.38 \\
0.02 \\
T \\
0.03 \\
0.26\end{array}$ & $\begin{array}{l}0.01 \\
0.34 \\
0.05 \\
1.26 \\
0.23\end{array}$ & $\begin{array}{r}1.03 \\
2.72^{(a)} \\
0.19 \\
0.56 \\
0.23\end{array}$ & $\begin{array}{l}0.15 \\
0.39 \\
0.77 \\
0.41 \\
0.92\end{array}$ & $\begin{array}{l}0.58 \\
0.53 \\
1.84 \\
0.26 \\
0.64\end{array}$ & $\begin{array}{l}5.60 \\
8.29 \\
8.74 \\
7.07 \\
5.42\end{array}$ \\
\hline $\begin{array}{l}1961 \\
1962 \\
1963 \\
1964 \\
1965\end{array}$ & $\begin{array}{l}0.33 \\
0.13 \\
0.95 \\
0.37 \\
0.93\end{array}$ & $\begin{array}{c}2.10^{(a)} \\
0.90 \\
0.69 \\
0.01 \\
0.14\end{array}$ & $\begin{array}{l}1.02 \\
0.14 \\
0.53 \\
0.03 \\
0.03\end{array}$ & $\begin{array}{l}0.48 \\
0.34 \\
1.17 \\
0.11 \\
0.09\end{array}$ & $\begin{array}{l}0.80 \\
1.35 \\
0.43 \\
0.04 \\
0.15\end{array}$ & $\begin{array}{l}0.42 \\
0.12 \\
0.28 \\
0.90 \\
0.49\end{array}$ & $\begin{array}{r}0.15 \\
\mathrm{~T} \\
0.31 \\
0.04 \\
0.11\end{array}$ & $\begin{array}{l}0.09 \\
0.50 \\
0.01 \\
0.24 \\
0.03\end{array}$ & $\begin{array}{r}T \\
0.38 \\
0.02 \\
0.09 \\
0.11\end{array}$ & $\begin{array}{l}0.07 \\
0.95 \\
0.04 \\
0.28 \\
0.01\end{array}$ & $\begin{array}{l}0.49 \\
0.65 \\
0.74 \\
0.94 \\
1.17\end{array}$ & $\begin{array}{l}0.89 \\
0.60 \\
1.14 \\
2.34 \\
0.39\end{array}$ & $\begin{array}{l}6.84 \\
6.06 \\
6.31 \\
5.39 \\
3.65\end{array}$ \\
\hline $\begin{array}{l}1966 \\
1967 \\
1968 \\
1969 \\
1970\end{array}$ & $\begin{array}{r}0.68 \\
0.32 \\
0.88 \\
1.24 \\
2.47^{(a)}\end{array}$ & $\begin{array}{r}0.03 \\
T \\
0.58 \\
0.54 \\
0.75\end{array}$ & $\begin{array}{r}0.39 \\
0.14 \\
0.02^{(a)} \\
0.10 \\
0.27\end{array}$ & $\begin{array}{l}0.03 \\
0.90 \\
0.01 \\
1.22 \\
0.45\end{array}$ & $\begin{array}{l}0.05 \\
0.56 \\
0.06 \\
0.51 \\
0.54\end{array}$ & $\begin{array}{l}0.43 \\
0.57 \\
0.19 \\
0.75 \\
0.25\end{array}$ & $\begin{array}{r}0.81 \\
\mathrm{~T} \\
0.04 \\
\mathrm{~T} \\
0.01\end{array}$ & $\begin{array}{r}\mathrm{T} \\
\mathrm{T} \\
0.51 \\
\mathrm{~T} \\
\mathrm{~T}\end{array}$ & $\begin{array}{l}0.27 \\
0.05 \\
0.25 \\
0.48 \\
0.03\end{array}$ & $\begin{array}{l}0.39 \\
0.13 \\
0.93 \\
0.10 \\
0.24\end{array}$ & $\begin{array}{l}2.25 \\
0.16 \\
1.23 \\
0.13 \\
0.71\end{array}$ & $\begin{array}{l}0.60 \\
0.43 \\
1.25 \\
1.29 \\
0.61\end{array}$ & $\begin{array}{l}5.93 \\
3.26 \\
5.95 \\
6.36 \\
6.33\end{array}$ \\
\hline $\begin{array}{l}1971 \\
1972 \\
1973 \\
1974 \\
1975\end{array}$ & $\begin{array}{l}0.78 \\
0.19 \\
0.90 \\
0.90 \\
1.43\end{array}$ & $\begin{array}{l}0.10 \\
0.27 \\
0.21 \\
0.41 \\
0.98\end{array}$ & $\begin{array}{l}1.02 \\
0.58 \\
0.08 \\
0.52 \\
0.33\end{array}$ & $\begin{array}{r}0.07 \\
0.10 \\
T \\
0.46 \\
0.42\end{array}$ & $\begin{array}{r}0.56 \\
2.03^{(2)} \\
0.24 \\
0.28 \\
0.38\end{array}$ & $\begin{array}{l}0.71 \\
0.66 \\
0.01 \\
0.12 \\
0.24\end{array}$ & $\begin{array}{r}0.13 \\
0.16 \\
T \\
0.71 \\
0.32\end{array}$ & $\begin{array}{r}0.09 \\
0.56 \\
0.02 \\
T \\
1.16\end{array}$ & $\begin{array}{l}1.13 \\
0.02 \\
0.43 \\
0.01 \\
0.03\end{array}$ & $\begin{array}{r}0.18 \\
T \\
1.72 \\
0.21 \\
0.87\end{array}$ & $\begin{array}{l}0.46 \\
0.55 \\
2.64 \\
0.71 \\
0.60\end{array}$ & $\begin{array}{l}1.07 \\
1.27 \\
2.02 \\
0.97 \\
0.70\end{array}$ & $\begin{array}{l}6.30 \\
6.39 \\
8.27 \\
5.30 \\
7.46\end{array}$ \\
\hline $\begin{array}{l}1976 \\
1977 \\
1978 \\
1979 \\
1980\end{array}$ & $\begin{array}{r}0.56 \\
0.08^{(a)} \\
1.72 \\
0.54 \\
1.32\end{array}$ & $\begin{array}{l}0.36 \\
0.57 \\
0.92 \\
0.17 \\
1.30\end{array}$ & $\begin{array}{l}0.23 \\
0.41 \\
0.30 \\
0.54 \\
0.30\end{array}$ & $\begin{array}{r}0.41 \\
T \\
0.46 \\
0.52 \\
0.86\end{array}$ & $\begin{array}{l}0.08 \\
0.65 \\
0.41 \\
0.10 \\
1.41\end{array}$ & $\begin{array}{r}0.11 \\
0.37 \\
0.09 \\
T \\
0.96\end{array}$ & $\begin{array}{c}0.13 \\
0.06 \\
0.52 \\
0.09 \\
T^{(0)}\end{array}$ & $\begin{array}{r}0.96 \\
1.36^{(2)} \\
0.57 \\
0.38 \\
0.02\end{array}$ & $\begin{array}{r}T \\
0.66 \\
0.11 \\
0.20 \\
0.85\end{array}$ & $\begin{array}{r}0.04 \\
0.15 \\
T \\
0.67 \\
0.33\end{array}$ & $\begin{array}{r}\mathrm{T} \\
0.63 \\
1.21 \\
1.36 \\
0.44\end{array}$ & $\begin{array}{r}0.11^{(\mathbf{a})} \\
1.47 \\
0.26 \\
0.99 \\
1.89\end{array}$ & $\begin{array}{r}2.99^{(2)} \\
6.41 \\
6.57 \\
5.56 \\
9.68\end{array}$ \\
\hline $\begin{array}{l}1981 \\
1982 \\
1983 \\
1984 \\
1985\end{array}$ & $\begin{array}{l}0.56 \\
0.33 \\
1.44 \\
0.23 \\
0.34\end{array}$ & $\begin{array}{l}0.60 \\
0.57 \\
1.36 \\
0.94 \\
0.82\end{array}$ & $\begin{array}{l}0.70 \\
0.30 \\
1.00 \\
1.01 \\
0.36\end{array}$ & $\begin{array}{l}0.02 \\
0.75 \\
0.42 \\
0.60 \\
0.01\end{array}$ & $\begin{array}{l}0.99 \\
0.28 \\
0.52 \\
0.55 \\
0.12\end{array}$ & $\begin{array}{l}0.43 \\
0.75 \\
0.68 \\
0.99 \\
0.15\end{array}$ & $\begin{array}{l}0.19 \\
0.22 \\
0.31 \\
0.06 \\
0.12\end{array}$ & $\begin{array}{r}0.03 \\
0.20 \\
0.12 \\
T \\
0.01\end{array}$ & $\begin{array}{l}0.60 \\
0.55 \\
0.46 \\
0.42 \\
0.63\end{array}$ & $\begin{array}{l}0.39 \\
1.33 \\
0.52 \\
0.07 \\
0.46\end{array}$ & $\begin{array}{l}1.08 \\
0.91 \\
2.12 \\
1.83 \\
1.24\end{array}$ & $\begin{array}{l}1.45 \\
1.79 \\
2.12 \\
0.57 \\
0.84\end{array}$ & $\begin{array}{r}7.04 \\
7.98 \\
11.07 \\
7.27 \\
5.10\end{array}$ \\
\hline $\begin{array}{l}1986 \\
1987 \\
1988 \\
1989 \\
1990\end{array}$ & $\begin{array}{l}1.76 \\
0.80 \\
0.48 \\
0.21 \\
0.77\end{array}$ & $\begin{array}{l}1.37 \\
0.19 \\
T^{(0)} \\
1.67 \\
0.09\end{array}$ & $\begin{array}{l}0.76 \\
1.05 \\
0.39 \\
1.56 \\
0.10\end{array}$ & $\begin{array}{l}T^{(\mathbf{p})} \\
0.14 \\
1.12 \\
0.84 \\
0.40\end{array}$ & $\begin{array}{l}0.30 \\
0.17 \\
0.33 \\
0.59 \\
0.86\end{array}$ & $\begin{array}{c}T^{(0)} \\
0.11 \\
0.11 \\
0.01 \\
0.36\end{array}$ & $\begin{array}{l}0.21 \\
0.50 \\
0.13 \\
0.01 \\
0.14\end{array}$ & $\begin{array}{c}0.02 \\
0.07 \\
0^{(0)} \\
0.26 \\
0.83\end{array}$ & $\begin{array}{r}0.96 \\
0.01 \\
0.39 \\
0.02 \\
T\end{array}$ & $\begin{array}{c}0.29 \\
\mathbf{T}^{(0)} \\
0.01 \\
0.42 \\
0.78\end{array}$ & $\begin{array}{l}0.65 \\
0.40 \\
0.82 \\
1.04 \\
0.02\end{array}$ & $\begin{array}{l}0.77 \\
1.63 \\
0.40 \\
0.29 \\
0.72\end{array}$ & $\begin{array}{l}7.09 \\
5.07 \\
4.18 \\
6.92 \\
5.07\end{array}$ \\
\hline $\begin{array}{l}1991 \\
1992 \\
1993 \\
1994 \\
1995\end{array}$ & $\begin{array}{l}0.33 \\
0.44 \\
1.30 \\
0.44 \\
2.14\end{array}$ & $\begin{array}{l}0.19 \\
0.94 \\
1.17 \\
0.11 \\
0.69\end{array}$ & $\begin{array}{l}1.12 \\
0.09 \\
0.67 \\
0.03 \\
0.95\end{array}$ & $\begin{array}{r}0.45 \\
0.94 \\
0.71 \\
0.61 \\
\mathbf{1 . 5 4}\end{array}$ & $\begin{array}{c}0.49 \\
T^{(2)} \\
0.60 \\
1.27 \\
0.79\end{array}$ & $\begin{array}{l}1.44 \\
1.14 \\
0.12 \\
0.38 \\
0.77\end{array}$ & $\begin{array}{r}0.29 \\
0.38 \\
1.76^{(2)} \\
0.15 \\
0.34\end{array}$ & $\begin{array}{l}0.07 \\
0.20 \\
0.24 \\
0.08 \\
0.07\end{array}$ & $\begin{array}{r}0^{(2)} \\
0.27 \\
0.04 \\
0.08 \\
0.79\end{array}$ & $\begin{array}{l}0.53 \\
0.61 \\
0.09 \\
0.93 \\
0.87\end{array}$ & $\begin{array}{l}1.44 \\
1.07 \\
0.19 \\
0.68 \\
1.04\end{array}$ & $\begin{array}{l}0.40 \\
1.82 \\
0.94 \\
1.36 \\
2.32\end{array}$ & $\begin{array}{r}6.75 \\
7.90 \\
7.83 \\
6.12 \\
12.31^{(2)}\end{array}$ \\
\hline $\begin{array}{l}1996 \\
1997 \\
1998\end{array}$ & $\begin{array}{l}1.42 \\
1.51 \\
1.24\end{array}$ & $\begin{array}{l}1.22 \\
0.25 \\
1.15\end{array}$ & $\begin{array}{l}0.83 \\
0.70 \\
0.50\end{array}$ & $\begin{array}{l}0.43 \\
0.33 \\
0.07\end{array}$ & $\begin{array}{l}0.62 \\
0.33 \\
0.52\end{array}$ & $\begin{array}{l}0.05 \\
0.46 \\
0.48\end{array}$ & $\begin{array}{l}0.14 \\
0.19 \\
0.34\end{array}$ & $\begin{array}{l}0.02 \\
0.06 \\
0.04\end{array}$ & $\begin{array}{l}0.22 \\
0.32 \\
0.10\end{array}$ & $\begin{array}{l}0.88 \\
0.92 \\
0.28\end{array}$ & $\begin{array}{r}2.67^{(8)} \\
1.01 \\
1.29\end{array}$ & $\begin{array}{r}3.69^{(a)} \\
0.31 \\
0.44\end{array}$ & $\begin{array}{r}12.19 \\
6.39 \\
6.45\end{array}$ \\
\hline $\begin{array}{l}\text { Average } \\
\text { Normal }\end{array}$ & $\begin{array}{l}0.93 \\
0.79\end{array}$ & $\begin{array}{l}0.63 \\
0.62\end{array}$ & $\begin{array}{l}0.51 \\
0.47\end{array}$ & $\begin{array}{l}0.45 \\
0.41\end{array}$ & $\begin{array}{l}0.53 \\
0.51\end{array}$ & $\begin{array}{l}0.53 \\
0.38\end{array}$ & $\begin{array}{l}0.22 \\
0.18\end{array}$ & $\begin{array}{l}0.24 \\
0.27\end{array}$ & $\begin{array}{l}0.32 \\
0.31\end{array}$ & $\begin{array}{l}0.55 \\
0.39\end{array}$ & $\begin{array}{l}0.91 \\
0.91\end{array}$ & $\begin{array}{l}1.01 \\
1.03\end{array}$ & $\begin{array}{l}6.83 \\
6.26\end{array}$ \\
\hline
\end{tabular}

(a) Greatest and least values.

(b) Most recent of multiple occurrences.

$\mathrm{T}=$ Trace. 
Table 4.2. Seasonal Precipitation (in.)

\begin{tabular}{|c|c|c|c|c|}
\hline Year & $\begin{array}{l}\text { Winter }{ }^{(2)} \\
\text { Dec-Feb }\end{array}$ & $\begin{array}{c}\text { Spring } \\
\text { Mar-May }\end{array}$ & $\begin{array}{l}\text { Summer } \\
\text { Jun-Aug }\end{array}$ & $\begin{array}{l}\text { Autumn } \\
\text { Sep-Nov }\end{array}$ \\
\hline $\begin{array}{l}1946 \\
1947 \\
1948 \\
1949 \\
1950\end{array}$ & $\begin{array}{r}-\overline{-} \\
0.70^{(b)} \\
2.80 \\
1.92 \\
3.02\end{array}$ & $\begin{array}{l}.0 .14 \\
2.73 \\
1.30 \\
1.61\end{array}$ & $\begin{array}{r}-\overline{46} \\
2.26 \\
0.05 \\
2.99^{(0)}\end{array}$ & $\begin{array}{l}1.83 \\
4.35 \\
1.56 \\
1.80 \\
3.02\end{array}$ \\
\hline $\begin{array}{l}1951 \\
1952 \\
1953 \\
1954 \\
1955\end{array}$ & $\begin{array}{l}2.32 \\
1.85 \\
3.18 \\
2.25 \\
1.13\end{array}$ & $\begin{array}{l}1.42 \\
0.77 \\
1.22 \\
1.07 \\
1.16\end{array}$ & $\begin{array}{l}1.90 \\
1.15 \\
1.51 \\
0.74 \\
0.85\end{array}$ & $\begin{array}{l}1.63 \\
0.32 \\
1.29 \\
1.79 \\
2.71\end{array}$ \\
\hline $\begin{array}{l}1956 \\
1957 \\
1958 \\
1959 \\
1960\end{array}$ & $\begin{array}{l}4.30 \\
1.29 \\
3.75 \\
5.06 \\
1.35\end{array}$ & $\begin{array}{l}0.32 \\
3.06 \\
1.84 \\
1.10 \\
1.91\end{array}$ & $\begin{array}{l}1.24 \\
0.54 \\
0.83 \\
0.26 \\
0.40\end{array}$ & $\begin{array}{l}1.19 \\
3.45 \\
1.01 \\
2.23 \\
1.38\end{array}$ \\
\hline $\begin{array}{l}1961 \\
1962 \\
1963 \\
1964 \\
1965\end{array}$ & $\begin{array}{l}3.07 \\
1.92 \\
2.24 \\
1.52 \\
3.41\end{array}$ & $\begin{array}{l}2.30 \\
1.83 \\
2.13 \\
0.18 \\
0.27\end{array}$ & $\begin{array}{l}0.66 \\
0.62 \\
0.60 \\
1.18 \\
0.63\end{array}$ & $\begin{array}{l}0.56 \\
1.98 \\
0.80 \\
1.31 \\
1.29\end{array}$ \\
\hline $\begin{array}{l}1966 \\
1967 \\
1968 \\
1969 \\
1970\end{array}$ & $\begin{array}{l}1.10 \\
0.92 \\
1.89 \\
3.03 \\
4.51\end{array}$ & $\begin{array}{r}0.47 \\
1.60 \\
0.09^{(0)} \\
1.83 \\
1.26\end{array}$ & $\begin{array}{l}1.24 \\
0.57 \\
0.74 \\
0.75 \\
0.26\end{array}$ & $\begin{array}{l}2.91 \\
0.34 \\
2.41 \\
0.71 \\
0.98\end{array}$ \\
\hline $\begin{array}{l}1971 \\
1972 \\
1973 \\
1974 \\
1975\end{array}$ & $\begin{array}{l}1.49 \\
1.53 \\
2.38 \\
3.33 \\
3.65\end{array}$ & $\begin{array}{l}1.65 \\
2.71 \\
0.32 \\
1.26 \\
1.13\end{array}$ & $\begin{array}{r}0.93 \\
1.38 \\
0.03^{(0)} \\
0.83 \\
1.72\end{array}$ & $\begin{array}{r}1.77 \\
0.57 \\
4.79^{(0)} \\
0.93 \\
1.50\end{array}$ \\
\hline $\begin{array}{l}1976 \\
1977 \\
1978 \\
1979 \\
1980\end{array}$ & $\begin{array}{l}1.62 \\
0.76 \\
3.91 \\
0.97 \\
3.61\end{array}$ & $\begin{array}{l}0.72 \\
1.06 \\
1.17 \\
1.16 \\
2.57\end{array}$ & $\begin{array}{l}1.20 \\
1.79 \\
1.18 \\
0.47 \\
0.98\end{array}$ & $\begin{array}{r}0.04^{(0)} \\
1.44 \\
1.32 \\
2.23 \\
1.62\end{array}$ \\
\hline $\begin{array}{l}1981 \\
1982 \\
1983 \\
1984 \\
1985\end{array}$ & $\begin{array}{l}3.05 \\
2.35 \\
4.59 \\
3.29 \\
1.73\end{array}$ & $\begin{array}{l}1.71 \\
1.33 \\
1.94 \\
2.16 \\
0.49\end{array}$ & $\begin{array}{l}0.65 \\
1.17 \\
1.11 \\
1.05 \\
0.28\end{array}$ & $\begin{array}{l}2.07 \\
2.79 \\
3.10 \\
2.32 \\
2.33\end{array}$ \\
\hline $\begin{array}{l}1986 \\
1987 \\
1988 \\
1989 \\
1990\end{array}$ & $\begin{array}{l}3.97 \\
1.76 \\
2.11 \\
2.28 \\
1.15\end{array}$ & $\begin{array}{l}1.06 \\
1.36 \\
1.84 \\
2.99 \\
1.36\end{array}$ & $\begin{array}{l}0.23 \\
0.68 \\
0.24 \\
0.28 \\
1.33\end{array}$ & $\begin{array}{l}1.90 \\
0.41 \\
1.22 \\
1.48 \\
0.80\end{array}$ \\
\hline $\begin{array}{l}1991 \\
1992 \\
1993 \\
1994 \\
1995\end{array}$ & $\begin{array}{l}1.24 \\
1.78 \\
4.29 \\
1.49 \\
4.19\end{array}$ & $\begin{array}{r}2.06 \\
1.03 \\
1.98 \\
1.91 \\
3.28^{(0)}\end{array}$ & $\begin{array}{l}1.80 \\
1.72 \\
2.12 \\
0.61 \\
1.18\end{array}$ & $\begin{array}{l}1.97 \\
1.95 \\
0.32 \\
1.69 \\
2.70\end{array}$ \\
\hline $\begin{array}{l}1996 \\
1997 \\
1998\end{array}$ & $\begin{array}{c}4.96 \\
5.45^{(0)} \\
2.70\end{array}$ & $\begin{array}{l}1.88 \\
1.36 \\
1.09\end{array}$ & $\begin{array}{l}0.21 \\
0.71 \\
0.86\end{array}$ & $\begin{array}{l}3.77 \\
2.25 \\
1.67\end{array}$ \\
\hline $\begin{array}{l}\text { Average } \\
\text { Normal }\end{array}$ & $\begin{array}{l}2.58 \\
2.44\end{array}$ & $\begin{array}{l}1.48 \\
1.40\end{array}$ & $\begin{array}{l}0.98 \\
0.83\end{array}$ & $\begin{array}{l}1.77 \\
1.60\end{array}$ \\
\hline
\end{tabular}

(a) For the winter season, December is included in the previous year.

(b) Greatest and least values. 
Table 4.3. Average Number of Days with Precipitation of Specified Amount

\begin{tabular}{|c|c|c|c|c|c|c|}
\hline Month & $\begin{array}{c}\text { Trace or } \\
\text { more }\end{array}$ & $\begin{array}{l}0.01 \mathrm{in} . \\
\text { or more }\end{array}$ & $\begin{array}{l}0.10 \mathrm{in} . \\
\text { or more }\end{array}$ & $\begin{array}{l}0.25 \mathrm{in} . \\
\text { or more }\end{array}$ & $\begin{array}{l}0.50 \text { in. } \\
\text { or more }\end{array}$ & $\begin{array}{l}1.00 \mathrm{in} . \\
\text { or more }\end{array}$ \\
\hline Jan & 16 & 9 & 3 & 1 & (a) & 0 \\
\hline Feb & 12 & 7 & 2 & 1 & (a) & 0 \\
\hline Mar & 11 & 6 & 2 & (a) & (a) & 0 \\
\hline Apr & 10 & 5 & 2 & 1 & (a) & 0 \\
\hline May & 10 & 5 & 2 & 1 & (a) & 0 \\
\hline Jun & 9 & 5 & 2 & 1 & (a) & (a) \\
\hline Jul & 5 & 2 & 1 & (a) & (a) & (a) \\
\hline Aug & 5 & 3 & 1 & (a) & (a) & 0 \\
\hline Sep & 6 & 3 & 1 & (a) & (a) & 0 \\
\hline Oct & 9 & 5 & 2 & 1 & (a) & (a) \\
\hline Nov & 14 & 9 & 3 & 1 & (a) & (a) \\
\hline Dec & 16 & 10 & 4 & 1 & (a) & 0 \\
\hline Annual ${ }^{(b)}$ & 124 & 68 & 23 & 7 & $1^{(c)}$ & (a) \\
\hline
\end{tabular}

(a) Used to denote an average of $<1 / 2$ day.

(b) Annual totals may differ from summation of monthly events because of rounding.

(c) Although the number of days with $0.50 \mathrm{in}$. or more averages $<1 / 2$ day for any one month, 72 such days were recorded during 52 years of record.

Table 4.4. Monthly and Annual Averages and Extremes in Total Time with Precipitation Observed: July 1946 Through June 1971, July 1974 Through December 1998

\begin{tabular}{|c|c|c|c|c|c|c|c|c|}
\hline \multirow[b]{2}{*}{ Month } & \multicolumn{2}{|c|}{ Averages } & \multicolumn{3}{|c|}{ Greatest } & \multicolumn{3}{|c|}{ Least } \\
\hline & $\begin{array}{l}\text { No. of } \\
\text { Hours } \\
\end{array}$ & $\begin{array}{l}\% \text { of } \\
\text { Time } \\
\end{array}$ & $\begin{array}{l}\text { No. of } \\
\text { Hours }\end{array}$ & $\begin{array}{l}\% \text { of } \\
\text { Time } \\
\end{array}$ & Year & $\begin{array}{l}\text { No. of } \\
\text { Hours }\end{array}$ & $\begin{array}{l}\% \text { of } \\
\text { Time }\end{array}$ & Year \\
\hline $\operatorname{Jan}$ & 90.3 & 12.1 & 212.0 & 28.5 & 1969 & 29.2 & 3.9 & 1949 \\
\hline Feb & 56.2 & 8.3 & 151.6 & 22.6 & 1980 & 2.5 & 0.4 & 1988 \\
\hline Mar & 39.8 & 5.3 & 135.2 & 18.2 & 1957 & 6.4 & 0.9 & 1994 \\
\hline Apr & 29.5 & 4.1 & 69.2 & 9.6 & 1953 & 1.6 & 0.2 & 1985 \\
\hline May & 31.3 & 4.2 & 89.9 & 12.1 & 1948 & 1.2 & 0.2 & 1992. \\
\hline Jun & 27.1 & 3.8 & 80.8 & 11.2 & 1950 & 2.9 & 0.4 & 1986 \\
\hline Jul & 10.8 & 1.5 & 38.2 & 5.1 & 1966 & 0.5 & 0.1 & 1984 \\
\hline Aug & 12.1 & 1.6 & 61.7 & 8.3 & 1968 & 0.0 & 0.0 & $1988^{(2)}$ \\
\hline Sep & 15.9 & 2.2 & 66.4 & 9.2 & 1977 & 0.0 & 0.0 & 1991 \\
\hline Oct & 32.0 & 4.3 & 119.9 & 16.1 & 1947 & 0.4 & 0.1 & 1978 \\
\hline Nov & 61.4 & 8.5 & 146.5 & 20.3 & 1985 & 4.8 & 0.7 & 1976 \\
\hline Dec & 89.3 & 12.0 & 230.5 & 31.0 & 1985 & 15.8 & 2.1 & 1976 \\
\hline Annual & 495.6 & 5.7 & 738.0 & 8.4 & 1950 & 286.7 & 3.3 & 1990 \\
\hline
\end{tabular}

(a) Most recent of several occurrences. 
sensors and computer programs was initiated to help ascertain the beginning and ending times of precipitation events during periods when the HMS is not staffed. Table 4.5 lists total hours of precipitation by month for the period 1946 through 1998 . As previously noted, complete precipitation duration data for the period July 1971 through June 1974 are not available, and incomplete data are not included.

The months of November through February, which contribute more than half of the annual precipitation, received precipitation $10.3 \%$ of the time, $>3$ times the other 8 months of the year $(3.4 \%)$.

\subsection{Notable Wet Periods}

Nine periods are listed when precipitation was abnormally high:

\begin{tabular}{|c|c|c|c|c|c|}
\hline \multirow[b]{2}{*}{ Period } & \multicolumn{3}{|c|}{ Number of Days with Trace or More } & \multicolumn{2}{|c|}{ Total Amount, in. } \\
\hline & Altogether & $\begin{array}{c}\text { Greatest } \\
\text { Consecutive }\end{array}$ & $\begin{array}{l}\text { Measurable } \\
\text { Precipitation }\end{array}$ & $\begin{array}{c}\text { Water } \\
\text { Equivalent }\end{array}$ & $\begin{array}{c}\text { Snow- } \\
\text { fall }\end{array}$ \\
\hline Oct 7 - Nov 4, 1947 & 23 out of 29 & 10 & 17 & 2.21 & 0.00 \\
\hline $\operatorname{Jan} 3-28,1950$ & 21 out of 26 & 10 & 15 & 1.80 & 23.4 \\
\hline Nov 11 - Dec 19, 1950 & 33 out of 39 & 12 & 20 & 1.37 & 3.7 \\
\hline Nov 16 - Dec 22, 1955 & 31 out of 37 & 15 & 24 & 3.19 & 22.7 \\
\hline Oct $31-\operatorname{Dec} 7,1973$ & 32 out of 38 & 14 & 20 & 3.45 & 8.1 \\
\hline Nov 15 - Dec 7, 1985 & 17 out of 23 & 8 & 14 & 1.96 & 25.2 \\
\hline Dec 27, 1992 - Jan 23, 1993 & 26 out of 29 & 12 & 19 & 2.02 & 26.8 \\
\hline Nov $13-27,1996$ & 12 out of 15 & 7 & 10 & 2.66 & 11.9 \\
\hline Dec 20 - 31, 1996 & 11 out of 12 & 9 & 9 & 3.00 & 20.1 \\
\hline
\end{tabular}

From a precipitation standpoint, 1973 was an unusual year. Total precipitation for 1973 was 8.27 in., $132 \%$ of normal (6.26 in.). The period March 30 through September 18, 1973 was extremely dry, receiving only 0.29 in. of precipitation during that 173-day period; however, the period October 31 through December 7, 1973 was a notable wet period. During the months of October, November, and December 1973, $6.38 \mathrm{in}$. of precipitation were recorded, $289 \%$ of normal ( $2.21 \mathrm{in}$.) for those months. November and December 1996 received $6.36 \mathrm{in}$. of precipitation, $328 \%$ of normal (1.94 in.) for those months, which is greater than the normal precipitation amount for an entire year (6.26 in.).

\subsection{Notable Dry Periods}

The HMS is in a semiarid region, thus it experiences many dry periods. January, March, and December are the only months that have always received measurable precipitation (1946 through 1998). 
Table 4.5. Total Duration (h) of Precipitation by Month and Year

\begin{tabular}{|c|c|c|c|c|c|c|c|c|c|c|c|c|c|}
\hline Year & Jan & Feb & Mar & Apr & May & Jun & Jul & Aug & Sep & Oct & Nov & Dec & Annual \\
\hline $\begin{array}{l}1946 \\
1947 \\
1948 \\
1949 \\
1950\end{array}$ & $\begin{array}{r}34 . \overline{6} \\
70.7 \\
29.2^{(2)} \\
147.4\end{array}$ & $\begin{array}{r}-\overline{8} \\
29.8 \\
70.4 \\
69.9 \\
78.0\end{array}$ & $\begin{array}{r}30.2 \\
16.6 \\
63.5 \\
72.0\end{array}$ & $\begin{array}{r}32 . \overline{7} \\
43.8 \\
5.6 \\
23.3\end{array}$ & $\begin{array}{r}\overline{4} \\
89.9^{(2)} \\
13.2 \\
13.9\end{array}$ & $\begin{array}{r}- \\
38.4 \\
36.4 \\
3.2 \\
80.8^{(2)}\end{array}$ & $\begin{array}{r}6.0 \\
17.4 \\
15.2 \\
2.2 \\
5.0\end{array}$ & $\begin{array}{r}16.3 \\
12.6 \\
16.6 \\
1.6 \\
2.3\end{array}$ & $\begin{array}{r}12.2 \\
36.4 \\
15.8 \\
21.2 \\
1.3\end{array}$ & $\begin{array}{r}38.2 \\
119.9^{(2)} \\
19.7 \\
15.0 \\
112.3\end{array}$ & $\begin{array}{l}66.0 \\
48.1 \\
44.4 \\
98.7 \\
92.9\end{array}$ & $\begin{array}{r}31.5 \\
71.1 \\
81.9 \\
32.1 \\
108.8\end{array}$ & $\begin{array}{r}- \\
476.0 \\
521.4 \\
355.4 \\
738.0^{(2)}\end{array}$ \\
\hline $\begin{array}{l}1951 \\
1952 \\
1953 \\
1954 \\
1955\end{array}$ & $\begin{array}{r}66.3 \\
151.0 \\
89.3 \\
92.6 \\
116.4\end{array}$ & $\begin{array}{l}55.9 \\
57.8 \\
29.7 \\
90.0 \\
33.4\end{array}$ & $\begin{array}{l}34.8 \\
19.3 \\
32.3 \\
35.8 \\
20.0\end{array}$ & $\begin{array}{r}21.5 \\
22.1 \\
69.2^{(2)} \\
18.4 \\
57.2\end{array}$ & $\begin{array}{l}23.7 \\
25.4 \\
20.6 \\
33.7 \\
47.8\end{array}$ & $\begin{array}{l}60.8 \\
77.7 \\
37.1 \\
32.4 \\
10.2\end{array}$ & $\begin{array}{r}12.8 \\
4.3 \\
2.0 \\
17.8 \\
36.9\end{array}$ & $\begin{array}{r}17.7 \\
5.4 \\
25.2 \\
22.2 \\
0.0\end{array}$ & $\begin{array}{r}26.6 \\
7.1 \\
7.1 \\
15.1 \\
40.1\end{array}$ & $\begin{array}{r}66.5 \\
3.6 \\
23.6 \\
17.9 \\
42.3\end{array}$ & $\begin{array}{r}66.0 \\
71.2 \\
59.4 \\
74.3 \\
132.2\end{array}$ & $\begin{array}{r}86.7 \\
137.0 \\
32.2 \\
39.9 \\
141.0\end{array}$ & $\begin{array}{l}539.3 \\
581.9 \\
427.7 \\
490.1 \\
677.5\end{array}$ \\
\hline $\begin{array}{l}1956 \\
1957 \\
1958 \\
1959 \\
1960\end{array}$ & $\begin{array}{r}126.9 \\
140.6 \\
82.8 \\
129.5 \\
86.8\end{array}$ & $\begin{array}{r}74.4 \\
46.4 \\
106.8 \\
98.2 \\
48.0\end{array}$ & $\begin{array}{r}15.9 \\
135.2^{(a)} \\
37.5 \\
32.6 \\
49.9\end{array}$ & $\begin{array}{r}1.8 \\
19.5 \\
54.5 \\
17.5 \\
32.8\end{array}$ & $\begin{array}{l}35.8 \\
43.4 \\
24.2 \\
33.0 \\
47.2\end{array}$ & $\begin{array}{r}30.8 \\
20.8 \\
24.2 \\
29.8 \\
6.3\end{array}$ & $\begin{array}{l}4.2 \\
2.6 \\
1.2 \\
4.2 \\
3.5\end{array}$ & $\begin{array}{r}17.4 \\
5.7 \\
2.0 \\
15.9 \\
27.3\end{array}$ & $\begin{array}{r}6.6 \\
23.1 \\
13.4 \\
52.2 \\
15.8\end{array}$ & $\begin{array}{l}65.7 \\
72.0 \\
13.6 \\
27.2 \\
34.8\end{array}$ & $\begin{array}{l}71.0 \\
21.4 \\
58.5 \\
44.8 \\
64.1\end{array}$ & $\begin{array}{r}98.8 \\
49.3 \\
107.7 \\
51.8 \\
120.8\end{array}$ & $\begin{array}{l}549.3 \\
580.0 \\
526.4 \\
536.7 \\
537.3\end{array}$ \\
\hline $\begin{array}{l}1961 \\
1962 \\
1963 \\
1964 \\
1965\end{array}$ & $\begin{array}{r}91.8 \\
43.9 \\
56.3 \\
49.1 \\
153.3\end{array}$ & $\begin{array}{r}94.4 \\
58.8 \\
88.4 \\
5.2 \\
18.8\end{array}$ & $\begin{array}{r}60.7 \\
55.1 \\
31.2 \\
8.3 \\
14.2\end{array}$ & $\begin{array}{l}39.2 \\
24.8 \\
66.5 \\
15.7 \\
30.9\end{array}$ & $\begin{array}{r}48.7 \\
80.2 \\
51.3 \\
6.1 \\
15.2\end{array}$ & $\begin{array}{l}23.9 \\
13.9 \\
37.1 \\
46.8 \\
28.6\end{array}$ & $\begin{array}{r}4.2 \\
4.3 \\
20.9 \\
14.5 \\
6.8\end{array}$ & $\begin{array}{r}17.2 \\
24.9 \\
4.4 \\
14.1 \\
18.4\end{array}$ & $\begin{array}{r}2.0 \\
21.6 \\
11.2 \\
7.2 \\
11.2\end{array}$ & $\begin{array}{l}15.9 \\
71.5 \\
26.4 \\
19.2 \\
11.2\end{array}$ & $\begin{array}{r}57.2 \\
44.4 \\
61.0 \\
109.0 \\
89.1\end{array}$ & $\begin{array}{r}99.0 \\
139.6 \\
179.6 \\
149.0 \\
57.8\end{array}$ & $\begin{array}{l}554.2 \\
583.0 \\
634.3 \\
444.2 \\
455.5\end{array}$ \\
\hline $\begin{array}{l}1966 \\
1967 \\
1968 \\
1969 \\
1970\end{array}$ & $\begin{array}{r}51.7 \\
34.1 \\
99.1 \\
212.0^{(a)} \\
157.2\end{array}$ & $\begin{array}{r}12.4 \\
4.7 \\
42.0 \\
75.4 \\
72.9\end{array}$ & $\begin{array}{r}42.9 \\
30.6 \\
7.3 \\
9.7 \\
34.0\end{array}$ & $\begin{array}{r}9.1 \\
60.9 \\
18.6 \\
52.2 \\
19.2\end{array}$ & $\begin{array}{r}7.2 \\
52.9 \\
29.9 \\
51.9 \\
27.2\end{array}$ & $\begin{array}{l}30.4 \\
23.3 \\
38.3 \\
38.7 \\
31.1\end{array}$ & $\begin{array}{r}38.2^{(2)} \\
2.2 \\
5.6 \\
1.3 \\
6.9\end{array}$ & $\begin{array}{c}3.7 \\
1.7 \\
61.7^{(a)} \\
0.3 \\
2.3\end{array}$ & \begin{tabular}{r|}
15.9 \\
12.1 \\
17.2 \\
26.8 \\
5.3
\end{tabular} & $\begin{array}{l}26.3 \\
29.4 \\
45.3 \\
20.4 \\
32.2\end{array}$ & $\begin{array}{r}103.5 \\
27.0 \\
68.9 \\
44.1 \\
85.8\end{array}$ & $\begin{array}{r}75.6 \\
88.2 \\
134.2 \\
148.3 \\
83.9\end{array}$ & $\begin{array}{l}416.9 \\
367.1 \\
568.1 \\
681.1 \\
558.0\end{array}$ \\
\hline $\begin{array}{l}1971 \\
1972 \\
1973 \\
1974 \\
1975\end{array}$ & $\begin{array}{r}49.5 \\
\text { (b) } \\
(b) \\
(b) \\
105.7\end{array}$ & $\begin{array}{r}14.8 \\
\text { (b) } \\
(b) \\
(b) \\
103.8\end{array}$ & $\begin{array}{r}68.0 \\
(\text { b) } \\
(b) \\
(b) \\
42.3\end{array}$ & $\begin{array}{r}25.0 \\
(b) \\
(b) \\
(b) \\
29.5\end{array}$ & $\begin{array}{r}43.7 \\
\text { (b) } \\
\text { (b) } \\
(b) \\
29.2\end{array}$ & $\begin{array}{r}52.7 \\
(b) \\
(b) \\
(b) \\
20.8\end{array}$ & $\begin{array}{r}\text { (b) } \\
\text { (b) } \\
(b) \\
21.8 \\
13.5\end{array}$ & $\begin{array}{l}\text { (b) } \\
\text { (b) } \\
0.8^{(b)} \\
26.8\end{array}$ & $\begin{array}{l}\text { (b) } \\
\text { (b) } \\
\text { (b) } \\
1.3 \\
1.0\end{array}$ & $\begin{array}{r}\text { (b) } \\
\text { (b) } \\
\text { (b) } \\
26.2 \\
65.6\end{array}$ & $\begin{array}{r}\text { (b) } \\
\text { (b) } \\
\text { (b) } \\
58.9 \\
56.1\end{array}$ & $\begin{array}{r}\text { (b) } \\
\text { (b) } \\
\text { (b) } \\
64.9 \\
76.6\end{array}$ & $\begin{array}{r}\text { (b) } \\
\text { (b) } \\
(b) \\
(b) \\
570.9\end{array}$ \\
\hline $\begin{array}{l}1976 \\
1977 \\
1978 \\
1979 \\
1980\end{array}$ & $\begin{array}{r}79.8 \\
138.7 \\
147.9 \\
111.5 \\
118.5\end{array}$ & $\begin{array}{r}28.6 \\
37.1 \\
98.6 \\
73.7 \\
151.6^{(2)}\end{array}$ & $\begin{array}{l}35.2 \\
37.8 \\
46.2 \\
45.0 \\
35.4\end{array}$ & \begin{tabular}{r|}
36.8 \\
4.6 \\
53.4 \\
29.6 \\
33.8
\end{tabular} & $\begin{array}{l}16.7 \\
45.9 \\
45.2 \\
14.0 \\
60.2\end{array}$ & \begin{tabular}{r|}
10.1 \\
24.8 \\
8.1 \\
4.5 \\
45.0
\end{tabular} & $\begin{array}{r}15.1 \\
14.2 \\
27.6 \\
8.6 \\
1.5\end{array}$ & $\begin{array}{r}38.8 \\
28.9 \\
37.7 \\
27.4 \\
8.8\end{array}$ & $\begin{array}{r}2.2 \\
66.4^{(2)} \\
27.9 \\
10.0 \\
24.5\end{array}$ & $\begin{array}{l}10.7 \\
15.8 \\
0.4^{(2)} \\
47.3 \\
22.0\end{array}$ & $\begin{array}{l}4.8^{(2)} \\
77.1 \\
71.6 \\
86.9 \\
44.2\end{array}$ & \begin{tabular}{|r|}
$15.8^{(a)}$ \\
98.1 \\
43.7 \\
104.2 \\
121.2
\end{tabular} & $\begin{array}{l}294.6 \\
589.4 \\
608.3 \\
562.7 \\
666.7\end{array}$ \\
\hline $\begin{array}{l}1981 \\
1982 \\
1983 \\
1984 \\
1985\end{array}$ & $\begin{array}{r}72.0 \\
47.5 \\
72.5 \\
32.6 \\
151.2\end{array}$ & $\begin{array}{l}31.7 \\
29.2 \\
76.1 \\
58.1 \\
54.9\end{array}$ & $\begin{array}{l}32.0 \\
27.8 \\
59.4 \\
40.8 \\
26.0\end{array}$ & $\begin{array}{r}3.4 \\
33.6 \\
15.2 \\
35.2 \\
1.6^{(2)}\end{array}$ & $\begin{array}{r}28.4 \\
9.4 \\
13.2 \\
34.2 \\
7.0\end{array}$ & $\begin{array}{l}21.1 \\
21.8 \\
22.4 \\
37.9 \\
17.4\end{array}$ & $\begin{array}{r}7.5 \\
5.8 \\
17.9 \\
0.5^{(a)} \\
3.2\end{array}$ & $\begin{array}{r}1.0 \\
5.3 \\
13.7 \\
0.5 \\
1.2\end{array}$ & $\begin{array}{l}17.4 \\
23.2 \\
12.5 \\
20.5 \\
30.8\end{array}$ & $\begin{array}{r}24.8 \\
40.1 \\
19.8 \\
7.1 \\
17.2\end{array}$ & $\begin{array}{r}34.1 \\
42.8 \\
79.3 \\
97.6 \\
146.5^{(2)}\end{array}$ & $\begin{array}{r}91.8 \\
81.7 \\
133.2 \\
75.5 \\
230.5^{(2)}\end{array}$ & $\begin{array}{l}365.2 \\
368.2 \\
535.2 \\
440.5 \\
687.5\end{array}$ \\
\hline $\begin{array}{l}1986 \\
1987 \\
1988 \\
1989 \\
1990\end{array}$ & $\begin{array}{r}107.6 \\
64.6 \\
92.4 \\
35.2 \\
34.6\end{array}$ & $\begin{array}{r}68.9 \\
20.8 \\
2.5^{(a)} \\
114.4 \\
20.5\end{array}$ & $\begin{array}{r}47.4 \\
74.0 \\
24.8 \\
102.2 \\
18.2\end{array}$ & $\begin{array}{l}14.4 \\
10.8 \\
36.2 \\
36.8 \\
29.9\end{array}$ & $\begin{array}{l}22.3 \\
14.6 \\
18.3 \\
25.8 \\
31.0\end{array}$ & $\begin{array}{r}2.9^{(n)} \\
11.8 \\
17.4 \\
5.4 \\
8.6\end{array}$ & $\begin{array}{r}13.3 \\
16.1 \\
6.2 \\
3.8 \\
7.3\end{array}$ & $\begin{array}{c}1.1 \\
4.5 \\
0.0^{(2 x)} \\
14.4 \\
15.4\end{array}$ & $\begin{array}{r}28.2 \\
0.5 \\
13.8 \\
0.7 \\
0.1\end{array}$ & $\begin{array}{r}10.3 \\
1.8 \\
2.2 \\
23.1 \\
49.1\end{array}$ & $\begin{array}{r}31.3 \\
21.1 \\
55.8 \\
33.2 \\
7.1\end{array}$ & $\begin{array}{r}143.8 \\
125.6 \\
62.7 \\
57.9 \\
64.9\end{array}$ & $\begin{array}{r}491.5 \\
366.2 \\
332.3 \\
452.9 \\
286.7^{(2)}\end{array}$ \\
\hline $\begin{array}{l}1991 \\
1992 \\
1993 \\
1994 \\
1995\end{array}$ & $\begin{array}{r}57.5 \\
36.2 \\
171.0 \\
40.5 \\
113.8\end{array}$ & $\begin{array}{l}28.0 \\
56.2 \\
64.4 \\
55.8 \\
39.2\end{array}$ & $\begin{array}{r}43.8 \\
7.9 \\
65.2 \\
6.4^{(2)} \\
47.3\end{array}$ & $\begin{array}{l}15.8 \\
42.0 \\
57.0 \\
43.2 \\
56.2\end{array}$ & $\begin{array}{c}39.0 \\
1.2^{(\text {a) }} \\
38.7 \\
40.7 \\
27.3\end{array}$ & $\begin{array}{l}41.8 \\
35.9 \\
13.0 \\
21.2 \\
52.6\end{array}$ & $\begin{array}{r}9.8 \\
22.5 \\
35.2 \\
4.7 \\
8.3\end{array}$ & $\begin{array}{r}4.2 \\
6.6 \\
12.1 \\
3.8 \\
7.9\end{array}$ & $\begin{array}{r}0.0^{(2)} \\
23.6 \\
3.0 \\
8.9 \\
14.5\end{array}$ & $\begin{array}{r}42.9 \\
36.6 \\
6.8 \\
37.6 \\
33.0\end{array}$ & $\begin{array}{l}70.2 \\
53.0 \\
30.3 \\
52.9 \\
47.1\end{array}$ & $\begin{array}{l}48.2 \\
92.6 \\
58.2 \\
72.2 \\
62.2\end{array}$ & $\begin{array}{l}401.2 \\
414.3 \\
554.9 \\
387.9 \\
509.4\end{array}$ \\
\hline $\begin{array}{l}1996 \\
1997 \\
1998\end{array}$ & $\begin{array}{r}102.1 \\
69.5 \\
60.0\end{array}$ & $\begin{array}{l}73.0 \\
17.7 \\
72.3\end{array}$ & $\begin{array}{l}55.4 \\
36.1 \\
34.2\end{array}$ & $\begin{array}{r}23.2 \\
13.7 \\
8.6\end{array}$ & $\begin{array}{l}30.8 \\
19.2 \\
45.7\end{array}$ & $\begin{array}{r}3.6 \\
14.7 \\
13.7\end{array}$ & $\begin{array}{r}8.2 \\
12.7 \\
12.7\end{array}$ & $\begin{array}{c}3.3 \\
4.1 \\
1.8\end{array}$ & $\begin{array}{r}13.7 \\
19.7 \\
6.8\end{array}$ & $\begin{array}{l}46.8 \\
29.4 \\
14.8\end{array}$ & $\begin{array}{l}81.5 \\
43.6 \\
45.1\end{array}$ & $\begin{array}{r}124.4 \\
15.5 \\
54.1\end{array}$ & $\begin{array}{l}566.0 \\
295.9 \\
369.8\end{array}$ \\
\hline $\begin{array}{l}\text { Average } \\
\text { Normal }\end{array}$ & $\begin{array}{l}90.3 \\
89.3\end{array}$ & $\begin{array}{l}56.2 \\
54.0\end{array}$ & $\begin{array}{l}39.8 \\
39.9\end{array}$ & $\begin{array}{l}29.5 \\
28.4\end{array}$ & $\begin{array}{l}31.3 \\
30.7\end{array}$ & $\begin{array}{l}27.1 \\
23.9\end{array}$ & $\begin{array}{l}10.8 \\
10.7\end{array}$ & $\begin{array}{l}12.1 \\
13.9\end{array}$ & $\begin{array}{l}15.9 \\
15.2\end{array}$ & $\begin{array}{l}32.0 \\
25.2\end{array}$ & $\begin{array}{l}61.4 \\
60.7\end{array}$ & $\begin{array}{r}89.3 \\
101.8\end{array}$ & $\begin{array}{l}495.6 \\
495.4\end{array}$ \\
\hline
\end{tabular}

(a) Greatest and least values.

(b) Incomplete data not included. See Section 4.4

(c) Most recent of numerous occurrences. 
A total of 37 months during the period of record have been without measurable precipitation, with the months of July and August accounting for 20 of those months. The record number of consecutive days with no precipitation (not even a trace) occurred in 1988, when the period July 14 through September 17 (66 days) was totally dry. The following list indicates some long periods with small amounts of precipitation.

\begin{tabular}{|c|c|c|c|c|}
\hline \multicolumn{5}{|c|}{ Notable Dry Periods } \\
\hline Year & From & To & $\begin{array}{l}\text { Number } \\
\text { of Days }\end{array}$ & $\begin{array}{l}\text { Total Precipitation, } \\
\text { in. }\end{array}$ \\
\hline 1952 & Jun 30 & Nov 10 & 134 & 0.20 \\
\hline 1967 & Jun 22 & Nov 7 & 139 & 0.18 \\
\hline 1968 & Feb 24 & Aug 13 & 172 & 0.32 \\
\hline 1973 & Mar 30 & Sep 18 & 173 & 0.29 \\
\hline 1976 & Aug 26 & Dec 31 & 128 & 0.15 \\
\hline 1985 & Mar 31 & $\operatorname{Sep} 7$ & 161 & 0.43 \\
\hline 1986 & May 6 & Sep 12 & 129 & 0.30 \\
\hline 1987 & Jul 19 & Oct 31 & 105 & 0.08 \\
\hline 1988 & Jun 6 & Sep 17 & 105 & 0.13 \\
\hline
\end{tabular}

The driest year on record was 1976 , which had 2.99 in. recorded ( $<50 \%$ of normal). During the period September through December 1976, total precipitation was 0.15 in., which was $6 \%$ of normal ( 2.52 in.) for those months.

\subsection{Snowfall}

Snowfall, which includes all frozen precipitation, varied from a seasonal total of 0.3 to 56.1 in. in 1957-1958 and 1992-1993, respectively. Table 4.6 provides information on monthly and seasonal snowfall amounts, as well as the dates and amounts of earliest and latest snowfall each season. The earliest measurable snowfall $(0.3$ in.) was recorded on October 26,1957 ; the latest measurable snowfall (1.0 in.) was recorded on April 6, 1982. The average date of the first measurable snow is November 30; the last measurable snow date is February 11. Normal snowfall for the period 1961 through 1990 and averages for the entire period of record are noted on the table, as are monthly and seasonal extremes.

Table 4.7 lists the greatest single storm snowfall amounts by month for the period 1946 through 1998 . The greatest single snowstorm, on February 18-20, 1993, produced $12.4 \mathrm{in}$. of snow. During the winter of 1957-58 (actually the only snowfall was recorded in October - not yet winter), the greatest single snowstorm produced only 0.3 in.

Table 4.8 lists some miscellaneous snowfall statistics for the HMS for the period 1946 through 1998. Included in this table are average number of days per month with snow depth above certain threshold values, greatest number of days per month with snow depth above certain threshold values, record number of consecutive days with snow depth above certain threshold values, record monthly snow depth, and 24-h snowfall amounts. The record snow depth at the HMS is 15.6 in., recorded in December 1985. The record number of days with snow depth $\geq 6$ in. was 43 days in the winter of 1992-1993. 
Table 4.6. Monthly and Seasonal Snowfall (in.), Including First and Last Dates of Both Trace and Measurable Snowfalls

\begin{tabular}{|c|c|c|c|c|c|c|c|c|c|c|c|c|c|c|c|c|}
\hline \multirow[b]{2}{*}{ Season } & \multirow[b]{2}{*}{ Oct } & \multirow[b]{2}{*}{ Nov } & \multirow[b]{2}{*}{ Dec } & \multirow[b]{2}{*}{ Jan } & \multirow[b]{2}{*}{ Feb } & \multirow[b]{2}{*}{ Mar } & \multirow[b]{2}{*}{ Apr } & \multirow[b]{2}{*}{ Total } & \multicolumn{2}{|c|}{ First } & \multicolumn{2}{|c|}{ First Measurable } & \multicolumn{2}{|c|}{ Last Measurable } & \multicolumn{2}{|c|}{ Last } \\
\hline & & & & & & & & & Date & Amount & Date & Amount & Date & Amount & Date & Amount \\
\hline $\begin{array}{l}1945-1946 \\
1946-1947 \\
1947-1948 \\
1948-1949 \\
1949-1950\end{array}$ & $\begin{array}{l}\bar{T} \\
0 \\
0 \\
T\end{array}$ & $\begin{array}{r}7 . \overline{2} \\
\mathbf{T} \\
1.7 \\
0\end{array}$ & $\begin{array}{l}0.5 \\
3.0 \\
8.1 \\
0.7\end{array}$ & $\begin{array}{r}3.3 \\
2.6 \\
1.8 \\
23.4^{(a)}\end{array}$ & $\begin{array}{r}-- \\
T \\
5.5 \\
6.9 \\
3.1\end{array}$ & $\begin{array}{r}\overline{\mathrm{T}} \\
0.1 \\
\mathrm{~T} \\
1.5\end{array}$ & $\begin{array}{c}-- \\
\mathbf{T} \\
0 \\
\mathrm{~T}\end{array}$ & $\begin{array}{l}11.0 \\
11.2 \\
18.5 \\
28.7\end{array}$ & $\begin{array}{c}-- \\
11 / 14 \\
11 / 07 \\
10 / 18\end{array}$ & $\begin{array}{l}- \\
\mathrm{T} \\
\mathrm{T} \\
\mathrm{T}\end{array}$ & $\begin{array}{c}10 \\
11 / 17 \\
12 / 03 \\
11 / 18 \\
12 / 16\end{array}$ & $\begin{array}{l}-\overline{0.2} \\
0.1 \\
0.2 \\
0.1\end{array}$ & $\begin{array}{l}01 / 31 \\
03 / 09 \\
02 / 21 \\
03 / 13\end{array}$ & $\begin{array}{l}0.1 \\
0.2 \\
0.3\end{array}$ & $\begin{array}{l}04 / 07 \\
03 / 15 \\
04 / 02\end{array}$ & $\begin{array}{r}2.2 \\
\mathbf{T} \\
\mathbf{T} \\
\mathbf{T}\end{array}$ \\
\hline $\begin{array}{l}1950-1951 \\
1951-1952 \\
1952-1953 \\
1953-1954 \\
1954-1955\end{array}$ & $\begin{array}{l}0 \\
0 \\
0 \\
0 \\
0\end{array}$ & $\begin{array}{l}0.8 \\
0.5 \\
T \\
0 \\
0\end{array}$ & $\begin{array}{l}2.9 \\
4.4 \\
3.1 \\
1.0 \\
1.8\end{array}$ & $\begin{array}{r}5.3 \\
7.5 \\
2.7 \\
14.3 \\
6.0\end{array}$ & $\begin{array}{r}5.3 \\
3.1 \\
0 \\
1.6 \\
2.4\end{array}$ & $\begin{array}{r}4.2^{(a)} \\
T \\
T \\
T \\
0.7\end{array}$ & $\begin{array}{l}0 \\
0 \\
0 \\
0 \\
T\end{array}$ & $\begin{array}{r}18.5 \\
15.5 \\
5.8 \\
16.9 \\
10.9\end{array}$ & $\begin{array}{l}11 / 19 \\
11 / 22 \\
12 / 03\end{array}$ & $\begin{array}{l}\mathbf{T} \\
\mathbf{T}\end{array}$ & $\begin{array}{l}11 / 30 \\
11 / 25 \\
12 / 01 \\
12 / 08 \\
12 / 04\end{array}$ & $\begin{array}{l}0.8 \\
0.5 \\
0.3 \\
1.0 \\
1.8\end{array}$ & $\begin{array}{l}03 / 12 \\
02 / 24 \\
01 / 02 \\
02 / 11 \\
03 / 25\end{array}$ & $\begin{array}{l}0.1 \\
2.7 \\
1.6 \\
0.7\end{array}$ & $\begin{array}{l}03 / 20 \\
03 / 31 \\
03 / 10 \\
04 / 02\end{array}$ & $\begin{array}{l}1,1 \\
T \\
T \\
T \\
T\end{array}$ \\
\hline $\begin{array}{l}1955-1956 \\
1956-1957 \\
1957-1958 \\
1958-1959 \\
1959-1960\end{array}$ & $\begin{array}{r}0 \\
\mathrm{~T} \\
0.3 \\
0 \\
0\end{array}$ & $\begin{array}{r}12.7 \\
0.1 \\
0 \\
T \\
0.3\end{array}$ & $\begin{array}{r}13.4 \\
2.5 \\
T \\
0.9 \\
1.0\end{array}$ & $\begin{array}{r}10.2 \\
7.9 \\
T \\
4.5 \\
5.9\end{array}$ & $\begin{array}{r}2.2 \\
1.4 \\
0 \\
12.7 \\
T\end{array}$ & $\begin{array}{r}\mathrm{T} \\
4.0 \\
\mathrm{~T} \\
0 \\
1.5\end{array}$ & $\begin{array}{l}\mathbf{0} \\
\mathbf{T} \\
0 \\
0 \\
0\end{array}$ & $\begin{array}{r}38.5 \\
15.9 \\
0.3^{(a)} \\
18.1 \\
8.7\end{array}$ & $\begin{array}{l}10 / 26 \\
11 / 14 \\
11 / 04\end{array}$ & $\begin{array}{l}\mathbf{T} \\
\mathbf{T}\end{array}$ & $\begin{array}{l}11 / 02 \\
11 / 26 \\
10 / 26 \\
12 / 06 \\
11 / 15\end{array}$ & $\begin{array}{l}0.2 \\
0.1 \\
0.3 \\
0.4 \\
0.1\end{array}$ & $\begin{array}{l}02 / 23 \\
03 / 06 \\
10 / 26 \\
02 / 19 \\
03 / 05\end{array}$ & $\begin{array}{l}0.1 \\
1.7 \\
0.3\end{array}$ & $\begin{array}{l}03 / 26 \\
03 / 12 \\
03 / 16\end{array}$ & $\begin{array}{r}\mathrm{T} \\
\mathrm{T} \\
\mathrm{T} \\
1.2 \\
1.4\end{array}$ \\
\hline $\begin{array}{l}1960-1961 \\
1961-1962 \\
1962-1963 \\
1963-1964 \\
1964-1965\end{array}$ & $\begin{array}{l}0 \\
0 \\
0 \\
0 \\
0\end{array}$ & $\begin{array}{r}0 \\
0.5 \\
T \\
T \\
0.1\end{array}$ & $\begin{array}{r}3.3 \\
6.1 \\
T^{(2.0)} \\
6.4 \\
19.1\end{array}$ & $\begin{array}{l}1.9 \\
0.4 \\
7.1 \\
2.9 \\
6.6\end{array}$ & $\begin{array}{r}0 \\
2.4 \\
0.6 \\
\mathrm{~T} \\
\mathrm{~T}\end{array}$ & $\begin{array}{r}1.6 \\
0.9 \\
0 \\
T \\
T\end{array}$ & $\begin{array}{l}0 \\
0 \\
0 \\
\mathrm{~T} \\
0\end{array}$ & $\begin{array}{r}6.8 \\
10.3 \\
7.7 \\
9.3 \\
25.8\end{array}$ & $\begin{array}{l}12 / 09 \\
11 / 18 \\
11 / 29 \\
11 / 19 \\
11 / 21\end{array}$ & $\begin{array}{l}\mathrm{T} \\
\mathrm{T} \\
\mathrm{T} \\
\mathrm{T} \\
\mathrm{T}\end{array}$ & $\begin{array}{l}12 / 10 \\
11 / 23 \\
01 / 30 \\
12 / 08 \\
11 / 29\end{array}$ & $\begin{array}{l}0.1 \\
0.1 \\
0.4 \\
4.3 \\
0.1\end{array}$ & $\begin{array}{l}03 / 05 \\
03 / 09 \\
02 / 01 \\
01 / 24 \\
01 / 23\end{array}$ & $\begin{array}{l}0.1 \\
0.6 \\
1.5 \\
3.1\end{array}$ & $\begin{array}{l}03 / 11 \\
02 / 13 \\
03 / 22 \\
03 / 27\end{array}$ & $\begin{array}{r}1.6 \\
T \\
T \\
T \\
T\end{array}$ \\
\hline $\begin{array}{l}1965-1966 \\
1966-1967 \\
1967-1968 \\
1968-1969 \\
1969-1970\end{array}$ & $\begin{array}{l}0 \\
0 \\
0 \\
0 \\
0\end{array}$ & $\begin{array}{r}\mathrm{T} \\
0.4 \\
0 \\
\mathrm{~T} \\
\mathrm{~T}\end{array}$ & $\begin{array}{l}6.9 \\
2.8 \\
5.7 \\
9.7 \\
2.7\end{array}$ & $\begin{array}{r}2.6 \\
0.1 \\
4.5 \\
15.9 \\
6.6\end{array}$ & $\begin{array}{r}T \\
0 \\
0.3 \\
2.1 \\
T\end{array}$ & $\begin{array}{r}T \\
0 \\
0 \\
0 \\
0.2\end{array}$ & $\begin{array}{l}0 \\
0 \\
T \\
0 \\
0\end{array}$ & $\begin{array}{r}9.5 \\
3.3 \\
10.5 \\
27.7 \\
9.5\end{array}$ & $\begin{array}{l}11 / 23 \\
12 / 06 \\
11 / 16 \\
11 / 29\end{array}$ & $\begin{array}{l}\mathrm{T} \\
\mathrm{T} \\
\mathrm{T}\end{array}$ & $\begin{array}{l}12 / 23 \\
11 / 11 \\
12 / 109 \\
12 / 19 \\
12 / 08\end{array}$ & $\begin{array}{l}0.6 \\
0.2 \\
0.6 \\
0.1 \\
1.3\end{array}$ & $\begin{array}{l}01 / 22 \\
01 / 26 \\
02 / 17 \\
02 / 23 \\
03 / 01\end{array}$ & $\begin{array}{l}0.3 \\
2.0\end{array}$ & $\begin{array}{l}03 / 21 \\
\\
04 / 16 \\
02 / 28\end{array}$ & $\begin{array}{r}T \\
0.1 \\
T \\
T \\
0.2\end{array}$ \\
\hline $\begin{array}{l}1970-1971 \\
1971-1972 \\
1972-1973 \\
1973-1974 \\
1974-1975\end{array}$ & $\begin{array}{r}0 \\
0.6 \\
0 \\
1.5^{(a)} \\
0\end{array}$ & $\begin{array}{r}0.5 \\
T \\
T \\
6.6 \\
0\end{array}$ & $\begin{array}{l}4.4 \\
8.1 \\
7.2 \\
7.5 \\
0.7\end{array}$ & $\begin{array}{l}2.0 \\
4.9 \\
4.3 \\
3.9 \\
2.5\end{array}$ & $\begin{array}{r}\mathrm{T} \\
1.4 \\
1.7 \\
0 \\
12.1\end{array}$ & $\begin{array}{r}0.6 \\
0.1 \\
0 \\
\mathbf{T} \\
\mathbf{T}\end{array}$ & $\begin{array}{l}0 \\
\mathrm{~T} \\
0 \\
0 \\
\mathrm{~T}\end{array}$ & $\begin{array}{r}7.5 \\
15.1 \\
13.2 \\
19.5 \\
15.3\end{array}$ & $\begin{array}{l}11 / 22 \\
11 / 27 \\
12 / 02 \\
12 / 02\end{array}$ & $\begin{array}{l}\mathrm{T} \\
\mathrm{T} \\
\mathrm{T} \\
\mathrm{T}\end{array}$ & $\begin{array}{l}11 / 30 \\
11 / 29 \\
12 / 03 \\
10 / 31 \\
12 / 12\end{array}$ & $\begin{array}{l}0.5 \\
0.1 \\
1.7 \\
1.5 \\
0.3\end{array}$ & $\begin{array}{l}03 / 14 \\
02 / 05 \\
02 / 10 \\
01 / 12 \\
02 / 09\end{array}$ & $\begin{array}{l}0.1 \\
0.1 \\
1.7 \\
2.3 \\
1.7\end{array}$ & $\begin{array}{l}03 / 22 \\
04 / 12 \\
02 / 13 \\
03 / 06 \\
04 / 04\end{array}$ & $\begin{array}{l}T \\
T \\
T \\
T \\
T\end{array}$ \\
\hline $\begin{array}{l}1975-1976 \\
1976-1977 \\
1977-1978 \\
1978-1979 \\
1979-1980\end{array}$ & $\begin{array}{l}0 \\
0 \\
0 \\
0 \\
0\end{array}$ & $\begin{array}{r}1.7 \\
0 \\
2.1 \\
10.1 \\
5.6\end{array}$ & $\begin{array}{l}3.8 \\
0.2 \\
3.4 \\
1.4 \\
7.3\end{array}$ & $\begin{array}{r}6.0 \\
2.9 \\
2.9 \\
10.3 \\
8.7\end{array}$ & $\begin{array}{r}0.2 \\
T \\
0.9 \\
0.5 \\
4.5\end{array}$ & $\begin{array}{r}\mathrm{T} \\
\mathrm{T} \\
\mathrm{T} \\
0.1 \\
0.3\end{array}$ & $\begin{array}{l}\mathrm{T} \\
0 \\
0 \\
0 \\
0\end{array}$ & $\begin{array}{r}11.7 \\
3.1 \\
9.3 \\
22.6 \\
26.2\end{array}$ & $\begin{array}{l}12 / 04 \\
11 / 15 \\
11 / 15\end{array}$ & $\begin{array}{l}\mathrm{T} \\
\mathrm{T} \\
\mathrm{T}\end{array}$ & $\begin{array}{l}11 / 10 \\
12 / 23 \\
11 / 18 \\
11 / 18 \\
11 / 22\end{array}$ & $\begin{array}{l}0.6 \\
0.2 \\
0.1 \\
5.3 \\
1.4\end{array}$ & $\begin{array}{l}02 / 03 \\
01 / 31 \\
02 / 26 \\
03 / 03 \\
03 / 05\end{array}$ & $\begin{array}{l}0.2 \\
0.2 \\
0.1 \\
0.1 \\
0.3\end{array}$ & $\begin{array}{l}04 / 01 \\
03 / 27 \\
03 / 05\end{array}$ & $\begin{array}{l}T \\
T \\
T\end{array}$ \\
\hline $\begin{array}{l}1980-1981 \\
1981-1982 \\
1982-1983 \\
1983-1984 \\
1984-1985\end{array}$ & $\begin{array}{l}0 \\
0 \\
0 \\
0 \\
\mathbf{T}\end{array}$ & $\begin{array}{r}0.3 \\
0 \\
0.2 \\
T \\
4.9\end{array}$ & $\begin{array}{r}2.2 \\
12.1 \\
4.6 \\
17.8 \\
5.8\end{array}$ & $\begin{array}{r}T \\
2.4 \\
3.2 \\
1.5 \\
1.3\end{array}$ & $\begin{array}{r}T \\
T \\
2.3 \\
T \\
8.5\end{array}$ & $\begin{array}{r}0 \\
\mathrm{~T} \\
0 \\
0 \\
1.4\end{array}$ & $\begin{array}{r}0 \\
1.0^{(3)} \\
0 \\
0 \\
0\end{array}$ & $\begin{array}{r}2.5 \\
15.5 \\
10.3 \\
19.3 \\
21.9\end{array}$ & $\begin{array}{l}12 / 03 \\
11 / 12 \\
11 / 28 \\
10 / 23\end{array}$ & $\begin{array}{l}\mathrm{T} \\
\mathrm{T} \\
\mathrm{T} \\
\mathrm{T}\end{array}$ & $\begin{array}{l}11 / 14 \\
12 / 13 \\
11 / 26 \\
12 / 02 \\
11 / 24\end{array}$ & $\begin{array}{l}0.3 \\
2.5 \\
0.2 \\
0.5 \\
0.2\end{array}$ & $\begin{array}{l}12 / 06 \\
04 / 06 \\
02 / 09 \\
01 / 21 \\
03 / 04\end{array}$ & $\begin{array}{l}0.3 \\
1.0 \\
0.3 \\
1.5 \\
1.4\end{array}$ & $02 / 13$ & $\mathrm{~T}$ \\
\hline
\end{tabular}


Table 4.6. (contd)

\begin{tabular}{|c|c|c|c|c|c|c|c|c|c|c|c|c|c|c|c|c|}
\hline \multirow[b]{2}{*}{ Season } & \multirow[b]{2}{*}{ Oct } & \multirow[b]{2}{*}{ Nov } & \multirow[b]{2}{*}{ Dec } & \multirow[b]{2}{*}{ Jan } & \multirow[b]{2}{*}{ Feb } & \multirow[b]{2}{*}{ Mar } & \multirow[b]{2}{*}{ Apr } & \multirow[b]{2}{*}{ Total } & \multicolumn{2}{|c|}{ First } & \multicolumn{2}{|c|}{ First Measurable } & \multicolumn{2}{|c|}{ Last Measurable } & \multicolumn{2}{|c|}{ Last } \\
\hline & & & & & & & & & Date & Amount & Date & Amount & Date & Amount & Date & Amount \\
\hline $\begin{array}{l}1985-1986 \\
1986-1987 \\
1987-1988 \\
1988-1989 \\
1989-1990\end{array}$ & $\begin{array}{l}0 \\
0 \\
0 \\
0 \\
0\end{array}$ & $\begin{array}{r}18.3^{(\mathbf{a})} \\
0 \\
1.1 \\
0 \\
0\end{array}$ & $\begin{array}{l}7.6 \\
5.1 \\
4.7 \\
3.5 \\
1.4\end{array}$ & $\begin{array}{l}2.7 \\
3.3 \\
5.6 \\
0.2 \\
0.6\end{array}$ & $\begin{array}{r}5.5 \\
0 \\
0 \\
17.0^{(a)} \\
0.7\end{array}$ & $\begin{array}{r}0 \\
0 \\
0 \\
3.1 \\
T\end{array}$ & $\begin{array}{r}0 \\
0 \\
0.2 \\
T \\
0\end{array}$ & $\begin{array}{r}34.1 \\
8.4 \\
11.6 \\
23.8 \\
2.7\end{array}$ & $12 / 25$ & $\mathrm{~T}$ & $\begin{array}{l}11 / 10 \\
12 / 04 \\
11 / 30 \\
12 / 18 \\
12 / 26\end{array}$ & $\begin{array}{l}0.6 \\
0.4 \\
1.1 \\
0.3 \\
0.3\end{array}$ & $\begin{array}{l}02 / 21 \\
01 / 26 \\
01 / 20 \\
03 / 05 \\
02 / 17\end{array}$ & $\begin{array}{l}0.9 \\
0.1 \\
0.1 \\
0.2 \\
0.2\end{array}$ & $\begin{array}{l}01 / 31 \\
05 / 18\end{array}$ & $\begin{array}{l}\mathrm{T} \\
\mathrm{T}\end{array}$ \\
\hline $\begin{array}{l}1990-1991 \\
1991-1992 \\
1992-1993 \\
1993-1994 \\
1994-1995\end{array}$ & $\begin{array}{r}0 \\
1.2 \\
0 \\
0 \\
0\end{array}$ & $\begin{array}{r}0 \\
\mathrm{~T} \\
2.1 \\
1.4 \\
0.1\end{array}$ & $\begin{array}{r}6.1 \\
0.6 \\
21.0 \\
1.8 \\
4.2\end{array}$ & $\begin{array}{r}3.8 \\
0.3 \\
17.1 \\
0^{(\text {a) }} \\
2.7\end{array}$ & $\begin{array}{r}0^{(2, b)} \\
\mathrm{T} \\
12.4 \\
0.9 \\
\mathrm{~T}\end{array}$ & $\begin{array}{r}0.1 \\
0 \\
3.5 \\
0 \\
0^{(a, 0)}\end{array}$ & $\begin{array}{l}0 \\
0 \\
0 \\
0 \\
\mathbf{T}\end{array}$ & $\begin{array}{r}10.0 \\
2.1 \\
56.1^{(a)} \\
4.1 \\
7.0\end{array}$ & & & $\begin{array}{l}12 / 18 \\
10 / 28 \\
11 / 21 \\
11 / 22 \\
11 / 17\end{array}$ & $\begin{array}{l}0.1 \\
0.8 \\
0.2 \\
0.6 \\
0.1\end{array}$ & $\begin{array}{l}03 / 02 \\
01 / 05 \\
03 / 03 \\
02 / 26 \\
12 / 14\end{array}$ & $\begin{array}{l}0.1 \\
0.3 \\
1.5 \\
0.3 \\
0.7\end{array}$ & $\begin{array}{l}02 / 07 \\
03 / 16 \\
04 / 14\end{array}$ & $\begin{array}{l}\mathbf{T} \\
\mathbf{T}\end{array}$ \\
\hline $\begin{array}{l}1995-1996 \\
1996-1997 \\
1997-1998 \\
1998-\end{array}$ & $\begin{array}{r}0 \\
0 \\
0 \\
0^{(n, 0)}\end{array}$ & $\begin{array}{r}1.0 \\
11.9 \\
0 \\
0^{(2, b)}\end{array}$ & $\begin{array}{r}4.0 \\
22.6^{\text {(a) }} \\
1.8 \\
0.9\end{array}$ & $\begin{array}{r}16.7 \\
1.8 \\
6.3 \\
-\end{array}$ & $\begin{array}{r}5.9 \\
2.7 \\
\text { T } \\
-\end{array}$ & $\begin{array}{r}0.4 \\
1.5 \\
T \\
.-\end{array}$ & $\begin{array}{r}0 \\
0 \\
0 \\
0(x, b) \\
--\end{array}$ & $\begin{array}{r}28.0 \\
40.5 \\
8.1 \\
-.\end{array}$ & $12 / 05$ & $\mathrm{~T}$ & $\begin{array}{l}11 / 10 \\
11 / 19 \\
12 / 07 \\
12 / 24\end{array}$ & $\begin{array}{l}1.0 \\
6.2 \\
1.8 \\
0.9\end{array}$ & $\begin{array}{l}03 / 04 \\
03 / 15 \\
01 / 21\end{array}$ & $\begin{array}{l}0.4 \\
1.5 \\
0.2\end{array}$ & $\begin{array}{l}03 / 05 \\
03 / 31 \\
03 / 05\end{array}$ & $\begin{array}{l}\mathbf{T} \\
\mathbf{T} \\
\mathbf{T}\end{array}$ \\
\hline $\begin{array}{l}\text { Average } \\
\text { Normal }\end{array}$ & $\begin{array}{l}0.1 \\
0.1\end{array}$ & $\begin{array}{l}1.7 \\
1.7\end{array}$ & $\begin{array}{l}5.2 \\
5.7\end{array}$ & $\begin{array}{l}5.0 \\
3.9\end{array}$ & $\begin{array}{l}2.4 \\
2.0\end{array}$ & $\begin{array}{l}0.5 \\
0.3\end{array}$ & $\begin{array}{l}T \\
T\end{array}$ & $\begin{array}{l}15.2 \\
13.8\end{array}$ & $\begin{array}{l}11 / 21 \\
11 / 24\end{array}$ & & $\begin{array}{l}11 / 30 \\
12 / 03\end{array}$ & & $\begin{array}{l}02 / 11 \\
02 / 12\end{array}$ & & $\begin{array}{l}03 / 16 \\
03 / 08\end{array}$ & \\
\hline
\end{tabular}

(a) Greatest and least values.

(b) Most recent of multiple occurrences

$\mathrm{T}=$ Trace. 
Table 4.7. Snowfall (in.) - Greatest Amount from a Single Storm

\begin{tabular}{|c|c|c|c|c|c|c|c|c|c|c|c|c|c|}
\hline $\begin{array}{l}\text { Year } \\
1946-47 \\
1947-48 \\
1948-49 \\
1949-50\end{array}$ & $\begin{array}{l}\text { Jul } \\
0 \\
0 \\
0 \\
0\end{array}$ & $\begin{array}{l}\text { Aug } \\
0 \\
0 \\
0 \\
0\end{array}$ & $\begin{array}{l}\text { Sep } \\
0 \\
0 \\
0 \\
0\end{array}$ & $\begin{array}{l}\text { Oct } \\
T \\
0 \\
0 \\
T\end{array}$ & $\begin{array}{l}\text { Nov } \\
4.5 \\
T \\
1.7 \\
0\end{array}$ & $\begin{array}{l}\text { Dec } \\
0.3 \\
1.8 \\
1.9 \\
0.4\end{array}$ & $\begin{array}{l}\operatorname{Jan} \\
2.2 \\
2.6 \\
0.8 \\
5.1\end{array}$ & $\begin{array}{l}\text { Feb } \\
\text { T } \\
5.2 \\
4.4 \\
2.2\end{array}$ & $\begin{array}{l}\text { Mar } \\
T \\
0.1 \\
T \\
1.2\end{array}$ & $\begin{array}{l}\text { Apr } \\
0 \\
T \\
0 \\
T\end{array}$ & $\begin{array}{l}\text { May } \\
0 \\
0 \\
0 \\
0\end{array}$ & $\begin{array}{l}\text { Jun } \\
0 \\
0 \\
0 \\
0\end{array}$ & $\begin{array}{l}\text { Season } \\
4.5 \\
5.2 \\
4.4 \\
5.1\end{array}$ \\
\hline $\begin{array}{l}1950-51 \\
1951-52 \\
1952-53 \\
1953-54 \\
1954-55\end{array}$ & $\begin{array}{l}0 \\
0 \\
0 \\
0 \\
0\end{array}$ & $\begin{array}{l}0 \\
0 \\
0 \\
0 \\
0\end{array}$ & $\begin{array}{l}0 \\
0 \\
0 \\
0 \\
0\end{array}$ & $\begin{array}{l}0 \\
0 \\
0 \\
0 \\
0\end{array}$ & $\begin{array}{l}0.8 \\
0.5 \\
T \\
0 \\
0\end{array}$ & $\begin{array}{l}2.1 \\
2.1 \\
1.1 \\
1.0 \\
1.8\end{array}$ & $\begin{array}{l}2.3 \\
3.0 \\
2.7 \\
9.6^{(a)} \\
1.7\end{array}$ & $\begin{array}{l}3.5 \\
2.5 \\
0 \\
1.6 \\
2.2\end{array}$ & $\begin{array}{l}2.2 \\
T \\
T \\
T \\
0.7\end{array}$ & $\begin{array}{l}0 \\
0 \\
0 \\
0 \\
\mathbf{T}\end{array}$ & $\begin{array}{l}0 \\
0 \\
0 \\
0 \\
T\end{array}$ & $\begin{array}{l}0 \\
0 \\
0 \\
0 \\
0\end{array}$ & $\begin{array}{l}3.5 \\
3.0 \\
2.7 \\
9.6 \\
2.2\end{array}$ \\
\hline $\begin{array}{l}1955-56 \\
1956-57 \\
1957-58 \\
1958-59 \\
1959-60\end{array}$ & $\begin{array}{l}0 \\
0 \\
0 \\
0 \\
0\end{array}$ & $\begin{array}{l}0 \\
0 \\
0 \\
0 \\
0\end{array}$ & $\begin{array}{l}0 \\
0 \\
0 \\
0 \\
0\end{array}$ & $\begin{array}{l}0 \\
T \\
0.3 \\
0 \\
0\end{array}$ & $\begin{array}{l}4.8 \\
0.1 \\
0 \\
T \\
0.2\end{array}$ & $\begin{array}{l}3.4 \\
2.4 \\
T \\
0.4 \\
0.6\end{array}$ & $\begin{array}{l}4.4 \\
3.5 \\
T \\
2.7 \\
3.6\end{array}$ & $\begin{array}{l}0.6 \\
1.2 \\
0 \\
5.9 \\
T\end{array}$ & $\begin{array}{l}T \\
2.2 \\
T \\
0 \\
1.5\end{array}$ & $\begin{array}{l}0 \\
0 \\
0 \\
0 \\
0\end{array}$ & $\begin{array}{l}0 \\
0 \\
0 \\
0 \\
\mathrm{~T}\end{array}$ & $\begin{array}{l}0 \\
0 \\
0 \\
0 \\
0\end{array}$ & $\begin{array}{l}4.8 \\
3.5 \\
0.3^{(n)} \\
5.9 \\
3.6\end{array}$ \\
\hline $\begin{array}{l}1960-61 \\
1961-62 \\
1962-63 \\
1963-64 \\
1964-65\end{array}$ & $\begin{array}{l}0 \\
0 \\
0 \\
0 \\
0\end{array}$ & $\begin{array}{l}0 \\
0 \\
0 \\
0 \\
0\end{array}$ & $\begin{array}{l}0 \\
0 \\
0 \\
0 \\
0\end{array}$ & $\begin{array}{l}0 \\
0 \\
0 \\
0 \\
0\end{array}$ & $\begin{array}{l}0 \\
0.4 \\
0 \\
\mathrm{~T} \\
0.1\end{array}$ & $\begin{array}{l}2.0 \\
3.0 \\
T \\
4.3 \\
5.3\end{array}$ & $\begin{array}{l}1.9 \\
0.3 \\
7.1 \\
1.5 \\
3.2\end{array}$ & $\begin{array}{l}0 \\
2.0 \\
0.6 \\
T \\
T\end{array}$ & $\begin{array}{l}1.6 \\
0.8 \\
0 \\
\mathrm{~T} \\
\mathrm{~T}\end{array}$ & $\begin{array}{l}0 \\
0 \\
0 \\
T \\
0\end{array}$ & $\begin{array}{l}0 \\
0 \\
0 \\
\mathrm{~T} \\
0\end{array}$ & $\begin{array}{l}0 \\
0 \\
0 \\
0 \\
0\end{array}$ & $\begin{array}{l}2.0 \\
3.0 \\
7.1 \\
4.3 \\
5.3\end{array}$ \\
\hline $\begin{array}{l}1965-66 \\
1966-67 \\
1967-68 \\
1968-69 \\
1969-70\end{array}$ & $\begin{array}{l}0 \\
0 \\
0 \\
0 \\
0\end{array}$ & $\begin{array}{l}0 \\
0 \\
0 \\
0 \\
0\end{array}$ & $\begin{array}{l}0 \\
0 \\
0 \\
0 \\
0\end{array}$ & $\begin{array}{l}0 \\
0 \\
0 \\
0 \\
0\end{array}$ & $\begin{array}{l}T \\
0.4 \\
0 \\
T \\
T\end{array}$ & $\begin{array}{l}5.4 \\
0.3 \\
3.3 \\
3.6 \\
1.3\end{array}$ & $\begin{array}{l}1.9 \\
0.1 \\
2.9 \\
6.4 \\
3.0\end{array}$ & $\begin{array}{l}T \\
0 \\
0.3 \\
2.0 \\
T\end{array}$ & $\begin{array}{l}T \\
T \\
0 \\
0 \\
0.2\end{array}$ & $\begin{array}{l}0 \\
0 \\
\mathrm{~T} \\
0 \\
0\end{array}$ & $\begin{array}{l}0 \\
0 \\
0 \\
0 \\
0\end{array}$ & $\begin{array}{l}0 \\
0 \\
0 \\
0 \\
0\end{array}$ & $\begin{array}{l}5.4 \\
0.4 \\
3.3 \\
6.4 \\
3.0\end{array}$ \\
\hline $\begin{array}{l}1970-71 \\
1971-72 \\
1972-73 \\
1973-74 \\
1974-75\end{array}$ & $\begin{array}{l}0 \\
0 \\
0 \\
0 \\
0\end{array}$ & $\begin{array}{l}0 \\
0 \\
0 \\
0 \\
0\end{array}$ & $\begin{array}{l}0 \\
0 \\
0 \\
0 \\
0\end{array}$ & $\begin{array}{l}0 \\
0.6 \\
0 \\
1.5^{(u)} \\
0\end{array}$ & $\begin{array}{l}0.5 \\
\mathbf{T} \\
\mathbf{T} \\
3.9 \\
0\end{array}$ & $\begin{array}{l}3.1 \\
3.4 \\
4.0 \\
5.8 \\
0.4\end{array}$ & $\begin{array}{l}1.8 \\
3.9 \\
2.8 \\
2.3 \\
0.9\end{array}$ & $\begin{array}{l}T \\
1.3 \\
1.7 \\
0 \\
5.6\end{array}$ & $\begin{array}{l}0.5 \\
0.1 \\
0 \\
0 \\
\mathrm{~T}\end{array}$ & $\begin{array}{l}0 \\
T \\
0 \\
0 \\
T\end{array}$ & $\begin{array}{l}0 \\
0 \\
0 \\
0 \\
0\end{array}$ & $\begin{array}{l}0 \\
0 \\
0 \\
0 \\
0\end{array}$ & $\begin{array}{l}3.1 \\
3.9 \\
4.0 \\
5.8 \\
5.6\end{array}$ \\
\hline $\begin{array}{l}1975-76 \\
1976-77 \\
1977-78 \\
1978-79 \\
1979-80\end{array}$ & $\begin{array}{l}0 \\
0 \\
0 \\
0 \\
0\end{array}$ & $\begin{array}{l}0 \\
0 \\
0 \\
0 \\
0\end{array}$ & $\begin{array}{l}0 \\
0 \\
0 \\
0 \\
0\end{array}$ & $\begin{array}{l}0 \\
0 \\
0 \\
0 \\
0\end{array}$ & $\begin{array}{l}1.7 \\
0 \\
1.8 \\
9.1^{( \pm)} \\
3.4\end{array}$ & $\begin{array}{l}3.1 \\
0.2 \\
2.5 \\
1.0 \\
3.6\end{array}$ & $\begin{array}{l}2.4 \\
1.8 \\
2.2 \\
5.0 \\
6.4\end{array}$ & $\begin{array}{l}0.2 \\
T \\
0.8 \\
0.3 \\
4.5\end{array}$ & $\begin{array}{l}T \\
T \\
T \\
0 \\
0.3\end{array}$ & $\begin{array}{l}0 \\
0 \\
0 \\
0 \\
0\end{array}$ & $\begin{array}{l}0 \\
0 \\
0 \\
0 \\
0\end{array}$ & $\begin{array}{l}0 \\
0 \\
0 \\
0 \\
0\end{array}$ & $\begin{array}{l}3.1 \\
1.8 \\
2.5 \\
9.1 \\
6.4\end{array}$ \\
\hline $\begin{array}{l}1980-81 \\
1981-82 \\
1982-83 \\
1983-84 \\
1984-85\end{array}$ & $\begin{array}{l}0 \\
0 \\
0 \\
0 \\
0\end{array}$ & $\begin{array}{l}0 \\
0 \\
0 \\
0 \\
0\end{array}$ & $\begin{array}{l}0 \\
0 \\
0 \\
0 \\
0\end{array}$ & $\begin{array}{l}0 \\
0 \\
0 \\
0 \\
T\end{array}$ & $\begin{array}{l}0.3 \\
0 \\
0.2 \\
T \\
4.7\end{array}$ & $\begin{array}{l}1.9 \\
3.8 \\
2.6 \\
5.1 \\
2.4\end{array}$ & $\begin{array}{l}T \\
1.0 \\
2.0 \\
1.5 \\
1.3\end{array}$ & $\begin{array}{l}T \\
T \\
2.0 \\
T \\
2.9\end{array}$ & $\begin{array}{l}0 \\
\mathrm{~T} \\
0 \\
0 \\
0\end{array}$ & $\begin{array}{l}0 \\
1.0^{(n)} \\
0 \\
0 \\
0\end{array}$ & $\begin{array}{l}0 \\
0 \\
T \\
0 \\
0\end{array}$ & $\begin{array}{l}0 \\
0 \\
0 \\
0 \\
0\end{array}$ & $\begin{array}{l}1.9 \\
3.8 \\
2.6 \\
5.1 \\
4.7\end{array}$ \\
\hline $\begin{array}{l}1985-86 \\
1986-87 \\
1987-88 \\
1988-89 \\
1989-90\end{array}$ & $\begin{array}{l}0 \\
0 \\
0 \\
0 \\
0\end{array}$ & $\begin{array}{l}0 \\
0 \\
0 \\
0 \\
0\end{array}$ & $\begin{array}{l}0 \\
0 \\
0 \\
0 \\
0\end{array}$ & $\begin{array}{l}0 \\
0 \\
0 \\
0 \\
0\end{array}$ & $\begin{array}{l}8.8 \\
0 \\
1.1 \\
0 \\
0\end{array}$ & $\begin{array}{l}6.6^{(n)} \\
2.1 \\
4.4 \\
1.7 \\
1.1\end{array}$ & $\begin{array}{l}1.1 \\
0.8 \\
2.3 \\
0.2 \\
0.6\end{array}$ & $\begin{array}{l}2.7 \\
0 \\
0 \\
10.0 \\
0.7\end{array}$ & $\begin{array}{l}0 \\
0 \\
0 \\
2.7^{(a)} \\
\mathrm{T}\end{array}$ & $\begin{array}{l}0 \\
0 \\
0 \\
T \\
0\end{array}$ & $\begin{array}{l}0 \\
0 \\
0 \\
T^{(2 b)} \\
0\end{array}$ & $\begin{array}{l}0 \\
0 \\
0 \\
0 \\
0\end{array}$ & $\begin{array}{l}8.8 \\
2.1 \\
4.4 \\
10.0 \\
1.1\end{array}$ \\
\hline $\begin{array}{l}1990-91 \\
1991-92 \\
1992-93 \\
1993-94 \\
1994-95\end{array}$ & $\begin{array}{l}0 \\
0 \\
0 \\
0 \\
0\end{array}$ & $\begin{array}{l}0 \\
0 \\
0 \\
0 \\
0\end{array}$ & $\begin{array}{l}0 \\
0 \\
0 \\
0 \\
0\end{array}$ & $\begin{array}{l}0 \\
0.9 \\
0 \\
0 \\
0\end{array}$ & $\begin{array}{l}0 \\
T \\
1.6 \\
0.6 \\
0.1\end{array}$ & $\begin{array}{l}2.8 \\
0.6 \\
3.8 \\
1.0 \\
1.7\end{array}$ & $\begin{array}{l}2.1 \\
0.3 \\
\mathrm{~T} \\
0 \\
1.9\end{array}$ & $\begin{array}{l}0 \\
T \\
12.4^{(a)} \\
0.3 \\
T\end{array}$ & $\begin{array}{l}0.1 \\
0 \\
2.0 \\
0 \\
0\end{array}$ & $\begin{array}{l}0 \\
0 \\
0 \\
0 \\
T\end{array}$ & $\begin{array}{l}0 \\
0 \\
0 \\
0 \\
0\end{array}$ & $\begin{array}{l}0 \\
0 \\
0 \\
0 \\
0\end{array}$ & $\begin{array}{l}2.8 \\
0.9 \\
12.4^{(n)} \\
1.0 \\
1.9\end{array}$ \\
\hline $\begin{array}{l}1995-96 \\
1996-97 \\
1997-98 \\
1998-99\end{array}$ & $\begin{array}{l}0 \\
0 \\
0 \\
0\end{array}$ & $\begin{array}{l}0 \\
0 \\
0 \\
0\end{array}$ & $\begin{array}{l}0 \\
0 \\
0 \\
0\end{array}$ & $\begin{array}{l}0 \\
0 \\
0 \\
0\end{array}$ & $\begin{array}{l}1.0 \\
7.1 \\
0 \\
0\end{array}$ & $\begin{array}{l}2.7 \\
6.0 \\
1.8 \\
0.9\end{array}$ & $\begin{array}{l}3.5 \\
0.9 \\
3.3 \\
-\end{array}$ & $\begin{array}{l}4.0 \\
2.7 \\
T \\
-\end{array}$ & $\begin{array}{l}0.4 \\
1.5 \\
T \\
-\end{array}$ & $\begin{array}{l}0 \\
0 \\
0 \\
-\end{array}$ & $\begin{array}{l}0 \\
0 \\
0 \\
-\end{array}$ & $\begin{array}{l}0 \\
0 \\
0 \\
-\end{array}$ & $\begin{array}{l}4.0 \\
7.1 \\
3.3 \\
-\end{array}$ \\
\hline
\end{tabular}

(a) Greatest value.

(b) Most recent of multiple occurrences.

$\mathrm{T}=$ Trace 
Table 4.8. Miscellaneous Snowfall Statistics, 1946 Through 1998

\begin{tabular}{|c|c|c|c|c|c|c|}
\hline Oct & Nov & Dec & Jan & Feb & Mar & Season \\
\hline & & & & & & \\
\hline (a) & 1 & 7 & 9 & 4 & (a) & 22 \\
\hline 0 & 1 & 3 & 6 & 3 & (a) & 12 \\
\hline 0 & (a) & 1 & 3 & 1 & (a) & 5 \\
\hline 0 & 0 & (a) & (a) & 0 & 0 & (a) \\
\hline
\end{tabular}

Average Number

of Days of Given

Depth at 0400 PST

$\geq 1$ in.

$\geq 3$ in.

26 in.

$\geq 12$ in.

Record Greatest

Number of Days

of Given Depth

at 0400 PST

$\geq 1$ in

$0 \quad 12(1996)^{(\text {b) }} \quad 31(1985) \quad 31(1969) \quad 20(1989) \quad 7(1993) \quad 72(1992-93)$

$\geq 3$ in.

$0 \quad 12(1996)$

31 (1985)

27 (1993)

$16(1950)$

6 (1993)

$58(1985-86)$

26 in.

$0 \quad 9(1985)$

23 (1985) 25 (1993)

9 (1993)

5 (1993)

43 (1992-93)

$\geq 12$ in.

0

Record Greatest

$1.5(1973)$

4 (1964) 10 (1993)

$10(1992-93)$

Depth

Greatest in

1.5 (1973)

$8.8(1985)$

$6.6(1985)$

7.1 (1954)

$10.2(1993) \quad 2.7(1989)$

10.2 (Feb 1993)

$24 \mathrm{~h}$

Record Consecutive

Number of Days of

Given Depth at

Number

0400 PST

$\frac{\text { of Days }}{60}$

$\frac{\text { From }}{\text { November 20, } 1985}$

To

$\geq 1$ in.

57

November 22, 1985

January 18, 1986

$\geq 3$ in.

$\geq 6$ in.

32

December 20, 1964

January 17, 1986

212 in.

6

January 15, 1993

January 20, 1965

January 20, 1993

(a) Denotes $<1 / 2$ day.

(b) Year of occurrence in parentheses.

PST = Pacific Standard Time. 


\subsection{Normal and Maximum Daily Precipitation}

Table 4.9 contains annual maximum precipitation statistics for the time periods $1,2,3,6,12$, and $24 \mathrm{~h}$, including the dates of occurrence for each time period, for 1947 through 1998 . Table 4.10 contains normal and maximum values of precipitation (minimum values are not needed because every day of the year has a minimum value of 0 ). The normal precipitation values are based on the period 1961 through 1990; the daily maximum values are for the entire period of record (1945 through 1998). The maximum daily value for each month is noted on the table.

Climatologically speaking, the wettest period of the year is from December 14 through 24 , with each day having a normal precipitation value of 0.04 in.; the driest period is from July 23 through August 11, with each day having a normal value of only a trace. October 1,1957 , recorded the greatest 1 -day precipitation, $1.60 \mathrm{in}$. There have been only 5 days during the period of record that have never received measurable precipitation (all have received a trace however). 
Table 4.9. Maximum Precipitation (in.)

\begin{tabular}{|c|c|c|c|c|c|c|c|c|c|c|c|c|}
\hline Year & $1 \mathrm{~h}$ & Date & $2 \mathrm{~h}$ & Date & $3 \mathrm{~h}$ & Date & $6 \mathrm{~h}$ & Date & $12 \mathrm{~h}$ & Date & $24 \mathrm{~h}$ & Date \\
\hline $\begin{array}{l}1947 \\
1948 \\
1949 \\
1950\end{array}$ & $\begin{array}{l}0.48 \\
0.24 \\
0.18 \\
0.30\end{array}$ & $\begin{array}{l}08-29 \\
05-19 \\
11-23 \\
06-17\end{array}$ & $\begin{array}{l}0.51 \\
0.30 \\
0.28 \\
0.52\end{array}$ & $\begin{array}{l}06-07 \\
06-11 \\
11-23 \\
06-17\end{array}$ & $\begin{array}{l}0.54 \\
0.31 \\
0.41 \\
0.58\end{array}$ & $\begin{array}{l}06-07 \\
06 / 10-11 \\
11-23 \\
06-17\end{array}$ & $\begin{array}{l}0.67 \\
0.50 \\
0.60 \\
0.87\end{array}$ & $\begin{array}{l}09-15 \\
01 / 6-7 \\
11-23 \\
06-17\end{array}$ & $\begin{array}{l}0.75 \\
0.65 \\
0.63 \\
1.05\end{array}$ & $\begin{array}{l}09 / 16-17 \\
01-06 \\
11-23 \\
06-17\end{array}$ & $\begin{array}{l}0.88 \\
1.08 \\
0.65 \\
1.24\end{array}$ & $\begin{array}{l}10 / 19-20 \\
01 / 06-07 \\
11 / 23-24 \\
06 / 16-17\end{array}$ \\
\hline $\begin{array}{l}1951 \\
1952 \\
1953 \\
1954 \\
1955\end{array}$ & $\begin{array}{l}0.28 \\
0.27 \\
0.35 \\
0.16 \\
0.13\end{array}$ & $\begin{array}{l}04-28 \\
05-10 \\
08-26 \\
03-19 \\
12-31\end{array}$ & $\begin{array}{l}0.41 \\
0.27 \\
0.35 \\
0.19 \\
0.21\end{array}$ & $\begin{array}{l}04-28 \\
05-10 \\
08-26 \\
05-26 \\
12-21\end{array}$ & $\begin{array}{l}0.44 \\
0.27 \\
0.35 \\
0.27 \\
0.31\end{array}$ & $\begin{array}{l}04-28 \\
05-10 \\
08-26 \\
01-16 \\
12-21\end{array}$ & $\begin{array}{l}0.45 \\
0.29 \\
0.44 \\
0.52 \\
0.49\end{array}$ & $\begin{array}{l}06-06 \\
06-29 \\
01 / 08-09 \\
01-16 \\
12-21\end{array}$ & $\begin{array}{l}0.47 \\
0.39 \\
0.77 \\
0.72 \\
0.61\end{array}$ & $\begin{array}{l}06-06 \\
06-29 \\
01 / 08-09 \\
01 / 15-16 \\
12-21\end{array}$ & $\begin{array}{l}0.70 \\
0.48 \\
0.83 \\
0.77 \\
0.64\end{array}$ & $\begin{array}{l}06 / 05-06 \\
06-29 \\
01 / 08-09 \\
01 / 15-16 \\
11 / 26-27\end{array}$ \\
\hline $\begin{array}{l}1956 \\
1957 \\
1958 \\
1959 \\
1960\end{array}$ & $\begin{array}{l}0.16 \\
0.47 \\
0.43 \\
0.18 \\
0.22\end{array}$ & $\begin{array}{l}06-14 \\
10-01 \\
06-12 \\
05-17 \\
03-27\end{array}$ & $\begin{array}{c}0.22 \\
0.88^{(4)} \\
0.43 \\
0.18 \\
0.23\end{array}$ & $\begin{array}{l}06-14 \\
10-01 \\
06-12 \\
05-17^{(0)} \\
03-27\end{array}$ & $\begin{array}{c}0.27 \\
1.08^{\text {(a) }} \\
0.43 \\
0.23 \\
0.33\end{array}$ & $\begin{array}{l}06-14 \\
10-01 \\
06-12 \\
09-14^{(0)} \\
05 / 06-07\end{array}$ & $\begin{array}{c}0.28^{(4)} \\
1.68^{(4)} \\
0.65 \\
0.40 \\
0.43\end{array}$ & $\begin{array}{l}01-15 \\
10 / 01-02 \\
12 / 10-11 \\
01-11 \\
05 / 06-07\end{array}$ & $\begin{array}{c}0.44 \\
1.88^{(n)} \\
0.88 \\
0.54 \\
0.44\end{array}$ & $\begin{array}{l}01 / 14-15 \\
10 / 01-02 \\
12 / 10-11 \\
01 / 11-12 \\
05 / 06-07\end{array}$ & $\begin{array}{c}0.73 \\
1.91^{(4)} \\
1.00 \\
0.82 \\
0.44\end{array}$ & $\begin{array}{l}01 / 14-15 \\
10 / 01-02 \\
12 / 10-11 \\
01 / 11-12 \\
05 / 06-07\end{array}$ \\
\hline $\begin{array}{l}1961 \\
1962 \\
1963 \\
1964 \\
1965\end{array}$ & $\begin{array}{l}0.21 \\
0.19 \\
0.22 \\
0.16 \\
0.10^{(2)}\end{array}$ & $\begin{array}{l}02-01 \\
11-30 \\
01-31 \\
12-22 \\
05-19\end{array}$ & $\begin{array}{c}0.39 \\
0.27 \\
0.37 \\
0.20 \\
0.14^{(1) 1)}\end{array}$ & $\begin{array}{l}02-01 \\
11-30 \\
01-31 \\
06-08 \\
11-24^{(0)}\end{array}$ & $\begin{array}{c}0.42 \\
0.34 \\
0.44 \\
0.32 \\
0.18^{(a)}\end{array}$ & $\begin{array}{l}02-01 \\
02-09 \\
01-31 \\
12-21 \\
06-17\end{array}$ & $\begin{array}{l}0.46 \\
0.40 \\
0.54 \\
0.42 \\
0.29\end{array}$ & $\begin{array}{l}05 / 09-10 \\
10-12 \\
01-31 \\
12-21 \\
06-17\end{array}$ & $\begin{array}{l}0.72 \\
0.52 \\
0.94 \\
0.54 \\
0.39\end{array}$ & $\begin{array}{l}02-01 \\
10-12 \\
01-31 / 02-01 \\
12-21 \\
06-17\end{array}$ & $\begin{array}{l}0.72 \\
0.52 \\
0.98 \\
0.60 \\
0.48\end{array}$ & $\begin{array}{l}02-01 \\
10-12 \\
01-31 / 02-01 \\
12 / 21-22 \\
06-17\end{array}$ \\
\hline $\begin{array}{l}1966 \\
1967 \\
1968 \\
1969 \\
1970\end{array}$ & $\begin{array}{l}0.14 \\
0.15 \\
0.12 \\
0.55^{(2)} \\
0.15\end{array}$ & $\begin{array}{l}07-02 \\
04-18 \\
12-24 \\
06-12 \\
05-12\end{array}$ & $\begin{array}{l}0.17 \\
0.26 \\
0.21 \\
0.59 \\
0.29\end{array}$ & $\begin{array}{l}07-02 \\
06-21 \\
12-24 \\
06-12 \\
05-12\end{array}$ & $\begin{array}{l}0.22 \\
0.31 \\
0.28 \\
0.59 \\
0.37\end{array}$ & $\begin{array}{l}11-19 \\
06-21 \\
12-24 \\
06-12 \\
05-12\end{array}$ & $\begin{array}{l}0.37 \\
0.31 \\
0.36 \\
0.60 \\
0.47\end{array}$ & $\begin{array}{l}11-19 \\
06-21 \\
12-24 \\
06-12 \\
05-12\end{array}$ & $\begin{array}{c}0.74 \\
0.32^{(n)} \\
0.43 \\
0.60 \\
0.50\end{array}$ & $\begin{array}{l}11 / 19-20 \\
06-21 \\
10-11 \\
06 / 12-13 \\
05-12 .\end{array}$ & $\begin{array}{c}0.78 \\
0.37^{(x)} \\
0.54 \\
0.60 \\
0.61\end{array}$ & $\begin{array}{l}11 / 19-20 \\
04 / 17-18 \\
10 / 07-08 \\
06-12 \\
01 / 22-23\end{array}$ \\
\hline $\begin{array}{l}1971 \\
1972 \\
1973 \\
1974 \\
1975\end{array}$ & $\begin{array}{l}0.15 \\
0.18 \\
0.15 \\
0.45 \\
0.30\end{array}$ & $\begin{array}{l}03-15 \\
05-20 \\
10-31 \\
07-19 \\
08-18\end{array}$ & $\begin{array}{l}0.26 \\
0.32 \\
0.21 \\
0.45 \\
0.47\end{array}$ & $\begin{array}{l}03-25 \\
05-30 \\
10-31^{(0)} \\
07-19 \\
08-18\end{array}$ & $\begin{array}{l}0.35 \\
0.45 \\
0.30 \\
0.45 \\
0.55\end{array}$ & $\begin{array}{l}01-16 \\
05-20 \\
11-12 \\
07-19 \\
08-18\end{array}$ & $\begin{array}{l}0.48 \\
0.80 \\
0.53 \\
0.45 \\
0.69\end{array}$ & $\begin{array}{l}03 / 25-26 \\
05 / 20-21 \\
10-31 \\
07-19 \\
08-18\end{array}$ & $\begin{array}{l}0.53 \\
1.24 \\
0.64 \\
0.45 \\
0.69\end{array}$ & $\begin{array}{l}01-16 \\
5 / 20-21 \\
10-31 \\
07-19 \\
08-18\end{array}$ & $\begin{array}{l}0.53 \\
1.39 \\
0.64 \\
0.45 \\
0.69\end{array}$ & $\begin{array}{l}01-16 \\
05 / 20-21 \\
11 / 11-12 \\
07-19 \\
08-18\end{array}$ \\
\hline $\begin{array}{l}1976 \\
1977 \\
1978 \\
1979 \\
1980\end{array}$ & $\begin{array}{l}0.32 \\
0.16 \\
0.15 \\
0.11 \\
0.14\end{array}$ & $\begin{array}{l}08-07 \\
12-13 \\
04-27 \\
04-17 \\
12-25\end{array}$ & $\begin{array}{l}0.33 \\
0.28 \\
0.22 \\
0.18 \\
0.24\end{array}$ & $\begin{array}{l}08-07 \\
12-13 \\
04-27 \\
03-27 \\
04-20\end{array}$ & $\begin{array}{l}0.33 \\
0.36 \\
0.23 \\
0.22 \\
0.29\end{array}$ & $\begin{array}{l}08-07 \\
12-13 \\
04-27 \\
03-27 \\
04-20\end{array}$ & $\begin{array}{l}0.33 \\
0.61 \\
0.31 \\
0.29 \\
0.47\end{array}$ & $\begin{array}{l}08-07 \\
12-13 \\
11-18^{104} \\
03-27 \\
05 / 25-26\end{array}$ & $\begin{array}{l}0.33 \\
0.75 \\
0.58 \\
0.40 \\
0.74\end{array}$ & $\begin{array}{l}08-07 \\
12-13 \\
11 / 18-19 \\
03-27 \\
09-13\end{array}$ & $\begin{array}{l}0.40 \\
0.89 \\
0.67 \\
0.42 \\
0.90\end{array}$ & $\begin{array}{l}08 / 24-25 \\
08 / 29-30 \\
11 / 18-19 \\
11-16+ \\
05 / 25-26\end{array}$ \\
\hline $\begin{array}{l}1981 \\
1982 \\
1983 \\
1984 \\
1985\end{array}$ & $\begin{array}{l}0.22 \\
0.22 \\
0.24 \\
0.20 \\
0.14\end{array}$ & $\begin{array}{l}05-25 \\
07-07 \\
09-01 \\
06-28 \\
12-07\end{array}$ & $\begin{array}{l}0.34 \\
0.33 \\
0.31 \\
0.38 \\
0.22\end{array}$ & $\begin{array}{l}05-25 \\
11-18 \\
11-10 \\
03-20 \\
11-21\end{array}$ & $\begin{array}{l}0.38 \\
0.40 \\
0.39 \\
0.39 \\
0.29\end{array}$ & $\begin{array}{l}05-25 \\
11-18 \\
11-10 \\
03-20 \\
11-21\end{array}$ & $\begin{array}{l}0.73 \\
0.64 \\
0.45 \\
0.48 \\
0.46\end{array}$ & $\begin{array}{l}05-25 \\
10-28 \\
11 / 23-24 \\
03 / 20-21 \\
11-21\end{array}$ & $\begin{array}{l}0.74 \\
0.95 \\
0.60 \\
0.51 \\
0.52\end{array}$ & $\begin{array}{l}05-25 \\
10 / 28-29 \\
11 / 23-24 \\
03 / 20-21 \\
11 / 21-22\end{array}$ & $\begin{array}{l}0.74 \\
0.97 \\
0.66 \\
0.53 \\
0.52\end{array}$ & $\begin{array}{l}05-25 \\
10 / 28-29 \\
11-10 \\
03 / 20-21 \\
11 / 21-22\end{array}$ \\
\hline $\begin{array}{l}1986 \\
1987 \\
1988 \\
1989 \\
1990\end{array}$ & $\begin{array}{l}0.24 \\
0.21 \\
0.31 \\
0.16 \\
0.25\end{array}$ & $\begin{array}{l}09-15 \\
07-09 \\
04-28 \\
04-25 \\
06-06\end{array}$ & $\begin{array}{l}0.43 \\
0.24 \\
0.42 \\
0.25 \\
0.33\end{array}$ & $\begin{array}{l}09-15 \\
07-09 \\
04-28 \\
04-25 \\
08-21^{(0)}\end{array}$ & $\begin{array}{l}0.45 \\
0.27 \\
0.42 \\
0.26 \\
0.43\end{array}$ & $\begin{array}{l}09-15 \\
07-09 \\
04-28 \\
04-25 \\
08-21\end{array}$ & $\begin{array}{l}0.47 \\
0.31 \\
0.42 \\
0.31 \\
0.66\end{array}$ & $\begin{array}{l}09-15 \\
12-09 \\
04-28 \\
05-23 \\
08-21\end{array}$ & $\begin{array}{l}0.47 \\
0.34 \\
0.48 \\
0.38 \\
0.77\end{array}$ & $\begin{array}{l}09-15 \\
12-09 \\
04-28 \\
02 / 16-17 \\
08 / 20-21\end{array}$ & $\begin{array}{l}0.54 \\
0.55 \\
0.49 \\
0.56 \\
0.77\end{array}$ & $\begin{array}{l}09-15 \\
12-09 \\
04 / 27-28 \\
02 / 16-17 \\
08 / 20-21\end{array}$ \\
\hline $\begin{array}{l}1991 \\
1992 \\
1993 \\
1994 \\
1995\end{array}$ & $\begin{array}{l}0.49 \\
0.17 \\
0.32 \\
0.27 \\
0.48\end{array}$ & $\begin{array}{l}06-29 \\
06-12 \\
07-17 \\
05-15 \\
05-09\end{array}$ & $\begin{array}{l}0.50 \\
0.25 \\
0.45 \\
0.32 \\
0.53\end{array}$ & $\begin{array}{l}06-29 \\
06-12 \\
07-17 \\
10-14 \\
05-09\end{array}$ & $\begin{array}{l}0.51 \\
0.31 \\
0.55 \\
0.37 \\
0.53\end{array}$ & $\begin{array}{l}06-29 \\
06-12 \\
07-17 \\
05-15 \\
05-09\end{array}$ & $\begin{array}{l}0.51 \\
0.44 \\
0.82 \\
0.49 \\
0.55\end{array}$ & $\begin{array}{l}06-29 \\
06-12 \\
07-17 \\
05-15 \\
12-12\end{array}$ & $\begin{array}{l}0.53 \\
0.70 \\
1.01 \\
0.58 \\
0.65\end{array}$ & $\begin{array}{l}06-29 \\
06-12 \\
07 / 16-17 \\
05-15 \\
12-12\end{array}$ & $\begin{array}{l}0.59 \\
0.79 \\
1.39 \\
0.59 \\
1.04\end{array}$ & $\begin{array}{l}06 / 05-06 \\
06-12 \\
07 / 16-17 \\
05 / 14-15 \\
12 / 11-12\end{array}$ \\
\hline $\begin{array}{l}1996 \\
1997 \\
1998\end{array}$ & $\begin{array}{l}0.16 \\
0.27 \\
0.19\end{array}$ & $\begin{array}{l}12-31^{(0)} \\
10-08 \\
11-05\end{array}$ & $\begin{array}{l}0.29 \\
0.36 \\
0.29\end{array}$ & $\begin{array}{l}12-29 \\
10-08 \\
11-05\end{array}$ & $\begin{array}{l}0.40 \\
0.40 \\
0.36\end{array}$ & $\begin{array}{l}12-29 \\
10-08 \\
11-05\end{array}$ & $\begin{array}{l}0.65 \\
0.48 \\
0.49\end{array}$ & $\begin{array}{l}12-29 \\
01-31 \\
11-05\end{array}$ & $\begin{array}{l}0.90 \\
0.57 \\
0.62\end{array}$ & $\begin{array}{l}11-19 \\
11-07 \\
11-05\end{array}$ & $\begin{array}{l}1.70 \\
0.70 \\
0.62\end{array}$ & $\begin{array}{l}11 / 18-19 \\
01-17 \\
11-05\end{array}$ \\
\hline
\end{tabular}

(a) Greatest and least values.

(b) Last of multiple occurrences. 
Table 4.10. Normal and Maximum Daily Precipitation (in.)

\begin{tabular}{|c|c|c|c|c|c|c|c|}
\hline \multirow[b]{3}{*}{ Day } & \multicolumn{5}{|c|}{ Normal Period (1961-1990) } & \multicolumn{2}{|c|}{$\begin{array}{c}\text { Historical Period } \\
(1945-1998)\end{array}$} \\
\hline & \multicolumn{3}{|c|}{ Number of Years } & \multirow[b]{2}{*}{ Maximum } & \multirow{3}{*}{ Year } & \multirow{3}{*}{ Maximum } & \multirow{3}{*}{ Year } \\
\hline & Normal & W/Meas. & W/Trace & & & & \\
\hline \multicolumn{5}{|c|}{ January } & & & \\
\hline 1 & 0.02 & 5 & 10 & 0.20 & 1987 & 0.20 & 1987 \\
\hline 2 & 0.02 & 9 & 9 & 0.17 & 1983 & 0.27 & 1953 \\
\hline 3 & 0.02 & 8 & 7 & 0.28 & 1966 & 0.28 & 1966 \\
\hline 4 & 0.02 & 7 & 10 & 0.17 & 1976 & 0.25 & 1956 \\
\hline 5 & 0.02 & 9 & 8 & 0.23 & 1966 & 0.23 & 1966 \\
\hline 6 & 0.02 & 4 & 8 & 0.50 & 1983 & 0.87 & 1948 \\
\hline 7 & 0.02 & 8 & 5 & 0.31 & 1990 & 0.31 & 1990 \\
\hline 8 & 0.02 & 7 & 9 & 0.25 & 1978 & 0.59 & 1953 \\
\hline 9 & 0.02 & 8 & 9 & 0.20 & $1980^{(2)}$ & 0.41 & $1995^{(8)}$ \\
\hline 10 & 0.03 & 10 & 5 & 0.20 & $1979^{(x)}$ & 0.22 & 1995 \\
\hline 11 & 0.03 & 4 & 10 & 0.17 & 1980 & 0.48 & 1959 \\
\hline 12 & 0.03 & 9 & 10 & 0.32 & 1973 & 0.58 & 1958 \\
\hline 13 & 0.03 & 10 & 8 & 0.33 & 1980 & 0.37 & 1950 \\
\hline 14 & 0.03 & 10 & 3 & 0.43 & 1978 & 0.43 & 1978 \\
\hline 15 & 0.03 & 7 & 5 & 0.18 & 1968 & 0.34 & 1956 \\
\hline 16 & 0.03 & 10 & 6. & 0.53 & 1971 & 0.70 & 1954 \\
\hline 17 & 0.02 & 5 & 7 & 0.10 & 1986 & 0.31 & 1997 \\
\hline 18 & 0.02 & 7 & 8 & 0.25 & 1974 & 0.28 & 1996 \\
\hline 19 & 0.02 & 8 & 1 & 0.32 & 1970 & 0.39 & 1950 \\
\hline 20 & 0.02 & 5 & 6 & 0.26 & 1985 & 0.32 & 1953 \\
\hline 21 & 0.02 & 6 & 5 & 0.15 & 1984 & 0.16 & $1997^{(\mathrm{a})}$ \\
\hline 22 & 0.02 & 6 & 7 & 0.54 & 1970 & 0.54 & 1970 \\
\hline 23 & 0.03 & 8 & 9 & 0.27 & 1965 & 0.27 & 1965 \\
\hline 24 & 0.03 & 8 & 6 & 0.22 & 1970 & 0.26 & 1996 \\
\hline 25 & 0.03 & 6 & 6 & 0.72 & 1975 & 0.72 & 1975 \\
\hline 26 & 0.03 & 8 & 4 & 0.36 & 1970 & 0.36 & 1970 \\
\hline 27 & 0.03 & 6 & 6 & 0.20 & 1981 & 0.32 & 1954 \\
\hline 28 & 0.03 & 6 & 7 & 0.19 & 1986 & 0.19 & $1995^{(0)}$ \\
\hline 29 & 0.03 & 9 & 4 & 0.31 & 1965 & 0.33 & 1958 \\
\hline 30 & 0.03 & 8 & 8 & 0.12 & 1986 & 0.24 & 1995 \\
\hline 31 & 0.03 & 9 & 10 & 0.94 & 1963 & $0.94^{(0)}$ & $1963^{(0)}$ \\
\hline \multicolumn{8}{|c|}{ February } \\
\hline 1 & 0.03 & 8 & 4 & 0.72 & 1961 & 0.72 & 1961 \\
\hline 2 & 0.02 & 5 & 8 & 0.26 & 1963 & 0.26 & 1963 \\
\hline 3 & 0.02 & 5 & 6 & 0.06 & 1990 & 0.31 & 1998 \\
\hline 4 & 0.02 & 5 & 5 & 0.28 & 1975 & 0.28 & 1975 \\
\hline 5 & 0.02 & 7 & .7 & 0.07 & 1978 & 0.15 & 1953 \\
\hline 6 & 0.02 & 10 & 2 & 0.18 & 1961 & 0.18 & 1961 \\
\hline 7 & 0.02 & 5 & 4 & 0.27 & 1985 & 0.27 & 1985 \\
\hline 8 & 0.02 & 5 & 8 & 0.12 & 1985 & 0.12 & 1985 \\
\hline 9 & 0.02 & 9 & 5 & 0.37 & 1962 & 0.43 & 1959 \\
\hline 10 & 0.02 & 5 & 5 & 0.64 & 1961 & 0.64 & 1961 \\
\hline 11 & 0.02 & 10 & 3 & 0.30 & 1969 & 0.30 & 1969 \\
\hline 12 & 0.03 & 9 & 6 & 0.20 & 1986 & 0.42 & 1958 \\
\hline 13 & 0.02 & 10 & 6 & 0.21 & 1981 & 0.21 & 1981 \\
\hline 14 & 0.03 & 5 & 11 & 0.39 & 1986 & 0.39 & 1986 \\
\hline 15 & 0.03 & 12 & 7 & 0.30 & 1970 & 0.30 & 1970 \\
\hline 16 & 0.03 & 7 & 7 & 0.42 & 1989 & 0.42 & 1989 \\
\hline 17 & 0.03 & 10 & 7 & 0.42 & 1989 & 0.42 & 1989 \\
\hline 18 & 0.03 & 9 & 2 & 0.34 & 1983 & 0.34 & $1983^{(\mathbf{a})}$ \\
\hline 19 & 0.02 & 9 & 5 & 0.14 & 1989 & $0.78^{(b)}$ & $1993^{(b)}$ \\
\hline
\end{tabular}


Table 4.10. (contd)

\begin{tabular}{|c|c|c|c|c|c|c|c|}
\hline \multirow[b]{3}{*}{ Day } & \multicolumn{5}{|c|}{ Normal Period (1961-1990) } & \multicolumn{2}{|c|}{$\begin{array}{c}\text { Historical Period } \\
(1945-1998)\end{array}$} \\
\hline & \multirow[b]{2}{*}{ Normal } & \multicolumn{2}{|c|}{ Number of Years } & \multirow[b]{2}{*}{ Maximum } & \multirow[b]{2}{*}{ Year } & \multirow[b]{2}{*}{ Maximum } & \multirow[b]{2}{*}{ Year } \\
\hline & & W/Meas. & W/Trace & & & & \\
\hline 20 & 0.02 & 5 & 3 & 0.18 & 1984 & 0.18 & 1984 \\
\hline 21 & 0.02 & 8 & 3 & 0.20 & 1986 & 0.36 & 1956 \\
\hline 22 & 0.02 & 4 & 5 & 0.15 & 1989 & 0.21 & 1949 \\
\hline 23 & 0.02 & 5 & 4 & 0.22 & 1968 & 0.22 & 1968 \\
\hline 24 & 0.02 & 5 & 5 & 0.10 & 1981 & 0.33 & 1950 \\
\hline 25 & 0.02 & 9 & 3 & 0.22 & 1983 & 0.25 & 1948 \\
\hline 26 & 0.02 & 6 & 4 & 0.30 & 1976 & 0.30 & 1976 \\
\hline 27 & 0.02 & 9 & 3 & 0.24 & 1980 & 0.24 & 1980 \\
\hline 28 & 0.02 & 7 & 4 & 0.33 & 1977 & 0.33 & 1977 \\
\hline \multirow[t]{2}{*}{29} & 0.02 & 1 & 1 & 0.04 & 1984 & 0.04 & 1984 \\
\hline & \multicolumn{5}{|c|}{ March } & & \\
\hline 1 & 0.02 & 8 & 4 & 0.15 & 1972 & 0.15 & 1972 \\
\hline 2 & 0.01 & 4 & 8 & 0.07 & 1989 & 0.20 & $1991^{(a)}$ \\
\hline 3 & 0.01 & 3 & 7 & 0.15 & 1977 & 0.15 & $1991^{(2)}$ \\
\hline 4 & 0.01 & 9 & 6 & 0.16 & 1985 & 0.44 & 1957 \\
\hline 5 & 0.01 & 6 & 7 & 0.23 & 1989 & 0.23 & 1989 \\
\hline 6 & 0.02 & 7 & 3 & 0.07 & $1971^{(\mathrm{a})}$ & 0.24 & 1957 \\
\hline 7 & 0.02 & 6 & 4 & 0.21 & 1986 & 0.21 & 1986 \\
\hline 8 & 0.02 & 8 & 2 & 0.19 & 1988 & 0.23 & 1951 \\
\hline 9 & 0.02 & 6 & 6 & 0.31 & 1989 & 0.42 & 1995 \\
\hline 10 & 0.02 & 11 & 4 & 0.06 & $1980^{(2)}$ & 0.21 & 1995 \\
\hline 11 & 0.02 & 8 & 6 & 0.24 & 1989 & 0.24 & 1989 \\
\hline 12 & 0.02 & 9 & 6 & 0.42 & 1987 & 0.42 & 1987 \\
\hline 13 & 0.02 & 7 & 8 & 0.35 & 1983 & 0.35 & 1983 \\
\hline 14 & 0.02 & 9 & 3 & 0.14 & 1970 & 0.16 & 1995 \\
\hline 15 & 0.02 & 7 & 4 & 0.18 & 1987 & 0.25 & 1949 \\
\hline 16 & 0.01 & 7 & 5 & 0.22 & 1989 & 0.34 & $1997^{(2)}$ \\
\hline 17 & 0.01 & 3 & 7 & 0.05 & 1967 & 0.16 & 1949 \\
\hline 18 & 0.01 & 7 & 5 & 0.04 & 1989 & 0.25 & 1949 \\
\hline 19 & 0.01 & 3 & 4 & 0.12 & 1987 & 0.12 & 1987 \\
\hline 20 & 0.01 & 5 & 4 & 0.43 & 1984 & 0.43 & 1984 \\
\hline 21 & 0.01 & 5 & 2 & 0.10 & $1984^{(2)}$ & 0.18 & 1958 \\
\hline 22 & 0.02 & 4 & 8 & 0.22 & 1961 . & 0.22 & 1961 \\
\hline 23 & 0.02 & 7 & 4 & 0.26 & 1986 & 0.26 & 1986 \\
\hline 24 & 0.02 & 4 & 2 & 0.12 & 1961 & $0.52^{(b)}$ & $1991^{(b)}$ \\
\hline 25 & 0.02 & 9 & 3 & 0.43 & 1971 & 0.43 & 1971 \\
\hline 26 & 0.02 & 5 & 6 & 0.50 & 1981 & 0.50 & 1981 \\
\hline 27 & 0.02 & 5 & 4 & 0.42 & 1979 & 0.42 & 1979 \\
\hline 28 & 0.02 & 5 & 3 & 0.13 & 1982 & 0.13 & 1982 \\
\hline 29 & 0.01 & 8 & 1 & 0.15 & 1983 & 0.15 & 1983 \\
\hline 30 & 0.01 & 4 & 7 & 0.23 & 1974 & 0.23 & 1974 \\
\hline \multirow[t]{2}{*}{31} & 0.01 & 4 & 6 & 0.14 & 1976 & 0.26 & 1996 \\
\hline & & & \multicolumn{3}{|c|}{ April } & . & \\
\hline 1 & 0.01 & 3 & 12 & 0.18 & 1983 & 0.22 & 1958 \\
\hline 2 & 0.01 & 6 & 3 & 0.10 & 1979 & 0.18 & 1948 \\
\hline 3 & 0.01 & 2 & 3 & 0.03 & 1963 & 0.18 & 1947 \\
\hline 4 & 0.01 & 5 & 9 & 0.13 & 1984 & 0.18 & 1948 \\
\hline 5 & 0.01 & 6 & 6 & 0.44 & 1969 & 0.44 & 1969 \\
\hline 6 & 0.01 & 6 & 3 & 0.36 & 1982 & 0.36 & 1982 \\
\hline 7 & 0.01 & 3 & 1 & 0.22 & 1984 & 0.30 & 1953 \\
\hline 8 & 0.01 & 5 & 2 & 0.17 & 1976 & 0.18 & 1991 \\
\hline 9 & 0.01 & 3 & 10 & 0.12 & 1980 & 0.32 & 1992 \\
\hline 10 & 0.01 & 2 & 6 & 0.03 & 1969 & 0.10 & 1958 \\
\hline
\end{tabular}


Table 4.10. (contd)

Normal Period (1961-1990)

\begin{tabular}{|c|c|c|}
\hline \multirow[b]{2}{*}{ Day } & \multirow[b]{2}{*}{ Normal } & Number of Yea \\
\hline & & W/Meas. \\
\hline 11 & 0.01 & 4 \\
\hline 12 & 0.01 & 4 \\
\hline 13 & 0.01 & 1 \\
\hline 14 & 0.01 & 4 \\
\hline 15 & 0.01 & 3 \\
\hline 16 & 0.02 & 4 \\
\hline 17 & 0.02 & 6 \\
\hline 18 & 0.02 & 4 \\
\hline 19 & 0.02 & 7 \\
\hline 20 & 0.02 & 6 \\
\hline 21 & 0.02 & 2 \\
\hline 22 & 0.02 & 7 \\
\hline 23 & 0.01 & 6 \\
\hline 24 & 0.01 & 4 \\
\hline 25 & 0.02 & 3 \\
\hline 26 & 0.02 & 2 \\
\hline 27 & 0.02 & 7 \\
\hline 28 & 0.02 & 6 \\
\hline 29 & 0.02 & 2 \\
\hline 30 & 0.02 & 6 \\
\hline
\end{tabular}

\begin{tabular}{c}
\hline Trace \\
\hline 9 \\
5 \\
3 \\
4 \\
8 \\
7 \\
6 \\
6 \\
9 \\
6 \\
7 \\
6 \\
5 \\
8 \\
6 \\
7 \\
5 \\
5 \\
3 \\
4
\end{tabular}

Historical Period (1945-1998)

\begin{tabular}{l} 
Maximum \\
\hline 0.23 \\
0.08 \\
0.01 \\
0.17 \\
0.09 \\
0.08 \\
0.36 \\
0.31 \\
0.41 \\
0.56 \\
0.07 \\
0.12 \\
0.22 \\
0.22 \\
0.35 \\
0.04 \\
0.28 \\
0.48 \\
0.30 \\
0.12
\end{tabular}

\begin{tabular}{l} 
Year \\
\hline 1982 \\
1961 \\
1990 \\
$1975^{(a)}$ \\
1962 \\
$1979^{(a)}$ \\
1988 \\
1967 \\
1970 \\
1980 \\
1989 \\
1974 \\
1974 \\
1975 \\
1989 \\
1989 \\
$1989^{(a)}$ \\
1988 \\
1961 \\
1984
\end{tabular}

\begin{tabular}{ccc} 
Maximum & & Year \\
\cline { 3 - 3 } 0.23 & & 1982 \\
0.36 & & 1995 \\
0.39 & & 1992 \\
0.17 & & $1975^{(a)}$ \\
0.17 & & 1991 \\
0.11 & & 1948 \\
0.36 & & 1988 \\
0.31 & & 1967 \\
0.41 & & 1970 \\
$0.56^{(b)}$ & & $1980^{(b)}$ \\
0.07 & & 1989 \\
0.28 & & 1996 \\
0.22 & & 1974 \\
0.22 & & 1975 \\
0.35 & 1989 \\
0.25 & 1955 \\
0.34 & 1995 \\
0.51 & 1951 \\
0.30 & 1961 \\
0.12 & 1984 \\
&
\end{tabular}

May

\begin{tabular}{|c|c|c|c|c|c|c|c|}
\hline 1 & 0.02 & 5 & 6 & 0.19 & 1984 & 0.19 & 1984 \\
\hline 2 & 0.01 & 5 & 1 & 0.17 & 1975 & 0.17 & 1975 \\
\hline 3 & 0.01 & 4 & 3 & 0.29 & 1977 & 0.29 & 1977 \\
\hline 4 & 0.01 & 6 & 5 & 0.10 & 1967 & 0.10 & 1967 \\
\hline 5 & 0.02 & 8 & 3 & 0.28 & 1963 & 0.28 & 1963 \\
\hline 6 & 0.02 & 4 & 6 & 0.20 & 1986 & 0.20 & 1986 \\
\hline 7 & 0.02 & 3 & 1 & 0.39 & 1983 & 0.39 & 1983 \\
\hline 8 & 0.02 & 5 & 7 & 0.55 & 1972 & 0.55 & 1972 \\
\hline 9 & 0.02 & 5 & 5 & 0.25 & 1961 & 0.53 & 1995 \\
\hline 10 & 0.02 & 6 & 7 & 0.39 & 1961 & 0.39 & 1961 \\
\hline 11 & 0.02 & 4 & 4 & 0.19 & 1967 & 0.39 & 1951 \\
\hline 12 & 0.02 & 4 & 7 & 0.50 & 1970 & 0.50 & 1970 \\
\hline 13 & 0.01 & 4 & 2 & 0.11 & 1985 & 0.15 & 1952 \\
\hline 14 & 0.01 & 4 & 6 & 0.25 & 1978 & 0.25 & 1978 \\
\hline 15 & 0.01 & 3 & 6 & 0.06 & 1975 & 0.58 & 1994 \\
\hline 16 & 0.01 & 0 & 8 & $\mathbf{T}$ & $1988^{(a)}$ & 0.14 & 1991 \\
\hline 17 & 0.01 & 4 & 3 & 0.13 & 1982 & 0.25 & 1959 \\
\hline 18 & 0.01 & 5 & 2 & 0.13 & 1981 & 0.13 & 1981 \\
\hline 19 & 0.01 & 7 & 3 & 0.15 & 1965 & 0.55 & 1948 \\
\hline 20 & 0.02 & 4 & 1 & 0.70 & 1972 & 0.70 & 1972 \\
\hline 21 & 0.02 & 3 & 4 & 0.69 & 1972 & 0.69 & 1972 \\
\hline 22 & 0.02 & 6 & 5 & 0.12 & 1984 & 0.12 & 1984 \\
\hline 23 & 0.03 & 5 & 6 & 0.33 & 1990 & 0.33 & 1990 \\
\hline 24 & 0.03 & 4 & 5 & 0.51 & 1962 & 0.51 & 1962 \\
\hline 25 & 0.02 & 6 & 2 & 0.74 & 1981 & 0.74 & 1981 \\
\hline 26 & 0.02 & 4 & 6 & 0.79 & 1980 & $0.79^{(b)}$ & $1980^{(b)}$ \\
\hline 27 & 0.02 & 6 & 2 & 0.11 & 1990 & 0.11 & 1990 \\
\hline 28 & 0.02 & 5 & 7 & 0.28 & 1988 & 0.28 & 1988 \\
\hline 29 & 0.02 & 5 & 3 & 0.11 & 1961 & 0.11 & 1961 \\
\hline 30 & 0.01 & 6 & 1 & 0.14 & 1987 & 0.14 & 1987 \\
\hline 31 & 0.02 & 2 & 5 & 0.35 & 1971 & 0.35 & 1971 \\
\hline
\end{tabular}


Table 4.10. (contd)

\begin{tabular}{|c|c|c|c|c|c|c|c|}
\hline \multirow[b]{3}{*}{ Day } & \multicolumn{5}{|c|}{ Normal Period (1961-1990) } & \multicolumn{2}{|c|}{$\begin{array}{c}\text { Historical Period } \\
(1945-1998)\end{array}$} \\
\hline & \multicolumn{3}{|c|}{ Number of Years } & \multirow[b]{2}{*}{ Maximum } & \multirow{3}{*}{ Year } & \multirow{3}{*}{ Maximum } & \multirow{3}{*}{ Year } \\
\hline & Normal & W/Meas. & W/Trace & & & & \\
\hline \multicolumn{5}{|c|}{ June } & & & \\
\hline 1 & 0.02 & 3 & 5 & 0.29 & 1977 & 0.29 & 1977 \\
\hline 2 & 0.02 & 5 & 6 & 0.12 & 1966 & 0.12 & 1966 \\
\hline 3 & 0.02 & 6 & 9 & 0.30 & 1971 & 0.30 & 1971 \\
\hline 4 & 0.02 & 6 & 3 & 0.25 & 1984 & 0.45 & 1951 \\
\hline 5 & 0.02 & 4 & 4 & 0.14 & 1981 & 0.49 & 1991 \\
\hline 6 & 0.02 & 6 & 3 & 0.36 & 1990 & 0.54 & 1951 \\
\hline 7 & 0.01 & 5 & 5 & 0.15 & 1972 & 0.71 & 1947 \\
\hline 8 & 0.01 & 6 & 6 & 0.49 & 1964 & 0.49 & 1964 \\
\hline 9 & 0.02 & 3 & 4 & 0.07 & 1963 & 0.22 & 1948 \\
\hline 10 & 0.02 & 2 & 7 & 0.08 & 1983 & 0.14 & 1956 \\
\hline 11 & 0.01 & 3 & 5 & 0.13 & 1961 & 0.39 & 1950 \\
\hline 12 & 0.01 & 4 & 8 & 0.60 & 1969 & 0.79 & 1992 \\
\hline 13 & 0.01 & 6 & 4 & 0.35 & 1980 & 0.49 & 1948 \\
\hline 14 & 0.01 & 4 & 2 & 0.04 & 1983 & 0.37 & 1956 \\
\hline 15 & 0.01 & 4 & 1 & 0.15 & 1964 & 0.15 & 1964 \\
\hline 16 & 0.01 & 2 & 5 & 0.14 & 1980 & 0.18 & 1948 \\
\hline 17 & 0.01 & 2 & 5 & 0.48 & 1965 & $1.09^{(b)}$ & $1950^{(b)}$ \\
\hline 18 & 0.01 & 2 & 5 & 0.07 & 1983 & 0.09 & 1994 \\
\hline 19 & 0.01 & 1 & 3 & 0.01 & 1983 & 0.29 & 1998 \\
\hline 20 & 0.01 & 5 & 2 & 0.24 & 1984 & 0.24 & 1984 \\
\hline 21 & 0.01 & 5 & 2 & 0.32 & 1967 & 0.32 & 1967 \\
\hline 22 & 0.01 & 4 & 7 & 0.14 & 1971 & 0.14 & 1971 \\
\hline 23 & 0.01 & 7 & 2 & 0.17 & 1963 & 0.17 & 1963 \\
\hline 24 & 0.01 & 5 & 3 & 0.21 & 1972 & 0.21 & 1972 \\
\hline 25 & 0.01 & 3 & 4 & 0.02 & 1980 & 0.03 & 1954 \\
\hline 26 & 0.01 & 2 & 6 & 0.27 & 1982 & 0.27 & 1982 \\
\hline 27 & 0.01 & 4 & 3 & 0.37 & 1983 & 0.37 & 1983 \\
\hline 28 & 0.01 & 2 & 8 & 0.23 & 1984 & 0.24 & 1992 \\
\hline 29 & 0.01 & 4 & 2 & 0.16 & 1984 & 0.53 & 1991 \\
\hline 30 & 0.01 & 1 & 1 & 0.06 & 1976 & 0.06 & 1976 \\
\hline \multicolumn{8}{|c|}{ July } \\
\hline 1 & 0.01 & 5 & 3 & 0.31 & 1966 & 0.31 & 1966 \\
\hline 2 & 0.01 & 3 & 6 & 0.34 & 1966 & 0.34 & 1966 \\
\hline 3 & 0.01 & 3 & 3 & 0.31 & 1978 & 0.31 & 1978 \\
\hline 4 & 0.01 & 3 & 4 & 0.10 & 1986 & 0.10 & 1986 \\
\hline 5 & 0.01 & 1 & 3 & 0.19 & 1981 & 0.36 & 1951 \\
\hline 6 & 0.01 & 1 & 3 & 0.02 & 1979 & 0.04 & 1993 \\
\hline 7 & 0.01 & 3 & 2 & 0.30 & 1963 & 0.30 & 1963 \\
\hline 8 & 0.01 & 5 & 3 & 0.14 & 1974 & 0.20 & 1995 \\
\hline 9 & 0.01 & 3 & 2 & 0.27 & 1987 & 0.27 & 1987 \\
\hline 10 & 0.01 & 2 & 5 & 0.05 & 1972 & 0.16 & 1954 \\
\hline 11 & 0.01 & 2 & 4 & 0.04 & 1979 & 0.04 & 1979 \\
\hline 12 & $\mathrm{~T}$ & 0 & 4 & $\mathbf{T}$ & $1982^{(2)}$ & $\mathbf{T}$ & $1982^{(2)}$ \\
\hline 13 & $\mathrm{~T}$ & 2 & 5 & 0.28 & 1975 & 0.28 & 1975 \\
\hline 14 & $\mathrm{~T}$ & 1 & 2 & 0.03 & 1966 & 0.05 & 1957 \\
\hline 15 & $T$ & 2 & 2 & 0.04 & 1975 & 0.08 & 1991 \\
\hline 16 & $T$ & 4 & 3 & 0.07 & 1966 & 0.50 & 1993 \\
\hline 17 & $T$ & 3 & 1 & 0.05 & $1987^{(2)}$ & $0.89^{(0)}$ & $1993^{(b)}$ \\
\hline 18 & $\mathrm{~T}$ & 2 & 2 & 0.12 & 1987 & 0.12 & 1987 \\
\hline 19 & $\mathrm{~T}$ & 2 & 2 & 0.45 & 1974 & 0.45 & 1974 \\
\hline 20 & $T$ & 2 & 2 & 0.09 & 1965 & 0.09 & 1965 \\
\hline 21 & $T$ & 1 & 2 & 0.02 & 1965 & 0.02 & 1965 \\
\hline
\end{tabular}


Table 4.10. (contd)

\begin{tabular}{|c|c|c|}
\hline \multirow[b]{2}{*}{ Day } & \multicolumn{2}{|c|}{$\frac{\text { Normal Peri }}{\text { Number of Yea }}$} \\
\hline & Normal & W/Meas. \\
\hline 22 & $T$ & 0 \\
\hline 23 & $\mathrm{~T}$ & 2 \\
\hline 24 & $\mathrm{~T}$ & 2 \\
\hline 25 & $T$ & 3 \\
\hline 26 & $T$ & 0 \\
\hline 27 & $T$ & 1 \\
\hline 28 & $T$ & 1 \\
\hline 29 & $T$ & 1 \\
\hline 30. & $T$ & 0 \\
\hline 31 & $T$ & 1 \\
\hline
\end{tabular}

(1961-1990)

\section{Wears}

August

\begin{tabular}{|c|c|c|c|c|c|c|c|}
\hline 1 & $\mathbf{T}$ & 2 & 1 & 0.08 & $1976^{(a)}$ & 0.08 & $1976^{(a)}$ \\
\hline 2 & $\mathbf{T}$ & 1 & 3 & 0.01 & 1976 & 0.01 & $1996^{(2)}$ \\
\hline 3 & $\mathbf{T}$ & 1 & 2 & 0.29 & 1962 & 0.29 & 1962 \\
\hline 4 & $\mathbf{T}$ & 1 & 2 & 0.01 & 1985 & 0.04 & 1948 \\
\hline 5 & $\mathbf{T}$ & 0 & 3 & $\mathbf{T}$ & $1984^{(a)}$ & $\mathbf{T}$ & $1991^{(4)}$ \\
\hline 6 & $\mathbf{T}$ & 1 & 2 & 0.11 & 1976 & 0.11 & 1976 \\
\hline 7 & $\mathbf{T}$ & 2 & 1 & 0.33 & 1976 & 0.33 & 1976 \\
\hline 8 & $\mathbf{T}$ & 0 & 4 & $\mathbf{T}$ & $1989^{(a)}$ & 0.08 & 1952 \\
\hline 9 & $\mathbf{T}$ & 2 & 2 & 0.10 & 1982 & 0.10 & 1982 \\
\hline 10 & $\mathbf{T}$ & 0 & 3 & $T$ & $1984^{(a)}$ & 0.01 & 1947 \\
\hline 11 & $\mathbf{T}$ & 1 & 4 & 0.01 & 1983 & 0.11 & 1947 \\
\hline 12 & 0.01 & 3 & 5 & 0.18 & 1962 & 0.18 & 1962 \\
\hline 13 & 0.01 & 4 & 2 & 0.04 & $1987^{\text {(a) }}$ & 0.06 & 1995 \\
\hline 14 & 0.01 & 5 & 3 & 0.09 & 1979 & 0.09 & 1979 \\
\hline 15 & 0.01 & 3 & 1 & 0.42 & 1972 & 0.42 & 1972 \\
\hline 16 & 0.01 & 1 & 1 & 0.01 & 1968 & 0.24 & 1993 \\
\hline 17 & 0.01 & 0 & 3 & $\mathbf{T}$ & $1980^{(2)}$ & $\mathrm{T}$ & $1995^{(a)}$ \\
\hline 18 & 0.01 & 4 & 3 & 0.69 & 1975 & 0.69 & 1975 \\
\hline 19 & 0.01 & 3 & 5 & 0.05 & 1979 & 0.18 & 1954 \\
\hline 20 & 0.01 & 4 & 6 & 0.03 & 1978 & 0.22 & 1953 \\
\hline 21 & 0.02 & 3 & 4 & 0.76 & 1990 & $0.76^{(0)}$ & $1990^{(b)}$ \\
\hline 22 & 0.01 & 6 & 1 & 0.18 & 1978 & 0.18 & 1978 \\
\hline 23 & 0.02 & 4 & 1 & 0.14 & 1975 & 0.14 & 1975 \\
\hline 24 & 0.02 & 6 & 1 & 0.38 & 1977 & 0.38 & 1977 \\
\hline 25 & 0.01 & 2 & 6 & 0.29 & 1976 & 0.29 & 1976 \\
\hline 26 & 0.01 & 3 & 4 & 0.19 & 1968 & 0.38 & 1953 \\
\hline 27 & 0.01 & 5 & 3 & 0.14 & 1989 & 0.14 & 1989 \\
\hline 28 & 0.01 & 2 & 4 & 0.13 & 1975 & 0.13 & 1975 \\
\hline 29 & 0.01 & 6 & 1 & 0.28 & 1977 & 0.51 & 1947 \\
\hline 30 & 0.01 & 2 & 4 & 0.61 & 1977 & 0.61 & 1977 \\
\hline 31 & 0.01 & 2 & 3 & 0.02 & 1961 & 0.02 & $1961^{(a)}$ \\
\hline \multicolumn{8}{|c|}{ September } \\
\hline 1 & 0.01 & 7 & 2 & 0.43 & 1971 & 0.43 & 1971 \\
\hline 2 & 0.01 & 2 & 4 & 0.17 & 1971 & 0.17 & 1971 \\
\hline 3 & 0.01 & 3 & 1 & 0.13 & 1979 & 0.15 & 1997 \\
\hline 4 & 0.01 & 1 & 1 & 0.02 & 1977 & 0.19 & 1960 \\
\hline 5 & $\mathbf{T}$ & 3 & 2 & 0.19 & 1971 & 0.19 & 1971 \\
\hline 6 & $\mathbf{T}$ & 3 & 0 & 0.29 & 1971 & 0.48 & 1995 \\
\hline 7 & $\mathbf{T}$ & 2 & 0 & 0.04 & 1978 & 0.23 & 1947 \\
\hline 8 & $\mathrm{~T}$ & 2 & 3 & 0.10 & 1985 & 0.10 & 1985 \\
\hline 9 & $\mathbf{T}$ & 2 & 4 & 0.07 & 1985 & 0.07 & 1985 \\
\hline
\end{tabular}

Historical Period

(1945-1998)

\begin{tabular}{lll}
\cline { 1 - 1 } Maximum & & Year \\
\cline { 1 - 1 } $\mathbf{T}$ & & $1987^{(\mathbf{a})}$ \\
0.15 & & 1961 \\
0.06 & & 1990 \\
0.23 & & 1983 \\
$T$ & & 1978 \\
0.02 & & 1983 \\
0.06 & & 1984 \\
0.01 & & 1964 \\
$T$ & & $1987^{(a)}$ \\
0.12 & & 1985
\end{tabular}

\begin{tabular}{|c|c|}
\hline Maximum & Year \\
\hline $\mathrm{T}$ & $1993^{\text {(a) }}$ \\
\hline 0.28 & 1992 \\
\hline 0.07 & 1955 \\
\hline 0.23 & 1983. \\
\hline 0.22 & 1955 \\
\hline 0.31 & 1947 \\
\hline 0.28 & 1947 \\
\hline 0.05 & 1997 \\
\hline $\mathrm{T}$ & $1997^{(2)}$ \\
\hline
\end{tabular}


Table 4.10. (contd)

\begin{tabular}{|c|c|c|c|c|c|c|c|}
\hline \multirow[b]{3}{*}{ Day } & \multicolumn{5}{|c|}{ Normal Period (1961-1990) } & \multicolumn{2}{|c|}{$\begin{array}{l}\text { Historical Period } \\
\text { (1945-1998) }\end{array}$} \\
\hline & \multicolumn{3}{|c|}{ Number of Years } & \multirow[b]{2}{*}{ Maximum } & \multirow[b]{2}{*}{ Year } & \multirow[b]{2}{*}{ Maximum } & \multirow[b]{2}{*}{ Year } \\
\hline & Normal & W/Meas. & W/Trace & & & & \\
\hline 10 & 0.01 & 3 & 3 & 0.06 & 1985 & 0.06 & 1985 \\
\hline 11 & 0.01 & 4 & 1 & 0.10 & 1966 & 0.10 & 1966 \\
\hline 12 & 0.01 & 0 & 3 & $T$ & $1980^{(2)}$ & 0.03 & 1958 \\
\hline 13 & 0.01 & 5 & 2 & 0.79 & 1980 & $0.79^{(0)}$ & $1980^{(b)}$ \\
\hline 14 & 0.01 & 3 & 4 & 0.04 & 1985 & 0.41 & 1959 \\
\hline 15 & 0.01 & 2 & 5 & 0.54 & 1986 & 0.54 & 1986 \\
\hline 16 & 0.02 & 2 & 7 & 0.03 & 1985 & 0.66 & 1947 \\
\hline 17 & 0.01 & 4 & 5 & 0.26 & 1969 & 0.26 & 1969 \\
\hline 18 & 0.01 & 4 & 6 & 0.22 & 1983 & 0.41 & 1959 \\
\hline 19 & 0.01 & 6 & 4 & 0.26 & 1973 & 0.26 & 1973 \\
\hline 20 & 0.01 & 4 & 8 & 0.13 & 1988 & 0.13 & 1988 \\
\hline 21 & 0.01 & 2 & 4 & 0.03 & 1971 & 0.03 & 1971 \\
\hline 22 & 0.01 & 4 & 4 & 0.20 & 1984 & 0.20 & 1984 \\
\hline 23 & 0.01 & 4 & 2 & 0.21 & 1986 & 0.21 & 1986 \\
\hline 24 & 0.01 & 4 & 0 & 0.10 & 1977 & 0.10 & 1977 \\
\hline 25 & 0.01 & 3 & 3 & 0.25 & 1982 & 0.25 & 1982 \\
\hline 26 & 0.01 & 4 & 1 & 0.22 & 1981 & 0.22 & 1981 \\
\hline 27 & 0.01 & 4 & 2 & 0.38 & 1981 & 0.43 & 1955 \\
\hline 28 & 0.01 & 5 & 3 & 0.34 & 1962 & 0.34 & 1962 \\
\hline 29 & 0.01 & 3 & 1 & 0.07 & 1986 & 0.07 & 1986 \\
\hline 30 & 0.01 & 2 & 3 & 0.02 & 1969 & 0.03 & $1953^{(a)}$ \\
\hline \multicolumn{8}{|c|}{ October } \\
\hline 1 & $\mathbf{T}$ & 2 & 5 & 0.01 & $1969^{(2)}$ & $1.60^{(0)}$ & $1957^{(b)}$ \\
\hline 2 & $\mathbf{T}$ & 5 & 5 & 0.06 & 1967 & 0.31 & 1957 \\
\hline 3 & $\mathbf{T}$ & 2 & 1 & 0.04 & 1975 & 0.38 & 1995 \\
\hline 4 & $\mathbf{T}$ & 1 & 4 & 0.01 & 1970 & 0.15 & 1950 \\
\hline 5 & $\mathbf{T}$ & 0 & 9 & $T$ & $1981^{(a)}$ & 0.25 & 1950 \\
\hline 6 & $T$ & 5 & 4 & 0.22 & 1973 & 0.22 & 1973 \\
\hline 7 & 0.01 & 3 & 3 & 0.25 & 1985 & 0.25 & 1985 \\
\hline 8 & 0.01 & 1 & 5 & 0.06 & 1964 & 0.49 & 1950 \\
\hline 9 & 0.01 & 5 & 2 & 0.09 & 1968 & 0.32 & 1947 \\
\hline 10 & 0.01 & 5 & 4 & 0.09 & 1985 & 0.32 & 1959 \\
\hline 11 & 0.01 & 3 & 7 & 0.43 & 1968 & 0.43 & 1968 \\
\hline 12 & 0.01 & 6 & 3 & 0.52 & 1962 & 0.52 & 1962 \\
\hline 13 & 0.01 & 2 & 3 & 0.04 & 1980 & 0.16 & 1994 \\
\hline 14 & 0.01 & 4 & 3 & 0.11 & 1962 & 0.43 & 1950 \\
\hline 15 & 0.01 & 1 & 1 & 0.01 & 1980 & 0.15 & 1947 \\
\hline 16 & 0.01 & 0 & 1 & $T$ & 1975 & 0.24 & 1947 \\
\hline 17 & 0.01 & 2 & 3 & 0.16 & 1968 & 0.23 & 1950 \\
\hline 18 & 0.01 & 4 & 3 & 0.28 & 1979 & 0.28 & 1979 \\
\hline 19 & 0.02 & 5 & 4 & 0.12 & 1979 & 0.64 & 1947 \\
\hline 20 & 0.02 & 5 & 5 & 0.15 & 1968 & 0.37 & 1947 \\
\hline 21 & 0.02 & 8 & 3 & 0.45 & 1975 & 0.45 & 1975 \\
\hline 22 & 0.02 & 5 & 9 & 0.20 & 1983 & 0.23 & 1957 \\
\hline 23 & 0.02 & 4 & 2 & 0.39 & 1973 & 0.39 & 1973 \\
\hline 24 & 0.02 & 4 & 5 & 0.11 & 1979 & 0.12 & 1991 \\
\hline 25 & 0.02 & 5 & 3 & 0.22 & 1975 & 0.22 & 1975 \\
\hline 26 & 0.02 & 6 & 3 & 0.12 & 1989 & 0.18 & 1956 \\
\hline 27 & 0.02 & 7 & 4 & 0.09 & 1969 & 0.33 & 1956 \\
\hline 28 & 0.02 & 4 & 6 & 0.93 & 1982 & 0.93 & 1982 \\
\hline 29 & 0.02 & 7 & 8 & 0.18 & 1986 & 0.38 & 1950 \\
\hline 30 & 0.02 & 3 & 7 & 0.52 & 1990 & 0.52 & 1990 \\
\hline 31 & 0.02 & 3 & 6 & 0.64 & 1973 & 0.64 & 1973 \\
\hline
\end{tabular}


Table 4.10. (contd)

\begin{tabular}{|c|c|c|c|c|c|c|c|}
\hline \multirow[b]{3}{*}{ Day } & \multicolumn{5}{|c|}{ Normal Period (1961-1990) } & \multicolumn{2}{|c|}{$\begin{array}{c}\text { Historical Period } \\
(1945-1998)\end{array}$} \\
\hline & \multicolumn{3}{|c|}{ Number of Years } & \multirow[b]{2}{*}{ Maximum } & \multirow{3}{*}{ Year } & \multirow{3}{*}{ Maximum } & \multirow{3}{*}{ Year } \\
\hline & Normal & W/Meas. & W/Trace & & & & \\
\hline \multicolumn{5}{|c|}{ November } & & & \\
\hline 1 & 0.02 & 7 & 3 & 0.18 & 1987 & 0.26 & 1948 \\
\hline 2 & 0.02 & 6 & 2 & 0.25 & 1984 & 0.25 & 1984 \\
\hline 3 & 0.02 & 8 & 3 & 0.28 & 1965 & 0.28 & 1965 \\
\hline 4 & 0.02 & 9 & 5 & 0.19 & 1973 & 0.24 & 1991 \\
\hline 5 & 0.02 & 9 & 2 & 0.19 & 1963 & 0.62 & 1998 \\
\hline 6 & 0.02 & 8 & 4 & 0.30 & 1980 & 0.30 & $1980^{\text {(a) }}$ \\
\hline 7 & 0.03 & 5 & 7 & 0.23 & 1963 & 0.57 & 1967 \\
\hline 8 & 0.03 & 9 & 6 & 0.50 & 1968 & 0.50 & 1968 \\
\hline 9 & 0.04 & 8 & 7 & 0.16 & 1973 & 0.27 & 1949 \\
\hline 10 & 0.04 & 11 & 4 & 0.66 & 1983 & 0.66 & 1983 \\
\hline 11 & 0.04 & 12 & 3 & 0.26 & 1970 & 0.26 & 1970 \\
\hline 12 & 0.04 & 10 & 4 & 0.57 & 1973 & 0.57 & 1973 \\
\hline 13 & 0.04 & 7 & 4 & 0.47 & 1981 & 0.47 & 1981 \\
\hline 14 & 0.04 & 7 & 6 & 0.35 & 1966 & 0.35 & 1966 \\
\hline 15 & 0.04 & 12 & 5 & 0.17 & 1985 & 0.19 & 1994 \\
\hline 16 & 0.03 & 9 & 5 & 0.42 & 1979 & 0.42 & 1979 \\
\hline 17 & 0.03 & 9 & 7 & 0.12 & 1974 & 0.18 & 1955 \\
\hline 18 & 0.03 & 7 & 4 & 0.46 & 1982 & 0.51 & 1996 \\
\hline 19 & 0.03 & 7 & 5 & 0.37 & 1966 & $1.39^{(b)}$ & $1996^{(0)}$ \\
\hline 20 & 0.03 & 4 & 7 & 0.42 & 1966 & 0.42 & 1966 \\
\hline 21 & 0.03 & 6 & 8 & 0.50 & 1985 & 0.50 & 1985 \\
\hline 22 & 0.03 & 6 & 10 & 0.30 & 1979 & 0.30 & 1979 \\
\hline 23 & 0.03 & 12 & 5 & 0.42 & 1983 & 0.63 & 1949 \\
\hline 24 & 0.03 & 10 & 7 & 0.37 & 1965 & 0.37 & 1965 \\
\hline 25 & 0.03 & 5 & 10 & 0.25 & 1977 & 0.25 & 1977 \\
\hline 26 & 0.03 & 8 & 6 & 0.11 & $1986^{(a)}$ & 0.54 & 1955 \\
\hline 27 & 0.03 & 9 & 6 & 0.49 & 1984 & 0.49 & 1984 \\
\hline 28 & 0.02 & 9 & 7 & 0.19 & 1986 & 0.19 & 1986 \\
\hline 29 & 0.03 & 8 & 7 & 0.18 & 1978 & 0.18 & 1978 \\
\hline 30 & 0.03 & 9 & 5 & 0.30 & 1962 & 0.34 & 1998 \\
\hline \multicolumn{8}{|c|}{ December } \\
\hline .1 & 0.03 & 8 & 4 & 0.19 & 1966 & 0.29 & 1955 \\
\hline 2 & 0.04 & 8 & 8 & 0.34 & $1985^{(2)}$ & 0.34 & $1985^{\text {(a) }}$ \\
\hline 3 & 0.04 & 7 & 9 & 0.56 & 1980 & 0.56 & 1980 \\
\hline 4 & 0.03 & 13 & 3 & 0.28 & 1974 & 0.28 & 1974 \\
\hline 5. & 0.04 & 11 & 7 & 0.43 & 1963 & 0.43 & 1963 \\
\hline 6 & 0.04 & 12 & 8 & 0.18 & 1985 & 0.18 & 1985 \\
\hline 7 & 0.03 & 8 & 5 & 0.32 & 1983 & 0.36 & 1948 \\
\hline 8 & 0.03 & 5 & 7 & 0.36 & 1963 & 0.36 & 1963 \\
\hline 9 & 0.03 & 9 & 8 & 0.55 & 1987 & 0.55 & 1987 \\
\hline 10 & 0.03 & 9 & 4 & 0.25 & 1990 & 0.54 & 1958 \\
\hline 11 & 0.04 & 6 & 8 & 0.33 & 1969 & 0.53 & 1958 \\
\hline 12 & 0.04 & 10 & 5 & 0.29 & 1982 & 0.65 & 1995 \\
\hline 13 & 0.03 & 8 & 5 & 0.76 & 1977 & 0.76 & 1977 \\
\hline 14 & 0.04 & 12 & 5 & 0.25 & 1964 & 0.25 & 1964 \\
\hline 15 & 0.04 & 15 & 2 & 0.22 & 1981 & 0.22 & 1981 \\
\hline 16 & 0.04 & 9 & 9 & 0.30 & 1961 & 0.37 & 1994 \\
\hline 17 & 0.04 & 10 & 5 & 0.22 & 1973 & 0.22 & 1973 \\
\hline 18 & 0.04 & 11 & 8 & 0.22 & 1981 & 0.27 & 1960 \\
\hline 19 & 0.04 & 15 & 7 & 0.19 & 1964 & 0.20 & 1953 \\
\hline 20 & 0.04 & 13 & 7 & 0.33 & 1982 & 0.33 & 1982 \\
\hline 21 & 0.04 & 10 & 10 & 0.54 & 1964 & 0.61 & 1955 \\
\hline
\end{tabular}


Table 4.10. (contd)

Normal Period (1961-1990)

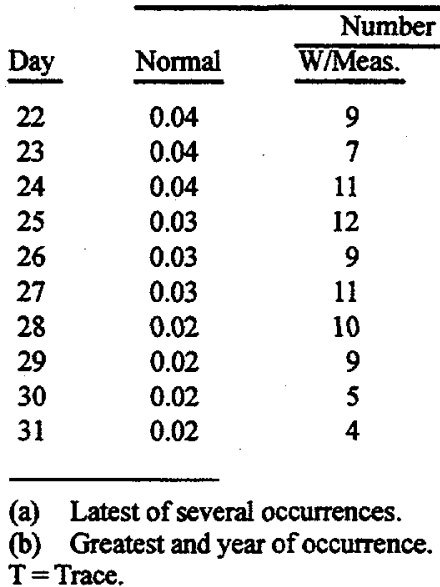

(b) Greatest and year of occurrence.
Historical Period (1945-1998)

\begin{tabular}{c} 
W/Trace \\
\hline 5 \\
7 \\
8 \\
6 \\
6 \\
7 \\
11 \\
7 \\
9 \\
9
\end{tabular}

\begin{tabular}{|c|c|}
\hline Maximum & Year \\
\hline 0.59 & 1964. \\
\hline 0.31 & 1975 \\
\hline 0.36 & 1968 \\
\hline 0.26 & 1980 \\
\hline 0.19 & 1973 \\
\hline 0.36 & 1973 \\
\hline 0.09 & $1990^{(2)}$ \\
\hline 0.55 & 1983 \\
\hline 0.15 & 1979 \\
\hline 0.29 & 1968 \\
\hline
\end{tabular}

\begin{tabular}{|c|c|}
\hline Maximum & Year \\
\hline 0.59 & 1964 \\
\hline 0.31 & 1975 \\
\hline 0.36 & 1968 \\
\hline 0.32 & 1996 \\
\hline 0.58 & 1996 \\
\hline 0.36 & 1973 \\
\hline 0.10 & 1951 \\
\hline $0.80^{(0)}$ & $1996^{(b)}$ \\
\hline 0.28 & 1995 \\
\hline 0.72 & 1996 \\
\hline
\end{tabular}




\subsection{Wind Climatology}

\subsection{Monthly and Annual Prevailing Wind Directions, Average Speeds, and Peak Gusts}

At the HMS, the prevailing wind direction for every month of the year is either WNW or NW (Table 5.1), and the peak gusts for every month are from the SSW, SW, or WSW. The highest monthly average wind speeds occur in June, the lowest in December. The variability in monthly average wind speeds is much greater in the winter months than during the remainder of the year. The highest January average $(10.3 \mathrm{mph})$ is $<3.5$ times greater than the lowest $(2.9 \mathrm{mph})$; however, in June, the highest average $(10.7 \mathrm{mph})$ is only 1.4 times greater than the lowest $(7.7 \mathrm{mph})$.

Table 5.1. Monthly and Annual Prevailing Wind Directions, Average Speeds, and Peak Gusts at 50-Foot Level, 1945 Through 1998

\begin{tabular}{|c|c|c|c|c|c|c|c|c|c|}
\hline \multirow[b]{2}{*}{ Month } & \multirow[b]{2}{*}{$\begin{array}{l}\text { Prevailing } \\
\text { Direction }\end{array}$} & \multirow{2}{*}{$\begin{array}{c}\text { Average } \\
\text { Speed, } \\
\text { mph }\end{array}$} & \multirow{2}{*}{$\begin{array}{c}\text { Highest } \\
\text { Average, } \\
\text { mph }\end{array}$} & \multirow{2}{*}{\multicolumn{2}{|c|}{$\begin{array}{c}\text { Lowest } \\
\text { Average, } \\
\text { mph }\end{array}$}} & & \multicolumn{3}{|c|}{ Peak Gusts } \\
\hline & & & & & & Year & $\begin{array}{c}\text { Speed, } \\
\text { mph }\end{array}$ & Direction & Year \\
\hline Jan & NW & 6.3 & 10.3 & 1972 & 2.9 & 1985 & 80 & SW & 1972 \\
\hline Feb & NW & 7.0 & 10.8 & 1976 & 4.6 & 1963 & 65 & SW & 1971 \\
\hline Mar & WNW & 8.2 & 10.7 & $1977^{(2)}$ & 5.9 & 1958 & 70 & SW & 1956 \\
\hline Apr & WNW & 8.9 & 11.1 & $1972^{(1)}$ & 7.4 & $1989^{(2)}$ & 73 & SSW & 1972 \\
\hline May & WNW & 8.8 & 10.7 & 1983 & 5.8 & 1957 & 71 & SSW & 1948 \\
\hline Jun & $\mathrm{NW}$ & 9.1 & 10.7 & $1983^{(a)}$ & 7.7 & $1950^{(2)}$ & 72 & SW & 1957 \\
\hline Jul & NW & 8.6 & 10.7 & 1983 & 6.8 & 1955 & 69 & WSW & 1979 \\
\hline Aug & WNW & 8.0 & 9.5 & 1996 & 6.0 & 1956 & 66 & SW & 1961 \\
\hline Sep & WNW & 7.5 & 9.2 & 1961 & 5.4 & 1957 & 65 & SSW & 1953 \\
\hline Oct & NW & 6.6 & 9.1 & 1946 & 4.4 & 1952 & 72 & SW & 1997 \\
\hline Nov & NW & 6.3 & 10.0 & 1990 & 2.9 & 1956 & 67 & WSW & 1993 \\
\hline Dec & NW & 6.0 & 8.3 & 1968 & 3.3 & 1985 & 71 & SW & 1955 \\
\hline Annual & NW & 7.6 & 8.4 & 1983 & 6.2 & 1989 & 80 & SW & $\operatorname{Jan} 1972$ \\
\hline
\end{tabular}

(a) Also in earlier years.

\subsection{Days with Peak Gusts Above or Below Specific Thresholds}

Table 5.2 lists the number of days by month and year with peak wind gusts (at $50 \mathrm{ft}$ ) above or below specific threshold wind speeds. June and July have the highest average number of days with gusts $\geq 25 \mathrm{mph}$ (nearly 20 each); however, January, March, and April have the highest average number of days with gusts $\geq 40 \mathrm{mph}$ (nearly 3 days) and January has the highest average number of days with gusts $\geq 50 \mathrm{mph}$ ( 0.8 day). January also has the record highest number of gusts $\geq 40$ and $\geq 50 \mathrm{mph}$ at 11 and 7 days, respectively, in 1990 . Calendar year 1990 recorded the most days with gusts $\geq 40$ and $\geq 50 \mathrm{mph}$ 
Table 5.2. Number of Days with Peak Gusts Above or Below Specific Thresholds at 50-Foot Level, 1945 Through 1998

\begin{tabular}{|c|c|c|c|c|c|}
\hline \multirow[b]{2}{*}{ Month } & \multicolumn{5}{|c|}{ Days with Peak Gusts $\leq 12 \mathrm{mph}$} \\
\hline & Avg & Max & Year & Min & Year \\
\hline Jan & 9.7 & 29 & 1985 & 3 & 1968 \\
\hline Feb & 6.5 & 16 & 1963 & 0 & 1990 \\
\hline Mar & 2.8 & 8 & 1992 & 0 & $1983^{(n)}$ \\
\hline Apr & 0.6 & 6 & 1951 & 0 & $1996^{(a)}$ \\
\hline May & 0.3 & 3 & 1955 & 0 & $1996^{(1)}$ \\
\hline Jun & 0.1 & 1 & $1980^{(a)}$ & $\mathbf{0}$ & $1998^{(\mathbf{a})}$ \\
\hline Jul & 0.1 & 1 & $1957^{(a)}$ & 0 & $1998^{(a)}$ \\
\hline Aug & 0.3 & 2 & 1972 & 0 & $1998^{(2)}$ \\
\hline Sep & 2.4 & 9 & 1987 & $\mathbf{0}$ & $1991^{(1)}$ \\
\hline Oct & 6.9 & 15 & 1974 & 2 & $1975^{(a)}$ \\
\hline Nov & 9.1 & 20 & $1956^{(a)}$ & 2 & $1977^{(2)}$ \\
\hline Dec & 11.0 & 23 & 1985 & 3 & 1968 \\
\hline Annual & 49.8 & 87 & 1952 & 28 & 1973 \\
\hline
\end{tabular}

\begin{tabular}{rrrrr}
\multicolumn{6}{c}{ Days with Peak Gusts 225 mph } \\
\hline \multicolumn{1}{c}{ Avg } & Max & Year & Min & Year \\
\hline 7.6 & 21 & 1953 & 0 & $1985^{(a)}$ \\
8.6 & 17 & $1976^{(a)}$ & 2 & $1952^{(a)}$ \\
12.9 & 21 & 1977 & 4 & 1992 \\
17.2 & 26 & 1954 & 8 & 1946 \\
18.5 & 26 & 1978 & 9 & 1945 \\
19.6 & 26 & 1963 & 11 & $1950^{(a)}$ \\
19.4 & 26 & 1995 & 11 & 1955 \\
15.6 & 22 & $1988^{(a)}$ & 7 & 1945 \\
11.2 & 17 & 1971 & 7 & $1975^{(a)}$ \\
8.8 & 17 & $1985^{(a)}$ & 3 & $1987^{(a)}$ \\
8.4 & 16 & 1990 & 0 & 1979 \\
7.5 & 15 & 1968 & 0 & 1985 \\
155.2 & 190 & 1953 & 123 & 1952
\end{tabular}

\begin{tabular}{cccccc}
\multicolumn{6}{c}{ Days with Peak Gusts $\geq 40$ mph } \\
\hline Avg & Max & Year & Min & Year \\
\hline 2.8 & 11 & $1990^{(a)}$ & 0 & $1995^{(a)}$ \\
2.4 & 10 & 1976 & 0 & $1993^{(a)}$ \\
2.8 & 9 & 1956 & 0 & $1998^{(a)}$ \\
2.8 & 8 & 1991 & 0 & $1998^{(a)}$ \\
2.2 & 6 & $1990^{(a)}$ & 0 & $1997^{(a)}$ \\
2.3 & 7 & 1985 & 0 & $1982^{(a)}$ \\
1.8 & 5 & $1995^{(a)}$ & 0 & $1981^{(a)}$ \\
1.2 & 5 & 1951 & 0 & $1993^{(a)}$ \\
1.3 & 4 & 1946 & 0 & $1998^{(a)}$ \\
1.8 & 8 & 1967 & 0 & $1993^{(a)}$ \\
2.4 & 8 & 1990 & 0 & $1982^{(a)}$ \\
2.5 & 8 & $1957^{(a)}$ & 0 & $1989^{(a)}$ \\
26.4 & 57 & 1990 & 10 & 1978
\end{tabular}

\begin{tabular}{cccccc}
\multicolumn{6}{c}{ Days with Peak Gusts $250 \mathrm{mph}$} \\
\hline Avg & Max & Year & Min & Year \\
\hline 0.8 & 7 & 1990 & 0 & $1998^{(\mathrm{a})}$ \\
0.6 & 4 & 1972 & 0 & $1995^{(\mathrm{a})}$ \\
0.6 & 4 & 1956 & 0 & $1998^{(\mathrm{a})}$ \\
0.4 & 2 & $1997^{(\mathrm{a})}$ & 0 & $1998^{(\mathrm{a})}$ \\
0.2 & 2 & $1993^{(\mathrm{a})}$ & 0 & $1996^{(\mathrm{a})}$ \\
0.3 & 2 & $1992^{(\mathrm{a})}$ & 0 & $1996^{(\mathrm{a})}$ \\
0.2 & 1 & $1995^{(\mathrm{a})}$ & 0 & $1998^{(\mathrm{a})}$ \\
0.1 & 1 & $1998^{(\mathrm{a})}$ & 0 & $1996^{(\mathrm{a})}$ \\
0.2 & 2 & 1953 & 0 & $1998^{(\mathrm{a})}$ \\
0.2 & 2 & 1967 & 0 & $1998^{(\mathrm{a})}$ \\
0.6 & 4 & $1998^{(\mathrm{a})}$ & 0 & $1996^{(\mathrm{a})}$ \\
0.7 & 3 & $1995^{(\mathrm{a})}$ & 0 & $1996^{(\mathrm{a})}$ \\
4.8 & 18 & 1990 & 0 & $1985^{1}$
\end{tabular}

(a) Most recent of multiple occurrences. 
at 57 and 18 days, respectively. Of particular interest is that previous records for these categories were 41 days $\geq 40 \mathrm{mph}$ in 1961 and 10 days $\geq 50 \mathrm{mph}$ in 1972 .

\subsection{Frequency of Monthly and Annual Wind Direction and Speed at 50-Foot Level}

Table 5.3 presents HMS data on the percent frequency of monthly and annual wind direction and wind speed at $50 \mathrm{ft}$. This table shows that for every month of the year the prevailing wind direction is either from the WNW or NW. Winds are relatively evenly distributed from the NNE through the SSW at between $2 \%$ and $4 \%$ on an annual average for each direction.

Table 5.3. Frequency (\%) of Monthly and Annual Wind Direction and Speed at 50-Foot Level, 1955 Through 1998

\begin{tabular}{|c|c|c|c|c|c|c|c|c|c|c|c|c|c|}
\hline Direction & Jan & Feb & Mar & Apr & May & Jun & Jul & Aug & Sep & Oct & Nov & Dec & Total \\
\hline $\begin{array}{l}\text { N } \\
\text { NNE } \\
\text { NE } \\
\text { ENE } \\
\text { E } \\
\text { ESE } \\
\text { SE } \\
\text { SSE } \\
\text { S }\end{array}$ & $\begin{array}{l}4.3 \\
3.7 \\
3.0 \\
2.3 \\
2.6 \\
2.8 \\
4.1 \\
3.4 \\
3.3\end{array}$ & $\begin{array}{l}4.8 \\
4.7 \\
3.8 \\
2.1 \\
2.2 \\
2.6 \\
3.7 \\
3.3 \\
3.3\end{array}$ & $\begin{array}{l}4.7 \\
4.5 \\
3.5 \\
2.1 \\
2.2 \\
2.7 \\
3.8 \\
3.4 \\
3.5\end{array}$ & $\begin{array}{l}4.1 \\
3.6 \\
3.4 \\
2.4 \\
2.5 \\
2.5 \\
3.0 \\
3.0 \\
3.1\end{array}$ & $\begin{array}{l}3.8 \\
3.5 \\
3.4 \\
2.5 \\
2.4 \\
2.6 \\
3.1 \\
3.0 \\
2.7\end{array}$ & $\begin{array}{l}3.6 \\
3.2 \\
3.2 \\
2.2 \\
2.5 \\
2.7 \\
2.9 \\
2.7 \\
2.7\end{array}$ & $\begin{array}{l}4.6 \\
4.2 \\
3.7 \\
2.6 \\
2.8 \\
2.8 \\
3.0 \\
2.5 \\
2.5\end{array}$ & $\begin{array}{l}4.7 \\
4.0 \\
3.5 \\
2.5 \\
3.1 \\
3.3 \\
3.5 \\
2.8 \\
2.6\end{array}$ & $\begin{array}{l}5.6 \\
5.5 \\
4.2 \\
2.4 \\
3.0 \\
3.3 \\
3.8 \\
3.2 \\
2.7\end{array}$ & $\begin{array}{l}5.0 \\
4.1 \\
3.5 \\
2.6 \\
2.9 \\
3.7 \\
5.0 \\
4.0 \\
3.6\end{array}$ & $\begin{array}{l}4.4 \\
3.5 \\
3.0 \\
2.5 \\
2.7 \\
3.2 \\
4.4 \\
3.9 \\
4.1\end{array}$ & $\begin{array}{l}4.3 \\
3.3 \\
2.9 \\
2.5 \\
2.7 \\
3.2 \\
4.5 \\
3.7 \\
3.6\end{array}$ & $\begin{array}{c}4.5 \\
4.0 \\
3.4 \\
2.4 \\
2.6 \\
3.0 \\
3.7 \\
3.2 \\
3.1\end{array}$ \\
\hline $\begin{array}{l}\text { SSW } \\
\text { SW } \\
\text { WSW } \\
\text { W } \\
\text { WNW } \\
\text { NW } \\
\text { NNW } \\
\text { Calm }\end{array}$ & $\begin{array}{r}4.7 \\
6.4 \\
6.5 \\
6.6 \\
15.4 \\
19.4 \\
7.4 \\
4.2\end{array}$ & $\begin{array}{r}4.4 \\
7.7 \\
7.3 \\
8.2 \\
14.7 \\
17.9 \\
6.9 \\
2.4\end{array}$ & $\begin{array}{r}5.0 \\
9.1 \\
10.3 \\
9.6 \\
14.6 \\
14.4 \\
5.8 \\
0.8\end{array}$ & $\begin{array}{r}4.3 \\
8.8 \\
11.8 \\
11.6 \\
16.5 \\
14.3 \\
4.6 \\
0.5\end{array}$ & $\begin{array}{r}3.6 \\
6.7 \\
10.4 \\
11.5 \\
18.4 \\
17.5 \\
4.3 \\
0.5\end{array}$ & $\begin{array}{r}3.4 \\
6.6 \\
9.7 \\
10.9 \\
19.1 \\
19.4 \\
4.8 \\
0.4\end{array}$ & $\begin{array}{r}2.7 \\
5.7 \\
8.4 \\
9.6 \\
19.4 \\
20.0 \\
5.3 \\
0.3\end{array}$ & $\begin{array}{r}3.0 \\
6.0 \\
8.8 \\
10.8 \\
18.0 \\
17.7 \\
5.4 \\
0.5\end{array}$ & $\begin{array}{r}3.3 \\
5.9 \\
9.3 \\
11.3 \\
15.1 \\
14.7 \\
5.9 \\
1.0\end{array}$ & $\begin{array}{r}4.2 \\
6.9 \\
8.6 \\
10.3 \\
13.2 \\
13.5 \\
6.5 \\
2.2\end{array}$ & $\begin{array}{r}5.3 \\
7.7 \\
7.8 \\
8.3 \\
12.6 \\
15.8 \\
7.1 \\
3.8\end{array}$ & $\begin{array}{r}4.6 \\
6.8 \\
7.2 \\
7.2 \\
13.7 \\
18.1 \\
7.1 \\
4.8\end{array}$ & $\begin{array}{r}4.1 \\
7.0 \\
8.8 \\
9.6 \\
15.9 \\
16.9 \\
5.9 \\
1.8\end{array}$ \\
\hline Total & 100.0 & 100.0 & 100.0 & 100.0 & 100.0 & 100.0 & 100.0 & 100.0 & 100.0 & 100.0 & 100.0 & 100.0 & 100.0 \\
\hline Speed, mph & Jan & Feb & Mar & Apr & May & Jun & Jul & Aug & Sep & Oct & Nov & Dec & Total \\
\hline $\begin{array}{l}\text { Calm } \\
1-3 \\
4-7 \\
8-12 \\
13-18 \\
19-24 \\
25-31 \\
32-38 \\
39-46 \\
>46\end{array}$ & $\begin{array}{r}4.2 \\
30.8 \\
34.4 \\
20.1 \\
6.8 \\
2.5 \\
1.0 \\
0.3 \\
0.0 \\
0.0\end{array}$ & $\begin{array}{r}2.4 \\
25.1 \\
35.3 \\
23.9 \\
8.7 \\
3.1 \\
1.2 \\
0.3 \\
0.0 \\
0.0\end{array}$ & $\begin{array}{r}0.8 \\
17.1 \\
36.5 \\
27.4 \\
12.0 \\
4.5 \\
1.4 \\
0.2 \\
0.0 \\
0.0\end{array}$ & $\begin{array}{r}0.5 \\
13.3 \\
34.7 \\
28.5 \\
15.7 \\
5.8 \\
1.5 \\
0.1 \\
0.0 \\
0.0\end{array}$ & $\begin{array}{r}0.5 \\
11.9 \\
35.1 \\
30.5 \\
15.6 \\
5.4 \\
0.9 \\
0.0 \\
0.0 \\
0.0\end{array}$ & $\begin{array}{r}0.4 \\
9.7 \\
35.8 \\
30.2 \\
16.6 \\
6.2 \\
1.1 \\
0.0 \\
0.0 \\
0.0\end{array}$ & $\begin{array}{r}0.3 \\
10.9 \\
39.2 \\
29.2 \\
14.3 \\
5.3 \\
0.9 \\
0.0 \\
0.0 \\
0.0\end{array}$ & $\begin{array}{r}0.5 \\
13.6 \\
42.0 \\
27.6 \\
11.8 \\
4.0 \\
0.6 \\
0.0 \\
0.0 \\
0.0\end{array}$ & $\begin{array}{r}1.0 \\
18.2 \\
41.3 \\
25.8 \\
9.9 \\
3.2 \\
0.6 \\
0.0 \\
0.0 \\
0.0\end{array}$ & $\begin{array}{r}2.2 \\
26.6 \\
38.9 \\
21.5 \\
7.6 \\
2.6 \\
0.6 \\
0.0 \\
0.0 \\
0.0\end{array}$ & $\begin{array}{r}3.8 \\
29.7 \\
35.4 \\
20.0 \\
7.0 \\
2.8 \\
1.0 \\
0.2 \\
0.0 \\
0.0\end{array}$ & $\begin{array}{r}4.8 \\
33.3 \\
33.5 \\
18.3 \\
6.4 \\
2.5 \\
0.9 \\
0.2 \\
0.0 \\
0.0\end{array}$ & $\begin{array}{r}1.8 \\
20.0 \\
36.8 \\
25.2 \\
11.0 \\
4.0 \\
1.0 \\
0.1 \\
0.0 \\
0.0\end{array}$ \\
\hline Total & 100.0 & 100.0 & 100.0 & 100.0 & 100.0 & 100.0 & 100.0 & 100.0 & 100.0 & 100.0 & 100.0 & 100.0 & 100.0 \\
\hline
\end{tabular}

The wind speed class with the highest frequency of occurrence is 4 to $7 \mathrm{mph}$, with winds in that category $37 \%$ of the time. The speed class with the second highest frequency is 8 to $12 \mathrm{mph}$, at $25 \%$. Winds averaging $>25 \mathrm{mph}$ occur only $1 \%$ of the time on an annual basis, with the highest frequency occurring in March and April (1.5\%). 


\subsection{Composite Wind Roses and Joint Frequency Distributions for the Hanford Meteorological Monitoring Network}

Figure 5.1 and Table 5.4 contain composite wind roses and joint frequency distributions at the 10-m level for the entire HMMN (see Table 1.1 and Figure 1.1) for the period 1982 through 1998.

Figure 5.2 and Table 5.5 contain composite wind roses and joint frequency distributions at the $60-\mathrm{m}$ level for stations 9, 11, 13, and 21 for the period 1986 through 1998. 


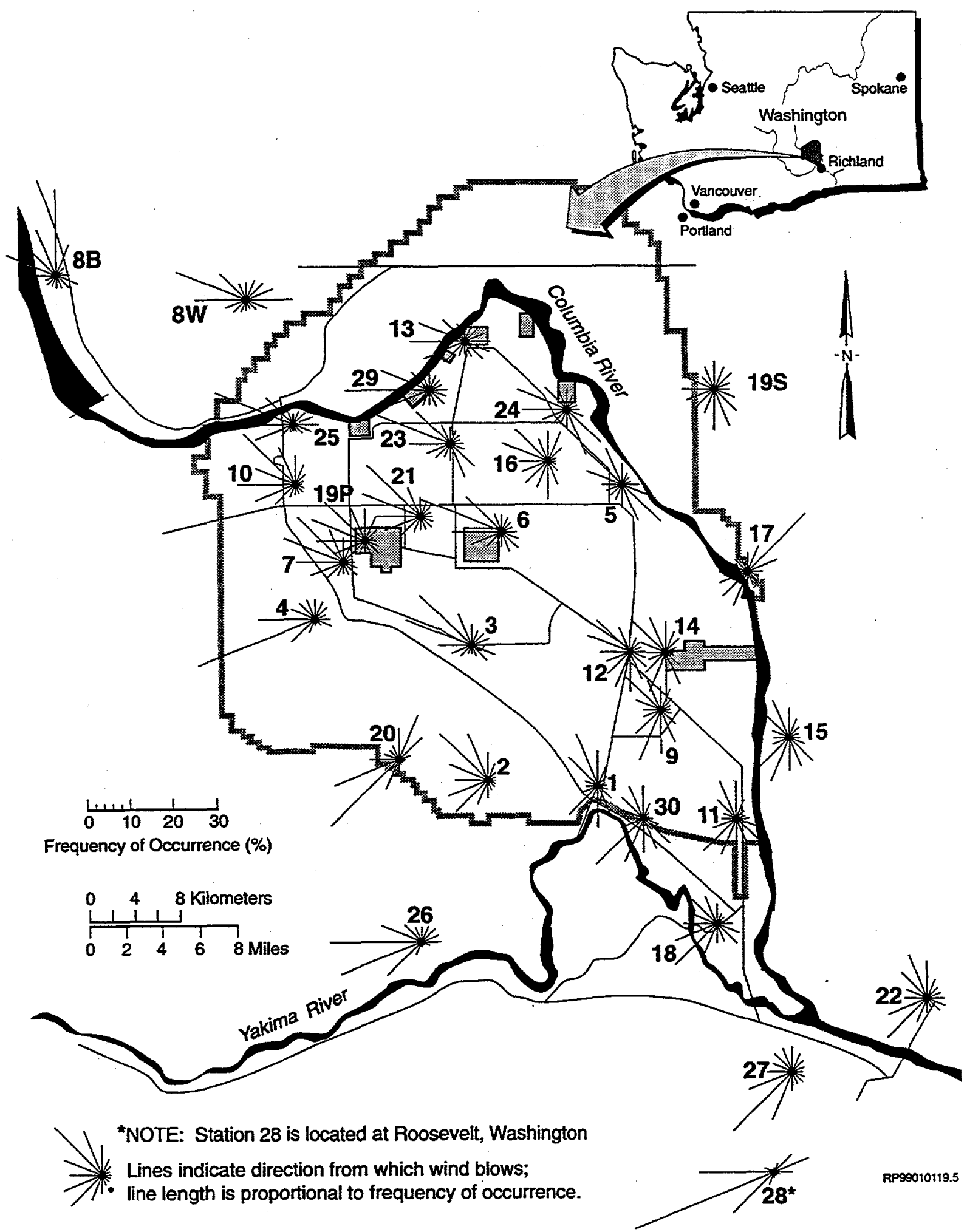

Figure 5.1. Hanford Meteorological Monitoring Network Wind Roses at 10-Meter Level, 1982 Through 1998 
Table 5.4. Joint Frequency Distributions (\%) for Hanford Meteorological Monitoring Network Wind Stations at 10-Meter Level, 1982 Through 1998

Station: ( 1) PROS

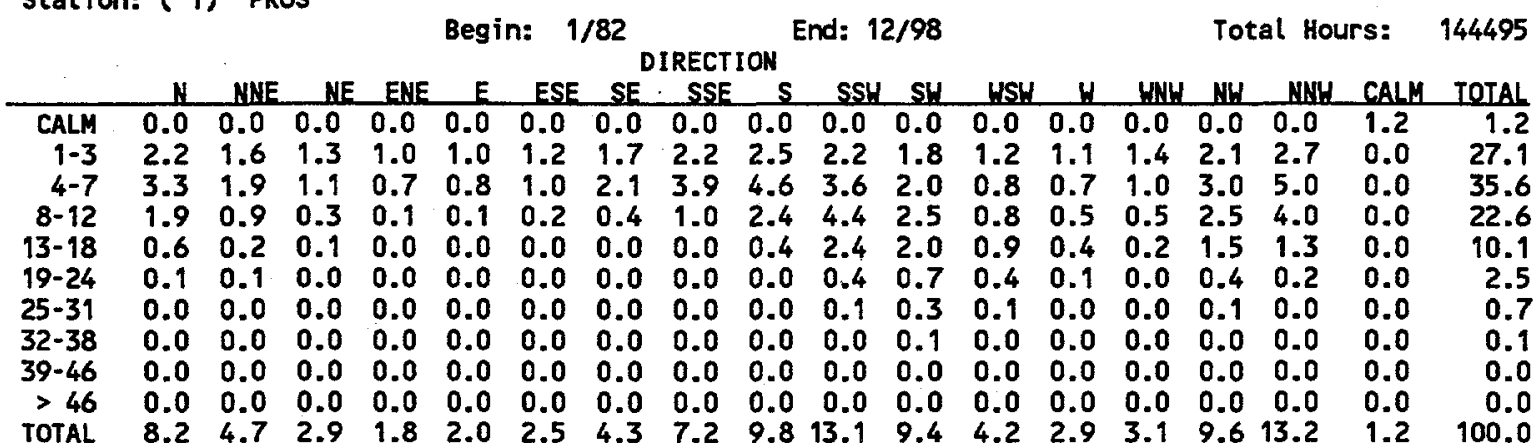

Station: ( 2) EOC

Begin: $1 / 82 \quad$ End: $12 / 98$

Total Hours: 145356

DIRECTION

H NNE HE ENE E ESE SE SSE S SSH SH WSH $W$ WNH NH NHH CALH TOTAL

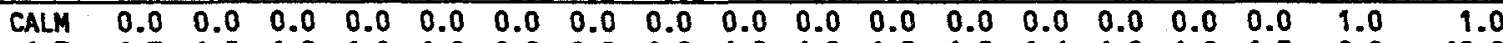

$\begin{array}{lllllllllllllllllll}1-3 & 1.3 & 1.2 & 1.2 & 1.0 & 1.0 & 0.9 & 0.8 & 0.9 & 1.2 & 1.2 & 1.2 & 1.2 & 1.6 & 1.8 & 1.8 & 1.5 & 0.0 & 19.8\end{array}$

$\begin{array}{lllllllllllllllllll}4-7 & 2.8 & 1.9 & 1.2 & 0.9 & 1.0 & 1.1 & 1.1 & 1.2 & 1.9 & 2.1 & 1.5 & 1.2 & 2.0 & 3.6 & 4.2 & 3.4 & 0.0 & 31.1\end{array}$

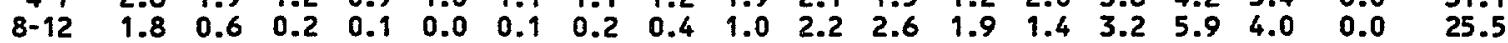

$\begin{array}{lllllllllllllllllll}13-18 & 0.6 & 0.2 & 0.1 & 0.0 & 0.0 & 0.0 & 0.0 & 0.0 & 0.1 & 0.6 & 2.1 & 2.2 & 1.4 & 0.7 & 3.1 & 2.4 & 0.0 & 13.6\end{array}$

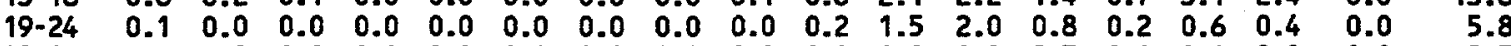

$\begin{array}{lllllllllllllllllll}25-31 & 0.0 & 0.0 & 0.0 & 0.0 & 0.0 & 0.0 & 0.0 & 0.0 & 0.0 & 0.0 & 1.0 & 1.0 & 0.3 & 0.1 & 0.1 & 0.0 & 0.0 & 2.5\end{array}$

$\begin{array}{lllllllllllllllllll}32-38 & 0.0 & 0.0 & 0.0 & 0.0 & 0.0 & 0.0 & 0.0 & 0.0 & 0.0 & 0.0 & 0.3 & 0.2 & 0.1 & 0.0 & 0.0 & 0.0 & 0.0 & 0.5\end{array}$

$\begin{array}{lllllllllllllllllll}39-46 & 0.0 & 0.0 & 0.0 & 0.0 & 0.0 & 0.0 & 0.0 & 0.0 & 0.0 & 0.0 & 0.0 & 0.0 & 0.0 & 0.0 & 0.0 & 0.0 & 0.0 & 0.1\end{array}$

$\begin{array}{lllllllllllllllllll}>46 & 0.0 & 0.0 & 0.0 & 0.0 & 0.0 & 0.0 & 0.0 & 0.0 & 0.0 & 0.0 & 0.0 & 0.0 & 0.0 & 0.0 & 0.0 & 0.0 & 0.0 & 0.0\end{array}$

$\begin{array}{lllllllllllllllllll}\text { TOTAL } & 6.7 & 4.0 & 2.7 & 2.0 & 2.0 & 2.0 & 2.2 & 2.5 & 4.2 & 6.3 & 10.4 & 9.7 & 7.6 & 9.5 & 15.5 & 11.7 & 1.0 & 100.0\end{array}$

Station: ( 3) ARMY

Begin: $1 / 82$

End: $12 / 98$

Total Hours:

145260

DIRECTION

\begin{tabular}{rrrllllllllllllllll} 
& N & NNE & NE & EHE & E & ESE & SE & SSE & S & SSH & SH & USH & H & HH & HH & NWH & CALH & TOTAL \\
\hline CALM & 0.0 & 0.0 & 0.0 & 0.0 & 0.0 & 0.0 & 0.0 & 0.0 & 0.0 & 0.0 & 0.0 & 0.0 & 0.0 & 0.0 & 0.0 & 0.0 & 1.0 & 1.0 \\
$1-3$ & 1.9 & 1.7 & 2.0 & 2.3 & 2.6 & 2.4 & 1.9 & 1.2 & 0.9 & 0.8 & 0.9 & 1.2 & 2.2 & 3.2 & 3.2 & 2.4 & 0.0 & 30.8 \\
$4-7$ & 2.1 & 1.4 & 1.5 & 1.9 & 2.5 & 2.8 & 2.1 & 0.9 & 0.6 & 0.5 & 0.6 & 0.9 & 2.4 & 7.2 & 7.6 & 3.4 & 0.0 & 38.5 \\
$8-12$ & 0.9 & 0.5 & 0.3 & 0.2 & 0.4 & 0.7 & 0.9 & 0.5 & 0.4 & 0.4 & 0.6 & 1.1 & 1.8 & 4.6 & 4.0 & 1.5 & 0.0 & 18.8 \\
$13-18$ & 0.2 & 0.2 & 0.1 & 0.0 & 0.0 & 0.1 & 0.2 & 0.2 & 0.2 & 0.3 & 0.6 & 1.1 & 1.0 & 1.1 & 1.5 & 0.4 & 0.0 & 7.1 \\
$19-24$ & 0.0 & 0.1 & 0.0 & 0.0 & 0.0 & 0.0 & 0.0 & 0.0 & 0.0 & 0.2 & 0.5 & 0.6 & 0.3 & 0.2 & 0.6 & 0.1 & 0.0 & 2.6 \\
$25-31$ & 0.0 & 0.0 & 0.0 & 0.0 & 0.0 & 0.0 & 0.0 & 0.0 & 0.0 & 0.1 & 0.3 & 0.2 & 0.1 & 0.0 & 0.1 & 0.0 & 0.0 & 0.9 \\
$32-38$ & 0.0 & 0.0 & 0.0 & 0.0 & 0.0 & 0.0 & 0.0 & 0.0 & 0.0 & 0.0 & 0.1 & 0.0 & 0.0 & 0.0 & 0.0 & 0.0 & 0.0 & 0.2 \\
$39-46$ & 0.0 & 0.0 & 0.0 & 0.0 & 0.0 & 0.0 & 0.0 & 0.0 & 0.0 & 0.0 & 0.0 & 0.0 & 0.0 & 0.0 & 0.0 & 0.0 & 0.0 & 0.0 \\
$>46$ & 0.0 & 0.0 & 0.0 & 0.0 & 0.0 & 0.0 & 0.0 & 0.0 & 0.0 & 0.0 & 0.0 & 0.0 & 0.0 & 0.0 & 0.0 & 0.0 & 0.0 & 0.0 \\
TOTAL & 5.1 & 3.8 & 3.9 & 4.4 & 5.6 & 6.0 & 5.1 & 2.9 & 2.1 & 2.2 & 3.7 & 5.1 & 7.8 & 16.3 & 17.0 & 7.9 & 1.0 & 100.0
\end{tabular}

Station: ( 4) RSPG

Begin: $1 / 82$

DIRECTION

End: $12 / 98$

Total Hours: 144288

N NINE ME ENE E ESE SE SSE S SSH SH HSH 4 HNH NH MMY CALM TOTAL

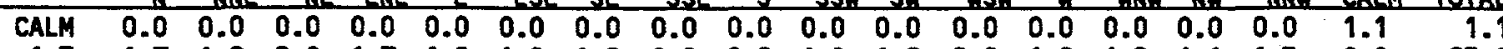

$\begin{array}{lllllllllllllllllll}1-3 & 1.7 & 1.9 & 2.0 & 1.7 & 1.8 & 1.8 & 1.2 & 0.8 & 0.8 & 1.0 & 1.9 & 2.8 & 1.9 & 1.2 & 1.1 & 1.3 & 0.0 & 25.0\end{array}$

$\begin{array}{lllllllllllllllllll}4-7 & 2.5 & 2.1 & 1.5 & 1.0 & 1.6 & 2.1 & 0.9 & 0.4 & 0.5 & 0.7 & 2.4 & 10.1 & 4.5 & 1.8 & 1.8 & 2.2 & 0.0 & 36.2\end{array}$

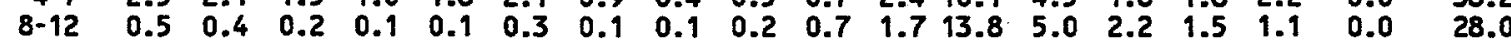

$\begin{array}{lllllllllllllllllll}13-18 & 0.0 & 0.1 & 0.1 & 0.0 & 0.0 & 0.0 & 0.0 & 0.0 & 0.1 & 0.5 & 1.0 & 2.0 & 1.7 & 0.9 & 0.7 & 0.4 & 0.0 & 7.5\end{array}$

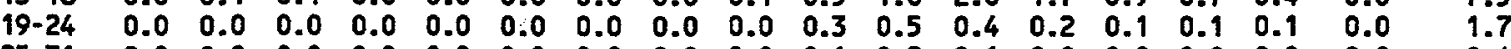

$\begin{array}{lllllllllllllllllll}25-31 & 0.0 & 0.0 & 0.0 & 0.0 & 0.0 & 0.0 & 0.0 & 0.0 & 0.0 & 0.1 & 0.2 & 0.1 & 0.0 & 0.0 & 0.0 & 0.0 & 0.0 & 0.4\end{array}$

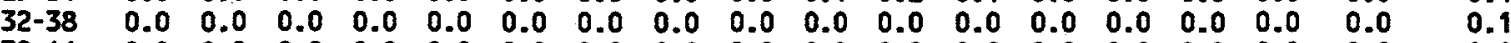

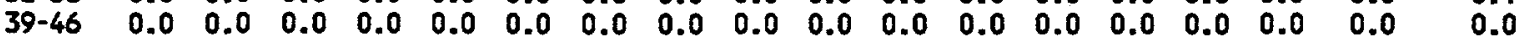

$\begin{array}{lllllllllllllllllll}>46 & 0.0 & 0.0 & 0.0 & 0.0 & 0.0 & 0.0 & 0.0 & 0.0 & 0.0 & 0.0 & 0.0 & 0.0 & 0.0 & 0.0 & 0.0 & 0.0 & 0.0 & 0.0\end{array}$

$\begin{array}{lllllllllllllllllll}\text { TOTAL } & 4.8 & 4.6 & 3.9 & 2.7 & 3.6 & 4.1 & 2.1 & 1.2 & 1.6 & 3.4 & 7.7 & 29.2 & 13.4 & 6.2 & 5.2 & 5.1 & 1.1 & 100.0\end{array}$ 
Table 5.4. (contd)

Station: ( 5) EDNA

Begin: $1 / 82$

End: $12 / 98$

Total Hours: 145531

DIRECTION

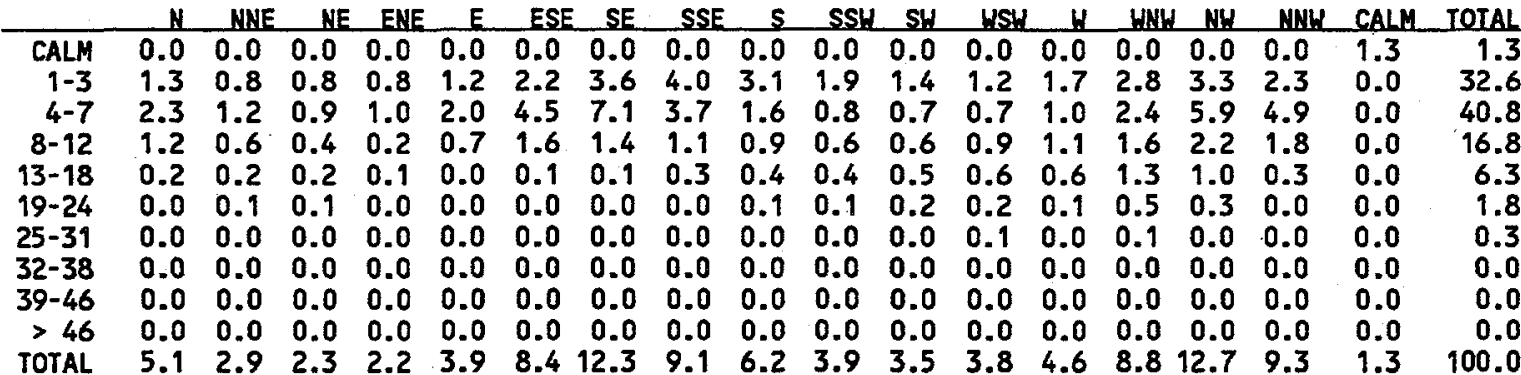

Station: (6) $200 E$

Begin: 1/82 End: 12/98

Total Hours: 145040 DIRECTION

\begin{tabular}{|c|c|c|c|c|c|c|c|c|c|c|c|c|c|c|c|c|c|c|c|c|}
\hline $\begin{array}{r}\text { CALM } \\
1-3 \\
4-7 \\
8-12 \\
13-18 \\
19-24 \\
25-31 \\
32-38 \\
39-46 \\
>46\end{array}$ & $\begin{array}{l}0.0 \\
1.6 \\
1.6 \\
0.7 \\
0.2 \\
0.0 \\
0.0 \\
0.0 \\
0.0 \\
0.0\end{array}$ & $\begin{array}{l}0.0 \\
1.4 \\
1.4 \\
0.7 \\
0.2 \\
0.1 \\
0.0 \\
0.0 \\
0.0 \\
0.0 \\
3.8\end{array}$ & $\begin{array}{l}0.0 \\
1.5 \\
1.0 \\
0.3 \\
0.2 \\
0.0 \\
0.0 \\
0.0 \\
0.0 \\
0.0 \\
3.1\end{array}$ & $\begin{array}{l}0.0 \\
1.6 \\
1.0 \\
0.1 \\
0.0 \\
0.0 \\
0.0 \\
0.0 \\
0.0 \\
0.0 \\
2.8\end{array}$ & $\begin{array}{l}0.0 \\
1.8 \\
1.5 \\
0.2 \\
0.0 \\
0.0 \\
0.0 \\
0.0 \\
0.0 \\
0.0 \\
3.5\end{array}$ & $\begin{array}{l}0.0 \\
1.7 \\
2.1 \\
0.4 \\
0.0 \\
0.0 \\
0.0 \\
0.0 \\
0.0 \\
0.0\end{array}$ & $\begin{array}{l}0.0 \\
1.7 \\
2.9 \\
0.8 \\
0.1 \\
0.0 \\
0.0 \\
0.0 \\
0.0 \\
0.0 \\
5.5\end{array}$ & $\begin{array}{l}0.0 \\
1.3 \\
2.3 \\
1.1 \\
0.2 \\
0.0 \\
0.0 \\
0.0 \\
0.0 \\
0.0\end{array}$ & $\begin{array}{l}0.0 \\
1.1 \\
1.5 \\
0.7 \\
0.2 \\
0.0 \\
0.0 \\
0.0 \\
0.0 \\
0.0\end{array}$ & $\begin{array}{l}0.0 \\
1.0 \\
1.1 \\
0.5 \\
0.3 \\
0.2 \\
0.0 \\
0.0 \\
0.0 \\
0.0 \\
3.1\end{array}$ & $\begin{array}{l}0.0 \\
1.0 \\
1.5 \\
1.0 \\
0.8 \\
0.5 \\
0.2 \\
0.0 \\
0.0 \\
0.0\end{array}$ & $\begin{array}{l}0 . \\
1 . \\
2 . \\
2 . \\
1 . \\
0 . \\
0 . \\
0 . \\
0 .\end{array}$ & & & $\begin{array}{l}0.0 \\
1.8 \\
6.2 \\
7.5 \\
3.5 \\
1.0 \\
0.2 \\
0.0 \\
0.0 \\
0.0\end{array}$ & & & $\begin{array}{l}0.0 \\
1.7 \\
2.2 \\
0.7 \\
0.1 \\
0.0 \\
0.0 \\
0.0 \\
0.0 \\
0.0 \\
4.7\end{array}$ & $\begin{array}{l}0.8 \\
0.0 \\
0.0 \\
0.0 \\
0.0 \\
0.0 \\
0.0 \\
0.0 \\
0.0 \\
0.0 \\
0.8\end{array}$ & $\begin{array}{r}0.8 \\
23.7\end{array}$ \\
\hline
\end{tabular}

Station: ( 7 ) $200 \mathrm{~W}$

Begin: $1 / 82$

End: $12 / 98$

Total Hours: 131354

DIRECTION

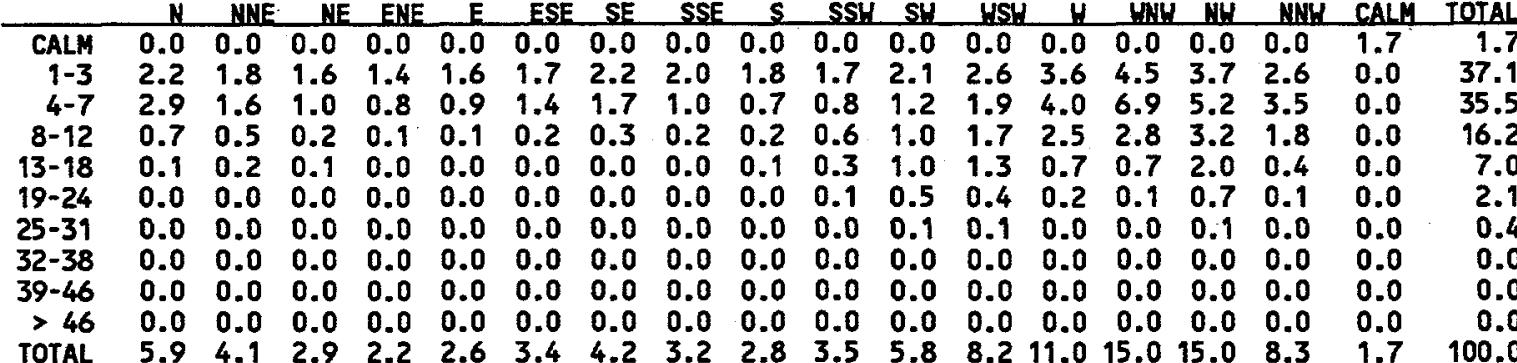

Station: ( 8) BVLY

Begin: 8/91 End: $12 / 98$

Total Hours: 64304

DIRECTION

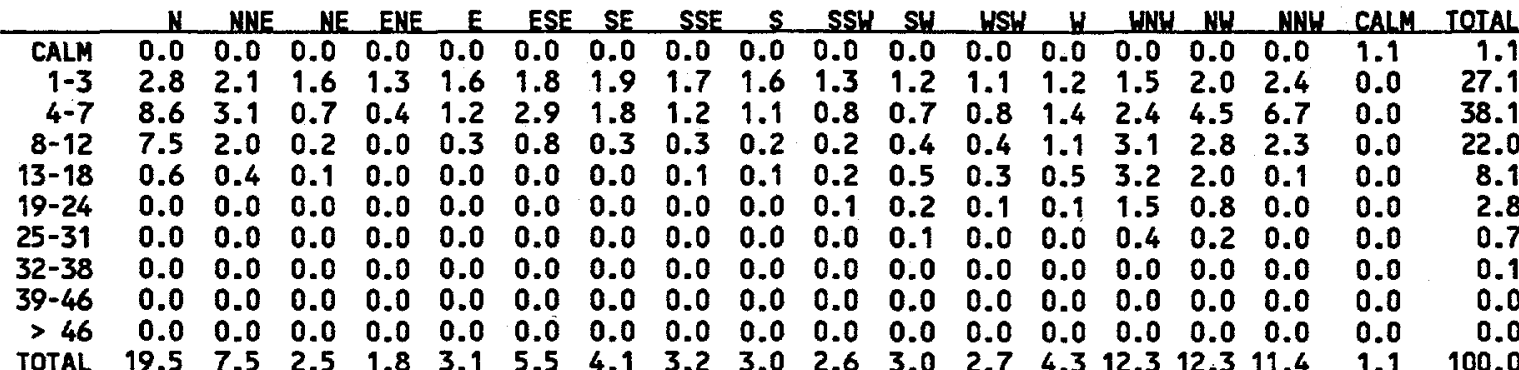


Table 5.4. (contd)

Station: (9) FFTF

Begin: 1/82 End: 12/98

Total Hours: 144673

DIRECTION

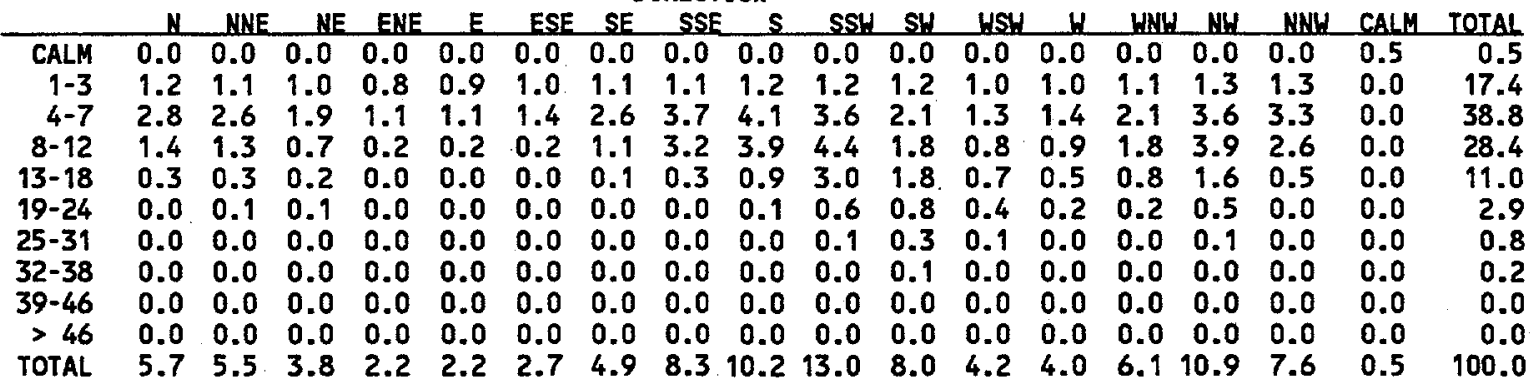

Station: (10) YAKB

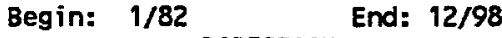

Total Hours: 145347

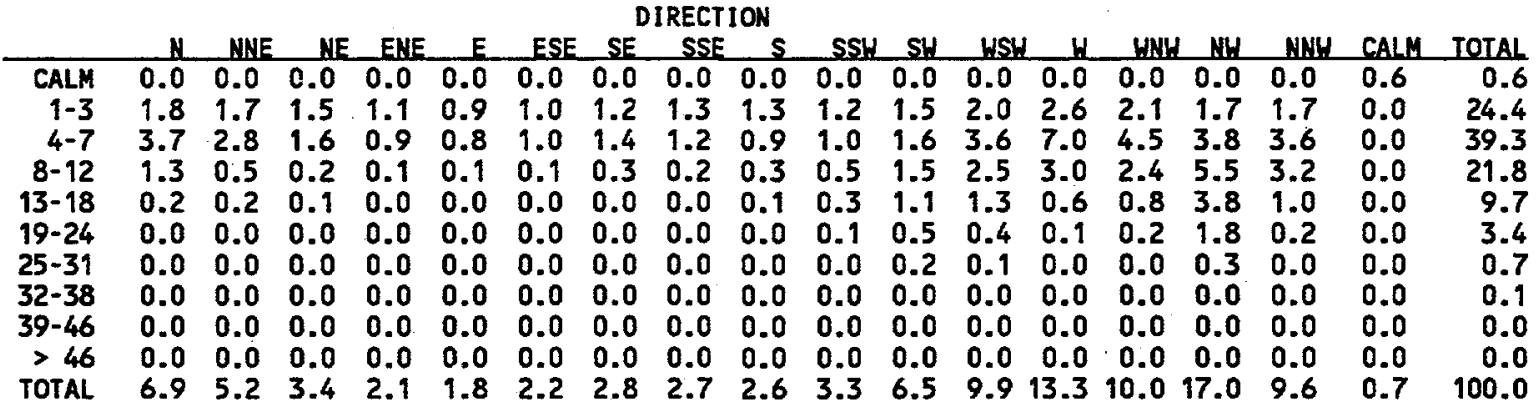

Station: (11) 300A

Begin: 1/82 End: 12/98

Total Hours: 132142

DIRECTION

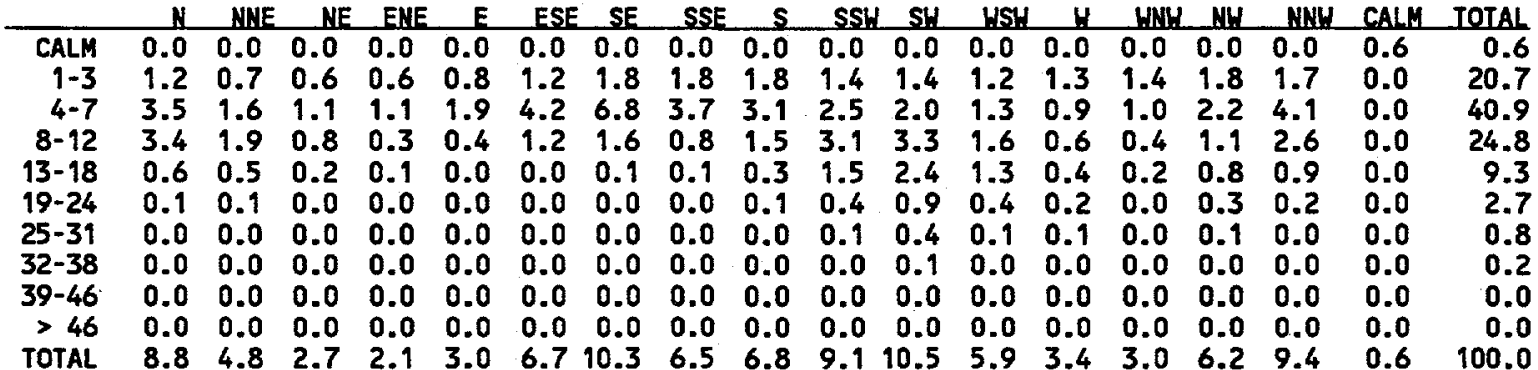

Station: (12) WYEB

$\begin{array}{ll}\text { Begin: 1/82 End: 12/98 } & \text { 12 }\end{array}$

Total Hours: 144681 DIRECTION

\begin{tabular}{|c|c|c|c|c|c|c|c|c|c|c|c|c|c|c|c|c|c|c|}
\hline & $N$ & NNE & NE & ENE & $E$ & ESE & & & & ;SH & & WSW & & WWI & & $\mathrm{I}$ is & ALM & OTAl \\
\hline $\begin{array}{r}\text { CALM } \\
1-3 \\
4-7 \\
8-12 \\
13-18 \\
19-24 \\
25-31 \\
32-38 \\
39-46 \\
>46 \\
\text { TOTAL }\end{array}$ & $\begin{array}{l}0.0 \\
1.3 \\
2.5 \\
1.2 \\
0.3 \\
0.0 \\
0.0\end{array}$ & $\begin{array}{l}0.0 \\
1.2 \\
1.6 \\
0.6 \\
0.2 \\
0.1 \\
0.0 \\
0.0 \\
0.0 \\
0.0 \\
3.7\end{array}$ & $\begin{array}{l}0.0 \\
1.2 \\
1.3 \\
0.4 \\
0.1 \\
0.0 \\
0.0 \\
0.0 \\
0.0 \\
0.0 \\
3.0\end{array}$ & $\begin{array}{l}0.0 \\
1.2 \\
1.3 \\
0.2 \\
0.0 \\
0.0 \\
0.0 \\
0.0 \\
0.0 \\
0.0\end{array}$ & $\begin{array}{l}0.0 \\
1.4 \\
2.0 \\
0.3 \\
0.0 \\
0.0 \\
0.0 \\
0.0 \\
0.0 \\
0.0 \\
3.7\end{array}$ & $\begin{array}{l}0.0 \\
1.5 \\
2.0 \\
0.3 \\
0.0 \\
0.0 \\
0.0 \\
0.0 \\
0.0 \\
0.0 \\
3.8\end{array}$ & $\begin{array}{l}0.0 \\
1.4 \\
2.6 \\
0.8 \\
0.1 \\
0.0 \\
0.0 \\
0.0 \\
0.0 \\
0.0 \\
5.0\end{array}$ & $\begin{array}{l}0.0 \\
1.5 \\
3.4 \\
1.8 \\
0.3 \\
0.0 \\
0.0 \\
0.0 \\
0.0 \\
0.0 \\
6.9\end{array}$ & $\begin{array}{l}0.0 \\
1.5 \\
3.9 \\
2.8 \\
1.0 \\
0.1 \\
0.0 \\
0.0 \\
0.0 \\
0.0 \\
9.3\end{array}$ & $\begin{array}{l}0.0 \\
1.3 \\
2.9 \\
2.5 \\
1.5 \\
0.5 \\
0.1 \\
0.0 \\
0.0 \\
0.0 \\
8.9\end{array}$ & $\begin{array}{l}0.0 \\
1.3 \\
2.5 \\
1.6 \\
1.1 \\
0.6 \\
0.2 \\
0.1 \\
0.0 \\
0.0 \\
7.4\end{array}$ & $\begin{array}{l}0.0 \\
1.2 \\
2.2 \\
1.3 \\
0.7 \\
0.3 \\
0.1 \\
0.0 \\
0.0 \\
0.0 \\
5.9\end{array}$ & $\begin{array}{l}0.0 \\
1.2 \\
2.6 \\
2.0 \\
0.8 \\
0.2 \\
0.1 \\
0.0 \\
0.0 \\
0.0 \\
6.9\end{array}$ & $\begin{array}{r}0.0 \\
1.2 \\
3.2 \\
4.1 \\
1.6 \\
0.3 \\
0.1 \\
0.0 \\
0.0 \\
0.0 \\
10.4\end{array}$ & $\begin{array}{r}0.0 \\
1.4 \\
3.8 \\
3.2 \\
1.4 \\
0.6 \\
0.1 \\
0.0 \\
0.0 \\
0.0 \\
10.6\end{array}$ & $\begin{array}{l}3 . \\
1 . \\
0 . \\
0 . \\
0 . \\
0 . \\
0 . \\
0 . \\
6 .\end{array}$ & & $\begin{array}{l}2.7 \\
0.8 \\
0.1 \\
0.0\end{array}$ \\
\hline
\end{tabular}


Table 5.4. (contd)

Station: (13) 100N

Begin: $1 / 82$

End: $12 / 98$

Total Hours: 144977

DIRECTION

\begin{tabular}{rrrrrrrrrrrrrrrrrrr} 
& N & NNE & NE & ENE & E & ESE & SE & SSE & S & SSH & SH & USH & W & WNH & NH & NNH & CALM & TOTAL \\
\hline CALM & 0.0 & 0.0 & 0.0 & 0.0 & 0.0 & 0.0 & 0.0 & 0.0 & 0.0 & 0.0 & 0.0 & 0.0 & 0.0 & 0.0 & 0.0 & 0.0 & 0.9 & 0.9 \\
$1-3$ & 2.1 & 1.8 & 2.0 & 2.3 & 3.1 & 3.2 & 2.6 & 1.9 & 1.8 & 1.7 & 2.3 & 2.8 & 3.2 & 3.3 & 3.1 & 2.5 & 0.0 & 39.5 \\
$4-7$ & 1.4 & 1.6 & 1.6 & 1.9 & 2.7 & 2.7 & 2.3 & 1.3 & 0.9 & 1.0 & 2.3 & 4.5 & 4.8 & 3.1 & 2.1 & 1.5 & 0.0 & 35.7 \\
$8-12$ & 0.4 & 0.8 & 0.6 & 0.2 & 0.3 & 0.4 & 0.8 & 0.5 & 0.3 & 0.5 & 1.2 & 2.1 & 3.1 & 2.2 & 0.7 & 0.4 & 0.0 & 14.4 \\
$13-18$ & 0.2 & 0.4 & 0.3 & 0.1 & 0.0 & 0.0 & 0.1 & 0.1 & 0.1 & 0.3 & 0.8 & 0.6 & 1.2 & 1.9 & 0.6 & 0.1 & 0.0 & 6.8 \\
$19-24$ & 0.0 & 0.1 & 0.1 & 0.0 & 0.0 & 0.0 & 0.0 & 0.0 & 0.0 & 0.1 & 0.3 & 0.2 & 0.2 & 0.6 & 0.4 & 0.0 & 0.0 & 2.2 \\
$25-31$ & 0.0 & 0.0 & 0.0 & 0.0 & 0.0 & 0.0 & 0.0 & 0.0 & 0.0 & 0.1 & 0.1 & 0.0 & 0.0 & 0.1 & 0.1 & 0.0 & 0.0 & 0.5 \\
$32-38$ & 0.0 & 0.0 & 0.0 & 0.0 & 0.0 & 0.0 & 0.0 & 0.0 & 0.0 & 0.0 & 0.0 & 0.0 & 0.0 & 0.0 & 0.0 & 0.0 & 0.0 & 0.0 \\
$39-46$ & 0.0 & 0.0 & 0.0 & 0.0 & 0.0 & 0.0 & 0.0 & 0.0 & 0.0 & 0.0 & 0.0 & 0.0 & 0.0 & 0.0 & 0.0 & 0.0 & 0.0 & 0.0 \\
$>46$ & 0.0 & 0.0 & 0.0 & 0.0 & 0.0 & 0.0 & 0.0 & 0.0 & 0.0 & 0.0 & 0.0 & 0.0 & 0.0 & 0.0 & 0.0 & 0.0 & 0.0 & 0.0 \\
TOTAL & 4.1 & 4.7 & 4.6 & 4.5 & 6.1 & 6.2 & 5.8 & 3.8 & 3.1 & 3.7 & 6.9 & 10.1 & 12.6 & 11.4 & 7.0 & 4.5 & 0.9 & 100.0
\end{tabular}

Station: (14) WPPS

$\begin{array}{ll}\text { Begin: 1/82 End: 12/98 } & \text { (1) }\end{array}$

Total Hours: 145379

DIRECTION

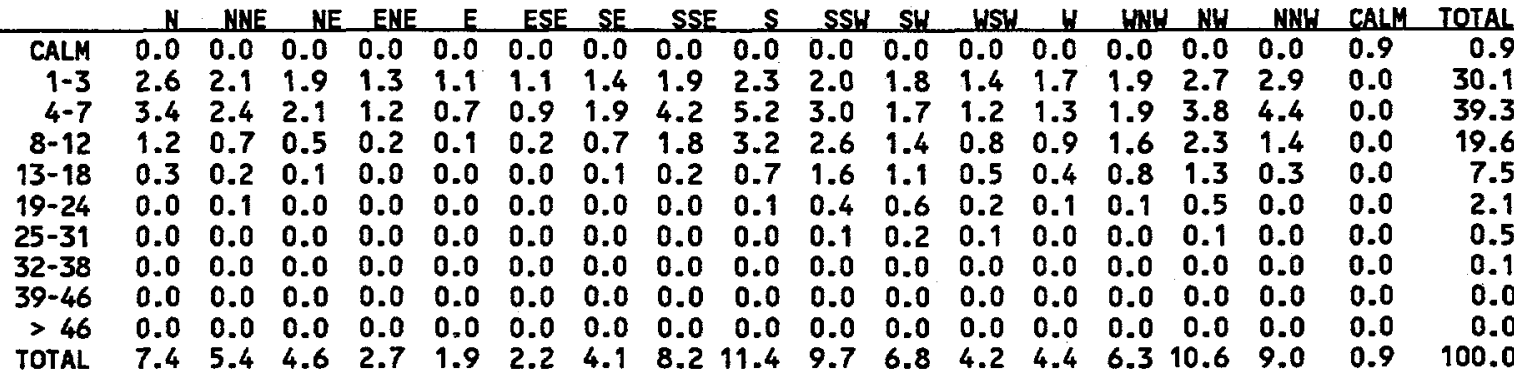

Station: (15) FRNK

Begin: 1/82 End: $12 / 98$

Total Hours: 144952

DIRECTION

\begin{tabular}{|c|c|c|c|c|c|c|c|c|c|c|c|c|c|c|c|c|c|c|c|}
\hline $\begin{array}{r}\text { CALM } \\
1-3 \\
4-7 \\
8-12 \\
13-18 \\
19-24 \\
25-31 \\
32-38 \\
39-46 \\
>46\end{array}$ & $\begin{array}{l}0.0 \\
1.2 \\
4.1 \\
1.7 \\
0.1 \\
0.0 \\
0.0 \\
0.0 \\
0.0 \\
0.0 \\
7.1\end{array}$ & $\begin{array}{l}0.0 \\
1.0 \\
2.7 \\
1.0 \\
0.2 \\
0.1 \\
0.0 \\
0.0 \\
0.0 \\
0.0 \\
4.9\end{array}$ & $\begin{array}{l}0.0 \\
1.0 \\
1.7 \\
0.6 \\
0.2 \\
0.1 \\
0.0 \\
0.0 \\
0.0 \\
0.0 \\
3.5\end{array}$ & $\begin{array}{l}0.0 \\
0.8 \\
1.1 \\
0.3 \\
0.1 \\
0.0 \\
0.0 \\
0.0 \\
0.0 \\
0.0 \\
2.4\end{array}$ & $\begin{array}{l}0.0 \\
0.9 \\
1.5 \\
0.3 \\
0.0 \\
0.0 \\
0.0 \\
0.0 \\
0.0 \\
0.0 \\
2.7\end{array}$ & $\begin{array}{l}0.0 \\
1.1 \\
2.3 \\
0.6 \\
0.0 \\
0.0 \\
0.0 \\
0.0 \\
0.0 \\
0.0 \\
4.1\end{array}$ & $\begin{array}{l}0.0 \\
1.5 \\
4.8 \\
1.6 \\
0.1 \\
0.0 \\
0.0 \\
0.0 \\
0.0 \\
0.0 \\
8.0\end{array}$ & $\begin{array}{l}0.0 \\
1.5 \\
4.2 \\
1.5 \\
0.1 \\
0.0 \\
0.0 \\
0.0 \\
0.0 \\
0.0 \\
7.4\end{array}$ & $\begin{array}{l}0.0 \\
1.3 \\
3.5 \\
2.4 \\
0.4 \\
0.0 \\
0.0 \\
0.0 \\
0.0 \\
0.0\end{array}$ & $\begin{array}{r}0.0 \\
1.1 \\
3.0 \\
4.7 \\
1.9 \\
0.3 \\
0.1 \\
0.0 \\
0.0 \\
0.0 \\
11.2\end{array}$ & $\begin{array}{l}0.0 \\
1.2 \\
2.5 \\
3.4 \\
2.0 \\
0.5 \\
0.2 \\
0.0 \\
0.0 \\
0.0 \\
9.8\end{array}$ & $\begin{array}{l}0.0 \\
1.2 \\
1.3 \\
1.0 \\
0.6 \\
0.2 \\
0.0 \\
0.0 \\
0.0 \\
0.0 \\
4.3\end{array}$ & $\begin{array}{l}0 . \\
1 . \\
1 . \\
0 . \\
0 . \\
0 . \\
0 . \\
0 . \\
0 . \\
0 . \\
3 .\end{array}$ & & & $\begin{array}{l}0.0 \\
1.6 \\
4.5 \\
2.1 \\
0.5 \\
0.1 \\
0.0 \\
0.0 \\
0.0 \\
0.0 \\
8.8\end{array}$ & $\begin{array}{l}0 \\
1 . \\
5 \\
2 . \\
0 \\
0 \\
0 \\
0\end{array}$ & $\begin{array}{l}0.9 \\
0.0 \\
0.0 \\
0.0 \\
0.0 \\
0.0 \\
0.0 \\
0.0 \\
0.0 \\
0.0 \\
0.9\end{array}$ & $\begin{array}{r}0.9 \\
19.4 \\
46.1 \\
24.8 \\
7.0 \\
1.3 \\
0.4 \\
0.1 \\
0.0 \\
0.0\end{array}$ \\
\hline
\end{tabular}

Station: (16) GABL

Begin: 1/82 End: $12 / 98$

Total Hours: 144441

DIRECTION

\begin{tabular}{rrrrrrrrrrrrrrrrrrrr} 
& N & MNE & NE & ENE & E & ESE & SE & SSE & S & SSH & SH & USH & H & WNH & NH & NHH & CALM & TOTAL \\
\hline CALM & 0.0 & 0.0 & 0.0 & 0.0 & 0.0 & 0.0 & 0.0 & 0.0 & 0.0 & 0.0 & 0.0 & 0.0 & 0.0 & 0.0 & 0.0 & 0.0 & 0.7 & 0.7 \\
$1-3$ & 1.0 & 0.9 & 0.9 & 0.7 & 0.6 & 0.6 & 0.7 & 0.8 & 1.0 & 1.0 & 1.0 & 0.8 & 0.8 & 0.8 & 0.8 & 0.9 & 0.0 & 13.3 \\
$4-7$ & 2.3 & 2.3 & 1.6 & 0.9 & 0.9 & 0.9 & 1.3 & 2.1 & 3.2 & 2.4 & 1.8 & 1.5 & 1.5 & 1.7 & 2.2 & 2.1 & 0.0 & 29.0 \\
$8-12$ & 2.1 & 2.2 & 1.0 & 0.3 & 0.4 & 0.5 & 0.9 & 1.7 & 2.4 & 1.5 & 1.5 & 1.6 & 1.7 & 2.2 & 2.9 & 1.8 & 0.0 & 24.6 \\
$13-18$ & 1.3 & 1.4 & 0.5 & 0.1 & 0.1 & 0.1 & 0.3 & 1.0 & 1.4 & 0.9 & 1.3 & 1.4 & 1.8 & 2.8 & 2.5 & 0.8 & 0.0 & 17.5 \\
$19-24$ & 0.4 & 0.5 & 0.3 & 0.0 & 0.0 & 0.0 & 0.0 & 0.3 & 0.6 & 0.5 & 0.9 & 0.8 & 0.9 & 2.7 & 1.6 & 0.2 & 0.0 & 9.7 \\
$25-31$ & 0.1 & 0.2 & 0.2 & 0.0 & 0.0 & 0.0 & 0.0 & 0.0 & 0.2 & 0.3 & 0.6 & 0.4 & 0.2 & 1.2 & 0.5 & 0.0 & 0.0 & 3.9 \\
$32-38$ & 0.0 & 0.0 & 0.1 & 0.0 & 0.0 & 0.0 & 0.0 & 0.0 & 0.1 & 0.1 & 0.3 & 0.1 & 0.0 & 0.2 & 0.0 & 0.0 & 0.0 & 1.0 \\
$39-46$ & 0.0 & 0.0 & 0.0 & 0.0 & 0.0 & 0.0 & 0.0 & 0.0 & 0.0 & 0.1 & 0.1 & 0.0 & 0.0 & 0.0 & 0.0 & 0.0 & 0.0 & 0.3 \\
$>46$ & 0.0 & 0.0 & 0.0 & 0.0 & 0.0 & 0.0 & 0.0 & 0.0 & 0.0 & 0.0 & 0.0 & 0.0 & 0.0 & 0.0 & 0.0 & 0.0 & 0.0 & 0.0 \\
TOTAL & 7.2 & 7.6 & 4.6 & 2.1 & 2.0 & 2.1 & 3.2 & 6.0 & 8.9 & 6.8 & 7.4 & 6.7 & 6.9 & 11.6 & 10.5 & 5.8 & 0.7 & 100.0
\end{tabular}


Table 5.4. (contd)

Station: (17) RING

\begin{tabular}{|c|c|c|c|c|c|c|c|c|c|c|c|c|c|c|c|c|c|c|c|}
\hline & & & & ENE & & & & SSE & & 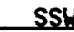 & & $\mathrm{WS}$ & & & WWH & $\mathrm{NH}$ & NWW & ALM & \\
\hline CALI & & 0.0 & 0.0 & 0.0 & 0.0 & 0.0 & 0.0 & 0.0 & 0.0 & 0.0 & 0.0 & 0.0 & 0. & & 1.0 & 0.0 & 0.0 & 1.2 & \\
\hline & & & & & & & & & & & & & & & & & & & \\
\hline $4-7$ & & & 10.4 & & 1.1 & & & & 1.8 & & & & & & & & & & \\
\hline & & & & & 0.1 & & & & & & & & & & & & & & \\
\hline & & 0. & 0 . & 0. & 0. & & 0. & & 0.1 & & & & & & & & & & \\
\hline & & & & & & & & & & & & & & & & & & & \\
\hline & & & & & & & & & & & & & & & & & & & \\
\hline & & & & & & & 0. & & 0. & & & & & & & & & & \\
\hline & 0 & & & 0. & 0 & & 0 & & 0 & & & & & & & & & & \\
\hline & & & & & & & & & & & & & & & & & & & \\
\hline & & & & & 3.5 & & & 3.3 & & & & & & & 0.5 & & & .2 & \\
\hline
\end{tabular}

Station: (18) RICH

Begin: $1 / 82$

End: $12 / 98$

Total Hours:

145637

DIRECTION

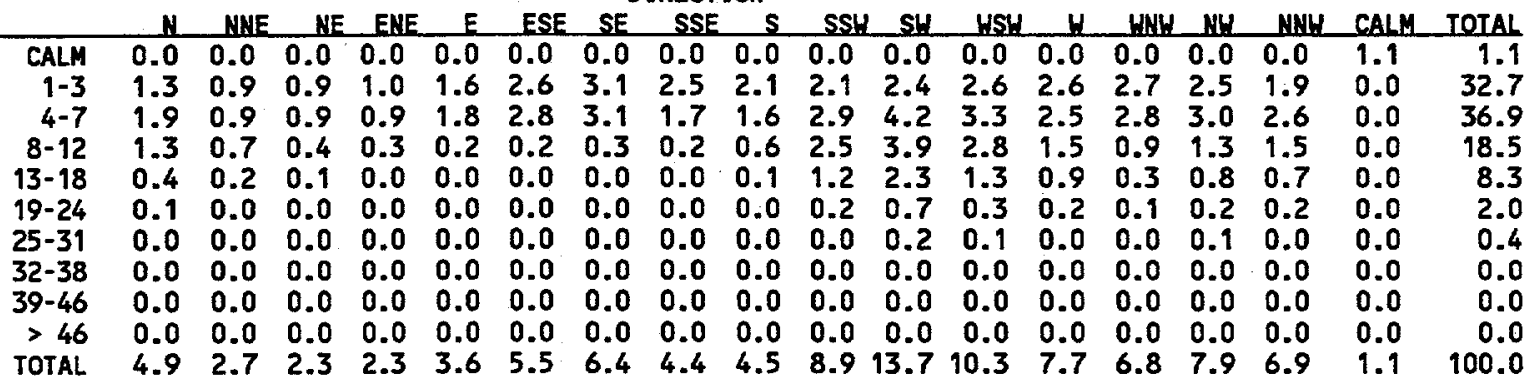

Station: (19) PFP

Begin: 2/94 End: 12/98

Total Hours:

42753 DIRECTION

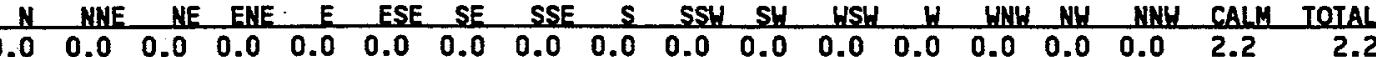

$\begin{array}{rllllllllllllllllll}\text { CALM } & 0.0 & 0.0 & 0.0 & 0.0 & 0.0 & 0.0 & 0.0 & 0.0 & 0.0 & 0.0 & 0.0 & 0.0 & 0.0 & 0.0 & 0.0 & 0.0 & 2.2 & 2.2 \\ 1-3 & 3.5 & 3.3 & 2.2 & 1.4 & 1.5 & 1.8 & 2.3 & 1.9 & 2.0 & 1.8 & 2.4 & 3.4 & 5.4 & 5.4 & 3.9 & 3.2 & 0.0 & 45.4\end{array}$

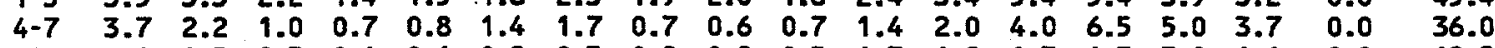

$\begin{array}{ccccccccccccccccccc}8-12 & 0.4 & 0.5 & 0.3 & 0.1 & 0.1 & 0.2 & 0.3 & 0.2 & 0.2 & 0.5 & 1.3 & 1.8 & 1.3 & 1.3 & 3.0 & 1.1 & 0.0 & 12.5\end{array}$

$\begin{array}{lllllllllllllllllll}13-18 & 0.0 & 0.1 & 0.1 & 0.0 & 0.0 & 0.0 & 0.0 & 0.0 & 0.1 & 0.2 & 0.7 & 0.8 & 0.3 & 0.1 & 1.0 & 0.2 & 0.0 & 3.6\end{array}$

$\begin{array}{lllllllllllllllllll}19-24 & 0.0 & 0.0 & 0.0 & 0.0 & 0.0 & 0.0 & 0.0 & 0.0 & 0.0 & 0.0 & 0.1 & 0.1 & 0.0 & 0.0 & 0.0 & 0.0 & 0.0 & 0.3\end{array}$

$\begin{array}{lllllllllllllllllll}25-31 & 0.0 & 0.0 & 0.0 & 0.0 & 0.0 & 0.0 & 0.0 & 0.0 & 0.0 & 0.0 & 0.0 & 0.0 & 0.0 & 0.0 & 0.0 & 0.0 & 0.0 & 0.0\end{array}$

$\begin{array}{lllllllllllllllllll}32-38 & 0.0 & 0.0 & 0.0 & 0.0 & 0.0 & 0.0 & 0.0 & 0.0 & 0.0 & 0.0 & 0.0 & 0.0 & 0.0 & 0.0 & 0.0 & 0.0 & 0.0 & 0.0\end{array}$

$\begin{array}{lllllllllllllllllll}39-46 & 0.0 & 0.0 & 0.0 & 0.0 & 0.0 & 0.0 & 0.0 & 0.0 & 0.0 & 0.0 & 0.0 & 0.0 & 0.0 & 0.0 & 0.0 & 0.0 & 0.0 & 0.0\end{array}$

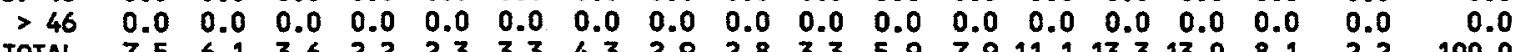

$\begin{array}{lllllllllllllllllll}\text { TOTAL } & 7.5 & 6.1 & 3.6 & 2.2 & 2.3 & 3.3 & 4.3 & 2.9 & 2.8 & 3.3 & 5.9 & 7.9 & 11.1 & 13.3 & 13.0 & 8.1 & 2.2 & 100.0\end{array}$

Station: (20) RMTN

Begin: $1 / 82 \quad$ End: $12 / 98$

Total Hours: 143035

DIRECTION

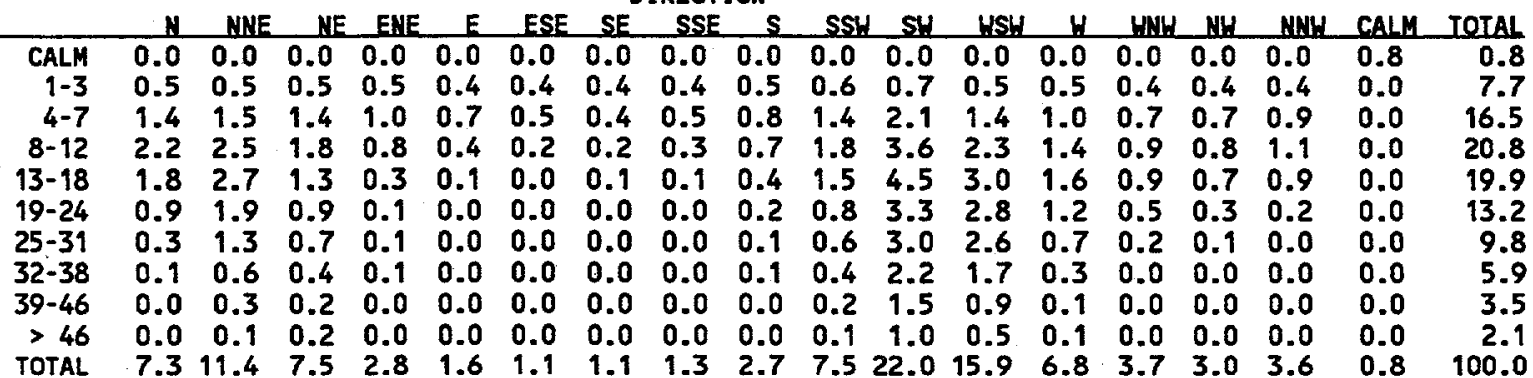


Table 5.4. (contd)

Station: (21) HMS

Begin: $1 / 82$

DIRECTION

End: $12 / 98$

Total Hours:

147741

$N$ NWE NE ENE E ESE SE SSE S SSH SH WSH W WNW NW NWH CALM TOTAL

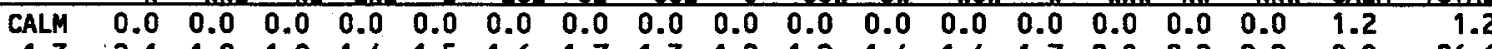

$\begin{array}{lllllllllllllllllll}1-3 & 2.1 & 1.8 & 1.8 & 1.4 & 1.5 & 1.6 & 1.7 & 1.3 & 1.2 & 1.2 & 1.4 & 1.4 & 1.7 & 2.0 & 2.2 & 2.2 & 0.0 & 26.6\end{array}$

$\begin{array}{lllllllllllllllllll}4-7 & 2.2 & 1.5 & 1.1 & 1.0 & 1.2 & 1.3 & 1.7 & 1.5 & 1.3 & 1.4 & 2.2 & 3.6 & 5.0 & 6.3 & 6.8 & 3.8 & 0.0 & 42.0\end{array}$

$\begin{array}{lllllllllllllllllll}8-12 & 0.5 & 0.5 & 0.3 & 0.1 & 0.1 & 0.1 & 0.2 & 0.4 & 0.4 & 0.6 & 1.3 & 2.3 & 2.4 & 4.3 & 5.2 & 1.2 & 0.0 & 19.9\end{array}$

$\begin{array}{lllllllllllllllllll}13-18 & 0.1 & 0.2 & 0.1 & 0.0 & 0.0 & 0.0 & 0.0 & 0.1 & 0.2 & 0.4 & 1.0 & 1.1 & 0.6 & 1.4 & 2.4 & 0.3 & 0.0 & 7.9\end{array}$

$\begin{array}{lllllllllllllllllll}19-24 & 0.0 & 0.0 & 0.0 & 0.0 & 0.0 & 0.0 & 0.0 & 0.0 & 0.0 & 0.2 & 0.4 & 0.3 & 0.1 & 0.2 & 0.6 & 0.0 & 0.0 & 2.0\end{array}$

$\begin{array}{lllllllllllllllllll}25-31 & 0.0 & 0.0 & 0.0 & 0.0 & 0.0 & 0.0 & 0.0 & 0.0 & 0.0 & 0.0 & 0.1 & 0.1 & 0.0 & 0.0 & 0.1 & 0.0 & 0.0 & 0.3\end{array}$

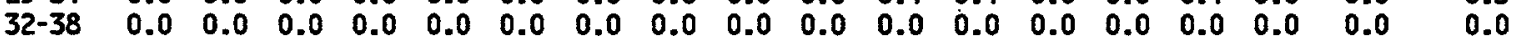

$\begin{array}{lllllllllllllllllll}39-46 & 0.0 & 0.0 & 0.0 & 0.0 & 0.0 & 0.0 & 0.0 & 0.0 & 0.0 & 0.0 & 0.0 & 0.0 & 0.0 & 0.0 & 0.0 & 0.0 & 0.0 & 0.0\end{array}$

$\begin{array}{lllllllllllllllllll}>46 & 0.0 & 0.0 & 0.0 & 0.0 & 0.0 & 0.0 & 0.0 & 0.0 & 0.0 & 0.0 & 0.0 & 0.0 & 0.0 & 0.0 & 0.0 & 0.0 & 0.0 & 0.0\end{array}$

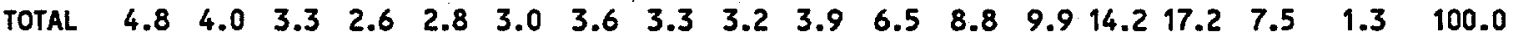

Station: (22) PASC

Begin: $10 / 87 \quad$ End: $12 / 98$

Total Hours:

93464

DIRECTION

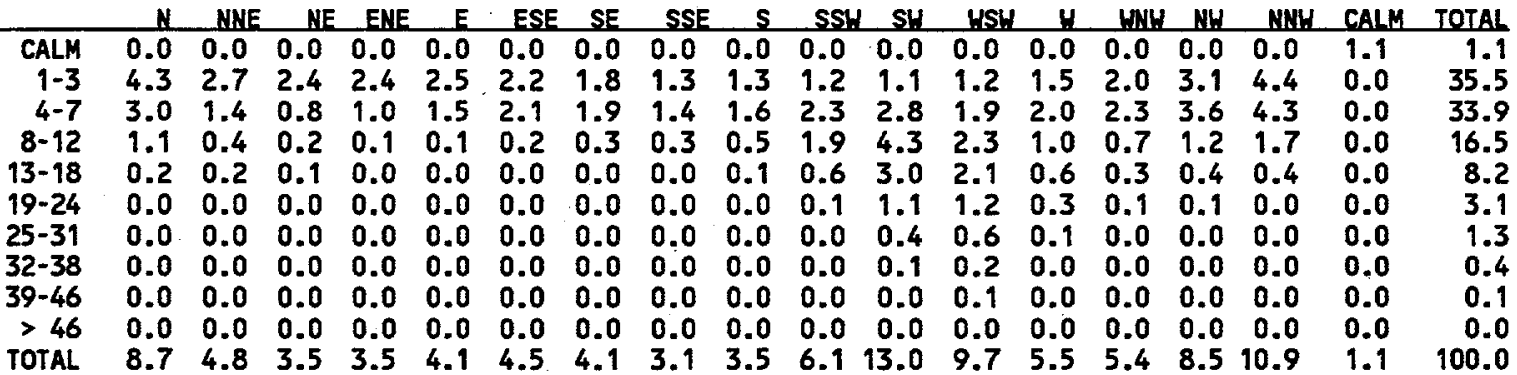

Station: (23) GABH

Begin: 3/86 End: $12 / 98$

Total Hours: 109669

DIRECTION

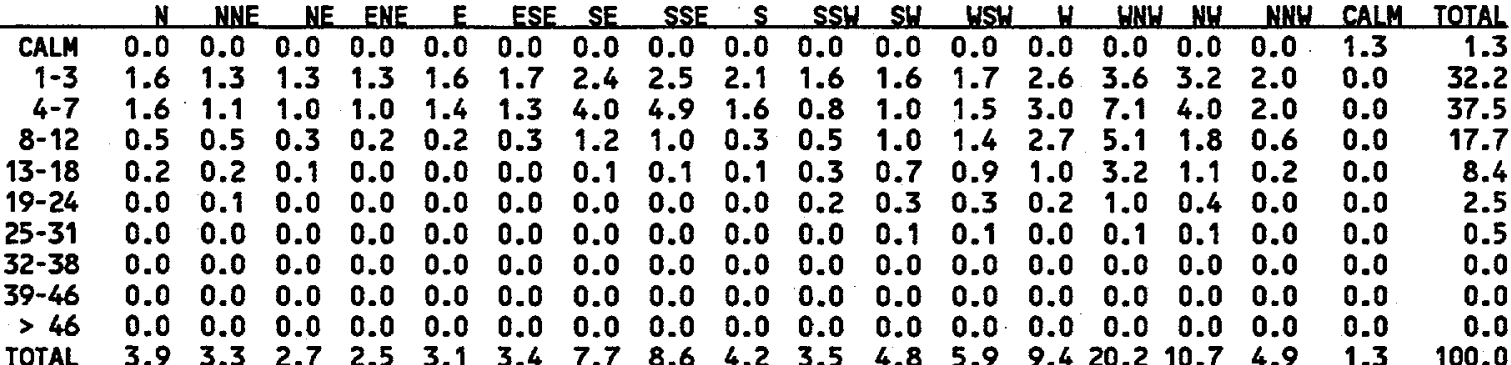

Station: (24) 100F

Begin: $3 / 86$

End: $12 / 98$

Total Hours:

109760

DIRECTION

\begin{tabular}{rrrrrrrrrrrrrrrrrrr} 
& N & NNE & NE & ENE & E & ESE & SE & SSE & S & SSH & SH & USH & U & NHY & NH & NNH & CALM & TOTAL \\
\hline CALM & 0.0 & 0.0 & 0.0 & 0.0 & 0.0 & 0.0 & 0.0 & 0.0 & 0.0 & 0.0 & 0.0 & 0.0 & 0.0 & 0.0 & 0.0 & 0.0 & 1.2 & 1.2 \\
$1-3$ & 1.8 & 1.4 & 1.2 & 1.2 & 1.5 & 1.9 & 2.8 & 2.8 & 2.3 & 1.8 & 1.9 & 2.4 & 3.6 & 4.1 & 3.5 & 2.4 & 0.0 & 36.7 \\
$4-7$ & 2.0 & 1.4 & 1.1 & 1.2 & 1.2 & 1.7 & 5.3 & 5.8 & 1.5 & 0.9 & 1.0 & 1.6 & 3.3 & 3.7 & 2.6 & 1.9 & 0.0 & 36.2 \\
$8-12$ & 1.0 & 0.7 & 0.3 & 0.3 & 0.2 & 0.5 & 2.5 & 2.5 & 0.6 & 0.5 & 0.8 & 1.2 & 2.5 & 2.4 & 0.6 & 0.5 & 0.0 & 17.1 \\
$13-18$ & 0.2 & 0.3 & 0.1 & 0.0 & 0.0 & 0.0 & 0.2 & 0.3 & 0.3 & 0.3 & 0.6 & 0.8 & 1.0 & 1.8 & 0.4 & 0.1 & 0.0 & 6.4 \\
$19-24$ & 0.0 & 0.1 & 0.1 & 0.0 & 0.0 & 0.0 & 0.0 & 0.0 & 0.1 & 0.1 & 0.3 & 0.3 & 0.2 & 0.6 & 0.2 & 0.0 & 0.0 & 1.9 \\
$25-31$ & 0.0 & 0.0 & 0.0 & 0.0 & 0.0 & 0.0 & 0.0 & 0.0 & 0.0 & 0.1 & 0.1 & 0.1 & 0.0 & 0.1 & 0.0 & 0.0 & 0.0 & 0.4 \\
$32-38$ & 0.0 & 0.0 & 0.0 & 0.0 & 0.0 & 0.0 & 0.0 & 0.0 & 0.0 & 0.0 & 0.0 & 0.0 & 0.0 & 0.0 & 0.0 & 0.0 & 0.0 & 0.0 \\
$39-46$ & 0.0 & 0.0 & 0.0 & 0.0 & 0.0 & 0.0 & 0.0 & 0.0 & 0.0 & 0.0 & 0.0 & 0.0 & 0.0 & 0.0 & 0.0 & 0.0 & 0.0 & 0.0 \\
$>46$ & 0.0 & 0.0 & 0.0 & 0.0 & 0.0 & 0.0 & 0.0 & 0.0 & 0.0 & 0.0 & 0.0 & 0.0 & 0.0 & 0.0 & 0.0 & 0.0 & 0.0 & 0.0 \\
TOTAL & 5.0 & 3.8 & 2.8 & 2.7 & 3.0 & 4.2 & 10.9 & 11.5 & 4.7 & 3.7 & 4.6 & 6.4 & 10.7 & 12.7 & 7.3 & 5.0 & 1.2 & 100.0
\end{tabular}


Table 5.4. (contd)

Station: (25) VERM

Begin: 2/88

End: $12 / 98$

Total Hours:

93874

DIRECTION

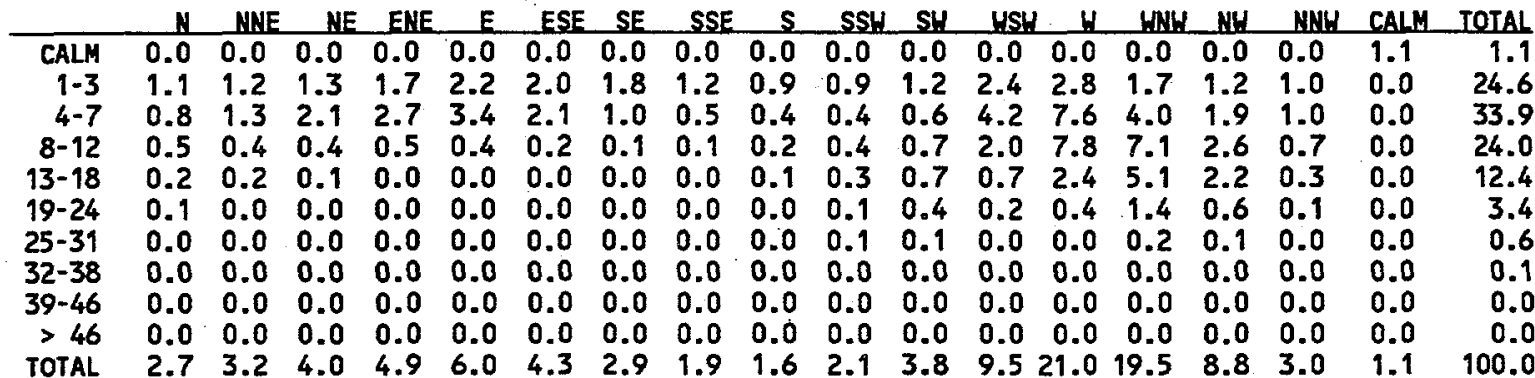

Station: (26) BENT

Begin: $2 / 95$

End: $12 / 98$

Total Hours:

34143

DIRECTION

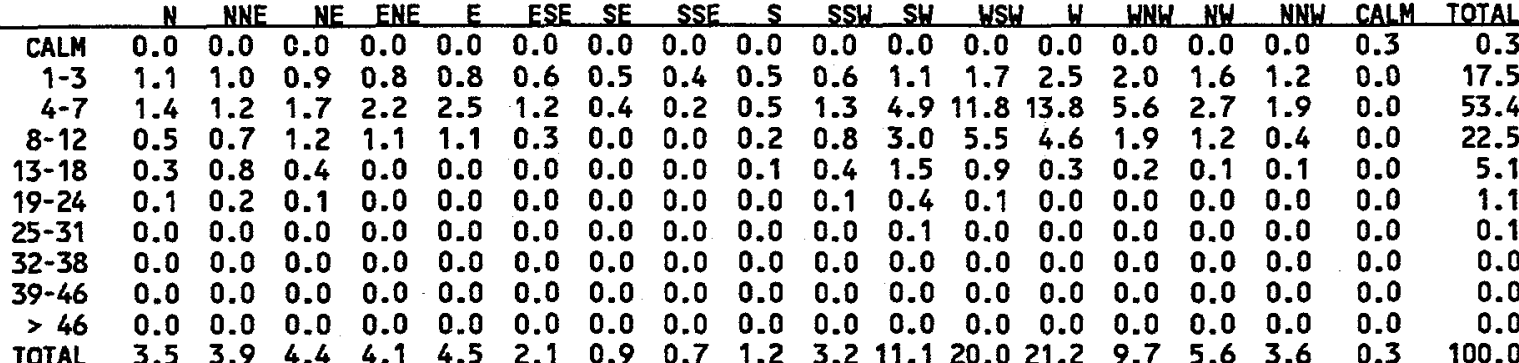

Station: (27) VSTA

Begin: $2 / 91$

End: $12 / 98$

Total Hours:

67863

DIRECTION

\begin{tabular}{rrrllllllllllllllll} 
& N & NNE & NE & ENE & E & ESE & SE & SSE & S & SSH & SW & WSW & W & WNH & NH & NNH & CALM & TOTAL \\
\hline CALM & 0.0 & 0.0 & 0.0 & 0.0 & 0.0 & 0.0 & 0.0 & 0.0 & 0.0 & 0.0 & 0.0 & 0.0 & 0.0 & 0.0 & 0.0 & 0.0 & 0.8 & 0.8 \\
$1-3$ & 2.2 & 2.3 & 2.1 & 1.6 & 1.6 & 1.8 & 2.0 & 1.7 & 1.9 & 2.4 & 2.7 & 2.5 & 2.3 & 1.8 & 1.8 & 1.7 & 0.0 & 32.6 \\
$4-7$ & 3.0 & 2.0 & 1.5 & 1.2 & 0.8 & 1.1 & 1.2 & 1.2 & 1.9 & 4.1 & 5.9 & 4.2 & 2.6 & 2.6 & 3.1 & 3.0 & 0.0 & 39.5 \\
$8-12$ & 0.6 & 0.3 & 0.1 & 0.0 & 0.0 & 0.1 & 0.1 & 0.2 & 0.6 & 3.8 & 6.0 & 2.7 & 0.8 & 0.4 & 0.9 & 1.2 & 0.0 & 17.8 \\
$13-18$ & 0.1 & 0.1 & 0.0 & 0.0 & 0.0 & 0.0 & 0.0 & 0.0 & 0.1 & 1.6 & 3.7 & 1.1 & 0.4 & 0.2 & 0.1 & 0.2 & 0.0 & 7.5 \\
$19-24$ & 0.0 & 0.0 & 0.0 & 0.0 & 0.0 & 0.0 & 0.0 & 0.0 & 0.0 & 0.2 & 1.0 & 0.3 & 0.0 & 0.0 & 0.0 & 0.0 & 0.0 & 1.6 \\
$25-31$ & 0.0 & 0.0 & 0.0 & 0.0 & 0.0 & 0.0 & 0.0 & 0.0 & 0.0 & 0.0 & 0.1 & 0.0 & 0.0 & 0.0 & 0.0 & 0.0 & 0.0 & 0.2 \\
$32-38$ & 0.0 & 0.0 & 0.0 & 0.0 & 0.0 & 0.0 & 0.0 & 0.0 & 0.0 & 0.0 & 0.0 & 0.0 & 0.0 & 0.0 & 0.0 & 0.0 & 0.0 & 0.0 \\
$39-46$ & 0.0 & 0.0 & 0.0 & 0.0 & 0.0 & 0.0 & 0.0 & 0.0 & 0.0 & 0.0 & 0.0 & 0.0 & 0.0 & 0.0 & 0.0 & 0.0 & 0.0 & 0.0 \\
$>46$ & 0.0 & 0.0 & 0.0 & 0.0 & 0.0 & 0.0 & 0.0 & 0.0 & 0.0 & 0.0 & 0.0 & 0.0 & 0.0 & 0.0 & 0.0 & 0.0 & 0.0 & 0.0 \\
TOTAL & 5.9 & 4.6 & 3.7 & 2.9 & 2.4 & 3.0 & 3.4 & 3.1 & 4.6 & 12.1 & 19.4 & 10.9 & 6.1 & 5.1 & 6.0 & 6.0 & 0.8 & 100.0
\end{tabular}

Station: (28) SURF

Begin: $9 / 94 \quad$ End: $12 / 98$

Total Hours:

37739 DIRECTION

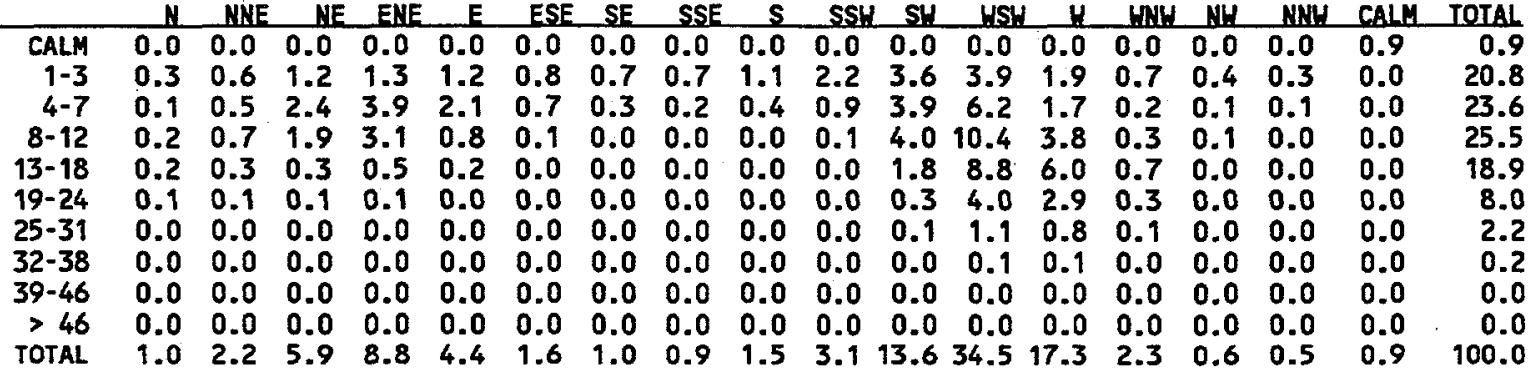


Table 5.4. (contd)

Station: (29) 100K

Begin: 3/96

End: $12 / 98$

Total Hours:

24400

DIRECTION

\begin{tabular}{|c|c|c|c|c|c|c|c|c|c|c|c|c|c|c|c|c|c|c|c|}
\hline $\begin{array}{r}\text { CALM } \\
1-3 \\
4-7 \\
8-12 \\
13-18 \\
19-24 \\
25-31 \\
32-38 \\
39-46 \\
>46 \\
\text { TOTAL }\end{array}$ & $\begin{array}{l}0.0 \\
2.1 \\
1.8 \\
0.4 \\
0.1 \\
0.0 \\
0.0 \\
0.0 \\
0.0 \\
0.0 \\
4.5\end{array}$ & $\begin{array}{l}0.0 \\
1.7 \\
1.3 \\
0.6 \\
0.3 \\
0.0 \\
0.0 \\
0.0 \\
0.0 \\
0.0 \\
3.8\end{array}$ & $\begin{array}{l}0.0 \\
1.7 \\
0.9 \\
0.3 \\
0.1 \\
0.0 \\
0.0 \\
0.0 \\
0.0 \\
0.0 \\
3.1\end{array}$ & $\begin{array}{l}0.0 \\
1.9 \\
1.0 \\
0.1 \\
0.0 \\
0.0 \\
0.0 \\
0.0 \\
0.0 \\
0.0 \\
3.0\end{array}$ & $\begin{array}{l}0.0 \\
2.2 \\
1.6 \\
0.2 \\
0.0 \\
0.0 \\
0.0 \\
0.0 \\
0.0 \\
0.0 \\
4.0\end{array}$ & $\begin{array}{l}0.0 \\
2.1 \\
1.6 \\
0.4 \\
0.0 \\
0.0 \\
0.0 \\
0.0 \\
0.0 \\
0.0\end{array}$ & $\begin{array}{l}0.0 \\
2.2 \\
1.7 \\
0.7 \\
0.1 \\
0.0 \\
0.0 \\
0.0 \\
0.0 \\
0.0 \\
4.7\end{array}$ & $\begin{array}{l}0.0 \\
1.8 \\
1.5 \\
0.7 \\
0.1 \\
0.0 \\
0.0 \\
0.0 \\
0.0 \\
0.0 \\
4.0\end{array}$ & $\begin{array}{l}0.0 \\
1.7 \\
1.1 \\
0.5 \\
0.2 \\
0.0 \\
0.0 \\
0.0 \\
0.0 \\
0.0 \\
3.0\end{array}$ & $\begin{array}{l}0.0 \\
1.7 \\
1.0 \\
0.6 \\
0.4 \\
0.1 \\
0.0 \\
0.0 \\
0.0 \\
0.0 \\
3.9\end{array}$ & $\begin{array}{l}0.0 \\
2.2 \\
1.0 \\
1.0 \\
0.5 \\
0.3 \\
0.0 \\
0.0 \\
0.0 \\
0.0\end{array}$ & $\begin{array}{l}0.0 \\
3.8 \\
4.8 \\
3.2 \\
0.8 \\
0.0 \\
0.0 \\
0.0 \\
0.0 \\
0.0\end{array}$ & & & $\begin{array}{l}0.0 \\
3.2 \\
3.5 \\
2.4 \\
2.0 \\
0.7 \\
0.1 \\
0.0 \\
0.0 \\
0.0\end{array}$ & $\begin{array}{l}0 . \\
2 . \\
2 . \\
0 . \\
0 . \\
0 . \\
0 .\end{array}$ & & $\begin{array}{l}0.0 \\
2.1 \\
1.7 \\
0.3 \\
0.1 \\
0.0 \\
0.0 \\
0.0 \\
0.0 \\
0.0\end{array}$ & $\begin{array}{l}1.5 \\
0.0 \\
0.0 \\
0.0 \\
0.0 \\
0.0 \\
0.0 \\
0.0 \\
0.0 \\
0.0 \\
1.5\end{array}$ \\
\hline
\end{tabular}

Station: (30) HAMR

Begin: 1/98 End: 12/98

Total Hours:

8711

DIRECTION

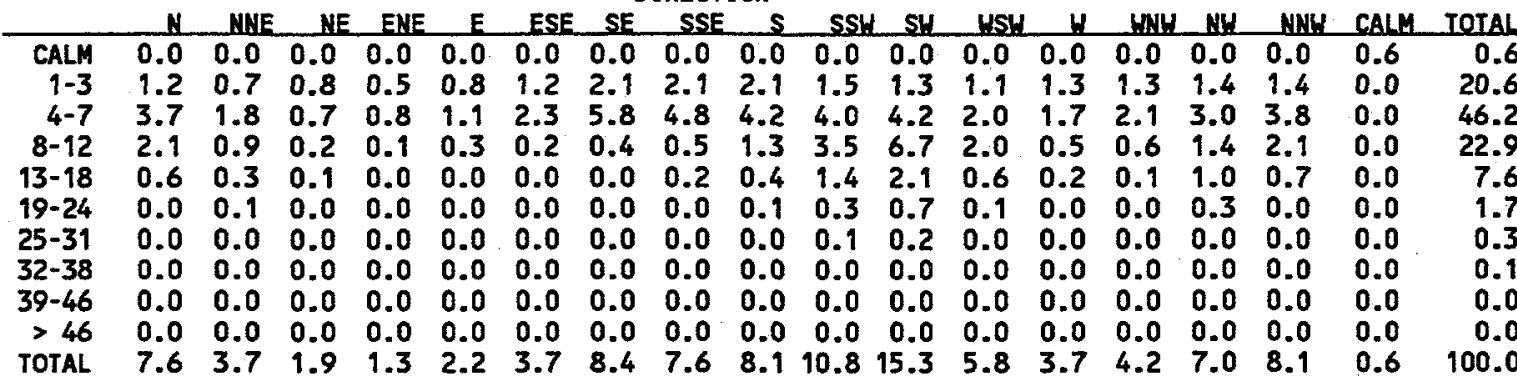

Station: (8W) WAHL

Begin: 1/82

End: $7 / 91$

Total Hours:

79579

DIRECTION

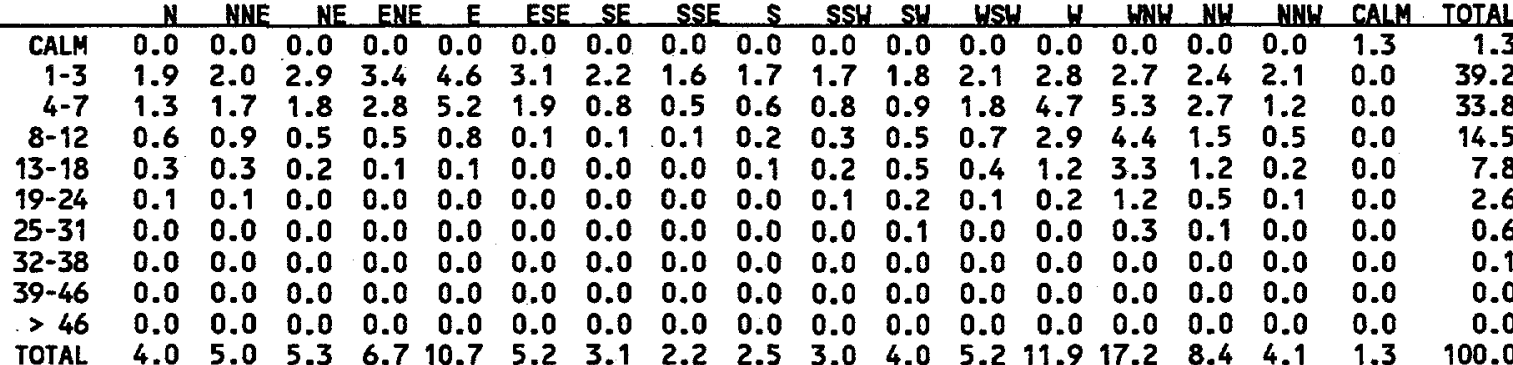

Station: (19S) SAGE

Begin: $3 / 82$

DIRECTION

End: $12 / 92$

Total Hours:

91756

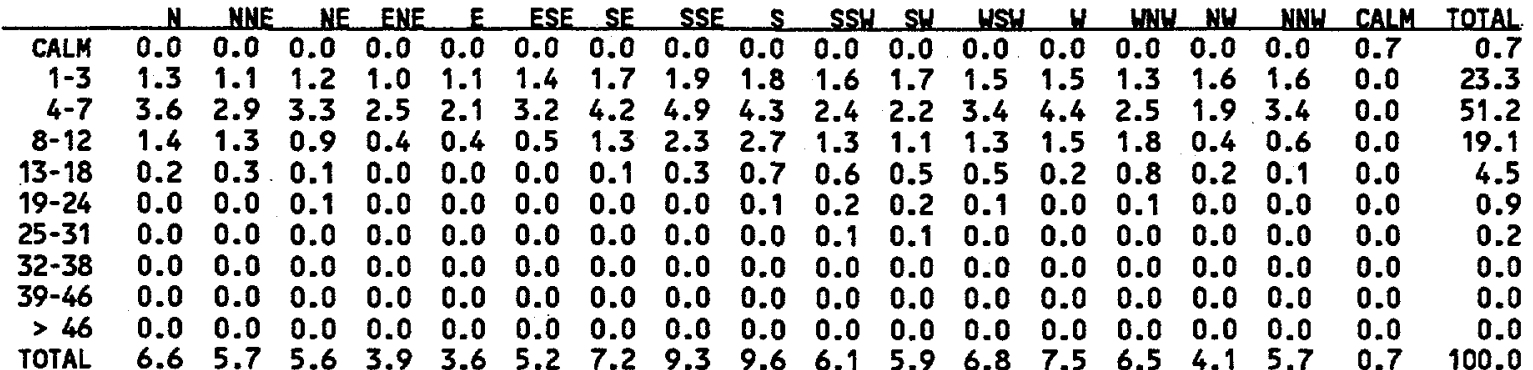




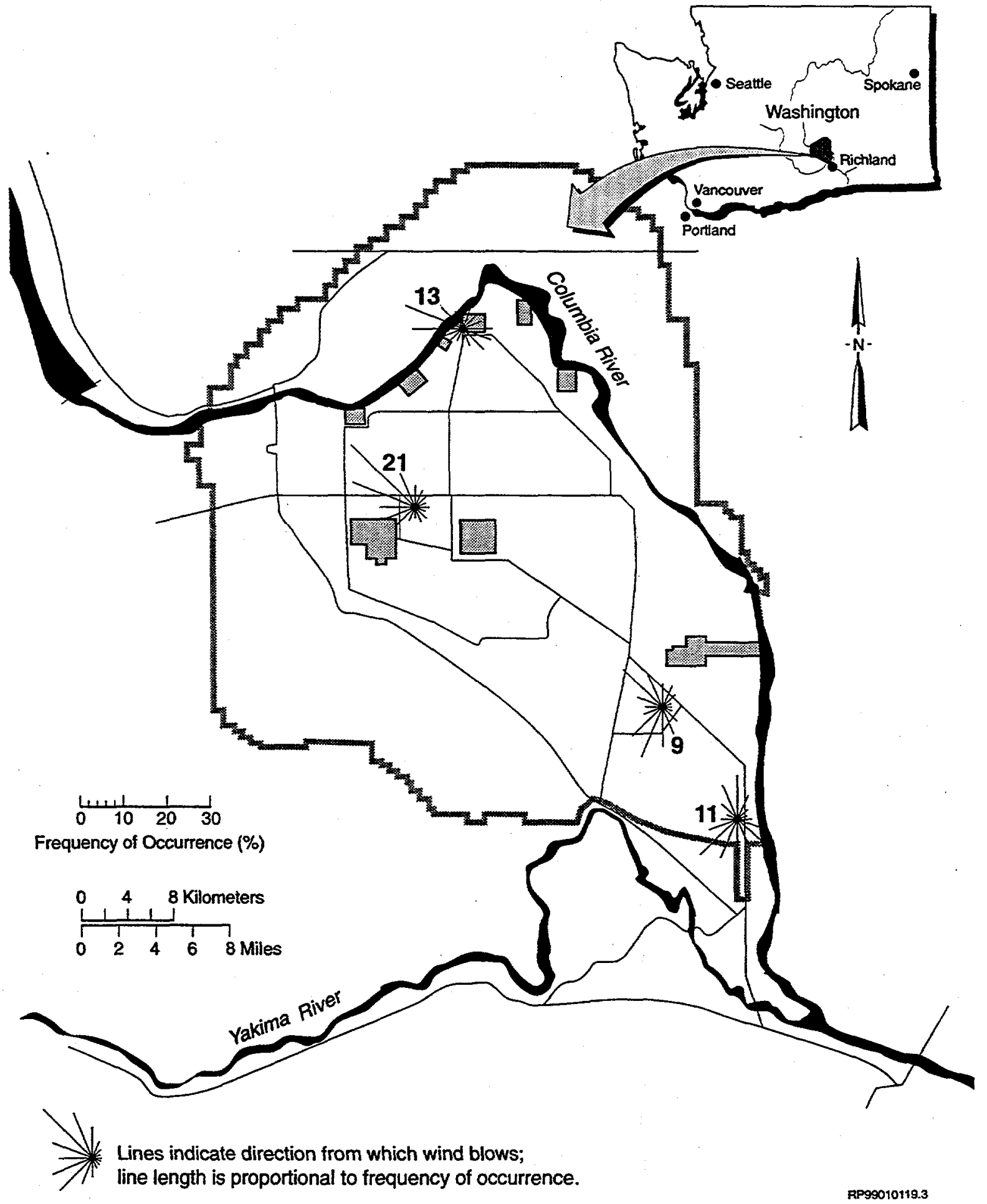

Figure 5.2. Hanford Meteorological Monitoring Network Wind Roses at 60-Meter Level, 1986 Through 1998 
Table 5.5. Joint Frequency Distributions (\%) for Hanford Meteorological Monitoring Network Wind Stations at 60-Meter Level, 1986 Through 1998

Tower: 100 Area

Begin: $1 / 86 \quad$ End: $12 / 98$

Total Hours: 108295

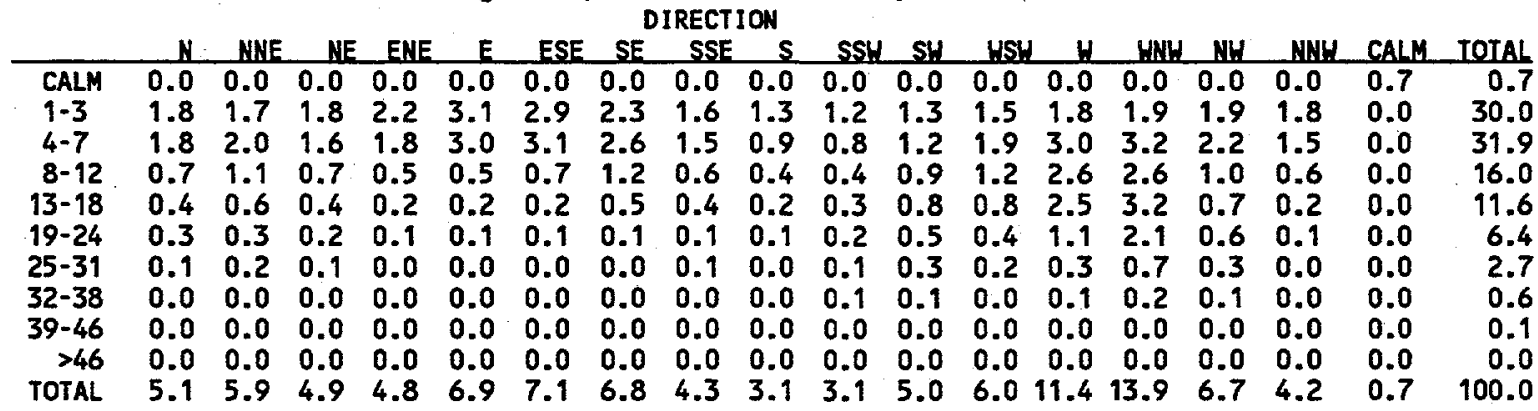

Tower: 200 Area

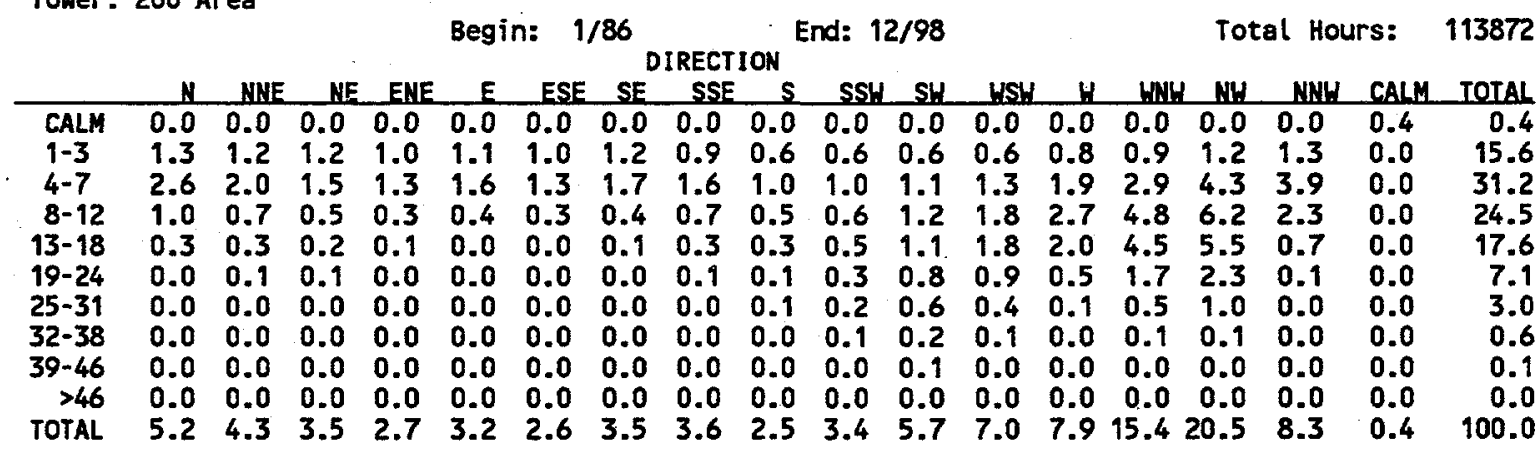

Tower: 300 Area

Begin: 1/86 End: 12/98

DIRECTION

\begin{tabular}{ccccccccccccccccccc} 
& N & NNE & NE & ENE & E & ESE & SE & SSE & S & SSH & SH & HSH & H & WNH & NH & NNH & CALM & TOTAL \\
\hline CALM & 0.0 & 0.0 & 0.0 & 0.0 & 0.0 & 0.0 & 0.0 & 0.0 & 0.0 & 0.0 & 0.0 & 0.0 & 0.0 & 0.0 & 0.0 & 0.0 & 0.6 & 0.6 \\
$1-3$ & 1.0 & 0.8 & 0.7 & 0.6 & 0.8 & 1.0 & 1.2 & 1.2 & 1.1 & 0.9 & 0.8 & 0.6 & 0.7 & 0.7 & 0.9 & 1.0 & 0.0 & 14.4 \\
$4-7$ & 3.1 & 1.9 & 1.2 & 1.2 & 1.8 & 2.8 & 4.1 & 3.1 & 2.7 & 2.4 & 1.9 & 1.3 & 0.9 & 0.8 & 1.2 & 2.4 & 0.0 & 33.1 \\
$8-12$ & 3.5 & 2.1 & 0.9 & 0.4 & 0.4 & 1.3 & 2.6 & 1.4 & 1.8 & 3.3 & 3.2 & 1.7 & 0.8 & 0.5 & 1.0 & 2.4 & 0.0 & 27.8 \\
$13-18$ & 2.0 & 0.9 & 0.2 & 0.1 & 0.0 & 0.2 & 0.4 & 0.3 & 0.5 & 2.0 & 3.2 & 1.9 & 0.6 & 0.4 & 1.0 & 1.8 & 0.0 & 15.7 \\
$19-24$ & 0.3 & 0.2 & 0.1 & 0.0 & 0.0 & 0.0 & 0.0 & 0.0 & 0.1 & 0.7 & 1.4 & 0.9 & 0.3 & 0.1 & 0.7 & 0.5 & 0.0 & 5.5 \\
$25-31$ & 0.0 & 0.1 & 0.0 & 0.0 & 0.0 & 0.0 & 0.0 & 0.0 & 0.0 & 0.2 & 0.8 & 0.3 & 0.1 & 0.0 & 0.2 & 0.1 & 0.0 & 2.2 \\
$32-38$ & 0.0 & 0.0 & 0.0 & 0.0 & 0.0 & 0.0 & 0.0 & 0.0 & 0.0 & 0.1 & 0.3 & 0.1 & 0.0 & 0.0 & 0.0 & 0.0 & 0.0 & 0.6 \\
$39-46$ & 0.0 & 0.0 & 0.0 & 0.0 & 0.0 & 0.0 & 0.0 & 0.0 & 0.0 & 0.0 & 0.2 & 0.0 & 0.0 & 0.0 & 0.0 & 0.0 & 0.0 & 0.2 \\
$>46$ & 0.0 & 0.0 & 0.0 & 0.0 & 0.0 & 0.0 & 0.0 & 0.0 & 0.0 & 0.0 & 0.0 & 0.0 & 0.0 & 0.0 & 0.0 & 0.0 & 0.0 & 0.0 \\
TOTAL & 9.9 & 6.0 & 3.1 & 2.4 & 3.0 & 5.3 & 8.5 & 6.1 & 6.4 & 9.5 & 11.7 & 6.8 & 3.5 & 2.6 & 4.9 & 8.2 & 0.6 & 100.0
\end{tabular}

Tower: 400 Area

$\begin{array}{lllllllllllllllllll}\text { CALM } & 0.0 & 0.0 & 0.0 & 0.0 & 0.0 & 0.0 & 0.0 & 0.0 & 0.0 & 0.0 & 0.0 & 0.0 & 0.0 & 0.0 & 0.0 & 0.0 & 0.3 & 0.3\end{array}$

$\begin{array}{lllllllllllllllllll}1-3 & 0.8 & 0.7 & 0.7 & 0.7 & 0.8 & 0.8 & 0.9 & 1.0 & 0.9 & 0.8 & 0.8 & 0.7 & 0.7 & 0.6 & 0.7 & 0.8 & 0.0 & 12.4\end{array}$

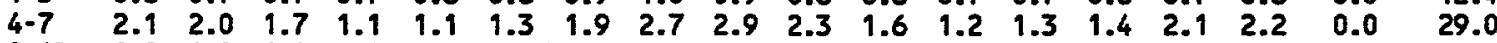

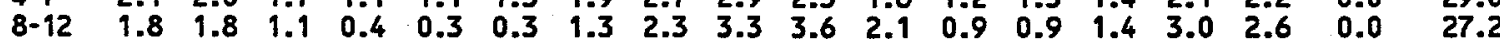

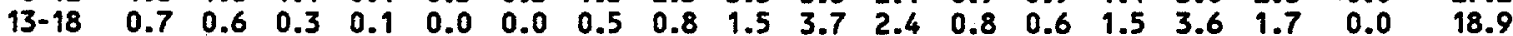

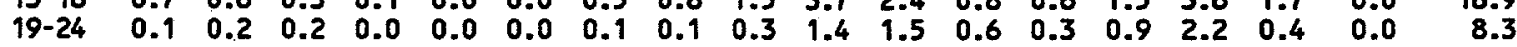

$\begin{array}{lllllllllllllllllll}25-31 & 0.0 & 0.1 & 0.1 & 0.0 & 0.0 & 0.0 & 0.0 & 0.0 & 0.1 & 0.4 & 0.8 & 0.3 & 0.1 & 0.2 & 0.6 & 0.1 & 0.0 & 2.9\end{array}$

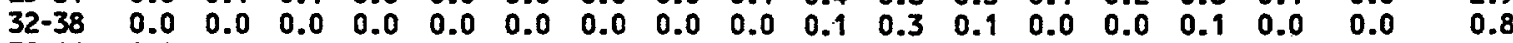

$\begin{array}{lllllllllllllllllll}39-46 & 0.0 & 0.0 & 0.0 & 0.0 & 0.0 & 0.0 & 0.0 & 0.0 & 0.0 & 0.1 & 0.1 & 0.0 & 0.0 & 0.0 & 0.0 & 0.0 & 0.0 & 0.2\end{array}$

$\begin{array}{lllllllllllllllllll}>46 & 0.0 & 0.0 & 0.0 & 0.0 & 0.0 & 0.0 & 0.0 & 0.0 & 0.0 & 0.0 & 0.1 & 0.0 & 0.0 & 0.0 & 0.0 & 0.0 & 0.0 & 0.1\end{array}$

$\begin{array}{lllllllllllllllllll}\text { TOTAL } & 5.6 & 5.5 & 4.1 & 2.3 & 2.2 & 2.5 & 4.8 & 6.9 & 9.1 & 12.5 & 9.6 & 4.5 & 3.9 & 6.0 & 12.3 & 7.9 & 0.3 & 100.0\end{array}$ 


\subsection{Miscellaneous Climatological Statistics}

\subsection{Sky Cover}

The term sky cover is used to express the portion of the celestial dome that is 1) covered, but not necessarily hidden, by clouds or obscuring phenomena aloft; 2) hidden by an obscuring phenomenon on the ground (such as fog or smoke); or 3 ) a combination of both 1 and 2 . The sky cover is determined hourly by scanning the sky and estimating the number of tenths that are covered ( 0 denotes clear and 10 denotes overcast). Average monthly sunrise-to-sunset sky covers for the period 1946 through 1998 are shown in Table 6.1. Also shown in Table 6.1 are the number of clear, partly cloudy, and cloudy days for the period 1954 through 1998. The number of clear, partly cloudy, and cloudy days is the result of assigning each day to one of the following categories based on its average sky cover for that day:

\begin{tabular}{lll}
\multicolumn{1}{c}{ Category } & & $\begin{array}{c}\text { Average } \\
\text { Sky Cover }\end{array}$ \\
Clear & & $0-3$ tenths \\
Partly cloudy & & $4-7$ tenths \\
Cloudy & & $8-10$ tenths
\end{tabular}

During the period of record (1954 through 1998), an average of 200 sunny days (the sum of the clear and partly cloudy days) was recorded per year at the HMS.

\subsection{Fog and Dense Fog}

Table 6.2 shows the average monthly and annual number of days with fog and dense fog. Fog is reported any time horizontal visibility is reduced to $6 \mathrm{mi}$ or less because of the suspension of water droplets in the surface layer of the atmosphere. Dense fog is reported when horizontal visibility is reduced to $0.25 \mathrm{mi}$ or less. Most fog at the HMS is radiation fog, a common type of fog that forms on nights characterized by light wind, clear sky, and moist air in the lower levels of the atmosphere. Nearly $90 \%$ of both fog and dense fog at the HMS occurs during the late autumn and winter months, though fog is observed every month of the year.

\subsection{Psychrometric Data}

Psychrometric data include observations of dry bulb, wet bulb, dew point temperatures, and relative humidity. The dry bulb temperature is the temperature of the ambient air; the wet bulb temperature is the lowest temperature to which a parcel of air, under constant pressure, can be cooled by evaporating water into it. The dew point temperature is the temperature to which a given parcel of air, under constant 
Table 6.1. Average Sky Cover (sunrise to sunset), 1946 Through 1998, and Number of Days Clear, Partly Cloudy, and Cloudy, 1954 Through 1998

\begin{tabular}{|c|c|c|c|c|c|c|c|c|c|c|c|c|c|c|c|c|}
\hline \multirow[b]{2}{*}{ Month } & \multicolumn{5}{|c|}{ Sky Cover (Scale 0-10) } & \multicolumn{5}{|c|}{ Number of Clear Days } & \multirow{2}{*}{$\begin{array}{c}\text { Number of } \\
\text { Partly Cloudy } \\
\text { Days } \\
\end{array}$} & \multicolumn{5}{|c|}{ Number of Cloudy Days } \\
\hline & Avg & Max & Year & Min & Year & Avg & Max & Year & Min & Year & & Avg & $\operatorname{Max}$ & Year & Min & Year \\
\hline Jan & 7.9 & 9.2 & 1978 & 4.3 & 1949 & 3.5 & 9 & 1984 & 0 & $1955^{(3)}$ & 5.3 & 22.3 & 28 & 1978 & 17 & 1963 \\
\hline Feb & 7.5 & 9.3 & 1980 & 5.9 & 1996 & 4.3 & 9 & $1991^{(a)}$ & 0 & $1984^{(\mathrm{a})}$ & 5.3 & 18.7 & 26 & $1980^{(\mathrm{s})}$ & 12 & 1964 \\
\hline Mar & 6.8 & 8.5 & 1978 & 4.9 & 1965 & 6.3 & 12 & $1979^{(a)}$ & 1 & $1978^{(a)}$ & 8.2 & 16.5 & 24 & 1993 & 9 & $1979^{(a)}$ \\
\hline Apr & 6.4 & 8.1 & 1963 & 3.7 & 1951 & 6.4 & 12 & 1962 & 1 & 1963 & 9.1 & 14.5 & 21 & $1979^{(a)}$ & 6 & 1956 \\
\hline May & 5.9 & 8.1 & 1993 & 3.6 & 1992 & 8.4 & 18 & 1992 & 1 & 1977 & 10.4 & 12.2 & 19 & $1977^{(a)}$ & 3 & 1992 \\
\hline Jun & 5.2 & 7.0 & 1950 & 2.8 & 1961 & 10.4 & 21 & 1961 & 5 & $1972^{(1)}$ & 9.9 & 9.7 & 15 & $1983^{(\mathrm{a})}$ & 5 & $1979^{(a)}$ \\
\hline Jul & 3.1 & 5.0 & 1983 & 0.9 & 1953 & 18.8 & 26 & 1960 & 12 & $1987^{(2)}$ & 7.7 & 4.6 & 12 & 1976 & 0 & $1996^{(a)}$ \\
\hline Aug & 3.3 & 5.9 & 1968 & 0.6 & 1955 & 18.4 & 30 & 1955 & 9 & 1978 & 7.6 & 5.0 & 13 & $1983^{(a)}$ & 0 & $1994^{(a)}$ \\
\hline Sep & 3.9 & 6.7 & 1978 & 1.4 & $1990^{(2)}$ & 15.4 & 27 & 1975 & 6 & 1978 & 7.5 & 7.1 & 16 & 1977 & 0 & 1990 \\
\hline Oct & 5.7 & 8.0 & 1975 & 3.3 & 1987 & 10.2 & 20 & 1987 & 1 & 1975 & 7.9 & 12.9 & 22 & 1973 & 6 & 1986 \\
\hline Nov & 7.5 & 9.1 & 1972 & 5.2 & 1993 & 4.7 & 12 & 1993 & 1 & $1973^{(2)}$ & 5.7 & 19.6 & 25 & $1973^{(\text {(a) }}$ & 13 & 1993 \\
\hline Dec & 8.0 & 9.3 & 1985 & 6.4 & 1978 & 3.8 & 9 & 1978 & 1 & $1985^{(a)}$ & 4.5 & 22.7 & 29 & 1985 & 17 & 1978 \\
\hline Annual & 5.9 & 6.6 & $1978^{(a)}$ & 5.1 & 1949 & 110.9 & 144 & 1998 & 80 & 1977 & 89.1 & 165.4 & 193 & 1978 & 146 & 1991 \\
\hline
\end{tabular}

(a) Most recent of multiple occurrences. 
Table 6.2. Monthly and Annual Number of Days with Fog and Dense Fog, 1945 Through 1998

\begin{tabular}{|c|c|c|c|c|c|c|c|c|c|c|}
\hline \multirow[b]{2}{*}{ Month } & \multicolumn{5}{|c|}{ Days with Fog (Visibility } & \multicolumn{3}{|c|}{ Days with Dense Fog (Visibility } & \multicolumn{2}{|c|}{$0.25 \mathrm{mi}$ ) } \\
\hline & Avg & $\operatorname{Max}$ & Year & Min & Year & Avg & Max & Year & Min & Year \\
\hline Jan & 11.6 & 25 & 1979 & 0 & 1949 & 6.1 & 15 & $1994^{(a)}$ & 0 & 1949 \\
\hline Feb & 6.6 & 20 & 1963 & 0 & $1988^{(2)}$ & 3.3 & 11 & 1963 & 0 & $1988^{(2)}$ \\
\hline Mar & 2.1 & 10 & 1993 & 0 & $1994^{(2)}$ & 0.8 & 5 & $1993^{(2)}$ & 0 & $1998^{(2)}$ \\
\hline Apr & 0.5 & 3 & 1992 & 0 & $1997^{(\mathrm{a})}$ & 0.1 & 1 & $1993^{(a)}$ & 0 & $1997^{(2)}$ \\
\hline May & 0.2 & 3 & 1948 & 0 & $1997^{(2)}$ & $<0.1$ & 1 & 1958 & 0 & $1997^{(a)}$ \\
\hline Jun & 0.1 & 2 & 1971 & 0 & $1998^{(a)}$ & $<0.1$ & 1 & 1971 & 0 & $1998^{(2)}$ \\
\hline Jul & $<0.1$ & 1 & 1966 & 0 & $1998^{(2)}$ & 0 & 0 & 0 & & \\
\hline Aug & 0.1 & 1 & $1985^{(2)}$ & 0 & $1998^{(2)}$ & $<0.1$ & 1 & $1985^{(a)}$ & 0 & $1998^{(2)}$ \\
\hline Sep & 0.3 & 2 & $1985^{(2)}$ & 0 & $1997^{(2)}$ & 0.1 & 1 & $1995^{(2)}$ & 0 & $1997^{(2)}$ \\
\hline Oct & 2.0 & 9 & 1962 & 0 & $1989^{(a)}$ & 1.0 & 7 & 1980 & 0 & $1998^{(2)}$ \\
\hline Nov & 9.8 & 19 & $1985^{(3)}$ & 0 & 1990 & 5.6 & 13 & 1965 & 0 & $1990^{(2)}$ \\
\hline Dec & 14.1 & 25 & $1989^{(a)}$ & 2 & 1968 & 7.5 & 17 & 1950 & 2 & $1996^{(2)}$ \\
\hline Annual & 47.4 & 84 & $1985-86$ & 22 & $1948-49$ & 24.6 & 42 & $1950-51$ & 9 & $1948-49$ \\
\hline
\end{tabular}

(a) Most recent of multiple occurrences.

Longest duration of fog: $113.7 \mathrm{~h}$, December 16-20, 1985.

Longest duration of dense fog: $47.0 \mathrm{~h}$, December 1957.

pressure and water-vapor content, must be cooled to attain saturation. Relative humidity is the ratio of the actual water-vapor content of the air to the one where saturation would occur if the pressure and temperature remained unchanged.

Table 6.3 presents monthly averages and extremes of dry bulb, wet bulb, dew point temperatures, and relative humidity from the HMS for the period 1950 through 1998. These parameters are collected hourly and are averaged on a monthly (as opposed to a daily) basis. Prior to 1975 , wet bulb temperatures $\geq 75^{\circ} \mathrm{F}$ had never been observed at the HMS. On July 8, 9, and 10, 1975, 7 hourly observations were made of wet bulb temperatures $\geq 75^{\circ} \mathrm{F}$.

\subsection{Solar Radiation}

Table 6.4 presents average and extreme daily solar radiation values by month for the period 1953 through 1998. These data are reported in langleys (a langley is a unit defined as 1 gram calorie per square centimeter.)

The highest daily values occur with a clear sky and clean air; the lowest commonly occur on days overcast with low stratus clouds. The lowest midday values of hourly solar radiation occurred on May 18, 1980, as the dense ash cloud from the morning eruption of Mount St. Helens passed over eastern Washington. Hourly solar radiation values dropped to 0 at $1100 \mathrm{~h}$ and remained at 0 for the rest of that day. 
Table 6.3. Monthly Averages and Extremes of Psychrometric Data, 1950 Through 1998

\begin{tabular}{|c|c|c|c|c|c|c|c|c|c|c|c|c|c|}
\hline \multirow{2}{*}{ Category (a) } & \multicolumn{13}{|c|}{ Monthly Averages } \\
\hline & Jan & Feb & Mar & Apr & May & Jun & Jul & Aug & Sep & Oct & Nov & Dec & Annual \\
\hline Dry bulb & 31.0 & 37.5 & 45.1 & 53.2 & 62.2 & 69.9 & 77.3 & 75.7 & 66.5 & 53.0 & 40.1 & 32.4 & 53.7 \\
\hline Wet bulb & 29 & 34 & 38 & 44 & 50 & 55 & 58 & 58 & 53 & 45 & 36 & 30 & 44 \\
\hline Rel. hum. & 77.3 & 70.5 & 56.6 & 47.3 & 43.0 & 39.6 & 33.3 & 35.5 & 42.2 & 56.4 & 73.6 & 80.1 & 54.6 \\
\hline \multirow[t]{2}{*}{ Dew point } & 24.2 & 27.8 & 28.9 & 31.7 & 37.1 & 41.6 & 43.7 & 43.9 & 40.5 & 36.1 & 31.4 & 26.4 & 34.4 \\
\hline & \multicolumn{13}{|c|}{ Extremes of Monthly Averages } \\
\hline Dry Bulb & & & & & & & & & & & & & \\
\hline Highest & 43.0 & 44.6 & 51.6 & 58.6 & 68.7 & 77.3 & 83.3 & 82.5 & 72.7 & 59.5 & 46.3 & 38.8 & 56.6 \\
\hline Year & 1953 & 1991 & 1992 & 1987 & 1958 & 1992 & 1985 & 1967 & 1990 & 1988 & 1990 & 1953 & 1992 \\
\hline Lowest & 12.9 & 25.8 & 39.6 & 48.3 & 57.0 & 64.2 & 71.3 & 70.6 & 58.9 & 48.1 & 25.7 & 21.9 & 50.2 \\
\hline Year & 1950 & 1956 & 1955 & 1955 & 1984 & 1953 & 1986 & 1964 & 1985 & 1984 & 1985 & 1985 & 1985 \\
\hline \multicolumn{14}{|l|}{ Wet Bulb } \\
\hline Highest & 39 & 41 & 44 & 47 & 55 & 59 & 63 & & 56 & 50 & 42 & & 47 \\
\hline Year & 1953 & 1956 & 1992 & 1992 & 1958 & $1992^{(0)}$ & 1998 & $1991^{(b)}$ & $1995^{(0)}$ & 1988 & 1954 & $1991^{(0)}$ & 1992 \\
\hline Lowest & 12 & 23 & 33 & 39 & 45 & 51 & 56 & 55 & 48 & 40 & 24 & 21 & 41 \\
\hline Year & 1950 & 1956 & 1955 & 1955 & 1959 & $1983^{(0)}$ & $1986^{(b)}$ & $1980^{(6)}$ & 1970 & 1984 & 1985 & 1985 & 1985 \\
\hline \multicolumn{14}{|c|}{ Relative Humidity } \\
\hline Highest & 88.8 & 86.9 & 69.1 & 64.5 & 61.9 & 53.5 & 45.6 & 47.8 & 55.5 & 74.2 & 88.7 & 90.5 & 58.9 \\
\hline Year & 1960 & 1963 & 1993 & 1963 & 1948 & 1950 & 1993 & 1976 & 1977 & 1962 & 1979 & 1950 & 1978 \\
\hline Lowest & 60.0 & 54.0 & 44.0 & 36.9 & 31.2 & 30.0 & 21.9 & 24.5 & 33.2 & 42.5 & 62.8 & 69.0 & 49.4 \\
\hline Year & 1963 & 1967 & 1965 & 1966 & 1966 & 1949 & 1959 & 1967 & 1974 & 1952 & 1976 & 1968 & 1967 \\
\hline \multicolumn{14}{|l|}{ Dew Point } \\
\hline Highest & 34.4 & 36.7 & 37.2 & 37.1 & 43.9 & 47.5 & 50.1 & 48.4 & 45.4 & 43.5 & 38.3 & 34.3 & 37.7 \\
\hline Year & 1953 & $1992^{(b)}$ & 1986 & $1992^{(0)}$ & 1998 & 1958 & 1975 & 1976 & 1963 & 1962 & 1954 & 1950 & 1958 \\
\hline Lowest & 6.5 & .17 .3 & 20.8 & 26.0 & 30.4 & 37.5 & 35.4 & 38.4 & 33.8 & 30.2 & 19.4 & 15.1 & 31.5 \\
\hline Year & 1950 & 1956 & $1965^{(0)}$ & 1982 & 1964 & 1954 & 1959 & 1955 & 1970 & 1984 & 1985 & 1983 & 1955 \\
\hline
\end{tabular}

(a) Dry bulb, wet bulb, and dew point temperatures in ${ }^{\circ} \mathrm{F}$, relative humidity in $\%$

(b) Most recent of multiple occurrences.

Table 6.4. Average and Extreme Solar Radiation Daily Values (langley), 1953 Through 1998

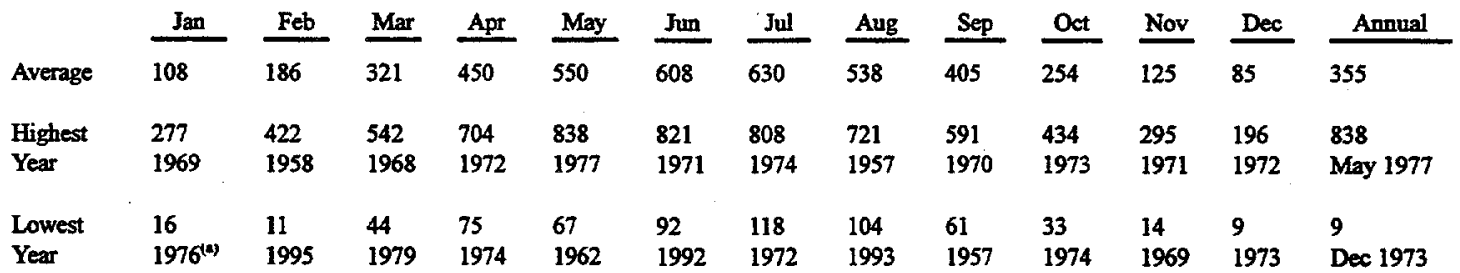

(a) Most recent of multiple occurrences. 


\subsection{Thunderstorms}

A thunderstorm day is one in which thunder is heard at the observing station one or more times during a calendar day. If a thunderstorm were to begin before midnight and continue until after midnight, it is possible to have two thunderstorm days from a single storm.

Table 6.5 shows that thunderstorms occurred in every month of the year, except January and November. The thunderstorm season is essentially from April through September. The average number of thunderstorm days per year is 10; however, the total varies from a low of 3 in 1949 to a high of 23 in 1948. The largest number of thunderstorms in any single month was 8 in June 1972, July 1983, and August 1953.

Table 6.5. Average Number of Days of Various Meteorological Phenomena, 1945 Through 1998

\begin{tabular}{|c|c|c|c|c|c|c|c|c|c|c|c|c|c|}
\hline Phenomenon & Jan & Feb & Mar & Apr & May & Jun & Jul & Aug & Sep & Oct & Nov & Dec & Annual \\
\hline Thunderstorm & 0 & $\leq 0.1$ & 0.1 & 0.8 & 1.6 & 2.3 & 2.2 & 1.9 & 0.8 & 0.2 & 0 & $\leq 0.1$ & 10.0 \\
\hline Dust or blowing dust & 0.4 & 0.4 & 0.5 & 0.6 & 0.7 & 0.4 & 0.4 & 0.3 & 0.5 & 0.3 & 0.2 & 0.2 & 4.7 \\
\hline Glaze & 2.2 & 0.7 & 0.1 & 0 & 0 & 0 & 0 & 0 & 0 & 0 & 0.8 & 2.4 & 6.2 \\
\hline
\end{tabular}

\subsection{Dust and Blowing Dust}

The criterion for both dust and blowing dust is that horizontal visibility be reduced to $6 \mathrm{mi}$ or less. Dust is carried into the area from a distant source and may occur without strong winds. Blowing dust occurs when dust is being picked up locally and occurs with stronger winds. Both dust and blowing dust occurred at the HMS; however, in most cases, it is blowing dust. Table 6.5 presents the average number of days per month and year of dust and blowing dust during the period 1945 through 1998 . The average number of days per year with dust or blowing dust is 5 . The greatest number of such days in any year was 20 in 1980; the fewest was 0 in 1987 and earlier years. The greatest number of days with dust or blowing dust in any month was 9 in May 1980. This peak in the number of days with dust or blowing dust resulted from the eruption of Mount St. Helens on May 18, 1980 and subsequent dates.

\subsection{Glaze}

Glaze is a coating of ice formed when rain or drizzle freezes on contact with any surface having a temperature that is below freezing. Table 6.5 provides data on the number of days per month and year with glaze for the period 1945 through 1998. The average number of days with freezing rain or freezing drizzle is 6 . The highest number of days with glaze in any winter season was 18 during the winter of 1969-1970; the least, 1 day during the winter of 1987-1988 and earlier winters. The greatest number of such days in any single month was 9 in January 1970. 


\subsection{Atmospheric Pressure}

Table 6.6 contains atmospheric pressure data for the period 1955 through 1998. This table lists both station and sea-level pressure, including extremes and years of occurrence. Atmospheric pressure may be indicated in several different units, including inches of mercury, millimeters of mercury, millibars; or Pascals; however, in this table, pressure is stated in inches of mercury. Station pressure is the barometric pressure measured at the HMS (at an elevation of $733 \mathrm{ft}$ ); sea-level pressure is the station pressure adjusted to sea level. Most are familiar with barometric pressure adjusted to sea level, which allows atmospheric pressures for all locations to be compared, regardless of the elevation of the station where the data are measured.

The highest sea-level pressure ever recorded at the HMS was 31.12 in. in January 1979; the lowest was 28.94 in. in December 1995 and on previous occasions.

Some rapid pressure changes occurred on November 3,1958 , falling $0.492 \mathrm{in}$. over a 6 -h period $(0.082 \mathrm{in} . \mathrm{h})$, including a $1-\mathrm{h}$ fall of $0.160 \mathrm{in}$. On the same day, the pressure rose $0.554 \mathrm{in}$. during a $6-\mathrm{h}$ period $(0.090 \mathrm{in} . \mathrm{h})$, including a $1-\mathrm{h}$ rise of $0.145 \mathrm{in}$. The greatest sea-level pressure change during a 1-day period was 1.02 in. (December 8, 1971). 
Table 6.6. Average and Extreme Station and Sea-Level Pressure Data, 1955 Through 1998 (inches of mercury)

\begin{tabular}{|c|c|c|c|c|c|c|c|c|c|c|}
\hline \multirow[b]{2}{*}{ Month } & \multicolumn{6}{|c|}{ Station Pressure } & \multicolumn{4}{|c|}{ Sea-Level Pressure } \\
\hline & Average & High & Year & Low & Year & $\begin{array}{c}\text { Greatest Daily } \\
\text { Range }\end{array}$ & High & Year & Low & Year \\
\hline Jan & 29.33 & 30.23 & $1979^{(a)}$ & 28.18 & 1980 & 0.77 & 31.12 & 1979 & 28.94 & 1964 \\
\hline Feb & 29.27 & $30: 08$ & 1956 & 28.23 & $1958^{(a)}$ & 0.79 & 30.97 & $1956^{(a)}$ & 28.98 & $1958^{(1)}$ \\
\hline Mar & 29.20 & 29.92 & 1955 & 28.34 & 1995 & 0.85 & 30.79 & 1955 & 29.11 & 1995 \\
\hline Apr & 29.19 & 29.84 & $1973^{(\mathbf{a})}$ & 28.49 & $1962^{(a)}$ & 0.81 & 30.64 & 1973 & 29.26 & 1962 \\
\hline May & 29.16 & 29.68 & $1970^{(8)}$ & 28.62 & $1957^{(\mathrm{a})}$ & 0.46 & 30.48 & $1970^{(a)}$ & 29.38 & $1957^{(\text {a) }}$ \\
\hline Jun & 29.13 & 29.60 & $1987^{(a)}$ & 28.67 & $1992^{(a)}$ & 0.54 & 30.40 & 1987 & 29.42 & 1992 \\
\hline Jul & 29.14 & 29.56 & $1993^{(a)}$ & 28.80 & $1979^{(a)}$ & 0.37 & 30.34 & $1993^{(8)}$ & 29.55 & $1979^{(a)}$ \\
\hline Aug & 29.14 & 29.55 & 1968 & 28.75 & 1980 & 0.39 & 30.32 & 1968 & 29.52 & 1980 \\
\hline Sep & 29.18 & 29.79 & $1983^{(\mathrm{a})}$ & 28.48 & $1986^{(a)}$ & 0.56 & 30.60 & 1983 & 29.25 & 1986 \\
\hline Oct & 29.25 & 29.82 & $1993^{(a)}$ & 28.39 & 1962 & 0.73 & 30.63 & $1993^{(a)}$ & 29.15 & 1962 \\
\hline Nov & 29.28 & 30.06 & $1979^{(a)}$ & 28.36 & $1982^{(s)}$ & 0.78 & 30.90 & $1979^{(a)}$ & 29.13 & 1982 \\
\hline Dec & 29.32 & 30.20 & 1978 & 28.16 & 1995 & 1.02 & 31.07 & 1978 & 28.94 & $1995^{(2)}$ \\
\hline Annual & 29.22 & 30.23 & Jan $1979^{(a)}$ & 28.16 & Dec 1995 & 1.02 & 31.12 & Jan 1979 & 28.94 & $\operatorname{Dec} 1995^{(0)}$ \\
\hline
\end{tabular}

(a) Most recent of several occurrences. 


\subsection{Extreme Values}

Extreme values are generally described in terms of probability of occurrence or in terms of return period. For low probability events, the return period is simply the reciprocal of the probability when the probability is expressed as the likelihood of the event occurring in a given year. As with all estimate extreme values, the uncertainty in the estimates increases as the return period increases.

\subsection{Annual Temperature Extremes}

Annual maximum and minimum temperatures with return periods from 2 to 1000 years are listed in Table 7.1. The probabilities of exceeding various maximum and minimum temperatures are shown in Figures 7.1 and 7.2 along with the maximum and minimum temperatures observed at the HMS from 1945 through 1998. The curves were estimated by assuming that the annual extreme temperatures may be fit using a normal distribution and calculating distribution parameters from the observed data.

\subsection{Precipitation Rates}

Maximum precipitation rates for return periods of 2 to 1000 years are listed in Table 7.2. The corresponding precipitation amounts are listed in Table 7.3. The precipitation rate estimates are based on precipitation measurements made at the HMS from 1947 through 1998. The precipitation rates were estimated for each return period assuming a lognormal distribution and distribution parameters calculated from the data. Figure 7.3 shows the predicted rates for $1,3,6$, and 12 hours duration along with the observed data.

Table 7.1. Return Periods for Annual Maximum and Minimum Temperatures

$\begin{array}{cccc}\begin{array}{c}\text { Return Period } \\ (\mathrm{yr})\end{array} & & \begin{array}{c}\text { Maximum Temperature } \\ \left({ }^{\circ} \mathrm{F}\right)\end{array} & \begin{array}{c}\text { Minimum Temperature } \\ \left({ }^{\circ} \mathrm{F}\right)\end{array} \\ \begin{array}{c}106.1 \\ 5\end{array} & 107.3 & -0.9 \\ 10 & 108.7 & -9.2 \\ 20 & 110.1 & -13.5 \\ 50 & 111.2 & -17.1 \\ 100 & 113.3 & -21.2 \\ 200 & 114.0 & -23.9 \\ 500 & 115.0 & -26.3 \\ 1000 & 115.6 & -29.3 \\ & & -31.4\end{array}$




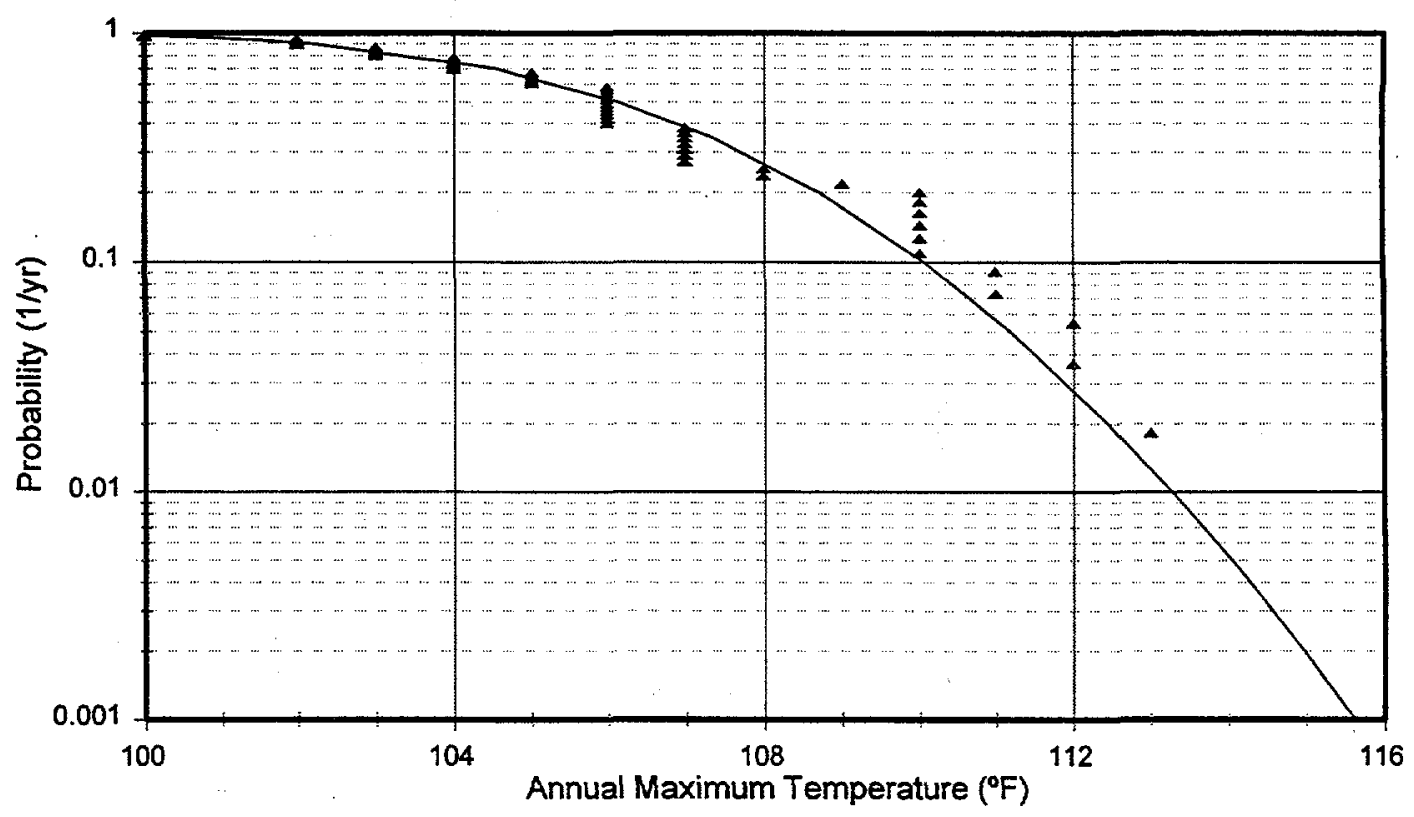

Figure 7.1. Probability of an Annual Maximum Temperature Exceeding a Given Value

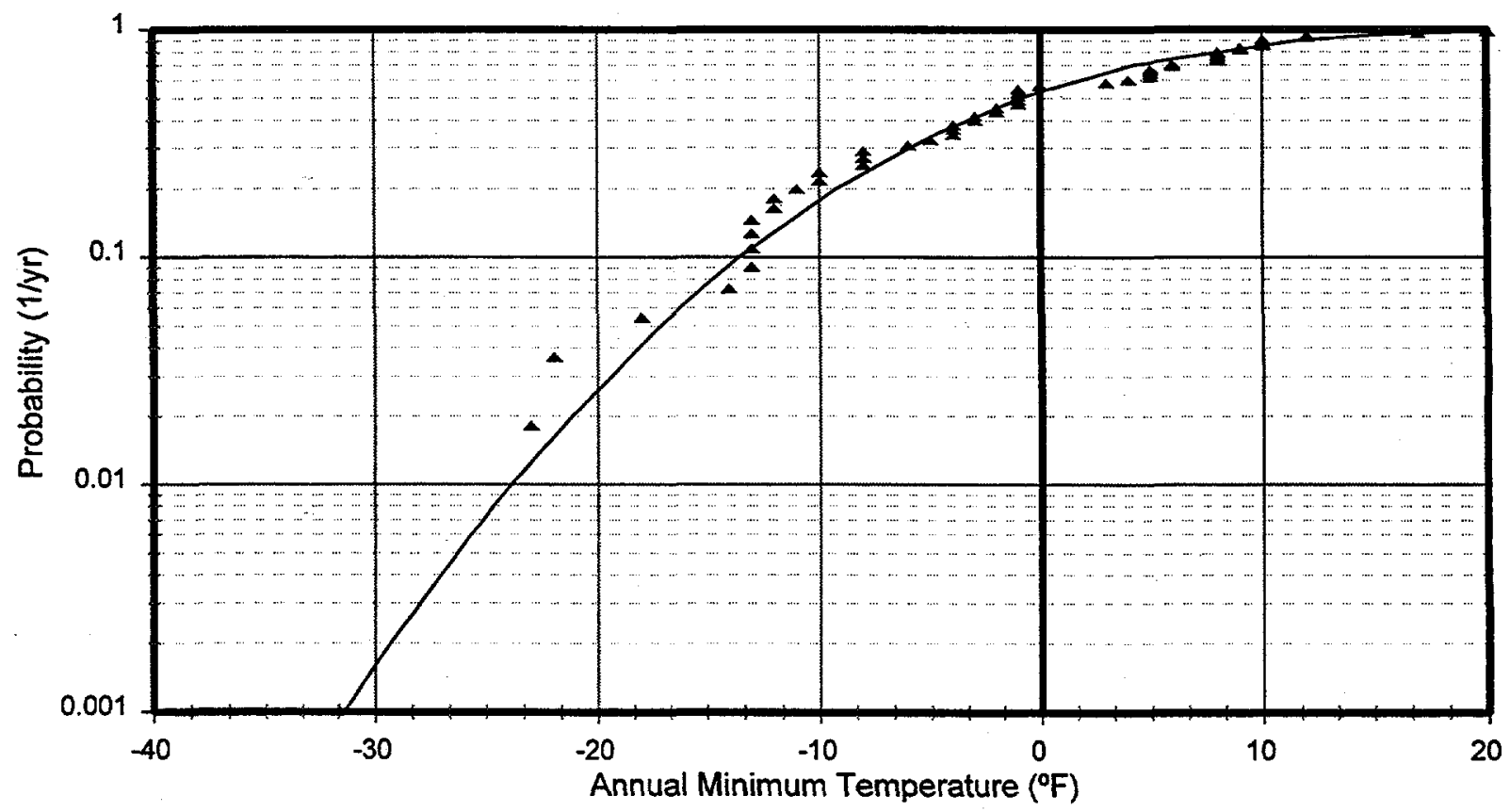

Figure 7.2. Probability of an Annual Minimum Temperature Being Less Than a Given Value 
Table 7.2. Precipitation Rates (in./h) for 1 to 24 Hours Duration and Return Periods from 2 to 1000 Years

\begin{tabular}{|c|c|c|c|c|c|c|}
\hline \multirow{2}{*}{$\begin{array}{c}\text { Return } \\
\text { Period (yr) }\end{array}$} & \multicolumn{6}{|c|}{ Duration } \\
\hline & $1 \mathrm{~h}$ & $2 \mathrm{~h}$ & $3 \mathrm{~h}$ & $6 \mathrm{~h}$ & $12 \mathrm{~h}$ & $24 \mathrm{~h}$ \\
\hline 2 & 0.22 & 0.15 & 0.12 & 0.081 & 0.051 & 0.029 \\
\hline 5 & 0.31 & 0.21 & 0.16 & 0.11 & 0.067 & 0.039 \\
\hline 10 & 0.38 & 0.24 & 0.18 & 0.12 & 0.078 & 0.046 \\
\hline 20 & 0.44 & 0.28 & 0.20 & 0.14 & 0.089 & 0.052 \\
\hline 50 & 0.52 & 0.32 & 0.23 & 0.16 & 0.10 & 0.061 \\
\hline 100 & 0.58 & 0.36 & 0.25 & 0.18 & 0.11 & .0067 \\
\hline 200 & 0.65 & 0.39 & 0.27 & 0.19 & 0.12 & 0.073 \\
\hline 500 & 0.73 & 0.43 & 0.30 & 0.21 & 0.14 & 0.081 \\
\hline 1000 & 0.80 & 0.47 & 0.32 & 0.22 & 0.15 & 0.088 \\
\hline
\end{tabular}

Table 7.3. Precipitation Amounts (in.) for 1 to 24 Hours in Periods and Return Periods from 2 to 1000 Years

\begin{tabular}{|c|c|c|c|c|c|c|}
\hline \multirow{2}{*}{$\begin{array}{c}\text { Return } \\
\text { Period (yr) }\end{array}$} & \multicolumn{6}{|c|}{ Duration } \\
\hline & $1 \mathrm{~h}$ & $2 \mathrm{~h}$ & $3 \mathrm{~h}$ & $6 \mathrm{~h}$ & $12 \mathrm{~h}$ & $24 \mathrm{~h}$ \\
\hline 2 & 0.22 & 0.31 & 0.36 & 0.48 & 0.61 & 0.70 \\
\hline 5 & 0.31 & 0.42 & 0.47 & 0.64 & 0.81 & 0.95 \\
\hline 10 & 0.38 & 0.49 & 0.54 & 0.74 & 0.94 & 1.11 \\
\hline 20 & 0.44 & 0.56 & 0.61 & 0.84 & 1.07 & 1.26 \\
\hline 50 & 0.52 & 0.65 & 0.69 & 0.96 & 1.23 & 1.46 \\
\hline 100 & 0.58 & 0.71 & 0.75 & 1.05 & 1.35 & 1.61 \\
\hline 200 & 0.65 & 0.78 & 0.81 & 1.14 & 1.47 & 1.75 \\
\hline 500 & 0.73 & 0.87 & 0.89 & 1.26 & 1.63 & 1.95 \\
\hline 1000 & 0.80 & 0.94 & 0.95 & 1.35 & 1.75 & 2.11 \\
\hline
\end{tabular}

\subsection{Snow}

Snow extremes for return periods from 2 to 1000 years are listed in Table 7.4. The estimates are based on data from the HMS for the 1946-1947 through 1997-1998 snow seasons. The values in the tables were estimated assuming a Type 1 (Gumbel) extreme value distribution (Johnson et al. 1995) using maximum-likelihood estimates (Kinnison 1985) of the distribution parameter values calculated from the HMS data. Figures 7.4, 7.5, and 7.6 show the probabilities of seasonal maximum snowfall, maximum single storm snowfall, and maximum snow depth, respectively with the corresponding HMS data. 


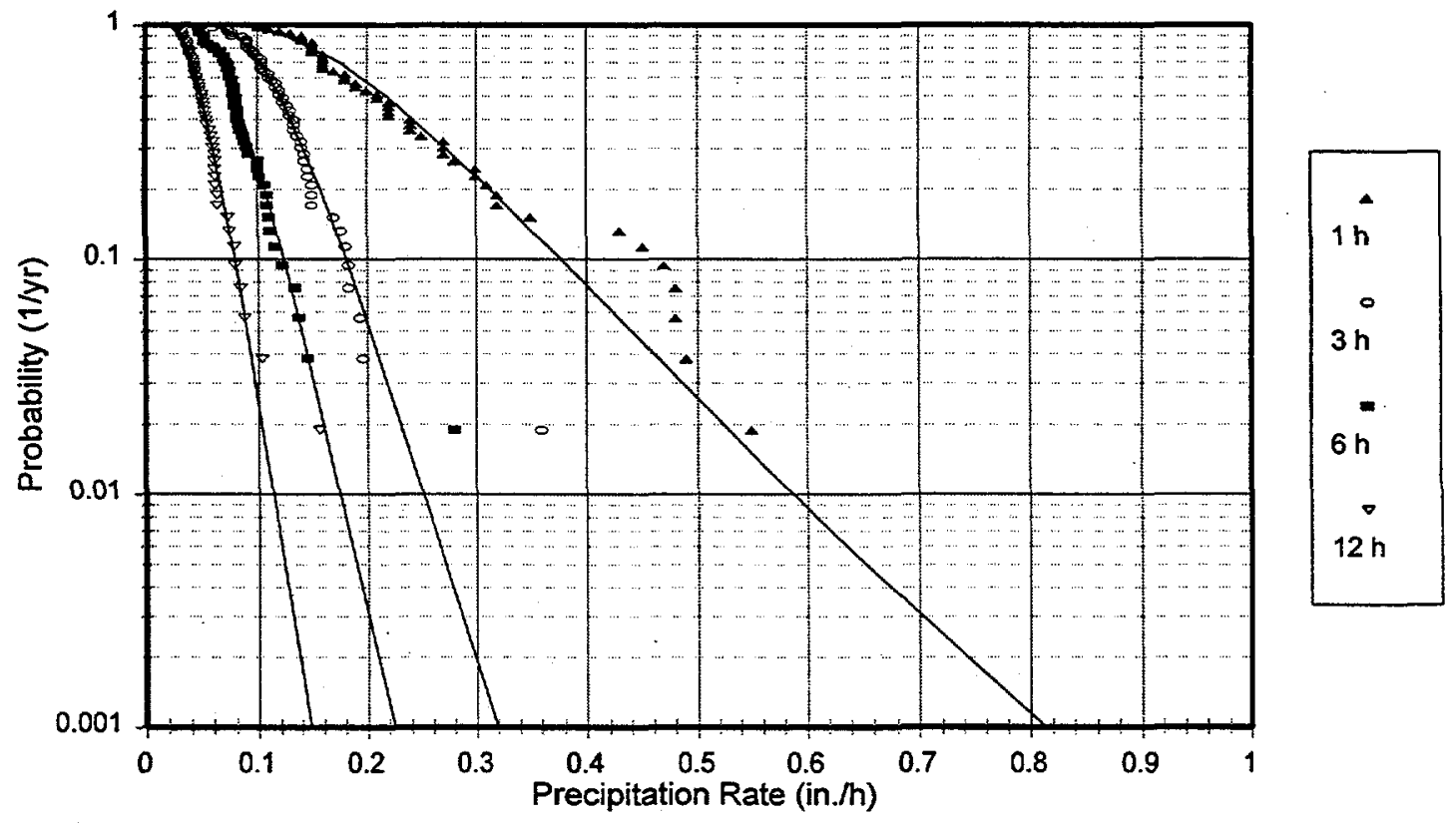

Figure 7.3. Probability of Precipitation Rate Exceeding Given Values by Duration

Table 7.4. Snowfall Extremes for Return Periods from 2 to 1000 Years

\begin{tabular}{|c|c|c|c|}
\hline $\begin{array}{c}\text { Return } \\
\text { Period (yr) }\end{array}$ & $\begin{array}{l}\text { Seasonal Total } \\
\text { (in.) }\end{array}$ & $\begin{array}{l}\text { Single Storm } \\
\text { (in.) }\end{array}$ & $\begin{array}{l}\text { Maximum on } \\
\text { Ground (in.) }\end{array}$ \\
\hline 2 & 13.6 & 3.8 & 5.1 \\
\hline 5. & 23.3 & 6.0 & 8.2 \\
\hline 10 & 29.6 & 7.5 & 10.3 \\
\hline 20 & 35.7 & 8.9 & 12.3 \\
\hline 50 & 43.7 & 10.7 & 14.9 \\
\hline 100 & 49.6 & 12.1 & 16.9 \\
\hline 200 & 55.5 & 13.4 & 18.8 \\
\hline 500 & 63.3 & 15.2 & 21.4 \\
\hline 1000 & 69.2 & 16.5 & 23.3 \\
\hline
\end{tabular}




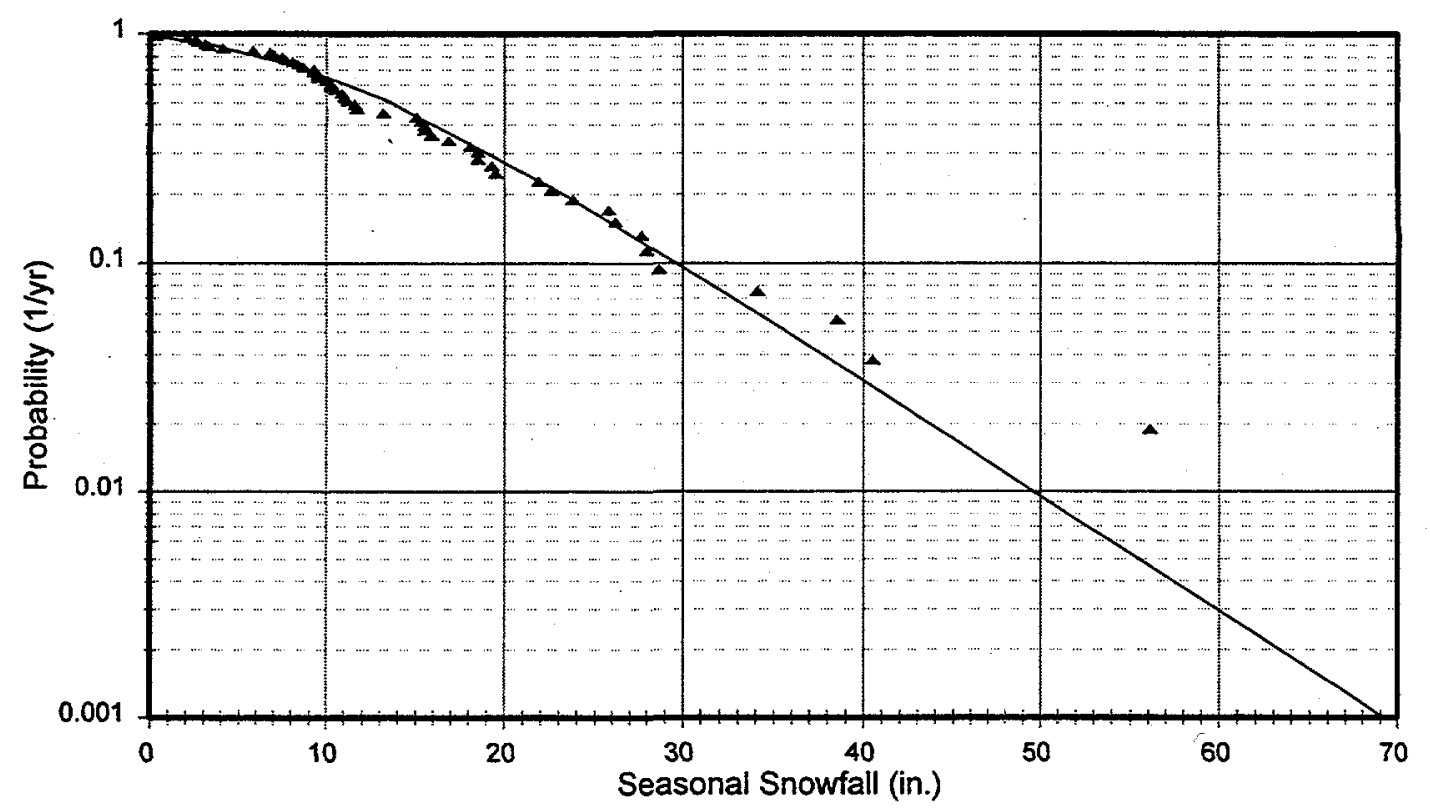

Figure 7.4. Probability of Exceeding a Given Seasonal Snowfall

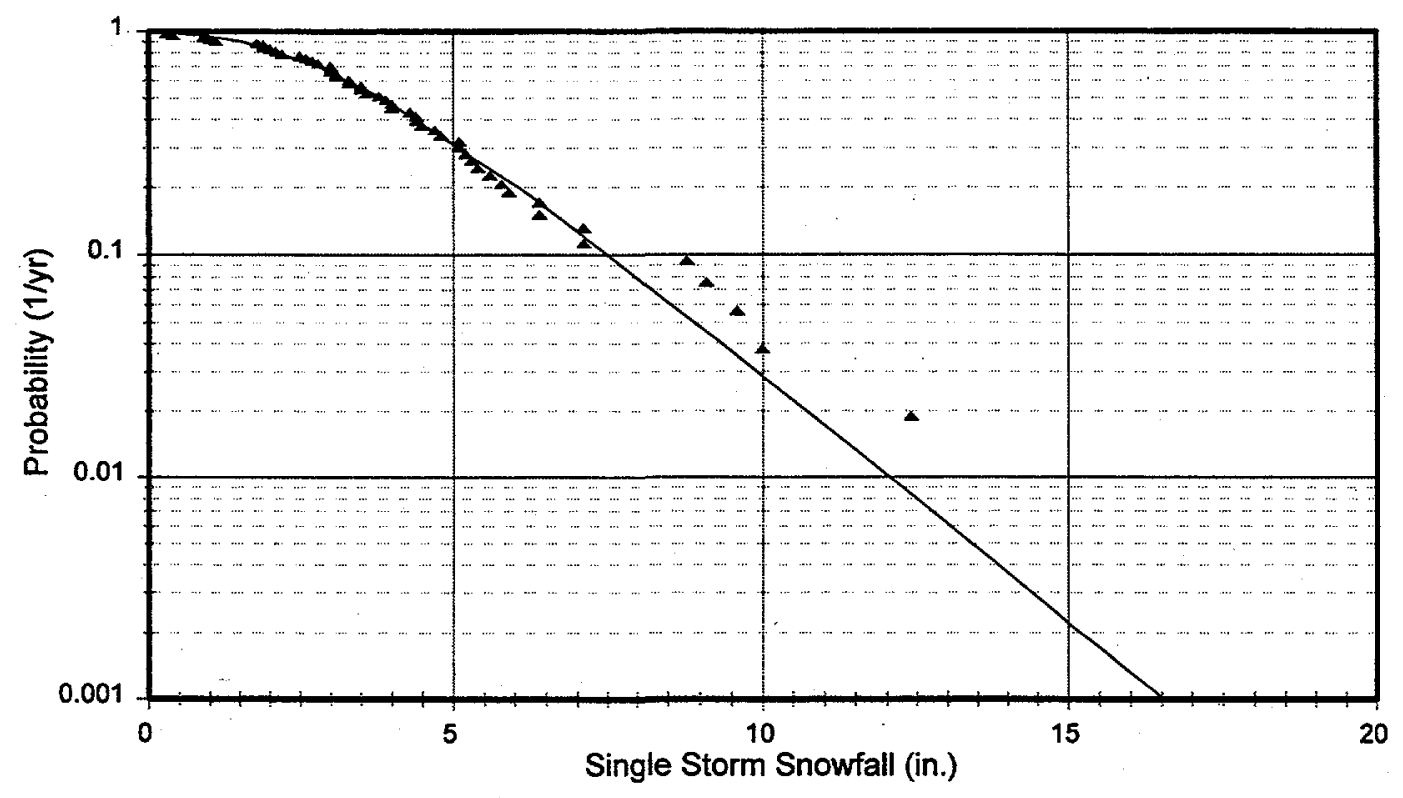

Figure 7.5. Probability of Exceeding a Given Snowfall in a Single Storm

\subsection{Peak Wind Gusts}

Peak wind gusts for return periods of 2 to 10,000 years are listed in Table 7.5 for heights of $30.5,50$, 200 , and $400 \mathrm{ft}$ above ground. The peak wind gust estimates are based on wind measurements made at the 50,200, 400-ft levels of the tower at the HMS. The peak wind gusts for each return period for these 


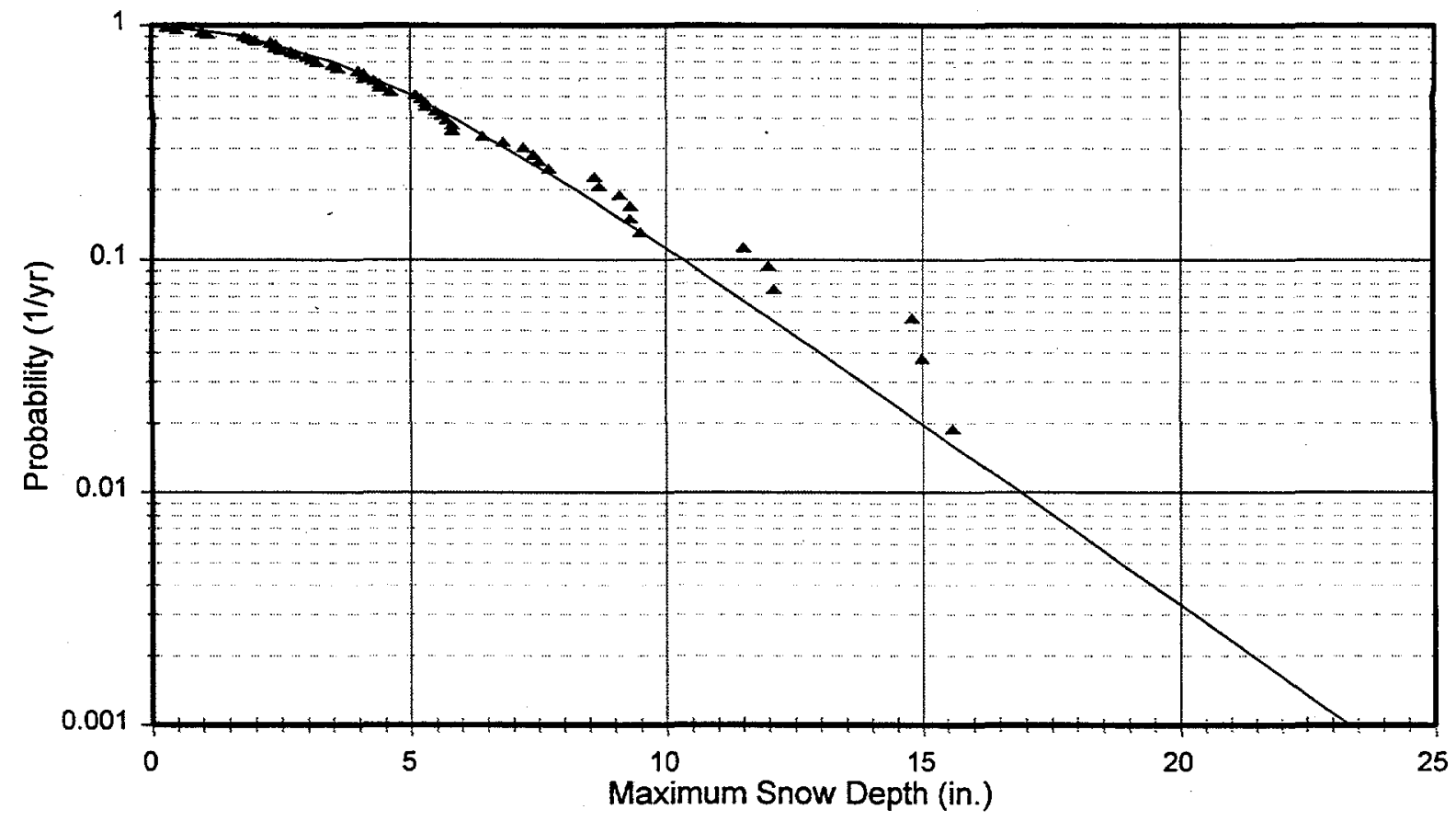

Figure 7.6. Probability of Exceeding a Given Snow Depth

Table 7.5. Peak Wind Gusts (mph) for Return Periods from 2 to 10,000 Years

\begin{tabular}{|c|c|c|c|c|}
\hline \multirow{2}{*}{$\begin{array}{c}\text { Return } \\
\text { Period (yr) }\end{array}$} & \multicolumn{4}{|c|}{ Height Above Ground } \\
\hline & $10 \mathrm{~m}$ & $50 \mathrm{ft}$ & $200 \mathrm{ft}$ & $400 \mathrm{ft}$ \\
\hline 2 & 57.4 & 60.2 & 67.5 & 71.6 \\
\hline 5 & 63.6 & 66.7 & 75.3 & 80.5 \\
\hline 10 & 67.8 & 71.0 & 80.5 & 86.4 \\
\hline 20 & 71.7 & 75.2 & 85.4 & 92.1 \\
\hline 50 & 76.9 & 80.5 & 91.8 & 99.4 \\
\hline 100 & 80.7 & 84.6 & 96.6 & 104.8 \\
\hline 200 & 84.5 & 88.6 & 101.4 & 110.3 \\
\hline 500 & 89.6 & 93.9 & 107.7 & 117.5 \\
\hline 1,000 & 93.4 & 97.9 & 112.4 & 122.9 \\
\hline 2,000 & 97.2 & 101.9 & 117.2 & 128.4 \\
\hline 5,000 & 102.2 & 107.1 & 123.5 & 135.6 \\
\hline 10,000 & 106.1 & 111.1 & 128.2 & 141.0 \\
\hline
\end{tabular}

levels were estimated assuming a Type 1 extreme value distribution and maximum likelihood distribution parameters calculated from the HMS data. The peak wind gusts for the $30.5-\mathrm{ft}(10-\mathrm{m})$ level were made by first adjusting the peak gusts observed at $50 \mathrm{ft}$ to $30.5 \mathrm{ft}$ using the technique described by Peterka and 
Shahid (1998) and then calculating the distribution parameters using maximum likelihood techniques. Figure 7.7 shows the probabilities of peak wind gusts at all four levels along with the HMS peak wind gust data for 50,200 , and $400 \mathrm{ft}$.

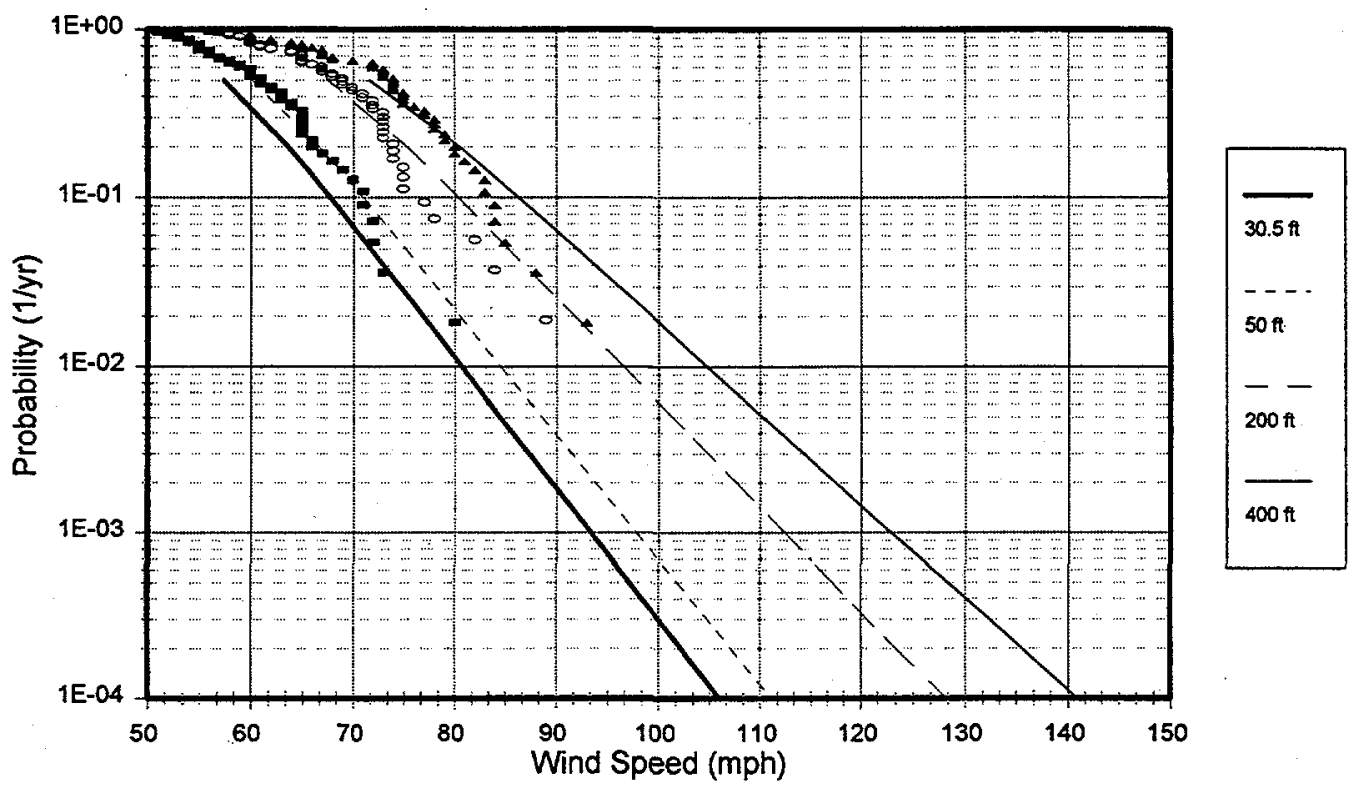

Figure 7.7. Probabilities of Peak Wind Gusts Exceeding Given Values 


\subsection{References}

Glantz, C. S., and M. M. Islam. 1988. The Data Collection Component of the Hanford Meteorology Monitoring Program. PNL-6684, Pacific Northwest Laboratory, Richland, Washington.

Hoitink, D. J., and K. W. Burk. 1994. Climatological Data Summary 1993, with Historical Data. PNL-9809, Pacific Northwest Laboratory, Richland, Washington.

Hoitink, D. J., and K. W. Burk. 1995. Climatological Data Summary 1994, with Historical Data. PNL-10553, Pacific Northwest Laboratory, Richland, Washington.

Hoitink, D. J., and K. W. Burk. 1996. Climatological Data Summary 1995, with Historical Data. PNNL-11107, Pacific Northwest National Laboratory, Richland, Washington.

Hoitink, D. J., and K. W. Burk. 1997. Climatological Data Summary 1996, with Historical Data. PNNL-11471, Pacific Northwest National Laboratory, Richland, Washington.

Hoitink, D. J., and K. W. Burk. 1998. Climatological Data Summary 1997, with Historical Data. PNNL-11794, Pacific Northwest National Laboratory, Richland, Washington.

Jenne, D. E., and R. E. Kerns. 1959. A Climatological Study of the Hanford Area. HW-57722, General Electric, Hanford Atomic Products Operation, Richland, Washington.

Johnson, N. L., S. Kotz, and N. Balakrishnan. 1995. Continuous Univariate Distributions, Vol. 2, John Wiley \& Sons, New York.

Kinnison, R. R. 1985. Applied Extreme Value Statistics. Macmillan, New York.

Peterka, J. A., and S. Shahid. 1998. "Design Gust Wind Speeds in the United States." Journal of Structural.Engineering, 124:207-214.

Stone, W. A., D. E. Jenne, and J. M. Thorp. 1972. Climatography of the Hanford Area. BNWL-1605, Pacific Northwest Laboratory, Richland, Washington.

Stone, W. A., J. M. Thorp, O. P. Gifford, and D. J. Hoitink. 1983. Climatological Summary for the Hanford Area. PNL-4622, Pacific Northwest Laboratory, Richland, Washington. 


\subsection{Bibliography}

Andrews, G. L. 1988. The Hanford Meteorological Data Collection System and Data Base. PNL-6509, Pacific Northwest Laboratory, Richland, Washington.

Andrews, G. L., and J. W. Buck. 1987. Hanford Meteorological Station Computer Codes: Volume 2 The PROD Computer Code. PNL-6279 Vol. 2, Pacific Northwest Laboratory, Richland, Washington.

Andrews, G. L., and J. W. Buck. 1987. Hanford Meteorological Station Computer Codes: Volume 4 The SUM Computer Code. PNL-6279 Vol. 4, Pacific Northwest Laboratory, Richland, Washington.

Andrews, G. L., and J. W. Buck. 1987. Hanford Meteorological Station Computer Codes: Volume 6 The SFC Computer Code. PNL-6279 Vol. 6, Pacific Northwest Laboratory, Richland, Washington.

Andrews, G. L., and J. W. Buck. 1988. Hanford Meteorological Station Computer Codes: Volume 7 The RIVER Computer Code. PNL-6279 Vol. 7, Pacific Northwest Laboratory, Richland, Washington.

Andrews, G. L., and K. W. Burk. 1988. Hanford Meteorological Station Computer Codes: Volume 8 The REVIEW Computer Code. PNL-6279 Vol. 8, Pacific Northwest Laboratory, Richland, Washington.

Andrews, G. L., and K. W. Burk. 1989. Hanford Meteorological Station Computer Codes: Volume 10 The ARCHIVE Computer Code. PNL-6279 Vol. 10, Pacific Northwest Laboratory, Richland, Washington.

Buck, J. W., and G. L. Andrews. 1987. Hanford Meteorological Station Computer Codes: Volume IThe GEN Computer Code. PNL-6279 Vol. 1, Pacific Northwest Laboratory, Richland, Washington.

Buck, J. W., and G. L. Andrews. 1987. Hanford Meteorological Station Computer Codes: Volume 3 The TANK Computer Code. PNL-6279 Vol. 3, Pacific Northwest Laboratory, Richland, Washington.

Buck, J. W., and G. L. Andrews. 1987. Hanford Meteorological Station Computer Codes: Volume 5 The TOWER Computer Code. PNL-6279 Vol. 5, Pacific Northwest Laboratory, Richland, Washington

Burk, K. W., and G. L. Andrews. 1989. Hanford Meteorological Station Computer Codes: 'Volume 9 The Quality Assurance Computer Codes. PNL-6279 Vol. 9, Pacific Northwest Laboratory, Richland, Washington.

Glantz, C. S., M. N. Schwartz, K. W. Burk, R. B. Kasper, M. W. Ligotke, and P. J. Perrault. 1990. Climatological Summary of Wind and Temperature Data for the Hanford Meteorological Monitoring Network. PNL-7471, Pacific Northwest Laboratory, Richland, Washington.

Stage, S. A., J. V. Ramsdell, Jr., C. A. Simonen, K. W. Burk, and L. K. Berg. 1993. Final Report on the Meteorological Database, December 1944 - 1949. PNWD-2200 HEDR, Battelle Pacific Northwest Laboratories, Richland, Washington. 
Appendix

1998 Wind Climatology 


\section{Appendix}

\section{Wind Climatology}

This appendix provides the 1998 station-specific wind roses (Figures A.1[a] and A.2[a]) from the Hanford Meteorological Monitoring Network. Each petal of the wind rose represents the proportional amount of time that the wind blew from that direction. The width of the petal corresponds to each wind speed category. Starting from the center of the rose, the narrowest petal represents winds in the 1- to 3-mph class, the next widest represents the 4- to 7-mph class, and so forth. The length of each of these petals is proportional to the frequency of occurrence for each speed class.

The wind speed histograms (Figures A.1[b] and A.2[b]) represent the proportional amount of time in each speed class.

Table A.1 lists joint frequency distributions (at $10 \mathrm{~m}$ ) of wind direction versus wind speed class for the individual stations (see Figure 2.1 in text for locations). Table A.2 lists joint frequency distributions (at $60 \mathrm{~m}$ ) for stations $9,11,13$, and 21 (see Figure 2.2 in text for locations). 


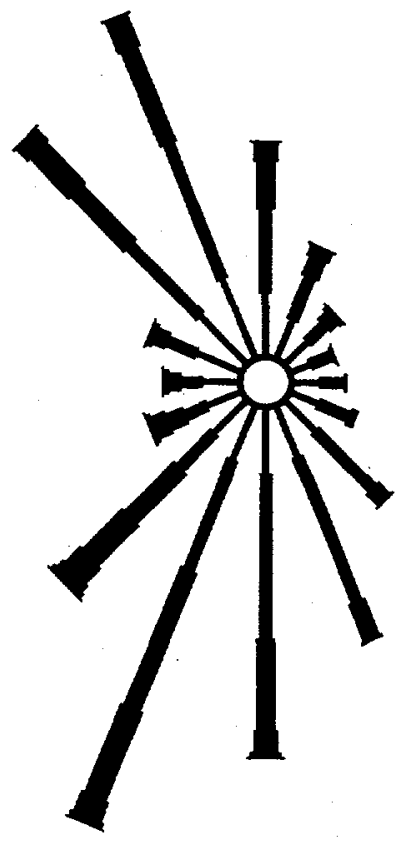

(a) Wind Rose

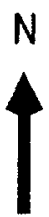

Station \# 1 - PROS

Period: $1 / 98-12 / 98$

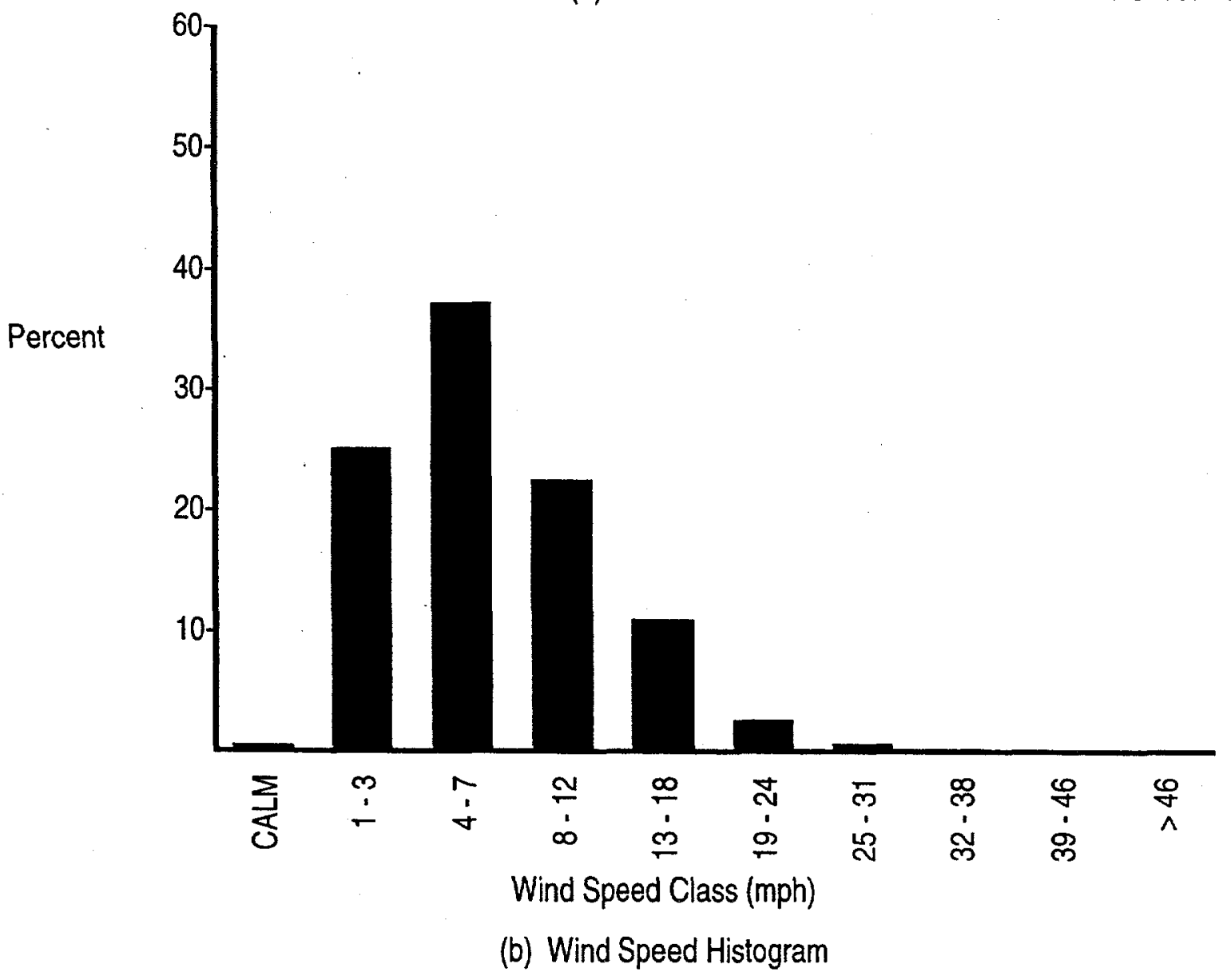

Figure A.1. Wind Rose and Wind Speed Histogram, 10 Meters 


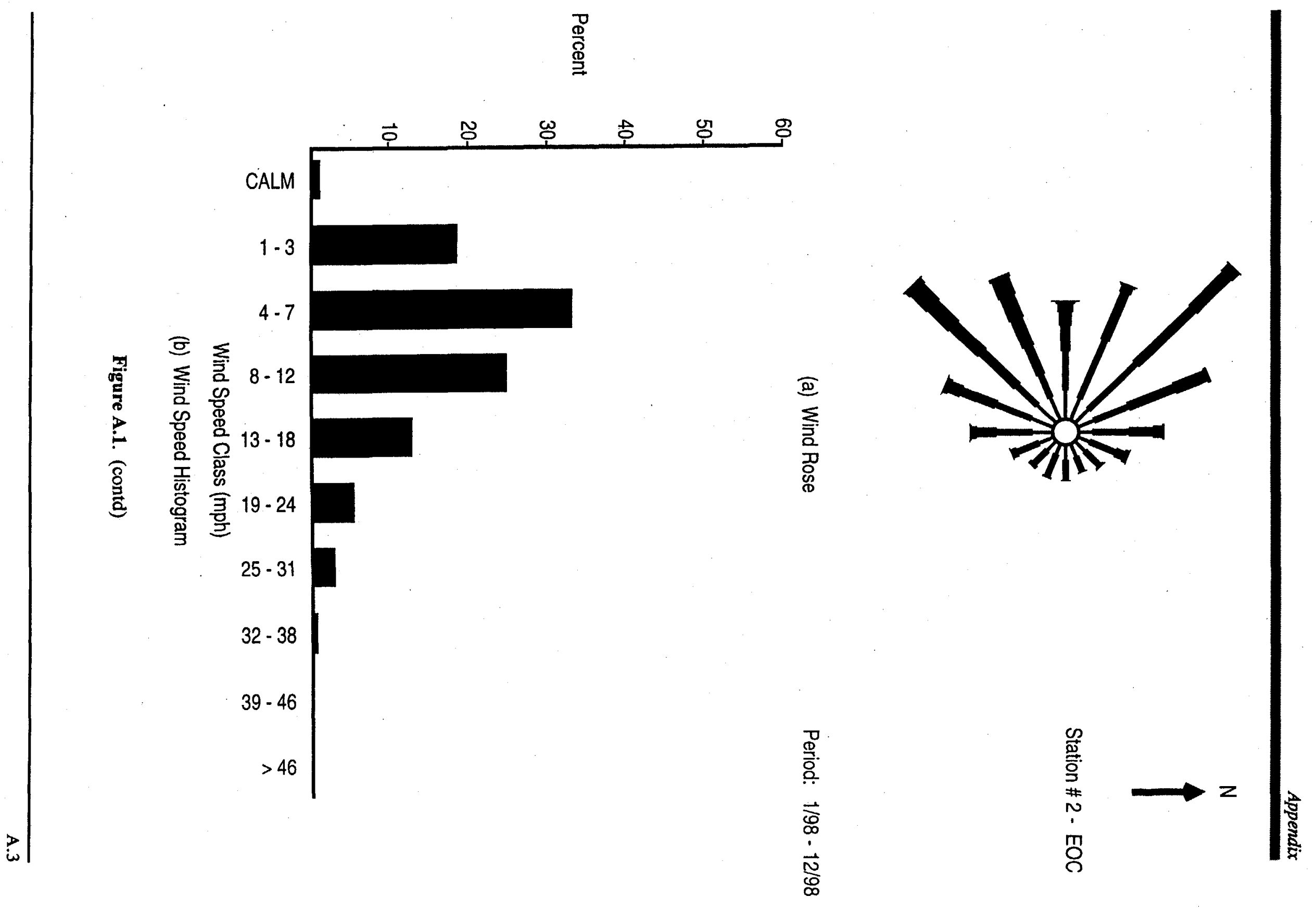



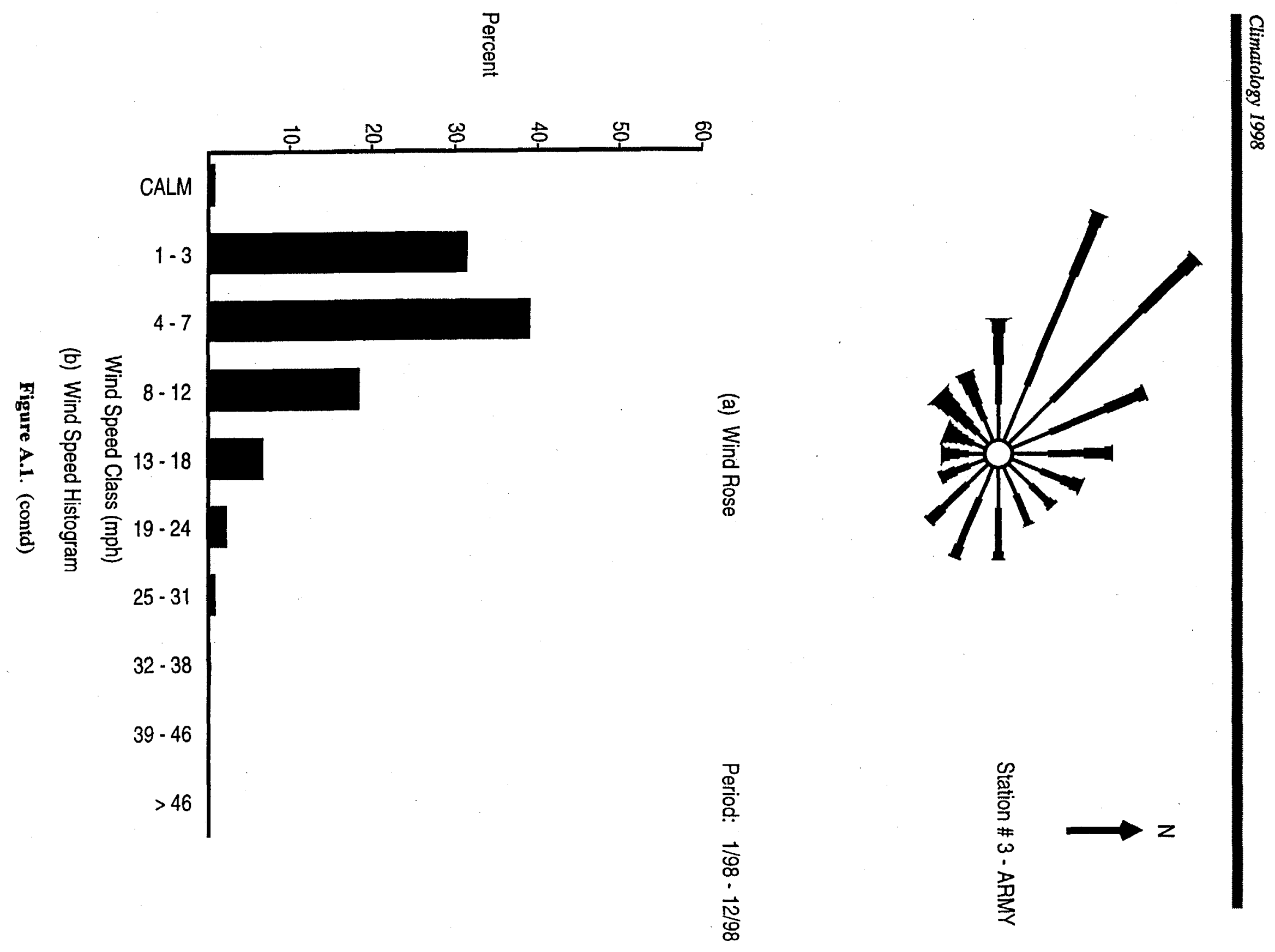


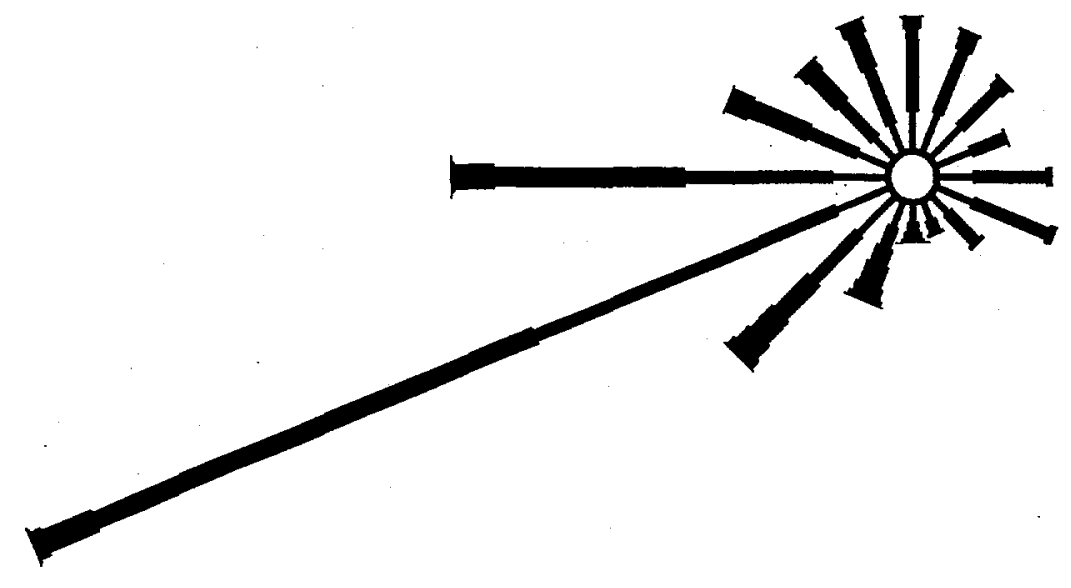

(a) Wind Rose

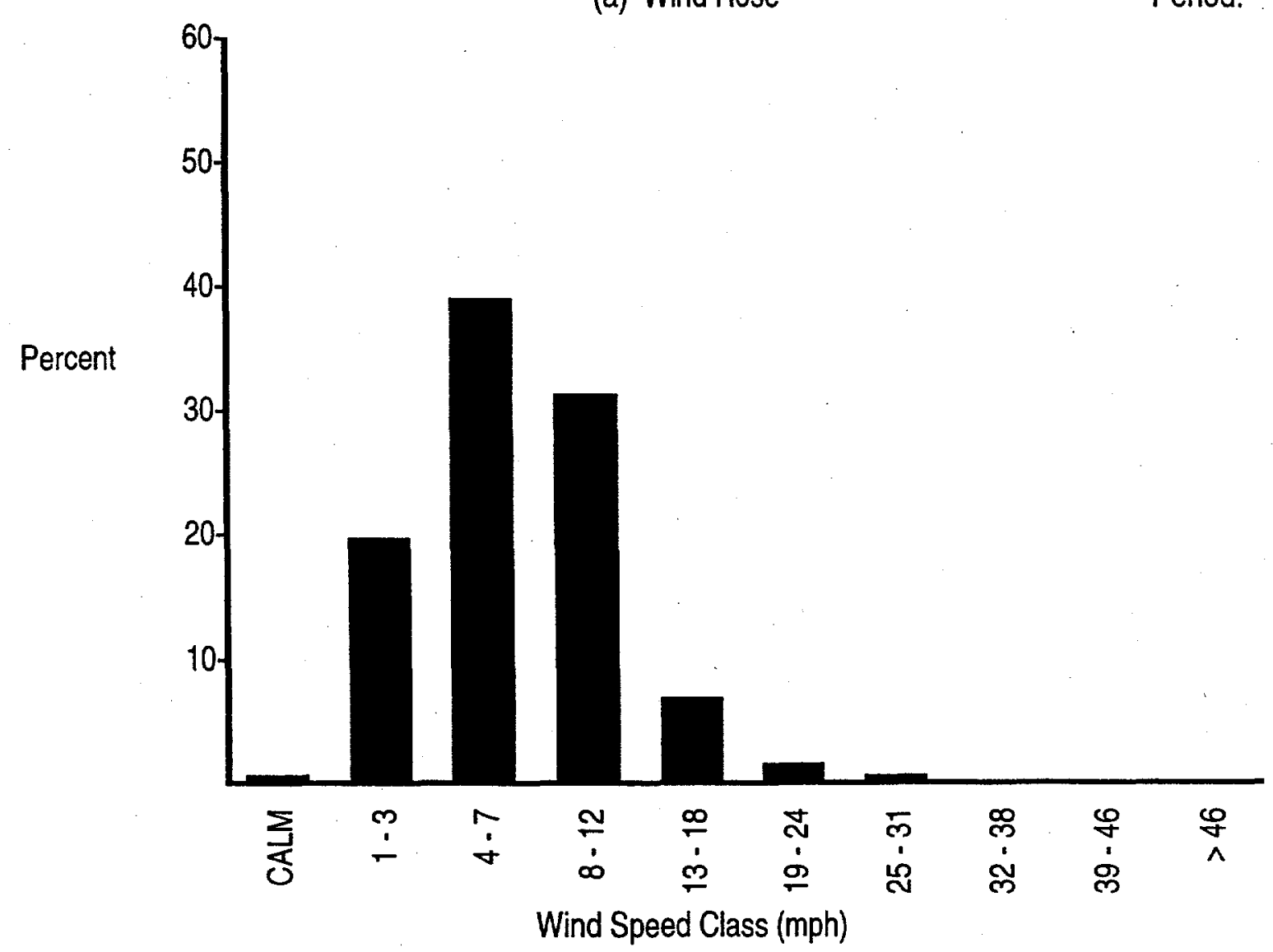

(b) Wind Speed Histogram

Figure A.1. (contd)

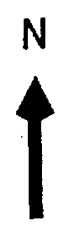

Station \# 4 - RSPG

Period: $1 / 98-12 / 98$ 


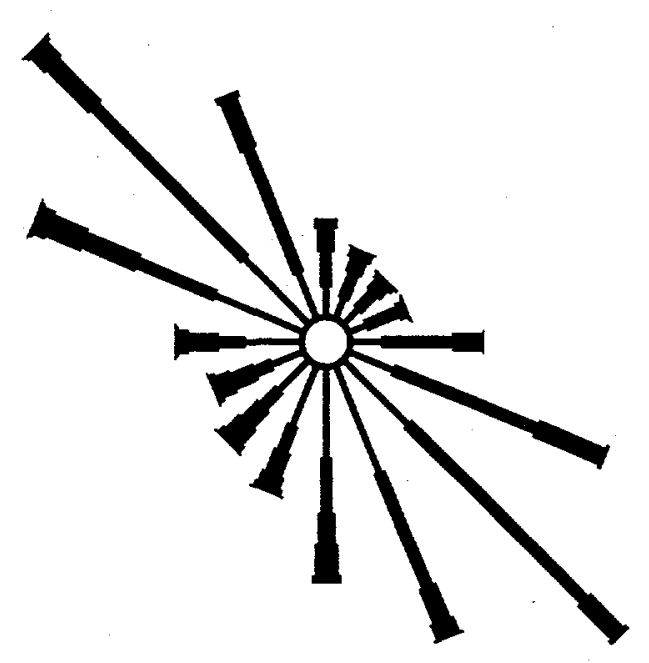

(a) Wind Rose

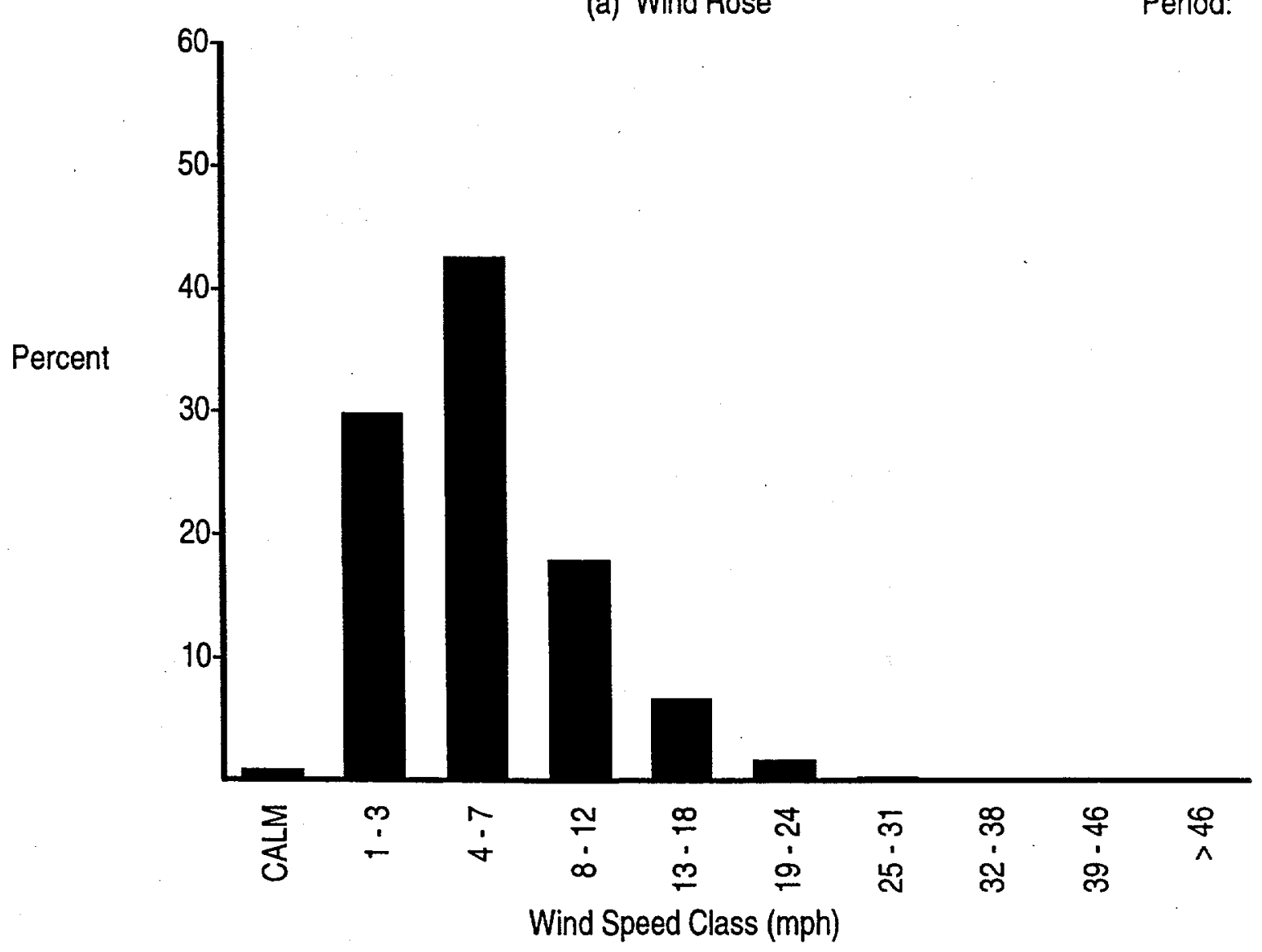

(b) Wind Speed Histogram

Figure A.1. (contd) 


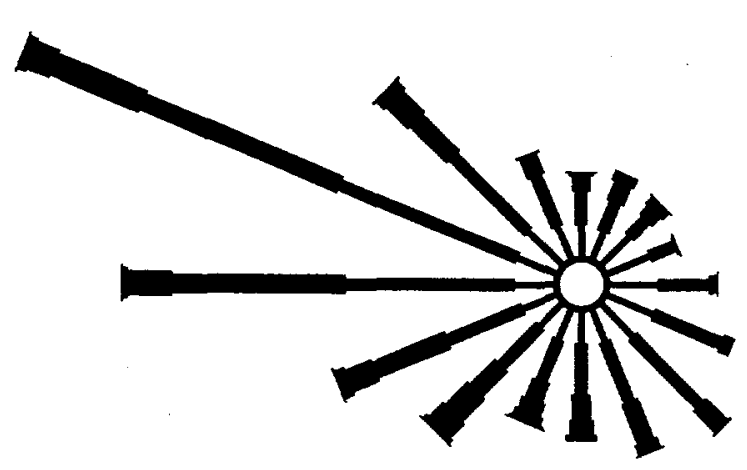

(a) Wind Rose

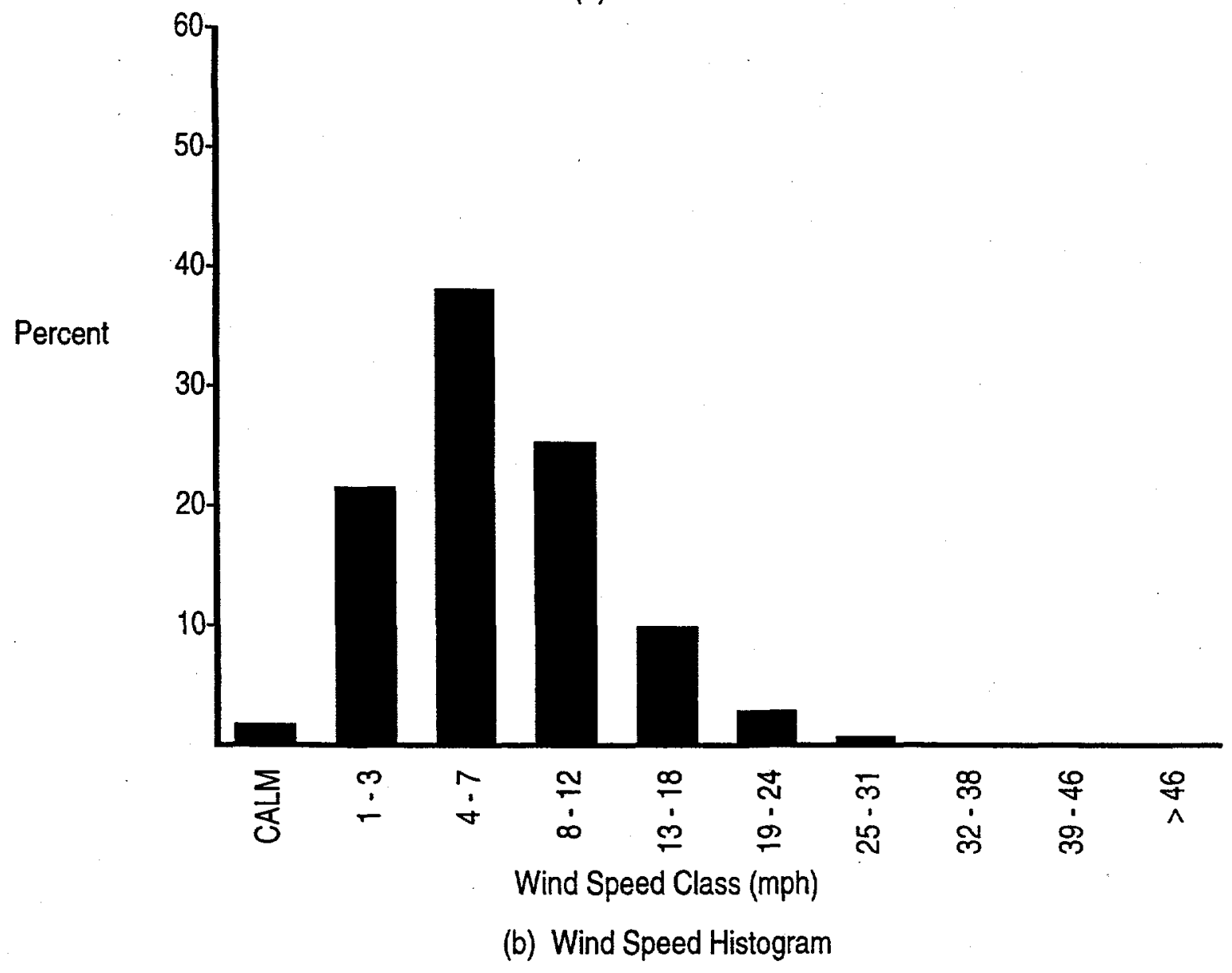

Figure A.1. (contd)

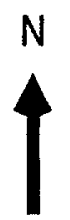

Station \# 6 - 200E

Period: $1 / 98-12 / 98$ 

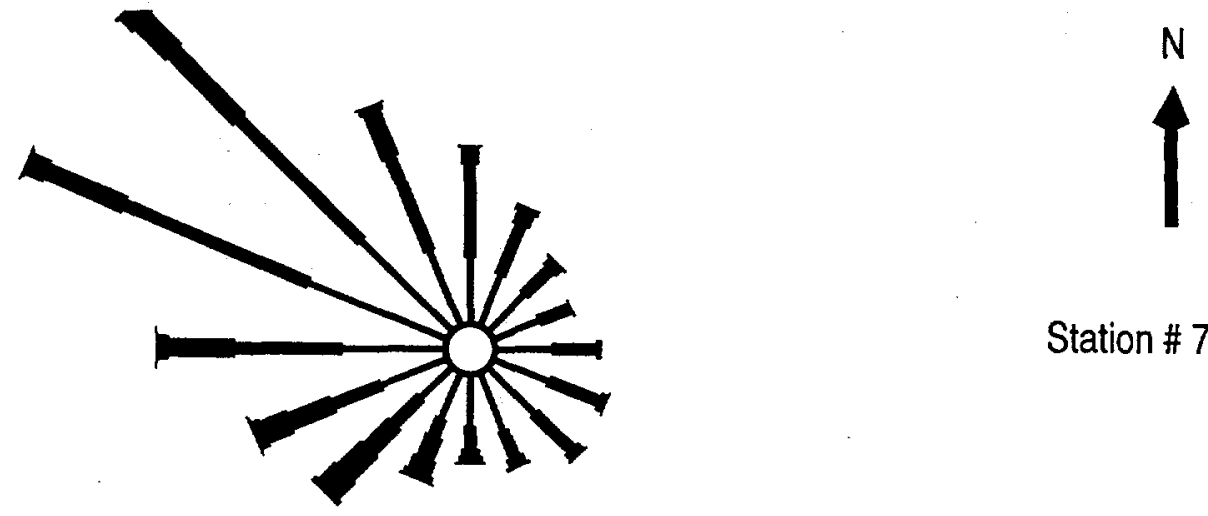

Station \# 7 - 200W

(a) Wind Rose

Period: $1 / 98-12 / 98$

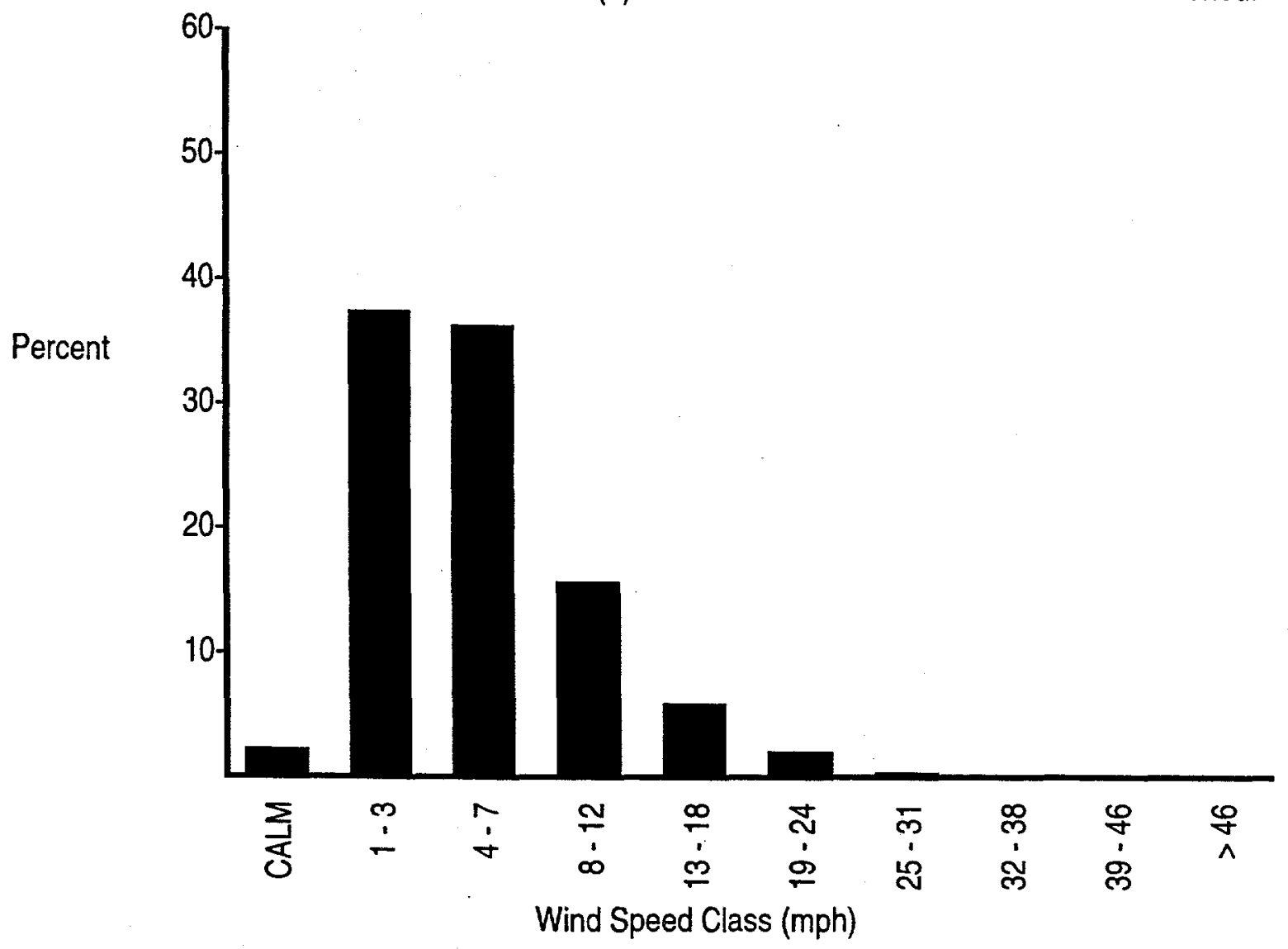

(b) Wind Speed Histogram

Figure A.1. (contd) 


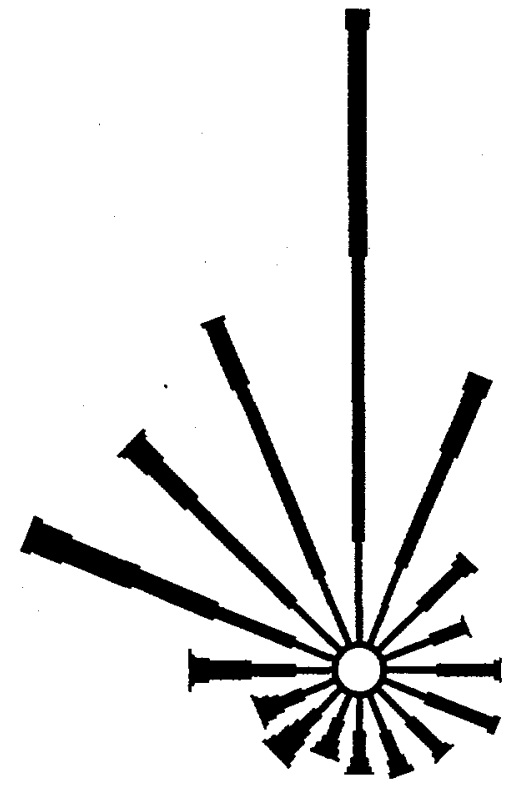

(a) Wind Rose

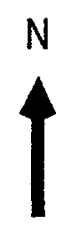

Station \# 8 - BVLY

Period: $1 / 98-12 / 98$

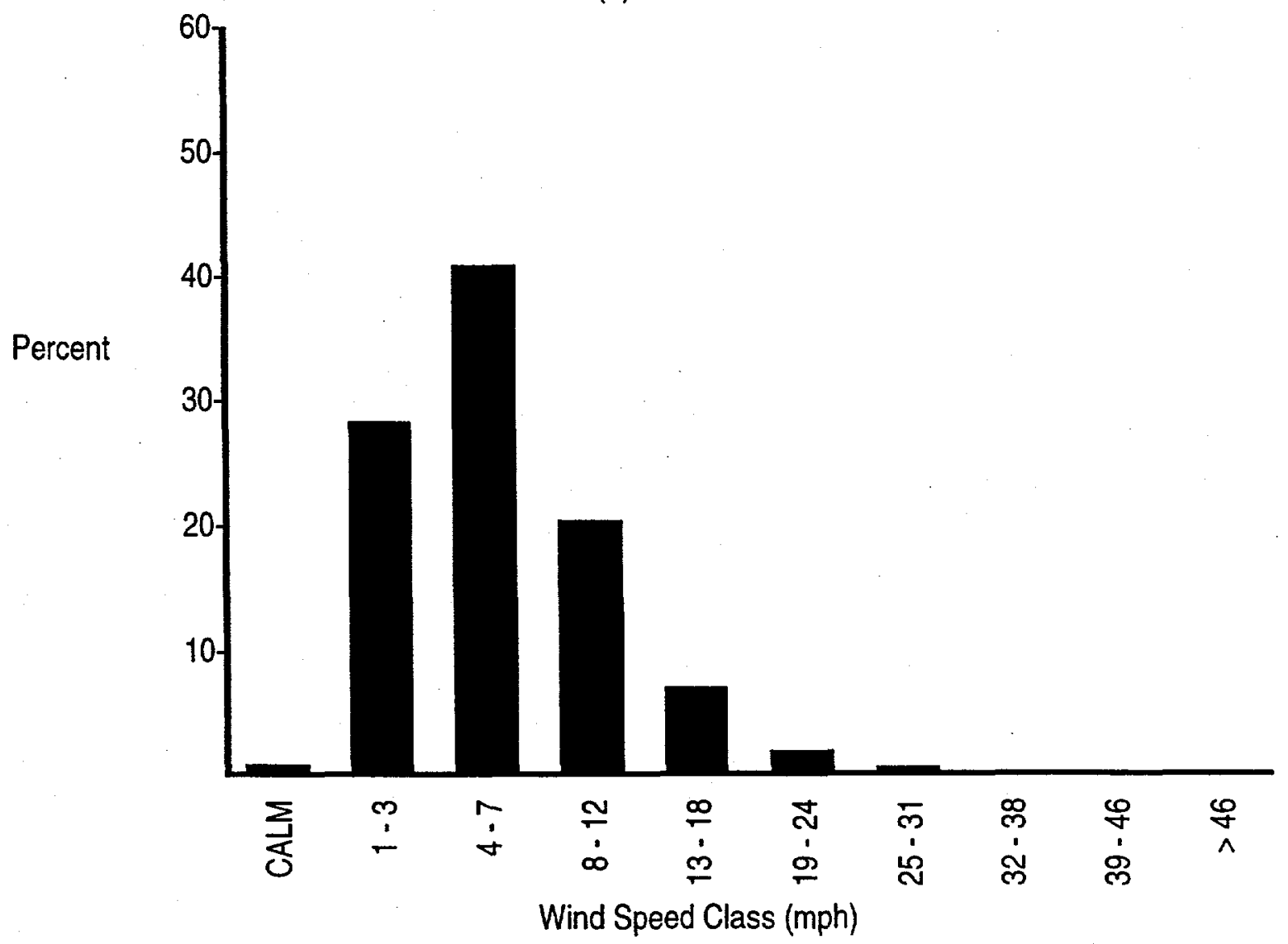

(b) Wind Speed Histogram

Figure A.1. (contd) 


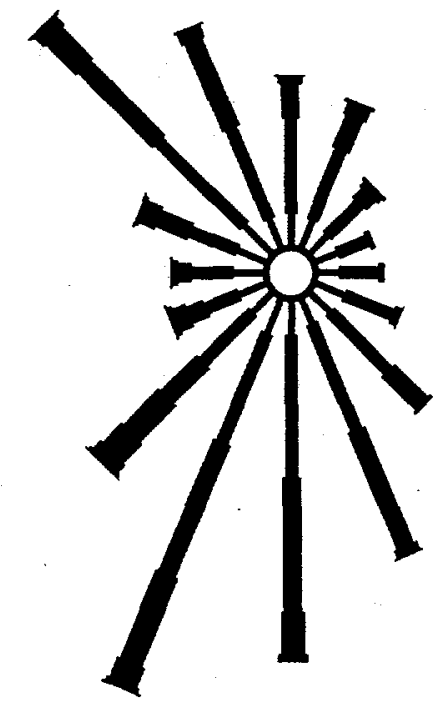

(a) Wind Rose

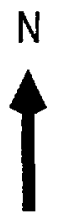

Station \# 9 - FFTF

Period: $1 / 98-12 / 98$

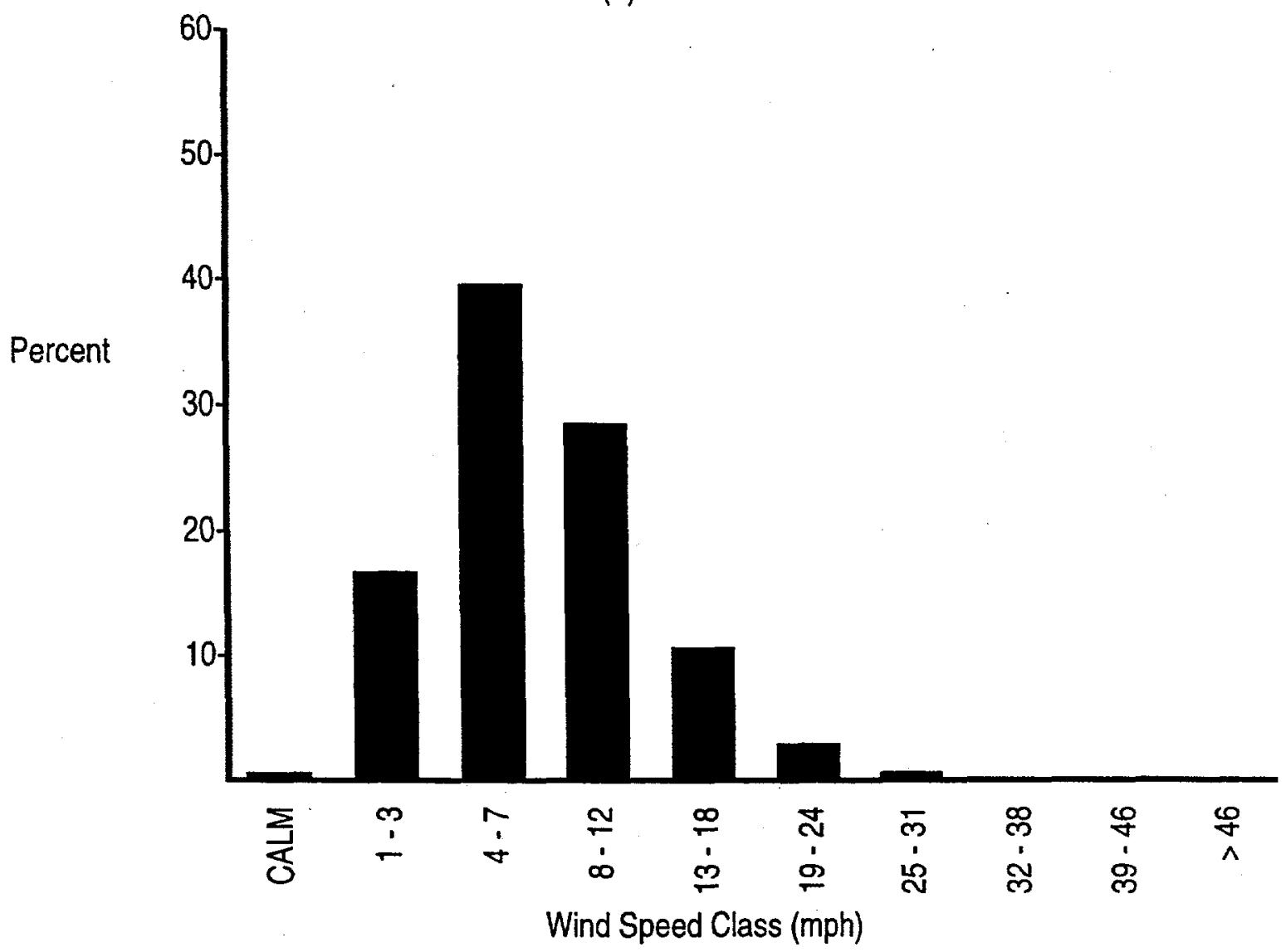

(b) Wind Speed Histogram

Figure A.1. (contd) 


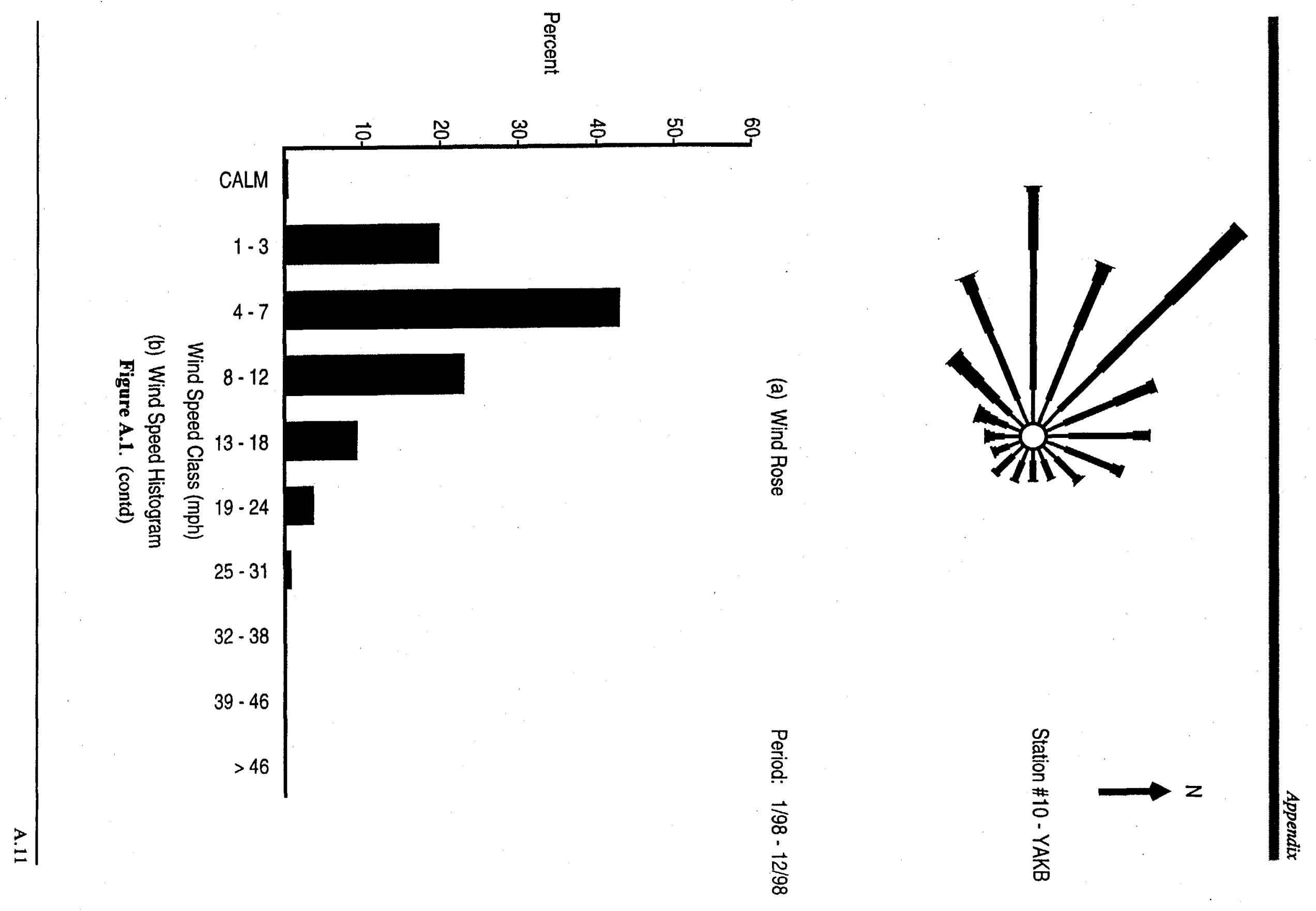




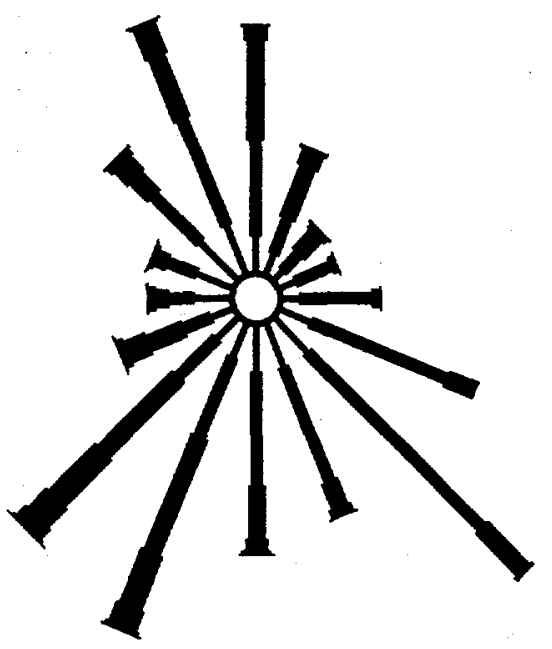

(a) Wind Rose

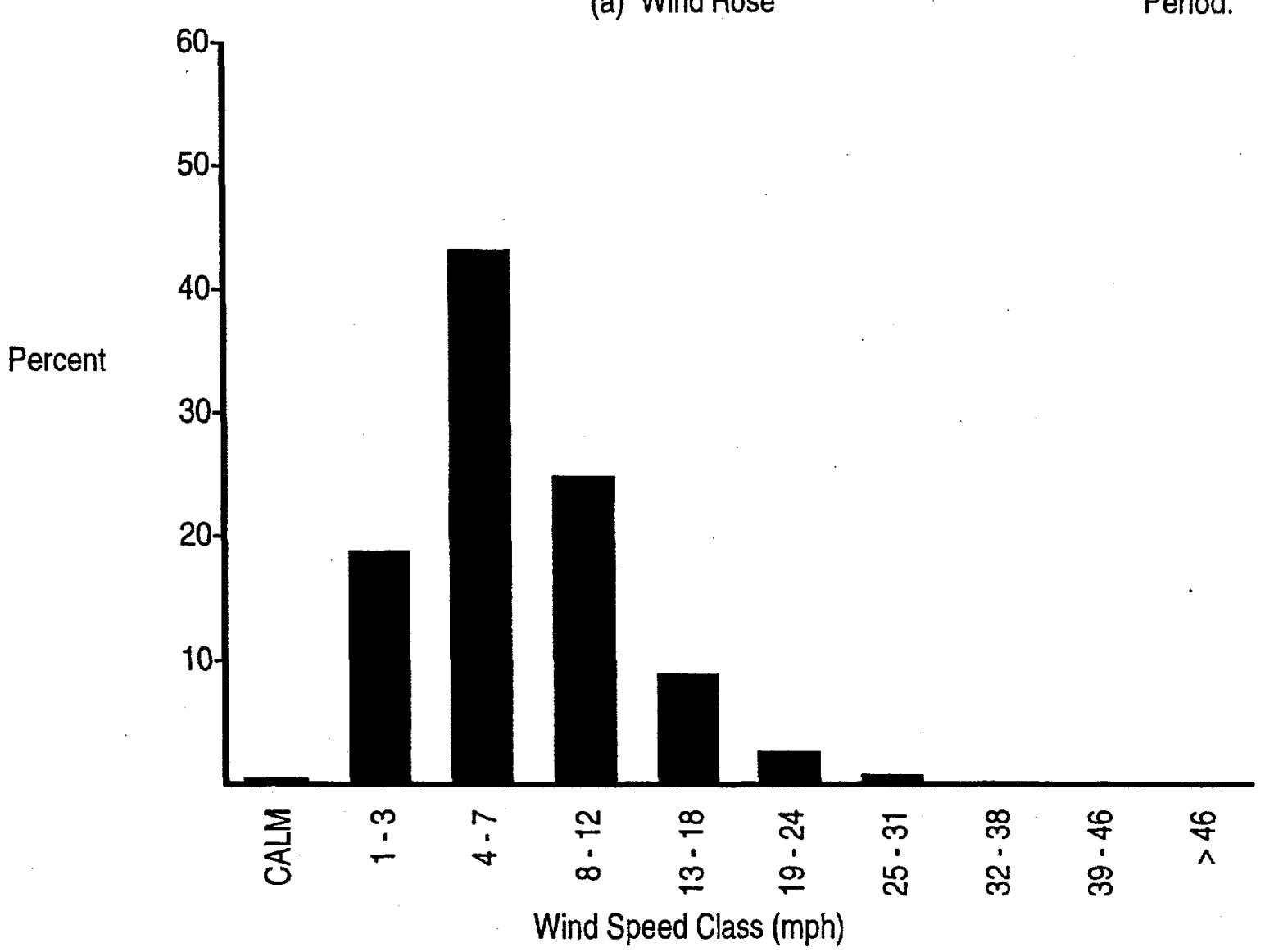

(b) Wind Speed Histogram

Figure A.1. (contd)

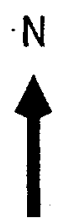

Station \#11-300A

Period: $1 / 98-12 / 98$ 


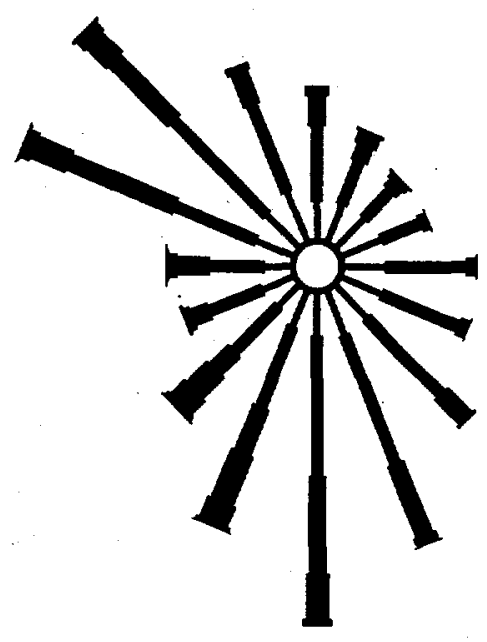

(a) Wind Rose

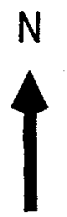

Station \#12 - WYEB

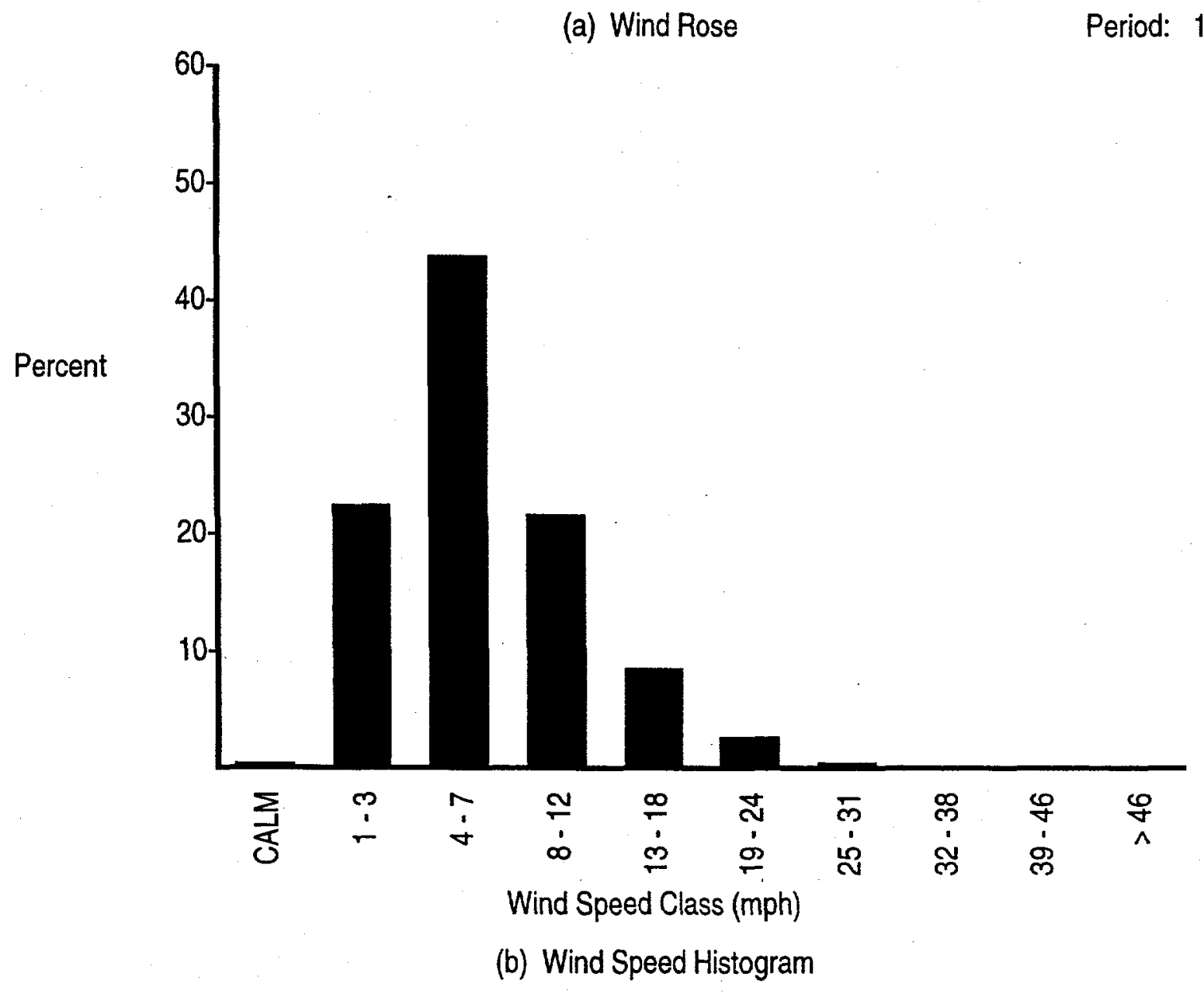

Figure A.1. (contd) 


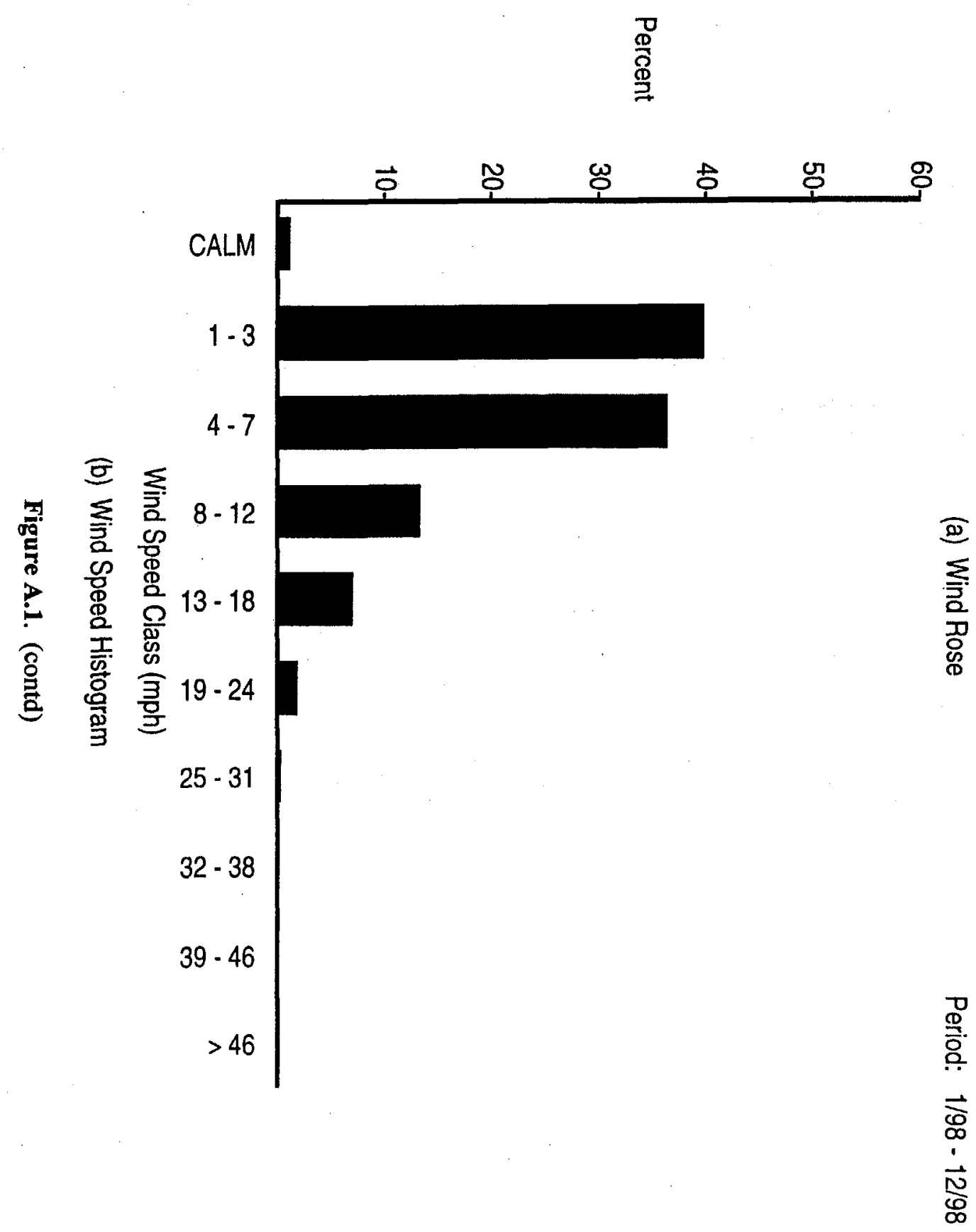

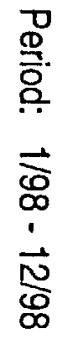

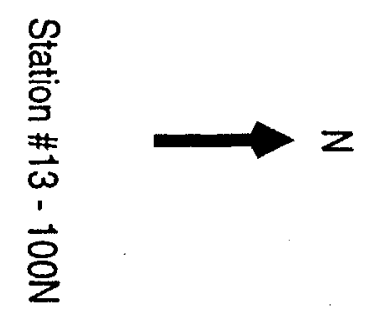




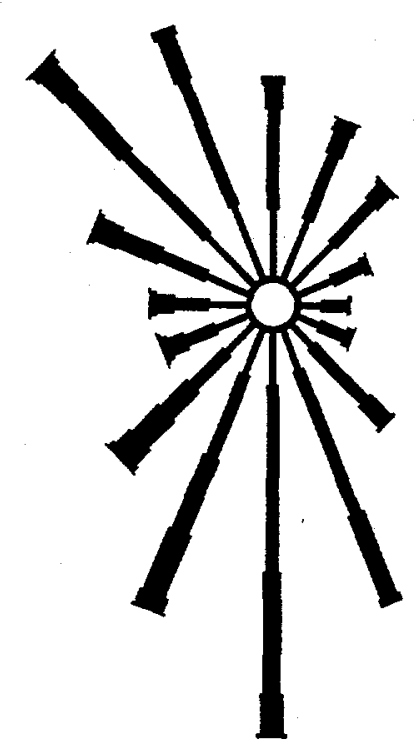

(a) Wind Rose

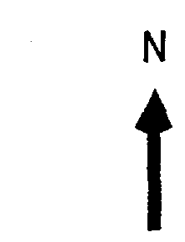

Station \#14 - WPPS

Period: $1 / 98-12 / 98$

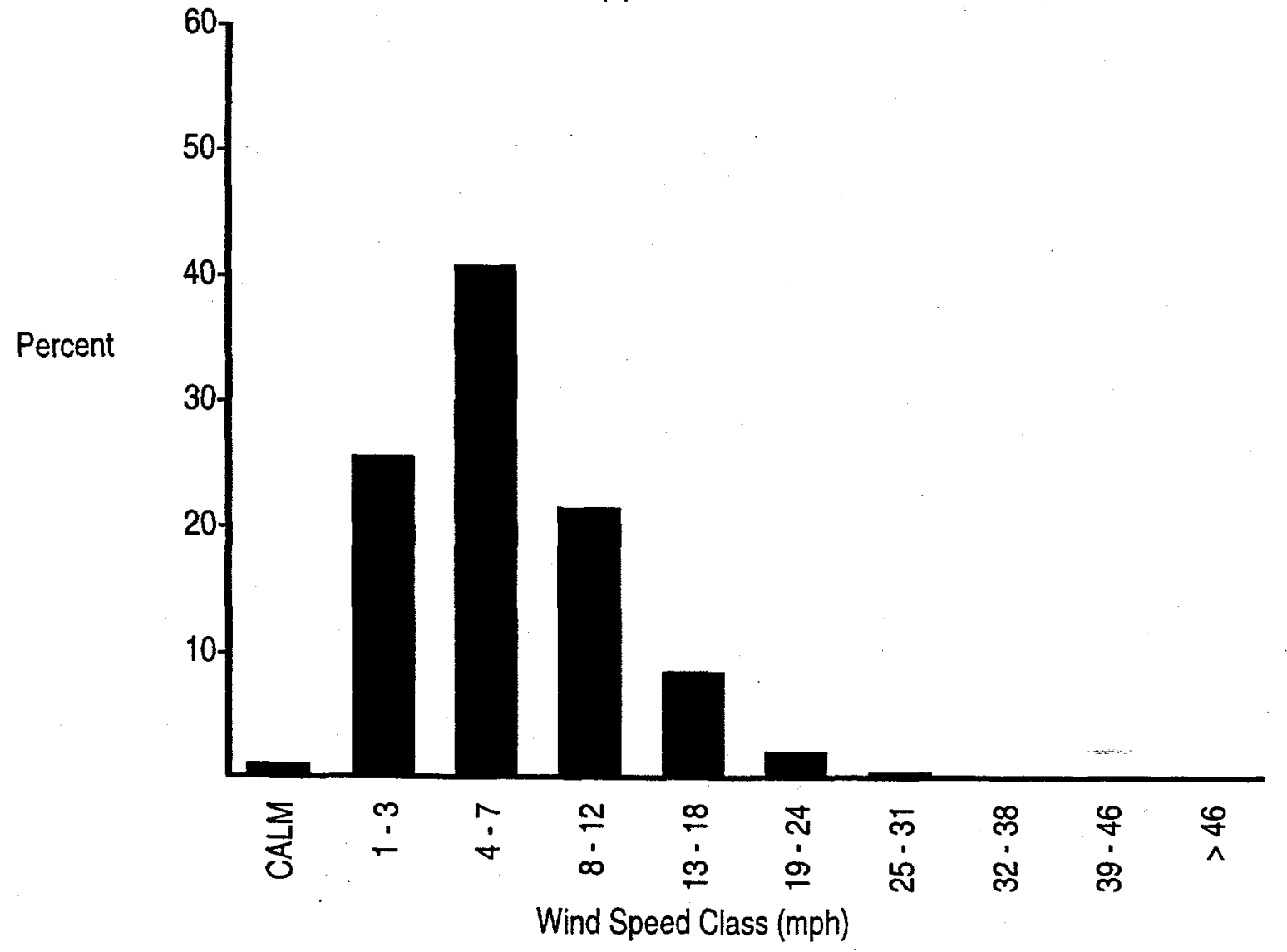

(b) Wind Speed Histogram

Figure A.1. (contd) 

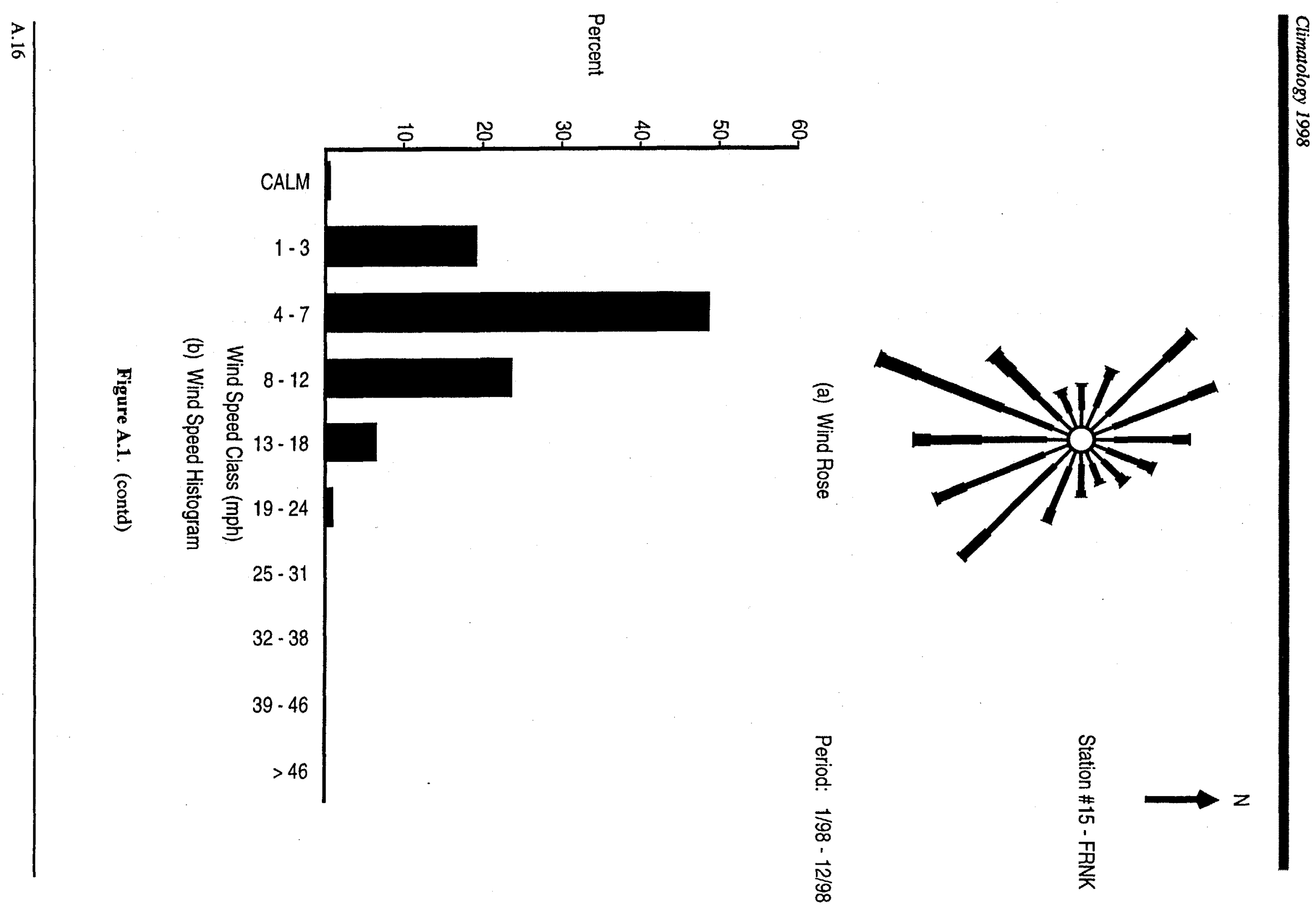

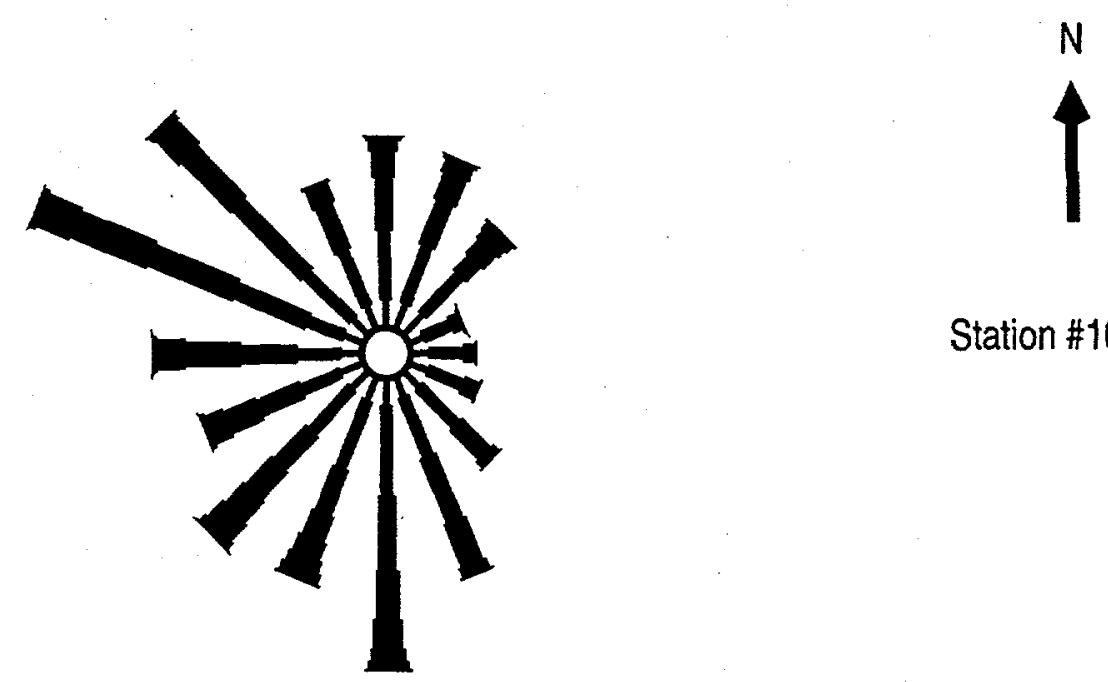

Station \#16 - GABL

(a) Wind Rose

Period: $1 / 98-12 / 98$

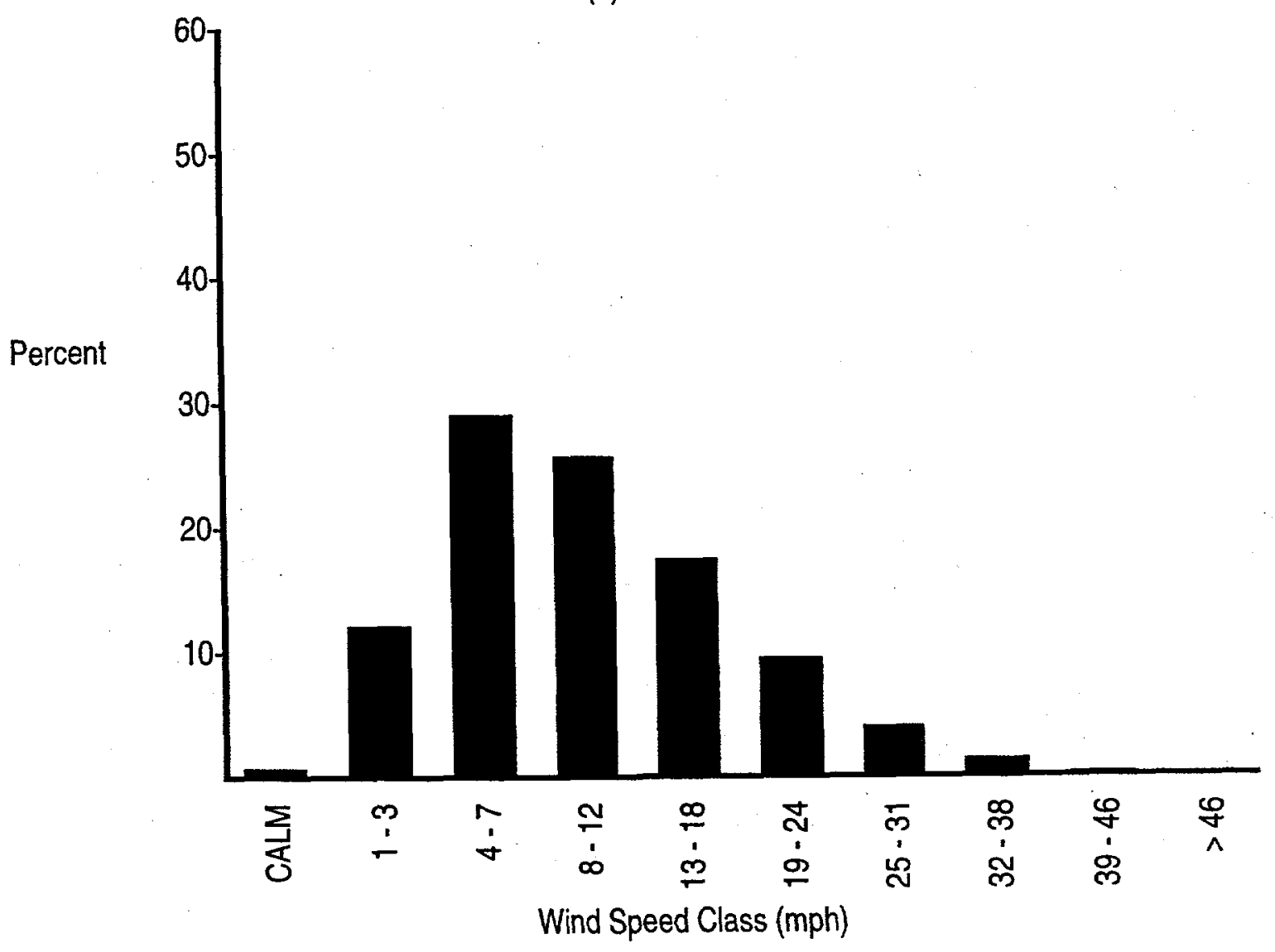

(b) Wind Speed Histogram

Figure A.1. (contd) 

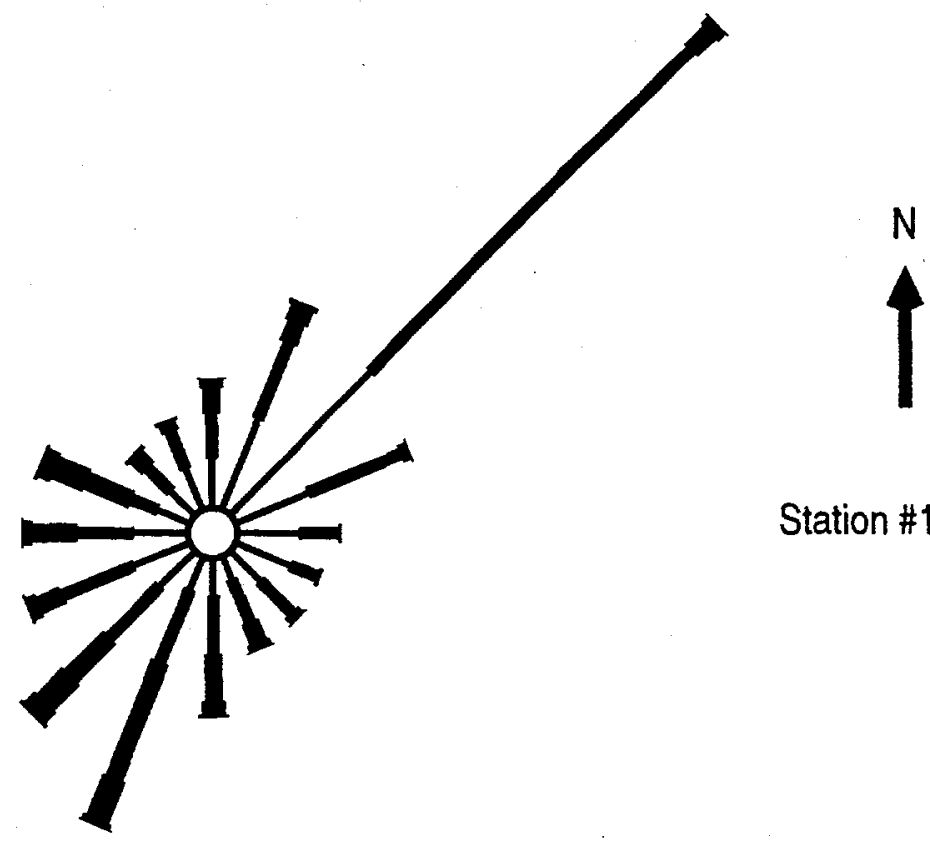

Station \#17 - RING

(a) Wind Rose

Period: $1 / 98-12 / 98$

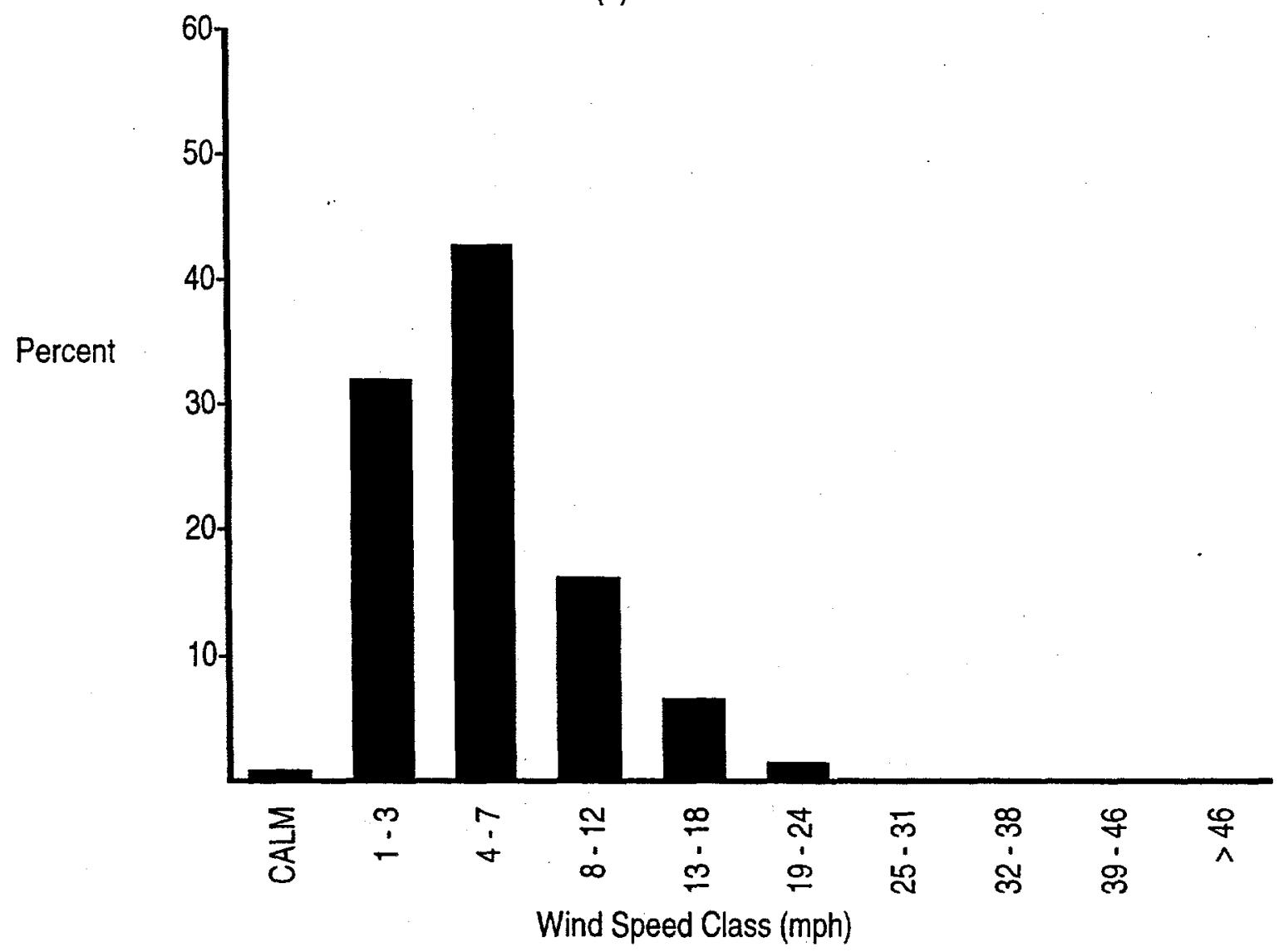

(b) Wind Speed Histogram

Figure A.1. (contd) 


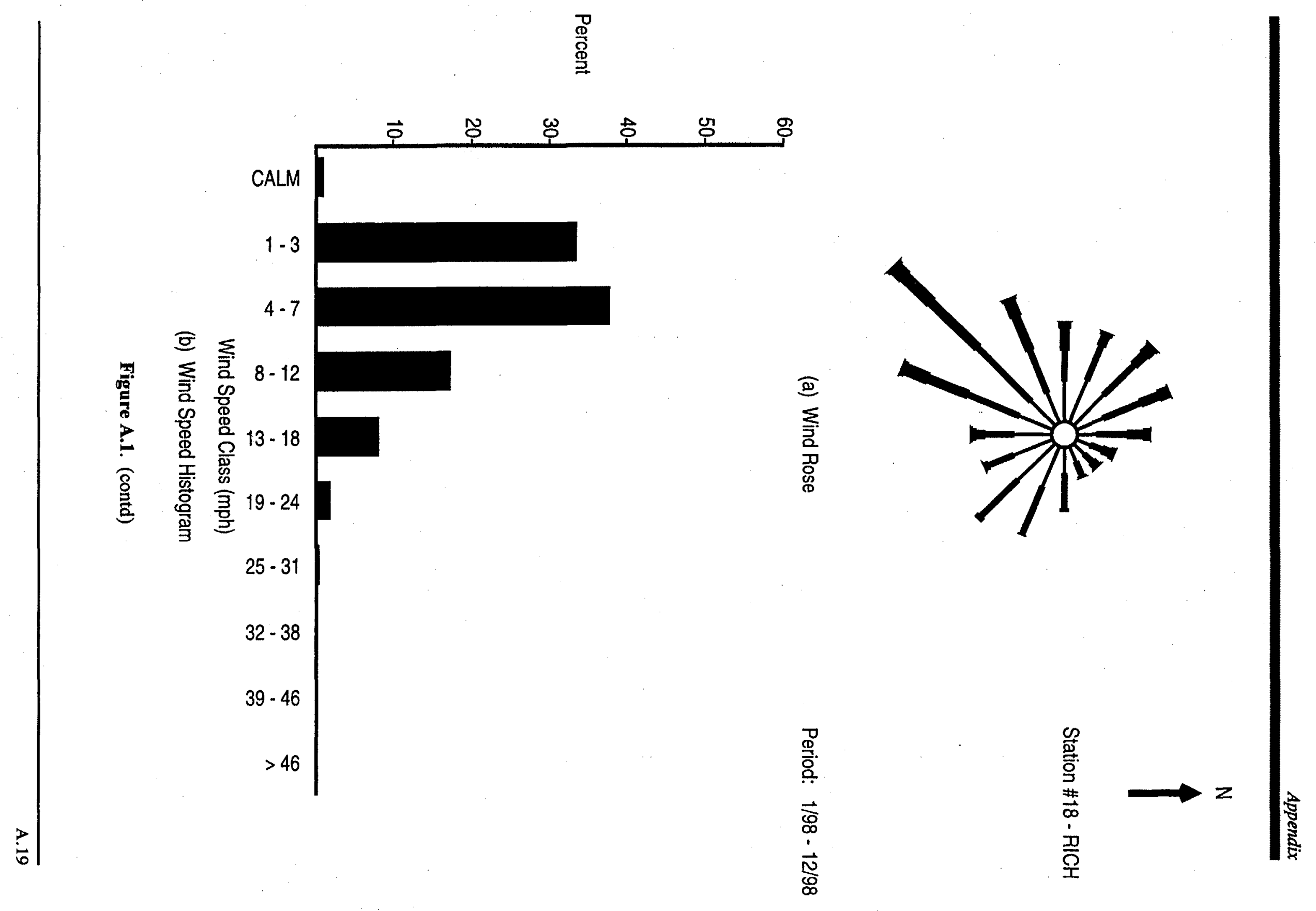




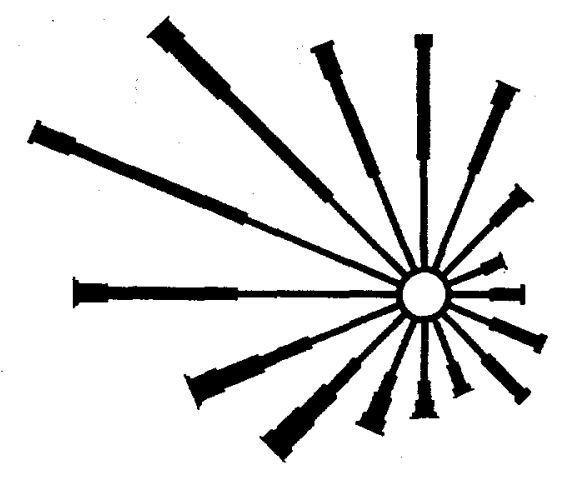

(a) Wind Rose

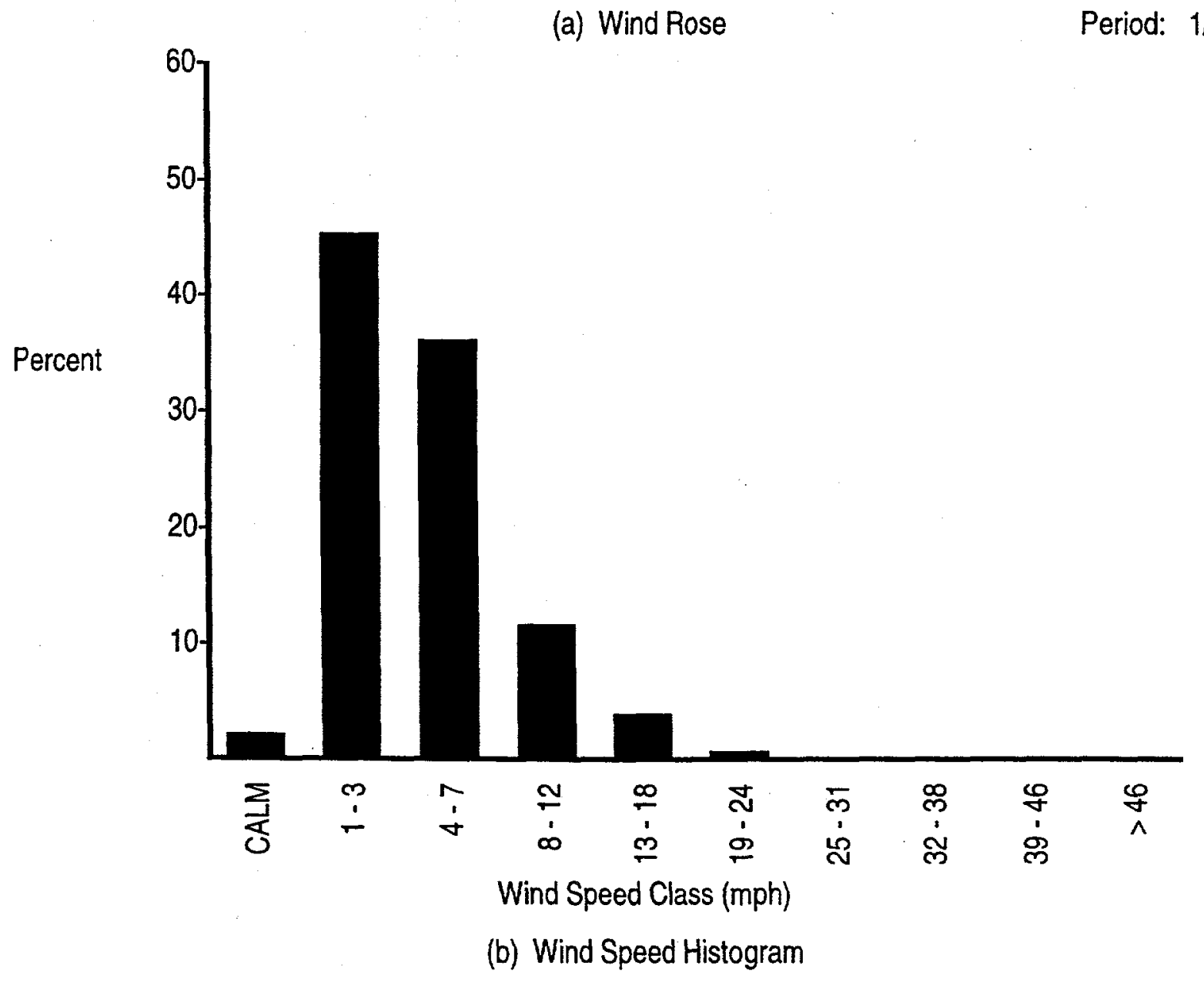

Figure A.1. (contd)

Station \#19 - PFP

Period: $1 / 98-12 / 98$ 


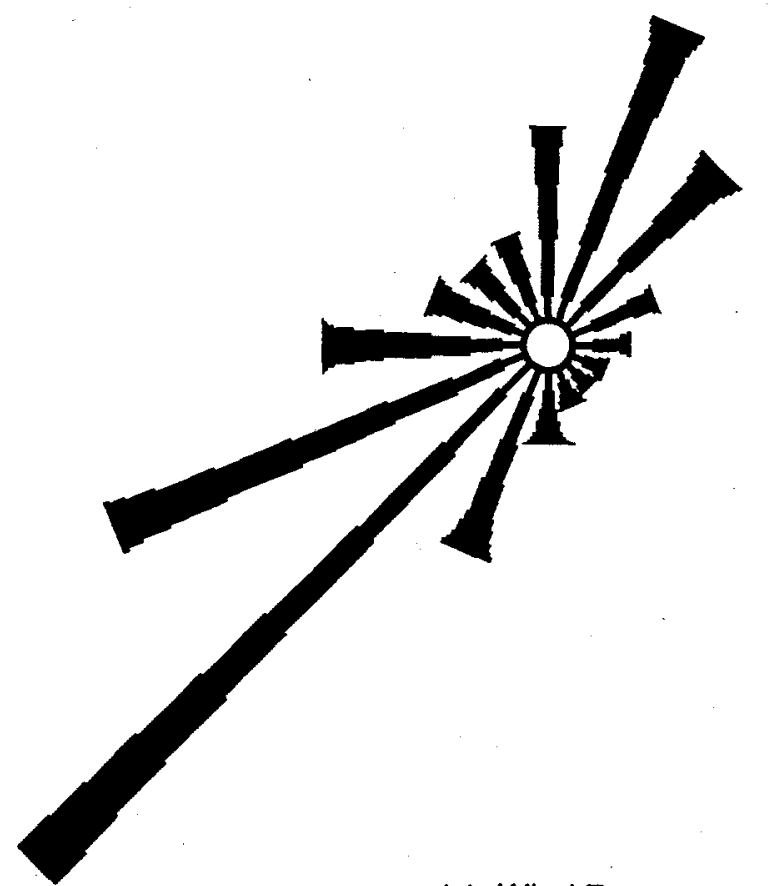

(a) Wind Rose

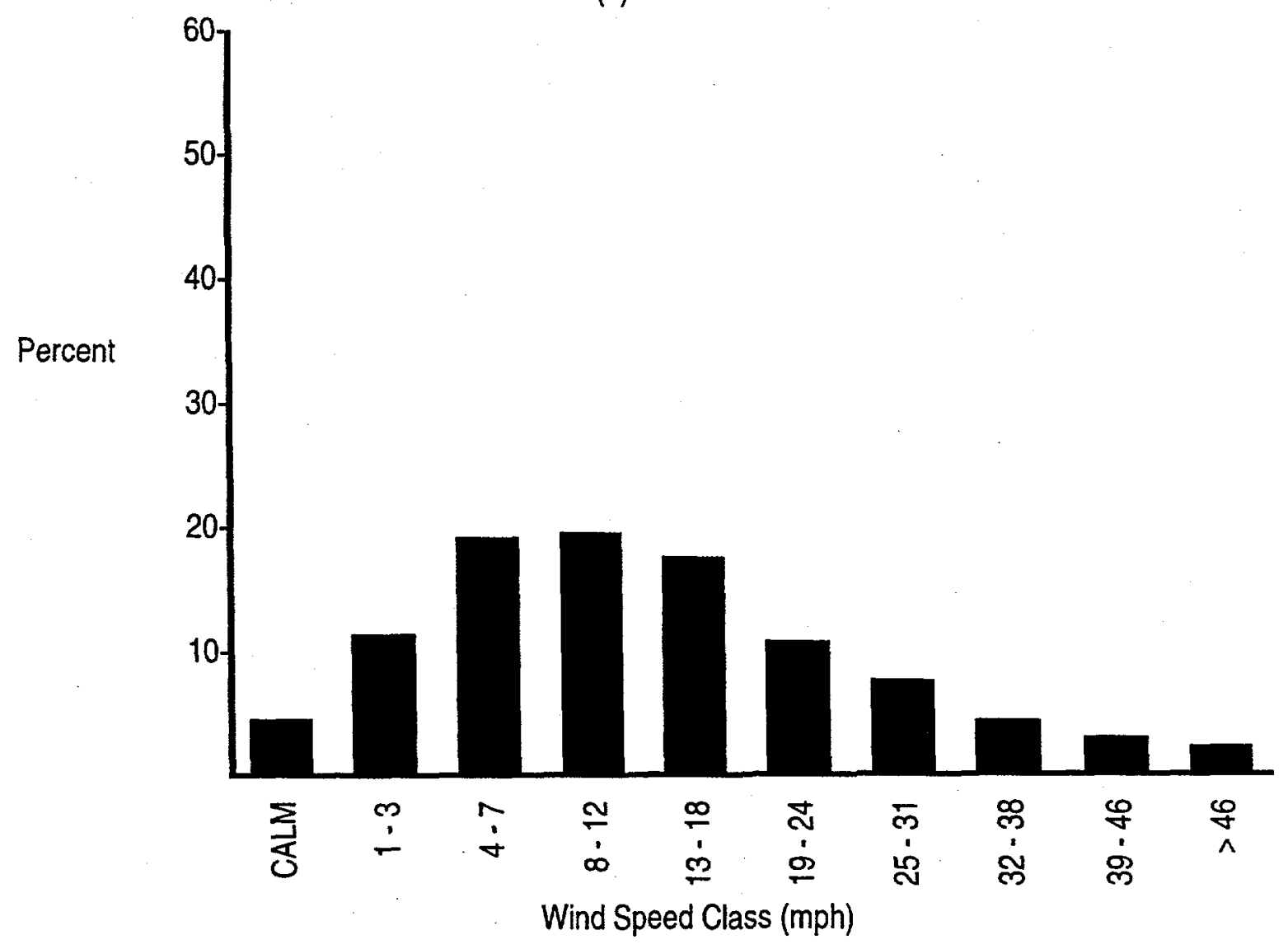

(b) Wind Speed Histogram

Figure A.1. (contd)
Station \#20 - RMTN

Period: $1 / 98-12 / 98$ 

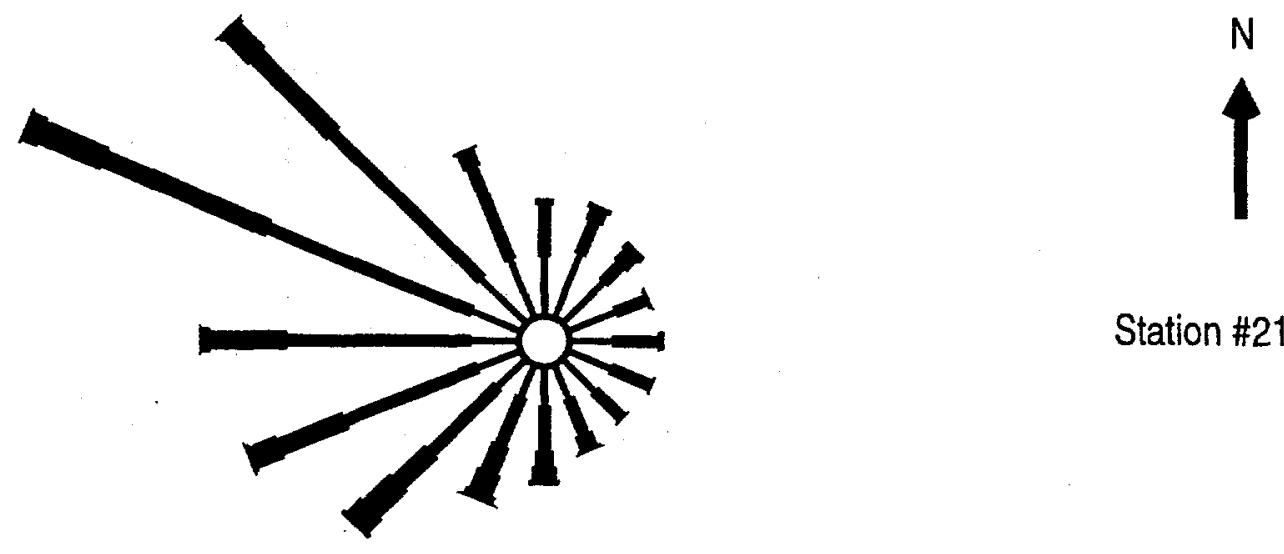

Station \#21 - HMS

(a) Wind Rose

Period: $1 / 98-12 / 98$

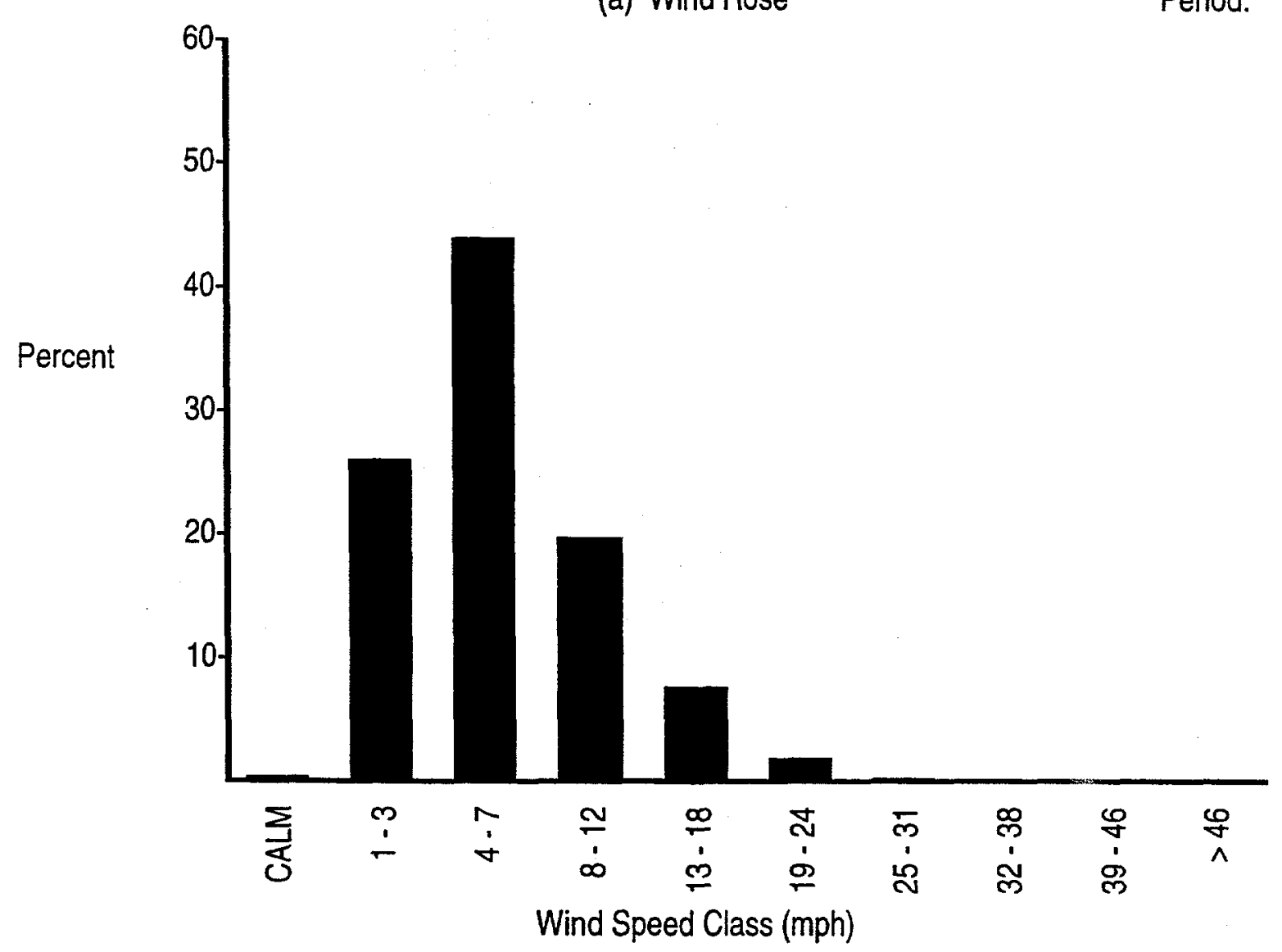

(b) Wind Speed Histogram

Figure A.1. (contd) 


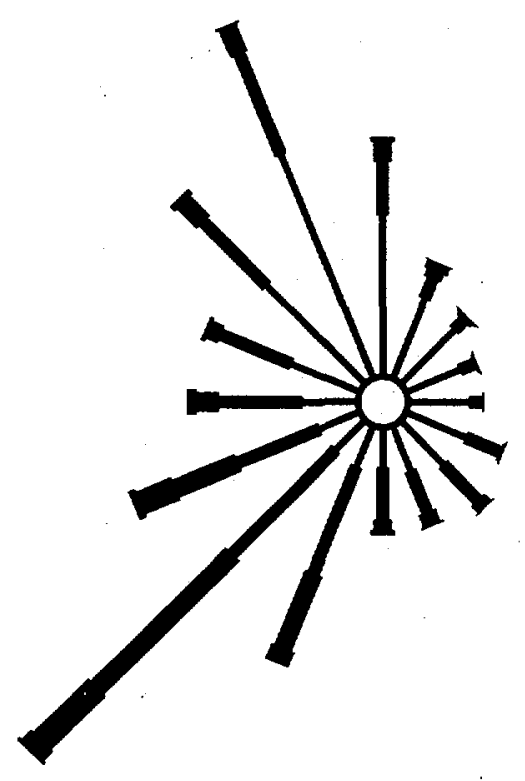

(a) Wind Rose

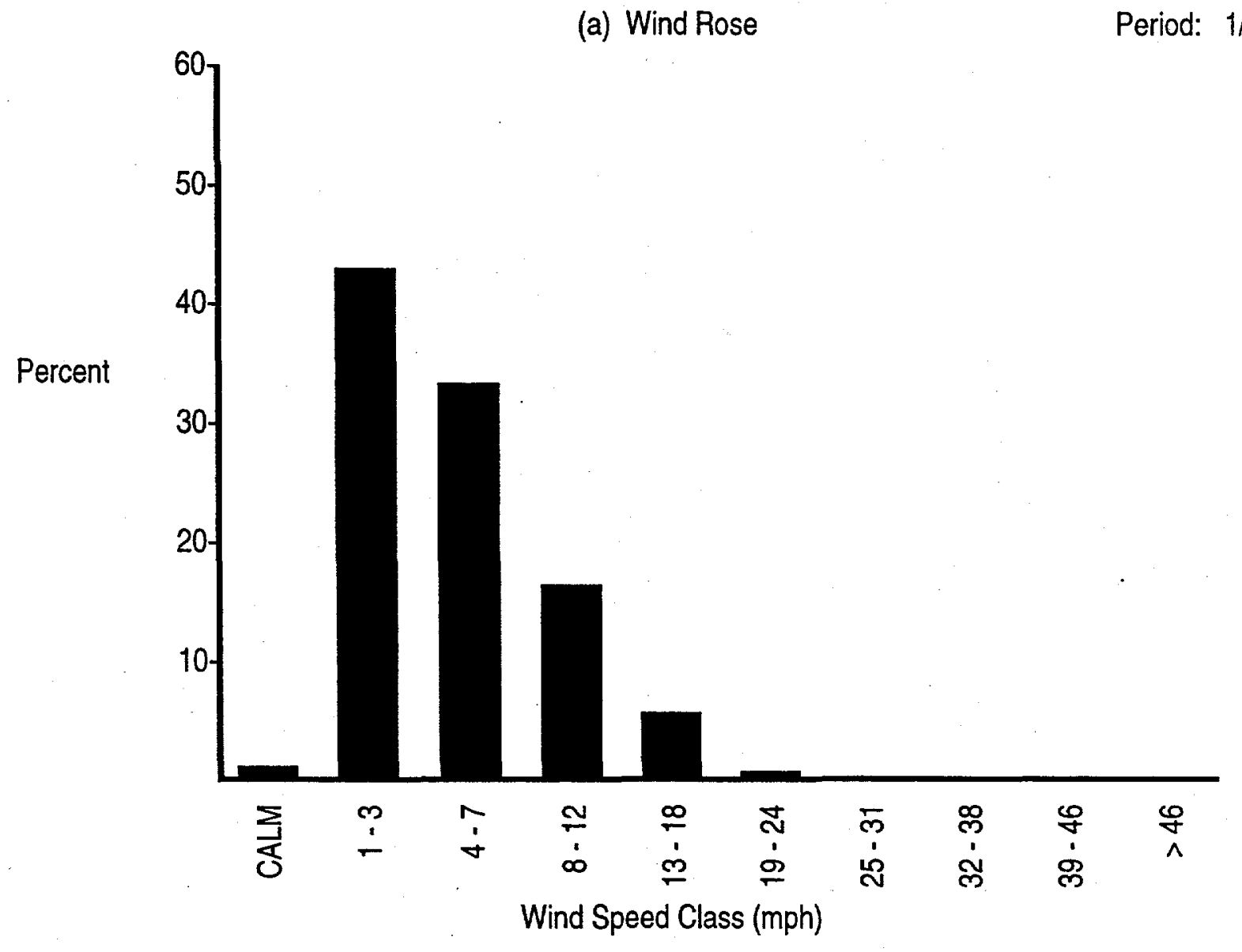

(b) Wind Speed Histogram

Figure A.1. (contd)

\section{Station \#22 - PASC}

Period: $1 / 98-12 / 98$ 


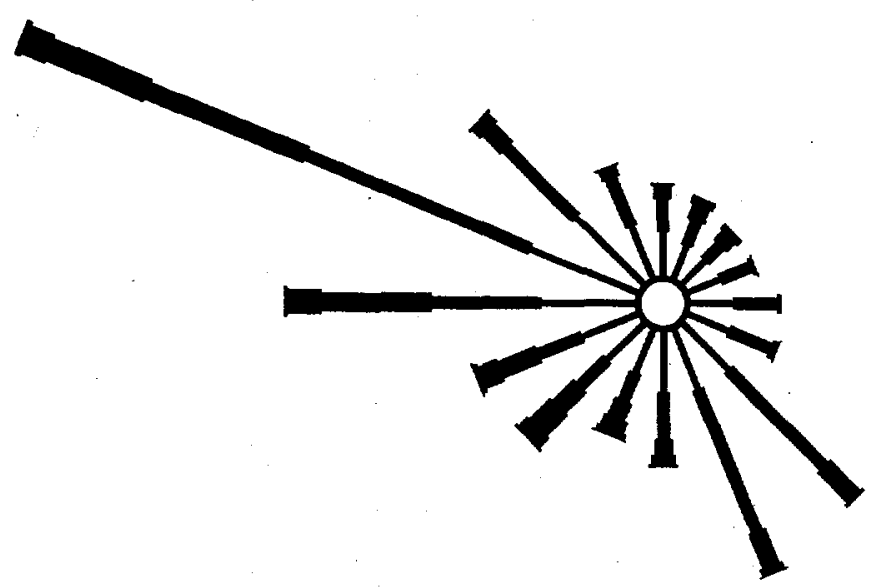

(a) Wind Rose

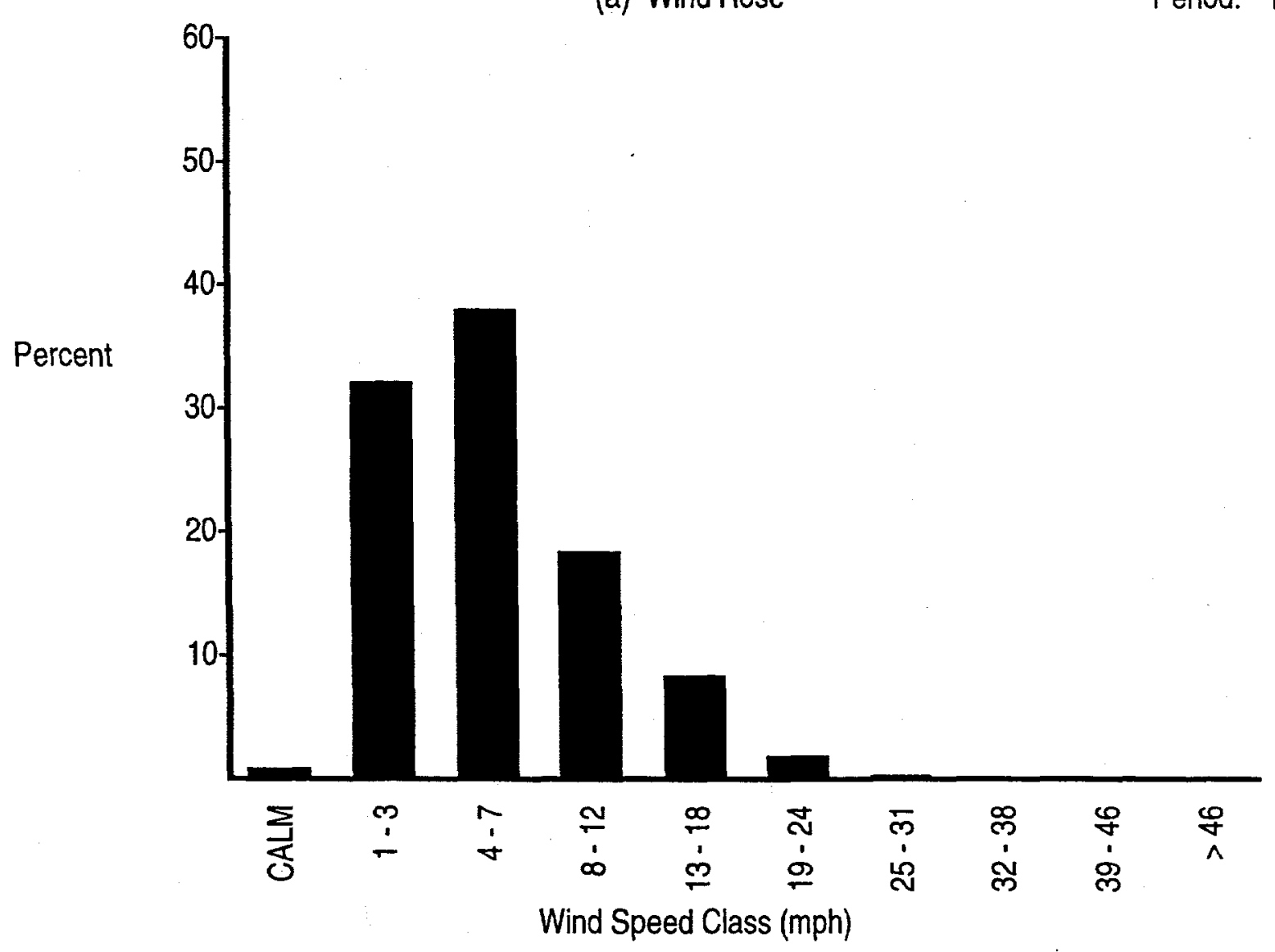

(b) Wind Speed Histogram

Figure A.1. (contd) 


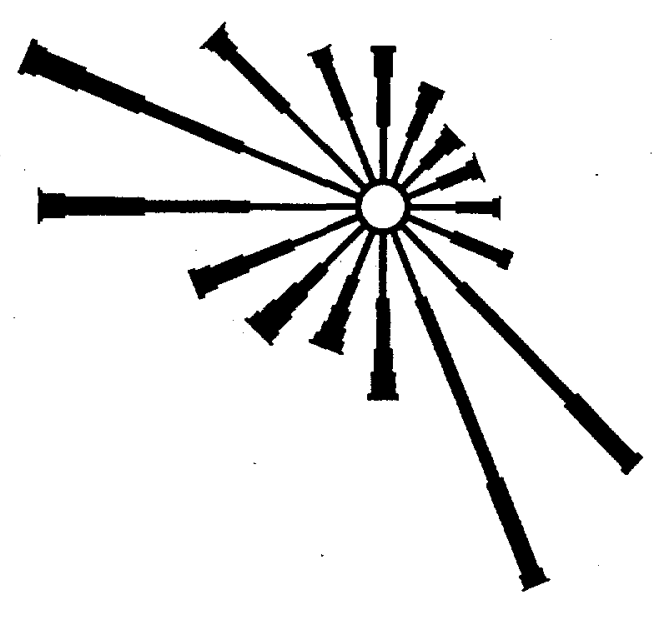

(a) Wind Rose

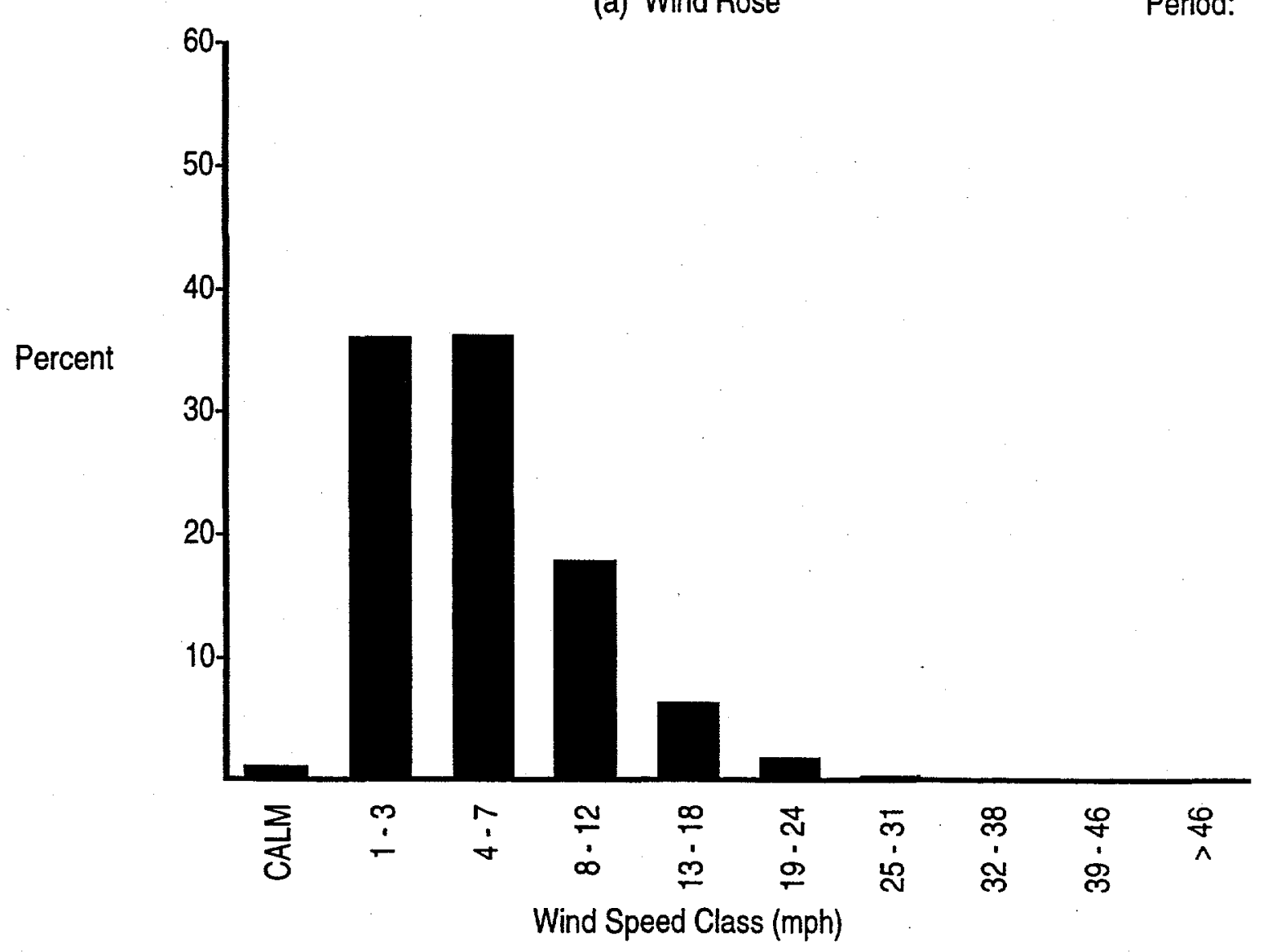

(b) Wind Speed Histogram

Figure A.1. (contd)
Station \#24 - 100F

Period: $1 / 98-12 / 98$ 

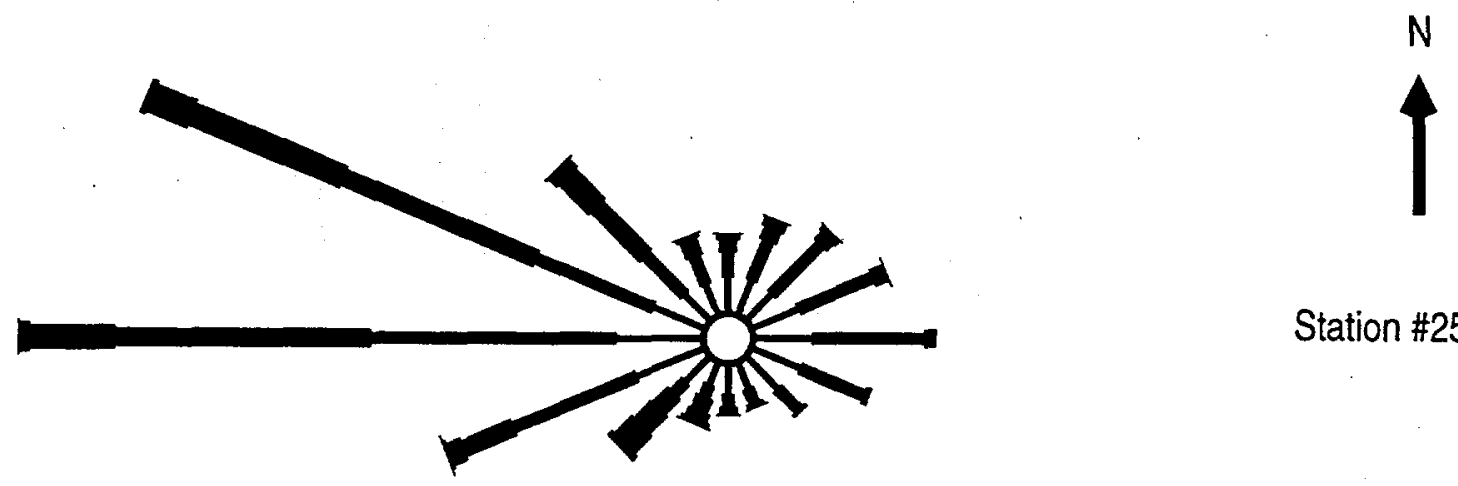

Station \#25 - VERN

(a) Wind Rose

Period: $1 / 98-12 / 98$

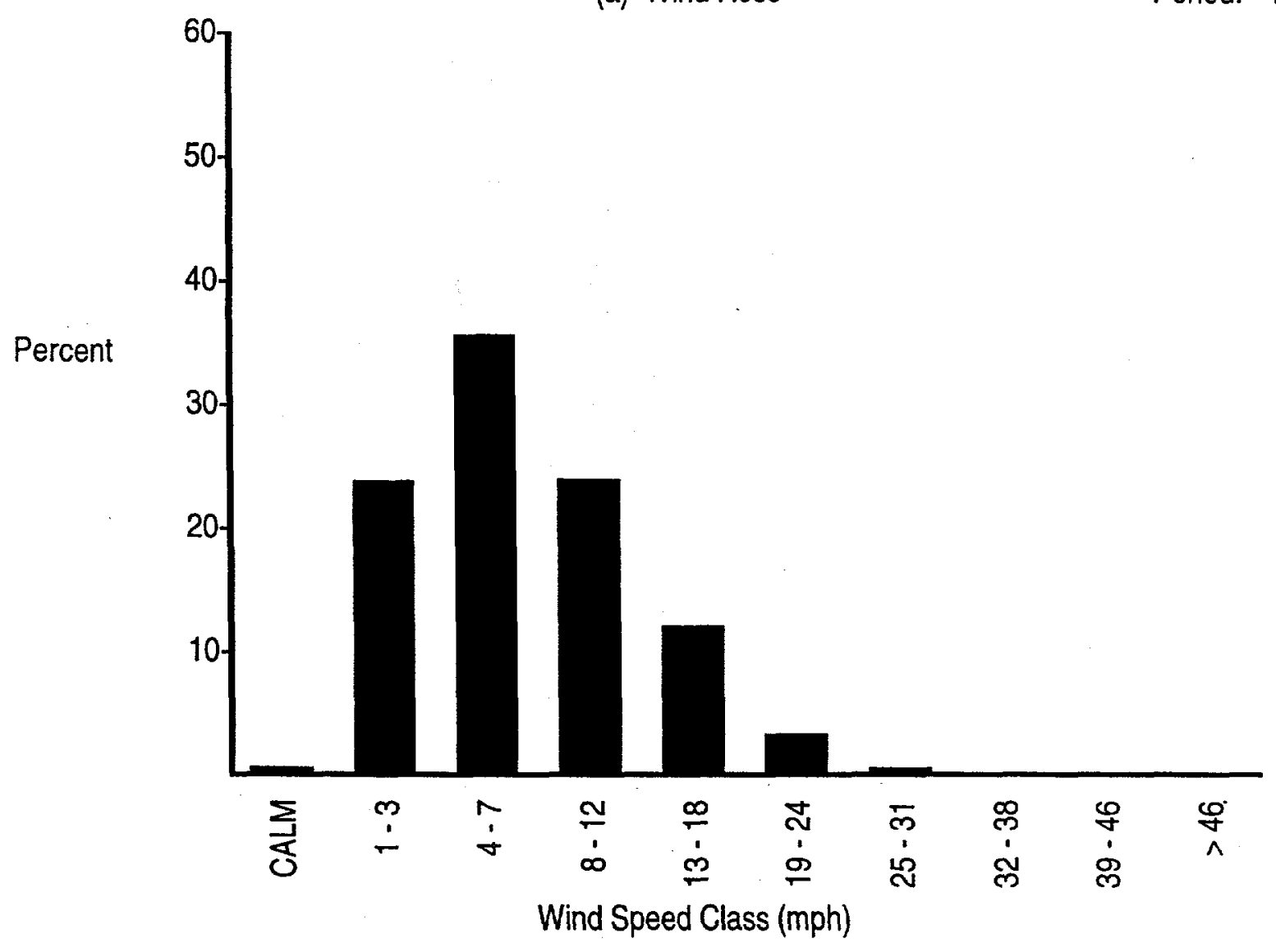

(b) Wind Speed Histogram

Figure A.1. (contd) 

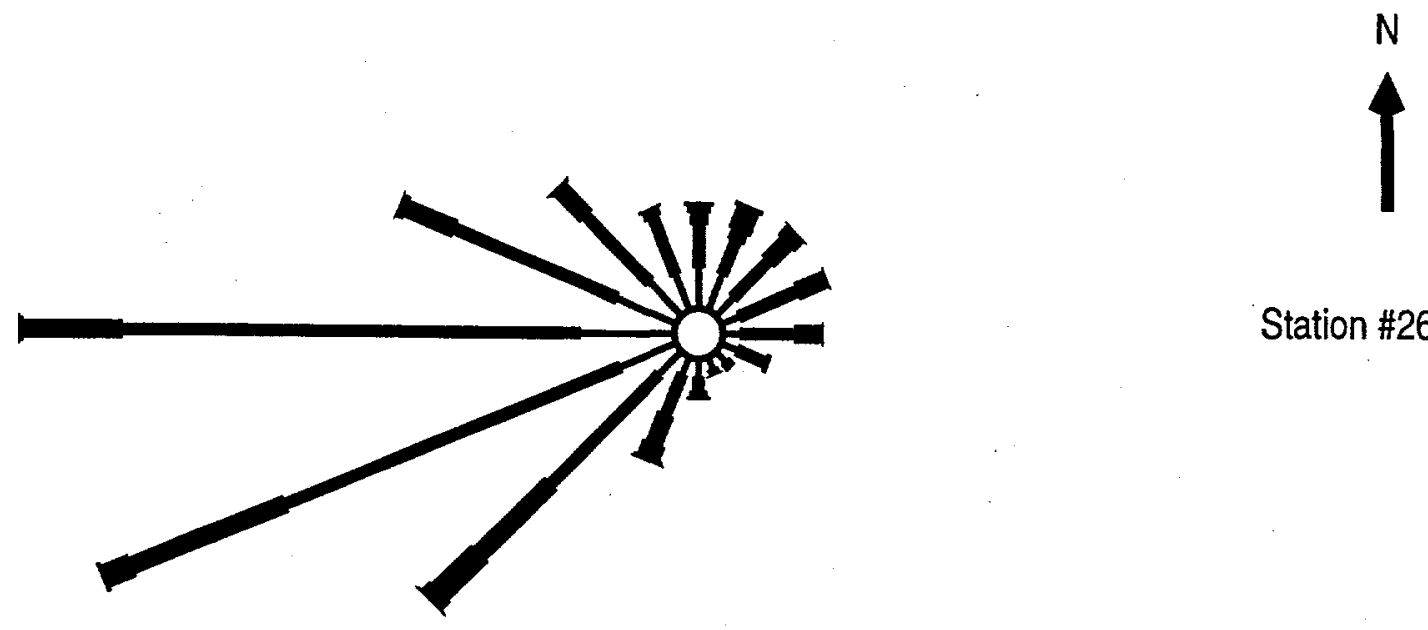

Station \#26 - BENT

(a) Wind Rose

Period: $1 / 98-12 / 98$

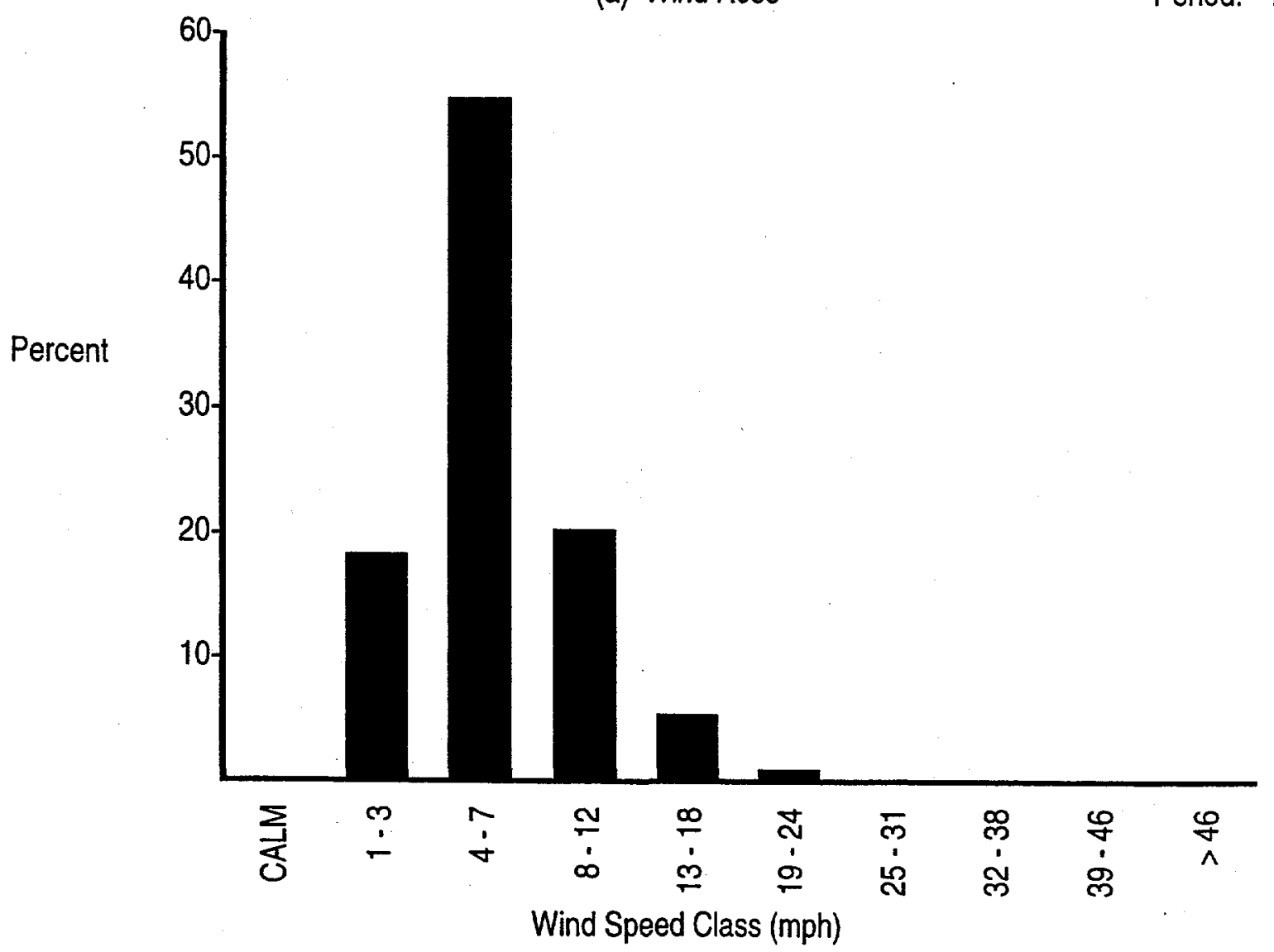

(b) Wind Speed Histogram

Figure A.1. (contd) 


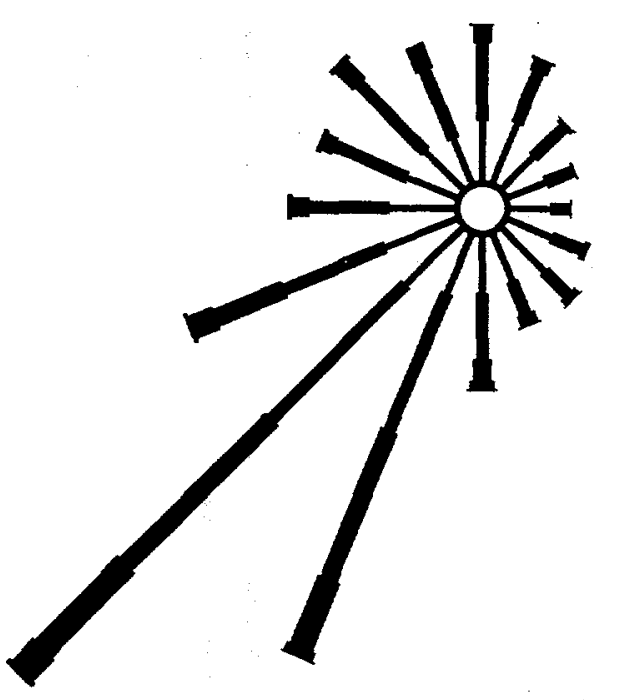

(a) Wind Rose

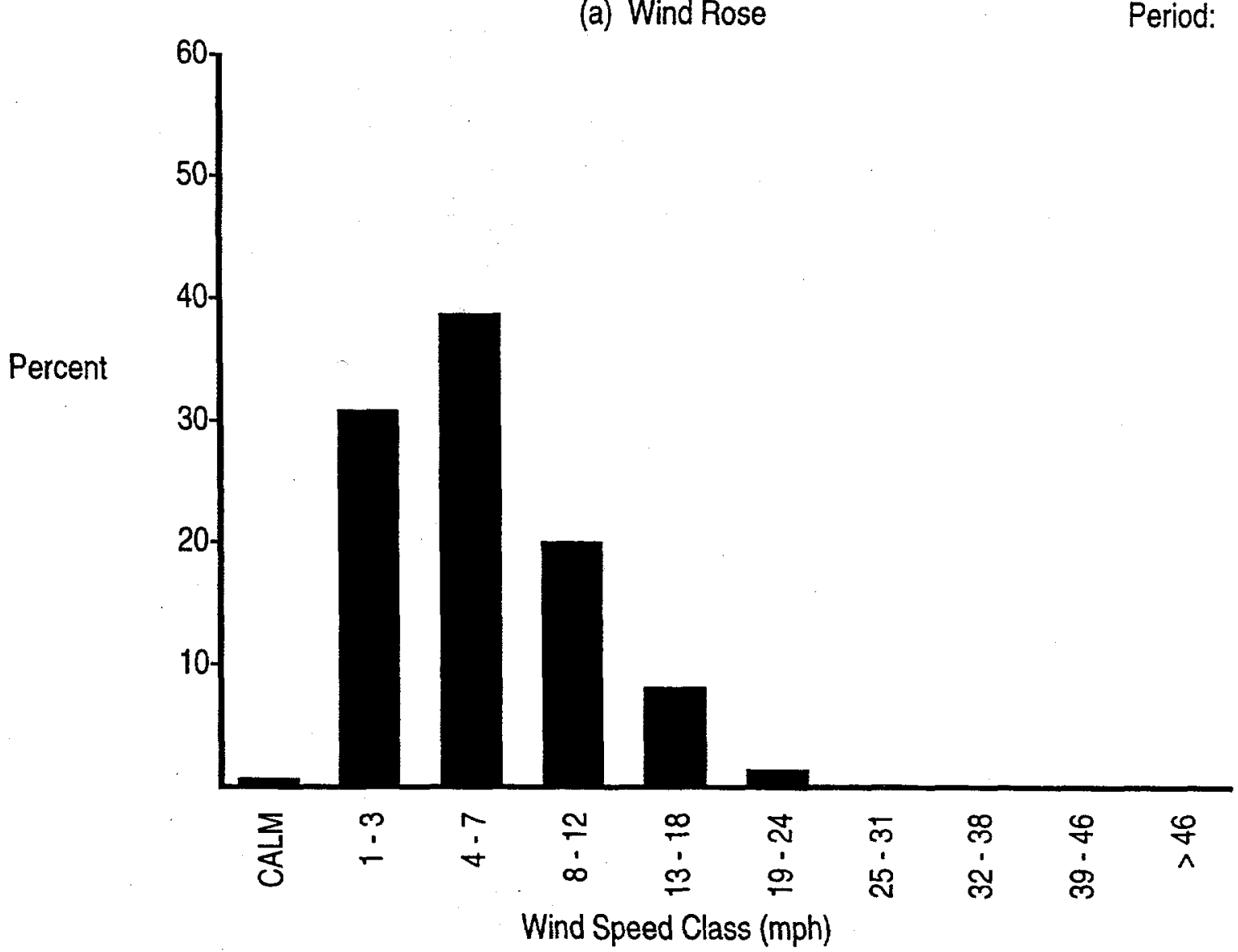

(b) Wind Speed Histogram

Figure A.1. (contd) 

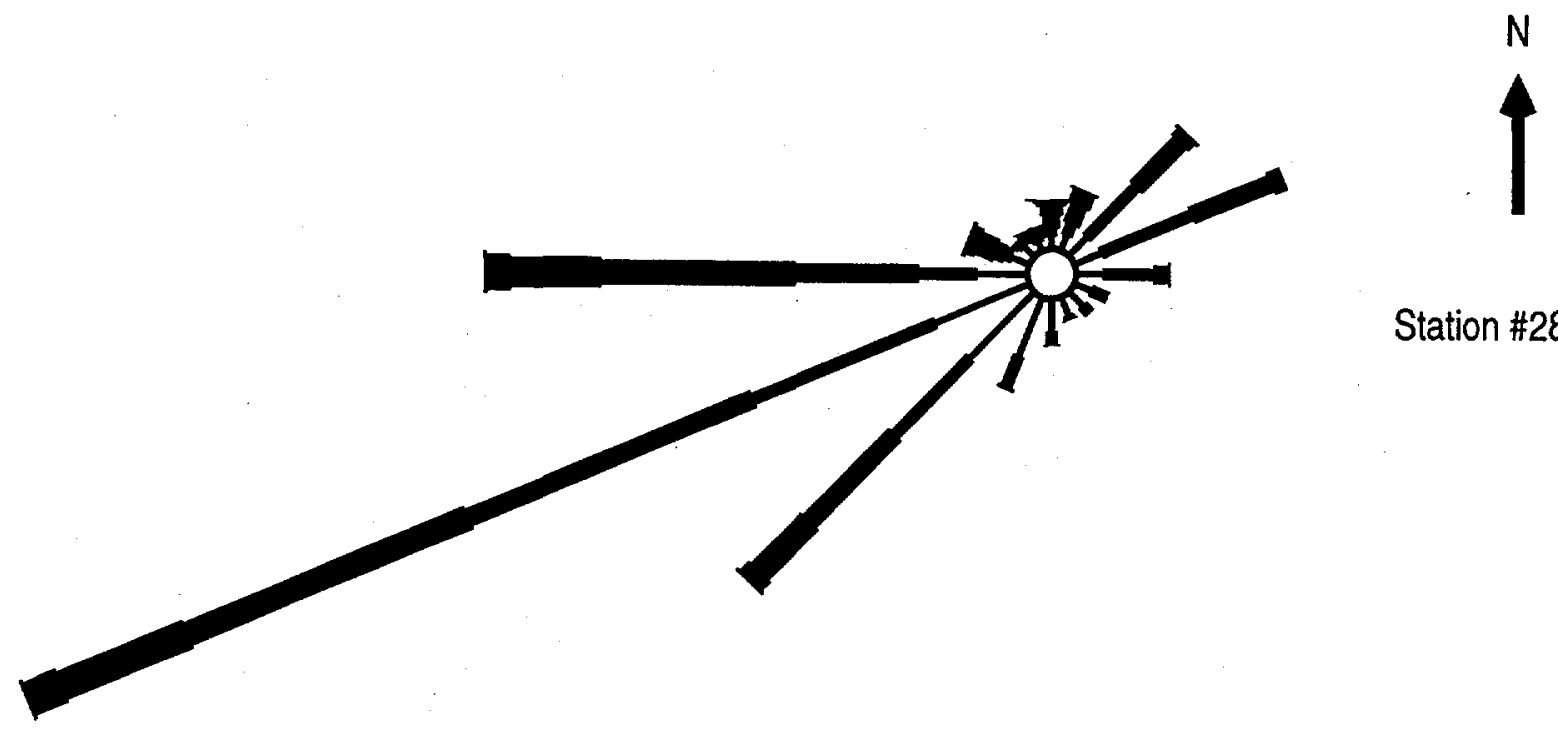

Station \#28 - SURF

(a) Wind Rose

Period: $1 / 98-12 / 98$

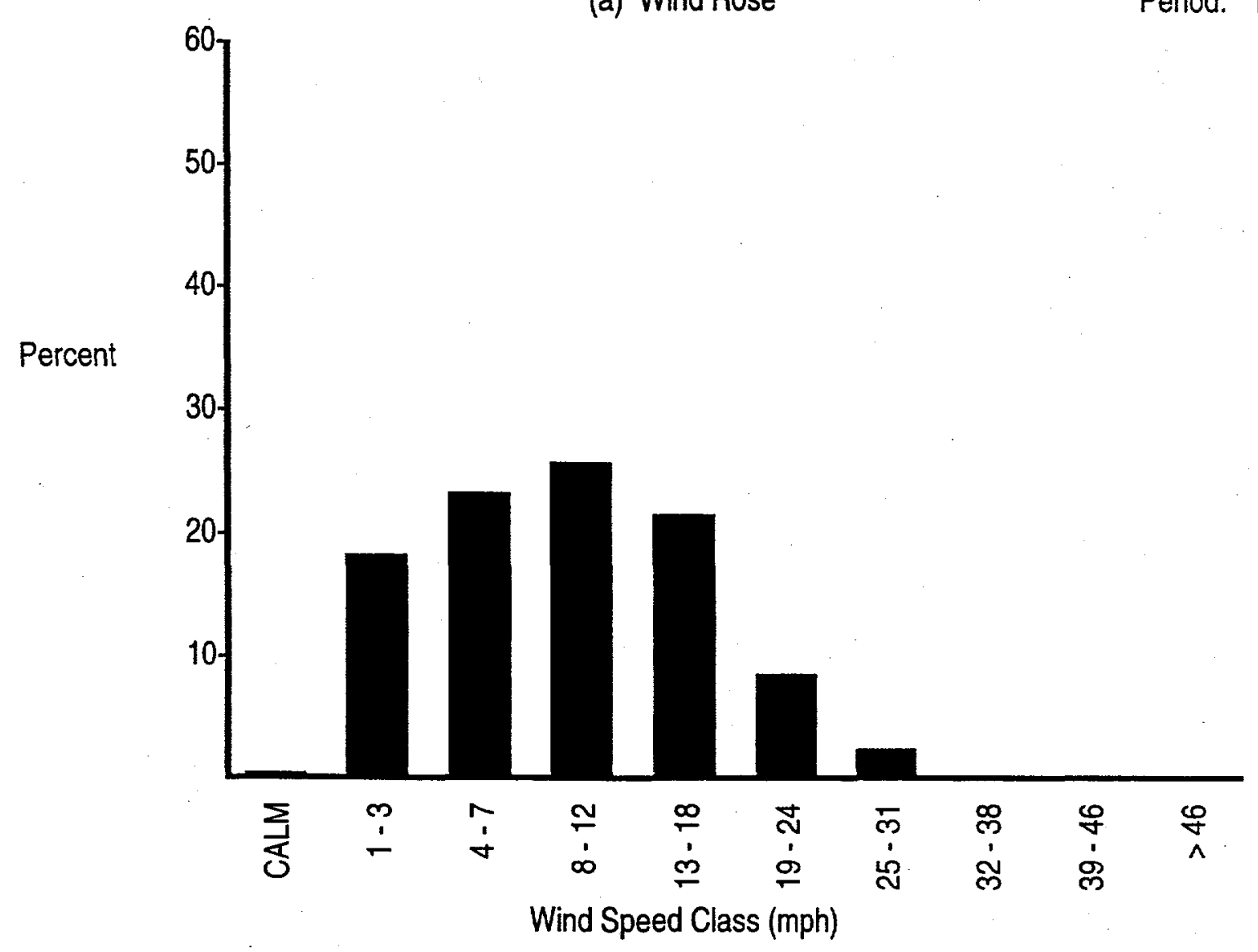

(b) Wind Speed Histogram

Figure A.1. (contd) 


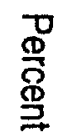
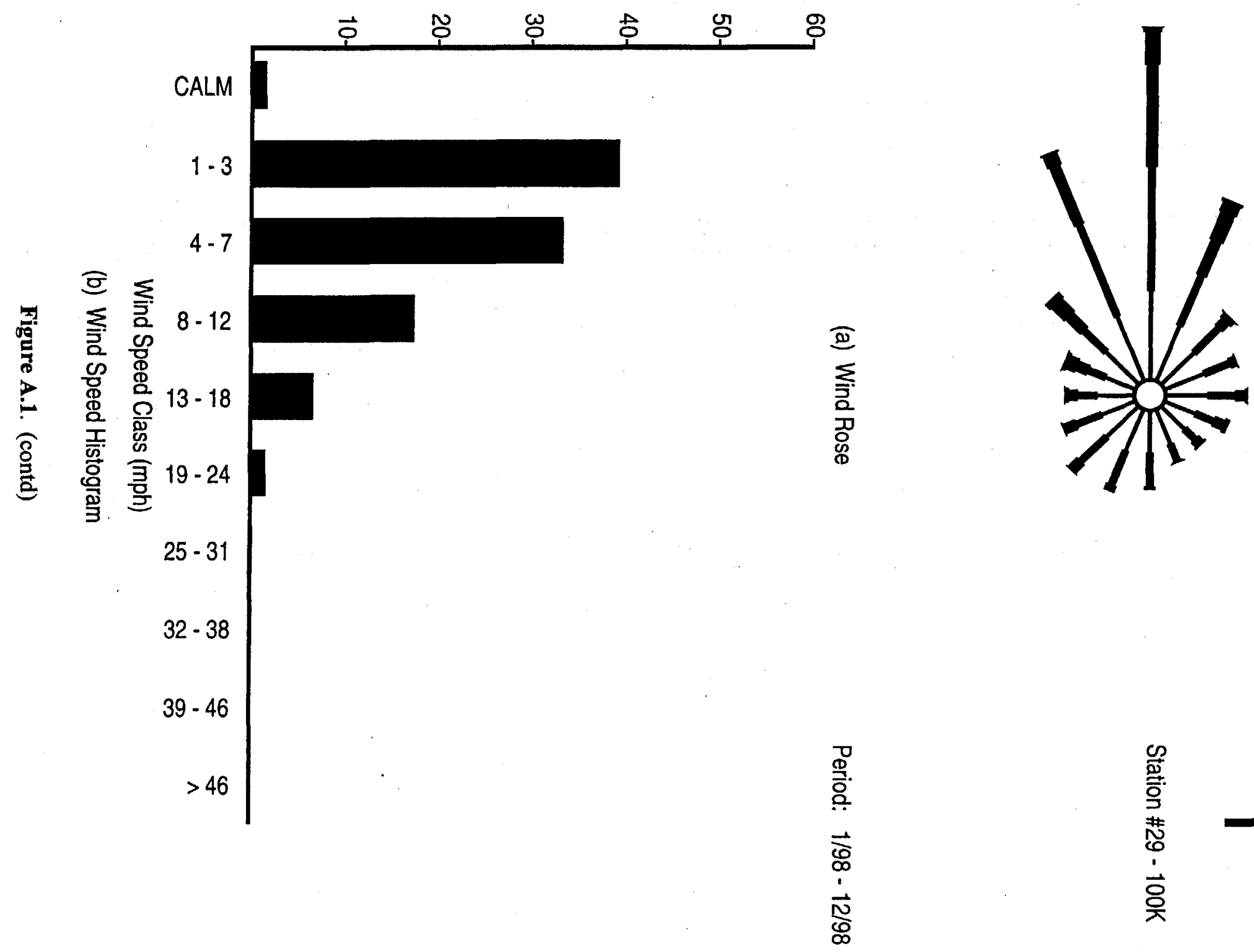

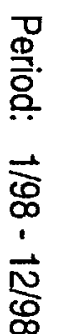

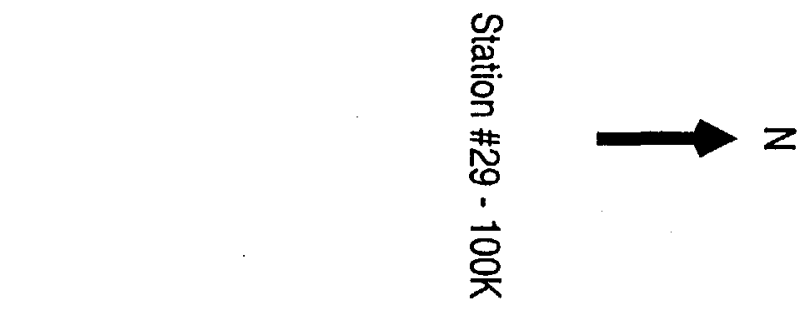

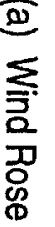




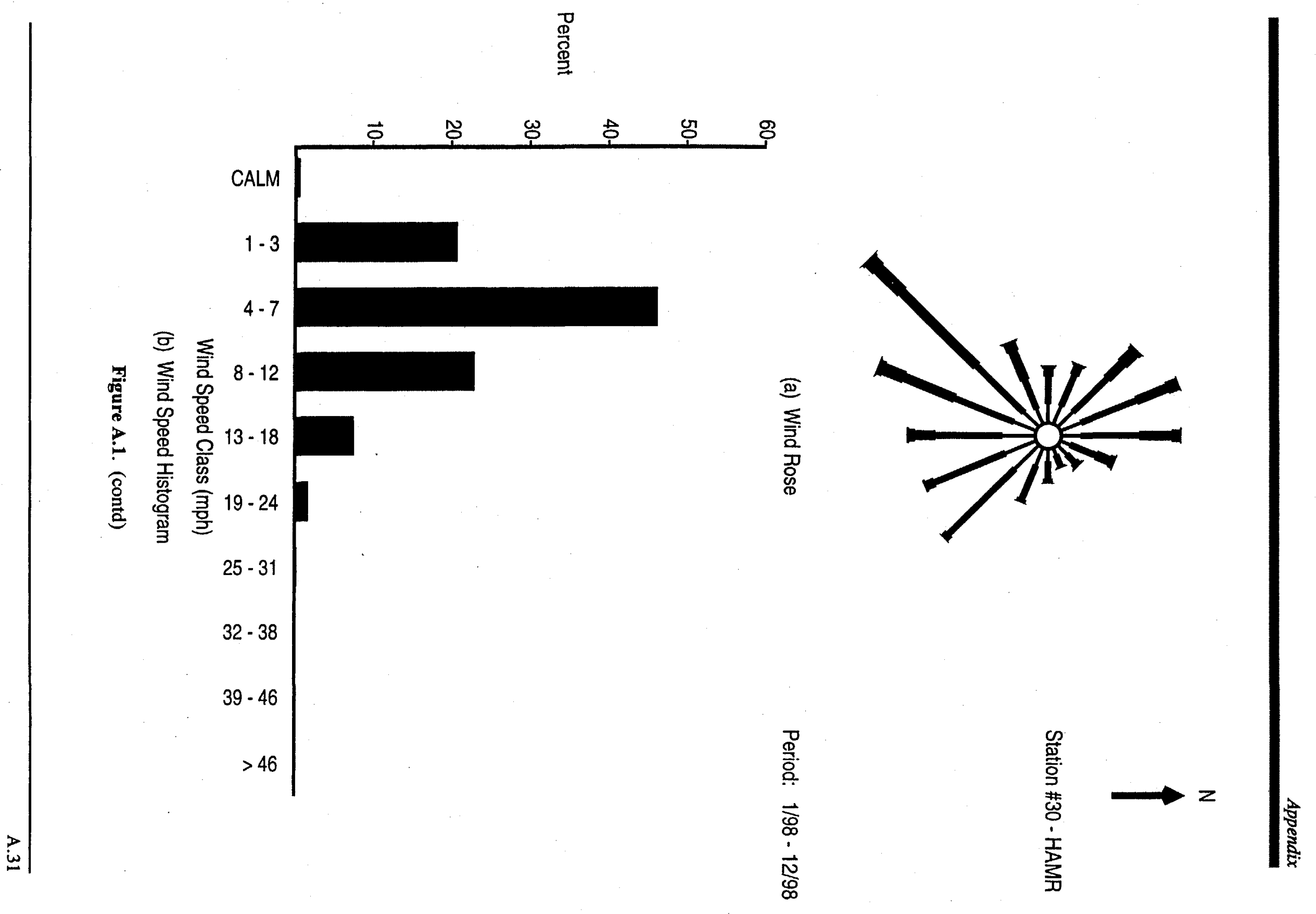




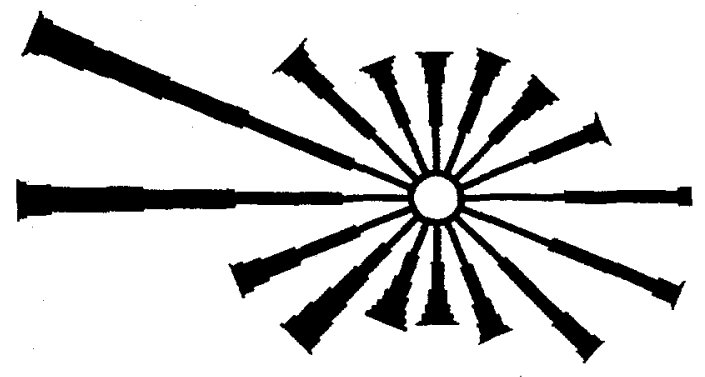

(a) Wind Rose

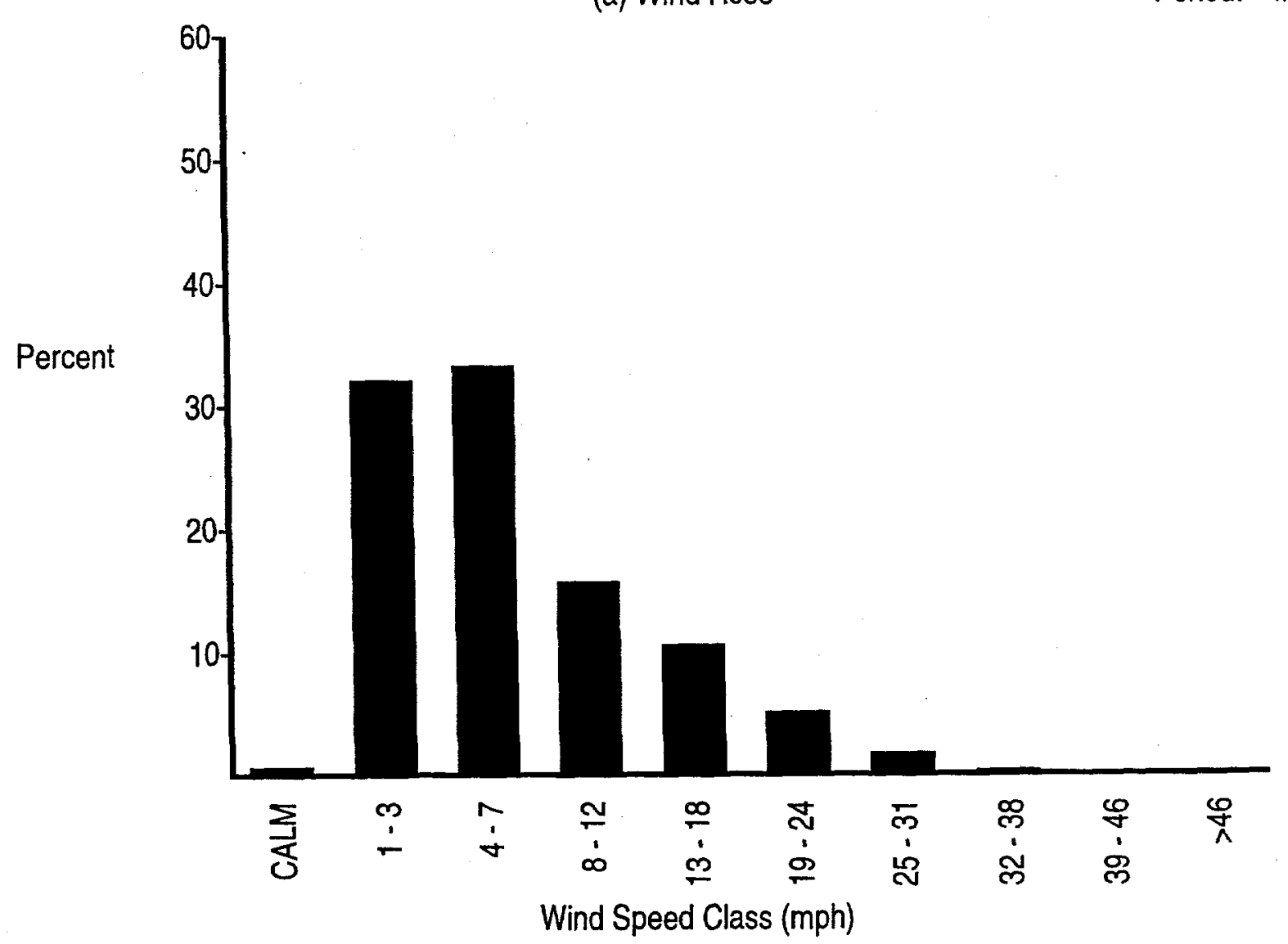

(b) Wind Speed Histogram

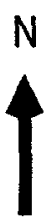

100 Area Tower

Period: $1 / 98-12 / 98$ 


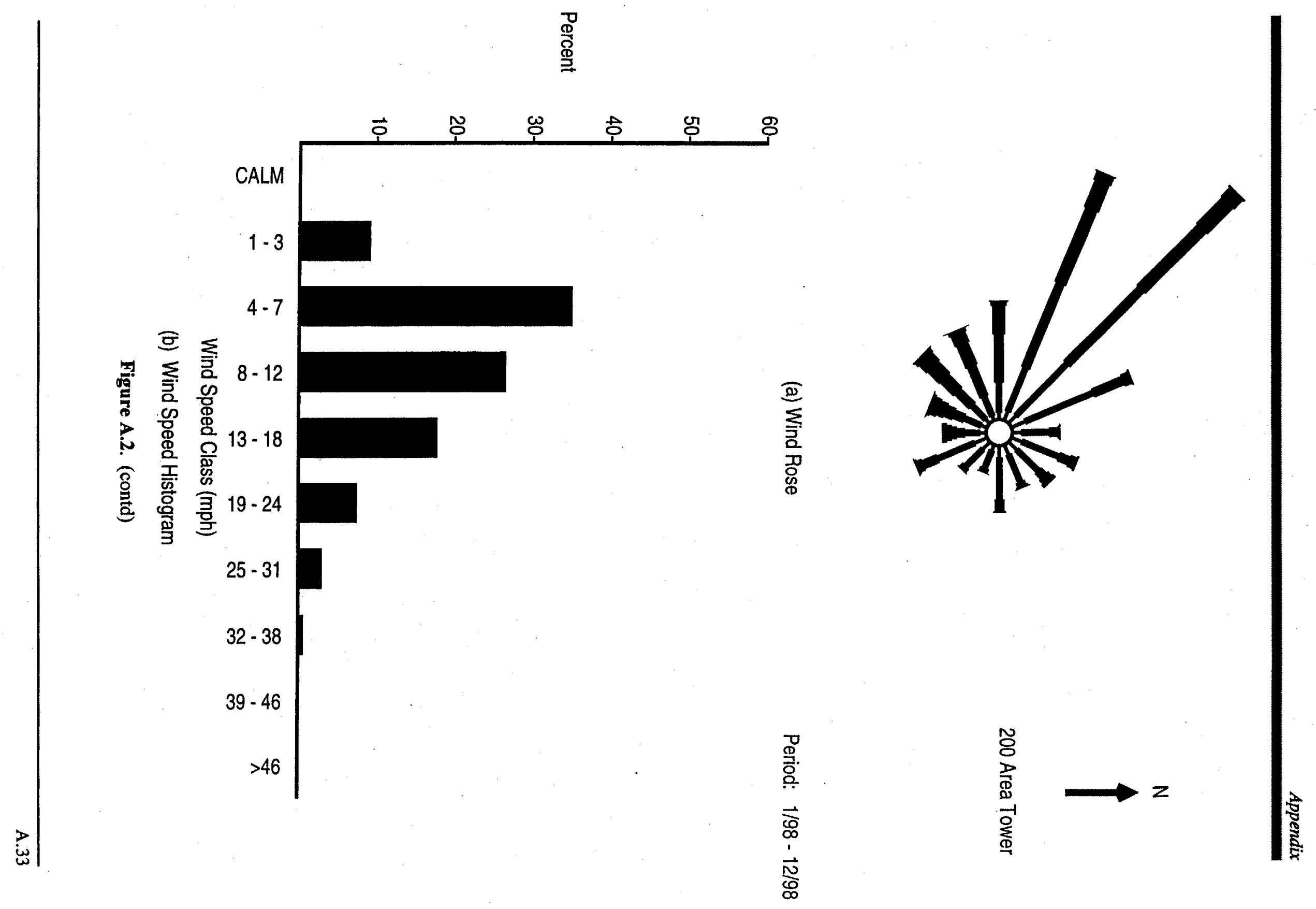




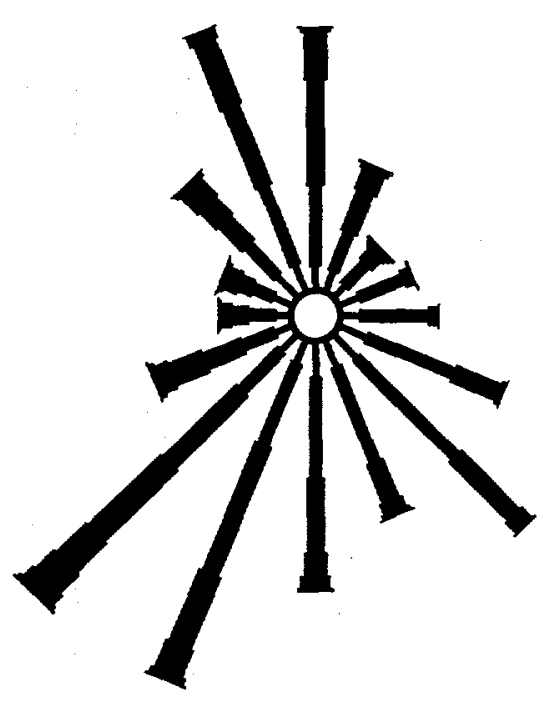

(a) Wind Rose

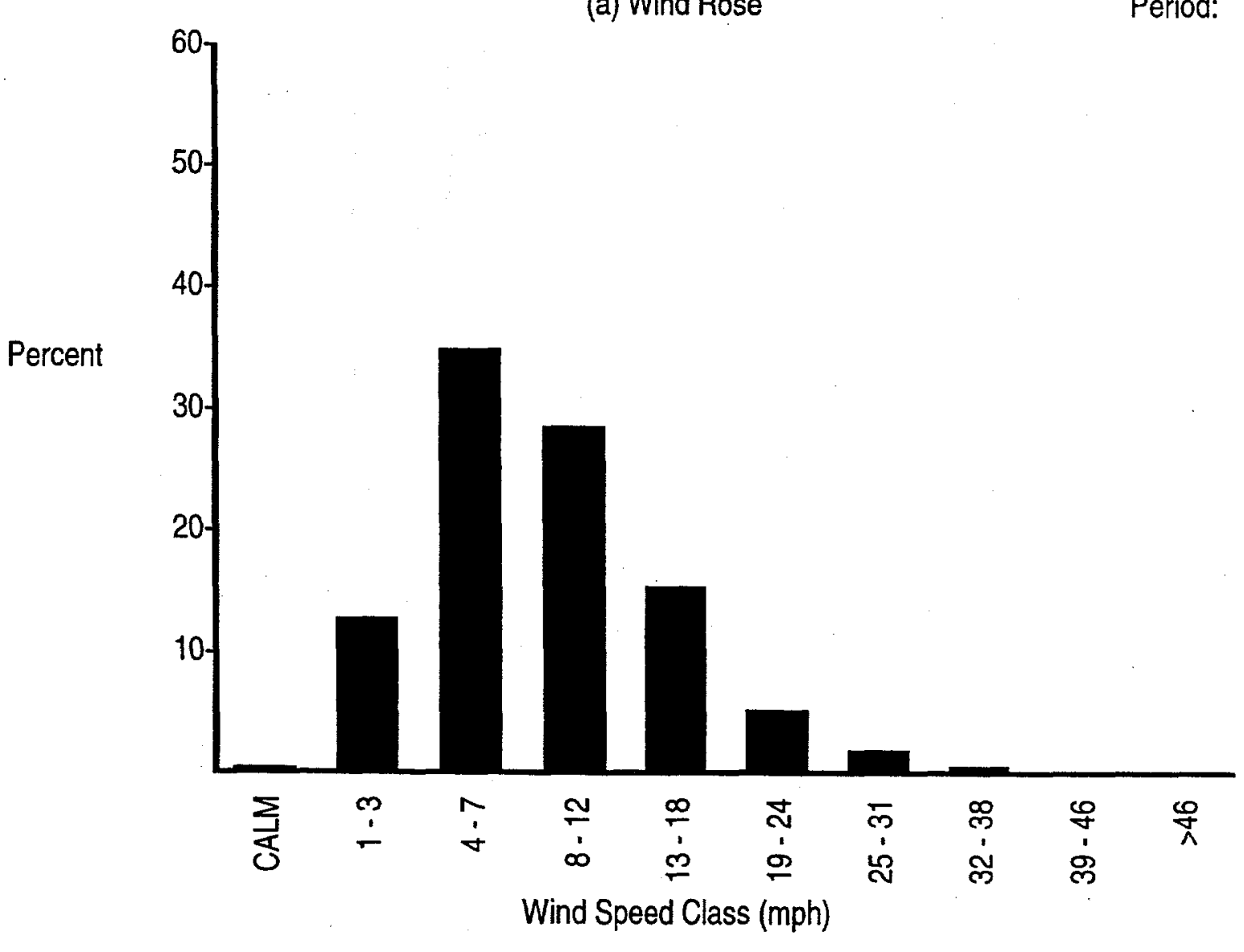

(b) Wind Speed Histogram

Figure A.2. (contd) 


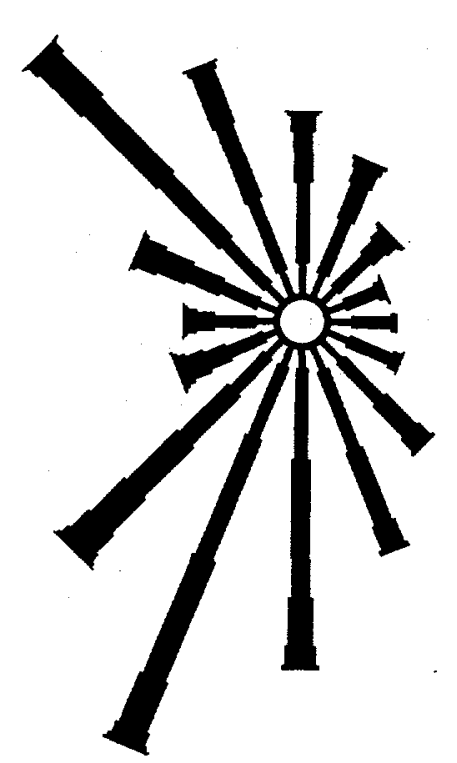

(a) Wind Rose

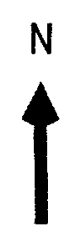

400 Area Tower

Period: $1 / 98-12 / 98$

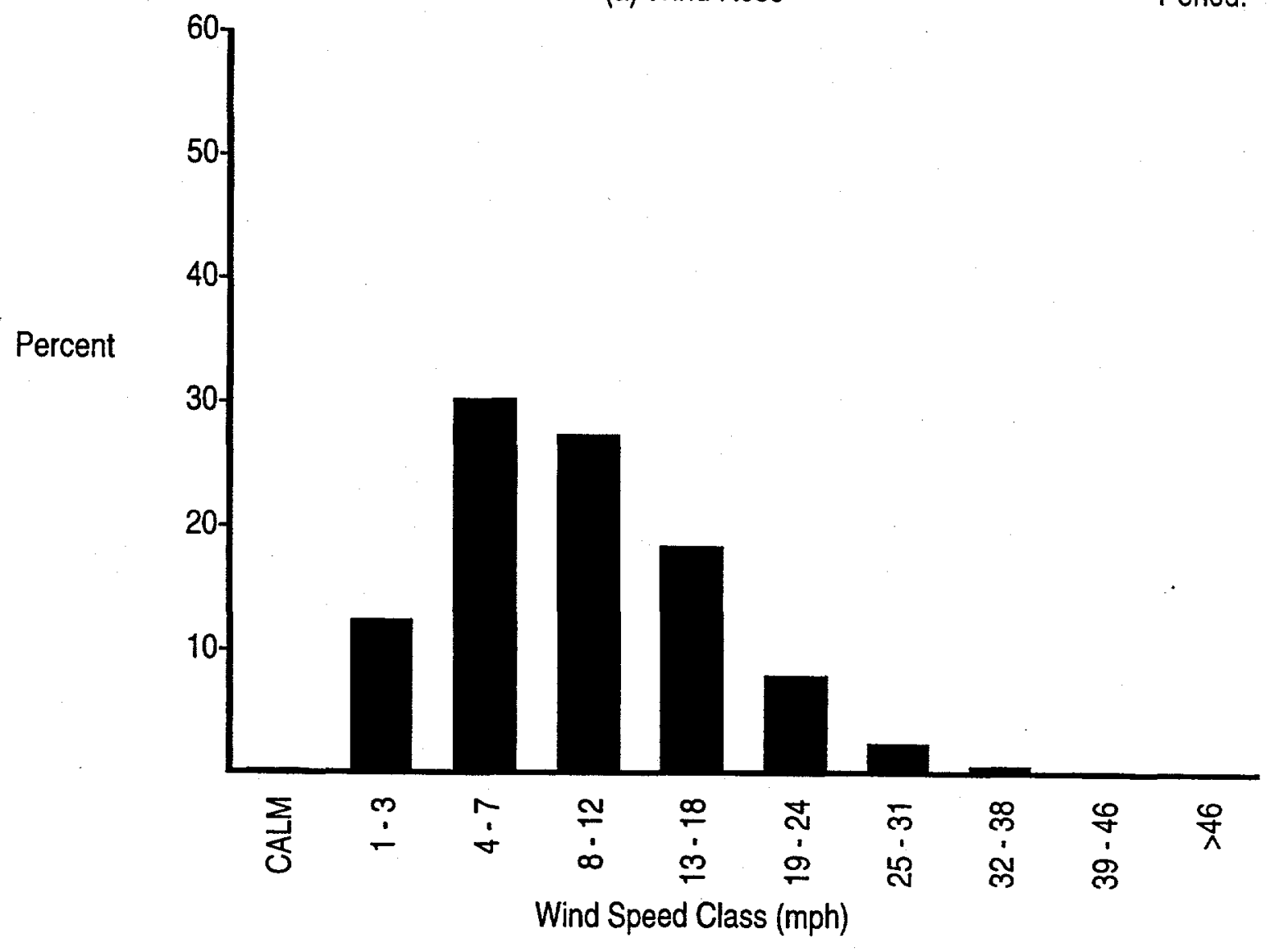

(b) Wind Speed Histogram

Figure A.2. (contd) 
Table A.1. Joint Frequency Distributions (\%) for Hanford Meteorological Monitoring Network Wind Stations at 10-Meter Level, 1998

Station: ( 1) PROS

Begin: $1 / 98 \quad$ End: $12 / 98$

Total Hours:

8760

DIRECTION

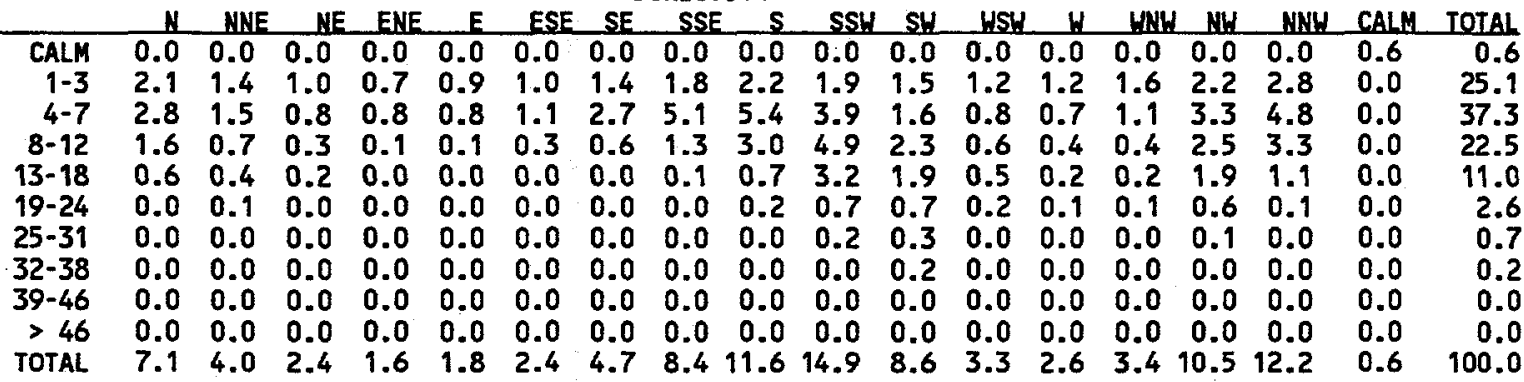

Station: ( 2) EOC

Begin: 1/98 End: 12/98

Total Hours:

8756

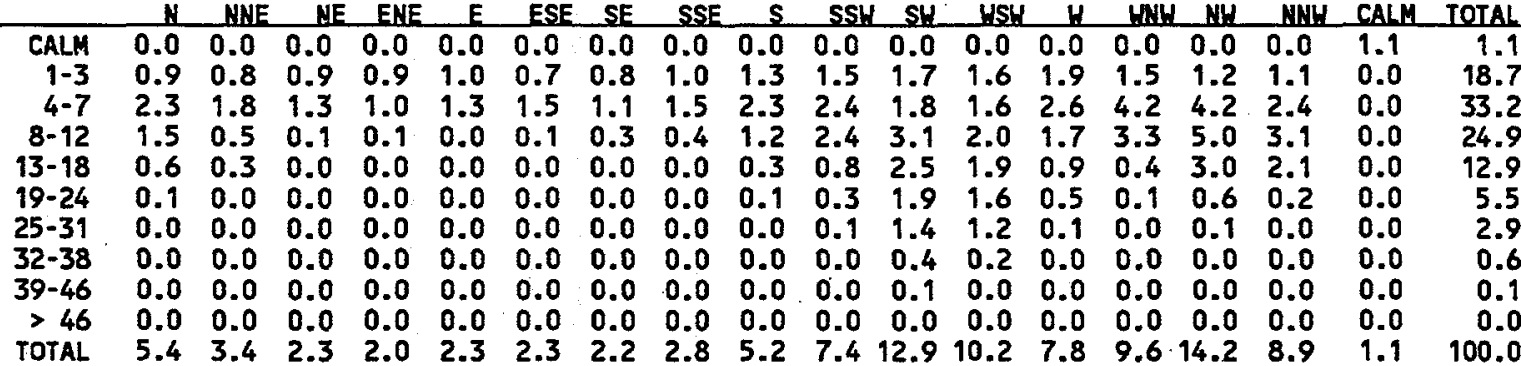

Station: ( 3) ARMY

Begin: $1 / 98$

End: $12 / 98$

Total Hours:

8760

DIRECTION

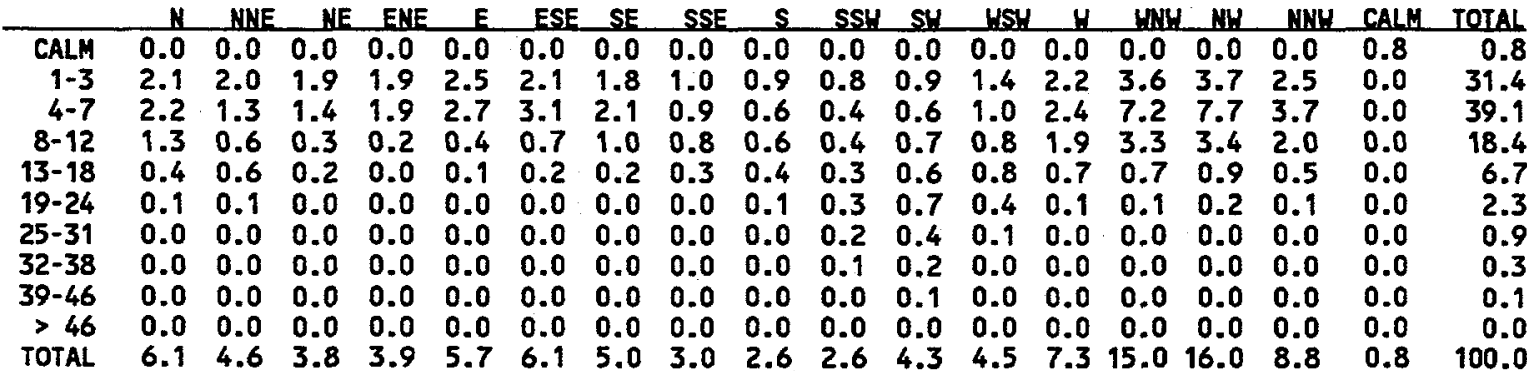

Station: (4) RSPC

Begin: $1 / 98$

End: $12 / 98$

Total Hours:

8760

DIRECTION

\begin{tabular}{|c|c|c|c|c|c|c|c|c|c|c|c|c|c|c|c|c|c|c|}
\hline & $\mathrm{N}$ & NME & NE & ENE & $E$ & ESE & SE & SSE & $s$ & SS! & SH & WS & $\begin{array}{r}H \\
\end{array}$ & HNW & $\mathrm{NH}$ & NHH & CALM & TOTAL \\
\hline $\begin{array}{r}\text { CALM } \\
1-3 \\
4-7 \\
8-12 \\
13-18 \\
19-24 \\
25-31 \\
32-38 \\
39-46 \\
>46 \\
\text { TOTAL }\end{array}$ & $\begin{array}{l}0.0 \\
1.3 \\
2.7 \\
0.4 \\
0.0 \\
0.0 \\
0.0 \\
0.0 \\
0.0 \\
0.0 \\
4.5\end{array}$ & $\begin{array}{l}0.0 \\
1.4 \\
2.3 \\
0.6 \\
0.1 \\
0.0 \\
0.0 \\
0.0 \\
0.0 \\
0.0 \\
4.3\end{array}$ & $\begin{array}{l}0.0 \\
1.4 \\
1.7 \\
0.4 \\
0.1 \\
0.0 \\
0.0 \\
0.0 \\
0.0 \\
0.0 \\
3.6\end{array}$ & $\begin{array}{l}0.0 \\
1.3 \\
1.2 \\
0.1 \\
0.0 \\
0.0 \\
0.0 \\
0.0 \\
0.0 \\
0.0 \\
2.6\end{array}$ & $\begin{array}{l}0.0 \\
1.2 \\
2.4 \\
0.2 \\
0.0 \\
0.0 \\
0.0 \\
0.0 \\
0.0 \\
0.0 \\
3.9\end{array}$ & $\begin{array}{l}0.0 \\
1.3 \\
2.7 \\
0.2 \\
0.0 \\
0.0 \\
0.0 \\
0.0 \\
0.0 \\
0.0 \\
4.3\end{array}$ & $\begin{array}{l}0.0 \\
0.8 \\
1.3 \\
0.1 \\
0.0 \\
0.0 \\
0.0 \\
0.0 \\
0.0 \\
0.0 \\
2.3\end{array}$ & $\begin{array}{l}0.0 \\
0.7 \\
0.4 \\
0.1 \\
0.0 \\
0.0 \\
0.0 \\
0.0 \\
0.0 \\
0.0 \\
1.2\end{array}$ & $\begin{array}{l}0.0 \\
0.7 \\
0.3 \\
0.2 \\
0.1 \\
0.0 \\
0.0 \\
0.0 \\
0.0 \\
0.0\end{array}$ & $\begin{array}{l}0.0 \\
0.9 \\
0.7 \\
0.6 \\
0.8 \\
0.4 \\
0.2 \\
0.0 \\
0.0 \\
0.0 \\
3.5\end{array}$ & $\begin{array}{l}0.0 \\
1.7 \\
2.2 \\
1.6 \\
1.0 \\
0.6 \\
0.4 \\
0.0 \\
0.0 \\
0.0 \\
7.5\end{array}$ & $\begin{array}{r}0.0 \\
2.0 \\
10.8 \\
16.0 \\
1.8 \\
0.4 \\
0.1 \\
0.0 \\
0.0 \\
0.0\end{array}$ & $\begin{array}{l}0.0 \\
1.8 \\
4.9 \\
6.3 \\
1.4 \\
0.1 \\
0.0 \\
0.0 \\
0.0 \\
0.0\end{array}$ & $\begin{array}{l}0.0 \\
1.2 \\
1.8 \\
2.1 \\
0.8 \\
0.0 \\
0.0 \\
0.0 \\
0.0 \\
0.0 \\
5.9\end{array}$ & $\begin{array}{l}0.0 \\
0.9 \\
1.6 \\
1.3 \\
0.4 \\
0.1 \\
0.0 \\
0.0 \\
0.0 \\
0.0 \\
4.3\end{array}$ & $\begin{array}{l}0.0 \\
1.0 \\
2.0 \\
1.2 \\
0.4 \\
0.1 \\
0.0 \\
0.0 \\
0.0 \\
0.0 \\
4.7\end{array}$ & $\begin{array}{l}0.7 \\
0.0 \\
0.0 \\
0.0 \\
0.0 \\
0.0 \\
0.0 \\
0.0 \\
0.0 \\
0.0 \\
0.7\end{array}$ & $\begin{array}{r}0.7 \\
19.7 \\
39.1 \\
31.3 \\
6.9 \\
1.6 \\
0.6 \\
0.1 \\
0.0\end{array}$ \\
\hline
\end{tabular}


Table A.1. (contd)

Station: ( 5) EDNA

Begin: 1/98 End: 12/98

Total Hours:

8760

DIRECTION

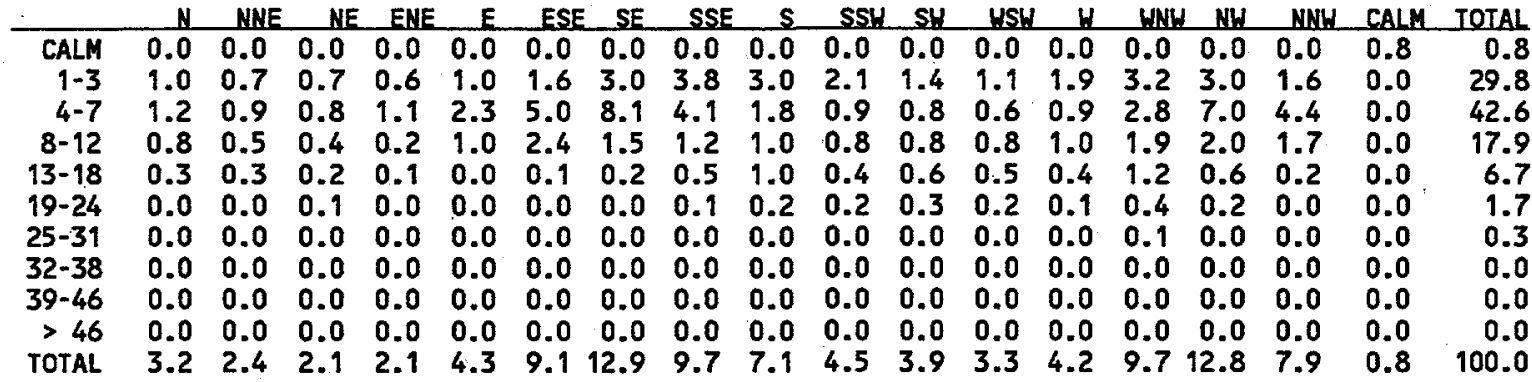

Station: ( 6) 200E

Begin: 1/98 End: $12 / 98$

Total Hours:

8760

DIRECTION

\begin{tabular}{|c|c|c|c|c|c|c|c|c|c|c|c|c|c|c|c|c|c|c|}
\hline & & NE & yE & WE & & ES & SE & SSE & & SSH & $3 \mathrm{~L}$ & HSY & & tWK & NW & NNy & $A L M$ & \\
\hline$A L$ & 0.0 & 0.0 & 0.0 & 0.0 & 0.0 & 0.0 & 0.0 & 0.0 & 0.0 & 0.0 & 0.0 & 0.0 & 0.0 & 0.0 & 0.0 & 0.0 & 1.7 & \\
\hline & 1.1 & 1.0 & 14 & & 1.7 & & & & & & & & & & & & & \\
\hline & & 1.0 & & 0.9 & 1.7 & 2. & & 2. & i. & & 1.8 & & & & & & & \\
\hline & & & & 0.1 & 0.3 & & & & & & & & & & & & & \\
\hline & & & & & & & & & & & & & & & & & & \\
\hline & & 0. & 0. & & 0. & & & & & & & & & & & & & \\
\hline & & 0. & & & 0. & & & & & & & & & & & & & \\
\hline & 0.0 & 0.0 & 0.0 & 0.0 & 0.0 & 0. & & & 0 . & & 0 . & & & & & & & \\
\hline & & 0. & & 0. & & & & & & & & & & & & & & \\
\hline & 0.0 & & 0.0 & & 0. & & & & & & & & & & & & & \\
\hline & & & & & & & & & & & & & & & .3 & 3.8 & & \\
\hline
\end{tabular}

Station: ( 7) $200 \mathrm{~W}$

Begin: 1/98 End: $12 / 98$

Total Hours:

8731

DIRECTION

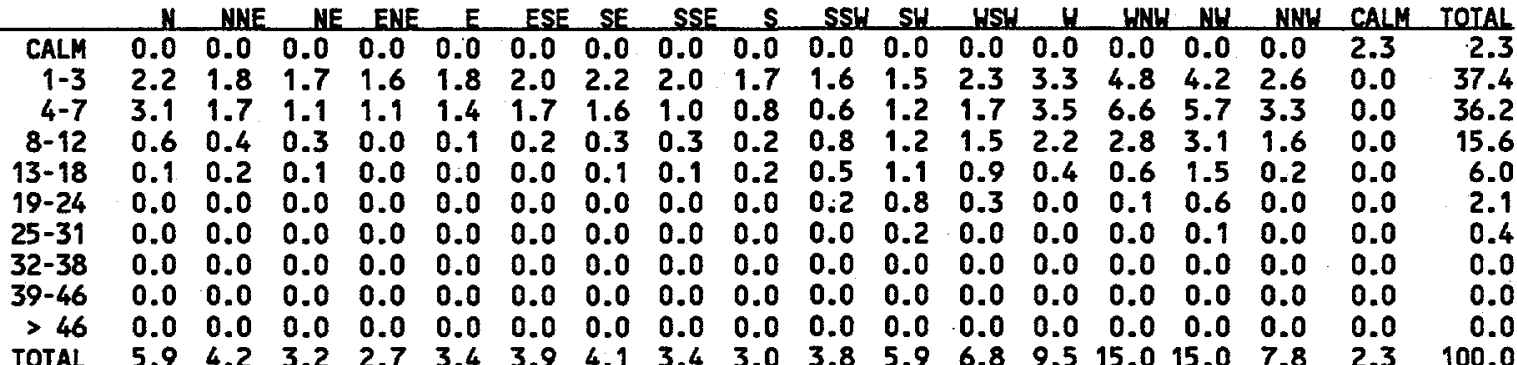

Station: ( 8) BVLY

Begin: $1 / 98 \quad$ End: $12 / 98$

Total Hours:

8760

DIRECTION

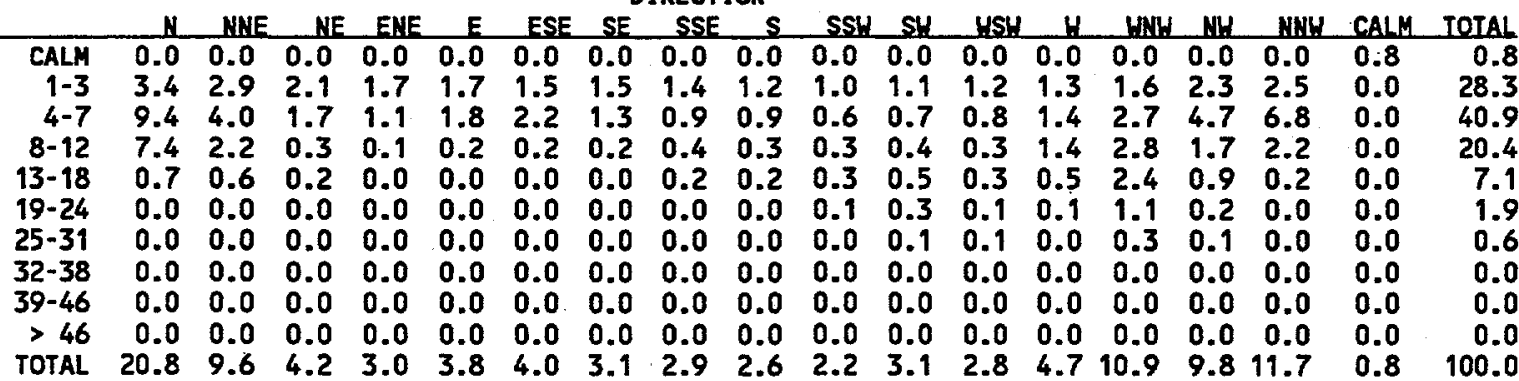


Table A.1. (contd)

Station: (9) FFTF

Begin: $1 / 98$

End: $12 / 98$

Total Hours:

8751

DIRECTION

\begin{tabular}{|c|c|c|c|c|c|c|c|c|c|c|c|c|c|c|c|c|c|c|}
\hline & $\mathbf{N}$ & NWE & NE & ENE & $E$ & ESE & SE & SS: & $S$ & SSL & SH & MSH & U & WNW & $\mathrm{NW}$ & NWW & CALM & TOTA \\
\hline $\begin{array}{r}\text { CALM } \\
1-3 \\
4-7 \\
8-12 \\
13-18 \\
19-24 \\
25-31 \\
32-38 \\
39-46 \\
>46 \\
\text { TOTAL }\end{array}$ & $\begin{array}{l}0.0 \\
1.1 \\
3.1 \\
1.1 \\
0.3 \\
0.0 \\
0.0 \\
0.0 \\
0.0\end{array}$ & $\begin{array}{l}0.0 \\
1.0 \\
2.5 \\
1.1 \\
0.4 \\
0.1 \\
0.0 \\
0.0 \\
0.0 \\
0.0 \\
5.2\end{array}$ & $\begin{array}{l}0.0 \\
0.9 \\
1.5 \\
0.4 \\
0.2 \\
0.1 \\
0.0 \\
0.0 \\
0.0 \\
0.0 \\
3.1\end{array}$ & $\begin{array}{l}0.0 \\
0.8 \\
1.1 \\
0.2 \\
0.0 \\
0.0 \\
0.0 \\
0.0 \\
0.0 \\
0.0 \\
2.1\end{array}$ & $\begin{array}{l}0.0 \\
0.8 \\
1.3 \\
0.2 \\
0.0 \\
0.0 \\
0.0 \\
0.0 \\
0.0 \\
0.0 \\
2.2\end{array}$ & $\begin{array}{l}0.0 \\
1.1 \\
1.7 \\
0.3 \\
0.0 \\
0.0 \\
0.0 \\
0.0 \\
0.0 \\
0.0 \\
3.0\end{array}$ & $\begin{array}{l}0.0 \\
1.0 \\
2.7 \\
1.5 \\
0.1 \\
0.0 \\
0.0 \\
0.0 \\
0.0 \\
0.0 \\
5.3\end{array}$ & $\begin{array}{l}4.0 \\
4.2 \\
0.3 \\
0.0 \\
0.0 \\
0.0\end{array}$ & $\begin{array}{r}0.0 \\
1.2 \\
4.7 \\
4.4 \\
1.4 \\
0.2 \\
0.0 \\
0.0 \\
0.0 \\
0.0 \\
12.0\end{array}$ & $\begin{array}{l}0.0 \\
1.3 \\
3.9 \\
4.7 \\
3.2 \\
0.7 \\
0.2 \\
0.1 \\
0.0 \\
0.0 \\
14.0\end{array}$ & $\begin{array}{l}1.7 \\
1.7 \\
0.9 \\
0.4 \\
0.1 \\
0.0 \\
0.0\end{array}$ & $\begin{array}{l}0.0 \\
1.0 \\
1.2 \\
0.6 \\
0.4 \\
0.2 \\
0.9 \\
0.0\end{array}$ & $\begin{array}{l}0.0 \\
1.1 \\
1.3 \\
0.4 \\
0.2 \\
0.1 \\
0.0 \\
0.0 \\
0.0 \\
0.0 \\
3.1\end{array}$ & $\begin{array}{l}0.0 \\
1.1 \\
1.6 \\
1.1 \\
0.5 \\
0.1 \\
0.0 \\
0.0 \\
0.0 \\
0.0\end{array}$ & $\begin{array}{r}0.0 \\
1.4 \\
3.9 \\
3.5 \\
1.4 \\
0.4 \\
0.1 \\
0.0 \\
0.0 \\
0.0 \\
10.7\end{array}$ & $\begin{array}{l}3.1 \\
0.4 \\
0.0 \\
0.0 \\
0.0 \\
0.0 \\
0.0 \\
7.9\end{array}$ & $\begin{array}{l}0.0 \\
0.0 \\
0.0 \\
0.0 \\
0.6\end{array}$ & $\begin{array}{l}0 \\
0 \\
0 \\
0\end{array}$ \\
\hline
\end{tabular}

Station: (10) YAKB

Begin: $1 / 98$

End: $12 / 98$

Total Hours:

8717 DIRECTION

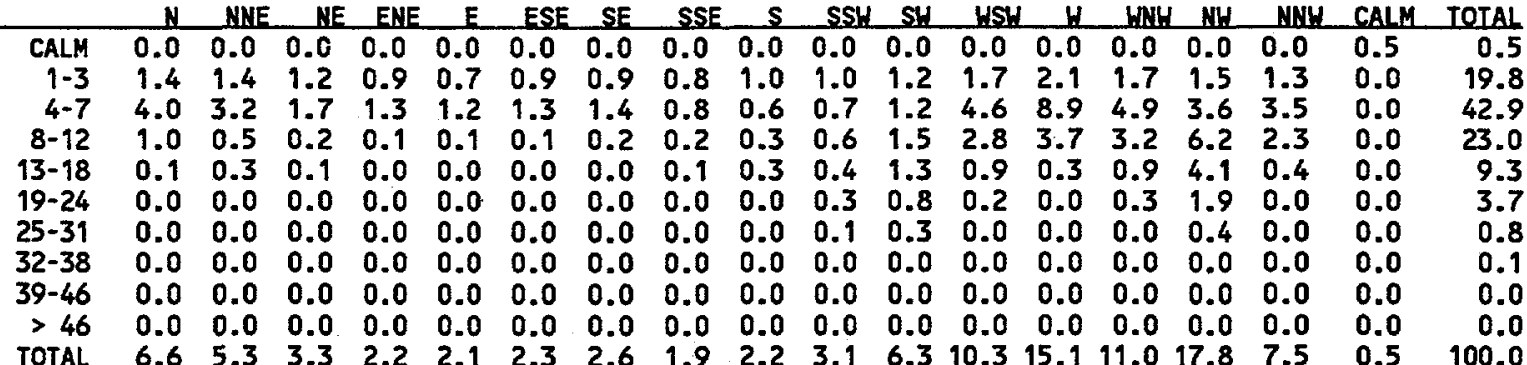

Station: (11) 300A

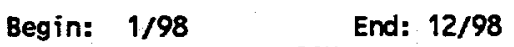

Total Hours:

8759

DIRECTION

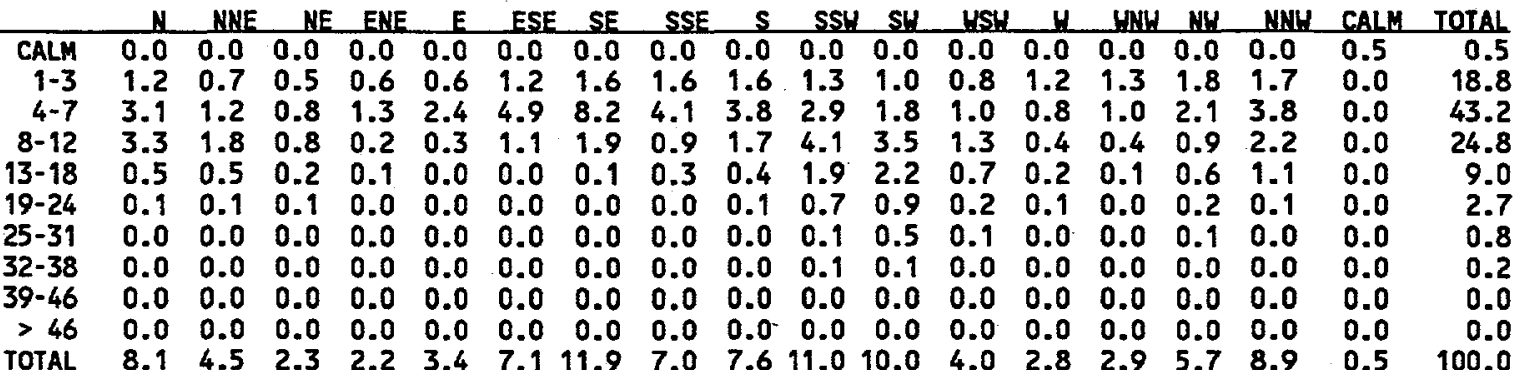

Station: (12) WYEB

Begin: 1/98 End: 12/98

Total Hours:

8759

DIRECTION

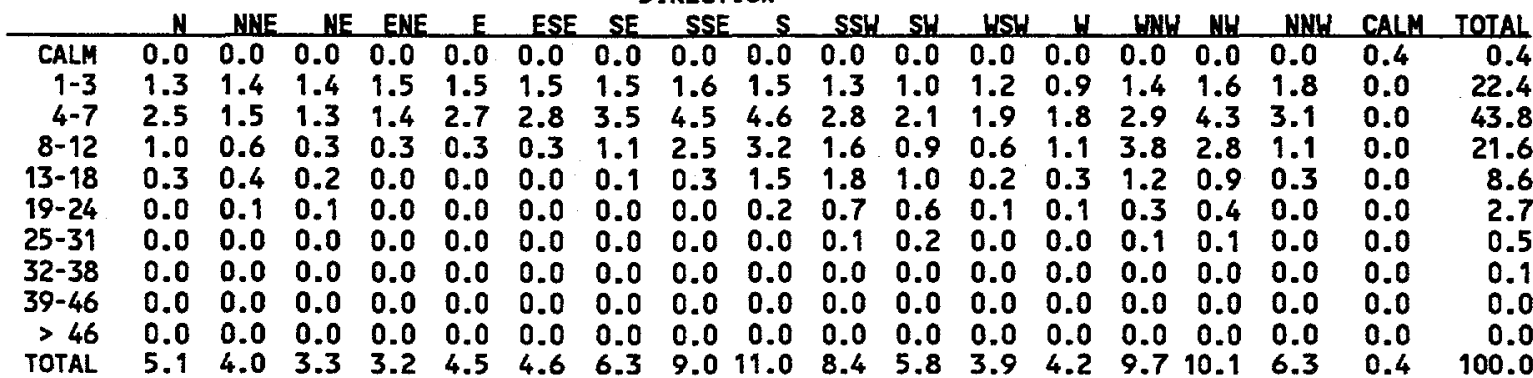


Table A.1. (contd)

Station: (13) $100 \mathrm{~N}$

End: $12 / 98$

Total Hours:

8596

DIRECTION

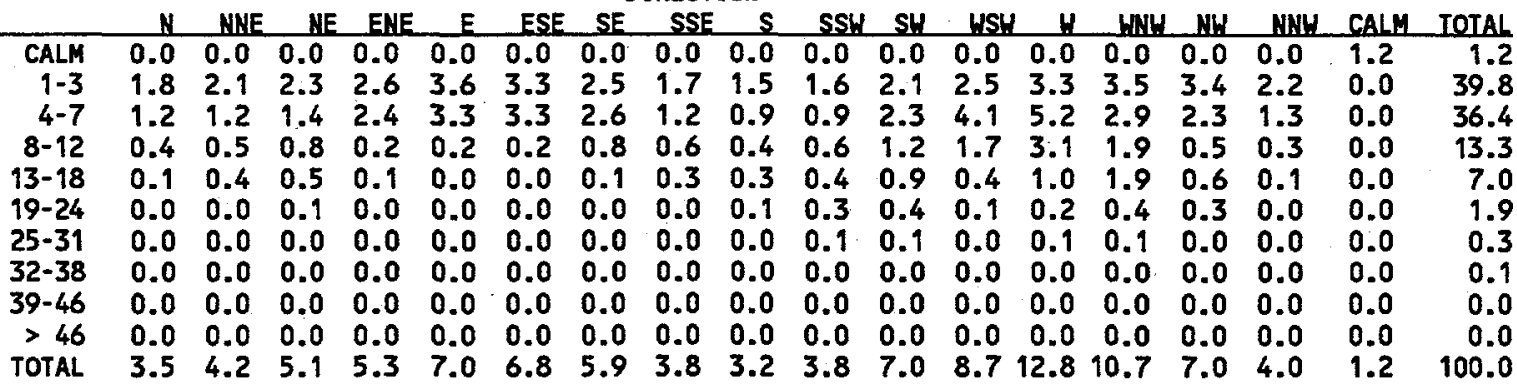

Station: (14) UPPS

Begin: $1 / 98$ End: 12/98

Total Hours:

8760

DIRECTION

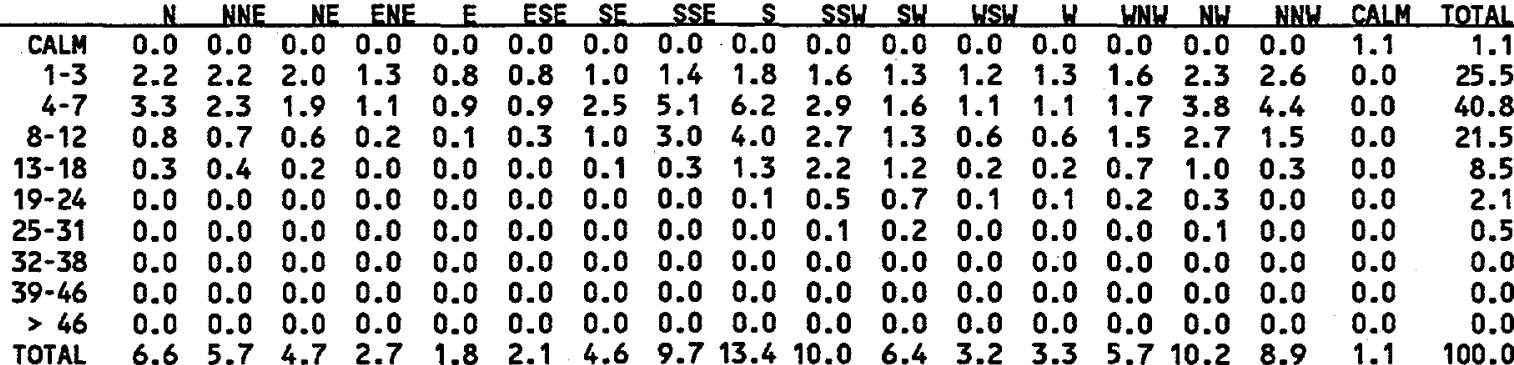

Station: (15) FRNK

Begin: 1/98 End: 12/98

Total Hours:

8757

DIRECTION

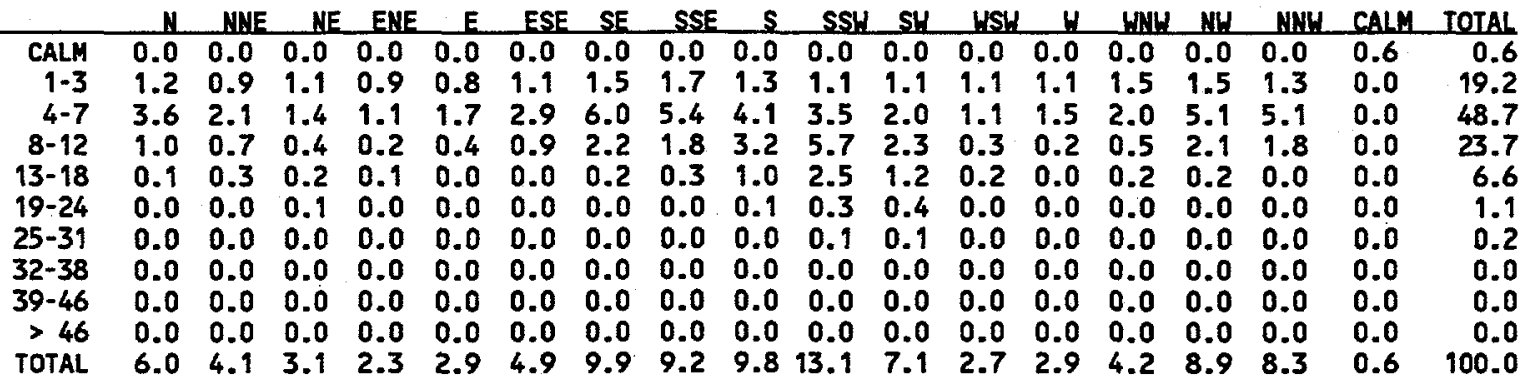

Station: (16) GABL

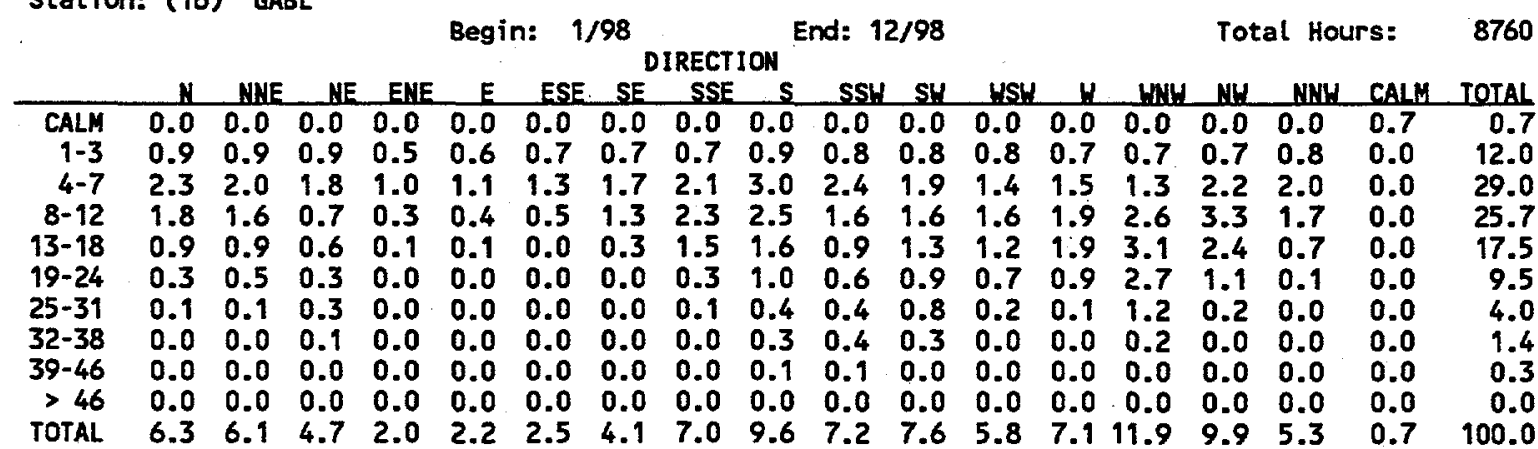


Table A.1. (contd)

Station: (17) RING

Begin: $1 / 98$ End: $12 / 98$

Total Hours:

8760

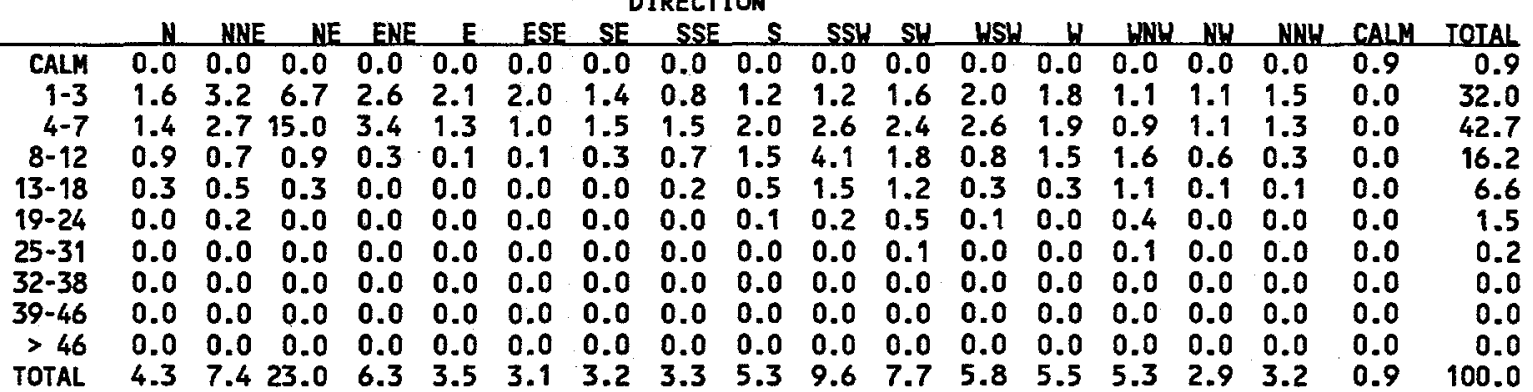

Station: (18) RICH

Begin: $1 / 98$

End: $12 / 98$

Total Hours:

8748

DIRECTION

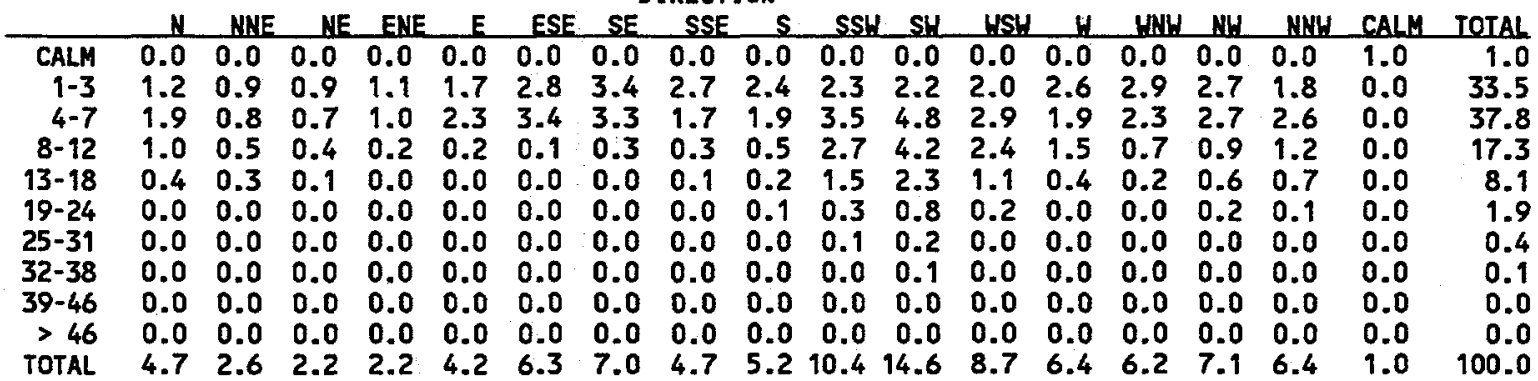

Station: (19) PFP

Begin: 1/98 End: 12/98

Total Hours:

8760

DIRECTION

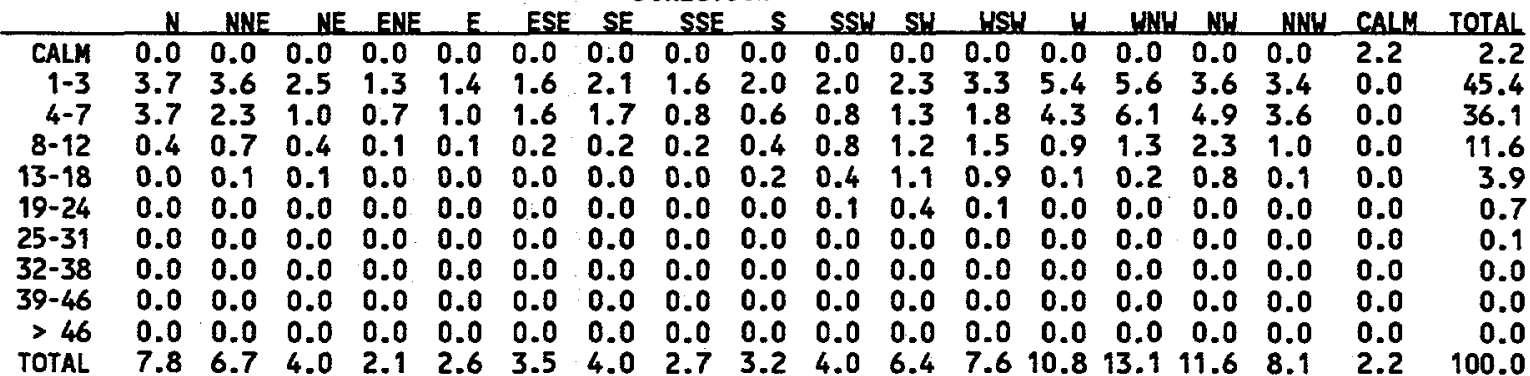

Station: (20) RMTM

Begin: $1 / 98$

DIRECTION

End: $12 / 98$

Total Hours:

8741

N NWE NE ENE E ESE SE SSE S SSH SH WSH $N$ HWH WH NWH CALM TOTAL

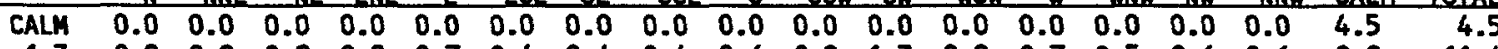

$\begin{array}{lllllllllllllllllll}1-3 & 0.8 & 0.8 & 0.9 & 0.8 & 0.7 & 0.4 & 0.4 & 0.4 & 0.6 & 0.9 & 1.3 & 0.8 & 0.7 & 0.5 & 0.6 & 0.6 & 0.0 & 11.4\end{array}$

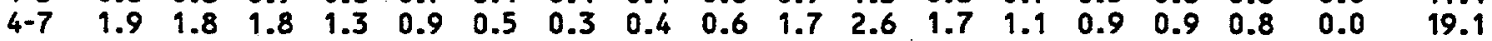

$\begin{array}{lllllllllllllllllll}8-12 & 1.8 & 2.5 & 1.7 & 0.6 & 0.2 & 0.3 & 0.2 & 0.2 & 0.4 & 1.4 & 4.0 & 2.3 & 1.3 & 0.8 & 0.7 & 1.0 & 0.0 & 19.5\end{array}$

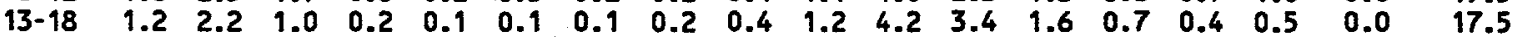

$\begin{array}{lllllllllllllllllll}19-24 & 0.6 & 1.5 & 0.8 & 0.0 & 0.0 & 0.0 & 0.0 & 0.1 & 0.2 & 0.4 & 2.9 & 2.7 & 1.0 & 0.3 & 0.1 & 0.1 & 0.0 & 10.8\end{array}$

$\begin{array}{lllllllllllllllllll}25-31 & 0.1 & 0.8 & 0.4 & 0.0 & 0.0 & 0.0 & 0.0 & 0.0 & 0.1 & 0.4 & 2.9 & 2.0 & 0.7 & 0.2 & 0.0 & 0.0 & 0.0 & 7.6\end{array}$

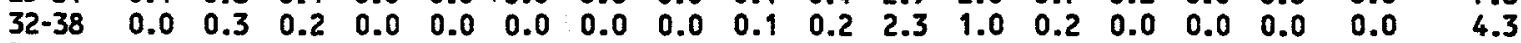

$\begin{array}{lllllllllllllllllll}39-46 & 0.0 & 0.2 & 0.3 & 0.0 & 0.0 & 0.0 & 0.0 & 0.0 & 0.0 & 0.1 & 1.6 & 0.5 & 0.1 & 0.0 & 0.0 & 0.0 & 0.0 & 3.0\end{array}$

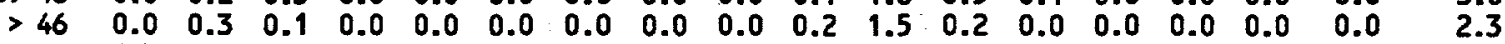

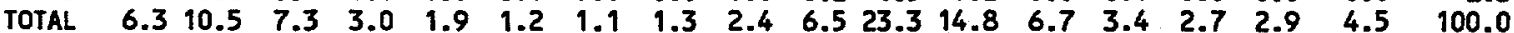


Table A.1. (contd)

Station: (21) HMS

Begin: 1/98 End: 12/98

Total Hours:

8760

DIRECTION

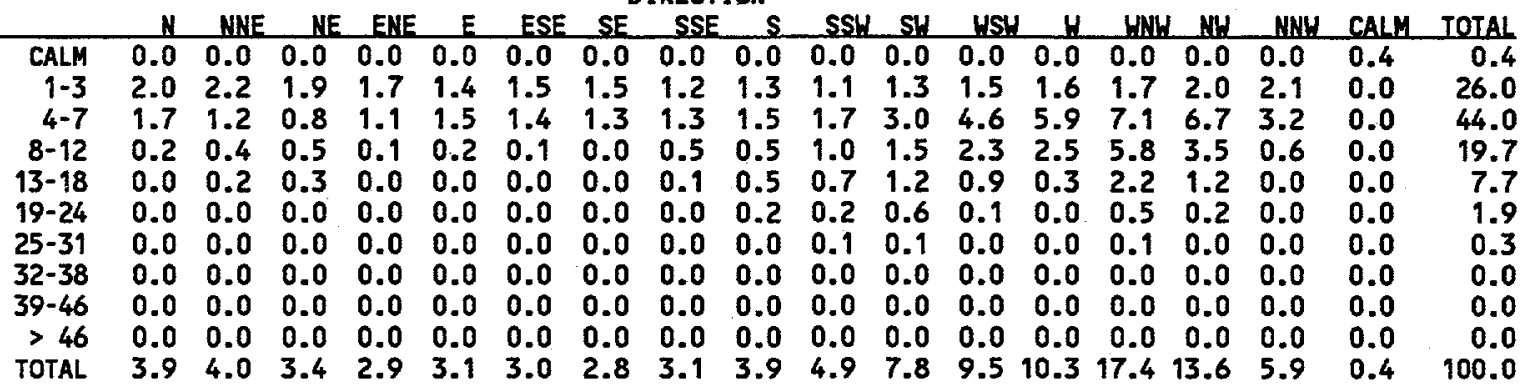

Station: (22) PASC

Begin: 1/98 End: 12/98

Total Hours:

8760

DIRECTION

$N$ NHE ME ENE E ESE SE SSE S SSH SH HSH H WNH NH NNH CALM TOTAL

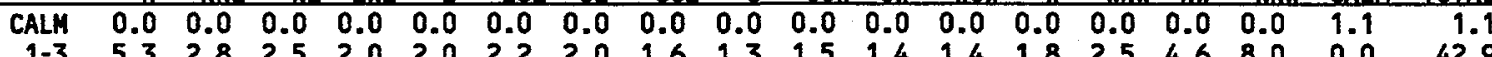

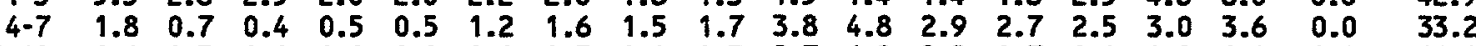

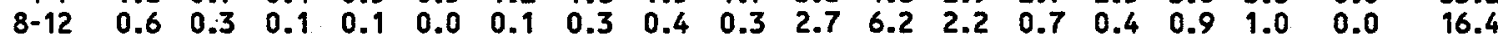

$\begin{array}{lllllllllllllllllll}13-18 & 0.1 & 0.3 & 0.1 & 0.0 & 0.0 & 0.0 & 0.0 & 0.1 & 0.2 & 0.5 & 2.5 & 1.2 & 0.3 & 0.1 & 0.2 & 0.0 & 0.0 & 5.6\end{array}$

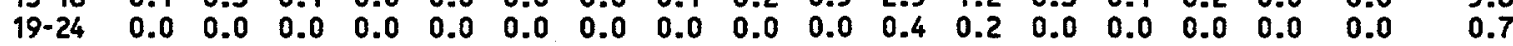

$\begin{array}{lllllllllllllllllll}25-31 & 0.0 & 0.0 & 0.0 & 0.0 & 0.0 & 0.0 & 0.0 & 0.0 & 0.0 & 0.0 & 0.1 & 0.0 & 0.0 & 0.0 & 0.0 & 0.0 & 0.0 & 0.1\end{array}$

$\begin{array}{lllllllllllllllllll}32-38 & 0.0 & 0.0 & 0.0 & 0.0 & 0.0 & 0.0 & 0.0 & 0.0 & 0.0 & 0.0 & 0.0 & 0.0 & 0.0 & 0.0 & 0.0 & 0.0 & 0.0 & 0.0\end{array}$

$\begin{array}{lllllllllllllllllll}39-46 & 0.0 & 0.0 & 0.0 & 0.0 & 0.0 & 0.0 & 0.0 & 0.0 & 0.0 & 0.0 & 0.0 & 0.0 & 0.0 & 0.0 & 0.0 & 0.0 & 0.0 & 0.0\end{array}$

$\begin{array}{rllllllllllllllllll}>46 & 0.0 & 0.0 & 0.0 & 0.0 & 0.0 & 0.0 & 0.0 & 0.0 & 0.0 & 0.0 & 0.0 & 0.0 & 0.0 & 0.0 & 0.0 & 0.0 & 0.0 & 0.0\end{array}$

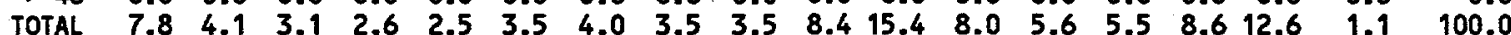

Station: (23) GABH

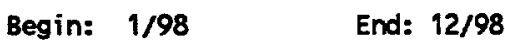

Total Hours:

8760

DIRECTION

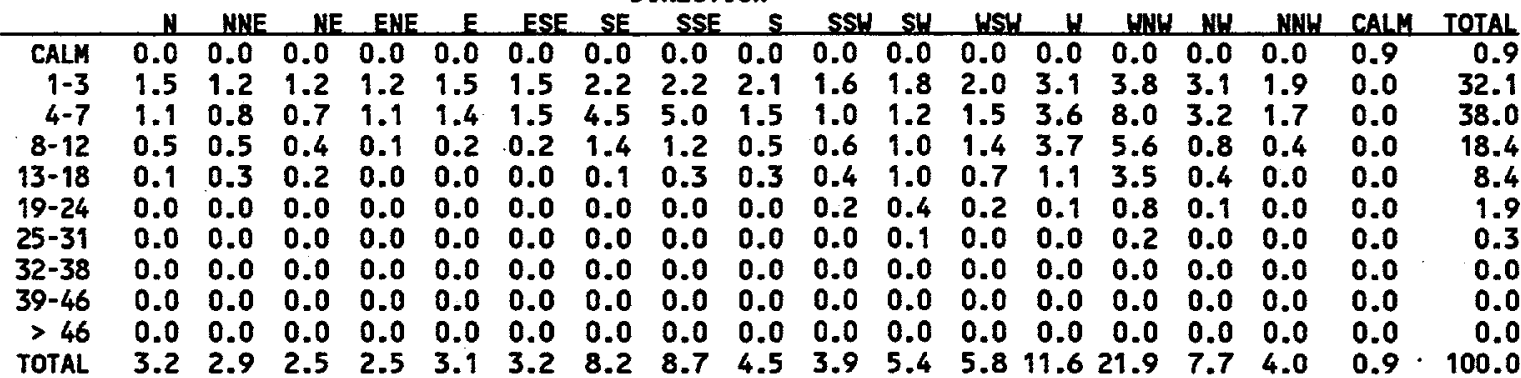

Station: (24) 100F

Total Hours: $\quad 8759$

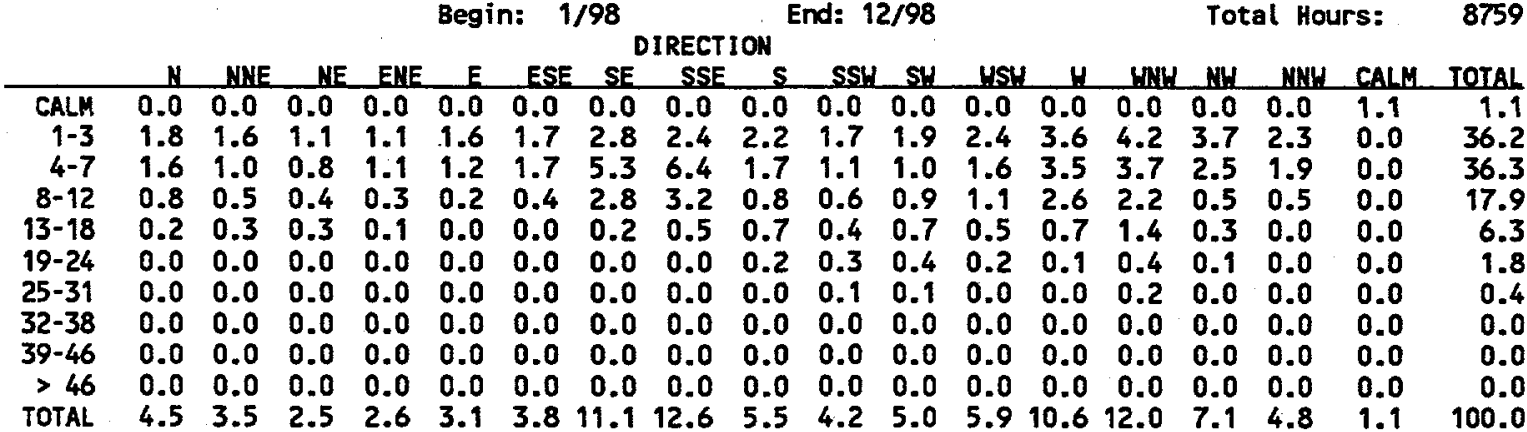


Table A.1. (contd)

Station: (25) VERN

$$
\text { Begin: 1/98 End: } 12 / 98
$$

Total Hours:

8759

$\begin{array}{rlllllllllllllllllll}\text { CALM } & 0.0 & 0.0 & 0.0 & 0.0 & 0.0 & 0.0 & 0.0 & 0.0 & 0.0 & 0.0 & 0.0 & 0.0 & 0.0 & 0.0 & 0.0 & 0.0 & 0.6 & 0.6 \\ 1-3 & 1.2 & 1.2 & 1.2 & 1.7 & 2.0 & 1.8 & 1.5 & 0.9 & 1.0 & 0.8 & 1.2 & 2.4 & 2.9 & 1.8 & 1.2 & 1.1 & 0.0 & 23.8 \\ 4-7 & 0.8 & 1.4 & 2.3 & 2.7 & 3.7 & 2.3 & 1.0 & 0.5 & 0.4 & 0.4 & 0.5 & 4.4 & 8.2 & 4.2 & 1.8 & 1.0 & 0.0 & 35.7 \\ 8-12 & 0.4 & 0.4 & 0.3 & 0.5 & 0.4 & 0.2 & 0.1 & 0.1 & 0.2 & 0.5 & 0.7 & 1.8 & 8.4 & 6.9 & 2.3 & 0.6 & 0.0 & 24.0 \\ 13-18 & 0.2 & 0.3 & 0.2 & 0.0 & 0.0 & 0.0 & 0.0 & 0.0 & 0.1 & 0.3 & 0.8 & 0.5 & 2.7 & 5.4 & 1.4 & 0.1 & 0.0 & 12.1 \\ 19-24 & 0.0 & 0.0 & 0.0 & 0.0 & 0.0 & 0.0 & 0.0 & 0.0 & 0.0 & 0.2 & 0.8 & 0.1 & 0.4 & 1.4 & 0.4 & 0.0 & 0.0 & 3.3 \\ 25-31 & 0.0 & 0.0 & 0.0 & 0.0 & 0.0 & 0.0 & 0.0 & 0.0 & 0.0 & 0.1 & 0.2 & 0.0 & 0.0 & 0.2 & 0.1 & 0.0 & 0.0 & 0.5 \\ 32-38 & 0.0 & 0.0 & 0.0 & 0.0 & 0.0 & 0.0 & 0.0 & 0.0 & 0.0 & 0.0 & 0.0 & 0.0 & 0.0 & 0.0 & 0.0 & 0.0 & 0.0 & 0.0 \\ 39-46 & 0.0 & 0.0 & 0.0 & 0.0 & 0.0 & 0.0 & 0.0 & 0.0 & 0.0 & 0.0 & 0.0 & 0.0 & 0.0 & 0.0 & 0.0 & 0.0 & 0.0 & 0.0 \\ \text { >46 } & 0.0 & 0.0 & 0.0 & 0.0 & 0.0 & 0.0 & 0.0 & 0.0 & 0.0 & 0.0 & 0.0 & 0.0 & 0.0 & 0.0 & 0.0 & 0.0 & 0.0 & 0.0 \\ \text { TOTAL } & 2.6 & 3.4 & 4.1 & 4.9 & 6.0 & 4.2 & 2.6 & 1.6 & 1.7 & 2.2 & 4.2 & 9.3 & 22.5 & 20.0 & 7.1 & 2.8 & 0.6 & 100.0\end{array}$

Station: (26) BENT

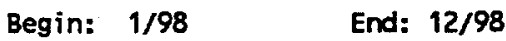

Total Hours:

8758

DIRECTION

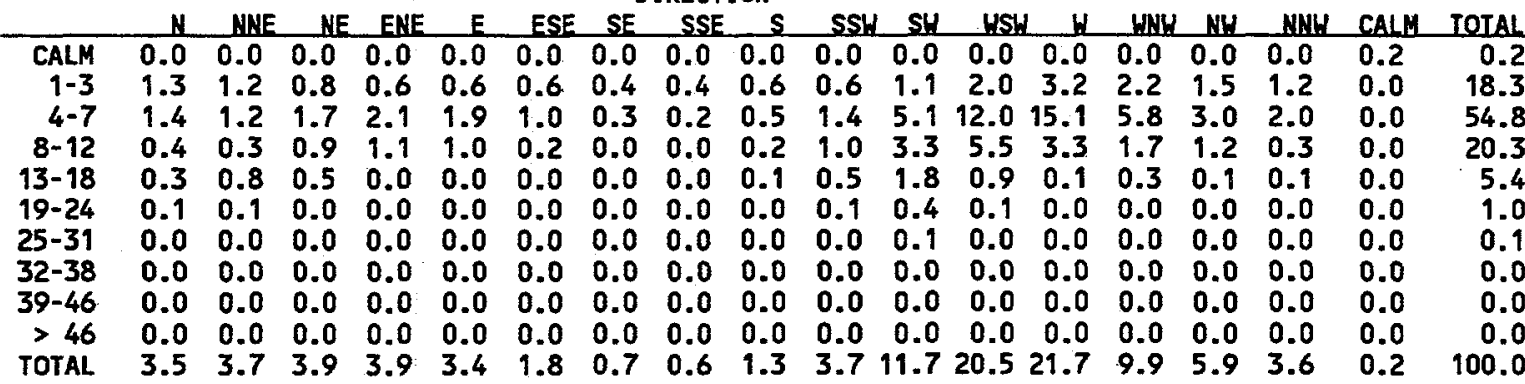

Station: (27) VSTA

Begin: 1/98 End: 12/98

Total Hours:

8748 DIRECTION

\begin{tabular}{rrrllllllllllllllll} 
& N & NNE & NE & ENE & E & ESE & SE & SSE & S & SSH & SH & HSH & H & HWH & NH & NNH & CALH & TOTAL \\
\hline CALM & 0.0 & 0.0 & 0.0 & 0.0 & 0.0 & 0.0 & 0.0 & 0.0 & 0.0 & 0.0 & 0.0 & 0.0 & 0.0 & 0.0 & 0.0 & 0.0 & 0.7 & 0.7 \\
$1-3$ & 2.1 & 2.2 & 1.6 & 1.4 & 1.4 & 1.6 & 2.1 & 1.7 & 1.9 & 2.2 & 2.7 & 2.6 & 2.2 & 1.8 & 1.8 & 1.6 & 0.0 & 30.8 \\
$4-7$ & 2.6 & 1.8 & 1.4 & 1.0 & 0.7 & 1.1 & 1.1 & 1.2 & 2.2 & 4.9 & 6.3 & 3.7 & 2.7 & 2.6 & 3.1 & 2.4 & 0.0 & 38.7 \\
$8-12$ & 0.6 & 0.4 & 0.1 & 0.0 & 0.0 & 0.2 & 0.2 & 0.4 & 0.7 & 5.3 & 6.8 & 2.4 & 0.6 & 0.4 & 0.9 & 0.9 & 0.0 & 20.0 \\
$13-18$ & 0.0 & 0.1 & 0.0 & 0.0 & 0.0 & 0.0 & 0.0 & 0.0 & 0.3 & 2.4 & 3.9 & 0.8 & 0.1 & 0.2 & 0.2 & 0.0 & 0.0 & 8.1 \\
$19-24$ & 0.0 & 0.0 & 0.0 & 0.0 & 0.0 & 0.0 & 0.0 & 0.0 & 0.0 & 0.3 & 1.0 & 0.1 & 0.0 & 0.0 & 0.0 & 0.0 & 0.0 & 1.4 \\
$25-31$ & 0.0 & 0.0 & 0.0 & 0.0 & 0.0 & 0.0 & 0.0 & 0.0 & 0.0 & 0.0 & 0.2 & 0.0 & 0.0 & 0.0 & 0.0 & 0.0 & 0.0 & 0.2 \\
$32-38$ & 0.0 & 0.0 & 0.0 & 0.0 & 0.0 & 0.0 & 0.0 & 0.0 & 0.0 & 0.0 & 0.0 & 0.0 & 0.0 & 0.0 & 0.0 & 0.0 & 0.0 & 0.0 \\
$39-46$ & 0.0 & 0.0 & 0.0 & 0.0 & 0.0 & 0.0 & 0.0 & 0.0 & 0.0 & 0.0 & 0.0 & 0.0 & 0.0 & 0.0 & 0.0 & 0.0 & 0.0 & 0.0 \\
$>46$ & 0.0 & 0.0 & 0.0 & 0.0 & 0.0 & 0.0 & 0.0 & 0.0 & 0.0 & 0.0 & 0.0 & 0.0 & 0.0 & 0.0 & 0.0 & 0.0 & 0.0 & 0.0 \\
TOTAL & 5.2 & 4.5 & 3.1 & 2.4 & 2.1 & 2.9 & 3.4 & 3.3 & 5.2 & 15.2 & 20.9 & 9.7 & 5.6 & 5.0 & 5.9 & 4.9 & 0.7 & 100.0
\end{tabular}

Station: (28) SURF

Begin: $1 / 98$

End: $12 / 98$

Total Hours:

8760 DIRECTION

\begin{tabular}{rrrrrrrrrrrrrrrrrrr} 
& N & NNE & NE & ENE & E & ESE & SE & \multicolumn{1}{c}{ SSE } & S & SSH & SH & HSW & H & WNW & NH & HNH & CALM & TOTAL \\
\hline CALM & 0.0 & 0.0 & 0.0 & 0.0 & 0.0 & 0.0 & 0.0 & 0.0 & 0.0 & 0.0 & 0.0 & 0.0 & 0.0 & 0.0 & 0.0 & 0.0 & 0.4 & 0.4 \\
$1-3$ & 0.4 & 0.5 & 0.9 & 1.0 & 0.9 & 0.6 & 0.6 & 0.6 & 1.1 & 2.1 & 3.1 & 3.4 & 1.7 & 0.6 & 0.4 & 0.3 & 0.0 & 18.2 \\
$4-7$ & 0.2 & 0.4 & 2.4 & 3.3 & 1.7 & 0.6 & 0.4 & 0.2 & 0.4 & 1.1 & 3.6 & 6.5 & 2.0 & 0.2 & 0.1 & 0.1 & 0.0 & 23.2 \\
$8-12$ & 0.5 & 0.6 & 2.1 & 2.8 & 0.5 & 0.0 & 0.0 & 0.0 & 0.0 & 0.1 & 4.0 & 10.2 & 4.1 & 0.4 & 0.1 & 0.2 & 0.0 & 25.6 \\
$13-18$ & 0.3 & 0.5 & 0.3 & 0.5 & 0.1 & 0.0 & 0.0 & 0.0 & 0.0 & 0.0 & 2.3 & 10.2 & 6.5 & 0.6 & 0.1 & 0.1 & 0.0 & 21.4 \\
$19-24$ & 0.1 & 0.1 & 0.1 & 0.0 & 0.0 & 0.0 & 0.0 & 0.0 & 0.0 & 0.0 & 0.4 & 4.5 & 3.0 & 0.3 & 0.0 & 0.1 & 0.0 & 8.5 \\
$25-31$ & 0.1 & 0.0 & 0.0 & 0.0 & 0.0 & 0.0 & 0.0 & 0.0 & 0.0 & 0.0 & 0.1 & 1.1 & 0.8 & 0.1 & 0.1 & 0.1 & 0.0 & 2.4 \\
$32-38$ & 0.0 & 0.0 & 0.0 & 0.0 & 0.0 & 0.0 & 0.0 & 0.0 & 0.0 & 0.0 & 0.0 & 0.1 & 0.1 & 0.0 & 0.0 & 0.0 & 0.0 & 0.2 \\
$39-46$ & 0.0 & 0.0 & 0.0 & 0.0 & 0.0 & 0.0 & 0.0 & 0.0 & 0.0 & 0.0 & 0.0 & 0.0 & 0.0 & 0.0 & 0.0 & 0.0 & 0.0 & 0.0 \\
$>46$ & 0.0 & 0.0 & 0.0 & 0.0 & 0.0 & 0.0 & 0.0 & 0.0 & 0.0 & 0.0 & 0.0 & 0.0 & 0.0 & 0.0 & 0.0 & 0.0 & 0.0 & 0.0 \\
TOTAL & 1.6 & 2.2 & 5.7 & 7.6 & 3.2 & 1.2 & 1.0 & 0.8 & 1.5 & 3.3 & 13.5 & 36.1 & 18.1 & 2.2 & 0.8 & 0.8 & 0.4 & 100.0
\end{tabular}


Table A.1. (contd)

Station: (29) $100 \mathrm{~K}$

Begin: 1/98 End: 12/98

Total Hours:

8694

DIRECTION

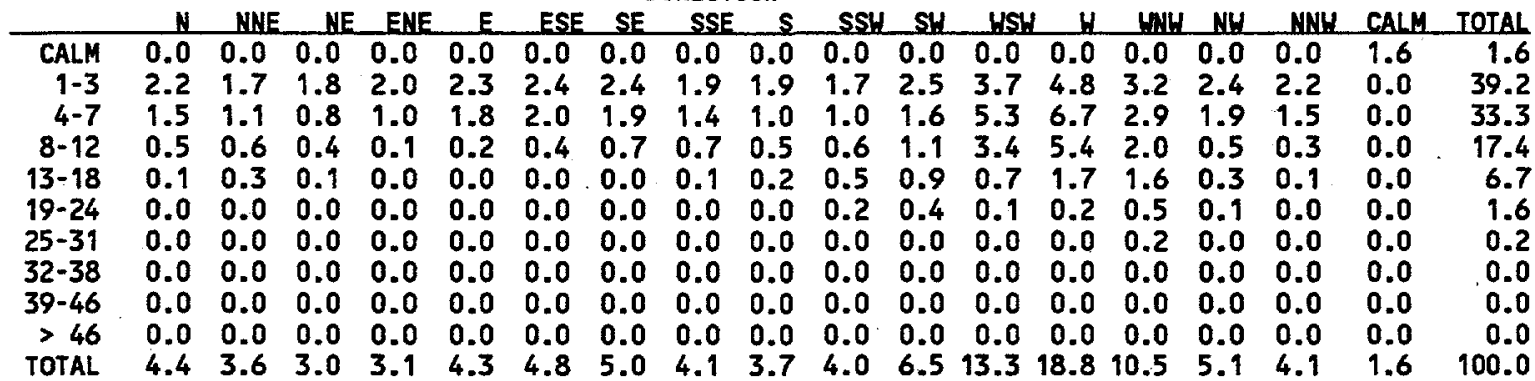

Station: (30) HAMR

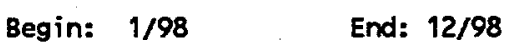

Total Hours: $\mathbf{8 7 1 1}$

DIRECTION

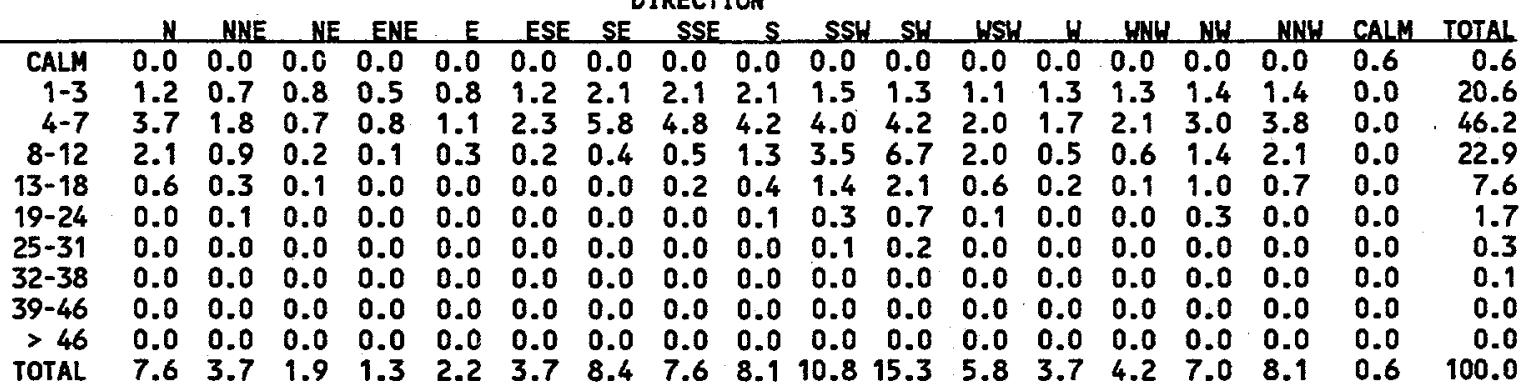


Table A.2. Joint Frequency Distributions (\%) for Hanford Meteorological Monitoring Network Wind Stations at 60-Meter Level, 1998

Tower: 100 Area

Total Hours: 8568

DIRECTION

\begin{tabular}{ccccccccccccccccccc} 
& N & NNE & NE & ENE & E & ESE & SE & SSE & S & SSW & SH & HSW & W & WHW NH & NNH & CALM & TOTAL \\
\hline CALM & 0.0 & 0.0 & 0.0 & 0.0 & 0.0 & 0.0 & 0.0 & $\mathbf{0 . 0}$ & 0.0 & 0.0 & 0.0 & 0.0 & $\mathbf{0 . 0}$ & 0.0 & 0.0 & 0.0 & 0.7 & 0.7 \\
$1-3$ & 1.6 & 1.6 & 1.8 & 2.6 & 3.4 & 3.2 & 2.2 & 1.6 & 1.3 & 1.2 & 1.5 & 2.0 & 2.2 & 2.1 & 2.1 & 1.8 & 0.0 & 32.1 \\
$4-7$ & 1.4 & 1.4 & 1.2 & 2.0 & 3.8 & 3.7 & 2.6 & 1.3 & 0.9 & 0.9 & 1.2 & 2.1 & 3.6 & 3.8 & 2.0 & 1.4 & 0.0 & 33.3 \\
$8-12$ & 0.6 & 0.7 & 0.6 & 0.4 & 0.4 & 0.7 & 1.2 & 0.7 & 0.5 & 0.5 & 1.1 & 1.2 & 3.0 & 2.8 & 0.9 & 0.5 & 0.0 & 15.8 \\
$13-18$ & 0.3 & 0.4 & 0.4 & 0.1 & 0.0 & 0.1 & 0.4 & 0.4 & 0.3 & 0.4 & 0.7 & 0.6 & 3.1 & 2.8 & 0.4 & 0.2 & 0.0 & 10.7 \\
$19-24$ & 0.1 & 0.2 & 0.3 & 0.0 & 0.0 & 0.0 & 0.0 & 0.2 & 0.2 & 0.3 & 0.7 & 0.4 & 0.8 & 1.5 & 0.3 & 0.1 & 0.0 & 5.2 \\
$25-31$ & 0.0 & 0.0 & 0.1 & 0.0 & 0.0 & 0.0 & 0.0 & 0.0 & 0.1 & 0.2 & 0.5 & 0.1 & 0.2 & 0.4 & 0.1 & 0.1 & 0.0 & 1.8 \\
$32-38$ & 0.0 & 0.0 & 0.0 & 0.0 & 0.0 & 0.0 & 0.0 & 0.0 & 0.0 & 0.1 & 0.1 & 0.0 & 0.0 & 0.1 & 0.0 & 0.0 & 0.0 & 0.4 \\
$39-46$ & 0.0 & 0.0 & 0.0 & 0.0 & 0.0 & 0.0 & 0.0 & 0.0 & 0.0 & 0.0 & 0.0 & 0.0 & 0.0 & 0.0 & 0.0 & 0.0 & 0.0 & 0.0 \\
$>46$ & 0.0 & 0.0 & 0.0 & 0.0 & 0.0 & 0.0 & 0.0 & 0.0 & 0.0 & 0.0 & 0.0 & 0.0 & 0.0 & 0.0 & 0.0 & 0.0 & 0.0 & 0.0 \\
TOTAL & 4.0 & 4.3 & 4.3 & 5.1 & 7.5 & 7.7 & 6.4 & 4.2 & 3.3 & 3.7 & 5.8 & 6.4 & 13.1 & 13.6 & 5.9 & 4.0 & 0.7 & 100.0
\end{tabular}

Tower: 200 Area

Total Hours:

8760

DIRECTION

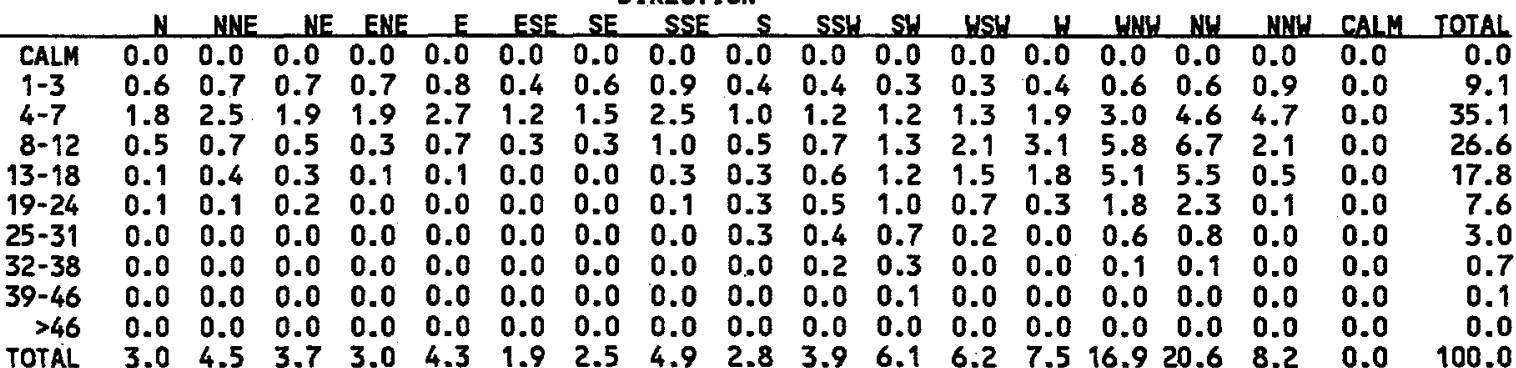

Tower: 300 Area

Begin: $1 / 98$

End: $12 / 98$

Total Hours:

8759 DIRECTION

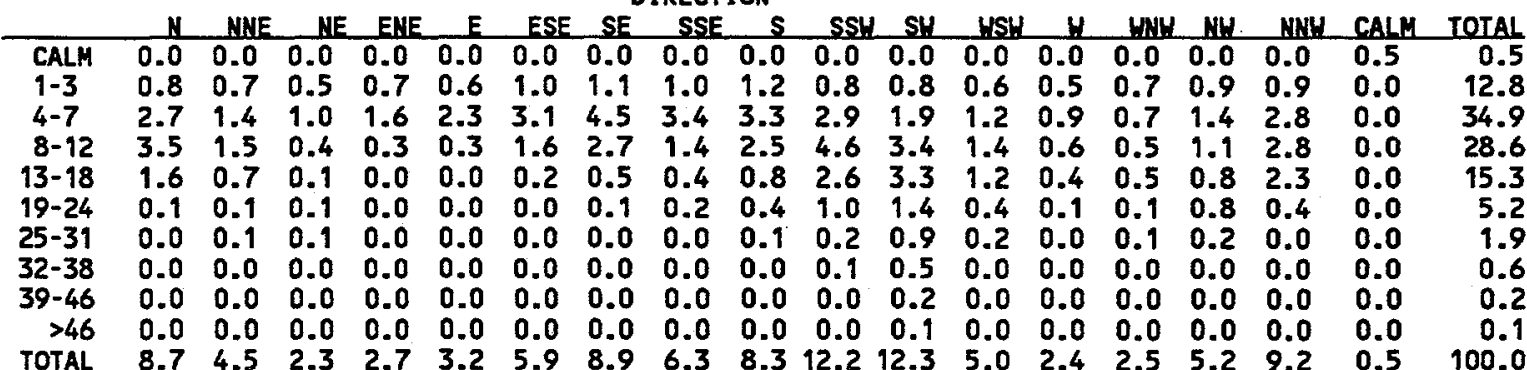

Tower: 400 Area

Begin: $1 / 98$ DIRECTION

End: $12 / 98$

Total Hours:

8751

4 NHE ME ENE E ESE SE SSE S SSY SH USH $Y$ WNH HW NHY CALM TOTAL

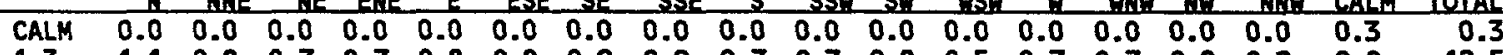

$\begin{array}{lllllllllllllllllll}1-3 & 1.1 & 0.8 & 0.7 & 0.7 & 0.8 & 0.8 & 0.9 & 0.9 & 0.7 & 0.7 & 0.8 & 0.5 & 0.7 & 0.7 & 0.8 & 0.9 & 0.0 & 12.5\end{array}$

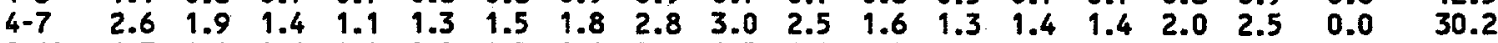

$\begin{array}{lllllllllllllllllll}8-12 & 1.7 & 1.2 & 0.6 & 0.2 & 0.2 & 0.3 & 1.4 & 2.6 & 4.5 & 4.3 & 2.3 & 0.8 & 0.6 & 1.2 & 2.9 & 2.6 & 0.0 & 27.4\end{array}$

$\begin{array}{lllllllllllllllllll}13-18 & 0.6 & 0.5 & 0.4 & 0.0 & 0.0 & 0.0 & 0.5 & 0.8 & 1.6 & 4.3 & 2.3 & 0.6 & 0.3 & 1.0 & 3.4 & 1.9 & 0.0 & 18.4\end{array}$

$\begin{array}{lllllllllllllllllll}19-24 & 0.1 & 0.2 & 0.1 & 0.0 & 0.0 & 0.0 & 0.1 & 0.2 & 0.6 & 1.8 & 1.6 & 0.3 & 0.1 & 0.7 & 2.0 & 0.2 & 0.0 & 7.9\end{array}$

$\begin{array}{lllllllllllllllllll}25-31 & 0.0 & 0.1 & 0.1 & 0.0 & 0.0 & 0.0 & 0.0 & 0.0 & 0.1 & 0.4 & 0.8 & 0.1 & 0.0 & 0.2 & 0.4 & 0.0 & 0.0 & 2.4\end{array}$

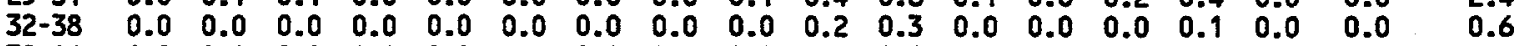

$\begin{array}{lllllllllllllllllll}39-46 & 0.0 & 0.0 & 0.0 & 0.0 & 0.0 & 0.0 & 0.0 & 0.0 & 0.0 & 0.1 & 0.1 & 0.0 & 0.0 & 0.0 & 0.0 & 0.0 & 0.0 & 0.3\end{array}$

$\begin{array}{lllllllllllllllllll}>46 & 0.0 & 0.0 & 0.0 & 0.0 & 0.0 & 0.0 & 0.0 & 0.0 & 0.0 & 0.0 & 0.0 & 0.0 & 0.0 & 0.0 & 0.0 & 0.0 & 0.0 & 0.0\end{array}$

$\begin{array}{lllllllllllllllllll}\text { TOTAL } & 6.1 & 4.9 & 3.2 & 2.1 & 2.3 & 2.6 & 4.8 & 7.3 & 10.6 & 14.3 & 9.9 & 3.6 & 3.1 & 5.1 & 11.5 & 8.2 & 0.3 & 100.0\end{array}$ 


\section{Distribution}

No. of

Copies

\section{OFFSITE}

Agricultural Chemical Branch

Washington State Department of Agriculture

2015 S. First Street

Yakima, WA 98903

B. Allen

611 Vaughan Road

Yakima, WA 98908

T. Allen

Power Resource Managers

2100 112th Ave. N.E., Suite 100

Bellevue, WA 98004

ASCS Office

Franklin County

1620 North Road 44

Pasco, WA 99301-2667

D. Austin

Raekes, Rettig, Osborne

6725 W. Clearwater

Kennewick, WA 99336

S. Bailey

Columbia Basin College Library

2600 N. 20th Street

Pasco, WA 99301

D. Batchelor

732 W. Park

Pasco, WA 99301

2 Benton County Public Utility District \#1

P.O. Box 6270

Kennewick, WA 99332

ATTN: K. Mey

M. Sheeran
No. of

Copies

Benton County Public Works 1709 S. Ely

Kennewick, WA 99320

R. C. Blewett

4409 Arlington Drive

West Richland, WA 99352

T. Brazil

Cascade Natural Gas

P.O. Box 6899

Kennewick, WA 99336

C. Burt

Smith Frozen Foods

205204 E. Bowles Road

Kennewick, WA 99337

M. Carrow

Director of Agricultural Services

2005 Saint Street

Richland, WA 99352

E. Charvet

McGregor Company

5251 Eltopia West Road

Eltopia, WA 99330

J. Cherry

Franklin County Planning

1016 N. 4th Street

Pasco, WA 99301

City of Benton City

ATTN: City Clerk

P.O. Box 70

Benton City, WA 99320 
No. of

Copies

4 City of Richland

P.O. Box 190

Richland, WA 99352

ATTN: L. D. Dunbar, C.E.M.

L. Herbert

M. E. Lippold

R. Overman

W. Clore

1317 Paterson Road

Prosser, WA 99350

W. T. Cobb

Cobb Consulting Services

815 S. Kellogg

Kennewick, WA 99336

Columbia National Wildlife Refuge

U.S. Fish \& Wildlife Service

U.S. Department of the Interior

P.O. Box F

Othello, WA 99344

Farmers Insurance Claims Office

P.O. Box 6998

Kennewick, WA 99336

D. Fiskum

Kadlec Medical Center

888 Swift Boulevard

Richland, WA 99352

E. Frimodt III

Franklin County Road Department 3416 Stearman Ave.

Pasco, WA 99301-7104

B. Hachey

Mid-Columbia Library - Pasco

1320 W. Hopkins

Pasco, WA 99301
No. of

Copies

P. M. Hamilton, Jr.

Assistant Director

Physical Plant

Central Washington University

Ellensburg, WA 98926

J. Hancock

U.S. Ecology

P.O. Box 638

Richland, WA 99352

L. Harms

Harms \& Associates

1632 W. Sylvester

Pasco, WA 99301

D. Harper

Wilbur-Ellis Company

P.O. Box 764

Pasco, WA 99301

A. Haymaker

1721 Cottonwood Drive

Pasco, WA 99301

Jacobs and Rhodes

P.O. Box 6881

Kennewick, WA 99336

B. Johnson

556 Columbia Center

Kennewick, WA 99336

B. Kelley

614 S. Kellogg Street

Kennewick, WA 99336

J. Kleckner

D. Hittle Associates

757 Columbia Drive, S.E.

Richland, WA 99352 
No. of

Copies

R. A. Kyle

Richland School District

615 Snow

Richland, WA 99352

R. LaFramboise

Central Washington Farm Crops

Association

P.O. Box 202

Yakima, WA 98907

K. Lettau

George Grant Construction Co.

P.O. Box 789

Richland, WA 99352

J. Mannix

Kennewick School District

622 N. Kellogg Street

Kennewick, WA 99336

R. Mazurkiewicz

Bonneville Power Administration

P.O. Box 968, MD-399

Richland, WA 99352

D. McBaugh

Division of Radiation Protection

State of Washington Department of Health

P.O. Box 47827

Olympia, WA 98504

W. A. McCullough

2402 Lariat Lane

Richland, WA 99352

J. McDonald

Washington Public Power

Supply System, MS 1025

P.O. Box 968

Richland, WA 99352
No. of

Copies

J. McKay

TRIDEC

901 N. Colorado

Kennewick, WA 99336

G. McMurphy

Valley Electric Co.

1010 E. Bruneau Ave.

Kennewick, WA 99336-3775

National Weather Service

2001 N.W. 56th Drive

Pendleton, OR 97801

R. Okarma

Kennewick High School

500 S. Dayton

Kennewick, WA 99336

M. Orgill, CCM

342 S. 200 West

Cedar City, UT 84720

Pioneer Hi-Bred

1040 Settler Road

Connell, WA 99326

T. Poe

All Seasons Heating \& Air

Conditioning, Inc.

P.O. Box 9006

Yakima, WA 98909-0006

Pasco School District

Attn: Kathy, Bldg. 210

Tri-Cities Airport

Pasco, WA 99301

Potato Growers of Washington

P.O. Box 563

Othello, WA 99344-0563 
No. of Copies

D. C. Powell 2110 Hudson

Richland, WA 99352

Professional Ag Services

P.O. Box 3053

Pasco, WA 99302

J. T. Raese

Tree Fruit Research Laboratory

1104 N. Western Avenue

Wenatchee, WA 98801

K. Redmond

Western Regional Climate Center

Desert Research Institute

P.O. Box 60220

Reno, NV 89506

R. R. Reid

1884 Rustic Road

Walla Walla, WA 99362

Richland Public Library

ATTN: Reference Librarian

955 Northgate

Richland, WA 99352

P. Roberts

7000 W. Van Giesen, \#632

West Richland, WA 99353

W. Sahli

P.O. Box 912

Mattawa, WA 99344

W. Sealock

622 N. Kellogg

Kennewick, WA 99336

J. St. Clair

Benton-Franklin County Clean

Air Authority

650 George Washington Way

Richland, WA 99352
No. of

Copies

Soil Conservation Service

1620 Road 44, North

Pasco, WA 99301-2667

N. Sorick

Franklin County Public Utility District \#1

P.O. Box 2407

Pasco, WA 99302

L. Stewart

Benton Rural Electric Association

P.O. Box 1150

Prosser, WA 99350

K. Tanaka

Siemens Power Corporation

P.O. Box 130

Richland, WA 99352

G. Taylor, Climatologist

Oregon Climate Service

Strand 326

Oregon State University

Corvallis, OR 97331-2209

J. Thompson

CENEX Land O'Lakes

Route 7, Box 430

Kennewick, WA 99337

J. Thorp

2110 Dover

Richland, WA 99352

Tri-City Sign \& Barricade, Inc.

307 N. Dayton

Kennewick, WA 99336

M. Troutman

1207 Sunnyvale Road

Kennewick, WA 99337 
No. of

Copies

2 UNOCAL

P.O. Box 5797

Kennewick, WA 99336

ATTN: J. Akers

G. Stroud

U.S. Army Corps of Engineers -

Hydrology Branch

201 S. 3rd Street

Walla Walla, WA 99362

R. W. Wallace

89 Waldron Street

Richland, WA 99352

J. Watson

County Extension Agent

1121 Dudley Avenue

Prosser, WA 99350

L. L. Wendell

Wind Energy Consultants

2328 Enterprise Drive

Richland, WA 99352

M. Wilson

Central Pre-Mix Concrete Co.

11919 Harris Road

Pasco, WA 99301

R. N. Wing

727 Airway Avenue

Lewiston, ID 83501

W. Ziebell

Prosser School District

823 Park Avenue

Prosser, WA 99350

L. Zeiher

405 South Dayton

Kennewick, WA 99336
No. of

Copies

\section{ONSITE}

3 DOE Richland Operations Office

J. B. Hall

A5-15

D. C. Ward

A5-15

Public Reading Room

$\mathrm{H} 2-53$

B\&W Protec, Inc.

R. T. Toyooka

G3-43

3 Bechtel Hanford, Inc.
R. L. Biggerstaff
HO-02
K. A. Gano
HO-02
C. J. Kemp
S3-20

Chapel Hill, Inc.

K. E. Cook

H9-02

COGEMA Engineering Corporation

S. I. Ryburn

S3-90

DE\&S Hanford, Inc.

K. D. Johnson

X3-61

DynCorp Tri-Cities Services, Inc.

J. W. Strege

L6-64

Fluor Daniel Hanford, Inc.

D. J. Washenfelder

S7-40 
No. of

Copies

4 Fluor Daniel Northwest, Inc.

T. E. Arndt

G3-17

P. G. Beaudet

S8-07

L. A. Gaddis

H5-57

T. L. Ostrander

S6-16

U.S. Environmental Protection Agency

D. R. Einan

B5-01

4 Waste Management Federal Services of Hanford, Inc.

E. G. Allen

T4-55

N. K. Myers

T3-28

J. R. Rosser

T4-03

R. W. Whitlock

T4-04

3 Waste Management Federal Services, Inc., Northwest Operations

R. B. Barmettlor

L6-04

A. R. Johnson

H1-13

R. C. Roos

H1-12

139 Pacific Northwest National Laboratory

O. B. Abbey

K9-30

O. P. Amacker

K8-58

D. L. Anderson

K9-72

G. L. Andrews

K8-84

W. R. Barchet

J. C. Barnard

K9-30

D. B. Barnett

L. E. Bisping

W. J. Bjorklund

K. W. Burk (10)

L. L. Cadwell
No. of

Copies

R. E. Clayton

K9-33

W. C. Cliff

T. S. Cress

K6-48

J. C. Doran

K9-38

K9-30

G. W. Dennis

K6-75

M. J. Fayer

K9-33

D. Felmy

K9-33

H. D. Freeman P8-38

G. W. Gee

E. R. Gerk

C. S. Glantz

G. L. Gower

D. L. Hadley

D. R. Haffner

C. W. Hall

S. W. Heaberlin

D. J. Hoitink (75)

K. A. Hoover

M. G. Horstman (BCO)

D. G. Horton

J. M. Hubbe

R. R. Kirkham

N. R. Larson

N. S. Laulainen

R. N. Lee

D. B. Mackey

J. P. McKinley

L. A. Monroe

L. F. Morasch

T. L. Page

J. V. Ramsdell

K. Rhoads

M. R. Sackschewsky

W. J. Shaw

S. F. Snyder

G. M. Stokes

A. L. Ward (BPO)

C. D. Whiteman

Technical Library

Information Release Office (7)
K9-33

P7-63

K6-80

P7-79

K5-16

K7-97

P7-57

K8-31

K9-30

K8-41

K4-10

K6-81

K9-30

K9-33

K9-55

K9-30

P7-07

P8-15

K3-61

K1-53

K6-86

K9-18

K9-30

K3-54

K6-84

K9-30

K3-54

K9-95

K9-33

K9-30

P8-55

K1-06 